\title{
Guía de escenarios regionalizados de cambio climático sobre España a partir de los resultados del IPCC-AR4
}
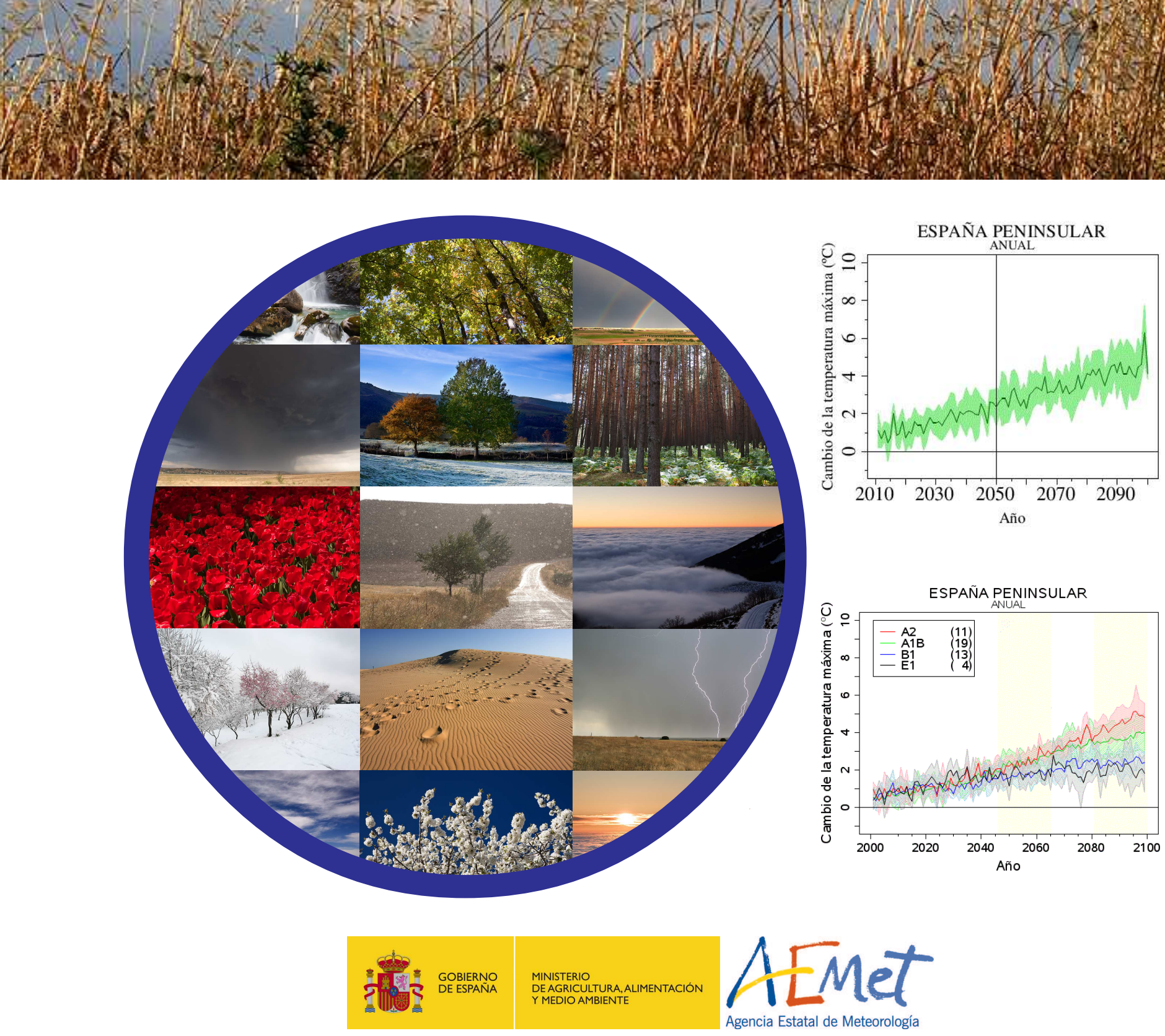

Madrid, 2014 


\section{Agradecimientos:}

Esta guía comprende parte de los resultados del trabajo que se lleva a cabo en la Agencia Estatal de Meteorología por un gran número de profesionales en el contexto de los estudios de Cambio Climático. La autora quiere expresar su gratitud a María Jesús Casado, José María Martín, Eduardo Petisco, Petra Ramos, Ernesto Rodríguez y Antonio Ángel Serrano por el excelente trabajo realizado.

\section{datos \\ abiertos}

Aviso Legal: los contenidos de esta publicación podrán ser reutilizados, citando la fuente y la fecha, en su caso, de la última actualización

\section{Edita:}

(C) Ministerio de Agricultura, Alimentación y Medio Ambiente Agencia Estatal de Meteorología Madrid, 2014

Catálogo de Publicaciones de la Administración General del Estado: https://cpage.mpr.gob.es

NIPO: 281-14-002-7

https://doi.org/10.31978/281-14-002-7

Agencia Estatal de Meteorología (AEMET)

$\mathrm{C} /$ Leonardo Prieto Castro, 8

28040 Madrid

http://www.aemet.es/

\section{@Aemet_Esp}

https://www.facebook.com/AgenciaEstataldeMeteorologia 


\section{Índice}

$\begin{array}{ll}\text { Capítulo 1. Introducción } & 1\end{array}$

Capítulo 2. Descripción de los datos y algoritmos de regionalización 4

2.1 Escenarios de emisiones ................................................................ 5

2.2 Modelos climáticos globales .........................................................

2.3 Datos y algoritmos de regionalización ................................................... 8

2.3.1 Regionalización estadística ........................................................ 8

- Método de análogos ....................................................... 9

- Método de regresión lineal ................................................... 11

2.3.2 Regionalización dinámica. Modelos climáticos regionales ......... 14

Capítulo 3. Resultados de los escenarios regionalizados sobre España 16

3.1 Cambios en la temperatura máxima .............................................. 17

3.1.1 Valores medios de temperatura máxima ................................. 17

3.1.2 Índices extremos de temperatura máxima ................................ 20

3.2 Cambios en la temperatura mínima .............................................. 22

3.2.1 Valores medios de temperatura mínima ................................... 22

3.2.2 Índices extremos de temperatura mínima .................................. 23

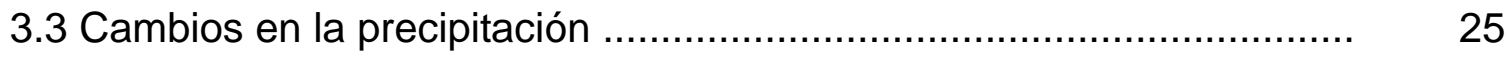

3.3.1 Valores medios de precipitación ............................................. 25

3.3.2 Índices extremos de precipitación ........................................... 28

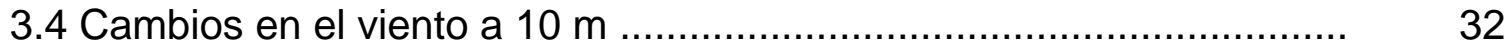


3.5 Cambios en la escorrentía y la evapotranspiración real

Capítulo 4. Conclusiones

Anexo A. Figuras por Comunidades Autónomas

Anexo B. Lista de Acrónimos

Anexo C. Referencias 


\section{Capítulo 1}

\section{Introducción}

Esta guía constituye el documento de acompañamiento a la segunda colección de proyecciones regionalizadas de cambio climático sobre España realizadas en la Agencia Estatal de Meteorología (AEMET). El trabajo en su conjunto, surge dentro del marco del Plan Nacional de Adaptación al Cambio Climático (PNACC) del Ministerio de Agricultura, Alimentación y Medio Ambiente con el objetivo de ofrecer los resultados más relevantes de los trabajos llevados a cabo en AEMET en el contexto de cambio climático a escala regional. El PNACC constituye el marco de referencia nacional para las actividades de evaluación de impactos, vulnerabilidad y adaptación al cambio climático y se desarrolla mediante Programas de Trabajo asignados a distintos grupos de trabajo para tareas identificadas en éstos programas.

En el Primer Programa de Trabajo (2006) se asignaron las primeras actividades al entonces Instituto Nacional de Meteorología (actual AEMET) que consistieron en desarrollar, documentar y poner a disposición del PNACC escenarios de cambio climático para España en la escala adecuada para los estudios regionales de impacto. Esta fase del trabajo se llevó a cabo a partir de los resultados del Tercer Informe del Grupo Intergubernamental de Expertos sobre el Cambio Climático (Third Assessment Report of the Intergovernmental Panel on Climate Change, IPCC-AR3). La siguiente tarea consistió en poner en funcionamiento un procedimiento de generación operativa y actualización de escenarios regionales de cambio climático para España. Esta primera fase 
quedó reflejada en el documento "Generación de Escenarios Regionalizados de Cambio Climático para España" (Brunet et al., 2009). En este documento se recoge la segunda fase del trabajo que ha consistido en aplicar nuevos métodos de regionalización dinámica y estadística a los resultados obtenidos a partir de las simulaciones climáticas integradas en el Programa Mundial de Investigación del Clima. Las simulaciones climáticas empleadas en esta segunda parte del trabajo se integran en la Fase 3 del Proyecto de Comparación de Modelos Acoplados (Coupled Model Intercomparison Project Phase 3, CMIP3) cuya contribución se incluye en Cuarto Informe del Grupo Intergubernamental de Expertos sobre el Cambio Climático (Fourth Assessment Report of the Intergovernmental Panel on Climate Change, IPCC-AR4; Meehl et al., 2007). En esta fase del trabajo, se emplea además otro grupo de modelos globales pertenecientes al Proyecto ENSEMBLES (ENSEMBLE-based Predictions of Climate Changes and their Impacts; van der Linden and Mitchell, 2009).

Las estimaciones de los cambios en la evolución futura del clima siempre están afectadas por diferentes fuentes de incertidumbre de diversas procedencias que deben ser tenidas en cuenta. Brunet et al. (2009) ofrece una explicación detallada de todas las fuentes de incertidumbre que se estudian en las proyecciones de cambio climático. La evolución de las emisiones de gases de efecto invernadero (GEI) constituye una de las mayores fuentes de incertidumbre de los estudios de cambio climático. Las emisiones de GEI son el resultado de una compleja dinámica de sistemas tales como crecimiento demográfico, socio-económico y el desarrollo tecnológico, cuyo origen deriva de la actividad humana. Para explorar el conjunto de posibles alternativas plausibles de la evolución del clima y su incertidumbre respecto a la emisión de GEI se plantean los escenarios de cambio climático como una serie de alternativas simplificadas de lo que podría suceder en el futuro (Nakicenovik et al., 2000). En el Informe Especial de Escenarios de Emisión (Special Report on Emissions Scenarios, SRES; Nakicenovik et al., 2000) se definen 40 escenarios de emisión internamente consistentes, divididos en cuatro posibles líneas evolutivas a las que se asigna la misma probabilidad. En su conjunto, los 40 escenarios SRES definidos describen una serie de posibles futuros divergentes que exploran una parte considerable de las incertidumbres relacionadas con la actividad humana. De cada una de las cuatro categorías deriva un grupo de supuestos futuros igualmente probables basados en la población mundial, el desarrollo económico y el cambio tecnológico, que en su conjunto completan los 40 escenarios. El planteamiento de los escenarios SRES responde a la necesidad de analizar la potencial influencia antropogénica sobre la evolución del clima en el futuro, para evaluar los posibles impactos y elaborar una serie de iniciativas de adaptación y mitigación. Para obtener la información que acote y cuantifique las incertidumbres asociadas a las simulaciones, en este trabajo se emplea un conjunto de simulaciones que permiten explorar las diferentes fuentes de incertidumbre.

Los estudios de cambio climático se llevan a cabo mediante modelos climáticos de circulación general que producen resultados aceptables a escalas hemisféricas y continentales; sin embargo, su escasa resolución espacial (de 
cientos de kilómetros) no permite su utilización para los estudios regionales de impacto que requieren mayor resolución (decenas de kilómetros). Para resolver este problema se recurre a técnicas de regionalización o downscaling que puede ser de naturaleza estadística o dinámica. En este trabajo se plantean y analizan varias técnicas de regionalización divididas en estadísticas y dinámicas que se detallan a lo largo del texto. Por otro lado, el clima en España está muy influido por sus características orográficas, los fuertes contrastes térmicos entre el mar y la tierra y efectos más locales, lo que determina microclimas que varían desde condiciones muy secas a frías y húmedas. Por esta razón, en este trabajo se incluye un análisis detallado por regiones que han sido definidas de acuerdo a la entidad territorial que componen las Comunidades Autónomas.

En este informe se analizan la evolución de los valores medios de distintas variables y de diversos índices de valores extremos asociados a ellas cuyo estudio es esencial a nivel regional. Las proyecciones regionalizadas de cambio climático que se muestran han sido realizadas con distintos escenarios de emisión, distintos modelos globales y distintas técnicas de regionalización. Esto permite analizar los resultados mediante intercomparaciones entre las distintas simulaciones y técnicas de regionalización, así como evaluar la sensibilidad de las estimaciones frente a cambios en las emisiones, los modelos y estudiar gran parte de las incertidumbres inherentes a las proyecciones de cambio climático. La similitud de buena parte de los resultados obtenidos puede considerarse como un índice de robustez y fiabilidad de las estimaciones de las proyecciones.

Este documento pretende servir como guía técnica de los resultados gráficos de escenarios regionalizados más relevantes que se han obtenido en la AEMET. Los resultados mostrados forman parte de un trabajo más amplio que se sigue llevando a cabo en la AEMET en el contexto de los estudios de cambio climático. Por tanto, en el presente informe se recoge sólo una parte del trabajo que se está realizando. La colección completa de resultados puede consultarse en la siguiente página web:

http://www.aemet.es/es/serviciosclimaticos/cambio_climat/

El documento se organiza de la siguiente manera: en el Capítulo 2 se ofrece una breve descripción de los algoritmos de regionalización y los modelos globales y regionales empleados. En el Capítulo 3 se analiza detalladamente los resultados obtenidos que se han considerado más representativos para el área en estudio. El Capítulo 4 recoge las conclusiones más relevantes. Finalmente, en el Anexo-A se recoge la colección de resultados gráficos generados para el estudio en su conjunto, y los Anexos-B y $C$ recogen respectivamente, el listado de acrónimos utilizados en el texto y las referencias consultadas. 


\title{
Capítulo 2
}

\section{Descripción de los datos y algoritmos de regionalización}

\begin{abstract}
En este Capítulo se explican los algoritmos o técnicas de regionalización empleados en este estudio. La Sección 2.1 recoge muy brevemente los escenarios de emisiones de GEl empleados y sus características principales. La Sección 2.2 describe los modelos globales utilizados para la generación de las proyecciones climáticas para el SXXI, así como los datos obtenidos a partir de ellos. En la sección 2.3 se describe la metodología estadística empleada para la regionalización sobre la Península Ibérica, Islas Baleares e Islas Canarias. Finalmente, en la sección 2.4 se describe los modelos regionales utilizados para las regionalizaciones dinámicas sobre la Península Ibérica.
\end{abstract}




\subsection{Escenarios de emisiones.}

Los escenarios de cambio climático empleados en este trabajo son un subconjunto extraído de los 40 escenarios planteados en el Informe Especial de Escenarios de Emisión (Special Report on Emissions Scenarios, SRES; Nakicenovik et al., 2000). Además se ha utilizado el escenario E1 de fuerte mitigación definido en el proyecto ENSEMBLES (ENSEMBLE-based Predictions of Climate Changes and their Impacts; van der Linden and Mitchell, 2009). Estos escenarios sirven de punto de partida para las simulaciones de cambio climático realizadas con los modelos climáticos que se describen en la sección siguiente. La Tabla 2.1 describe las principales características de los escenarios escogidos.

\begin{tabular}{|c|l|}
\hline Escenario & \multicolumn{1}{|c|}{ Características } \\
\hline A2 & $\begin{array}{l}\text { Emisiones altas. Mundo muy heterogéneo, basado en } \\
\text { tradiciones locales y modelo familiar. Desarrollo } \\
\text { económico y cambio tecnológico más lento que en otros } \\
\text { grupos de hipótesis. }\end{array}$ \\
\hline A1B & $\begin{array}{l}\text { Emisiones medias. Mundo con crecimiento económico } \\
\text { rápido basado en la utilización equilibrada de todo tipo de } \\
\text { fuentes de energía y tecnologías nuevas y eficientes. }\end{array}$ \\
\hline B1 & $\begin{array}{l}\text { Emisiones bajas. Mundo basado en la introducción de } \\
\text { tecnologías limpias y el aprovechamiento eficaz de los } \\
\text { recursos. Fomento de la sostenibilidad económica, social } \\
\text { y ambiental. }\end{array}$ \\
\hline E1 & $\begin{array}{l}\text { Fuerte mitigación. Objetivo de no sobrepasar } 2^{\circ} \mathrm{C} \text { de } \\
\text { calentamiento. }\end{array}$ \\
\hline
\end{tabular}

Tabla 2.1. Escenarios de emisión utilizados y sus características principales. 


\subsection{Modelos climáticos globales}

Los modelos climáticos constituyen la principal herramienta para el estudio de la dinámica del clima. Los modelos climáticos de circulación general resuelven a escala global, las ecuaciones de las leyes y principios de la Física que gobiernan los procesos que rigen el clima de la Tierra. La resolución numérica de estas ecuaciones permite obtener simulaciones plausibles del clima en la medida en la que son capaces de proporcionar simulaciones realistas de los campos de gran escala y de otras variables climáticas para diferentes horizontes temporales (Van Ulden and Van Oldenborgh, 2006). El sistema climático se divide en varios componentes: atmósfera, hidrosfera, criosfera, litosfera y biosfera. Cada componente se representa con un tipo de modelo de tal manera que todos ellos trabajan acoplados entre sí resolviendo la dinámica conjunta y los intercambios de energía y masa entre ellos. Estos modelos se conocen como modelos de circulación general acoplados atmósfera-océano (MACGAO; Coupled atmosphere-ocean circulation models).

El grupo de modelos globales empleados aquí son por un lado, los utilizados en la elaboración del Cuarto Informe del Grupo Intergubernamental de Expertos sobre el Cambio Climático y la Fase 3 del Proyecto de Comparación de Modelos Acoplados (Fourth Assessment Report of the Intergovernmental Panel on Climate Change / Phase 3 of the Coupled Model Intercomparison Project, IPCC-AR4/CMIP3; Meehl et al., 2007); por otro lado, se emplean además dos grupos más de modelos globales (ENSEMBLES-Stream1 (ES1) y ENSEMBLES-Stream2 (ES2)) pertenecientes a dos versiones del Proyecto ENSEMBLES (ENSEMBLE-based Predictions of Climate Changes and their Impacts; van der Linden and Mitchell, 2009). Los modelos de ES1, desarrollados en la primera parte del proyecto, se han utilizado para efectuar proyecciones para el SXXI con los escenarios de emisión SRES-A2, A1B y B1. Con los modelos de ES2 se han llevado a cabo proyecciones para el mismo período, con los escenarios A1B y E1. La Tabla 2.2 resume los modelos globales empleados en el presente estudio. 


\begin{tabular}{|c|c|c|c|}
\hline PROYECTO & MODELO & INSTITUCIÓN & REFERENCIA \\
\hline \multirow{9}{*}{ IPCC-AR4 } & GISS_AOM & $\begin{array}{l}\text { National Aeronautics and Space } \\
\text { Administration, NASA, USA }\end{array}$ & Russell et al. (1995) \\
\hline & GISS_MODEL_E_R & $\begin{array}{l}\text { Goddard Institute for Space Studies, } \\
\text { GISS, USA }\end{array}$ & Russell (2005) \\
\hline & INMCM3_0 & $\begin{array}{l}\text { Institute for Numerical Mathematics, } \\
\text { Russia }\end{array}$ & Diansky and Volodin (2002) \\
\hline & MIUB_ECHO_G & $\begin{array}{l}\text { Deutsches Klimarechenzentrum, } \\
\text { Germany }\end{array}$ & Legutke and Voss (1999) \\
\hline & MRI_CGCM2_3_2 & $\begin{array}{l}\text { Meteorological Research Institute, } \\
\text { Japan }\end{array}$ & Yukimoto et al. (2001) \\
\hline & MPI_ECHAM5 & $\begin{array}{l}\text { Max Plank Institute for Mateorology, } \\
\text { Germany }\end{array}$ & Roekner et al. (2003) \\
\hline & CCCMA_CGCM3_1T47 & $\begin{array}{l}\text { Canadian Centre for Climate } \\
\text { Modelling andAnalisys, Canada }\end{array}$ & Flato et al. (2005) \\
\hline & CCCMA_CGCM3_1T63 & $\begin{array}{l}\text { Canadian Centre for Climate } \\
\text { Modelling andAnalisys, Canada }\end{array}$ & Flato et al. (2005) \\
\hline & GFDL_CM2_1 & $\begin{array}{l}\text { US Departament of Commerce } \\
\text { National Oceanic and Atmosphic } \\
\text { Administration, NOAA, USA }\end{array}$ & Delworth et al. (2006) \\
\hline \multirow{6}{*}{$\begin{array}{l}\text { ENSEMBLES } \\
\text { ES1 }\end{array}$} & BCCR_BCM2 & $\begin{array}{l}\text { Bjerknes Centre for Climate Research, } \\
\text { Norway }\end{array}$ & Furevik et al. (2003) \\
\hline & CNR-CM3 & $\begin{array}{l}\text { Centre National de Recherches } \\
\text { Météorologiques, France }\end{array}$ & Royer (2006) \\
\hline & EGMAM & Freie Universität Berlin, Germany & Niehörster (2008) \\
\hline & INGV & $\begin{array}{l}\text { Istituto Nazionale di Geofisica e } \\
\text { Vulcanologia, Italy }\end{array}$ & Roeckner (1996) \\
\hline & IPCM4 & Institut Pierre Simon Laplace, France & Dufresne (2009) \\
\hline & MPEH5 & $\begin{array}{l}\text { Max Planck Institute for Meteorology, } \\
\text { Germany }\end{array}$ & Roeckner (2007) \\
\hline \multirow{6}{*}{$\begin{array}{l}\text { ENSEMBLES } \\
\text { ES2 }\end{array}$} & CNCM33 & $\begin{array}{l}\text { Centre National de Recherches } \\
\text { Météorologiques, France }\end{array}$ & Royer (2008) \\
\hline & DMIEH5C & $\begin{array}{l}\text { Danish Meteorological Institute, } \\
\text { Denmark }\end{array}$ & Roeckner et al. (2006) \\
\hline & EGMAM2 & Freie Universität Berlin, Germany & $\begin{array}{l}\text { Huebener and Koerper } \\
\text { (2008) }\end{array}$ \\
\hline & HADGEM2 & Met Office Hadley Centre, UK & Johns (2009) \\
\hline & IPCM4V2 & Institut Pierre Simon Laplace, France & Dufresne (2009) \\
\hline & MPEH5C & $\begin{array}{l}\text { Max Planck Institute for Meteorology, } \\
\text { Germany }\end{array}$ & Roeckner (2008) \\
\hline
\end{tabular}

Tabla 2.2. Modelos globales empleados. Nombre del proyecto; acrónimo del modelo; Institución y referencia.

La mejora en los modelos redunda en la mejor caracterización de los procesos que tienen lugar en el sistema climático. Sin embargo, siempre adolecerán de limitaciones asociadas tanto a la escala de los procesos como a las propias características de los modelos dando lugar a incertidumbres en la estimación de las posibles soluciones reales del sistema. 


\subsection{Datos y algoritmos de regionalización.}

Una de las principales limitaciones de los modelos climáticos es su escasa resolución espacio-temporal (cientos de kilómetros). Esta resolución es insuficiente para resolver variables a nivel local, limitando los resultados a valores promedio de estas variables en áreas muy extensas. Por tanto, es necesario incrementar la resolución de éstas variables con el objeto de estudiar la variabilidad regional del clima en pequeñas áreas para poder llevar a cabo estudios de impacto y adaptación en éstas regiones. Este aumento de resolución o descenso de escala se conoce como regionalización o downscaling y se puede llevar a cabo de dos maneras diferentes, estadística y dinámicamente. Actualmente, ambas técnicas de regionalización ofrecen niveles similares de habilidad bajo las mismas condiciones climáticas al estimar variables atmosféricas superficiales (Gershunov et al., 2000; Wilby and Dawson, 2004). Sin embargo, es necesario tener en cuenta que las relaciones estadísticas no son de causalidad por lo que no describen la física y la dinámica de las interacciones entre los diversos componentes del sistema climático. Cabe mencionar que las relaciones estadísticas deben estar sustentadas por un modelo conceptual físico. En este trabajo se aborda la tarea de aumento de resolución o descenso de escala de los resultados de los modelos globales mediante las dos metodologías mencionadas, estadística y dinámica.

\subsubsection{Regionalización estadística.}

La regionalización estadística o downscaling estadístico es una de las herramientas más utilizadas en los estudios de cambio climático. Esta metodología consiste en encontrar las relaciones empíricas existentes entre las variables de las simulaciones globales (variables predictoras) y las variables a escala regional (variables predictandos) en áreas con características climáticas conocidas. En los estudios de cambio climático es muy importante mantener las relaciones entre variables predictoras y variables predictando. Para ello, es razonable que los predictores seleccionados sean forzamientos físicos de los predictandos o estén relacionados físicamente con ellos y deben estar bien simulados por los MCGAO. Asimismo, es conveniente considerar como predictores variables en atmósfera libre en lugar de superficie y los campos completos en lugar de puntos de rejilla individuales.

La regionalización estadística se fundamenta en encontrar la función de transferencia adecuada entre el estado climático a gran escala y las características fisiográficas regionales, válida bajo condiciones climáticas alteradas (Wilby et al., 2004). En éste trabajo se aplican dos técnicas diferentes de regionalización estadística: una metodología de identificación de situaciones análogas y una metodología de regresión lineal.

La metodología de regionalización se ha aplicado a 373 estaciones termométricas y a 2324 estaciones pluviométricas procedentes de la base de datos generada por la Agencia Estatal de Meteorología para estudios climáticos. El conjunto de series seleccionadas para este estudio atiende a criterios de representatividad geográfica y continuidad en el tiempo. Asimismo, 
las series han sido sometidas a un estricto control de calidad, relleno de lagunas y homogeneización (Brunet et al., 2006; 2009). Para cada estación se toman los valores de estos predictores en el punto de rejilla más próximo. Como los diferentes modelos de circulación general han utilizado diferentes rejillas, ha sido necesaria la interpolación de todos los campos de los modelos de circulación general utilizados a una rejilla común. La rejilla empleada como referencia ha sido la utilizada en el re-análisis del NCEP/NCAR (Kalnay et al., 1996). El método de interpolación que se ha utilizado ha sido el método de interpolación bilineal (Schulzweida et al., 2012).

\section{- Método de análogos.}

La identificación automática de situaciones atmosféricas se ha llevado a cabo mediante un método de análogos sinópticos basado en la hipótesis de que configuraciones meteorológicas similares dan lugar a fenómenos meteorológicos similares. Este método consta de los siguientes pasos:

Selección de las variables predictoras: El dominio espacial escogido para la ventana de campos predictores se extiende desde $30^{\circ} \mathrm{N}$ hasta $55^{\circ} \mathrm{N}$ de latitud y desde $27.5^{\circ} \mathrm{W}$ hasta $15^{\circ} \mathrm{E}$ de longitud y contiene 198 nodos espaciales (Brunet et al., 2009). El periodo temporal es de 30 años comprendidos entre 1961 y 1990. Los campos predictores utilizados dependen de la variable predictando. Las variables predictando son la temperatura a $2 \mathrm{~m}$ y precipitación (Tablas 2.3 y 2.4).

\begin{tabular}{|l|l|}
\hline VARIABLE & UNIDADES \\
\hline Presión a nivel del mar & $\mathrm{Pa}$ \\
\hline Humedad específica en 700hPa & $\mathrm{Kg} \mathrm{kg}^{-1}$ \\
\hline Temperatura a 2m & $\mathrm{K}$ \\
\hline Temperatura a 2m del día anterior & $\mathrm{K}$ \\
\hline Temperatura del aire en 850hPa, 700hPa y 500hPa & $\mathrm{K}$ \\
\hline Componente zonal del viento geostrófico a nivel del mar & $\mathrm{ms}^{-1}$ \\
\hline Componente meridiana del viento geostrófico a nivel del mar & $\mathrm{ms}^{-1}$ \\
\hline Función de la insolación térmica correspondiente al día del año & \\
\hline
\end{tabular}

Tabla 2.3. Campos predictores utilizados para la temperatura con el método de análogos y unidades de las variables. 


\begin{tabular}{|l|l|}
\hline VARIABLE & UNIDADES \\
\hline Presión a nivel del mar & $\mathrm{Pa}$ \\
\hline Humedad específica en 700hPa & $\mathrm{Kg} \mathrm{kg}^{-1}$ \\
\hline Temperatura del aire en 500hPa & $\mathrm{K}$ \\
\hline $\begin{array}{l}\text { Componente zonal del viento geostrófico a nivel del mar y } \\
\text { 500hPa }\end{array}$ & $\mathrm{ms}^{-1}$ \\
\hline $\begin{array}{l}\text { Componente meridiana del viento geostrófico a nivel del mar y y } \\
\text { 500hPa }\end{array}$ & $\mathrm{ms}^{-1}$ \\
\hline Vorticidad geostrófica a nivel del mar y 500hPa & $\mathrm{ms}^{-1}$ \\
\hline Gradiente vertical de temperatura entre 800-500hPa & $\mathrm{K}^{2}$ \\
\hline Tendencia de la presión a nivel del mar & $\mathrm{m}^{2}{ }^{-3}$ \\
\hline
\end{tabular}

Tabla 2.4. Igual que la Tabla 2.3 utilizados para la precipitación.

Formulación matemática de la medida de similitud: Un aspecto clave en la aplicación práctica de este método es la forma matemática en la que se define la similitud entre dos configuraciones meteorológicas. En general, la optimización de esta medida de similitud es un proceso complejo que incluye la selección de variables predictoras eficaces y la formulación correcta de la medida que extraiga la máxima cantidad posible de información de los campos estudiados. Otro punto importante es determinar el número óptimo de situaciones análogas que es necesario identificar. No existe un criterio general acerca del número óptimo de análogos. En general, se suele fijar un valor máximo de la distancia y se seleccionan sólo los análogos que cumplen este criterio. En este trabajo, el criterio que se aplica es determinar el número de análogos que en cada caso minimiza el error cuadrático medio.

La medida de la similitud entre el patrón estimado y los patrones atmosféricos históricos se calcula mediante una función distancia definida por la Ecuación E2.1.

$$
d_{c}=\sqrt{\frac{\sum w_{i}\left(c_{t i}-c_{i}\right)^{2}}{\sum w_{i}}}, i=1, \ldots, 198
$$

Donde $c$ representa una componente cualquiera del viento $\left(u_{s l}, v_{s l}, u_{500}, v_{500}\right)$; $c_{i}$ representa el valor de la componente $c$ del viento en el punto de rejilla $i$ para una situación sinóptica de la base de datos de referencia; $c_{t i}$ representa el valor de la componente $c$ del viento en el punto de rejilla $i$ para la situación sinóptica del día problema; $w_{i}$ es la ponderación correspondiente al punto de rejilla $i$.

Simultáneamente, se tiene en cuenta la similitud de los valores tipificados de un conjunto de variables predictoras, calculados para el punto de rejilla más cercano al punto geográfico que se pretende estimar. Este valor, se obtiene mediante la Ecuación E2.2. 


$$
d_{p}=\sqrt{\frac{\sum_{1}^{J}\left(p_{t j}-p_{j}\right)^{2}}{J}}
$$

Donde $p_{t j} \mathrm{y} p_{j}$ son, respectivamente, los valores del campo predictor $j$ tipificado para el día problema y el elemento cuya similitud con el día problema se quiere analizar y $J$ es el número total de predictores utilizados.

Una vez calculadas las distancias $d_{c}$ y $d_{p}$ la medida de similitud que finalmente se aplica es el valor medio de ambas (Petisco et al., 2005).

\section{- Método de regresión lineal.}

El método de regionalización estadística o downscaling estadístico SDSM (Statistical Downscaling Method; Wilby et al., 2002; Wilby and Dawson, 2004) está basado en una metodología estadística robusta fundamentada en técnicas de regresión lineal. Este método permite establecer relaciones empíricas entre campos de variables predictoras a escala regional, obtenidos a partir de los modelos de circulación general y variables predictandos a escala local. Asimismo, esta metodología es adecuada para regiones heterogéneas con orografía compleja como la Península Ibérica. Sin embargo, aunque con esta metodología no se explica toda la variabilidad climática observada, la obtención de proyecciones climáticas futuras conlleva la aceptación de la hipótesis de que el modelo paramétrico ajustado tiene validez bajo condiciones futuras (Wilby et al., 2002). Este método consta de los siguientes pasos:

Selección de las variables predictoras: La selección de las variables predictoras se ha realizado mediante la aplicación de un método jerarquizado incorporando las variables una a una. Se comienza con la variable mejor correlacionada con el predictando y se van incorporando sucesivamente el resto de variables en función de la mejora en el coeficiente de determinación corregido con los grados de libertad que produce su introducción en el modelo de regresión. El proceso finaliza cuando la mejora obtenida en dicho coeficiente es inferior a un umbral establecido.

El dominio espacial escogido para la ventana de campos predictores es el mismo que se ha utilizado para el método de análogos. El periodo escogido en este caso es de 40 años de recorrido temporal comprendidos entre 1961 y 2000. Las variables analizadas pueden verse en la Tabla 2.1: 


\begin{tabular}{|l|l|}
\hline VARIABLE & UNIDADES \\
\hline Presión a nivel del mar & $\mathrm{Pa}$ \\
\hline Altura geopotencial (1) & $\mathrm{mgp}$ \\
\hline Temperatura máxima a 2m & $\mathrm{K}$ \\
\hline Temperatura mínima a 2m & $\mathrm{K}$ \\
\hline Temperatura del aire (1) & $\mathrm{K}$ \\
\hline Humedad específica (1) & $\mathrm{Kg} \mathrm{kg}^{-1}$ \\
\hline Componente zonal del viento (1+) & $\mathrm{m} \mathrm{s}^{-1}$ \\
\hline Componente meridiana del viento (1+) & $\mathrm{m} \mathrm{s}^{-1}$ \\
\hline
\end{tabular}

Tabla 2.4. Campos predictores utilizados con el método de de regresión lineal y unidades de las variables. Se indica: (1) niveles isobáricos $1000 \mathrm{hPa}$, $850 \mathrm{hPa}, 700 \mathrm{hPa}$ y $500 \mathrm{hPa}$. (1+) niveles isobáricos de (1) y superficie.

Obtención del modelo de regresión: Según las características de la variable predictando, se consideran dos tipos de modelo: condicional y no condicional. El modelo no condicional supone una relación directa entre predictores y predictando, mientras que en el modelo condicional esta relación se obtiene a través de una variable intermedia que en este estudio es la probabilidad de ocurrencia del fenómeno a predecir. Los parámetros de la regresión lineal múltiple se pueden obtener mediante dos métodos: estimación por mínimos cuadrados (MC) y minimización de la suma de los errores absolutos (MSAE) (Narula and Wellington, 1977).

El modelo condicional incluye un proceso estocástico adicional al determinar si un día concreto ha ocurrido o no el fenómeno que se pretende predecir. Para ello, compara la probabilidad obtenida a partir del modelo de regresión con un número pseudo-aleatorio extraído de una distribución uniforme con valores entre 0 y 1 . En general, se determina la probabilidad diaria de ocurrencia de precipitación a partir del modelo de regresión ajustado para probabilidad de ocurrencia menor o igual a $0.1 \mathrm{~mm}$. Si esta probabilidad es igual o superior al número aleatorio obtenido se considera la ocurrencia de precipitación y se cuantifica. El modelo no condicional aumenta la varianza de las series obtenidas añadiendo una componente residual aleatoria a la componente determinista.

Las proyecciones regionales de temperatura máxima y mínima se han llevado a cabo con modelos SDSM- no condicionales y mediante el ajuste de MSAE para la estimación de los parámetros. Sin embargo, para la precipitación se han utilizado modelos condicionales siguiendo el procedimiento indicado por Kilsby et al. (1998) mediante el ajuste de MC. 


\subsubsection{Regionalización dinámica. Modelos climáticos regionales.}

Las técnicas de regionalización dinámica o downscaling dinámico proporcionan una descripción de la atmósfera que considera todas las variables del modelo. Los modelos climáticos regionales (MCR) están diseñados para proporcionar resultados a mayor resolución espacio-temporal que los modelos globales y en un dominio espacial limitado, definido para cada estudio. Los MCR mejoran los resultados de las simulaciones globales en regiones donde existen forzamientos debidos a orografías complejas, contrastes tierra-mar y usos de suelo entre otros, permitiendo distinguir la señal de cambio climático de los forzamientos orográficos (Wang et al., 2004). Asimismo, los MCR permiten mejorar el conocimiento de procesos climáticos tales como el forzamiento radiativo de las nubes, los procesos convectivos nubosos y algunos procesos en superficie (Barros and Hwu, 2002). Los MCR generan representaciones regionales de clima realistas y consistentes con los MCGAO.

Los MCR son modelos de área limitada que trabajan integrados en un dominio de menor resolución perteneciente a un MCGAO que les proporciona condiciones de frontera o contorno como función del tiempo. Los resultados del MCGAO son interpolados para generar las condiciones iniciales y de contorno para el dominio de alta resolución. Esta metodología de trabajo se conoce como anidamiento. La Figura 2.1 esquematiza el dominio anidado del MCR en un modelo global.

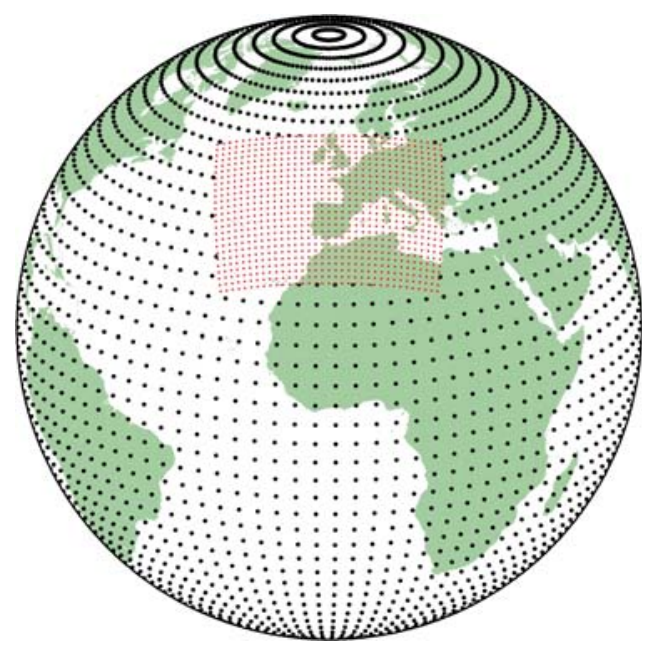

Figura 2.1 Esquema de anidamiento, en forma de puntos de rejilla, del dominio de un MCR (área punteada roja) en el dominio global de un MCGAO.

Los MRC que integran este trabajo son los utilizados en el proyecto ENSEMBLES (Tabla 2.5). Las proyecciones realizadas con los modelos de ENSEMBLES abarcan un área común sobre Europa, con una resolución horizontal de $25 \mathrm{~km}$ (Figura 2.2). 


\begin{tabular}{|c|l|l|}
\hline MCR & \multicolumn{1}{|c|}{ INSTITUCIÓN } & \multicolumn{1}{c|}{ REFERENCIA } \\
\hline RM4.5 & $\begin{array}{l}\text { Centre National de Recherches } \\
\text { Meteorologiques, CNRM, France }\end{array}$ & Gibelin and Déqué (2003) \\
\hline HIRHAM5 & $\begin{array}{l}\text { Danish Meteorological Institute, DMI, } \\
\text { Denmark }\end{array}$ & Christensen et al. (1996) \\
\hline CLM & $\begin{array}{l}\text { Swiss Institute of Technology, ETHZ, } \\
\text { Switzerland }\end{array}$ & $\begin{array}{l}\text { Steppeler et al. (2003) } \\
\text { Jaeger et al. (2008) }\end{array}$ \\
\hline RACMO2 & $\begin{array}{l}\text { Koninklijk Nederlands Meteorologisch } \\
\text { Instituut, KNMI, Netherland }\end{array}$ & Lenderink et al. (2003) \\
\hline HadRM3 & Hadley Center/UK Met Office, MetoHC, UK & Jones et al. (1995) \\
\hline RegCM3 & $\begin{array}{l}\text { Abdus Salam International Centre for } \\
\text { Theoretical Physics, ICTP, Italy }\end{array}$ & No disponible \\
\hline HIRHAM & $\begin{array}{l}\text { The Norwegian Meteorological Institute, } \\
\text { Met.NO, Norway }\end{array}$ & Christensen et al. (1996) \\
\hline REMO & $\begin{array}{l}\text { Max Planck Institute for Meteorology, MPI-M, } \\
\text { Germany }\end{array}$ & $\begin{array}{l}\text { Jacob and Podzun (1997) } \\
\text { Jacob (2001) }\end{array}$ \\
\hline RCA3.0 & $\begin{array}{l}\text { Swedish Meteorological and Hydrological } \\
\text { Institute, SMHI, Sweden }\end{array}$ & Kjellström et al. (2005) \\
\hline PROMES & $\begin{array}{l}\text { Universidad de Castilla la Mancha, UCLM, } \\
\text { España }\end{array}$ & Sánchez et al. (2004) \\
\hline RCA3.5 & $\begin{array}{l}\text { Agencia Estatal de Meteorología, AEMET, } \\
\text { España }\end{array}$ & Kjellström et al. (2005) \\
\hline
\end{tabular}

Tabla 2.5. Modelos climáticos regionales empleados. Acrónimo del modelo, Institución y referencia. El modelo HadRM3 cuenta con tres versiones: Q0, Q3 y Q16.

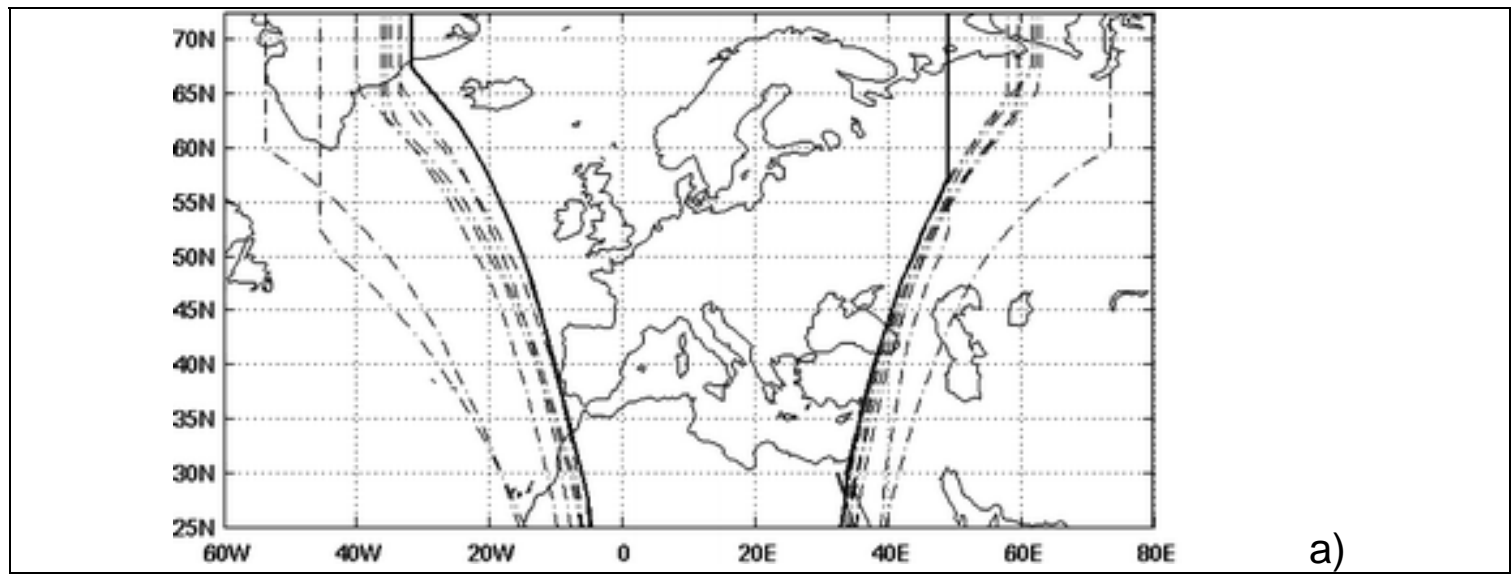

Figura 2.2. Dominios espaciales de las simulaciones de los MCR correspondientes al proyecto ENSEMBLES (Sánchez-Gómez et al., 2009). La línea continua indica el área común utilizada.

El escenario de emisiones utilizado para todas las regionalizaciones de cambio climático llevadas a cabo en ENSEMBLES es el SRES-A1B, de manera que únicamente se exploran las incertidumbres asociadas a los modelos globales y las técnicas de regionalización. Los forzamientos globales utilizados son los correspondientes a los MCGAO empleados en el IPCC-AR4. Sin embargo, solo se han llevado a cabo determinadas combinaciones de MCGAO-MCR con el fin de mantener un volumen manejable datos y un coste computacional asumible. 
La Tabla 2.6 resume las combinaciones realizadas con los modelos de ENSEMBLES, así como los períodos de cada simulación.

\begin{tabular}{|c|c|c|c|c|}
\hline & \multicolumn{4}{|c|}{ MCGAO } \\
\hline MCR & HadCM3Q0 & ECHAM5 & CNCM3 & BCM2 \\
\hline RM4.5 & & & + & \\
\hline HIRHAM5 & & $\mathrm{x}$ & + & $x$ \\
\hline CLM & +1 & & & \\
\hline RACMO2 & & + & & \\
\hline HadRM3 & + & & & \\
\hline RegCM3 & & + & & \\
\hline HIRHAM & $\mathrm{x}$ & & & +1 \\
\hline REMO & & + & & \\
\hline RCA3.0 & & $x$ & & + \\
\hline PROMES & +1 & & & \\
\hline
\end{tabular}

Tabla 2.6. Resumen de las combinaciones realizadas MCGAO-MCR para las simulaciones estudiadas. Los períodos de simulación y los forzamientos son: (+): 1961-2000 (CTRL) y 2001-2100 (SRES-A1B); (+1): 2001-2050 (SRESA1B); (x): simulaciones descartadas.

En el Capítulo 3 se analizan en detalle los resultados gráficos obtenidos con las simulaciones descritas en este Capítulo. Por brevedad, solamente se comentan las figuras que resultan más representativas para este estudio. La colección completa de resultados gráficos y numéricos puede consultarse en la siguiente página web: http://www.aemet.es/es/serviciosclimaticos/cambio_climat 


\section{Capítulo 3}

\section{Resultados de los escenarios regionalizados sobre España}

En este Capítulo se muestran los cambios potenciales bajo futuras condiciones de cambio climático a lo largo del SXXI de una serie de variables y un conjunto de índices extremos asociados a ellas. Distintos autores obtienen que los cambios esperados sobre estas variables conducen hacia un calentamiento más intenso en los meses de verano, ligado a aumentos en la intensidad y frecuencia de olas de calor sobre el sur de Europa y el Mediterráneo y a descenso de la precipitación (Kittel et al., 1998; Giorgi, 2001, 2006; Fischer and Schär, 2010). En el estudio del cambo climático además de la evolución de los valores medios y extremos de temperatura, tiene particular interés el conocimiento de los posibles cambios en los regímenes de precipitación. Esto se debe a que la precipitación es uno de los parámetros climáticos más relevantes, no solo como descriptor de las condiciones climáticas locales sino también como potencial indicador del impacto del cambio climático sobre el medio ambiente y los sistemas socioeconómicos (Giorgi, 2006). Desde el punto de vista social, económico y ecológico es fundamental el estudio de la evolución tanto de los valores medios de precipitación como de los índices extremos asociados a ella. Por otro lado, para comprender mejor las consecuencias del cambio climático es necesario tener en cuenta las interacciones entre los distintos subsistemas que componen el sistema climático. Por ello, además de las variables de temperatura y precipitación se analizan una serie de variables que junto con éstas, permiten obtener una descripción más completa del sistema climático y entender mejor la posible evolución del clima futuro. Las variables seleccionadas son viento a 10m, escorrentía, evapotranspiración real y nubosidad. 
En este Capítulo se muestran los resultados más representativos para el área en estudio. Las proyecciones regionalizadas se agrupan por técnicas de regionalización (dinámica/estadística) y por escenarios de emisión. Los escenarios de emisión representados corresponden a emisiones altas (A2), emisiones medias (A1B), emisiones bajas (B1) (Nakicenovic et al., 2000) y de fuerte mitigación (E1) (van der Linden and Mitchell, 2009). Las proyecciones dinámicas se han llevado a cabo para el escenario A1B. Se muestran, por un lado, la evolución del valor medio (línea continua) calculada como el promedio de su valor obtenido a partir de 72 simulaciones y por otro, la estimación de su incertidumbre (área sombreada) representada a partir de su desviación típica como +/una desviación típica.

Los cambios en la evolución de cada variable se representan promediados anual y estacionalmente, en forma de anomalías calculadas respecto a los valores observacionales de los periodos de control 1961-1990 para las proyecciones dinámicas y las estadísticas realizadas mediante regresión lineal y 1961-2000 para las estadísticas llevadas a cabo con el método de análogos. El período abarcado por las proyecciones regionalizadas comprende desde 2011 hasta 2100 para las regionalizaciones dinámicas y las estadísticas-regresión lineal y desde 2001 hasta 2100 para las estadísticas-análogos. La evolución temporal se muestra en un único gráfico mientras que los mapas de distribución espacial se dividen en dos períodos comprendidos entre los años 2046-2065 y 2081-2100. Por cuestión de brevedad, en este documento solamente se muestran los gráficos de evolución temporal y los de distribución espacial correspondientes al segundo período estudiado. Todas las Figuras no mostradas pueden consultarse en la siguiente página web:

http://www.aemet.es/es/serviciosclimaticos/cambio_climat

\subsection{Cambios en la temperatura máxima}

\subsubsection{Valores medios de temperatura máxima}

La Figura 3.1 muestra la evolución temporal de la temperatura máxima anual para el período completo obtenida mediante las dos técnicas de regionalización descritas en el Capítulo 2, promediada para todo el territorio peninsular. Los dos métodos de regionalización empleados ofrecen resultados similares. En general, se observa tendencia al aumento del valor medio de la temperatura máxima a lo largo de todo el período, manifestándose a final de siglo mayor incremento para los escenarios de mayores emisiones (Figura 3.1.b). Asimismo, se observa un aumento de la incertidumbre con el alcance de la proyección. Estos resultados son coherentes con los obtenidos por Nikulin et al. (2011) que encuentra intensificación estadísticamente significativa de las temperaturas máximas en el sur de Europa entre $4-6^{\circ} \mathrm{C}$ al final del SXXI. 


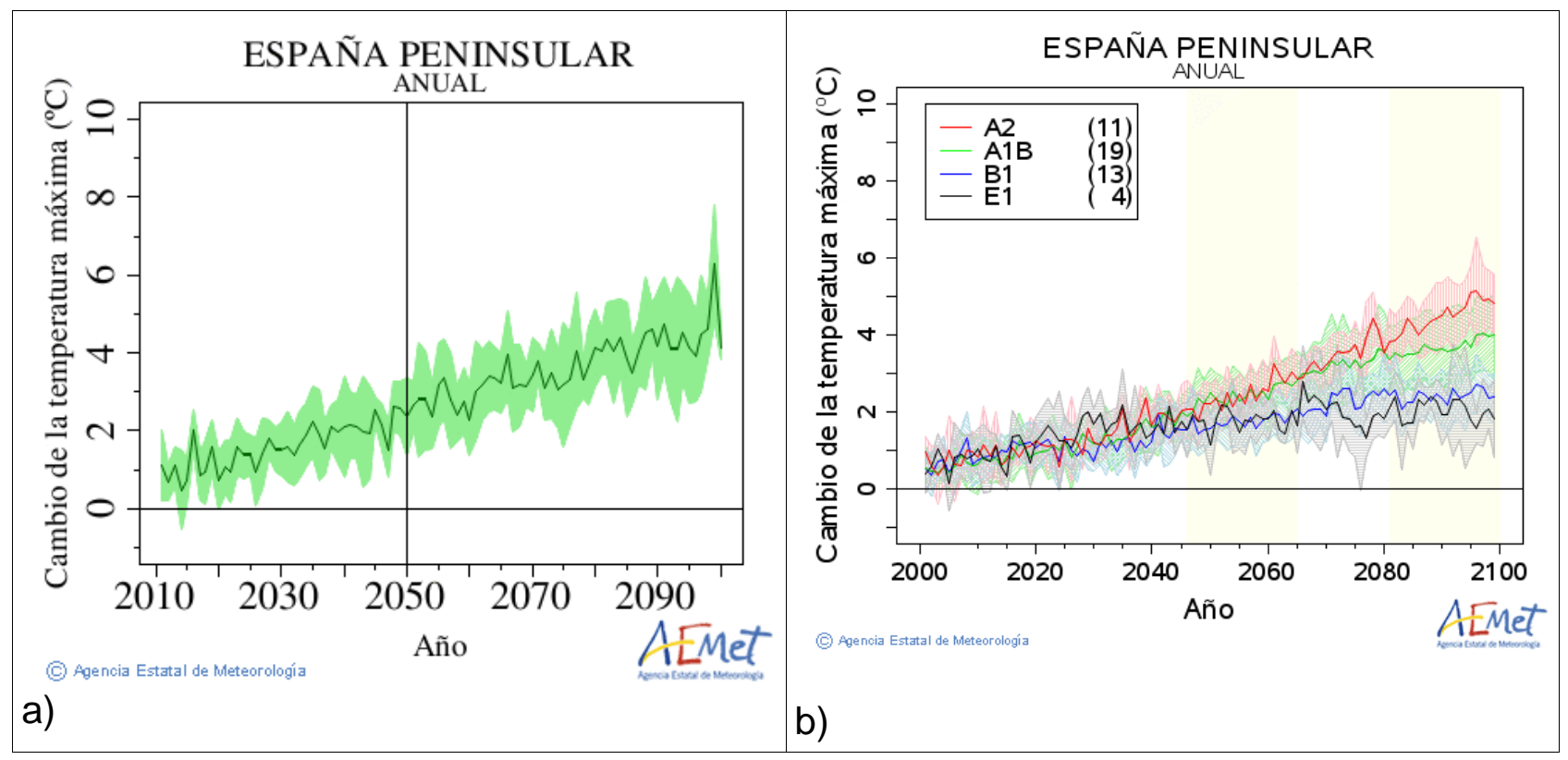

Figura 3.1. Cambios en el valor medio anual de la temperatura máxima $\left({ }^{\circ} \mathrm{C}\right)$ en España peninsular para el siglo XXI obtenida mediante técnicas de regionalización (a) dinámica y (b) estadística Entre paréntesis se recoge el número de proyecciones realizadas para cada escenario. En línea continua (sombreada) se representa el valor promedio (incertidumbre). En (b), las franjas verticales sombreadas indican las ventanas temporales en las que el número de regionalizaciones es máximo.

La Figura 3.2 (a y b) muestra la distribución espacial del valor medio anual de la temperatura máxima obtenida por métodos dinámicos y estadísticos, respectivamente, correspondiente al escenario de emisiones medias A1B. En general, en todas las regiones, se obtiene un comportamiento coherente con el anual. Se identifica una tendencia menos pronunciada al aumento de la temperatura en las regiones costeras, así como en Valle del Ebro e Islas Baleares y más acentuada en el Pirineo y las regiones del centro y sur peninsular. 


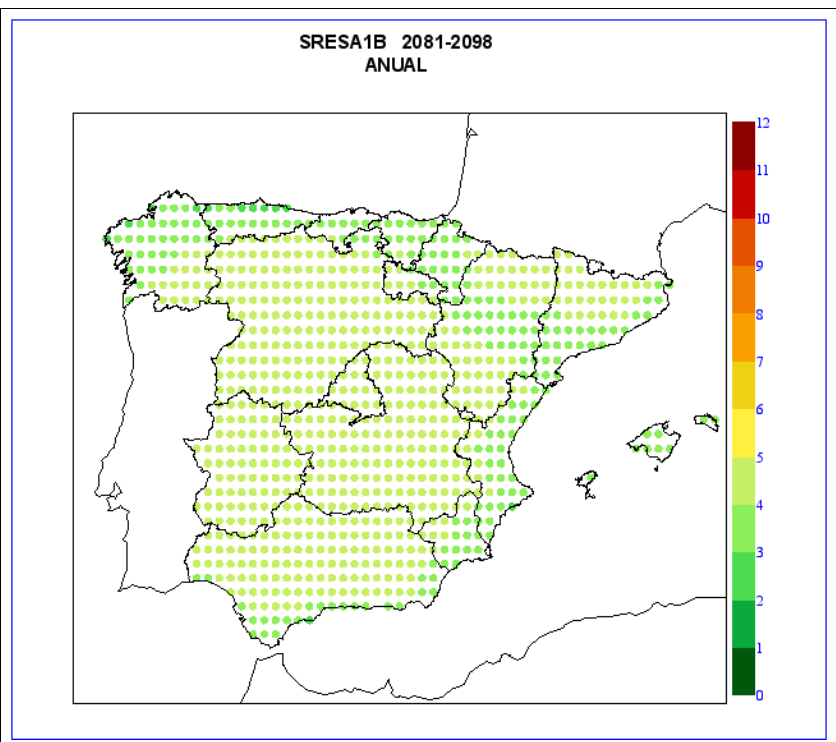

a)

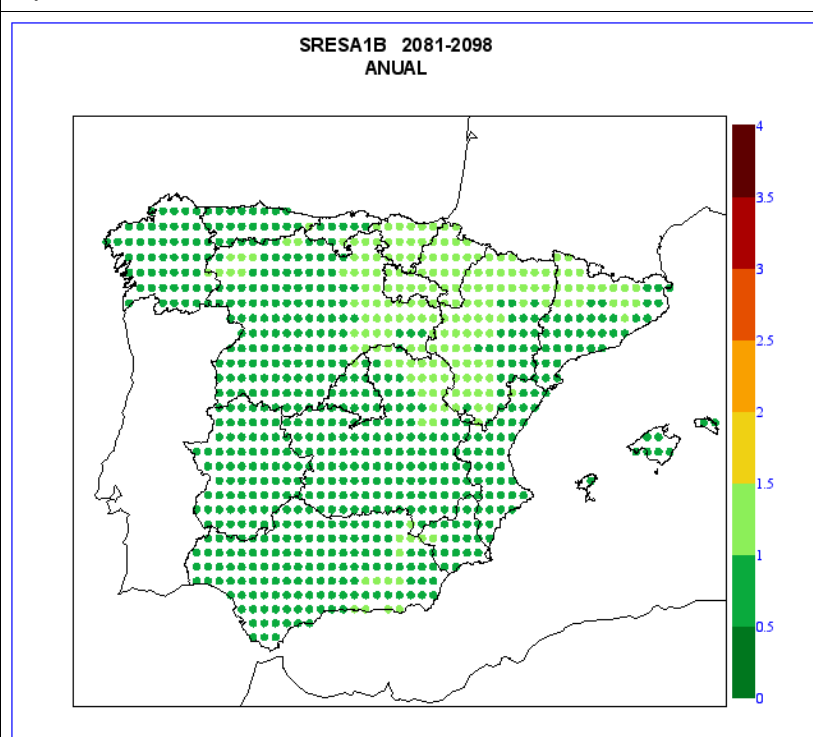

b)

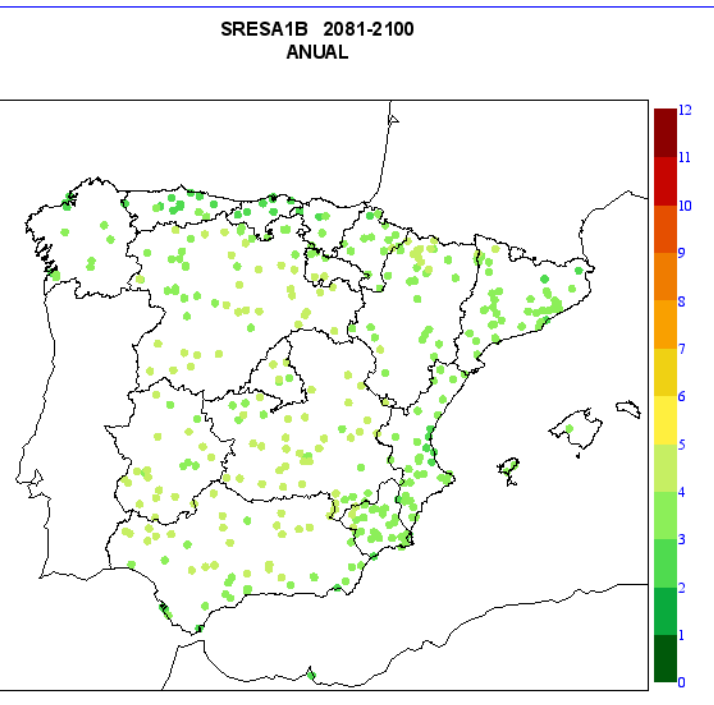

c)

d)

Figura 3.2. Izquierda: (a) Distribución espacial del cambio en el valor medio anual de la temperatura máxima obtenida mediante técnicas de regionalización dinámica del escenario A1B para el período 2081-2098 y (c) distribución espacial de su incertidumbre. Derecha: (b) Distribución espacial del valor medio anual de la temperatura máxima obtenida mediante técnicas de regionalización estadística del escenario A1B para el período 2081-2100 y (d) distribución espacial de su incertidumbre. Las escalas representan ${ }^{\circ} \mathrm{C}$.

Se analiza el comportamiento de la temperatura media por CCAA calculada por métodos dinámicos (Figura no mostrada), y se observa que la evolución de la temperatura es similar en todas las regiones, obteniéndose incrementos de temperatura mayores para las comunidades del centro y sur peninsular. El resultado es similar al obtenido mediante técnicas estadísticas (no mostrado) y coherente con los resultados estudiados. 
Por otra parte, se ha llevado a cabo segregación estacional se han obtenido distintos patrones espaciales en función de la estación. Estos resultados son similares a los encontrados por Giorgi y Coppola (2007) y Elguindi et al (2013). Así pues, en el estudio estacional se obtiene mayor incremento de la temperatura en los meses correspondientes a verano de hasta $5-6^{\circ} \mathrm{C}$, menos intenso en la zona cantábrica. En general, en invierno el valor medio esperado manifiesta un incremento de menor magnitud que en los meses de verano (Figuras no mostradas). Para las Islas Canarias solamente se tienen resultados de regionalización estadística; se observa un incremento de la temperatura de orden similar al de las regiones del norte peninsular, aunque sin variación estacional (Figuras no mostradas).

\subsection{2 Índices extremos de temperatura máxima}

Las simulaciones del clima futuro a escala global muestran que los extremos cálidos vienen ligados a la evolución de los valores medios de temperatura máxima de los meses más cálidos. De igual manera sucede con los extremos fríos y los valores medios de temperatura mínima en los meses más fríos (Kharin and Zwiers, 2004; Kharin et al., 2007). En general, tanto para extremos cálidos como fríos, los cambios obtenidos son mayores que los correspondientes a los valores medios (Schär et al., 2004; Rowell, 2005; Fischer and Schär, 2009).

La Figura 3.3 muestra el cambio esperado del porcentaje de días cálidos (DC) respecto al período de control y la duración de olas de calor (DOC). El resultado obtenido es similar para todas las zonas geográficas. Las series temporales de éstos índices muestran un comportamiento coherente con los valores medios previamente analizados. En la sección anterior se ha observado tendencia creciente en el valor medio de la temperatura máxima, por tanto parece lógico que el índice DC deba aumentar. Así pues, el porcentaje de días cálidos mantiene tendencia creciente constante a lo largo de todo el período (Figura 3.3a y b); esta tendencia es más marcada en el caso de los escenarios de mayores emisiones (A2 y A1B). Por otra parte, el índice DoC presenta incremento de en torno a 5 días hasta la mitad del período. Los resultados obtenidos, tanto por técnicas dinámicas como estadísticas, muestran a partir del año 2050 tendencia creciente que se hace más acusada en el caso de los escenarios A2 y A1B a partir del año 2050 hasta alcanzar valores próximos a los 40 días a finales del SXXI con el escenario A2 (Figura 3.3c y d). En todos los casos, la incertidumbre asociada a los valores medios aumenta con el alcance de la proyección y muestra fluctuaciones similares a las de la serie de valores medios correspondiente. Otros autores han Ilevado a cabo estudios similares con datos observacionales para el SXX a escala global y han obtenido resultados con tendencias análogas a las que se muestran en este estudio (Alexander et al., 2006). Este autor analiza, entre otros índices, el número de días cálidos y la duración de olas de calor encontrando tendencias estadísticamente significativas crecientes en ambos casos en la región de la Península Ibérica. 

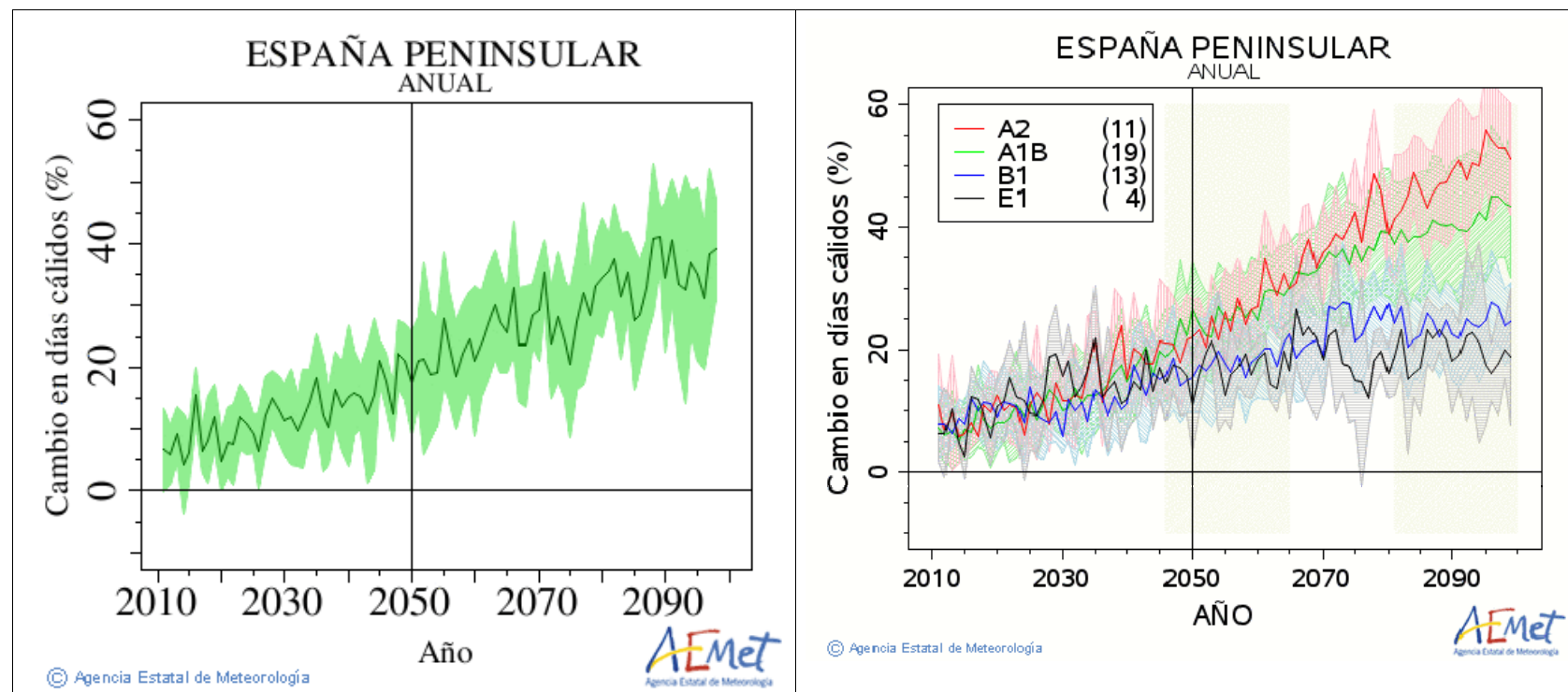

a)

b)

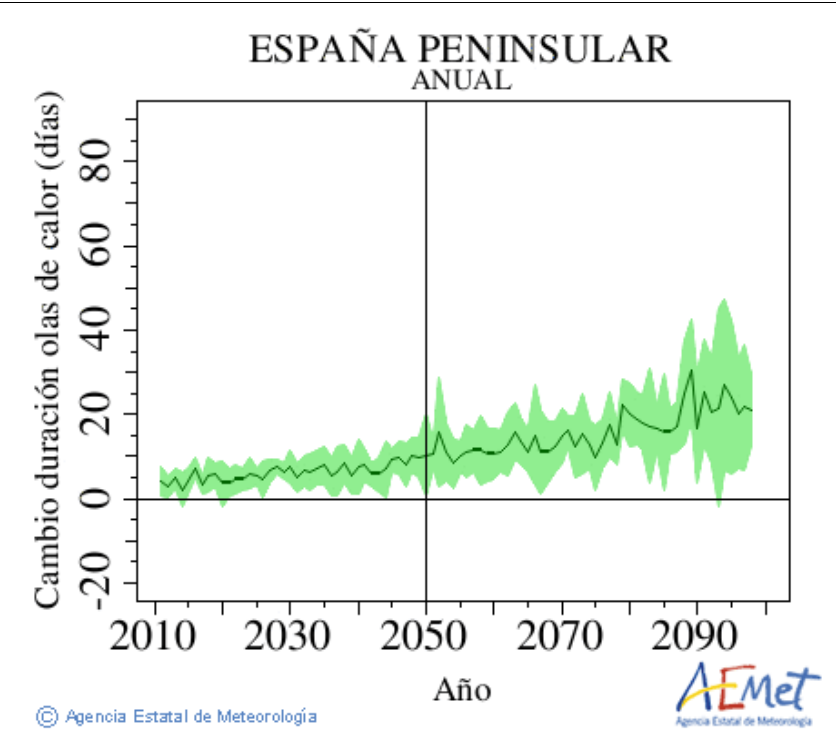

c)

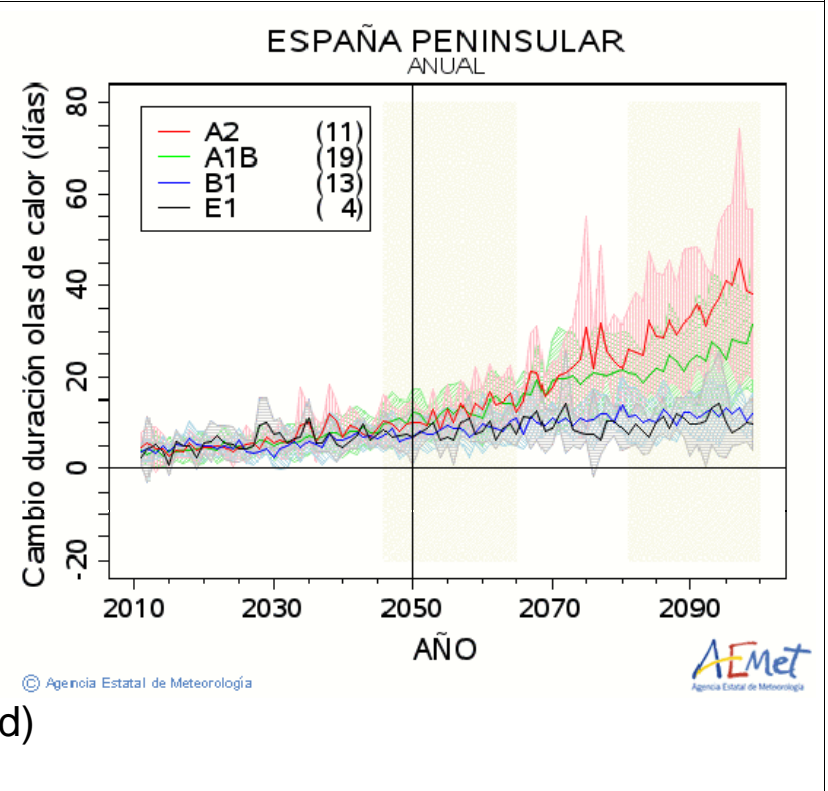

Figura 3.3. Cambios del valor medio anual de los índices extremos DOC (a)-(b) y DC (c)-(d) en la España peninsular para el siglo XXI obtenidos mediante técnicas de regionalización dinámica (izquierda) y estadística (derecha) Entre paréntesis se recoge el número de proyecciones realizadas para cada escenario. En línea continua (sombreada) se representa el valor promedio (incertidumbre). En (b) las franjas verticales sombreadas indican las ventanas temporales en las que el número de regionalizaciones es máximo. 


\subsection{Cambios en la temperatura mínima}

\subsubsection{Valores medios de temperatura mínima}

Los dos métodos de regionalización empleados presentan resultados coherentes entre sí. En general, se observa tendencia creciente del valor medio de la temperatura mínima anual que se mantiene constante a lo largo de todo el período (Figura 3.4a y b). Los resultados obtenidos a partir de técnicas dinámicas muestran un incremento de la temperatura más acusado que los obtenidos a partir de técnicas estadísticas, incluso para el mismo escenario de emisiones A1B. Asimismo, a partir de 2050, los escenarios con mayores concentraciones de GEI (A2 y A1B) dan cuenta de incrementos de temperatura mayores que los escenarios B1 y E1 cuyas series temporales mantienen una pendiente menos pronunciada que las anteriores (Figura 3.4b).

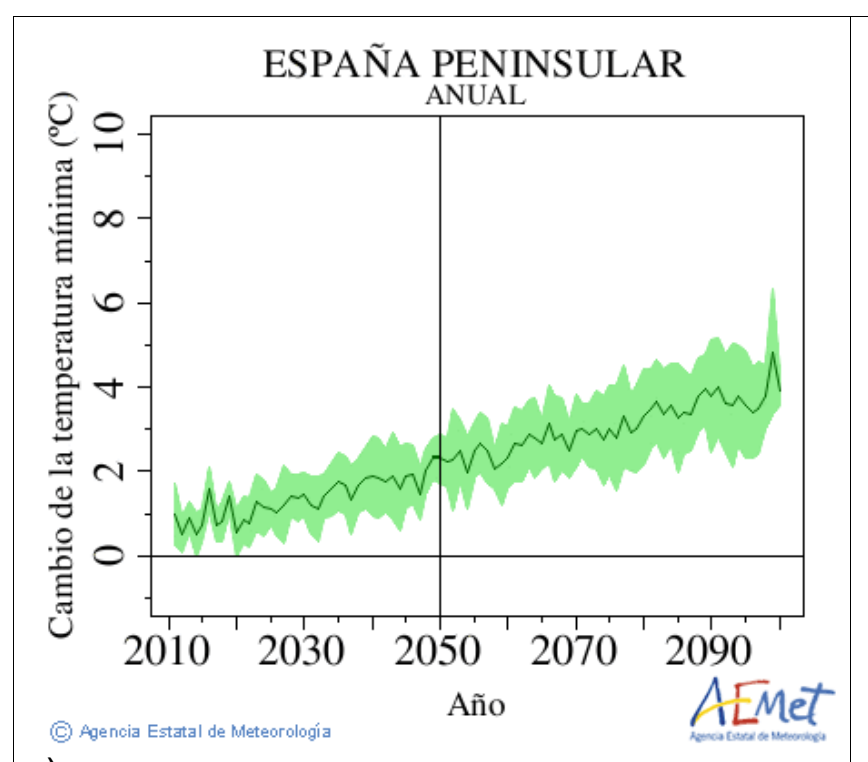

a)

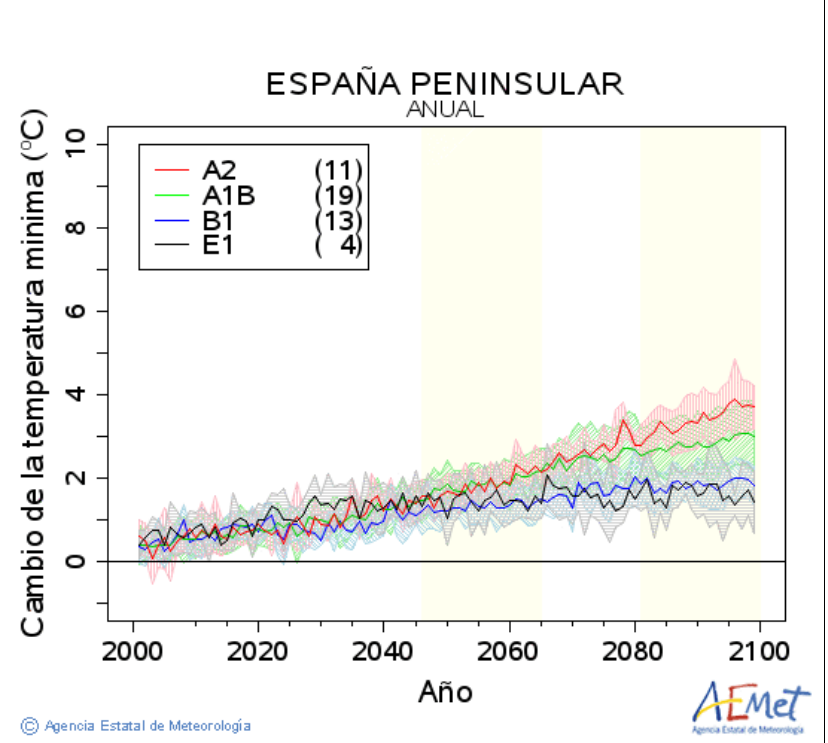

b)

Figura 3.4. Igual que la Figura 3.1 para la temperatura mínima $\left({ }^{\circ} \mathrm{C}\right)$.

La distribución espacial de los cambios en la temperatura mínima media anual correspondiente al escenario de emisiones A1B, muestra valores muy similares para toda el área estudiada (Figura 3.5a y b). Se identifica un aumento menos pronunciado en el norte peninsular, y más acentuado en el centro de Castilla-La Mancha y noreste de Andalucía (Figura 3.5a). Los valores de incertidumbre asociados muestran mayor variabilidad espacial que los valores medios, con máximos en el centro de la Península (Figura 3.5b). 


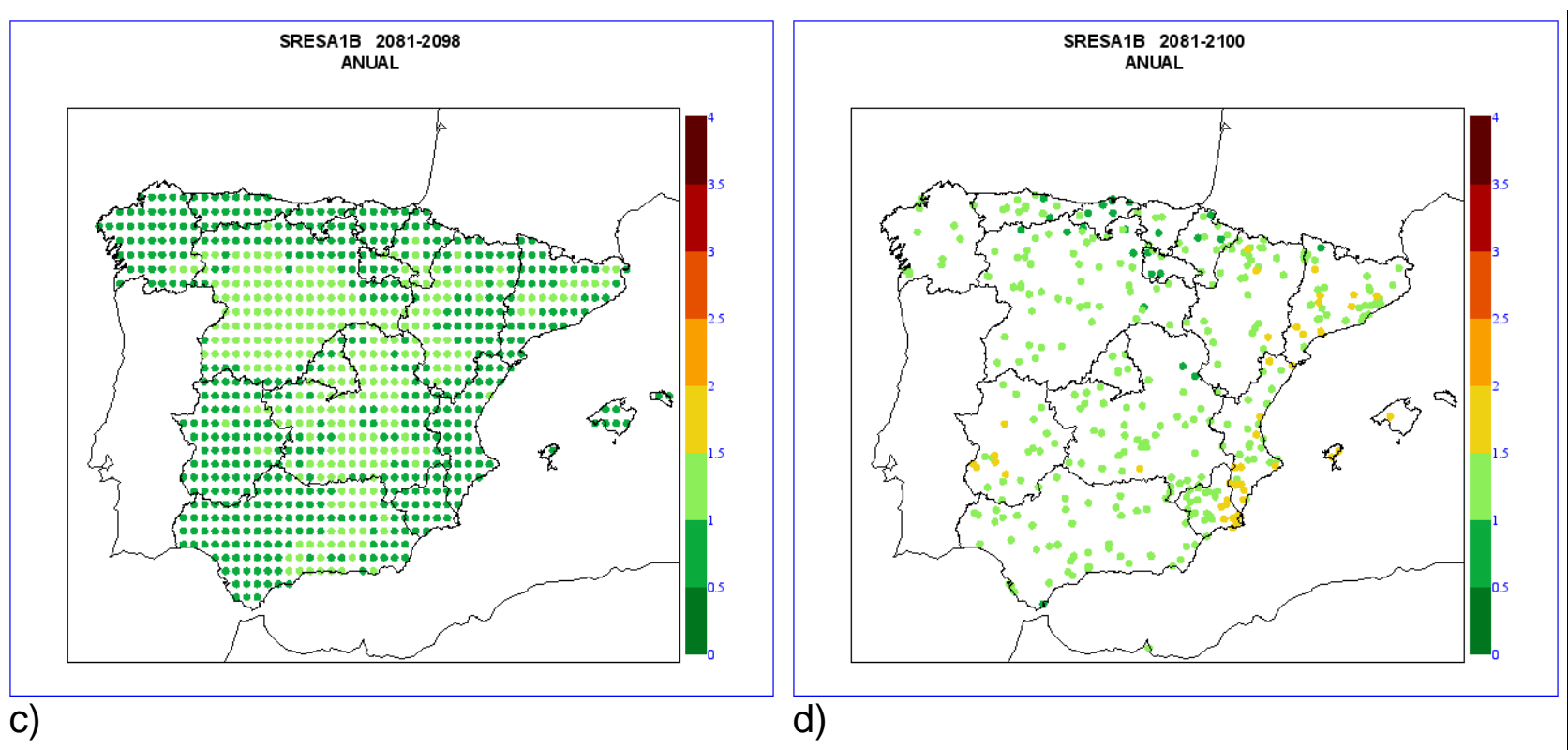

Figura 3.5. Igual que la Figura 3.2 para la temperatura mínima $\left({ }^{\circ} \mathrm{C}\right)$.

El estudio por CCAA muestra que el cambio en la evolución de la temperatura media es ligeramente más suave en el norte peninsular; el incremento se mantiene casi constante a lo largo de cada serie (Figura no mostrada). Este resultado es consecuente con el descrito anteriormente y con la evolución media peninsular mostrada en la Figura 3.4a. Asimismo, el resultado es similar al obtenido mediante técnicas estadísticas (no mostrado).

El estudio estacional muestra resultados análogos a los descritos en la sección anterior para la temperatura máxima que nuevamente concuerdan con los obtenidos por Giorgi y Coppola (2007) y Elguindi et al (2013). El incremento esperado de temperatura mínima es mayor en el sur peninsular en los meses de verano (Figuras no mostradas). En las estaciones de primavera y otoño se observa un incremento intermedio en todas las regiones estudiadas. Para Canarias solo se ha llevado a cabo regionalización estadística obteniéndose resultados que no muestran variación estacional (Figuras no mostradas).

\subsection{2 Índices extremos de temperatura mínima}

En esta sección se analizan los cambios en los promedios anuales peninsulares de los índices extremos: número de días de helada (DH) y porcentaje de noches cálidas (NC). La Figura 3.6 muestra la evolución media peninsular de los índices DH y NC, calculados respecto al período de referencia.

El principal resultado que se obtiene es disminución del índice $\mathrm{DH}$ y aumento del NC. El índice DH muestra un comportamiento similar con las dos técnicas de regionalización empleadas y los cuatro escenarios planteados (Figura 3.6a y b). Sin embargo, el índice NC muestra variaciones más acentuadas según el escenario de emisiones (Figura 3.6c y d). Como en casos anteriores, a partir de la década de los 50, las proyecciones realizadas con los escenarios $\mathrm{A} 2$ y $\mathrm{A} 1 \mathrm{~B}$ son las que muestran valores extremos más altos para los alcances más largos. Este comportamiento se produce de manera análoga, en todas las 
regiones (Figuras no mostradas). De nuevo, el estudio observacional para el SXX llevado a cabo por Alexander et al. (2006) obtiene resultados coherentes con los aquí mostrados para las proyecciones del SXXI. Alexander et al. (2006) encuentra que la evolución temporal del número de días fríos (noches cálidas) tiene tendencia estadísticamente significativa decreciente (creciente) en la región de la Península Ibérica.

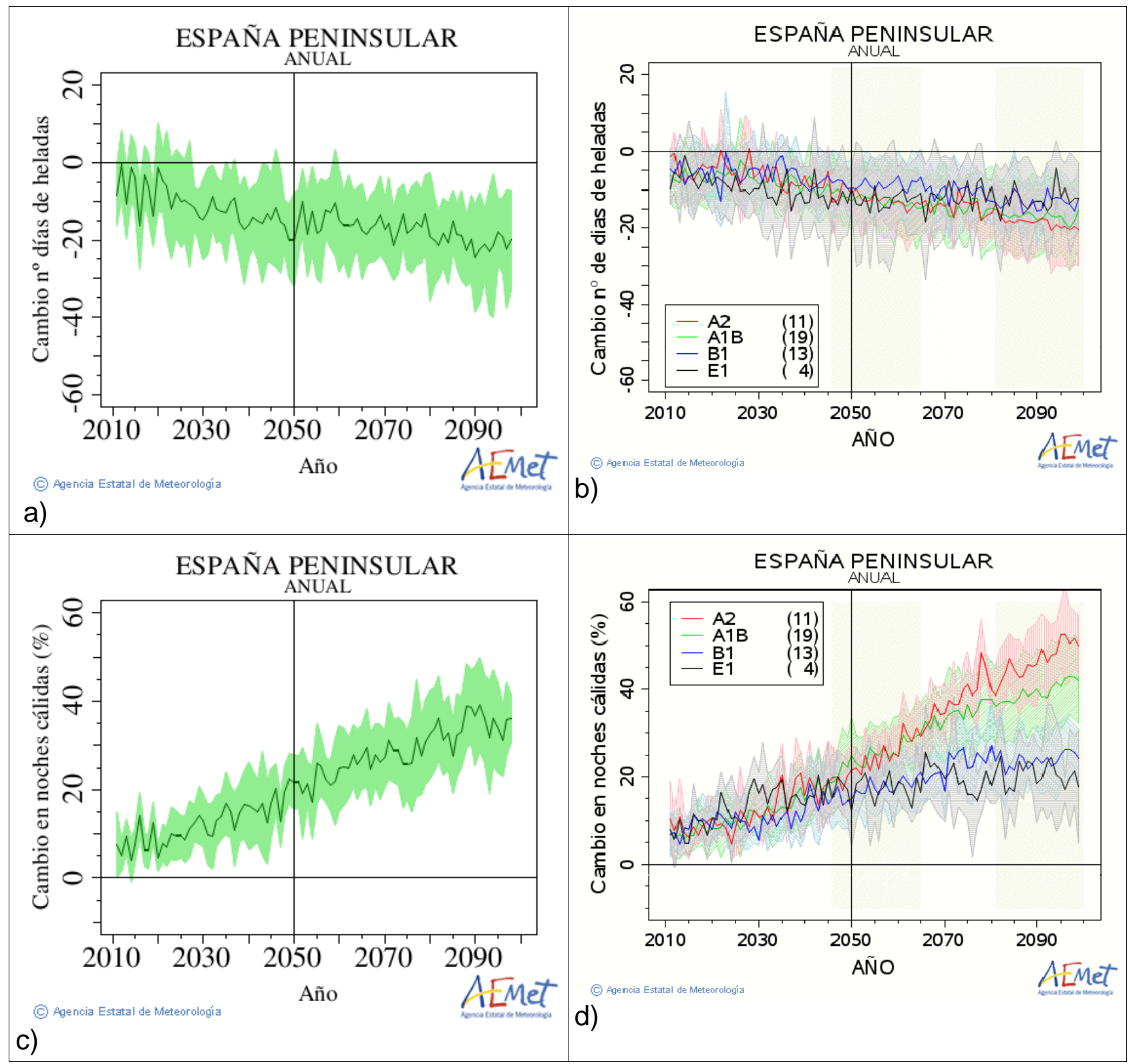

Figura 3.6. Igual que la Figura 3.3 para los índices extremos DH (a)-(b) y NC (c)-(d). 


\subsection{Cambios en la precipitación}

Las series precipitación media han sido sometidas a un algoritmo de media móvil aritmética centrada de 10 años. Esta técnica aplicada a series temporales, permite determinar ciertas características de la serie, tales como patrones estacionarios 0 tendencia, e identificar de manera más clara la señal.

\subsubsection{Valores medios de precipitación}

La Figura 3.7 muestra los cambios esperados de anomalías de precipitación (PCP) media anual promediada para todo el territorio peninsular, expresada en términos porcentuales. En general, se observa disminución la tasa de precipitación. Comparando ambas técnicas de regionalización para el escenario $A 1 B$, se obtiene que los resultados obtenidos mediante técnicas dinámicas (Figura 3.7a) dan cuenta de mayor disminución de precipitación que los obtenidos con técnicas estadísticas (Figura 3.7b). No obstante, la incertidumbre asociada a los resultados estadísticos es notablemente mayor que la obtenida para los resultados dinámicos. Por otro lado, los cuatro escenarios planteados para las regionalizaciones estadísticas ofrecen resultados muy similares; solamente la regionalización correspondiente al escenario E1 (fuerte mitigación) mantiene valores próximos o ligeramente superiores a cero a lo largo de todo período, mientras que el resto de escenarios muestran disminución de la precipitación.
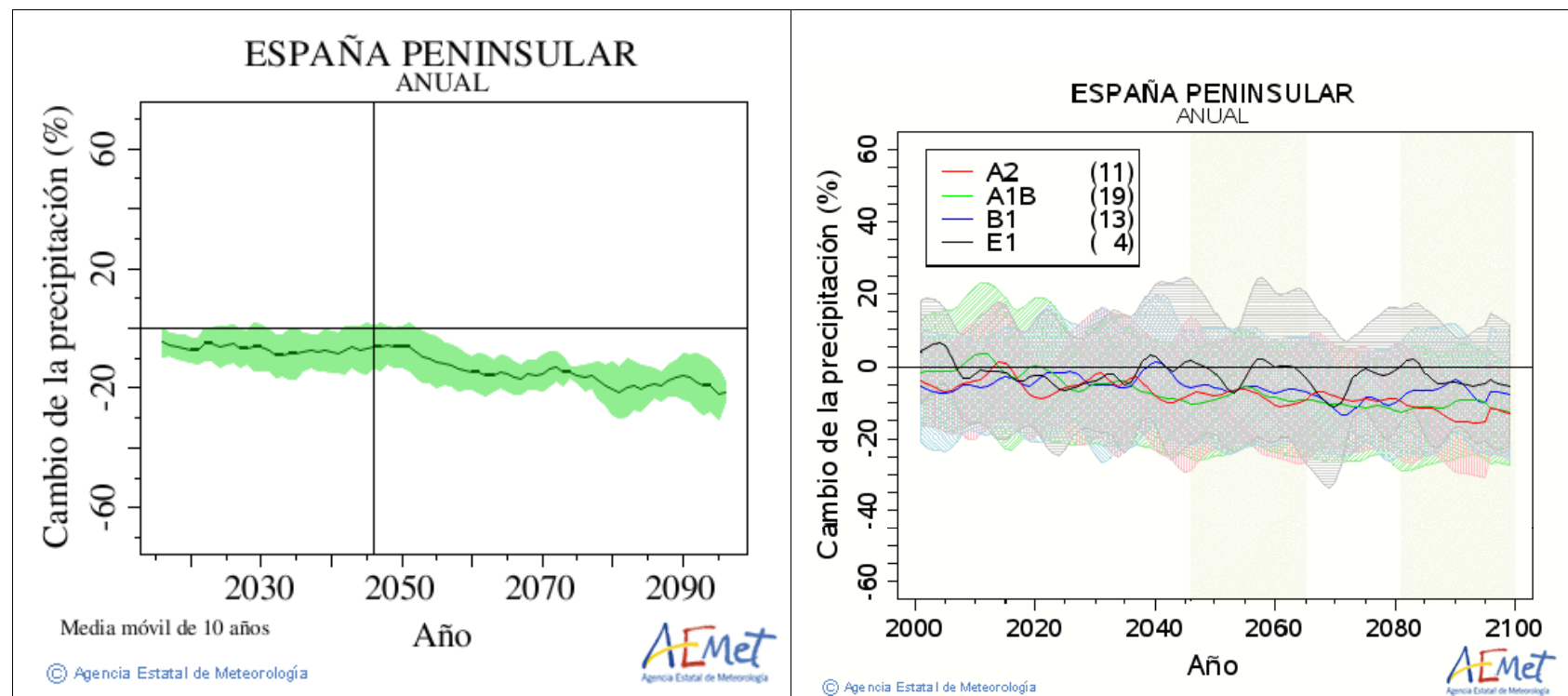

a)

b)

Figura 3.7. Igual que la Figura 3.1 para el valor medio anual de la tasa de PCP filtrada con media móvil de 10 años.

La Figura 3.8 ( $a$ y b) muestra la distribución espacial de los cambios esperados en el valor medio anual de anomalías de precipitación para los últimos años del período, correspondiente al escenario de emisiones medias A1B. En general, en todas las regiones se obtiene un comportamiento coherente con el comportamiento anual estudiado. En la 
mitad norte del área de estudio se identifica disminución de la precipitación media de entre 0-10\%, mientras que en el resto del área la disminución varía entre 10-20\%. Los valores de incertidumbre (Figura 3.8c y d). Los valores de incertidumbre más altos, entre 20-30\% se obtienen en las regiones de Extremadura, Andalucía y Comunidad Valenciana. Para el resto de regiones los valores de incertidumbre varían entre 10-20\%. En Canarias el cambio esperado en la precipitación es más acusado, alcanzando los 25 días anuales (Figuras no mostradas) y variando entre 30 días en invierno y 20 en verano.
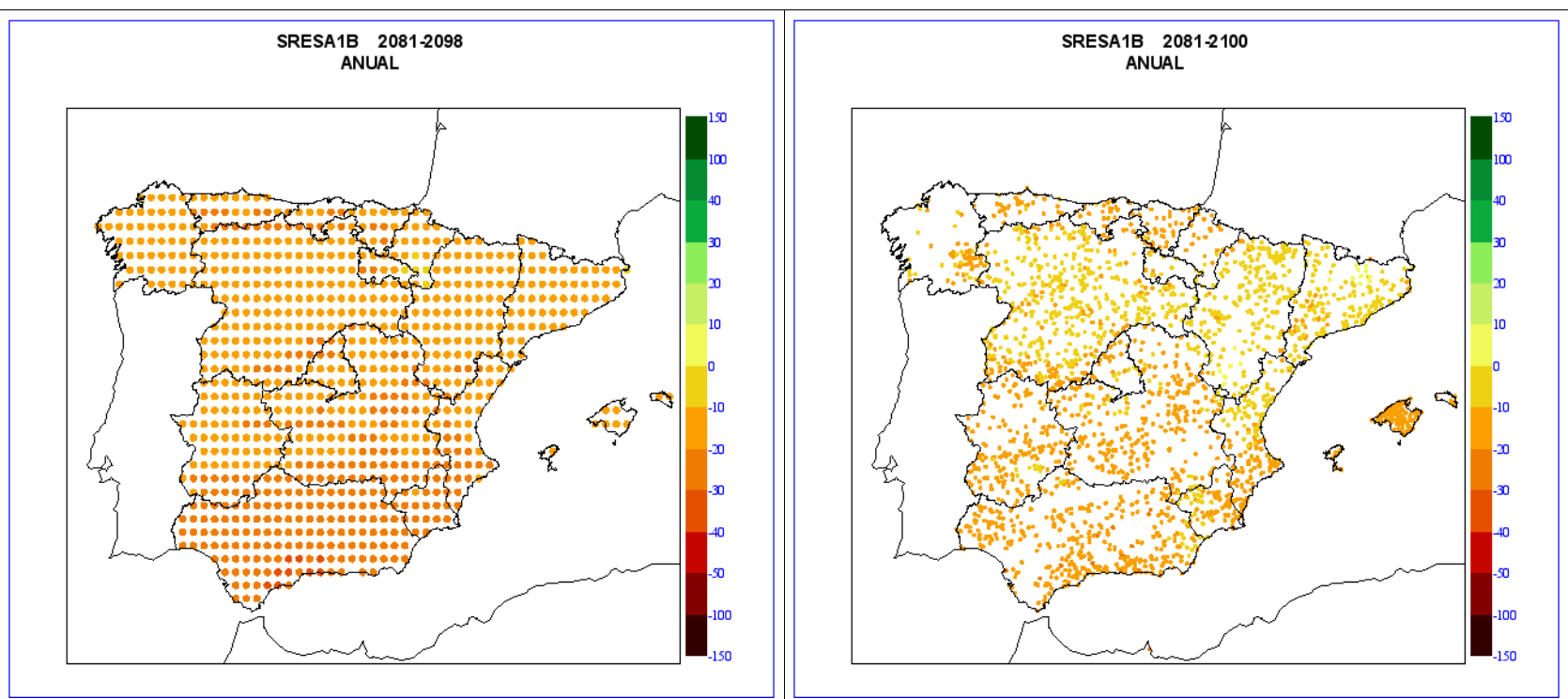

a)

b)

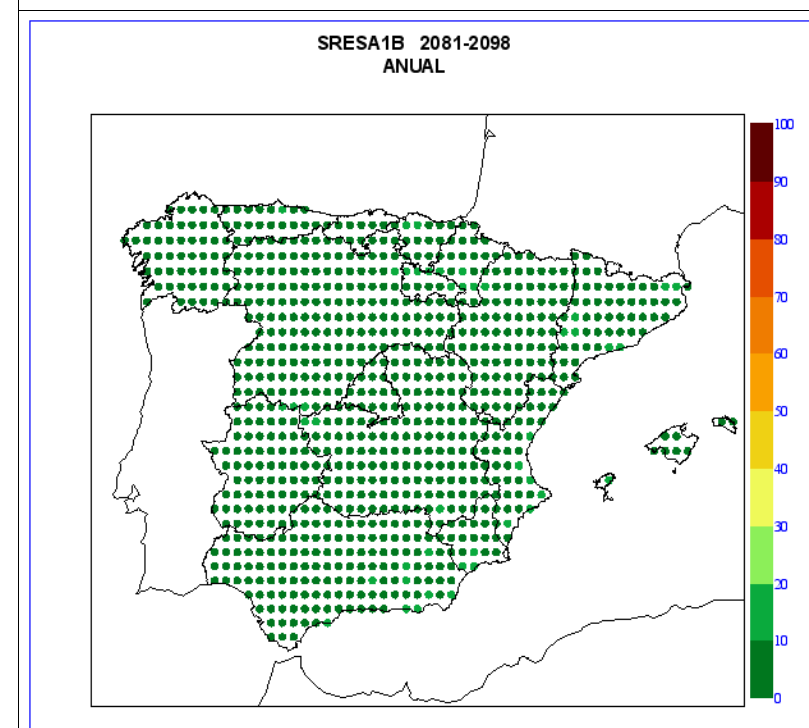

c)

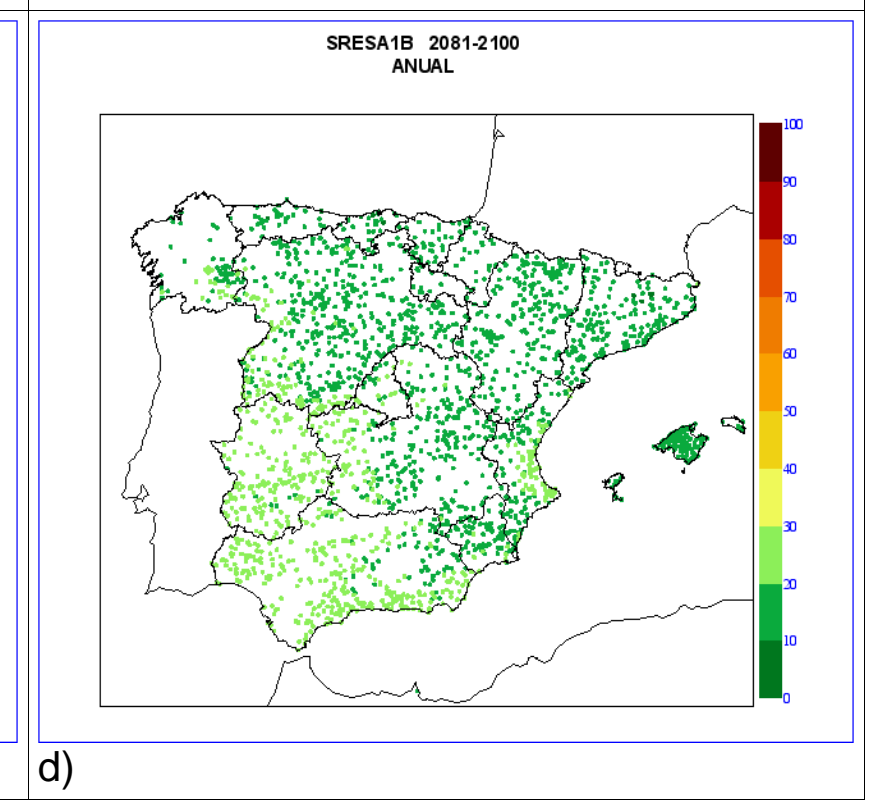

Figura 3.8. Igual que la Figura 3.2 para la PCP media $(\mathrm{mm})$.

Por CCAA, los cambios en la precipitación media correspondiente a las regionalizaciones dinámicas, es similar en todas las series (no mostradas). Aunque los valores promedio disminuyen en igual magnitud en todas las regiones, se aprecian mayores valores de incertidumbre en las regiones de Extremadura, Andalucía y Comunidad Valenciana. 
El estudio estacional no refleja comportamiento espacial homogéneo (Figuras no mostradas). Por otro lado, la evolución de la tasa de precipitación muestra mayor disminución en verano que en el resto de estaciones (Figura 3.9); en primavera y otoño se aprecia una disminución notable aunque menos extrema. No obstante, se observa que los mayores valores de incertidumbre, de alrededor del $40-50 \%$, corresponden a invierno y verano. Hertig et al. (2013) es su estudio para el campo de precipitación sobre la región mediterránea confirma éstos resultados.
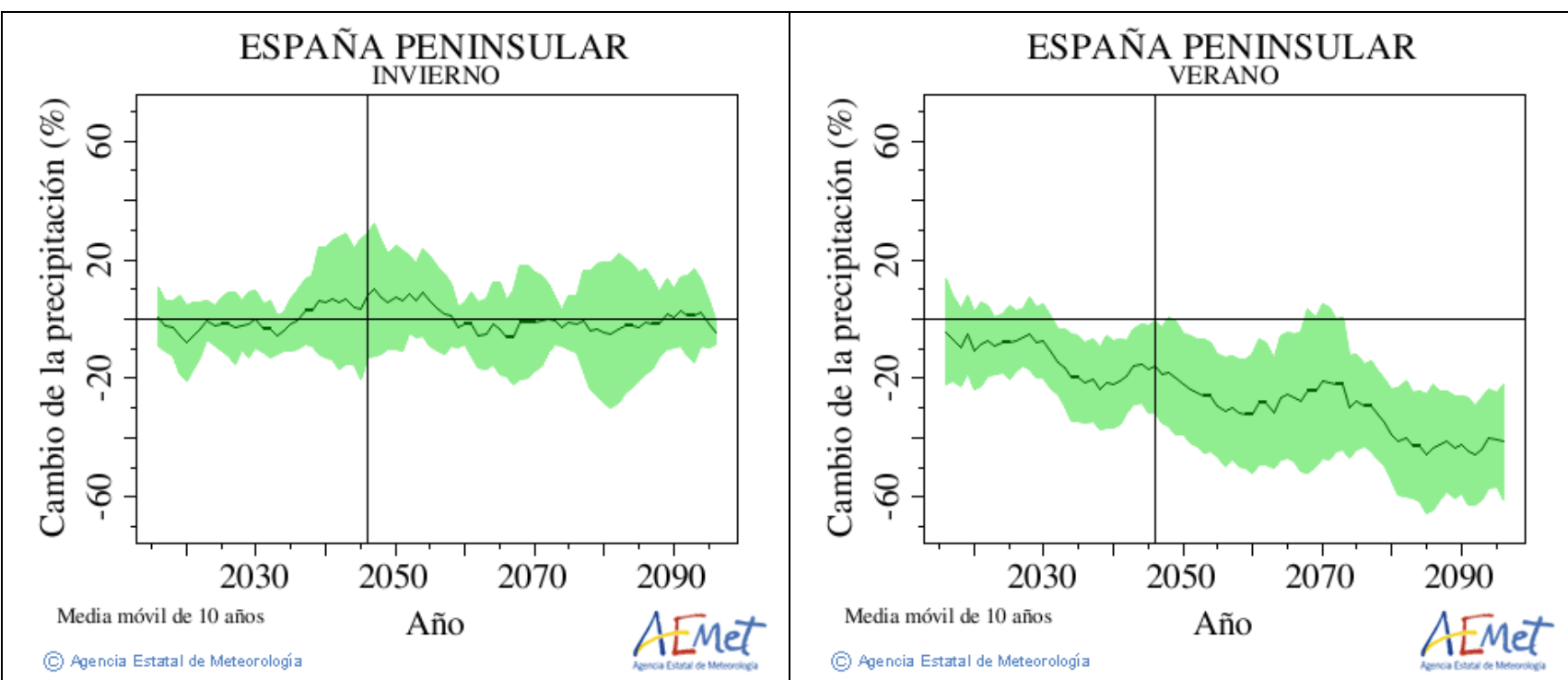

a)

b)
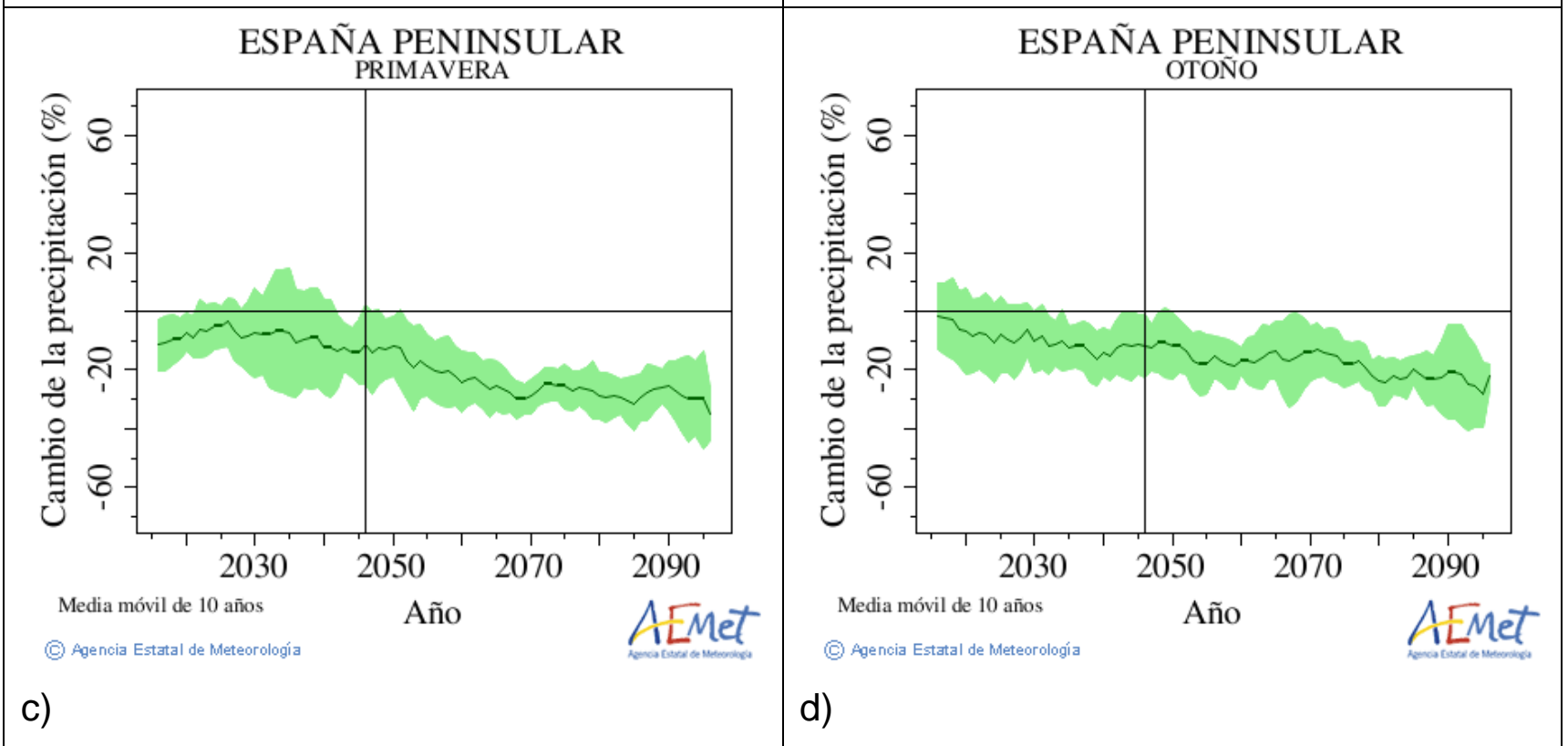

c)

Figura 3.9. Cambios en el valor medio estacional de la tasa de PCP media, filtrada con media móvil de 10 años, en la España peninsular para el siglo XXI obtenida mediante técnicas de regionalización dinámica para el escenario A1B. (a) Invierno; (b) verano; (c) primavera y (d) otoño. En línea continua (sombreada) se representa el valor promedio (incertidumbre). 


\subsection{2 Índices extremos de precipitación}

En esta sección se analizan los cambios en los promedios anuales peninsulares de los índices extremos asociados al campo de precipitación definidos en el capítulo anterior. Antes de comenzar la descripción de los resultados, conviene tener en cuenta los resultados de diversos autores que obtienen similares a los que a continuación se muestran, es decir, aumento en intensidad y frecuencia de precipitaciones extremas en regiones donde la precipitación media decrece (Costa et al., 2012; Hertig et al., 2013).

Los índices previamente escogidos para esta sección son los siguientes: número de días de lluvia (DL); duración de períodos secos (PS) y tasa de ocurrencia de precipitaciones intensas (PI). La Figura 3.10 muestra los cambios en los promedios anuales peninsulares de los índices DL, PS y PI para las proyecciones dinámicas y estadísticas. El resultado obtenido para el valor medio de cada índice es análogo para todas las zonas geográficas, y consistente con el comportamiento de las series promediadas espacialmente. 


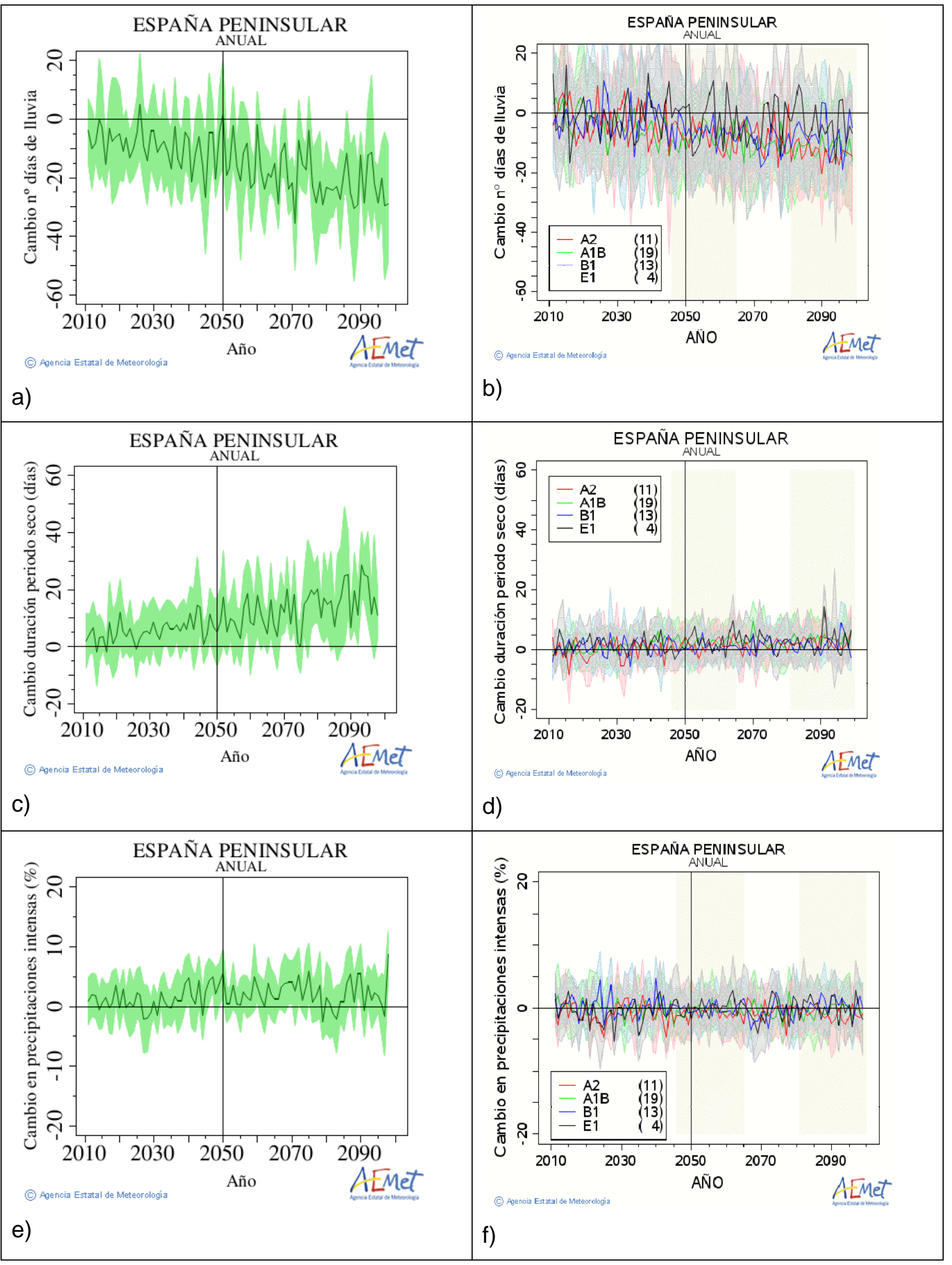

Figura 3.10. Igual que la Figura 3.3 para los índices extremos DL (a)-(b), PS (c)-(d) y PI (e)-(f). 
El índice DL muestra disminución en todas las regiones (Figura no mostrada). Como se ha comentado, la Figura 3.10 resume el comportamiento global de cada índice. Los resultados dinámicos muestran valores por debajo de cero durante todo el período, así como variabilidad decenal bastante clara (Figura 3.10a). La Figura 3.10b muestra los resultados obtenidos mediante técnicas estadísticas; se aprecia disminución menos clara de este índice. Para la mayor parte de los escenarios, los valores se mantienen próximos a cero, únicamente la regionalización correspondiente al escenario A2 presenta valores por debajo de cero durante la mayor parte del período. Los valores de incertidumbre son en general, similares para todos los escenarios; los valores más altos se localizan en las regiones del norte y centro peninsular, mientras que en las regiones del sur y levante son sensiblemente menores (no mostrado). En el contexto de la Península Ibérica, Costa et al. (2012) obtienen el mismo resultado para el SXXI mientras que Gallego et al. (2011) lo constata para el SXX.

Por otra parte, la duración de los períodos secos se encuentra lógicamente relacionada con el descenso del número de días de lluvia. El índice PS muestra tendencia creciente, en el caso de regionalización dinámica (Figura 3.10c). Es decir, la duración de los períodos secos tiende a ser más larga con el aumento del alcance de la proyección. Este resultado es coherente con el obtenido para DL para el que se aprecia disminución del número de días de lluvia y por tanto, se puede esperar mayor duración de los períodos secos lo que aumentaría el riesgo de sequías. Sillmann y Roeckner (2008) obtienen el mismo resultado para toda la región del sur europeo. En el estudio concluyen con incrementos de en torno a un mes en la longitud de períodos secos para el escenario A1B a finales del SXXI. Los resultados de las regionalizaciones estadísticas son menos claros; aparentemente no se observa tendencia; para todos los escenarios planteados se obtienen resultados similares (Figura 3.10d). La distribución espacial de los valores de incertidumbre asociada al índice PS muestra diferencias más notables que en el caso anterior. En este caso, los valores más altos de incertidumbre se localizan en el sur y levante peninsular para los últimos años de la proyección (Figura no mostrada). Por otro lado, el ciclo estacional podría hacerse más patente ya que se aprecia un aumento de PS mayor en verano y primavera que en invierno y otoño. No obstante, los valores de incertidumbre se encuentran directamente relacionados con el valor medio correspondiente, es decir, en las estaciones de verano y primavera la incertidumbre es apreciablemente mayor que en invierno y otoño (Figuras no mostradas).

Por último, se han estudiado los cambios esperados en el índice asociado a la frecuencia de ocurrencia de precipitaciones intensas, PI. La Figura 3.10 (e y f) muestra la evolución anual del índice PI promediada espacialmente. En el caso de regionalizaciones dinámicas la mayor parte de los valores se encuentran por encima de cero, es decir, se espera un aumento de la tasa de precipitaciones intensas, aunque sin tendencia aparente. Sin embargo con las regionalizaciones estadísticas los valores se encuentran en torno a cero aunque con mayor magnitud los de sentido positivo. Los valores de incertidumbre mayores se encuentran en las regiones del sur y levante. Por otro lado, no se observan cambios destacables en el ciclo estacional (Figuras no mostradas).

Los resultados aquí mostrados son consistentes con los obtenidos por Sillmann y Roeckner (2008) para la región sur de Europa. Por otro lado, Alexander et al. (2006) encuentra tendencias con significación estadística, similares a las de las proyecciones aquí mostradas para los índices extremos de PCP para el SXX. Sin embargo, todos los 
resultados mostrados son difíciles de cuantificar y su significación puede verse afectada debido a la naturaleza no gausiana de la precipitación.

\subsection{Cambios en el viento a 10 metros}

En esta sección se aborda el estudio del comportamiento del viento a 10 metros, tanto la velocidad como su variabilidad. Asimismo, se analiza el viento extremo a través del estudio de las rachas máximas diarias. Las variables que se analizan son las siguientes: valor medio de las componentes zonal (U10) y meridiana (V10), velocidad media (W10) y racha máxima diaria (R10).

La Figura 3.11 ( $\mathrm{a}$ y b) muestra los cambios esperados en U10 y V10 respectivamente, promediados anualmente para todo el territorio peninsular. Como puede observarse, ambas series muestran oscilaciones muy similares. La componente U10 muestra valores ligeramente por debajo de cero y disminución de la velocidad de la componente zonal del viento respecto al período de referencia estudiado. Por otra parte, los valores correspondientes a V10 oscilan en torno a cero, lo que supone poca o ninguna variación de la componente meridional respecto al período de control. La velocidad neta del viento W10 muestra variaciones muy próximas a cero y de menor magnitud que las componentes U10 y V10 (Figura 3.11c). Asimismo, la intensidad de las rachas máximas varía de manera desigual a lo largo del período esperándose los valores más bajos en la segunda mitad del SXXI. Diversos estudios confirman estos resultados (Yan et al., 2002; Pryor and Barthelmie, 2003; Pryor et al., 2005a; Pryor et al., 2005b). Por otra parte, analizando las simulaciones de cada modelo independientemente, se observan discrepancias entre los resultados obtenidos. En general, aunque en todas ellas se obtienen valores más altos y mayor frecuencia de vientos extremos a final de siglo, también se encuentran las mayores diferencias entre los resultados de cada modelo (Nikulin et al., 2011). Estas discrepancias en magnitud, frecuencia y distribución espacial se deben fundamentalmente las condiciones en las que cada modelos simula la circulación a gran escala sobre el Atlántico Norte y Europa (Räisänen et al., 2003; Leckebusch et al., 2006; Beniston et al., 2007; Rockel and Woth, 2007). 


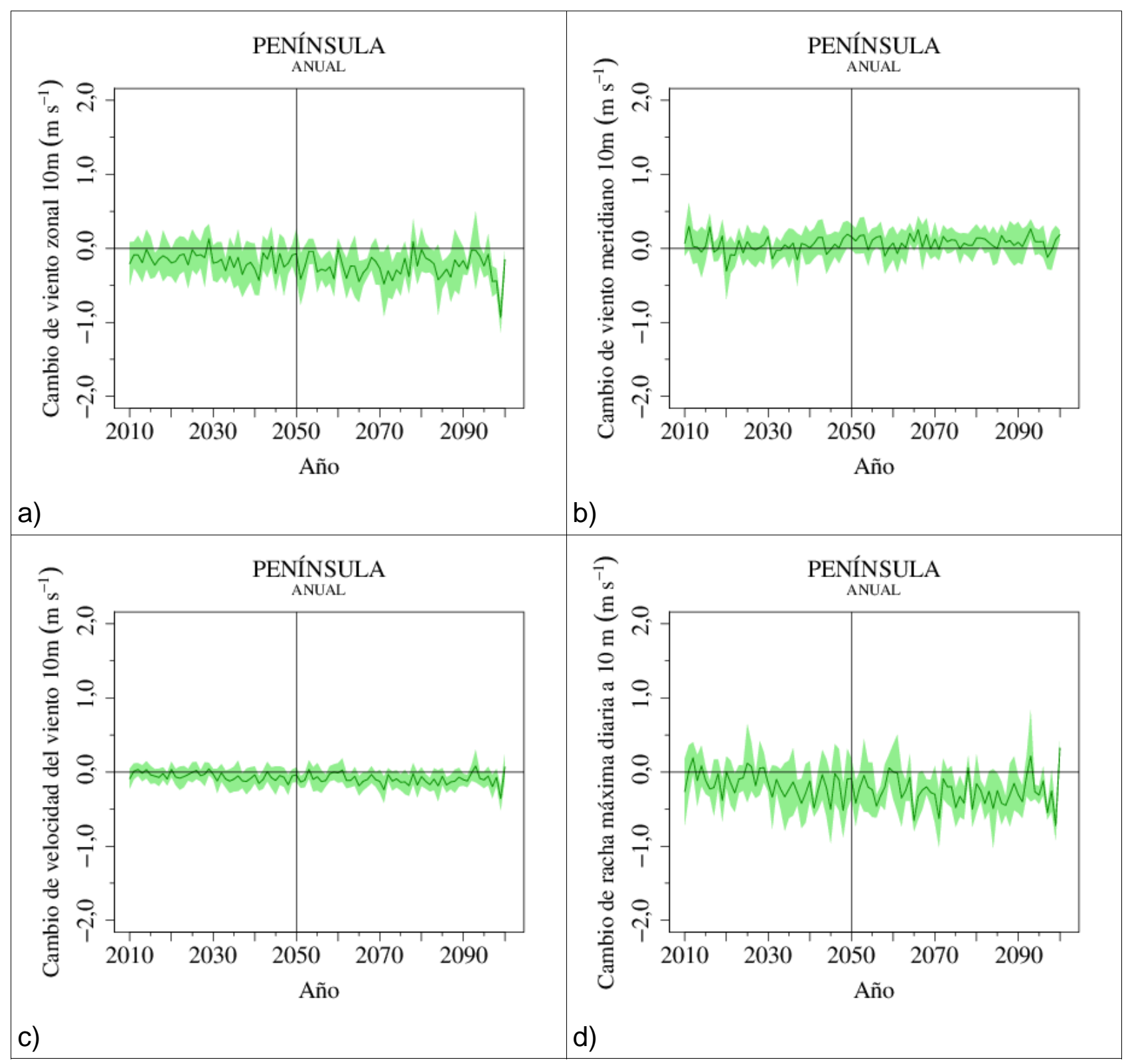

Figura 3.11. Cambios en el valor medio anual en España peninsular obtenida mediante técnicas de regionalización dinámica de: (a) componente U10 (m s$\left.~^{-1}\right)$; (b) componente V10 ( $\left.\mathrm{m} \mathrm{s}^{-1}\right)$; (c) velocidad W10 ( $\left.\mathrm{m} \mathrm{s}^{-1}\right)$; (d) racha máxima R10 $\left(\mathrm{m} \mathrm{s}^{-1}\right)$. En línea continua (área sombreada) se representa el valor promedio (incertidumbre).

La Figura 3.12 muestra la distribución espacial de los cambios en los valores medios anuales esperados para final del SXXI de velocidad del viento neta, por componentes zonal y meridional y racha máxima. Es importante destacar que aunque los patrones espaciales obtenidos por las simulaciones individuales no muestran coherencia entre sí, el patrón correspondiente a la media de las variaciones esperadas de las anomalías de velocidad del viento del conjunto de las simulaciones, muestra debilitamiento en todo el territorio estudiado. Este resultado es coherente con los encontrados por otros autores (Nikulin et al., 2011; Pryor et al., 2012). Nikulin et al. (2011) encuentra que en latitudes superiores a $45^{\circ} \mathrm{N}$ existe tendencia significativa al aumento de la velocidad neta mientras que en las regiones situadas al sur de esta latitud lo que se espera es debilitamiento significativo. 


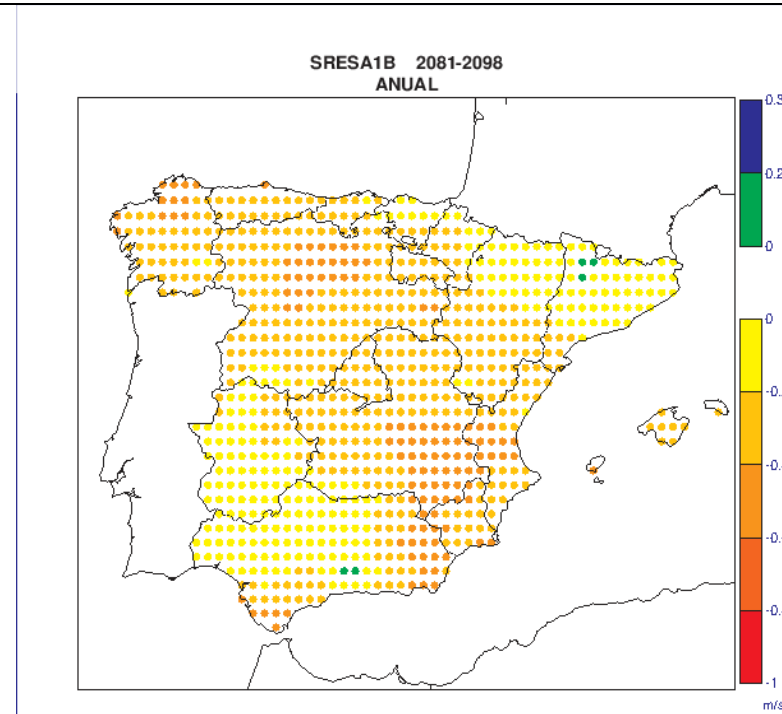

a)

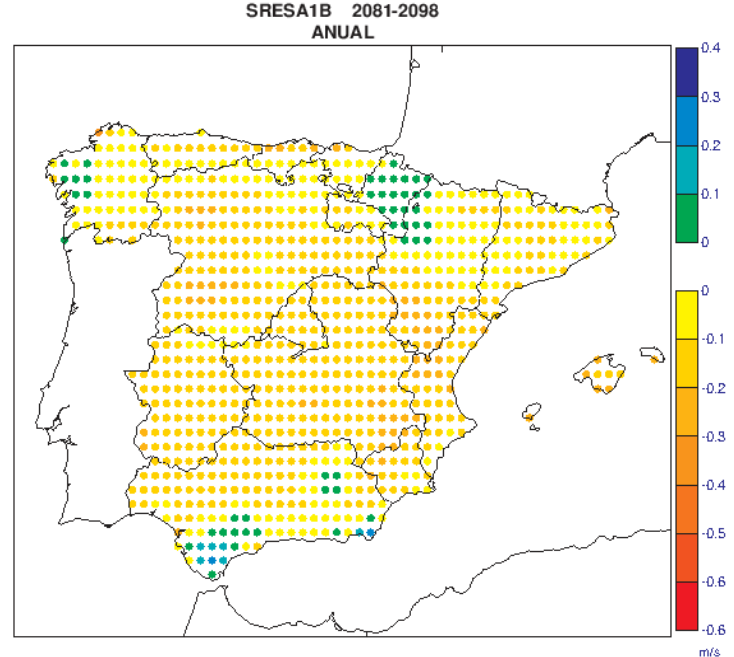

c)

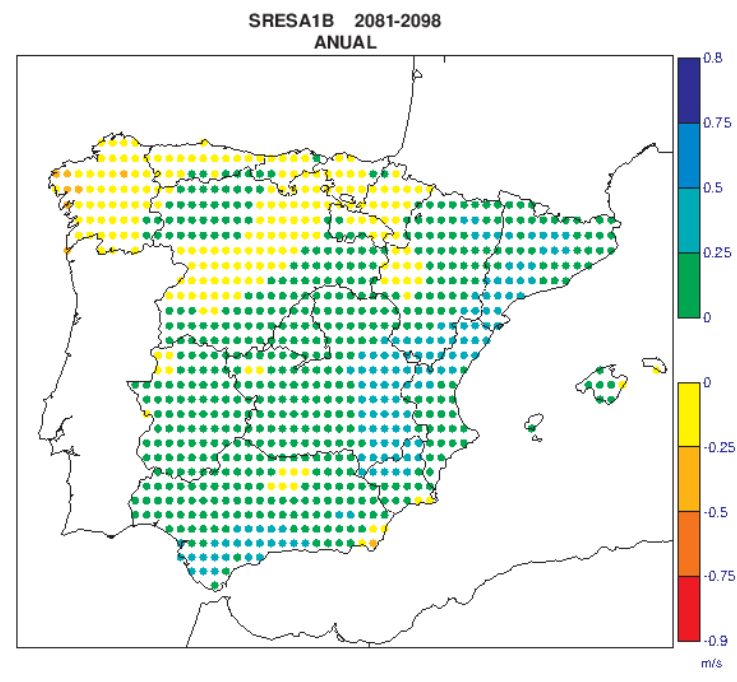

b)

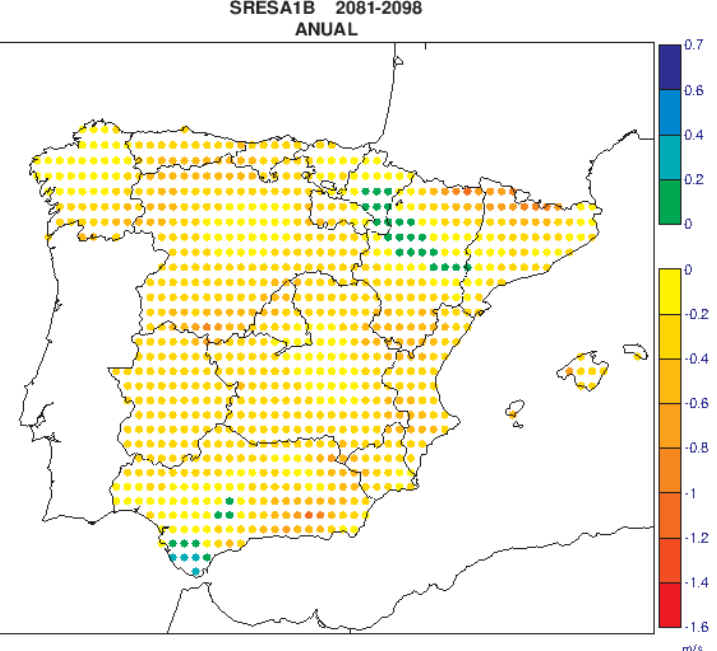

d)

Figura 3.12. Distribución espacial del valor medio anual de anomalías obtenidas mediante técnicas de regionalización dinámica del escenario A1B para el período 2081-2098 de: (a) componente U10 (m s $)^{-1}$; (b) componente V10 ( $\left.\mathrm{m} \mathrm{s}^{-1}\right)$; (c) velocidad W10 (m s$\left.)^{-1}\right)$; (d) racha máxima $\mathrm{R} 10\left(\mathrm{~m} \mathrm{~s}^{-1}\right)$.

En el estudio por CCAA el resultado es el siguiente: Tanto los valores de W10 como los de R10 muestran comportamiento decreciente en casi todas las CCAA ligeramente marcado en la segunda mitad del SXXI. Por otro lado, los valores de R10 muestran en general, mayor variabilidad e incertidumbre que los de W10.

La evolución estacional muestra coherencia entre los campos estudiados. Las Figuras 3.13 y 3.14 muestran la variabilidad estacional de las variables W10 y R10, respectivamente. En ambos casos puede observarse que el patrón correspondiente a la media de los cambios en la velocidad del viento del conjunto de las simulaciones, muestra 
debilitamiento en todo el territorio estudiado. El estudio de W10 por componentes muestra valores similares en signo y magnitud de U10 y V10 (Figuras no mostradas).
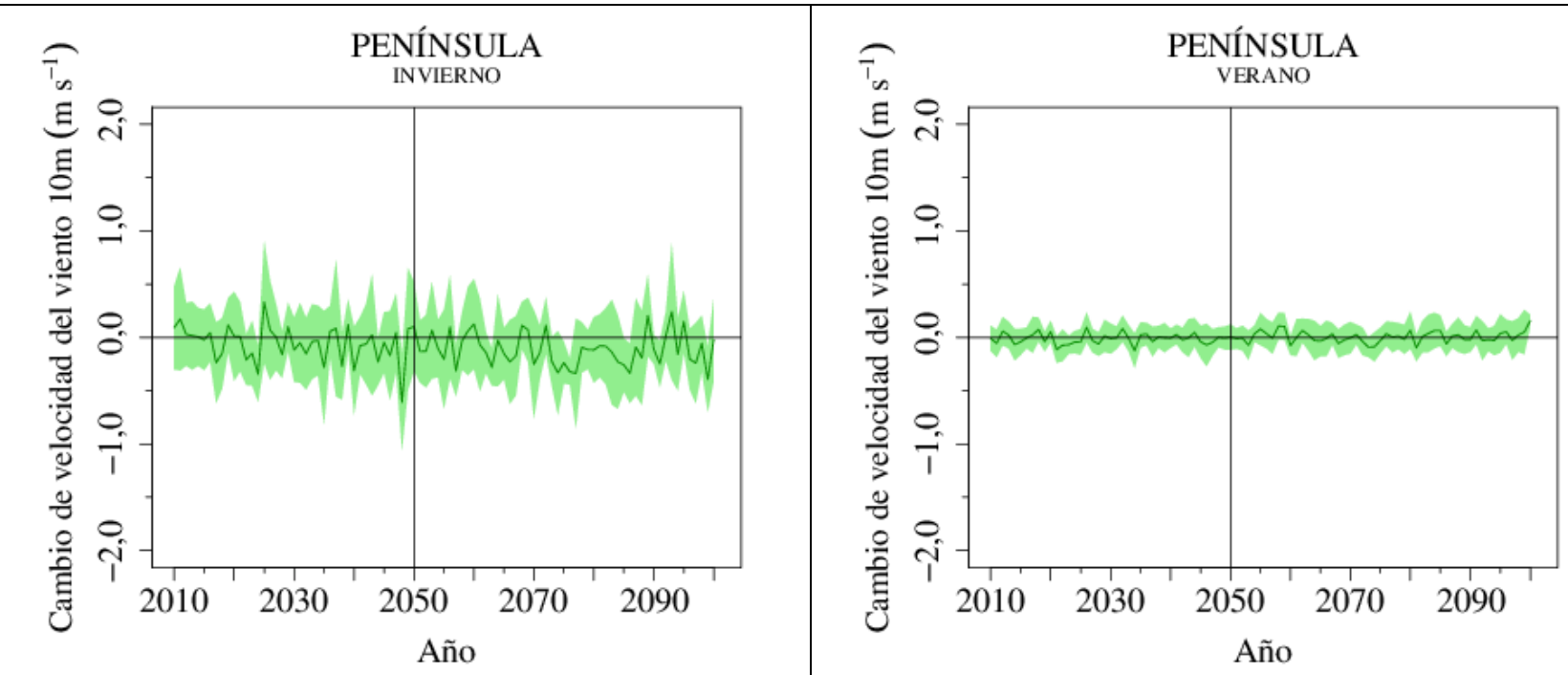

a)

b)

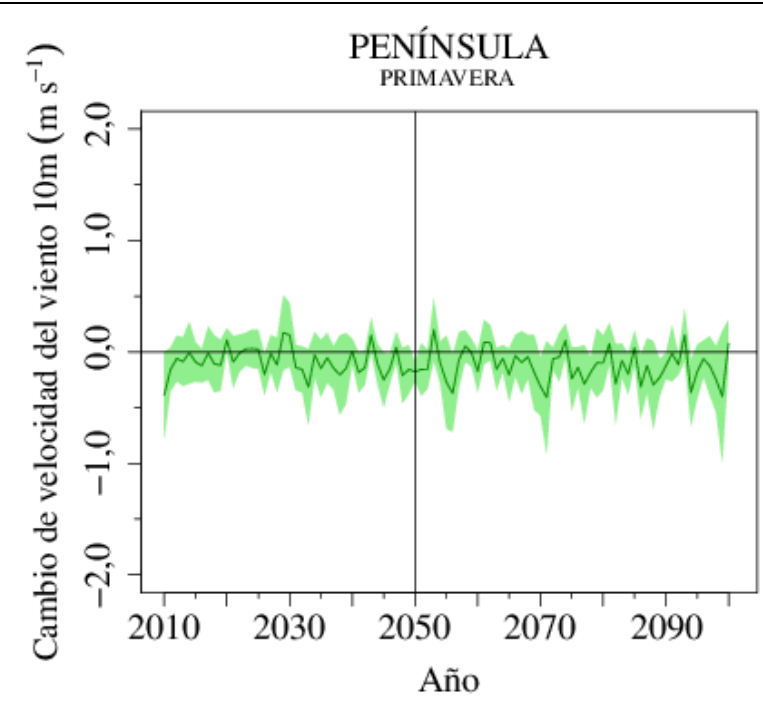

c)

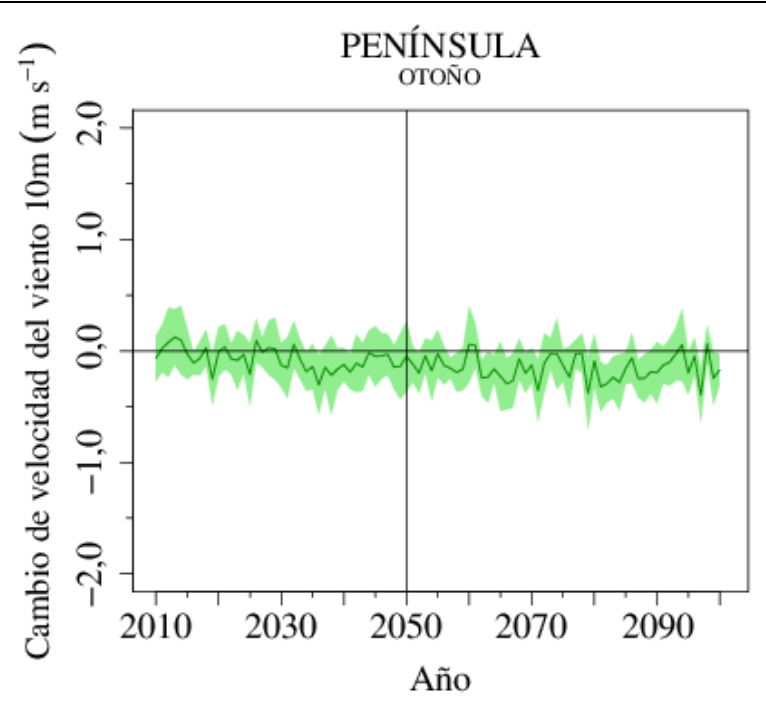

d)

Figura 3.13. Igual que la Figura 3.9 para W10. 


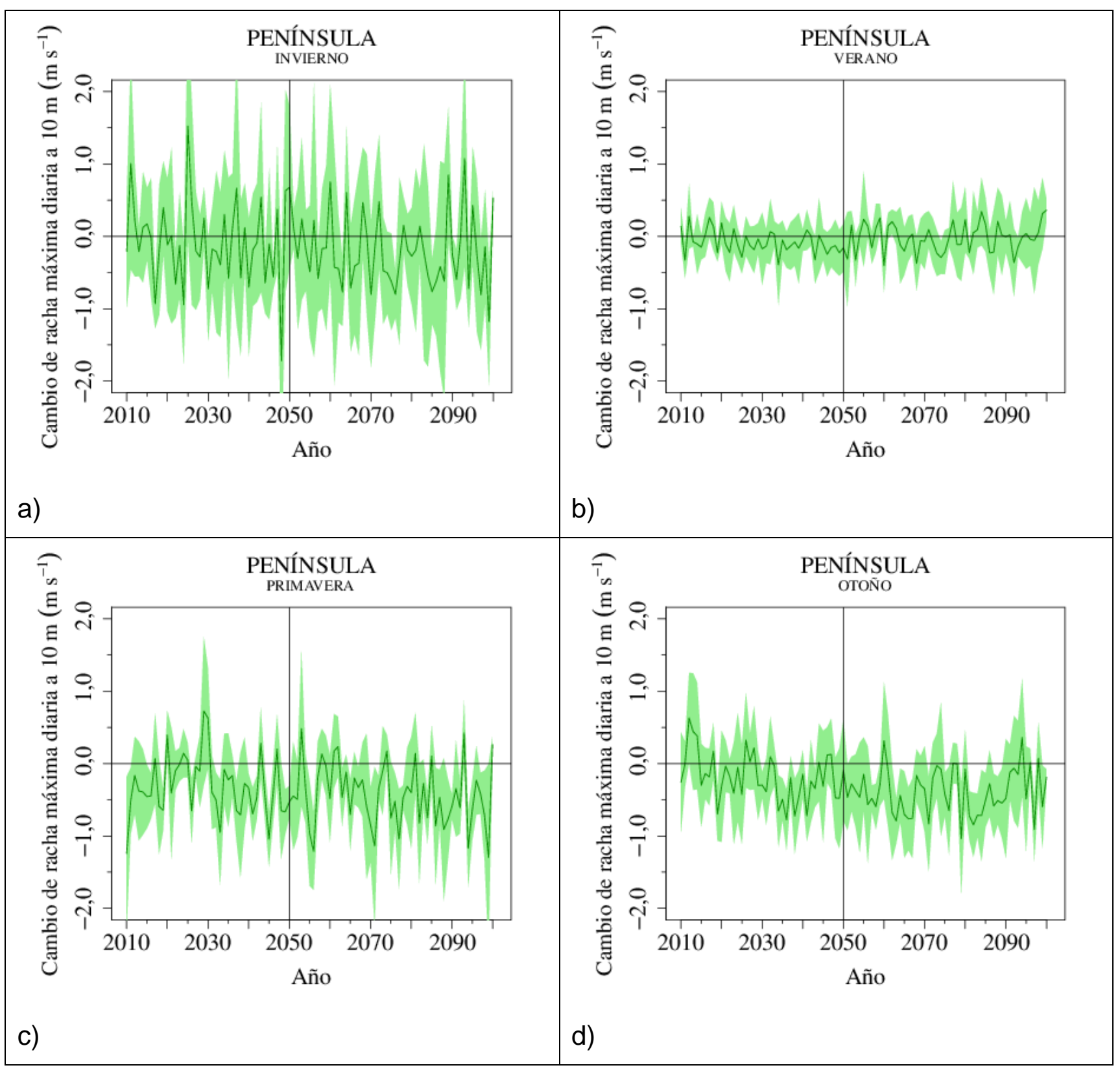

Figura 3.14. Igual que la Figura 3.9 para R10.

La modesta magnitud de los cambios esperados obtenidos por las proyecciones realizadas en intensidad del viento extremo y velocidad media es consistente con estudios realizados por otros autores que obtienen cambios similares en sentido y magnitud, con valores próximos a cero (Knote et al., 2010; Nikulin et al., 2011; Pryor et al., 2012). En general, los resultados obtenidos de estudios de cambio climático para las variables de viento son poco concluyentes. Estos resultados son el reflejo de que la señal de cambio climático no es clara para el viento en superficie, ya que la variabilidad que muestran estas variables es notablemente mayor que las tendencias (Weisse et al., 2005; Trenberth et al., 2007; Pryor et al., 2009; Pryor and Ledolter, 2010; Bärring and Fortuniak, 2009; Della-Marta et al., 2009; Pryor et al., 2012) 


\subsection{Cambios en la escorrentía y la evapotranspiración real}

El aumento de la concentración de GEl, además de influir sobre las variables atmosféricas, tiene efectos sobre el ciclo hidrológico y la fisiología de las plantas. La evapotranspiración es el resultado conjunto de la evaporación y la transpiración de las plantas y esta limitada por los factores atmosféricos y radiativos así como por el tipo de cultivo y la disponibilidad de humedad del suelo. Por otro lado, el contenido de agua del suelo se encuentra directamente vinculado a la cantidad de precipitación y la escorrentía. La escorrentía total depende de varios factores tales como las características geológicas del suelo, la vegetación y ocupación del mismo y el contenido de humedad en el momento de producirse la precipitación. Este último factor depende fundamentalmente de precipitaciones previas, temperatura y evapotranspiración. Así pues, el aumento de la concentración de $\mathrm{CO}_{2}$ influye directamente sobre las variables que participan en el ciclo del agua, que a su vez están interrelacionadas.

En esta sección se estudian en conjunto los cambios en la escorrentía total (Q) y la evapotranspiración real (ETR). Las series temporales han sido sometidas a un algoritmo de media móvil aritmética centrada de 10 años. La Figura 3.15 (a y b) muestra los cambios esperados en las tasas de anomalías de $\mathbf{Q}$ y ETR, respectivamente, promediadas anualmente para todo el territorio peninsular. Como puede observarse, ambas series muestran variabilidad conjunta con oscilaciones decenales. Asimismo, en ambos casos los valores que se observan se sitúan por debajo de cero a lo largo de todo el período, es decir se observa disminución con respecto al período de referencia. Estos resultados son consistentes con los obtenidos por otros autores (Hungate et al., 2002; Long et al., 2006). 


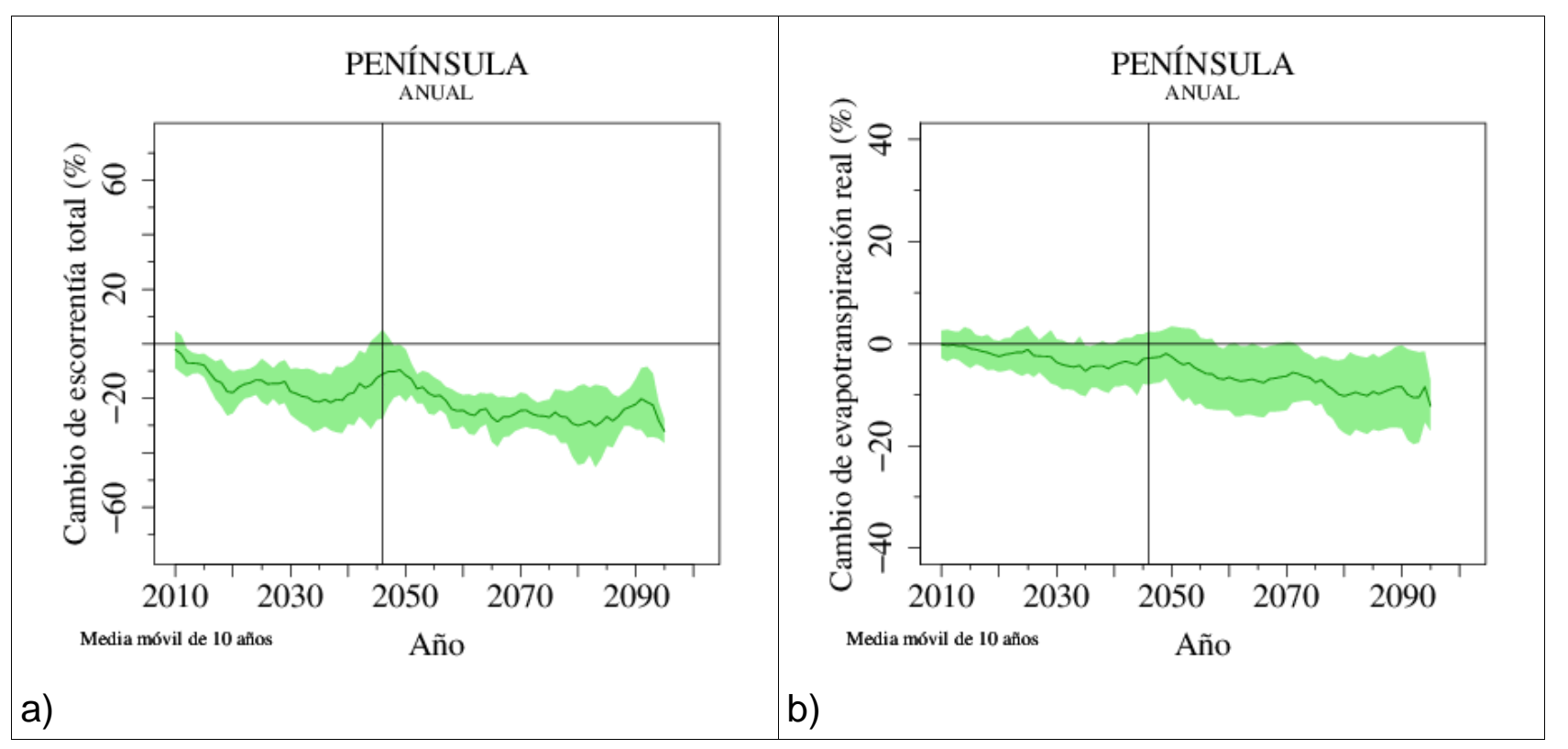

Figura 3.15. Cambios en el valor medio anual en la España peninsular obtenida mediante técnicas de regionalización dinámica de: (a) tasa de $\mathbf{Q}$ filtrada con media móvil de 10 años; (b) tasa de ETR filtrada con media móvil de 10 años. En línea continua (área sombreada) se representa el valor promedio (incertidumbre).

Como ya se ha mencionado, el cambio de $\mathrm{Q}$ se encuentra estrechamente ligado a los de la PCP y por tanto, a su incertidumbre. La Figura 3.9 muestra la evolución del valor medio anual de la tasa de PCP para el mismo período. Como puede observarse, la variabilidad es similar para las tres variables descritas (Q, ETR y PCP) con oscilaciones similares y máximos y mínimos relativos situados en los mismos intervalos de tiempo (Figuras 3.9a y $3.15 \mathrm{a}$ y b). Asimismo, todas ellas alcanzan valores más altos de decremento en la segunda mitad del período. No obstante, la amplitud de las oscilaciones difiere en cada variable. El comportamiento relativo de las serie de PCP muestra oscilaciones menos abruptas que las series de Q y ETR. Por otro lado, la tasa de decremento máxima alcanzada en cada serie es de aproximadamente el $20 \%$ en el caso de la PCP, 30\% para Q y $10 \%$ para ETR todas ellas en la segunda mitad del período.

Los patrones espaciales de Q y ETR vienen condicionados principalmente por el de precipitación. El patrón espacial de Q debe ser similar al de PCP aunque con mayor variabilidad debido a las características del terreno (Libro Digital del Agua, 2008). La Figura 3.16 muestra la distribución espacial de los cambios esperados en los valores medios anuales de anomalías de Q y ETR segregando los períodos para los que se tienen mayor número de proyecciones. Lo más destacable es que el patrón de $\mathrm{Q}$ no muestra variabilidad aparente a lo largo del SXXI, es decir, se mantiene prácticamente constante en los dos períodos diferenciados. Este resultado es coherente con la tendencia observada en las series temporales y los resultados analizados para PCP que mantienen un comportamiento casi constante. Este resultado también se observa en las series temporales correspondientes a cada Comunidad Autónoma (Figuras no mostradas). Por otro lado, el patrón espacial de ETR muestra aumento a lo largo del SXXI en diversas regiones del norte peninsular, como el Pirineo y el interior de Galicia y Asturias así como el norte de Castilla-León; y descenso en la mitad sur y sur-este al final del período (Figura 3.16c y d). 


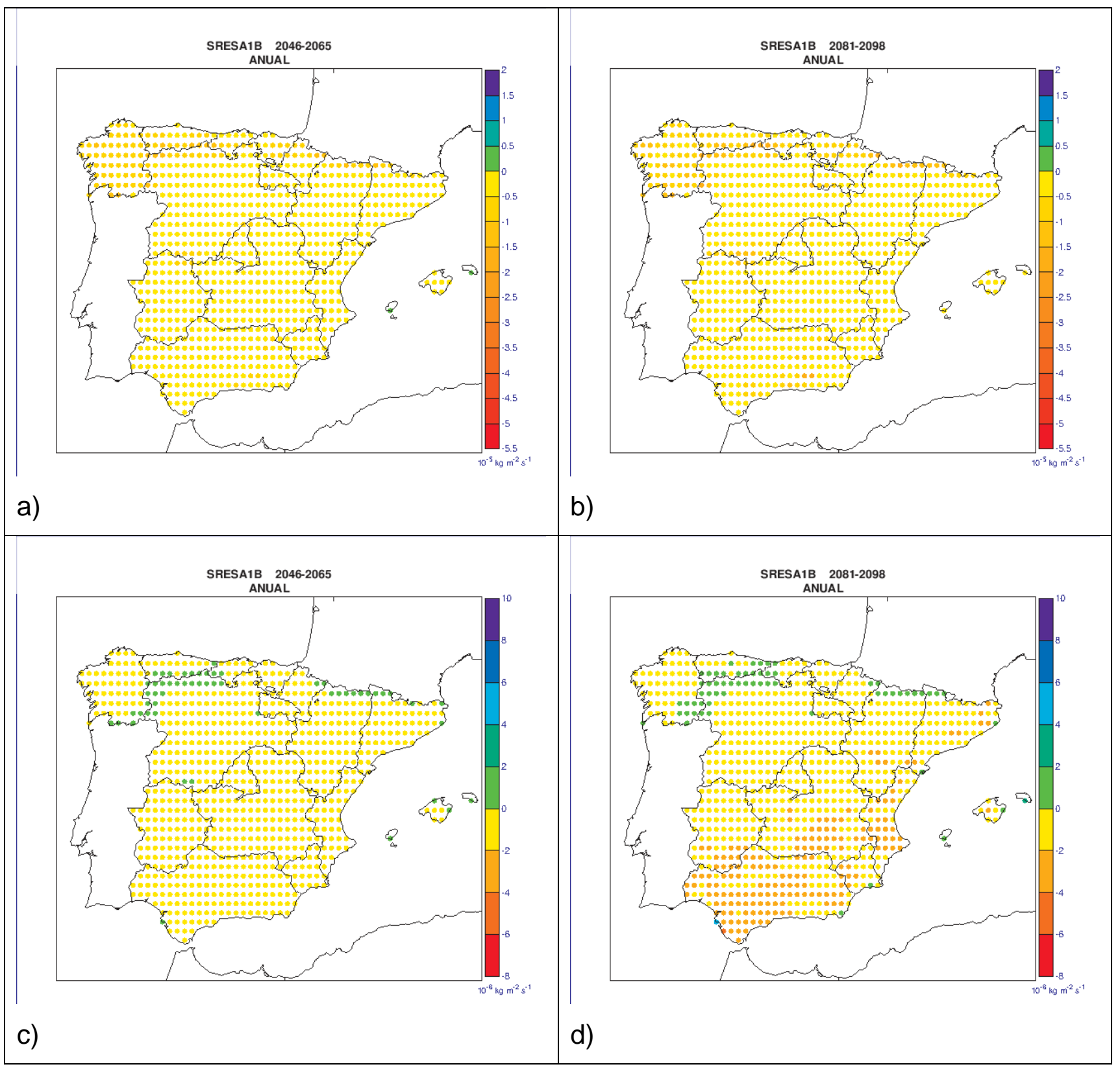

Figura 3.16. Arriba: Cambios en la distribución espacial del valor medio anual de anomalías de $\mathbf{Q}$ obtenidos mediante técnicas de regionalización dinámica para escenario A1B para los períodos: (a) 2046-2065 y (b) 2081-2098. Abajo: Cambios en la distribución espacial del valor medio anual de anomalías de ETR obtenidos mediante técnicas de regionalización dinámica para escenario A1B para los períodos: (c) 2046-2065 y (d) 20812098. Las escalas representan unidades de $\mathrm{kg} \mathrm{m}^{-2} \mathrm{~s}^{-1}$.

Si se comparan los cambios en la evolución por CCAA de ETR y PCP, se observa, que efectivamente ETR reproduce razonablemente bien el patrón de PCP. En general, el comportamiento es similar para ambas variables (Figuras no mostradas). Se observa comportamiento decreciente en casi todas las CCAA en ambos casos, salvo en los años centrales del siglo que en las CCAA de la mitad sur se observan valores crecientes que alcanzan el valor máximo en torno al año 2050. Por otro lado, para las CCAA del norte peninsular se obtienen valores negativos muy próximos a cero, es decir, se aprecia ligera 
disminución de los valores de $\mathrm{Q}$ respecto al período de control. Asimismo, en ambas figuras se observa que las CCAA para las que se obtiene mayor variabilidad y mayores valores de incertidumbre corresponden a las regiones de la mitad sur peninsular: Extremadura, Castilla - La Mancha, Comunidad Valenciana, Andalucía y Murcia.

La Figura 3.17 muestra el cambio estacional de la tasa de Q relativa al período de control. Al comparar estacionalmente con la evolución de la tasa de PCP se observa coherencia entre los resultados de ambas variables. Por otro lado, el comportamiento de ETR es similar (Figura 3.18). En los tres casos los decrementos mayores se obtienen en verano, aunque los valores más altos son los correspondientes a la variable Q. Las mayores diferencias entre ellas se observan en las estaciones de primavera y otoño. No obstante, todas ellas muestran tendencia decreciente. La estación de invierno es la que menos diferencias muestra respecto al período de control. Por otro lado, los valores más altos de incertidumbre se observan en las series correspondientes a verano, salvo en los primeros años del período; el resto de estaciones muestran valores similares que se mantienen a lo largo de toda la simulación. 


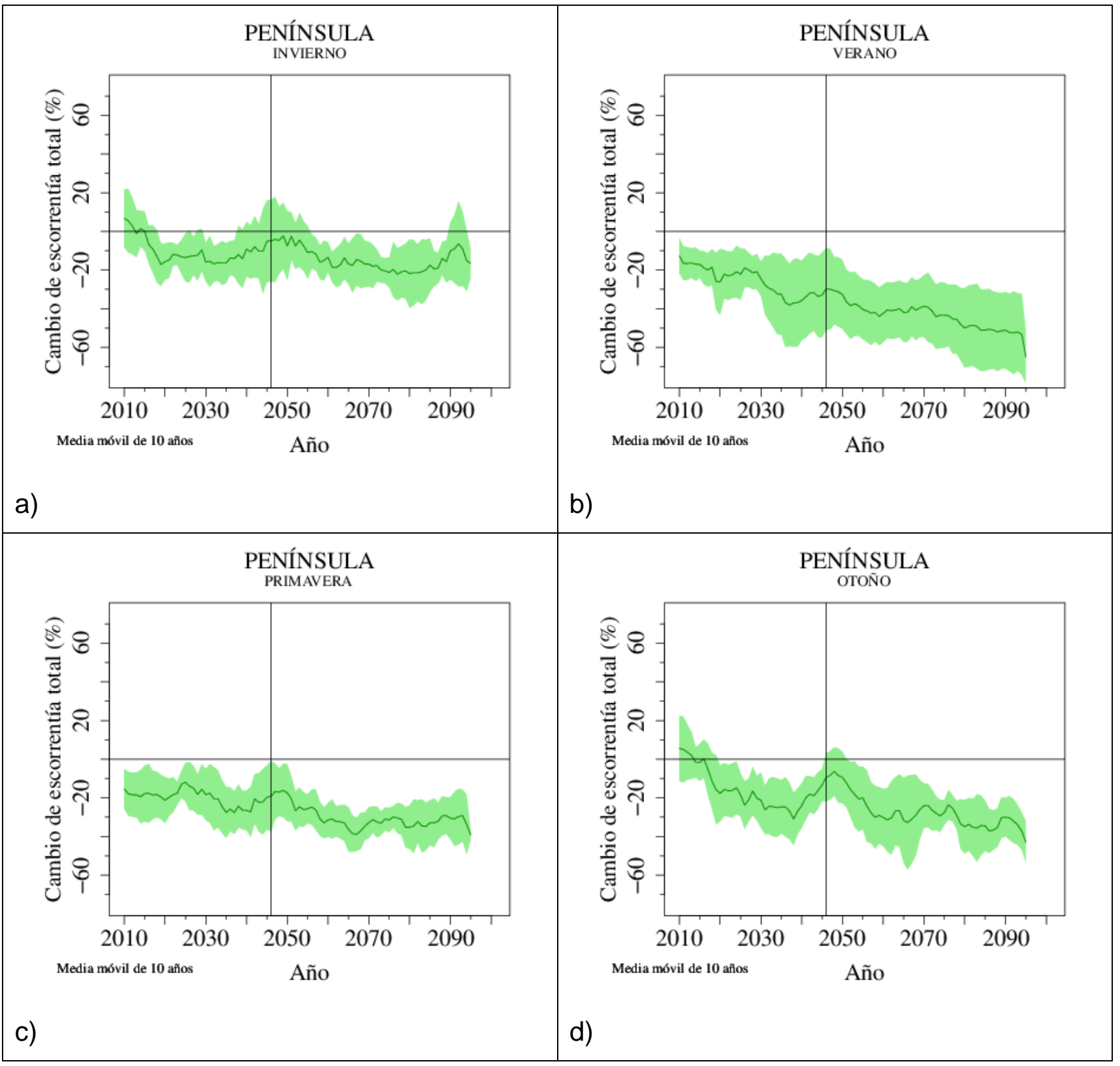

Figura 3.17. Igual que la Figura 3.9 para la tasa de Q media. 

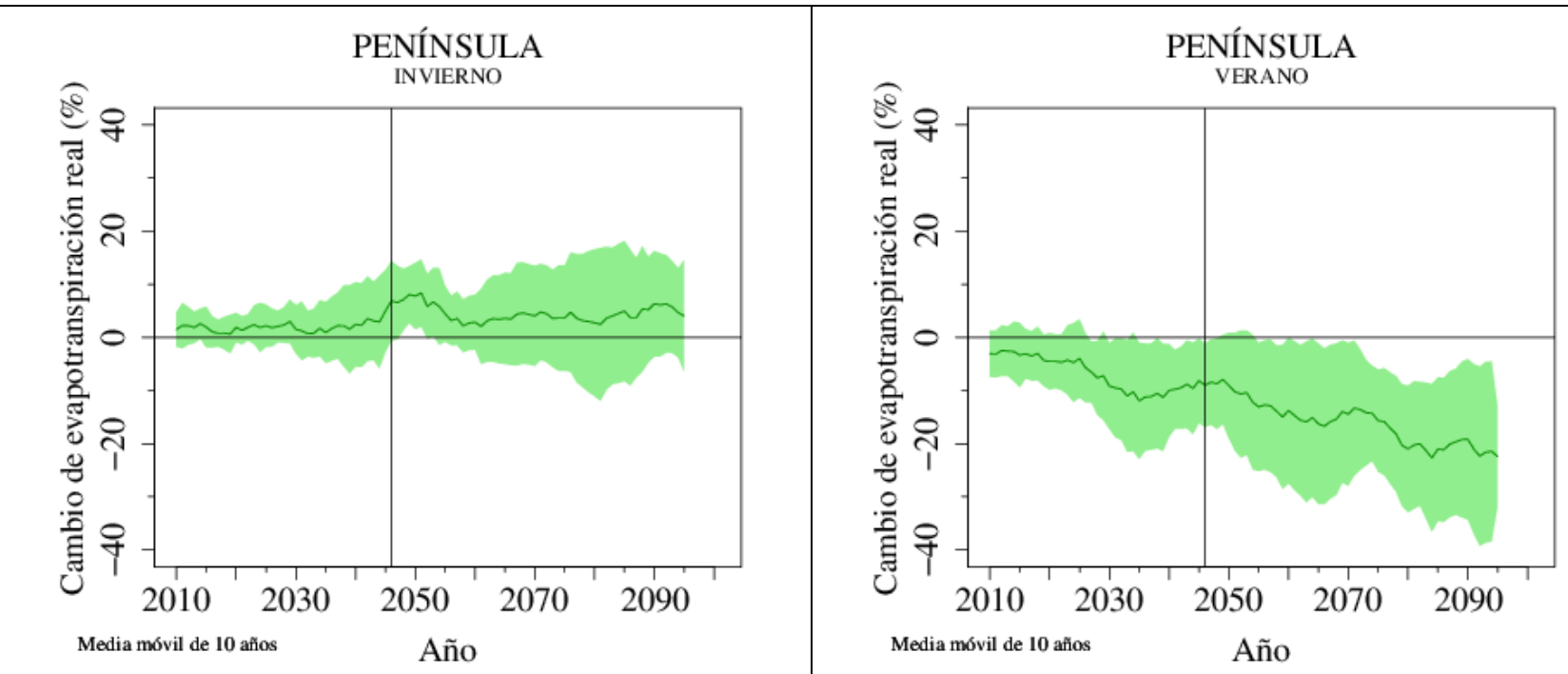

a)

b)
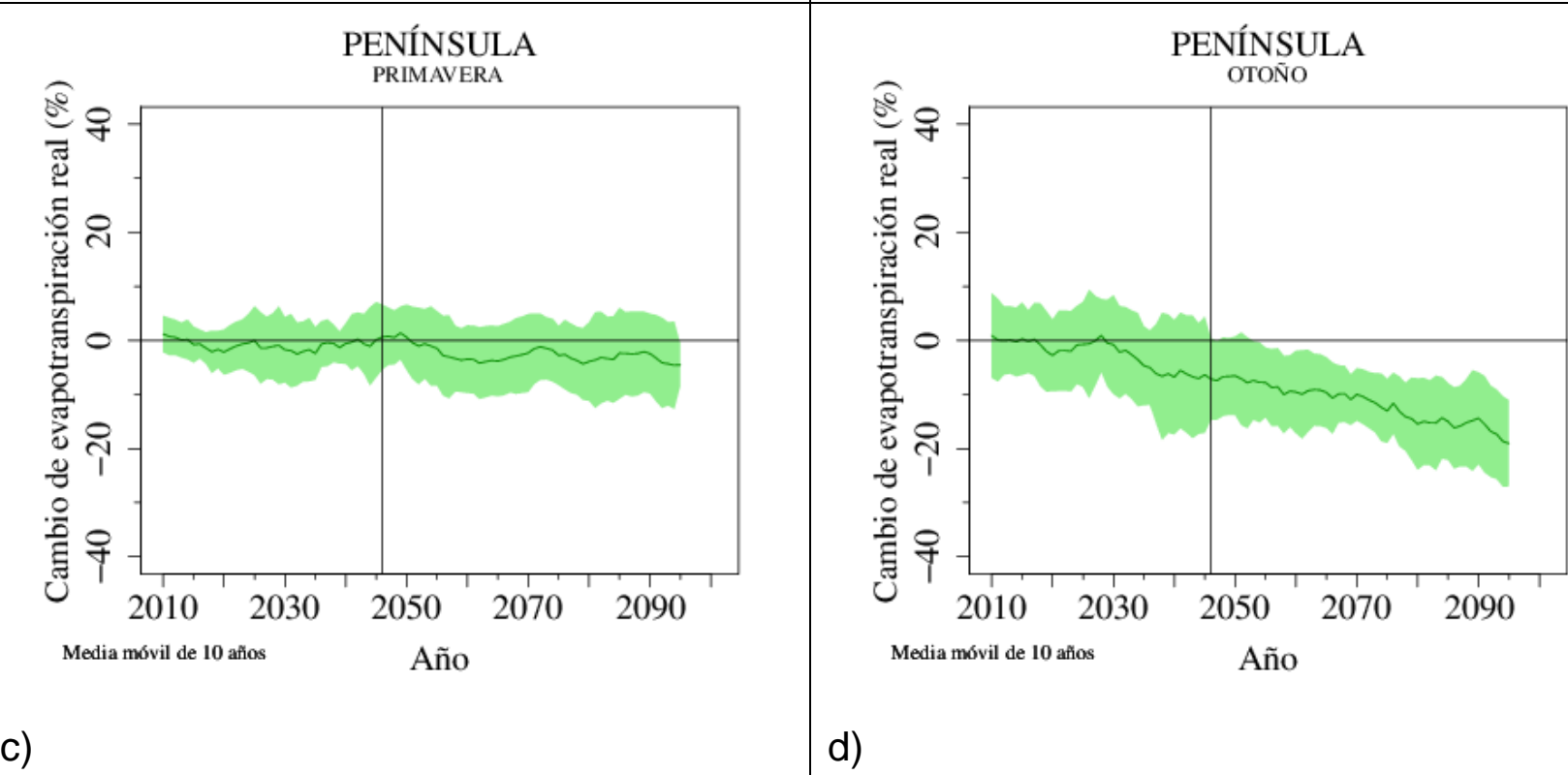

d)

Figura 3.18. Igual que la Figura 3.9 para la tasa de ETR media.

Diversos estudios han calculado el efecto del aumento de la concentración de CO2 sobre el ciclo hidrológico y han concluido que los modelos tienden a infraestimar los incrementos de $\mathrm{Q}$ y a sobreestimar los decrementos (Betts et al., 2007). Esto sugiere que las reservas de agua dulce podrían descender menos de lo previsto por los modelos climáticos a pesar de que se mantenga aumento en el riesgo de sequías. Este resultado es especialmente importante en las regiones donde la disponibilidad de agua es un recurso escaso. 


\subsection{Cambios en la nubosidad}

Diversos estudios han demostrado que la sensibilidad climática de la mayor parte de los modelos depende fuertemente de la representación de las nubes (Yao and Del Genio, 2002). La nubosidad total y la distribución de la tipología nubosa (no estudiada en este trabajo) están ligadas de manera directa al balance radiativo sobre la atmósfera que a su vez determina la variabilidad de las variables estudiadas hasta ahora. Las nubes dominan la variabilidad interanual y decenal de la radiación que alcanza la superficie terrestre y por tanto ejercen la influencia dominante sobre el balance radiativo global. Las nubes pueden contribuir al calentamiento de la atmósfera, debido a su capacidad para absorber radiación infrarroja o al enfriamiento por medio de su alto albedo. En general, es un hecho comúnmente aceptado que las nubes tienden a enfriar el sistema climático (Mace et al., 2006). Este resultado deriva de diversos estudios observacionales llevados a cabo sobre balance radiativo (Weilicki et al., 1998; Rossow and Schiffer, 1999).

Antes de comenzar el estudio de esta nueva variable, es esencial tener en cuenta que el forzamiento radiativo de las nubes está reconocido y aceptado como la mayor fuente de incertidumbre de las proyecciones de cambio climático (Houghton et al., 2001; Bony and Drufesne, 2005; Bony et al., 2006; Webb et al, 2006; Meehl et al., 2007; Randall et al., 2007; Andrews et al., 2012; Tsushima et al., 2012). Por otro lado, la comparación de las simulaciones de cambio climático de los distintos modelos utilizados, muestra que las diferencias entre ellos se atribuyen principalmente a la componente de onda corta del forzamiento radiativo, en particular a la respuesta de las nubes de la capa límite al calentamiento global (Colman, 2003; Bony and Drufesne, 2005; Soden and Held, 2006; Webb et al., 2006). Por tanto, las nubes modulan fuertemente el balance radiativo de la Tierra y los cambios que se produzcan en su distribución espacial y temporal producidos como respuesta al calentamiento global afectan de manera sustancial a otras variables tales como la temperatura (Bony et al., 2006). Sin embargo, el signo y la magnitud de los cambios producidos dependen de muchos factores y por tanto llevan asociada mucha incertidumbre.

La Figura 3.19 muestra los cambios esperados para el SXXI de la tasa de cobertura nubosa total (CTL), la de precipitación y las temperaturas máxima y mínima. Se observa que la evolución de las cuatro variables es consistente entre sí. Puede observarse la relación directa entre CLT-PCP e inversa entre CLT-TX y CLT-TI. Este resultado es coherente con estudios realizados sobre series de datos observacionales del SXX que han demostrado que variaciones en la cobertura nubosa, la humedad del suelo y la circulación atmosférica condicionan la evolución de la temperatura máxima y mínima en los períodos estudiados (Przybylak, 2000; Braganza et al., 2004). El aumento de la cobertura nubosa desde la década de los 50 es consistente con el aumento de la precipitación y el decrecimiento del rango diario de temperatura (Vose et al., 2005). Por el contrario, el decremento registrado de la cobertura nubosa total sobre las áreas de tierra desde finales de los 70 y principios de los 80 (Rossow and Dueñas, 2004; Warren et al., 2007) y el esperado para el SXXI pueden relacionarse con el descenso esperado en la precipitación y el aumento de temperatura. Sin embargo, la mayor parte de los modelos climáticos infraestima el promedio anual de la fracción nubosa total sobre todas las áreas del globo. Asimismo, los modelos sobreestiman la amplitud del ciclo estacional en los trópicos y latitudes medias. La variabilidad interanual promediada tiende a ser infraestimada, así como la variabilidad interanual de la amplitud del ciclo estacional (Probs et al., 2012). 


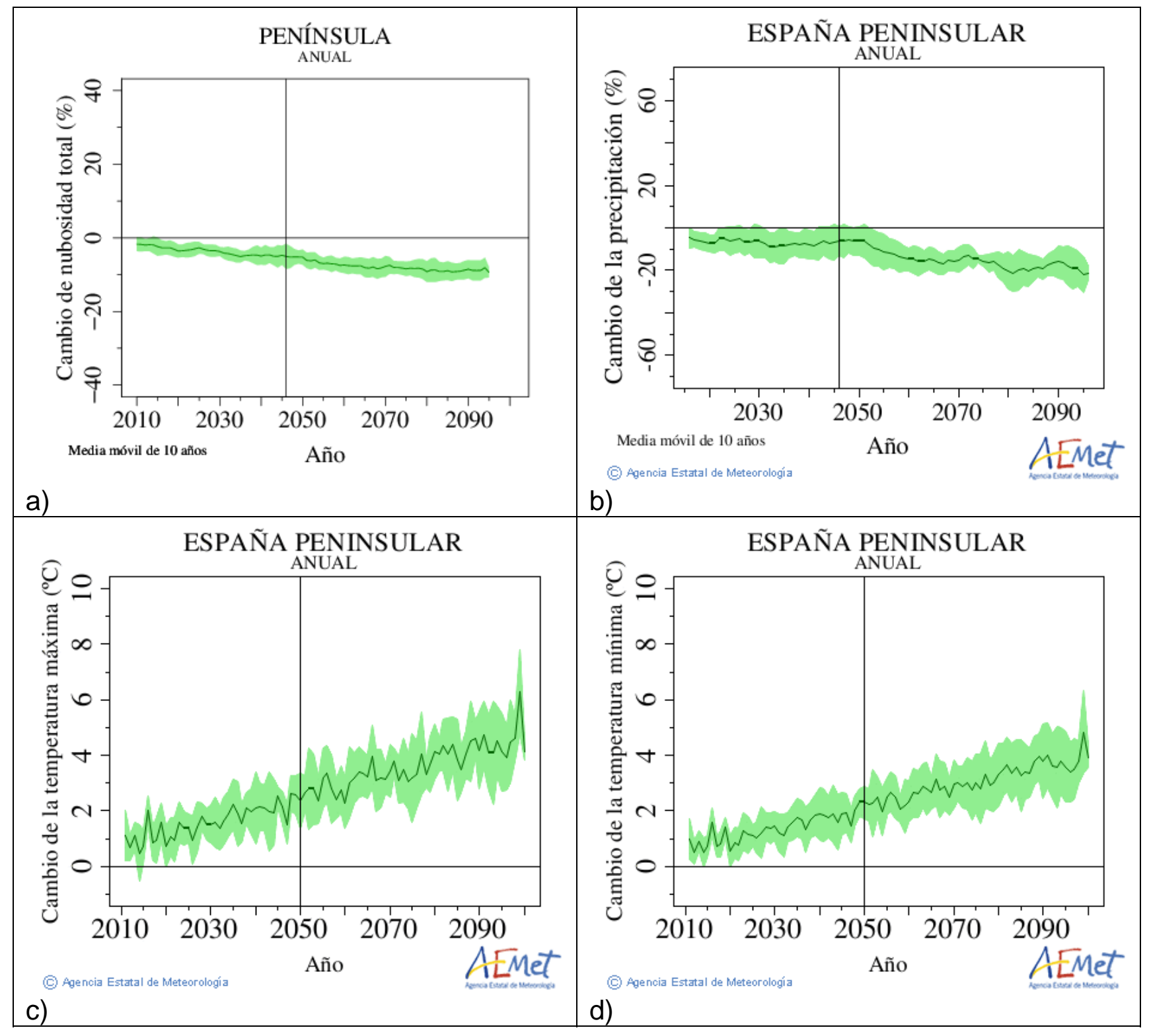

Figura 3.19. Cambios en el valor medio anual en la España peninsular obtenida mediante técnicas de regionalización dinámica para el escenario A1B de: (a) Tasa de cobertura nubosa total; (b) Tasa de precipitación; (c) Temperatura máxima ( $\left.{ }^{\circ} \mathrm{C}\right)$; (d) Temperatura mínima $\left({ }^{\circ} \mathrm{C}\right)$. En línea continua (área sombreada) se representa el valor promedio (incertidumbre). 
Los cambios en la evolución estacional mostrados en la Figura 3.20 son consistentes con los esperados para la evolución estacional de las variables TX, TI (Figuras no mostradas) y PCP (Figura 3.9), es decir, la nubosidad tiende a disminuir prácticamente en todas las estaciones en concordancia con el comportamiento anual. En invierno, sin embargo, se observa comportamiento constante y próximo a cero, que se observa también en la PCP.

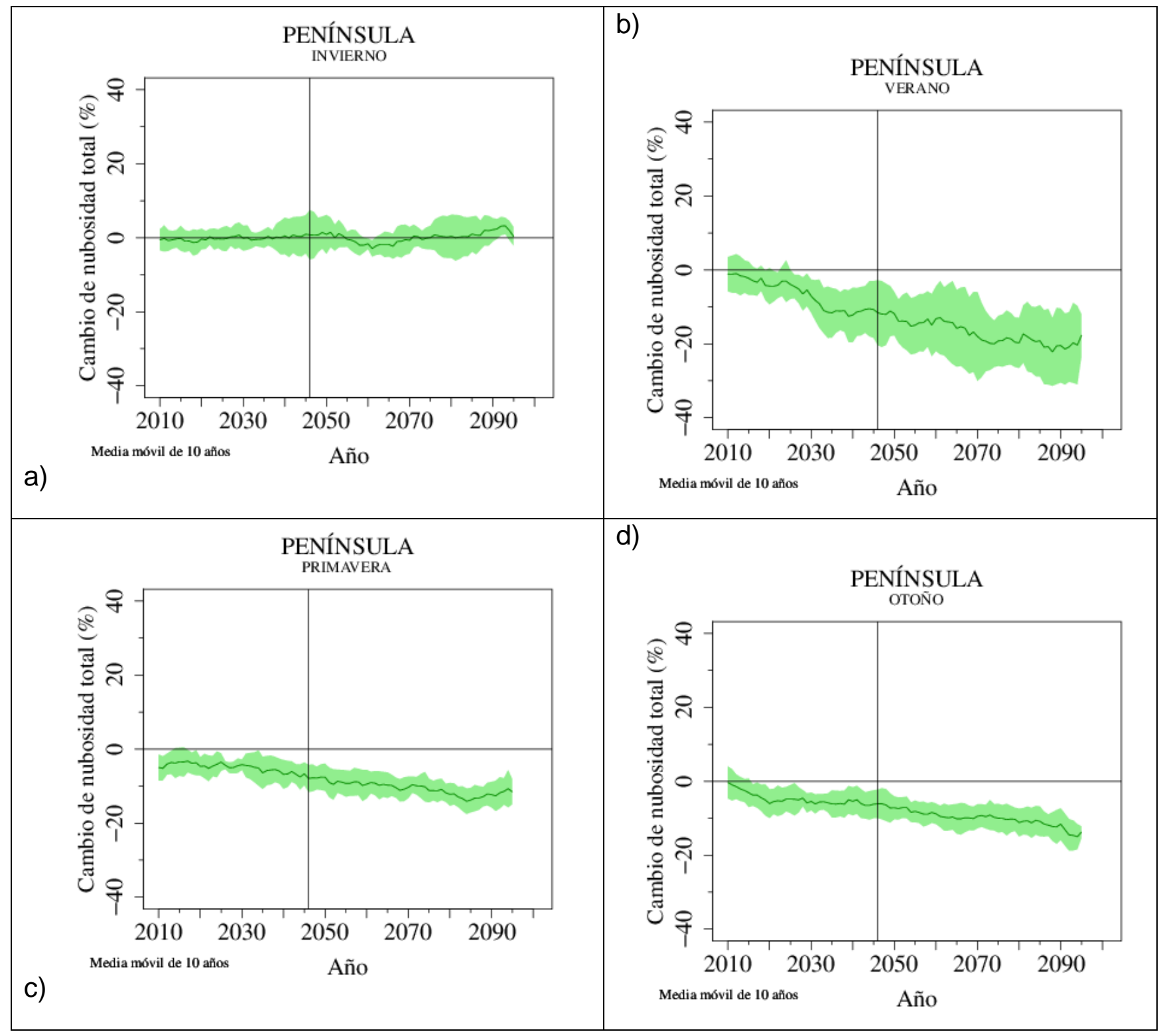

Figura 3.20. Cambios en el valor medio estacional de la tasa de cobertura nubosa total media, filtrada con media móvil de 10 años, en la España peninsular para el siglo XXI obtenida mediante técnicas de regionalización dinámica para el escenario A1B. (a) Invierno; (b) verano; (c) primavera y (d) otoño. En línea continua (sombreada) se representa el valor promedio (incertidumbre).

La alta variabilidad espacial y temporal de la cobertura nubosa supone la principal limitación para el estudio de su distribución espacial (Font, 2000; Calbó and SánchezLorenzo, 2009). Esto implica que el estudio regional, para pequeñas áreas, no ofrece resultados robustos en ningún caso. La Figura 3.21 muestra la distribución espacial del valor medio estacional esperado de nubosidad para la Península Ibérica en el segundo 
período estudiado. Las estaciones para las que se observa mayor variabilidad en la nubosidad son invierno y verano (Figura 3.21a y b, respectivamente). Por otro lado, las estaciones de primavera y otoño muestran patrones espaciales muy homogéneos (Figura $3.21 \mathrm{c}$ y d, respectivamente). Tanto en invierno como en verano, los mayores cambios en la cobertura nubosa se esperan en la mitad norte peninsular, observándose un claro gradiente norte-sur. Este resultado es coherente con las representaciones temporales de la evolución de la nubosidad total. Los patrones de incertidumbre (no mostrados) no muestran variabilidad espacial.

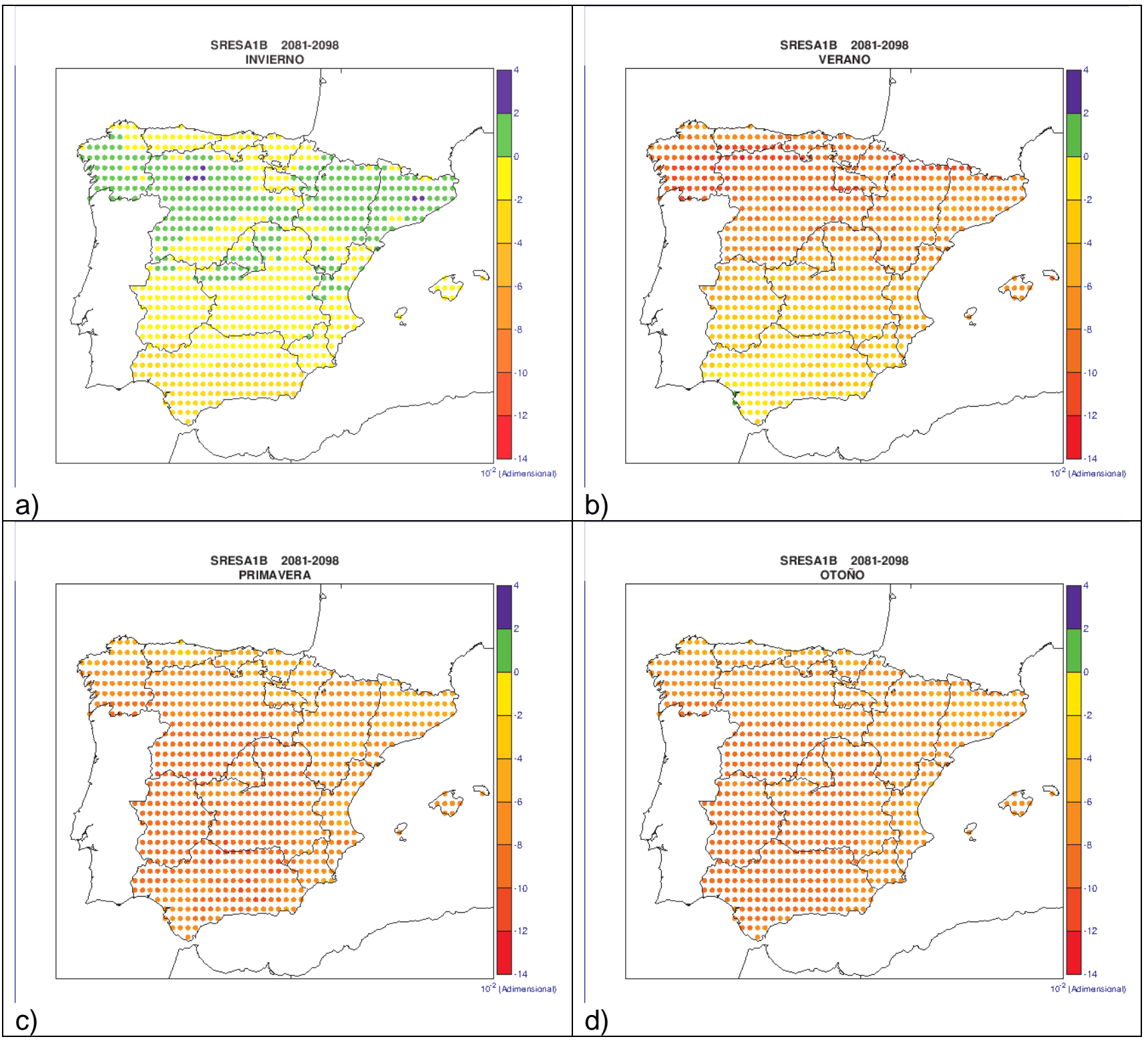

Figura 3.21. Cambios en la distribución espacial del valor medio estacional de anomalías de CLT obtenida mediante técnicas de regionalización dinámica para escenario A1B para el período 2081-2098. (a) invierno; (b) verano; (c) primavera y (d) otoño. La escala es adimensional entre [0.0-1.0].

Los cambios en los valores medios anuales para las dos ventanas temporales estudiadas muestran patrones espaciales homogéneos (no mostrados) de disminución de la 
nubosidad. La evolución temporal de los valores de nubosidad total por CCAA muestra, que efectivamente no existe variabilidad espacial importante en cada región. En todas las CCAA se espera disminución de la nubosidad en igual magnitud y con valores de incertidumbre similares, de manera que la evolución media peninsular sintetiza la evolución general en toda la zona (Figura no mostrada).

Con todo, el análisis climático de la cobertura nubosa es importante para el estudio del balance radiativo de la atmósfera, pero debido a las limitaciones de los modelos climáticos en general y sobre las variables nubosas en particular, estos resultados deben tomarse con mucha cautela (Przybylak, 2000; Houghton et al., 2001; Colman, 2003; Braganza et al., 2004; Bony and Drufesne, 2005; Bony et al., 2004; 2006; Ringer et al., 2006; Soden and Held, 2006; Webb et al, 2006; Meehl et al., 2007; Randall et al., 2007; Williams and Tselioudis, 2007; Andrews et al., 2012; Tsushima et al., 2012). 


\section{Capítulo 4}

\section{Conclusiones}

En este trabajo se ha generado un conjunto de escenarios regionalizados para el SXXI sobre España peninsular, Islas Baleares e Islas Canarias. Las variables estudiadas son temperatura, precipitación, viento a 10m, escorrentía, evapotranspiración real y nubosidad. Para las variables de temperatura y precipitación se han realizado regionalizaciones mediante dos diferentes técnicas estadísticas para España peninsular, Islas Baleares e Islas Canarias y técnicas dinámicas únicamente para la Península y Baleares. A continuación se resumen los resultados que correspondientes al escenario A2 en el caso de las regionalizaciones estadísticas y al A1B para las dinámicas. Estos resultados se representan en forma de anomalías calculadas respecto a los períodos de control 1961-1990 para las proyecciones dinámicas y las estadísticas realizadas mediante regresión lineal y 1961-2000 para las estadísticas llevadas a cabo con el método de análogos. El período abarcado por las proyecciones regionalizadas comprende desde 2011 hasta 2100 para las regionalizaciones dinámicas y las estadísticas-regresión lineal y desde 2001 hasta 2100 para las estadísticas-análogos. Las conclusiones más relevantes pueden resumirse como sigue: 


\section{Temperatura máxima:}

- Se observa tendencia al aumento del valor medio de la temperatura máxima a lo largo de todo el período, alcanzándose incrementos de entre $3-5^{\circ} \mathrm{C}$ a final del SXXI.

- Se identifica tendencia al aumento de la temperatura máxima menos pronunciada en las regiones costeras, Valle del Ebro e Islas Baleares y más acentuada en el Pirineo y las regiones del centro y sur peninsular.

- El mayor incremento de temperatura máxima corresponde a los meses de verano de hasta $5-7^{\circ} \mathrm{C}$, y es menos intenso en la zona cantábrica que en el resto de la Península. En invierno el valor medio esperado manifiesta un incremento con valores en torno a los $3^{\circ} \mathrm{C}$.

- Para las Islas Canarias se observa incremento de la temperatura máxima de orden similar al de las regiones del norte peninsular, aunque sin variación estacional

- El porcentaje de días cálidos mantiene tendencia creciente constante a lo largo de todo el período hasta alcanzar tasas en torno al 50\%. La duración de olas de calor presenta incremento de en torno a 5 días hasta la mitad del período; a partir del año 2050 la tendencia aumenta hasta alcanzar valores próximos a los 40 días a finales del SXXI.

\section{Temperatura mínima:}

- Se obtiene tendencia creciente del valor medio de la temperatura mínima anual que se mantiene constante a lo largo de todo el período con valores próximos a $3-4^{\circ} \mathrm{C}$ de aumento a finales del SXXI.

- Aumento menos pronunciado de las temperaturas mínimas en el norte peninsular, y más acentuado en el centro de Castilla-La Mancha y noreste de Andalucía.

- El incremento de temperatura mínima es mayor en el sur peninsular en los meses de verano alcanzando valores próximos a $4^{\circ} \mathrm{C}$. En invierno el aumento es de aproximadamente $2^{\circ} \mathrm{C}$. En 
primavera y otoño se observa incremento intermedio en todas las regiones.

- Los resultados para Canarias muestran comportamiento análogo a las regiones peninsulares pero sin variación estacional.

- En número de días de helada disminuye hasta valores entre 1015 días menos anuales; mientras que el porcentaje de noches cálidas aumenta entre el $40-50 \%$ para los últimos años del SXXI. 


\section{Precipitación:}

- En general, se observa disminución de la tasa de precipitación en toda el área peninsular. En la mitad norte de la península se observa disminución de la tasa de precipitación entre 0-10\%, mientras que en el resto del área la disminución varía entre 1020\%. Excepto Extremadura, Andalucía y Comunidad Valenciana que la disminución es entre $20-30 \%$.

- El número de días de lluvia disminuye en magnitud similar en todas las regiones alcanzando a final de siglo valores próximos a los 10-15 días, con mayor incertidumbre en las regiones del norte y centro peninsular.

- La duración de los períodos secos tiende a ser más larga con el aumento del alcance de la proyección con valores entre 5-10 días. Por otro lado, se aprecia disminución del número de días de lluvia y por tanto, se puede esperar mayor duración de los períodos secos lo que aumentaría el riesgo de sequías especialmente en la mitad sur peninsular.

- El ciclo estacional se hace más patente ya que se aprecia un aumento de los períodos secos mayor en verano y primavera que en invierno y otoño.

- En Canarias el cambio esperado en la precipitación es más acusado alcanzando los 25 días anuales, variando entre 30 días en invierno y 20 en verano.

- La tasa de precipitaciones intensas se encuentra por encima de cero aunque sin tendencia aparente y con valores de incertidumbre mayores en las regiones del sur y levante. No se observan cambios destacables en el ciclo estacional.

\section{Viento a $10 \mathrm{~m}$ :}

- Se observa disminución de la velocidad de la componente zonal del viento respecto al período de referencia estudiado. Los valores correspondientes a la velocidad de la componente meridiana muestran poca o ninguna variación respecto al período 
de referencia. La velocidad neta muestra variaciones muy próximas a cero y de menor magnitud que por componentes. La intensidad de las rachas máximas varía de manera desigual a lo largo del período con los valores más bajos en la segunda mitad del SXXI.

- El patrón espacial correspondiente a la media de las variaciones esperadas muestra debilitamiento en todo el territorio.

\section{Escorrentía y evapotranspiración real:}

- Ambas variables muestran variabilidad conjunta con oscilaciones decenales y evidente disminución con respecto al período de referencia. Los valores más altos de decremento se alcanzan en la segunda mitad del período. La tasa de decremento máxima alcanzada en cada serie es aproximadamente el 30\% en el caso de la escorrentía y el 10\% para la evapotranspiración real.

- El patrón espacial de escorrentía no muestra cambios a lo largo del SXXI. Mientras que el patrón espacial de evapotranspiración real muestra aumento en la mitad noreste de la Península y descenso en la mitad sur y sur-este al final del período.

- Los decrementos mayores de ambas variables se obtienen en verano siendo de mayor magnitud para la escorrentía con valores cercanos al 60\%; mientras que los valores de evapotranspiración real no superan el $20 \%$. La estación de invierno es la que menos decremento muestra respecto al período de control con valores del $20 \%$ para la escorrentía y aumento entre $5-10 \%$ de la evapotranspiración real.

\section{Nubosidad:}

- La nubosidad tiende a disminuir prácticamente en todas las estaciones en concordancia con el comportamiento anual. En invierno no se observan cambios apreciables. 
- Tanto en invierno como en verano, los mayores cambios en la cobertura nubosa se esperan en la mitad norte peninsular, observándose un claro gradiente norte-sur. Sin embargo, la alta variabilidad espacial y temporal de la nubosidad supone la principal limitación para el estudio de su distribución espacial en regiones más pequeñas. 


\section{Anexo A}

\section{Figuras por Comunidades Autónomas}

En este Anexo se recopila la colección de gráficos generados a lo largo de este estudio. En primer lugar se muestran las Figuras organizadas por CCAA; en la columna izquierda (derecha) se muestras las Figuras correspondientes a la regionalización dinámica (estadística-análogos). Por cuestión de brevedad, solamente se muestran los gráficos de evolución temporal. Para cada Comunidad Autónoma se muestra un conjunto de seis figuras que resumen el cambio potencial bajo futuras condiciones de cambio climático a lo largo del SXXI de las temperaturas máximas y mínimas, y la precipitación, en valores promediados anualmente y por estaciones; así como los índices extremos estudiados, asociados a estas variables. Los cambios en la evolución de cada variable se representan promediada anual y estacionalmente, en forma de anomalías calculadas respecto a los periodos de control 1961-1990 para las proyecciones dinámicas y 1961-2000 para las estadísticas. El período abarcado por las proyecciones regionalizadas comprende desde 2001 hasta 2100.

Se muestran las Figuras correspondientes a los cambios en los valores medios promediados espacialmente para la Península Ibérica de las temperaturas máximas y mínimas, la precipitación, los campos de viento estudiados en el texto, la evapotranspiración real, escorrentía y nubosidad. En primer lugar se muestran los cambios esperados en los valores promediados estacionalmente 
de las variables de temperatura y precipitación; a continuación los cambios en los promedios anuales correspondientes a regionalización dinámica (izquierda), estadística-análogos (centro) y estadística-sdsm (derecha). Por otra parte se muestran los cambios en los valores promediados anualmente de los campos de viento, la evapotranspiración real, escorrentía y nubosidad obtenidos mediante las técnicas de regionalización dinámica y estadística-análogos. Finalmente, se muestran los patrones espaciales de los cambios en los valores medios y su incertidumbre correspondientes a las proyecciones llevadas a cabo con el escenario A2 para el período 2081-2100 de las variables de temperatura y precipitación. La Tabla A.1 resume la organización de las Figuras.

\begin{tabular}{|l|l|}
\hline \multicolumn{1}{|c|}{ COMUNIDAD AUTÓNOMA } & \multicolumn{1}{c|}{ FIGURAS } \\
\hline Andalucía & Fig.A.1 - Fig.A.7 \\
\hline Aragón & Fig.A.8 - Fig.A.14 \\
\hline Asturias & Fig.A.15 - Fig.A.21 \\
\hline Illes Balears & Fig.A.22 - Fig.A.28 \\
\hline Islas Canarias & Fig.A.29 - Fig.A.35 \\
\hline Cantabria & Fig.A.36 - Fig.A.42 \\
\hline Castilla - La Mancha & Fig.A.43 - Fig.A.49 \\
\hline Castilla - León & Fig.A.50 - Fig.A.56 \\
\hline Cataluña & Fig.A.57 - Fig.A.63 \\
\hline Extremadura & Fig.A.64 - Fig.A.70 \\
\hline Galicia & Fig.A.71 - Fig.A.77 \\
\hline La Rioja & Fig.A.78 - Fig.A.84 \\
\hline Comunidad de Madrid & Fig.A.85 - Fig.A.91 \\
\hline Región de Murcia & Fig.A.92 - Fig.A.98 \\
\hline Comunidad Foral de Navarra & Fig.A.99 - Fig.A.105 \\
\hline País Vasco & Fig.A.106 - Fig.A.112 \\
\hline Comunidad Valenciana & Fig.A.113 - Fig.A.119 \\
\hline España peninsular estacional & Fig.A.120 - Fig.A.123 \\
\hline España peninsular anual & Fig.A.124 - Fig.A.131 \\
\hline
\end{tabular}

Tabla A.1. Resumen de las Figuras por regiones. Izquierda: Nombre de la región; derecha: Rango de Figuras correspondientes a cada región. 
ANDALUCÍA

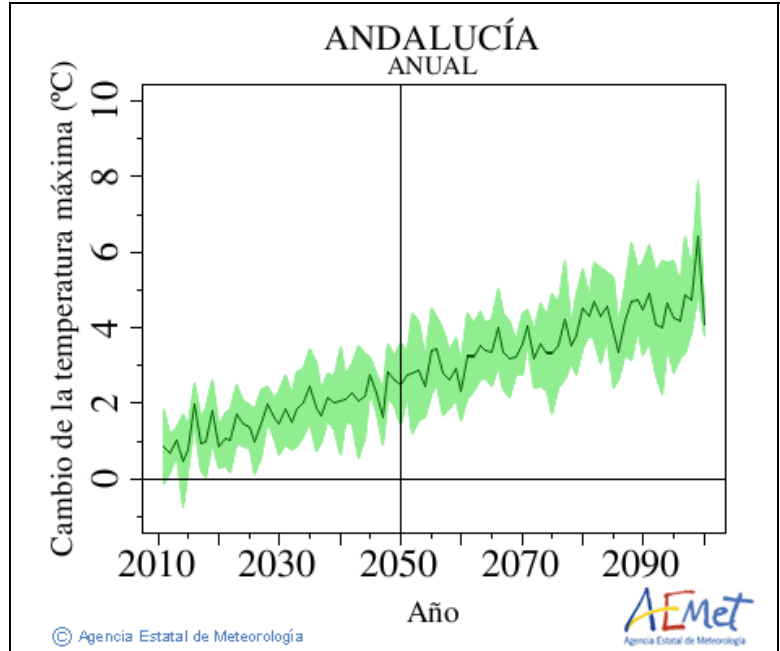

a)

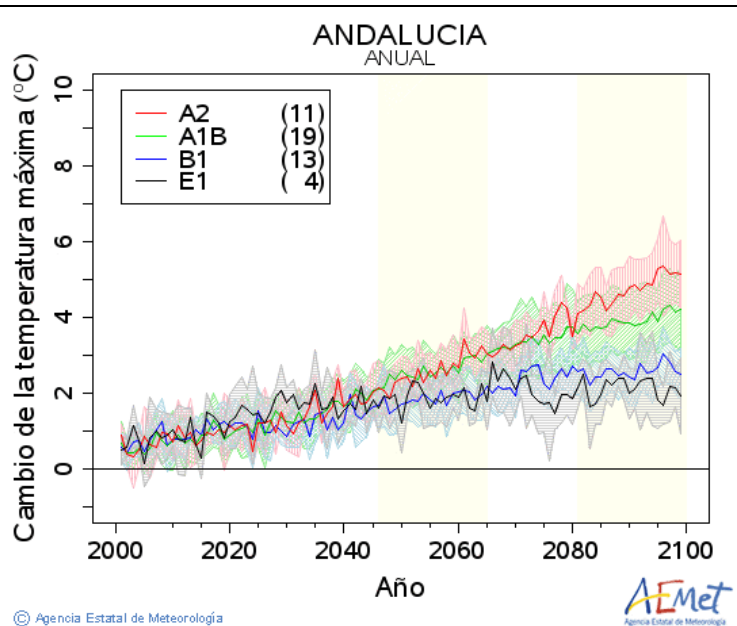

b)
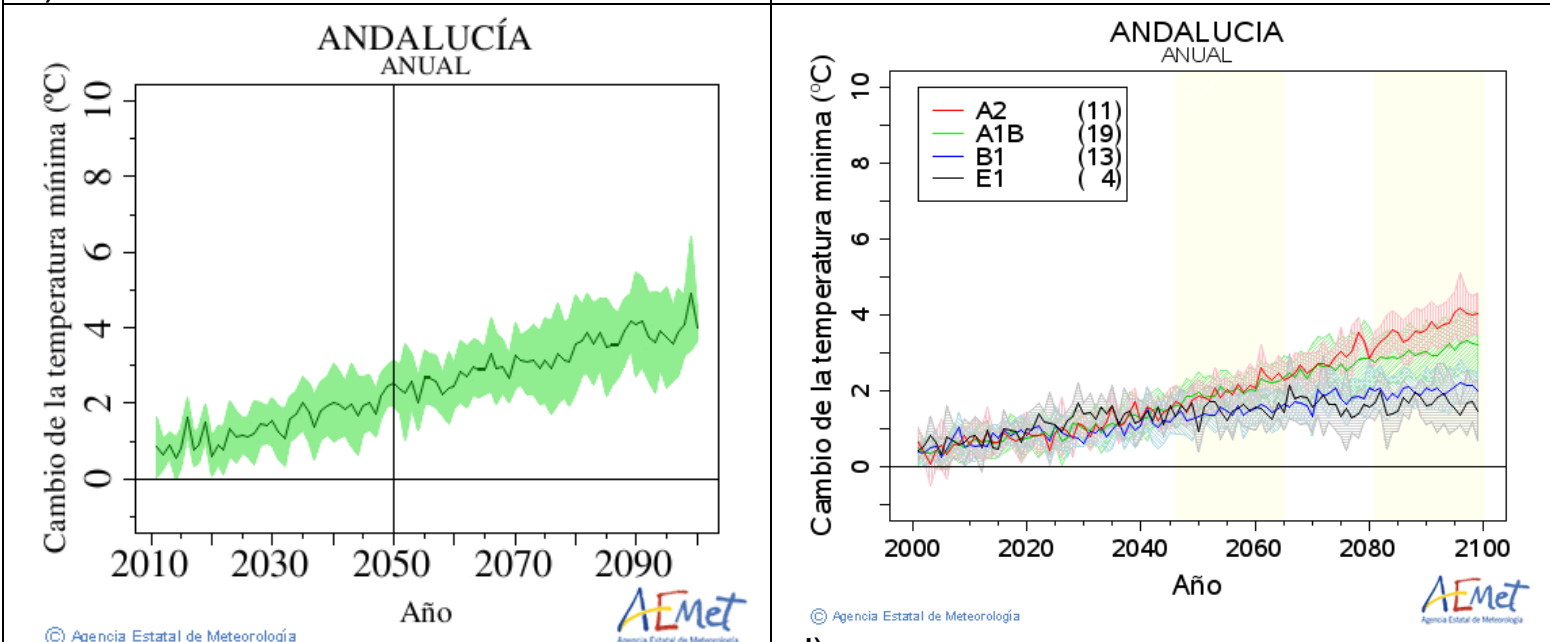

c)

d)

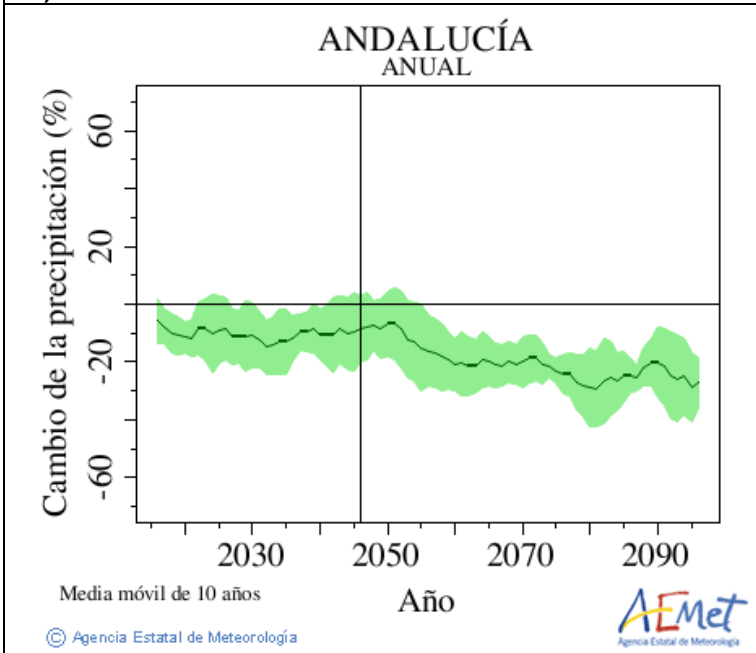

e)

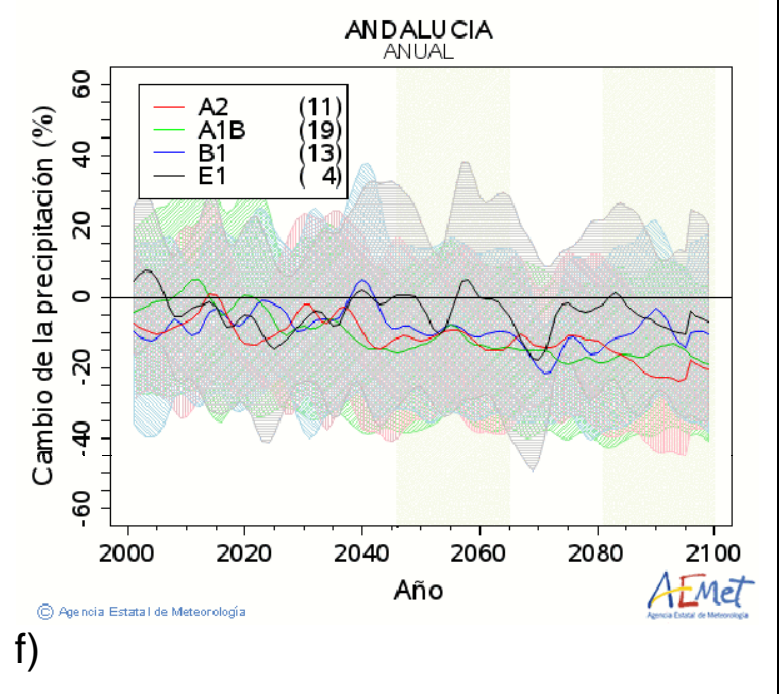

Figura A.1. Evolución temporal del valor medio anual para la Comunidad Autónoma de Andalucía de: (a)-(b) temperatura máxima $\left({ }^{\circ} \mathrm{C}\right)$; (c)-(d) temperatura mínima $\left({ }^{\circ} \mathrm{C}\right)$ y $(\mathrm{e})-(\mathrm{f})$ tasa de precipitación en la España peninsular para el siglo XXI obtenida mediante técnicas de regionalización dinámica (izquierda) y estadística (derecha) agrupadas por escenarios de emisiones: A2-altas (rojo); A1B-medias (verde); B1-bajas (azul) y E1-fuerte mitigación (negro). Entre paréntesis se recoge el número de proyecciones realizadas para cada escenario. En línea continua (sombreada) se representa el valor promedio (incertidumbre). En las figuras de la derecha, las franjas verticales sombreadas indican las ventanas temporales en las que el número de regionalizaciones es máximo. 


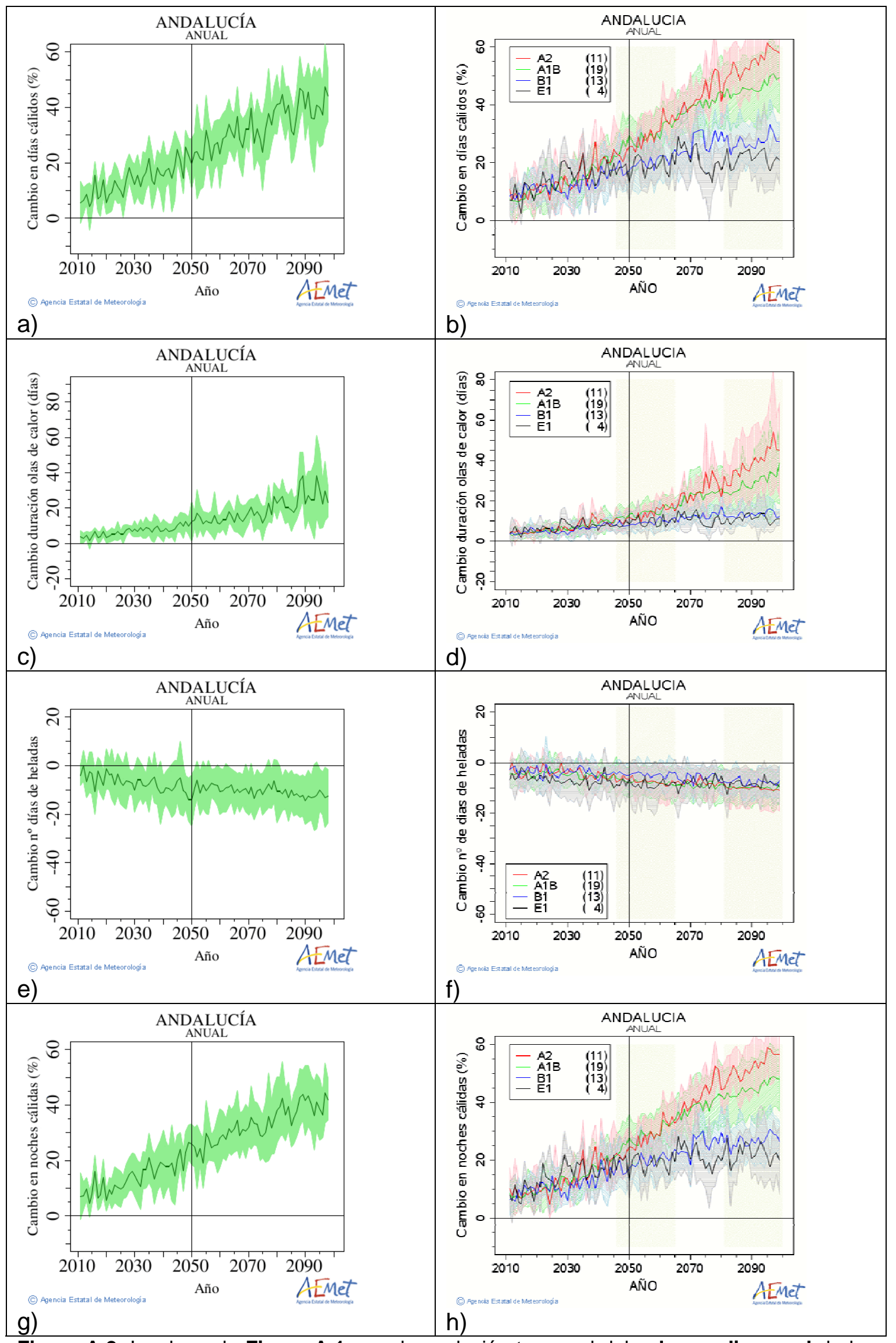

Figura A.2. Igual que la Figura A.1 para la evolución temporal del valor medio anual de los índices extremos (a)-(b) DC; (c)-(d) DOC; (e)-(f) DH y (g)-(h) NC. 

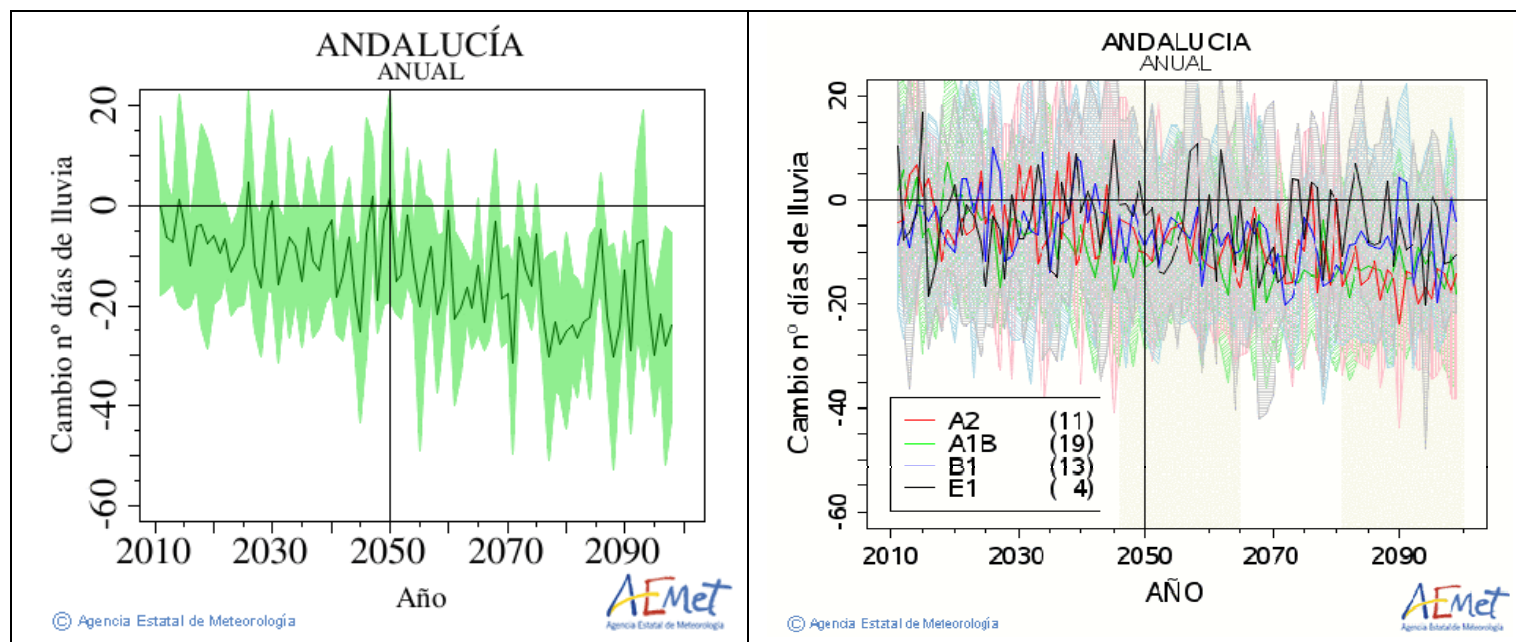

a)

b)
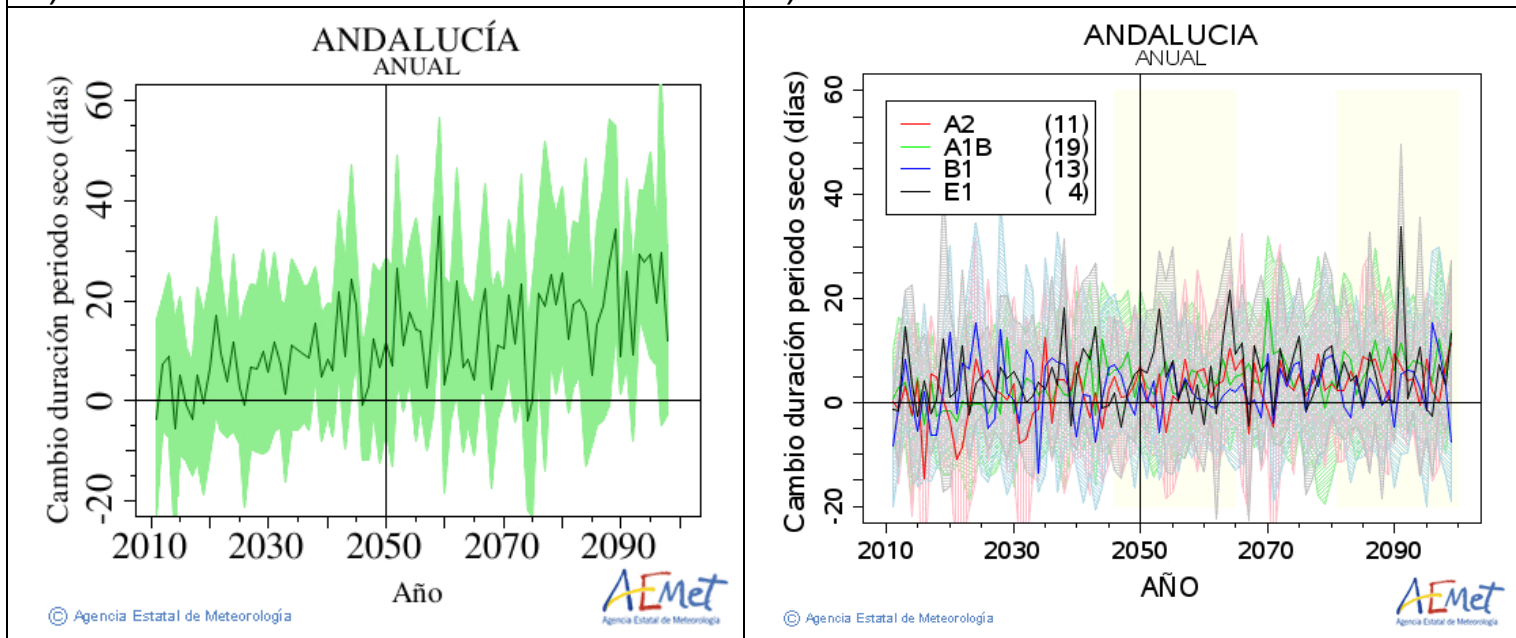

c)

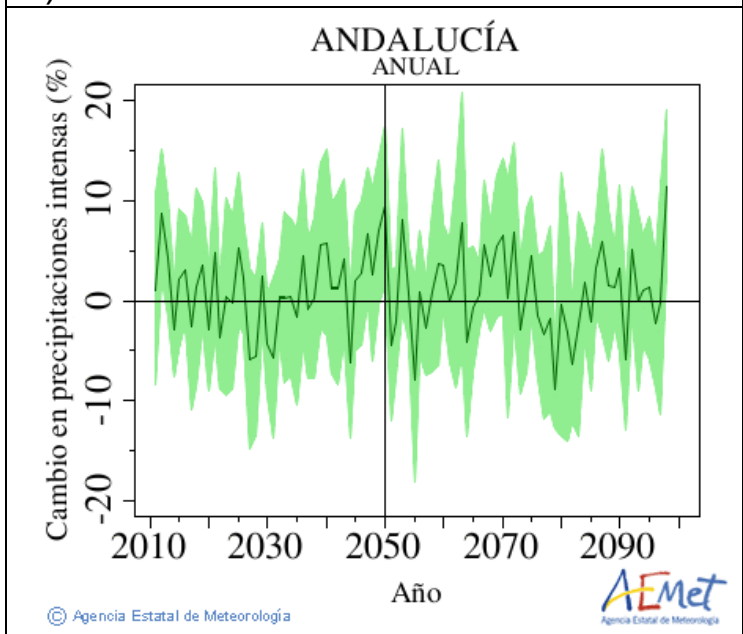

d)

e)

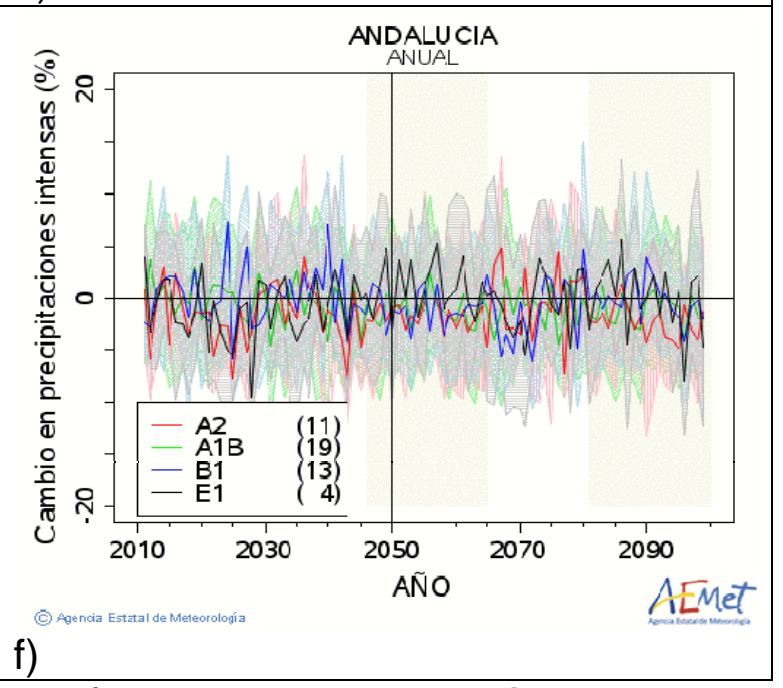

Figura A.3. Igual que la Figura A.1 para la evolución temporal del valor medio anual de los índices extremos (a)-(b) DL; (c)-(d) PS; (e)-(f) PI. 


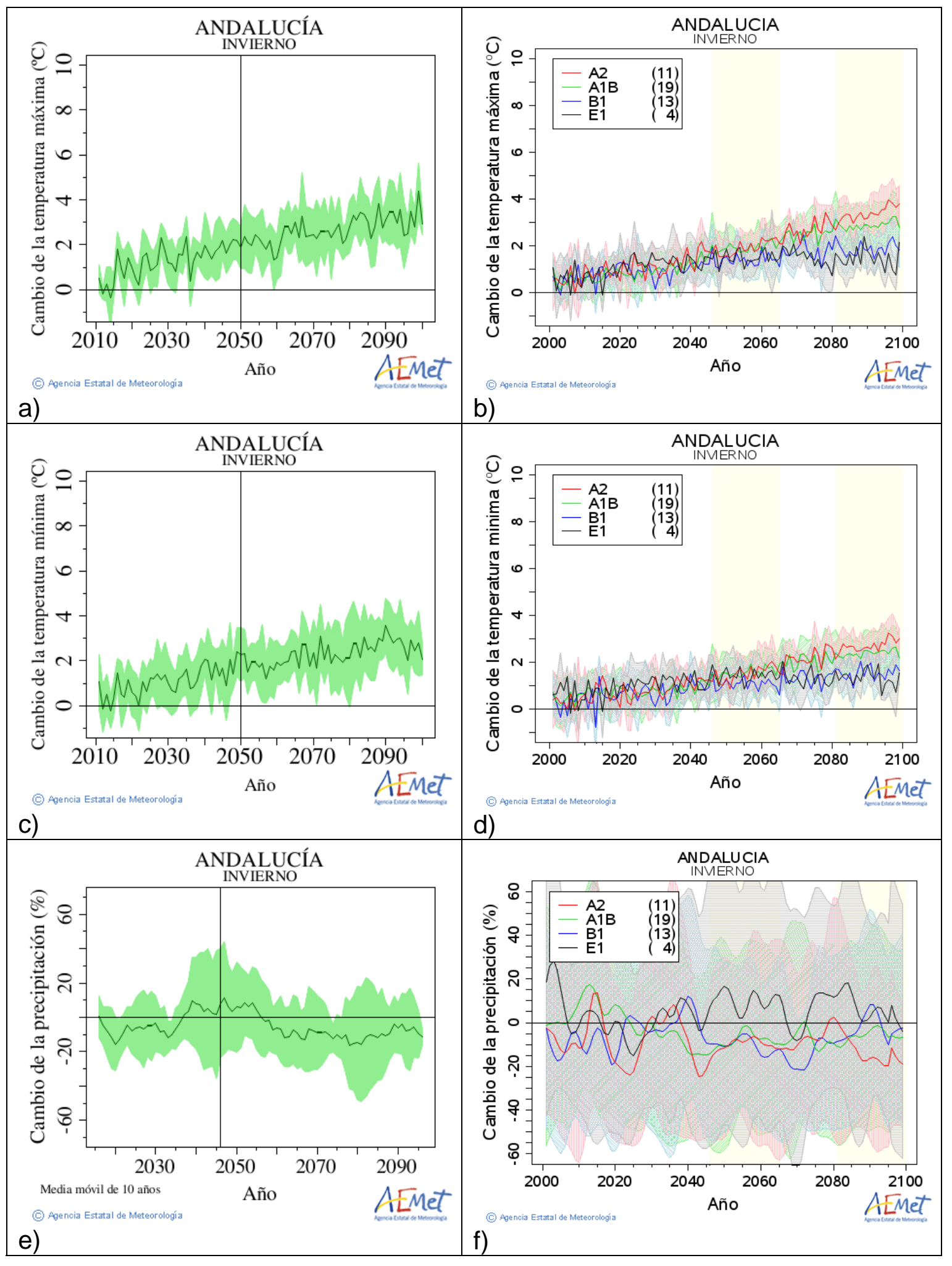

Figura A.4. Igual que la Figura A.1 para el valor medio de invierno. 

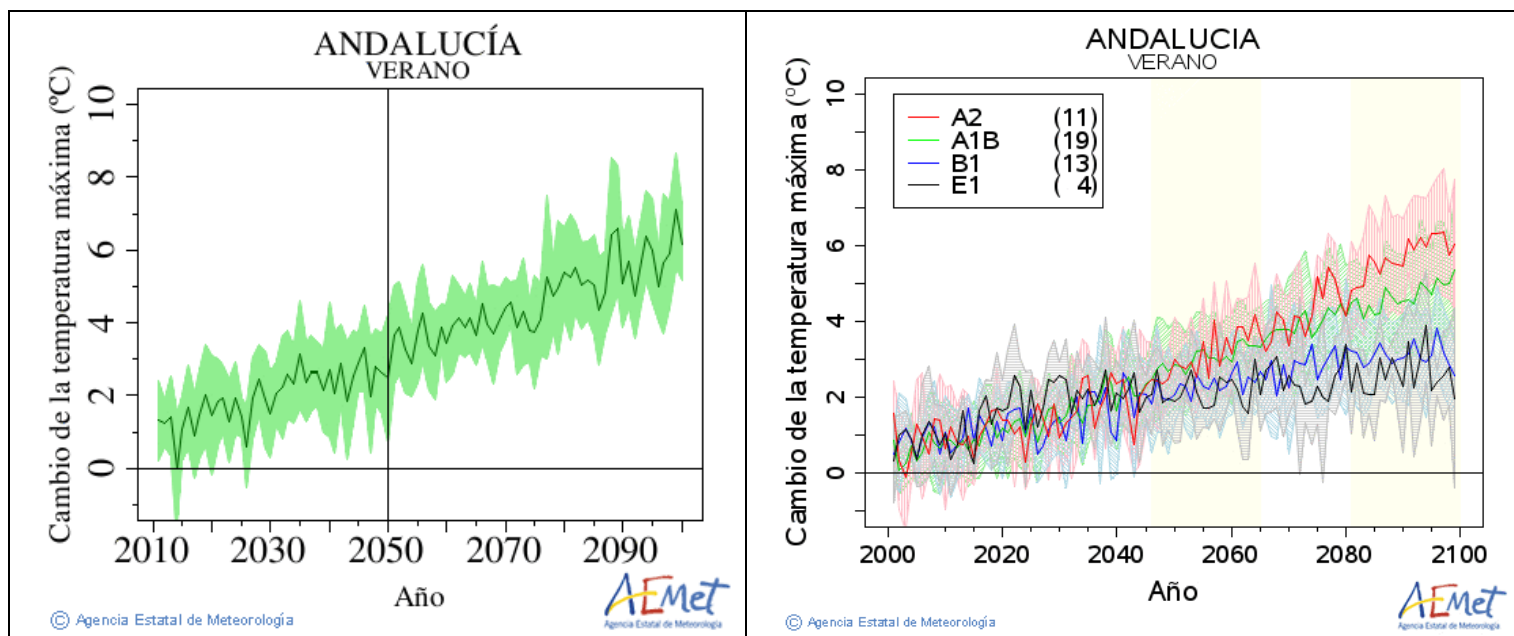

a)

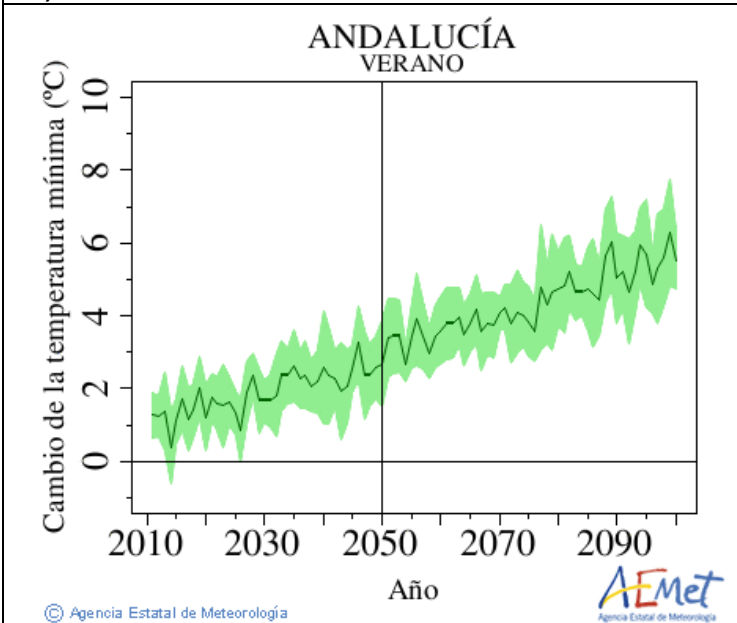

b)

c)

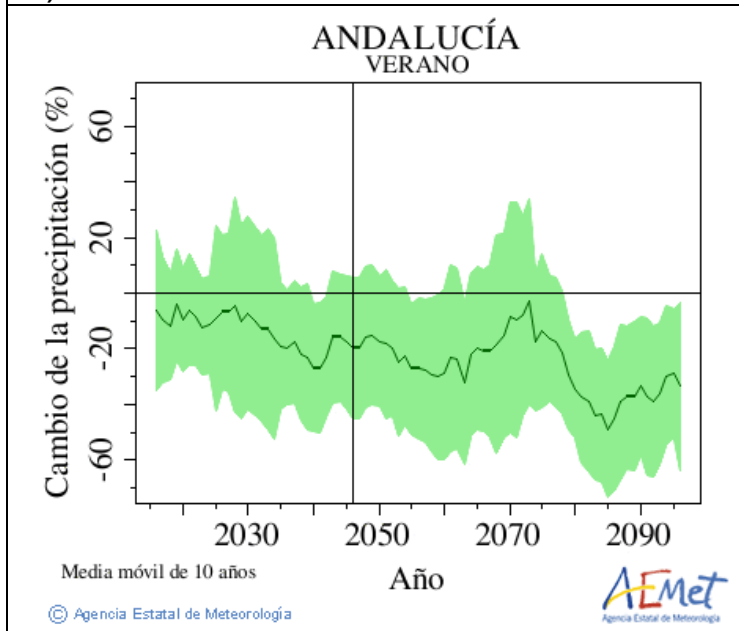

e)

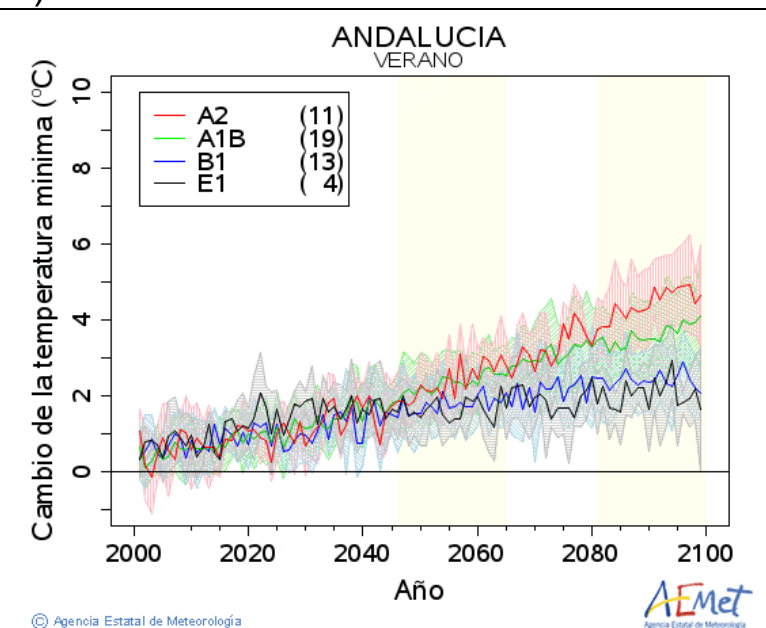

d)

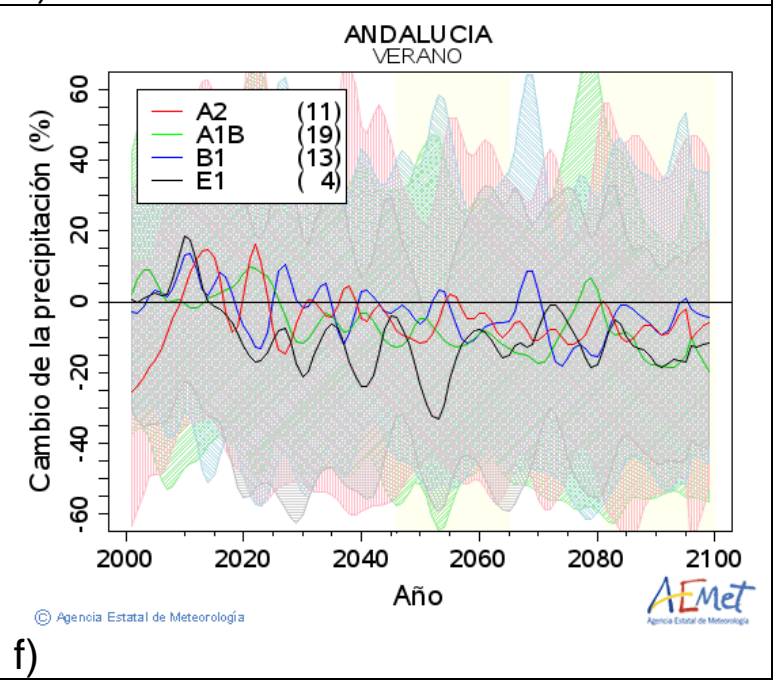

Figura A.5. Igual que la Figura A.1 para el valor medio de verano. 


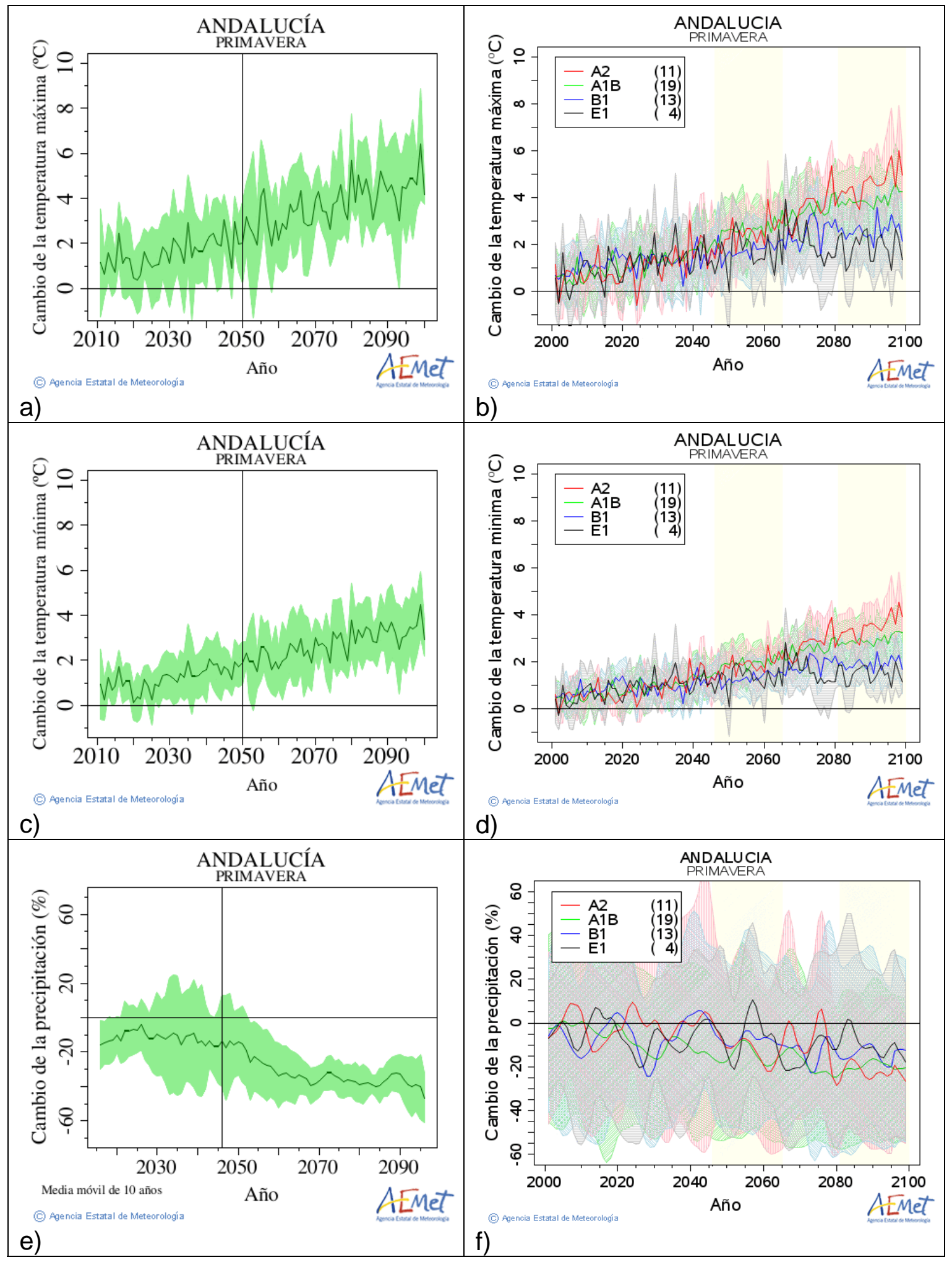

Figura A.6. Igual que la Figura A.1 para el valor medio de primavera. 

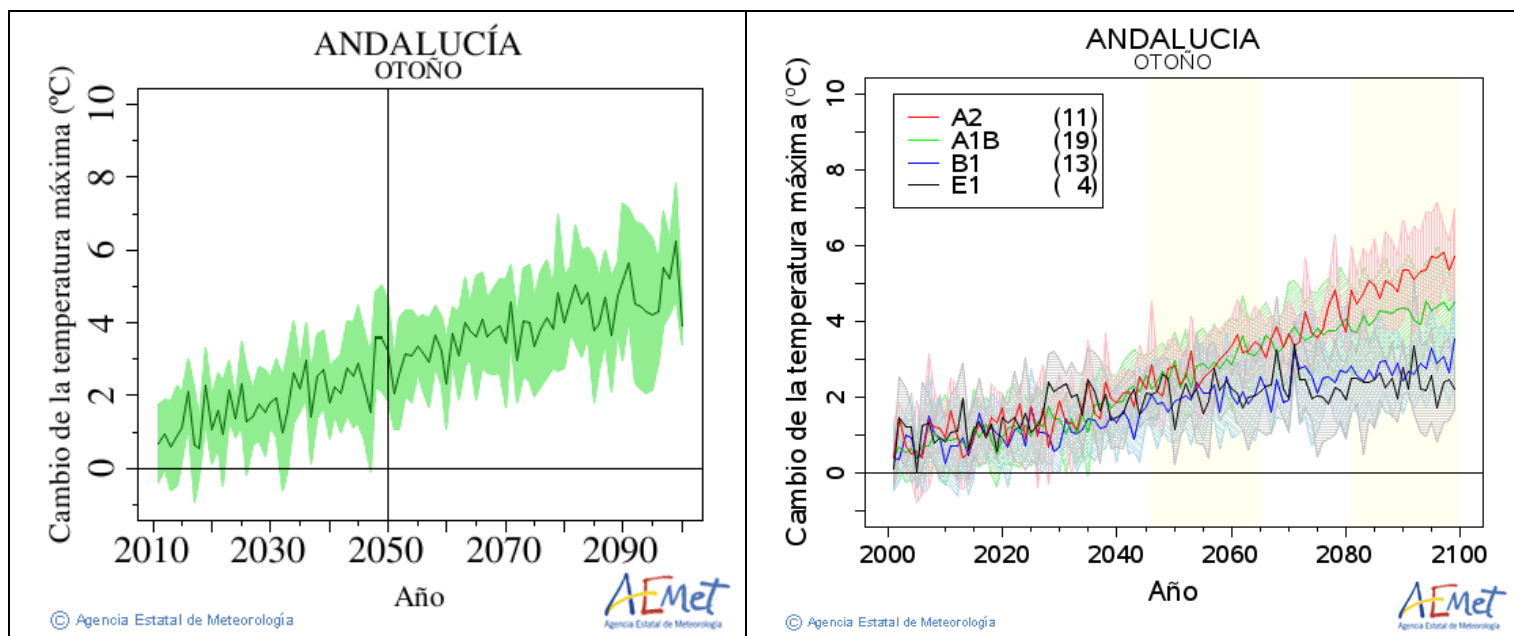

a)

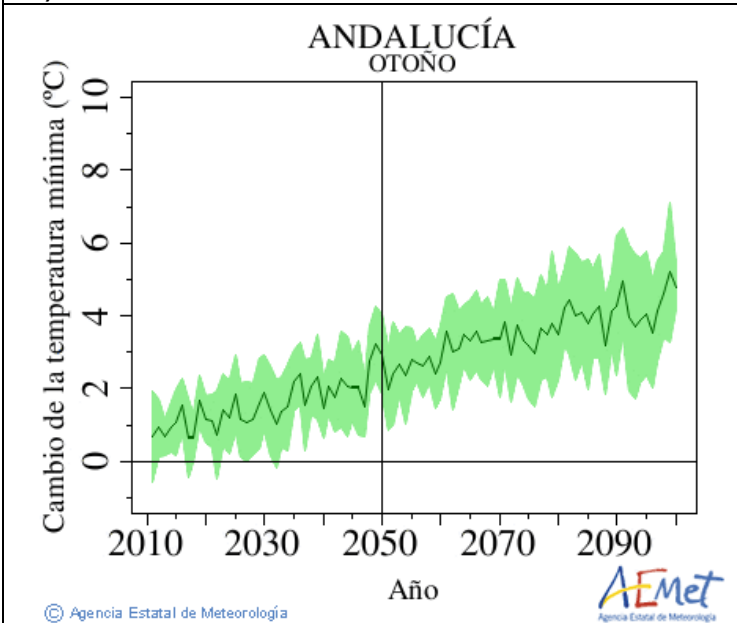

b)

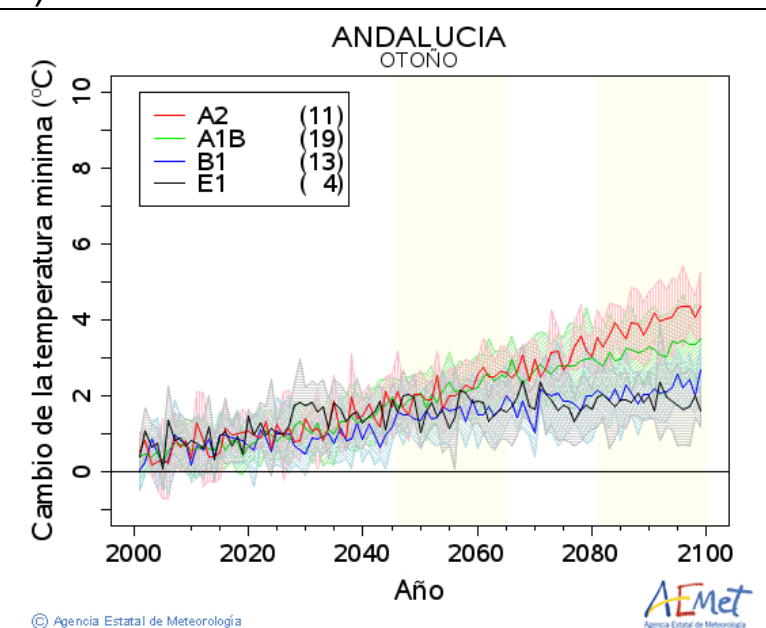

c)

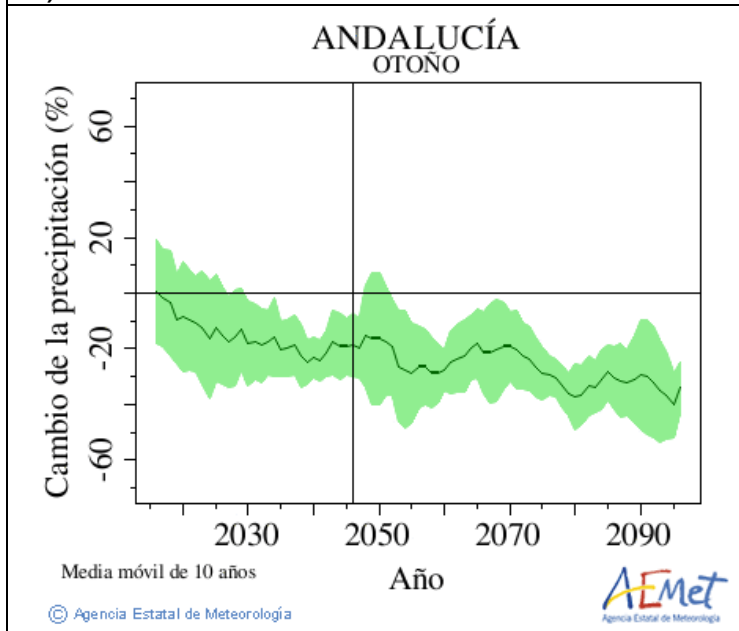

d)

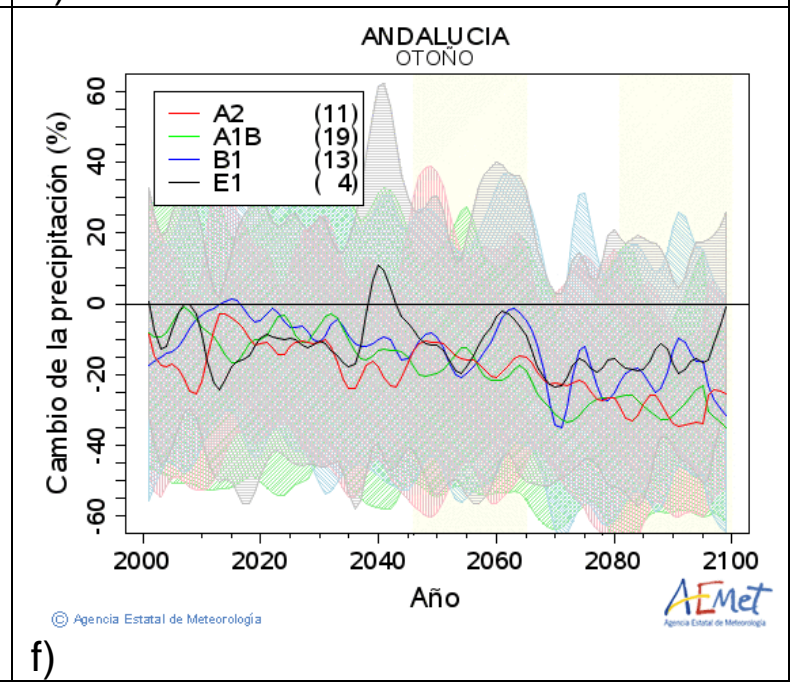

Figura A.7. Igual que la Figura A.1 para el valor medio de otoño. 
ARAGÓN

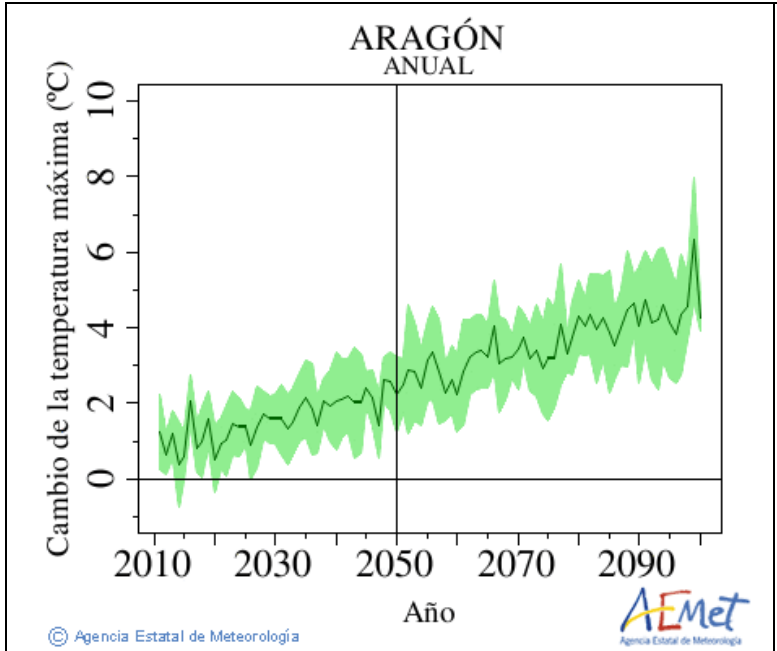

a)
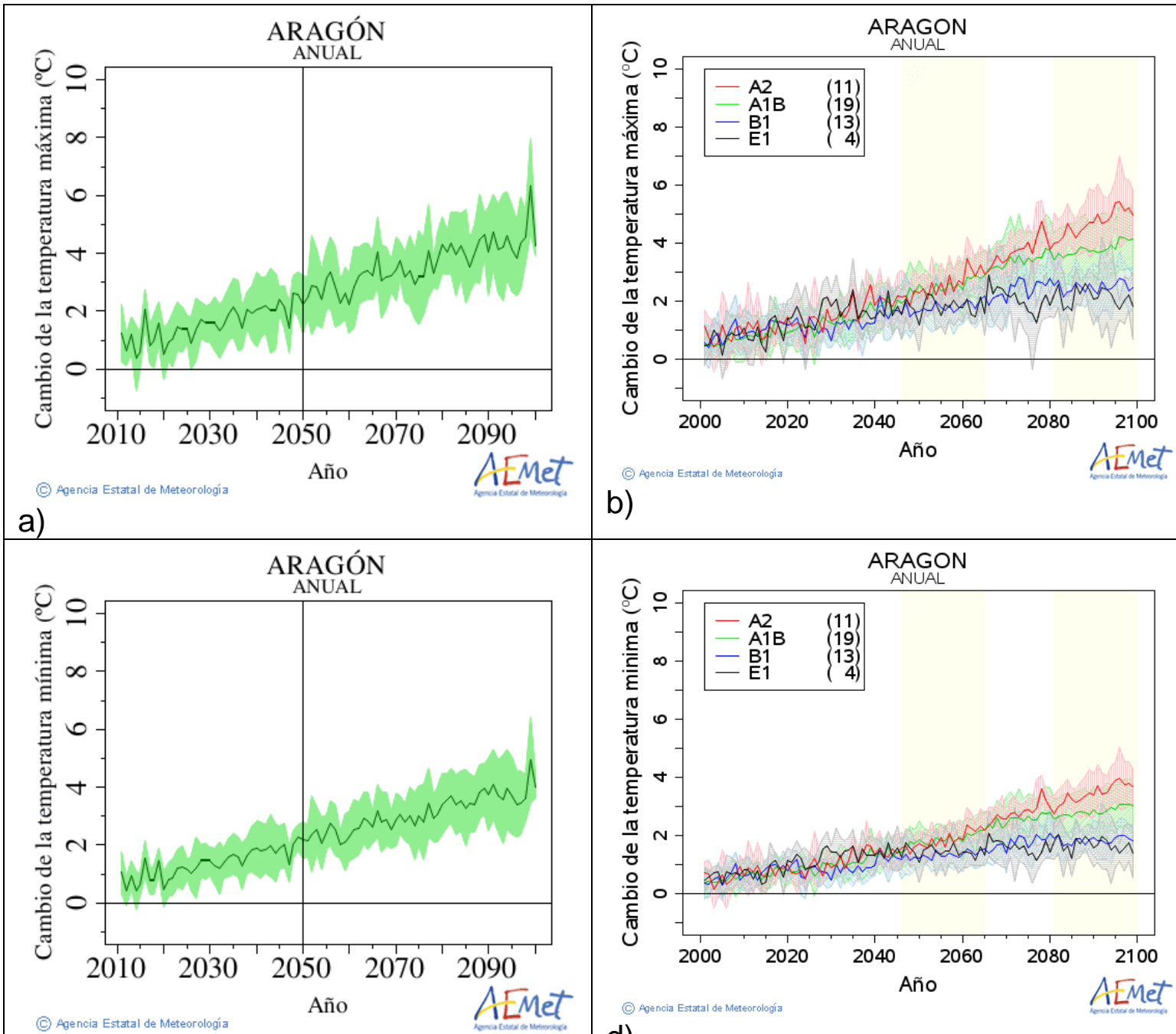

b)

c)

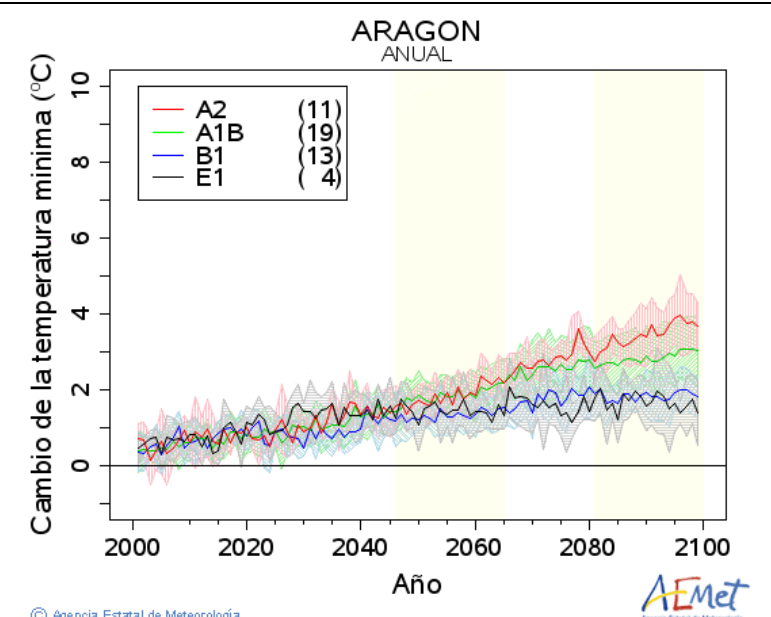

d)

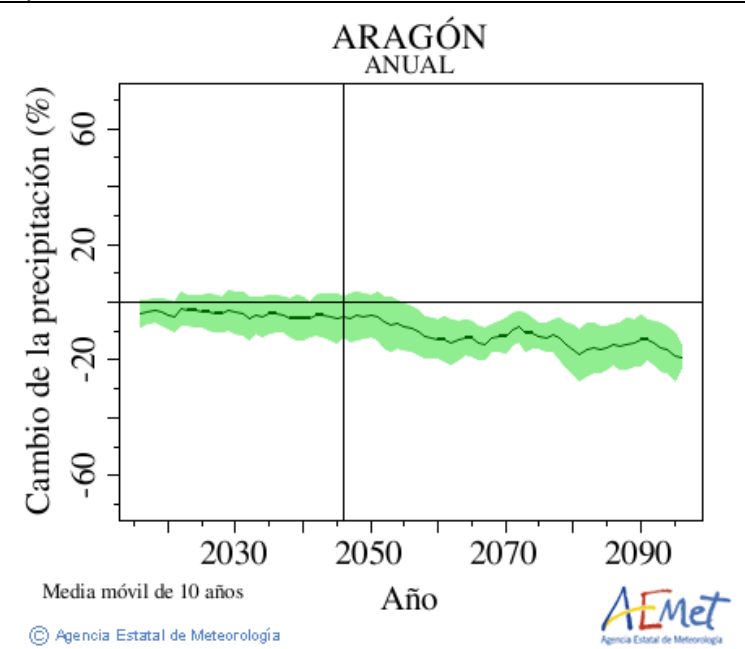

e)

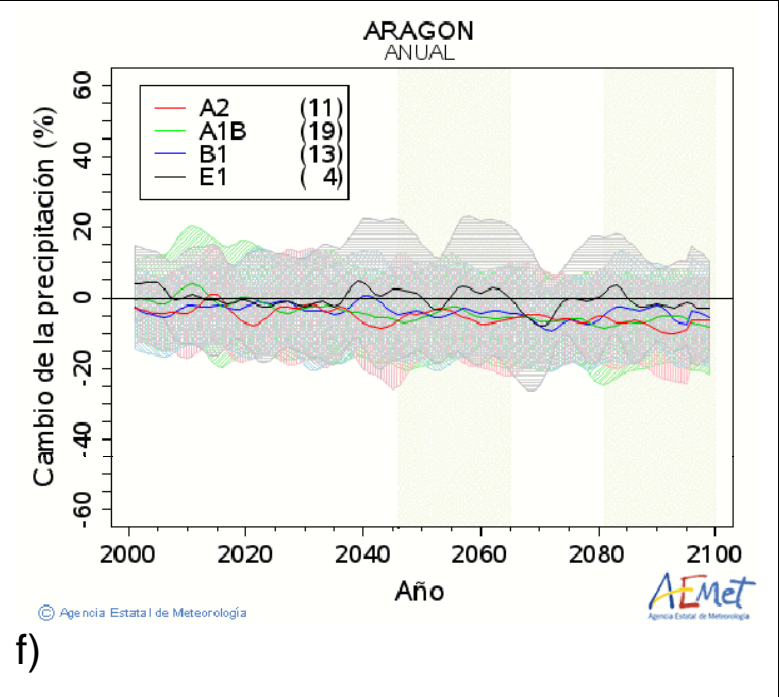

Figura A.8. Igual que la Figura A.1 para la Comunidad Autónoma de Aragón. 


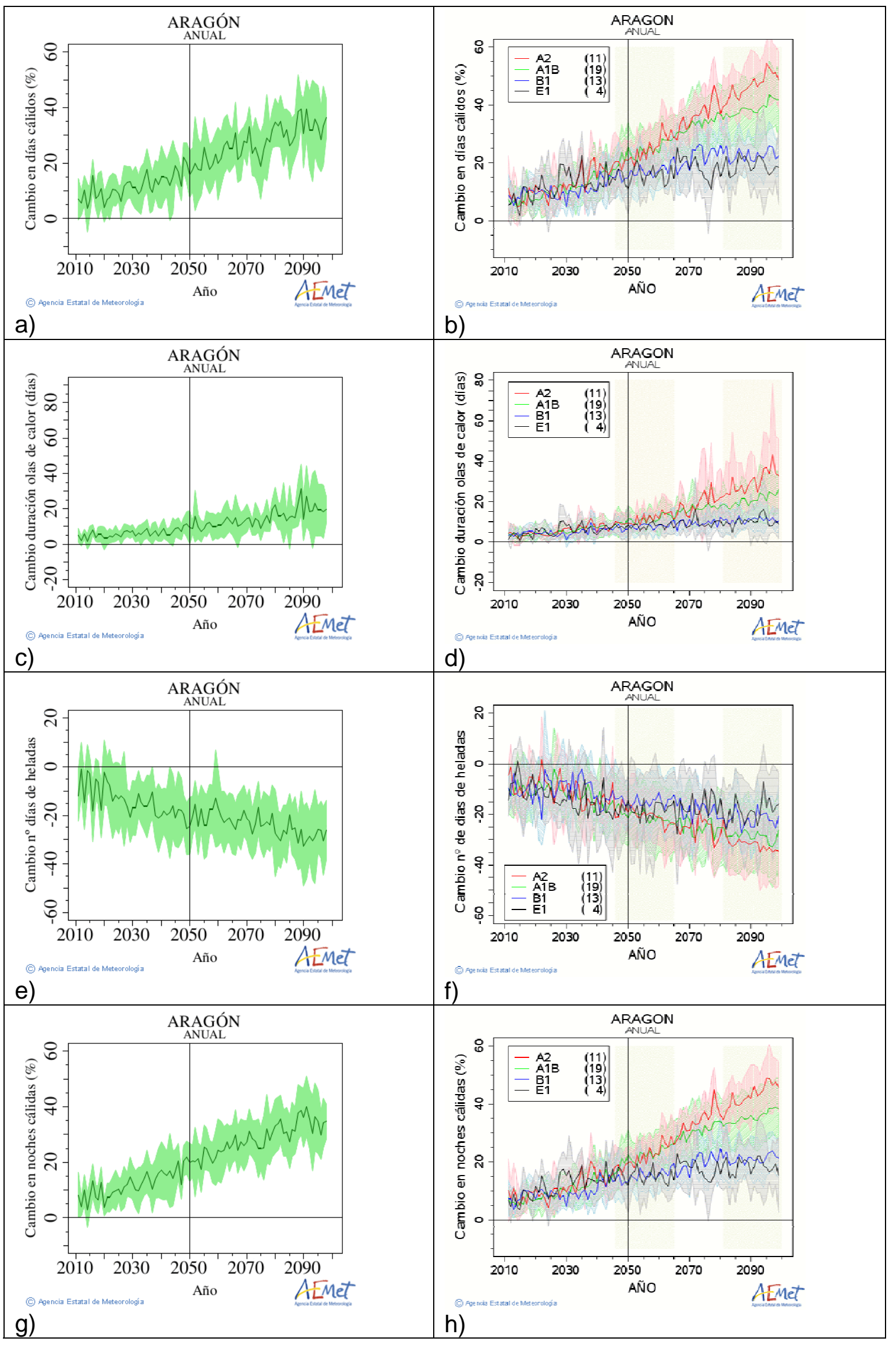

Figura A.9. Igual que la Figura A.2 para la Comunidad Autónoma de Aragón. 

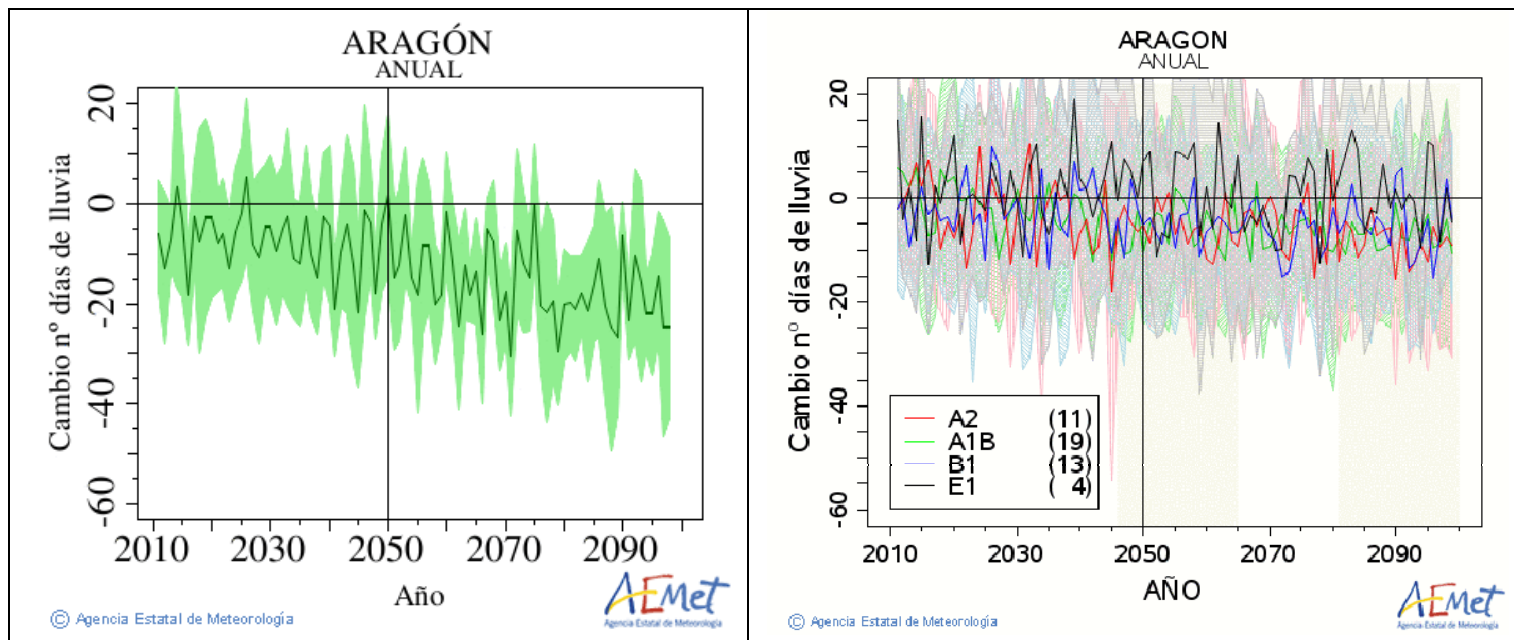

a)

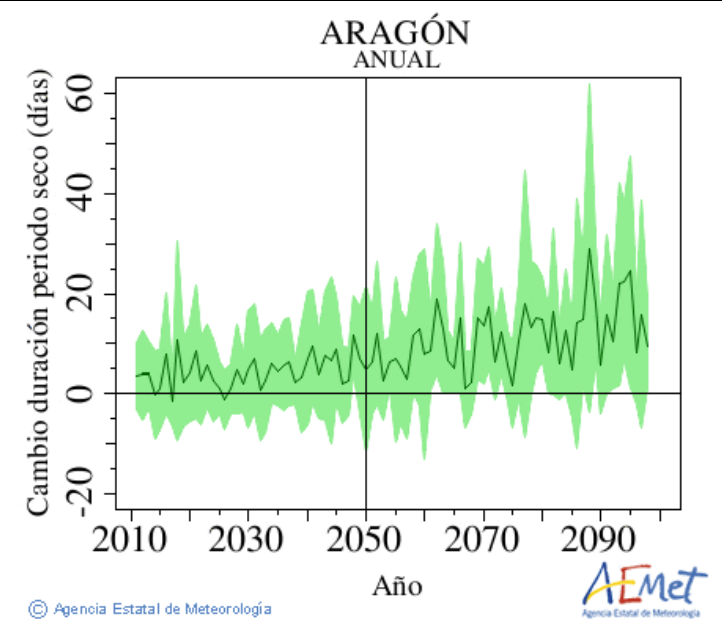

b)

c)

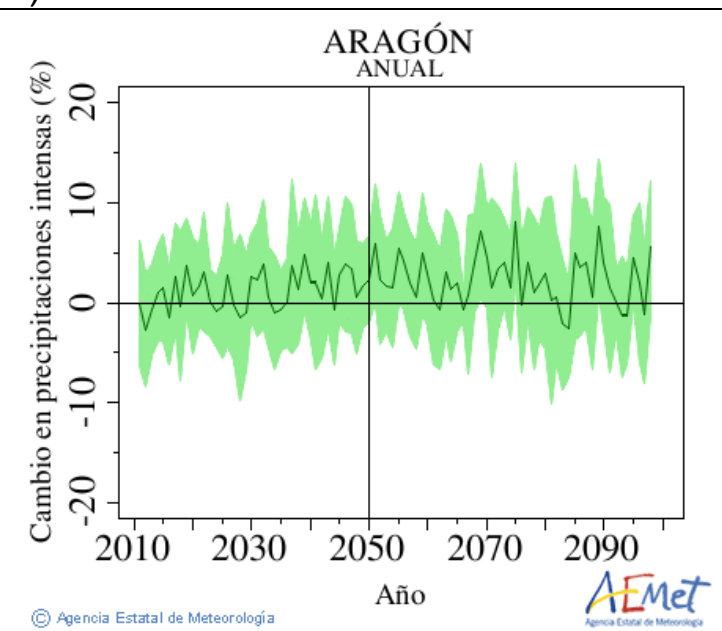

e)

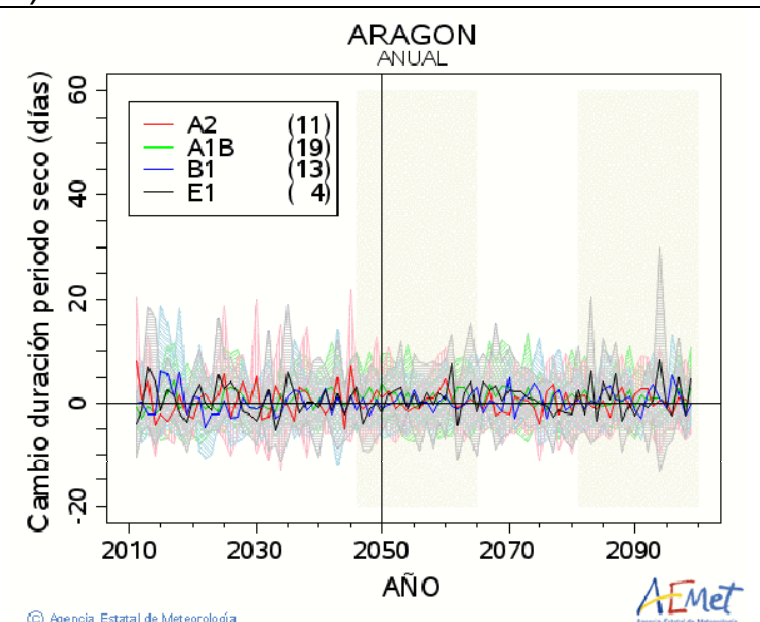

d)

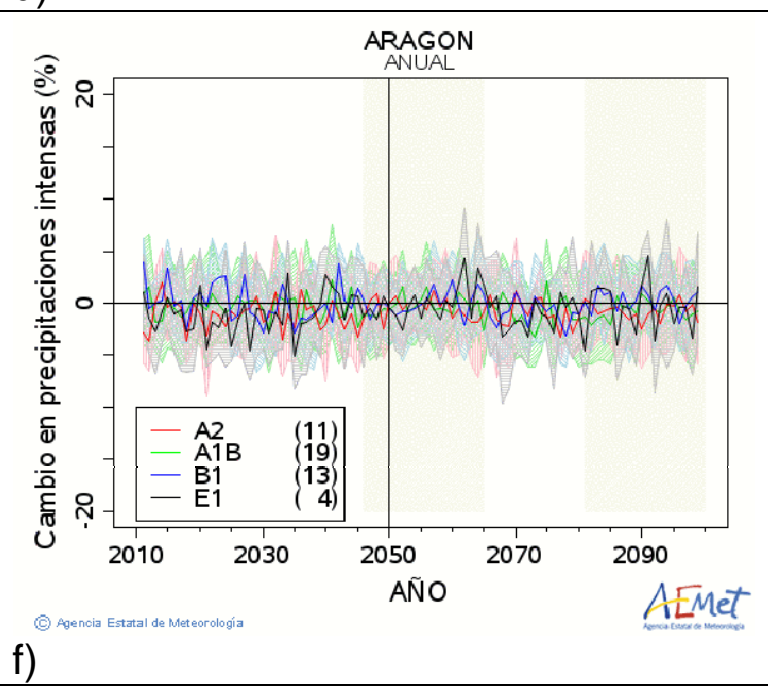

Figura A.10. Igual que la Figura A.3 para la Comunidad Autónoma de Aragón. 


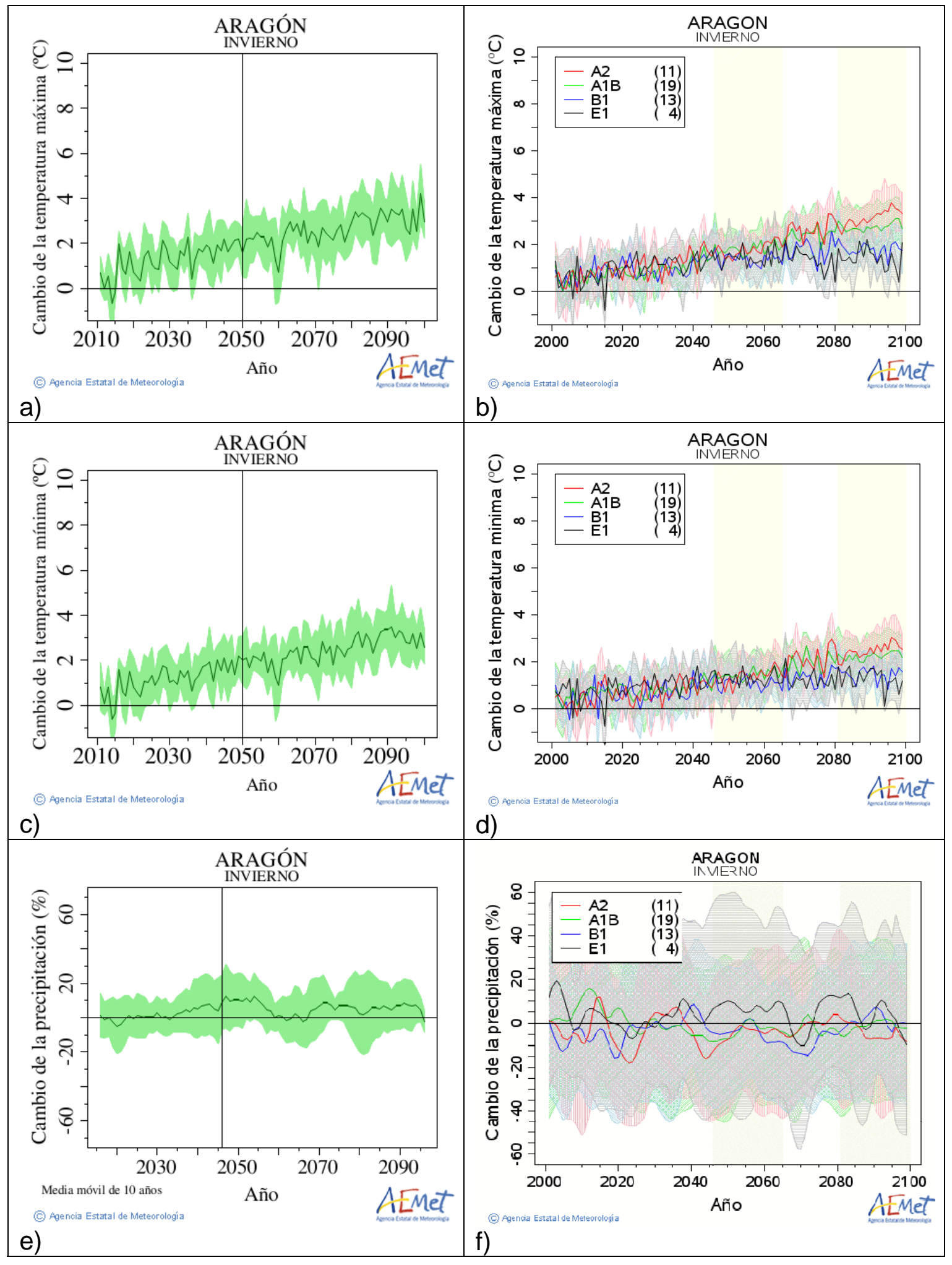

Figura A.11. Igual que la Figura A.4 para la Comunidad Autónoma de Aragón. 

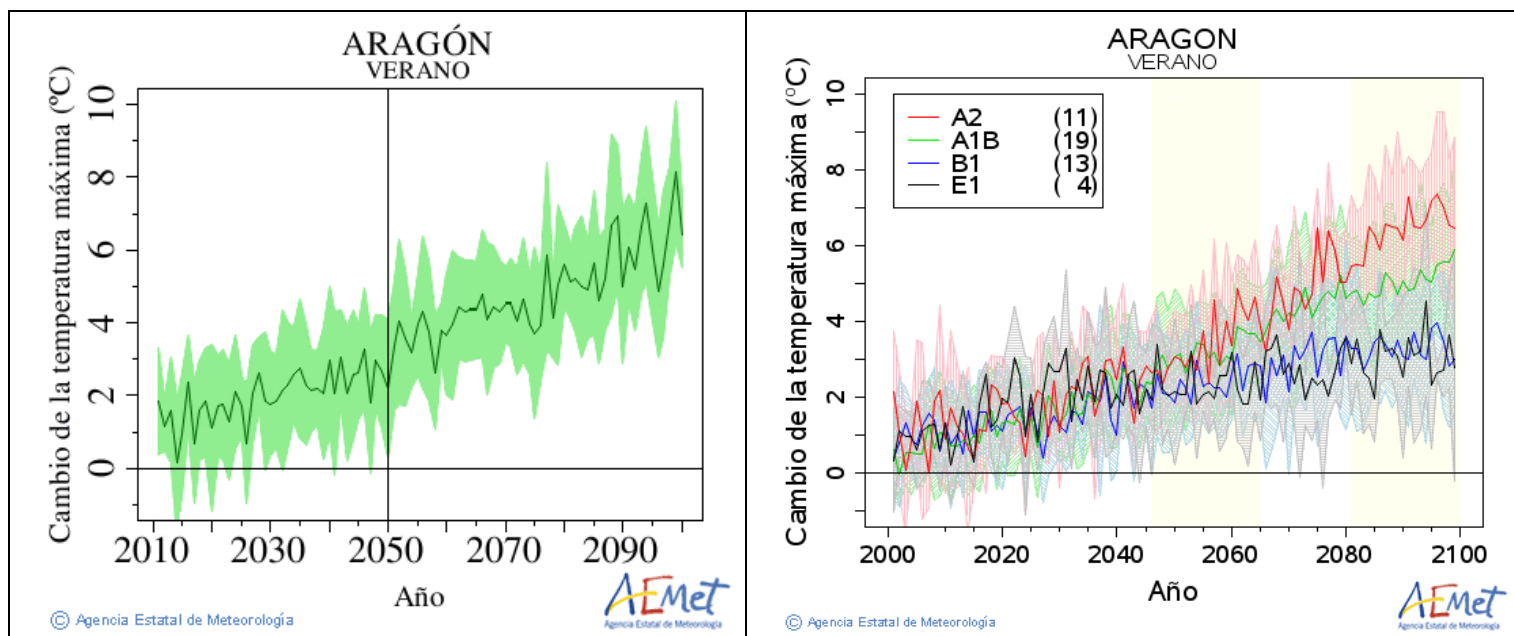

a)

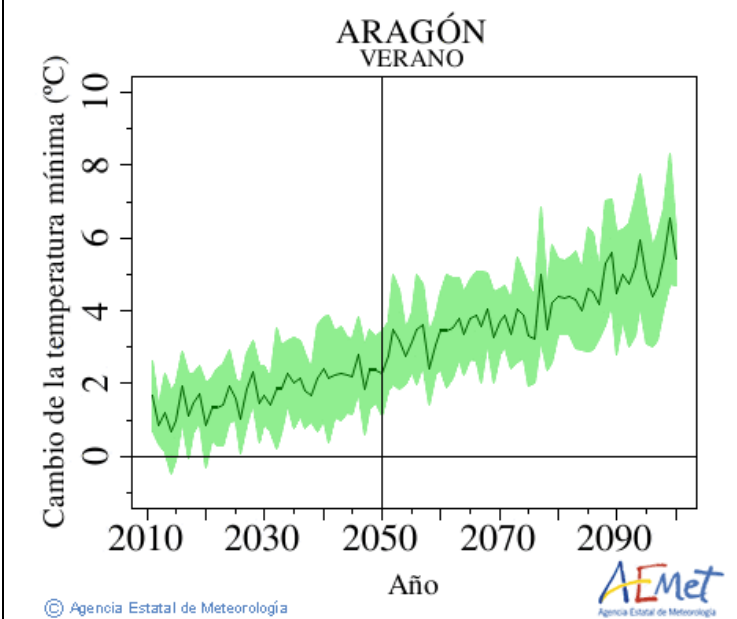

b)

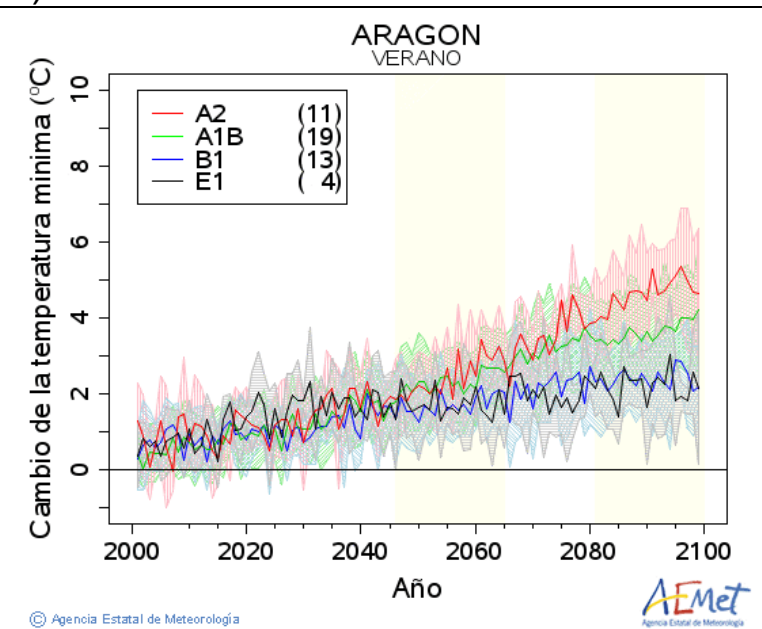

c)

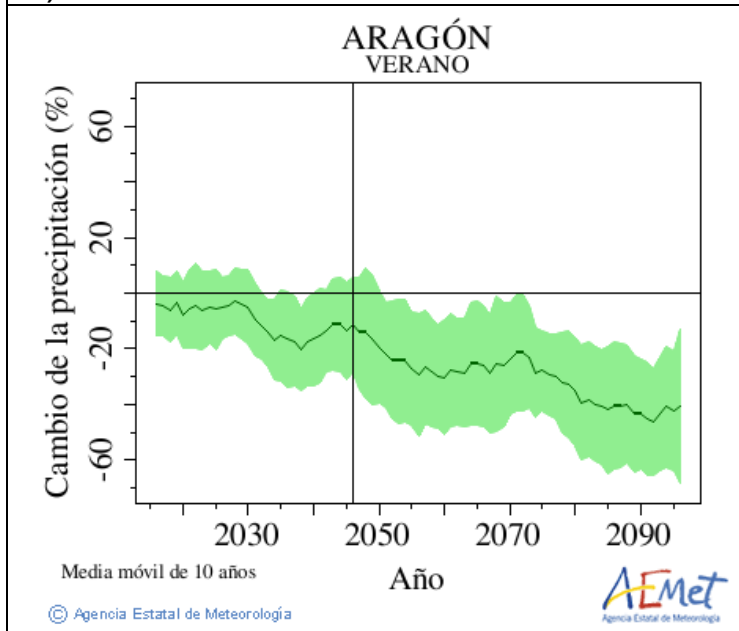

d)

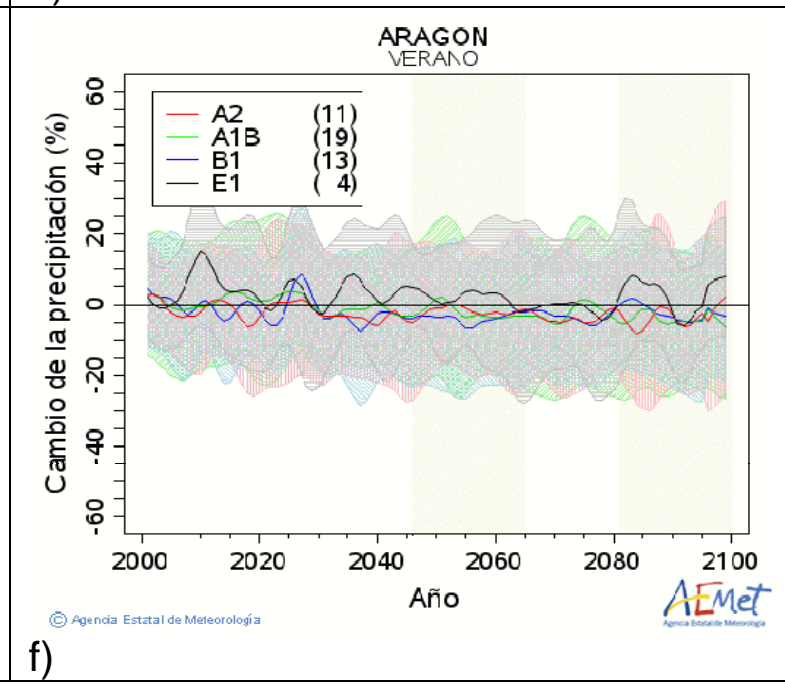

Figura A.12. Igual que la Figura A.5 para la Comunidad Autónoma de Aragón. 


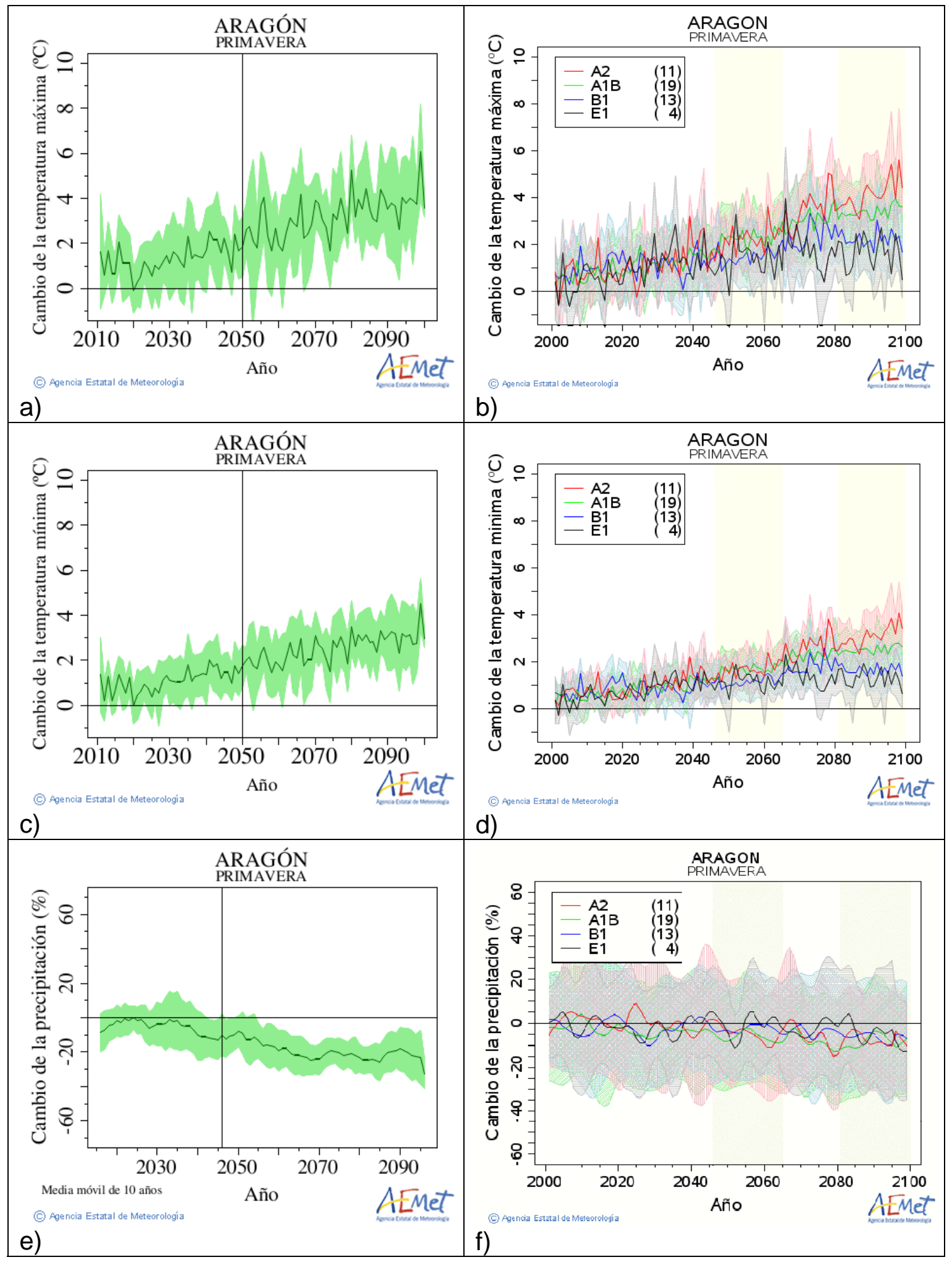

Figura A.13. Igual que la Figura A.6 para la Comunidad Autónoma de Aragón. 

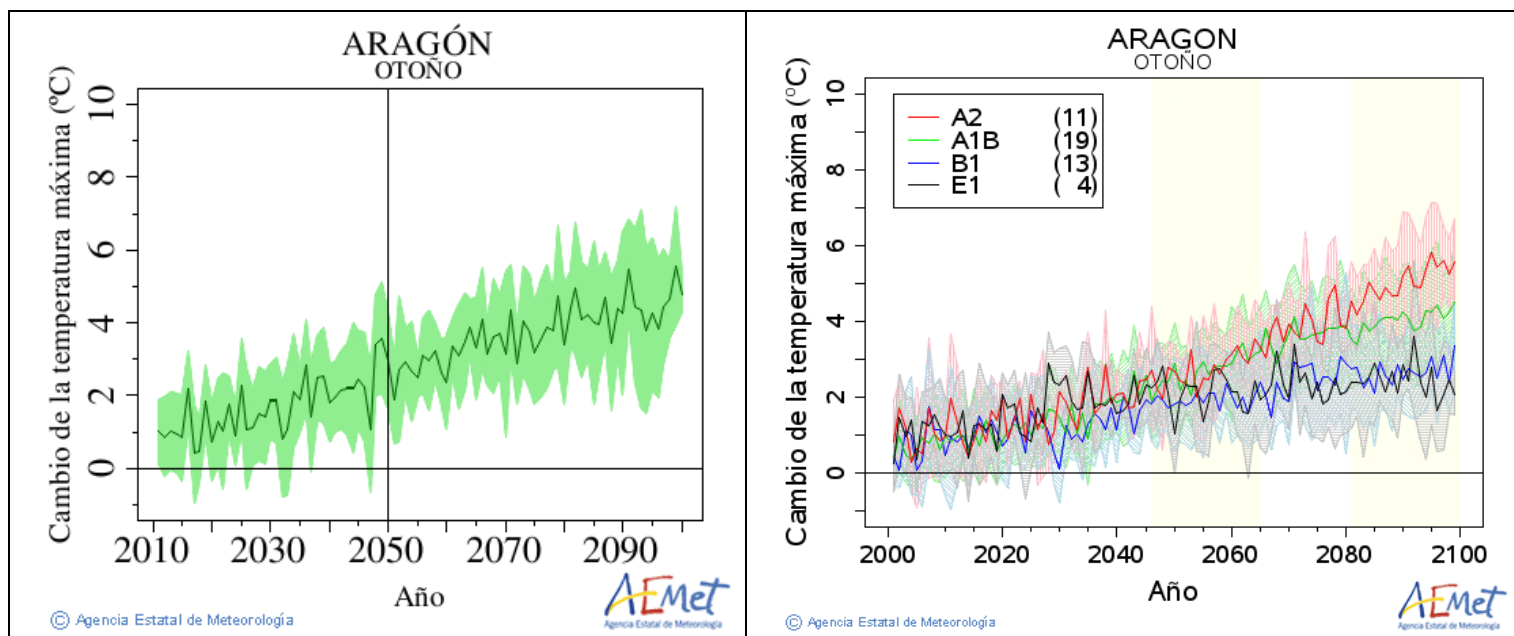

a)

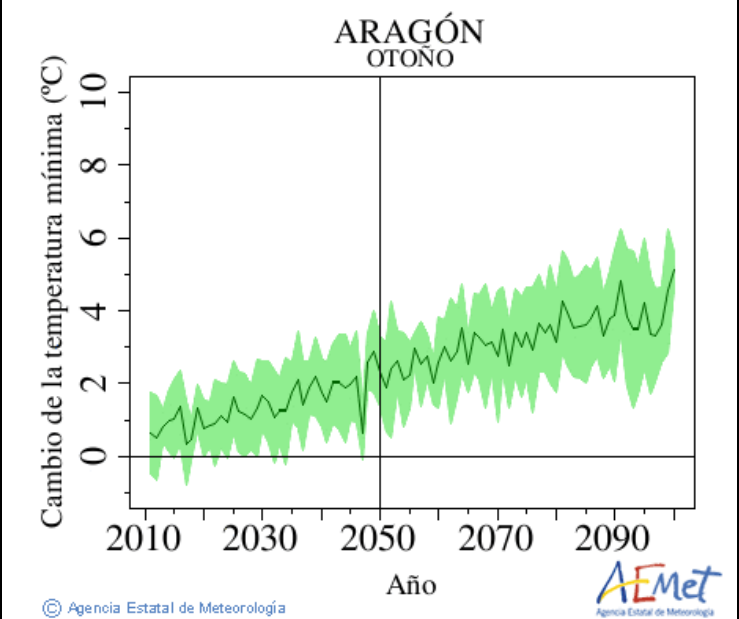

b)

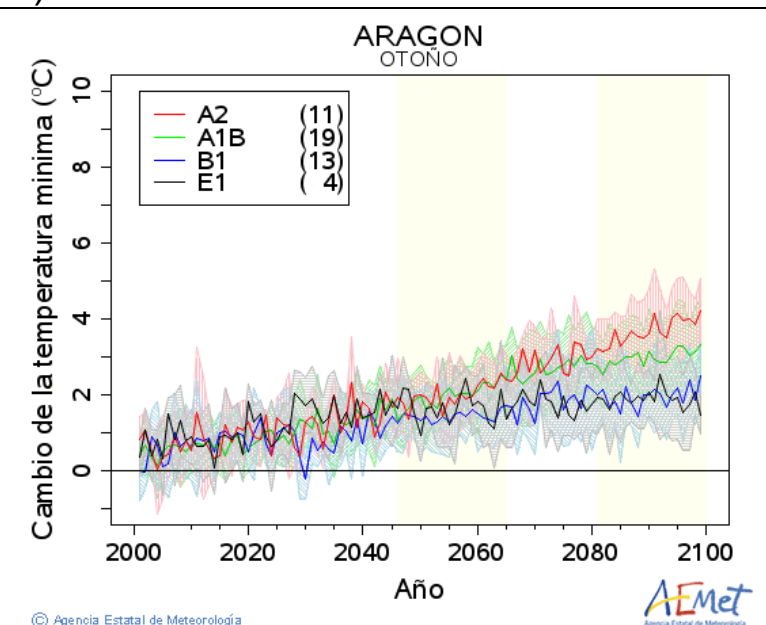

c)

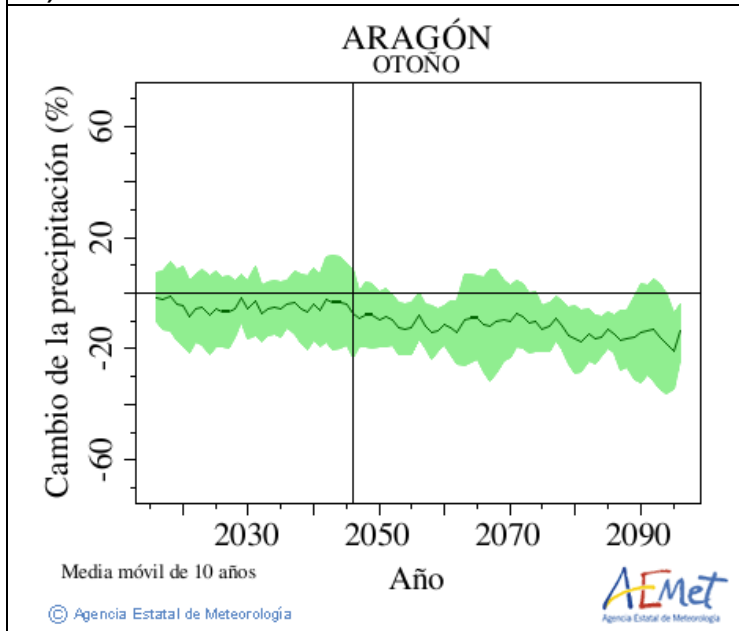

d)

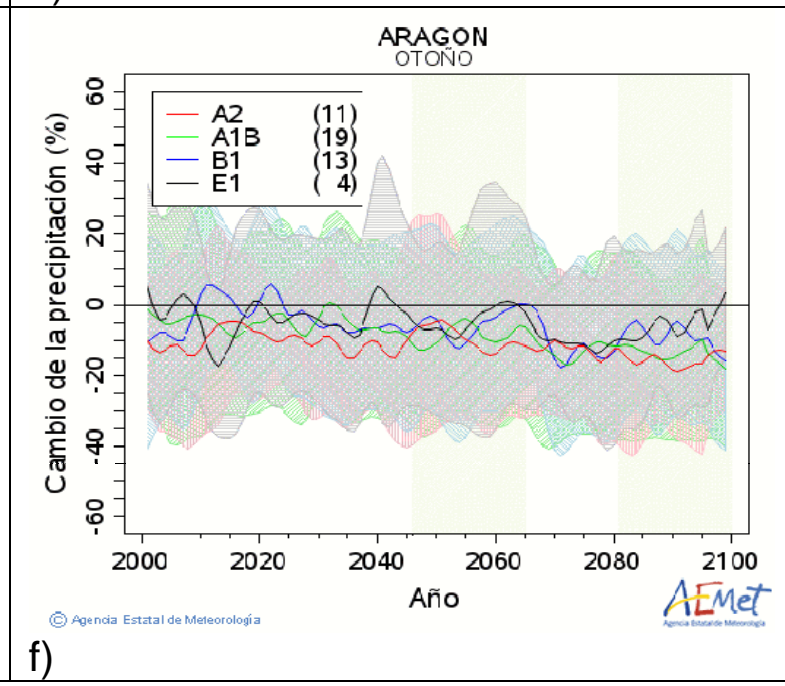

Figura A.14. Igual que la Figura A.7 para la Comunidad Autónoma de Aragón. 
PRINCIPADO DE ASTURIAS

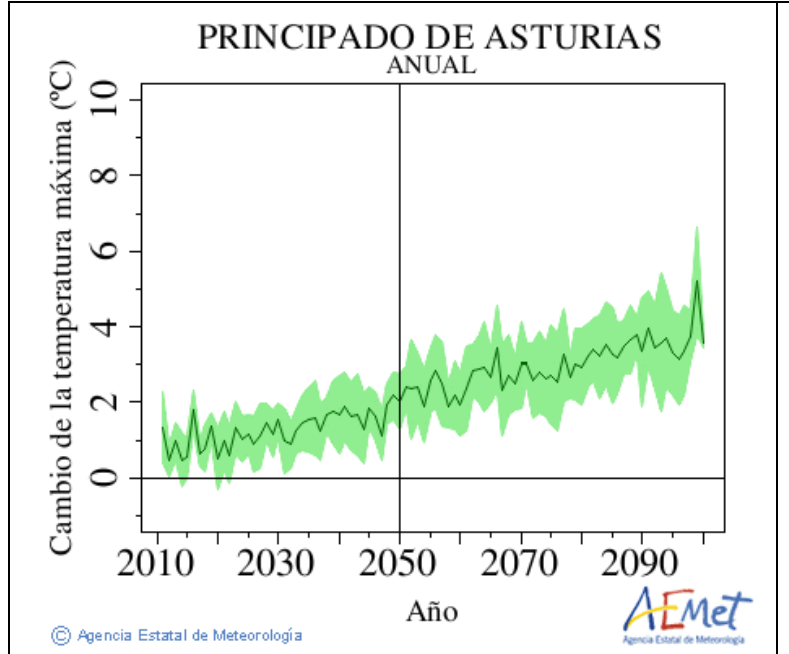

a)
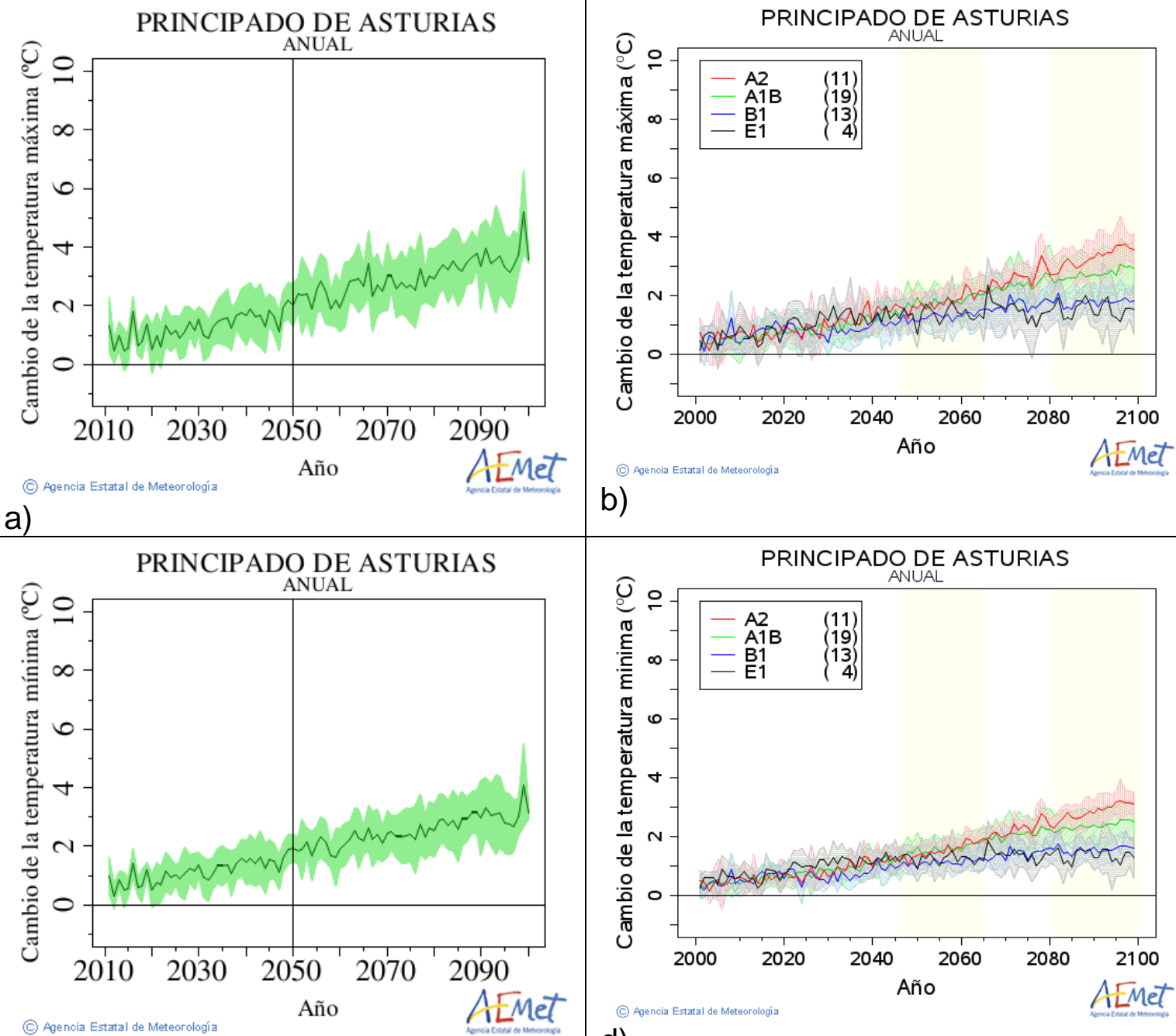

b)

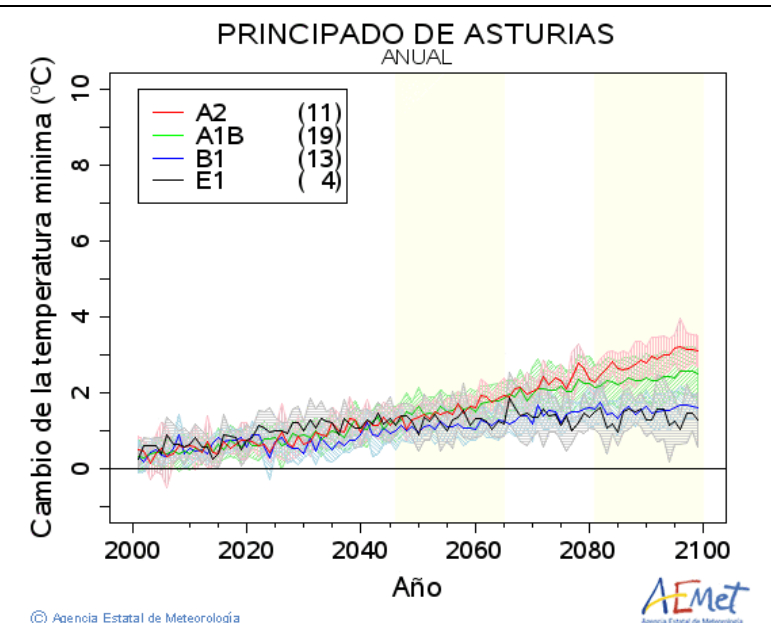

c)

d)

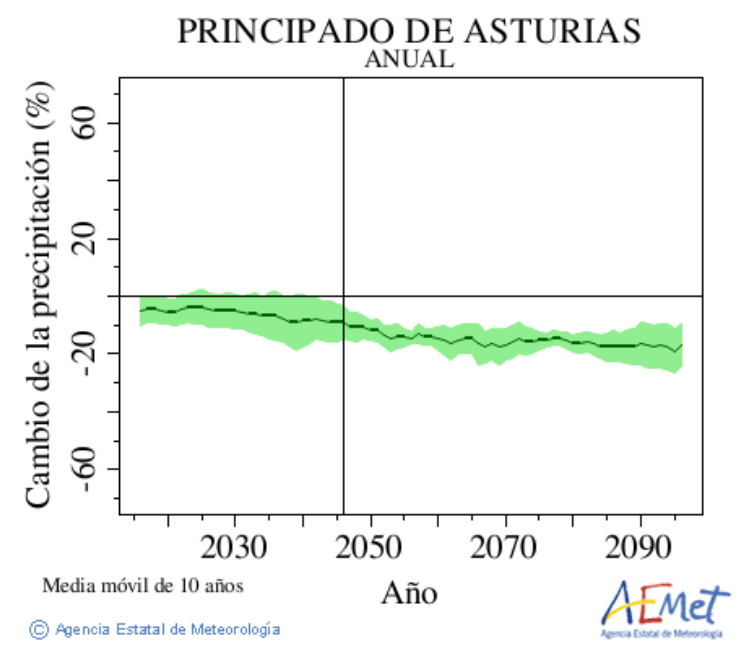

e)

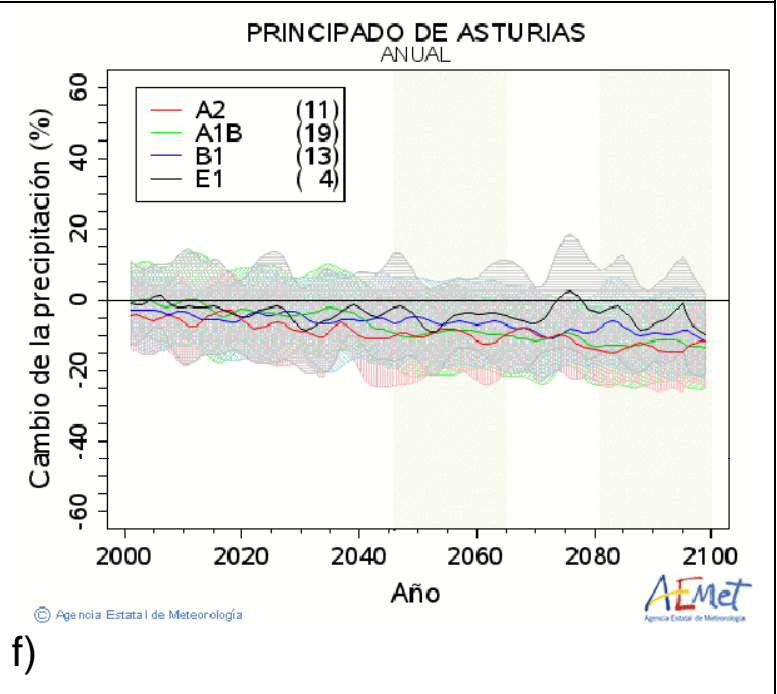

Figura A.15. Igual que la Figura A.1 para el Principado de Asturias. 


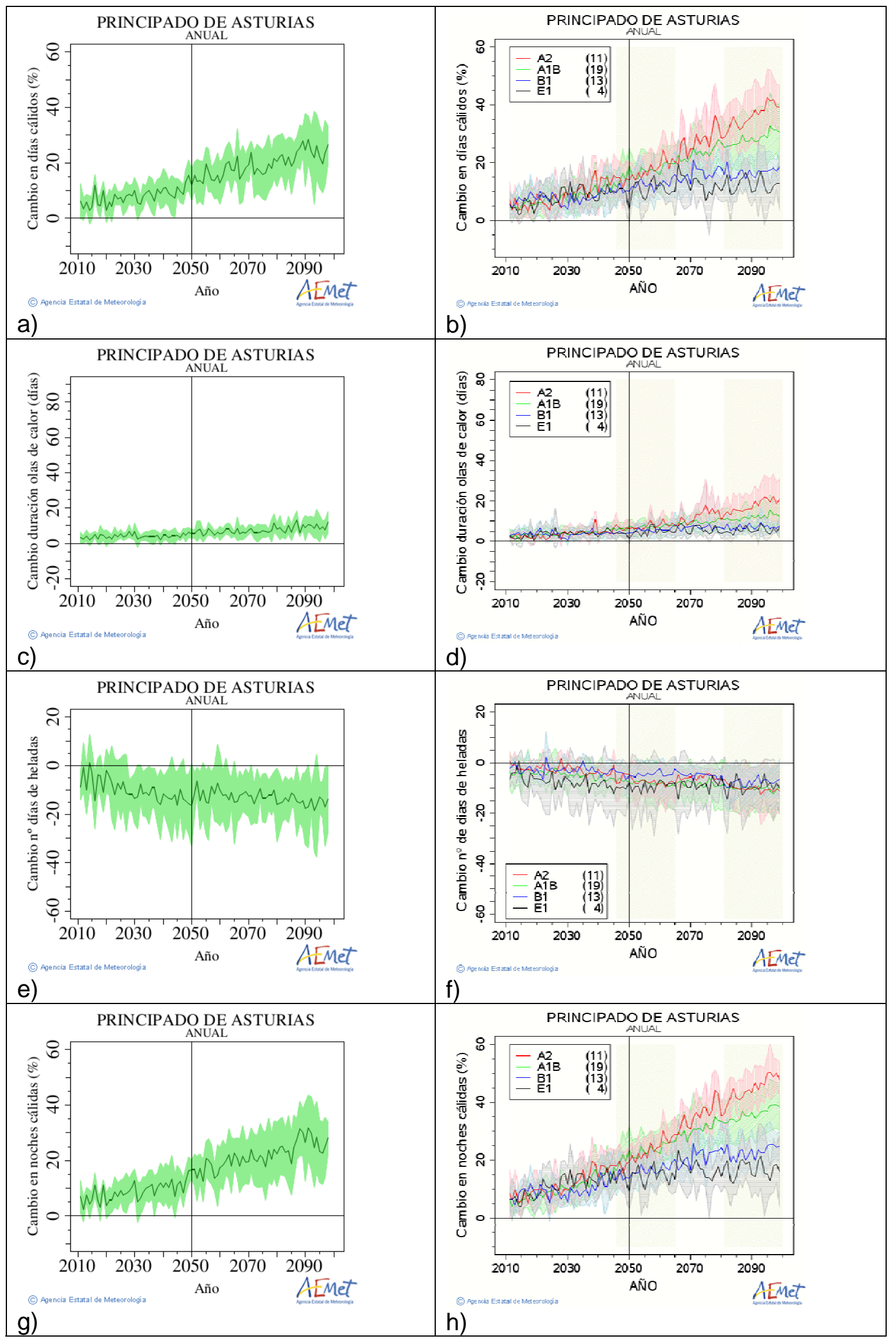

Figura A.16. Igual que la Figura A.2 para el Principado de Asturias. 

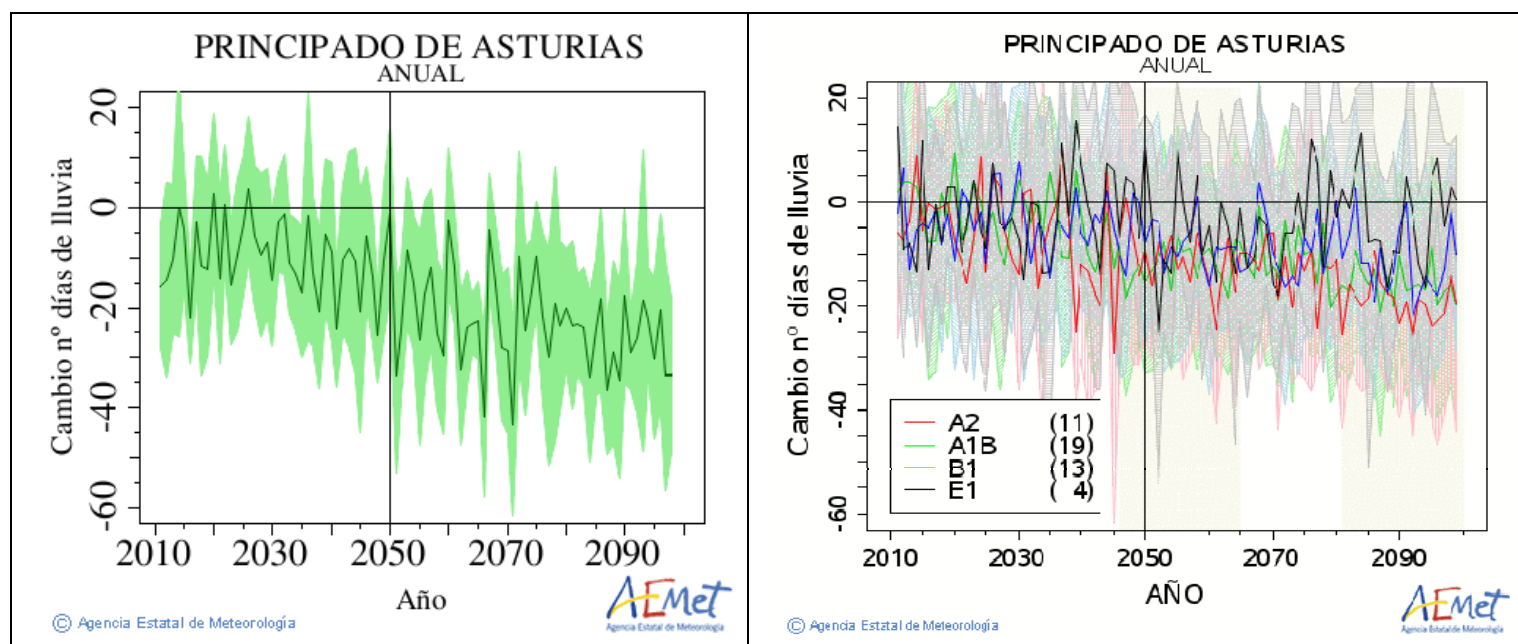

a)

b)
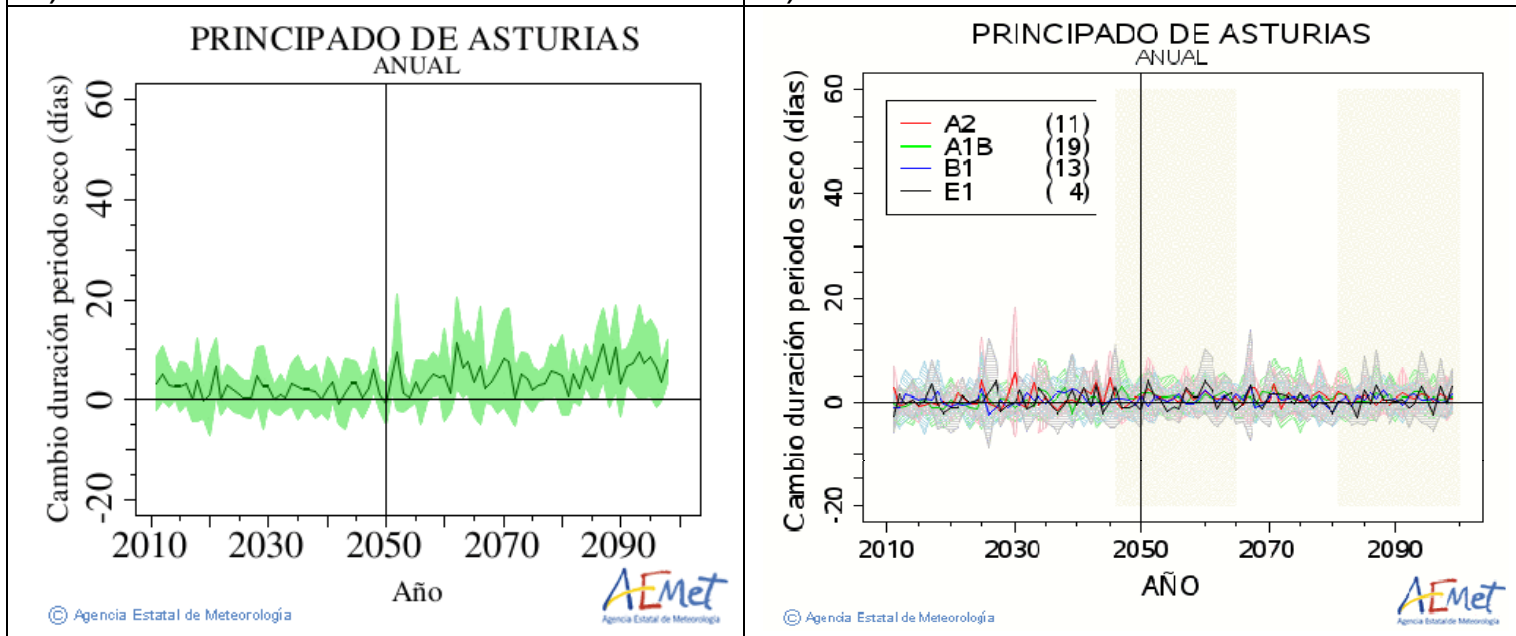

c)

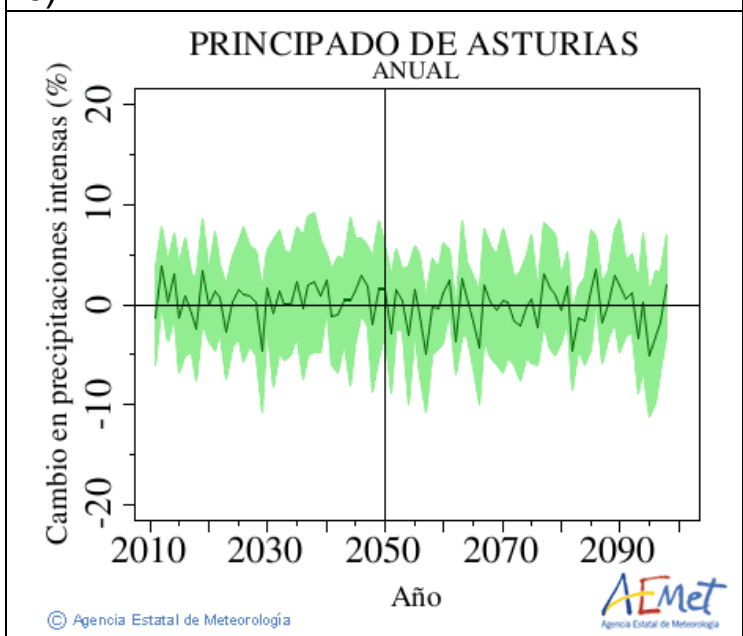

e)

d)

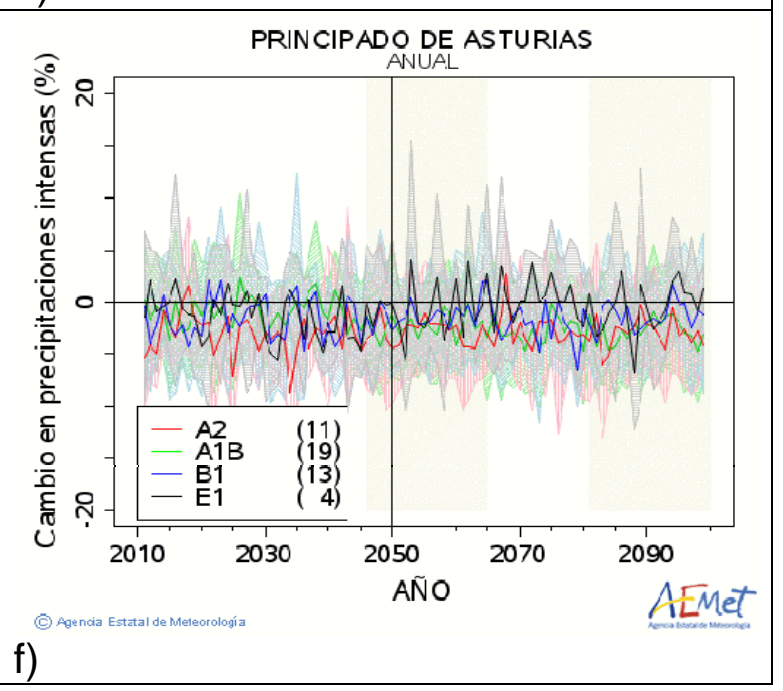

Figura A.17. Igual que la Figura A.3 para el Principado de Asturias. 

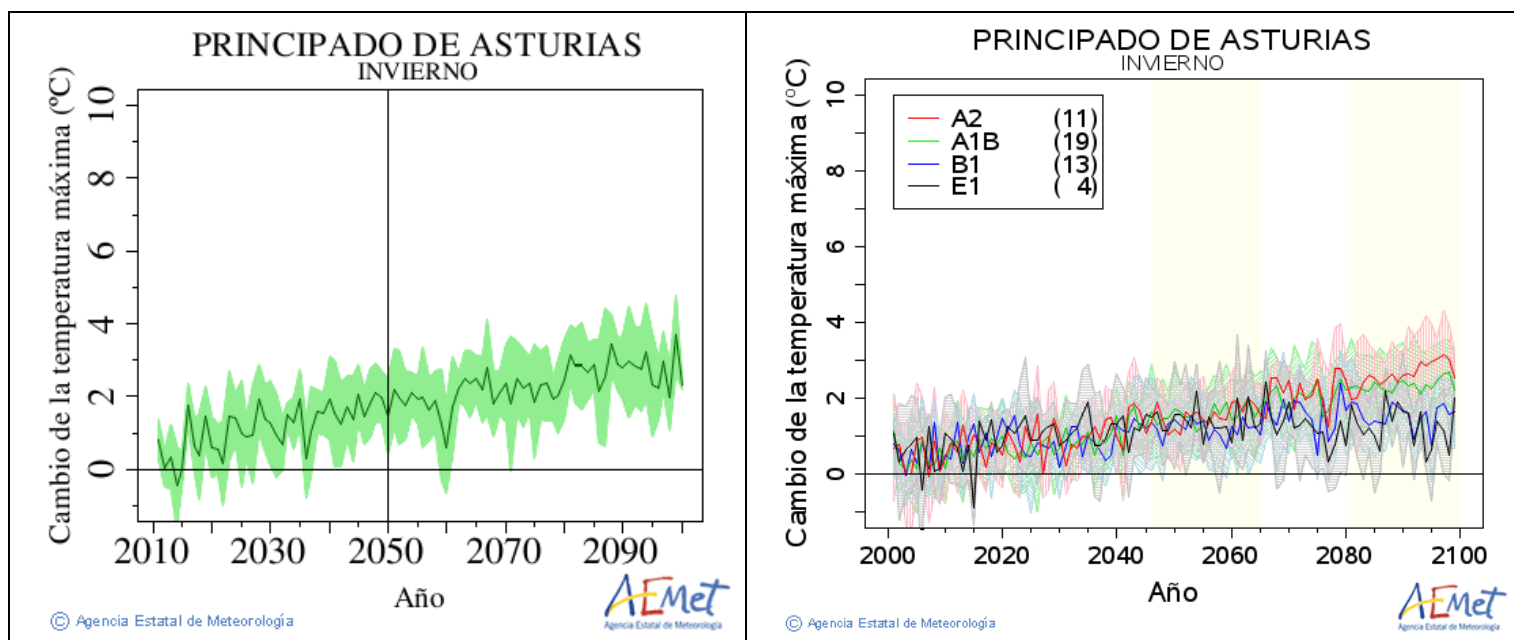

a)

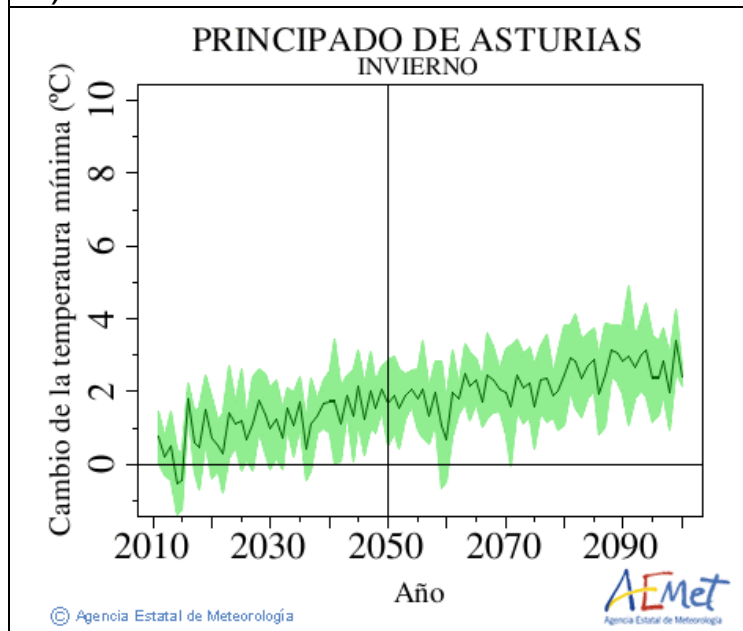

b)

c)

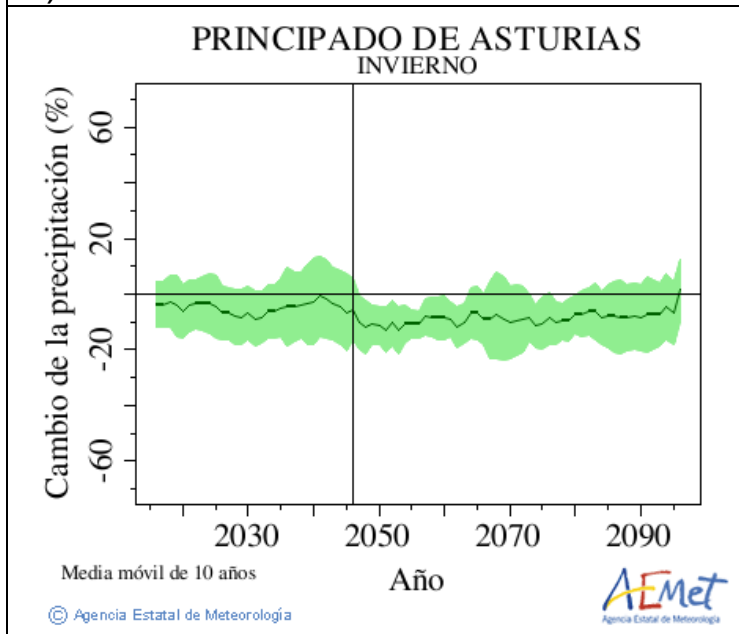

e)

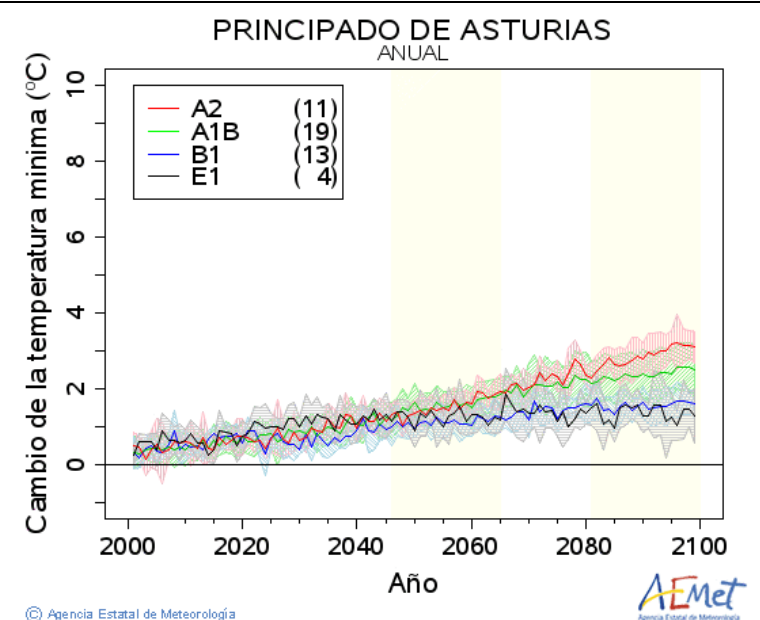

d)

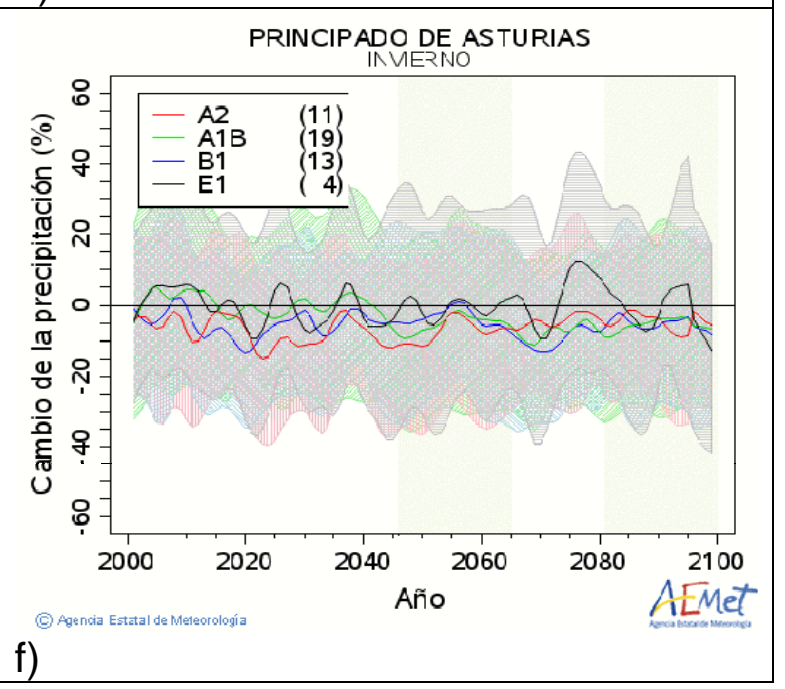

Figura A.18. Igual que la Figura A.4 para el Principado de Asturias. 

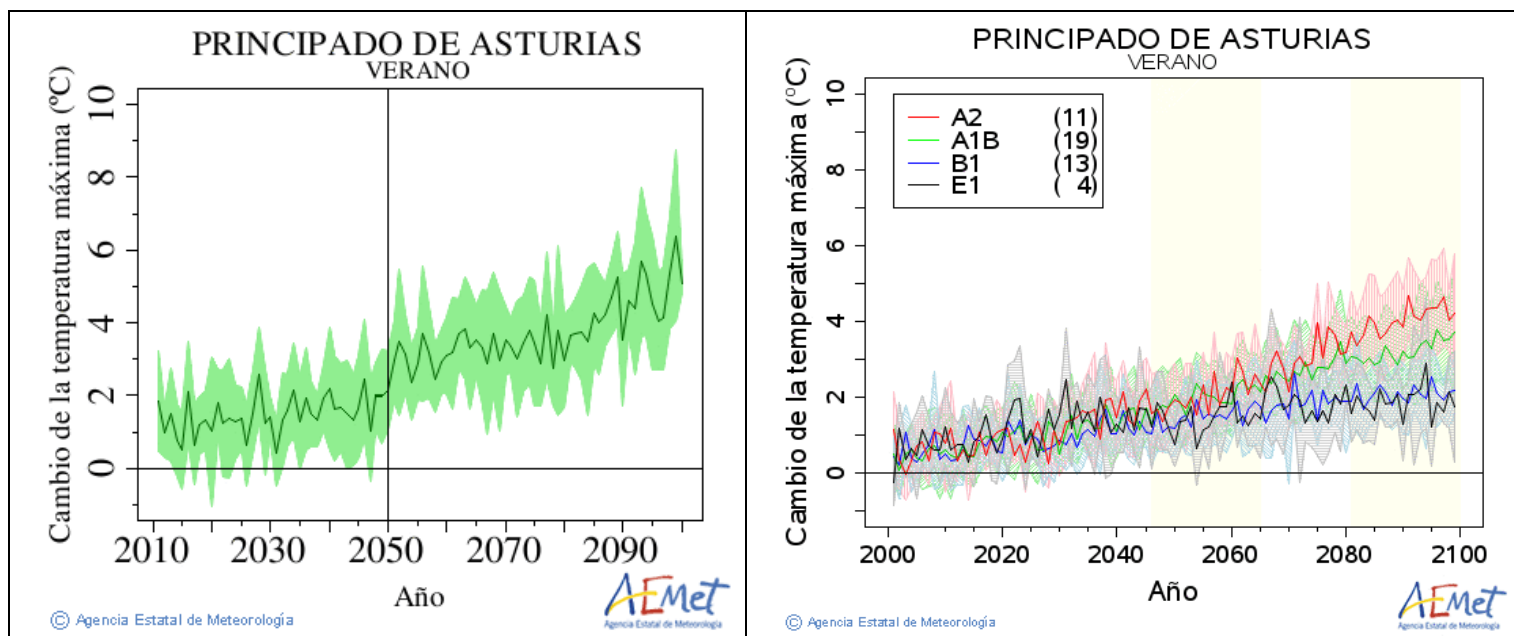

a)

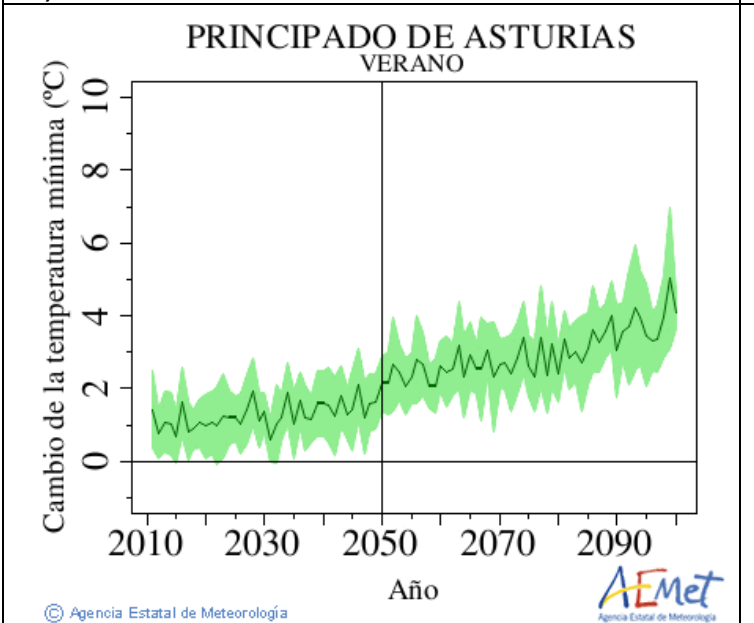
b)

c)

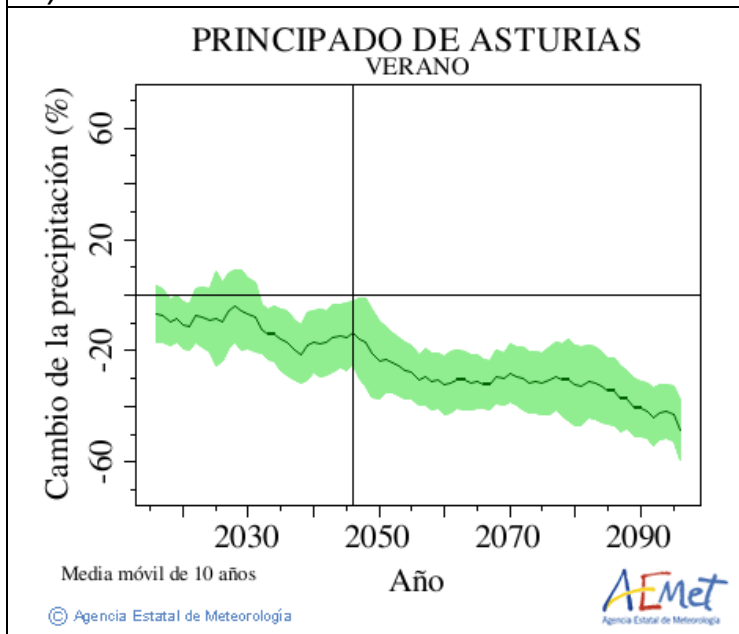

e)

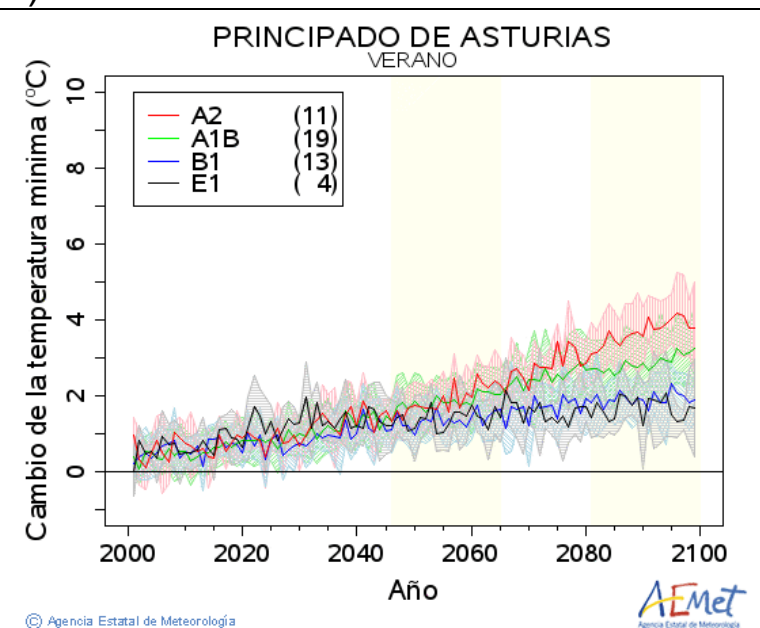

d)

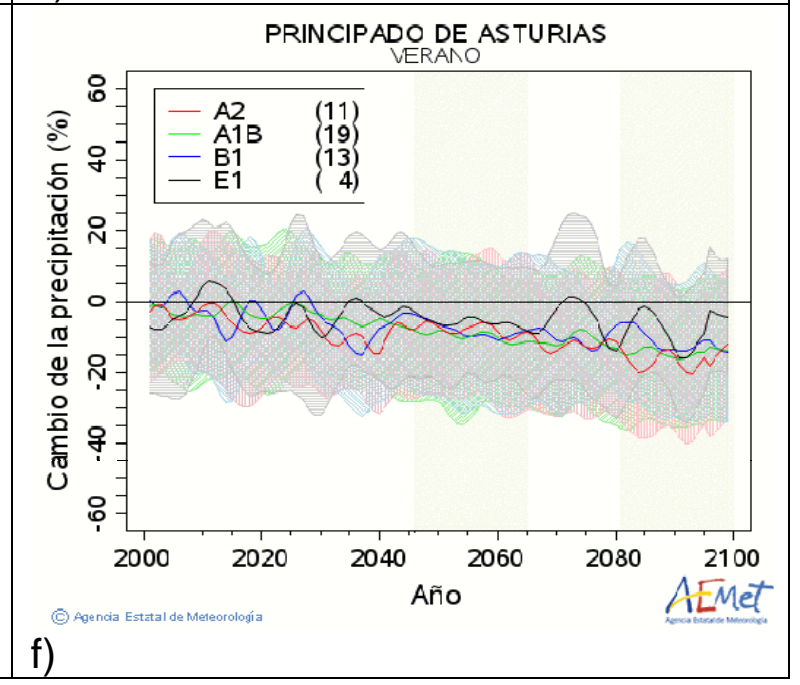

Figura A.19. Igual que la Figura A.5 para el Principado de Asturias. 

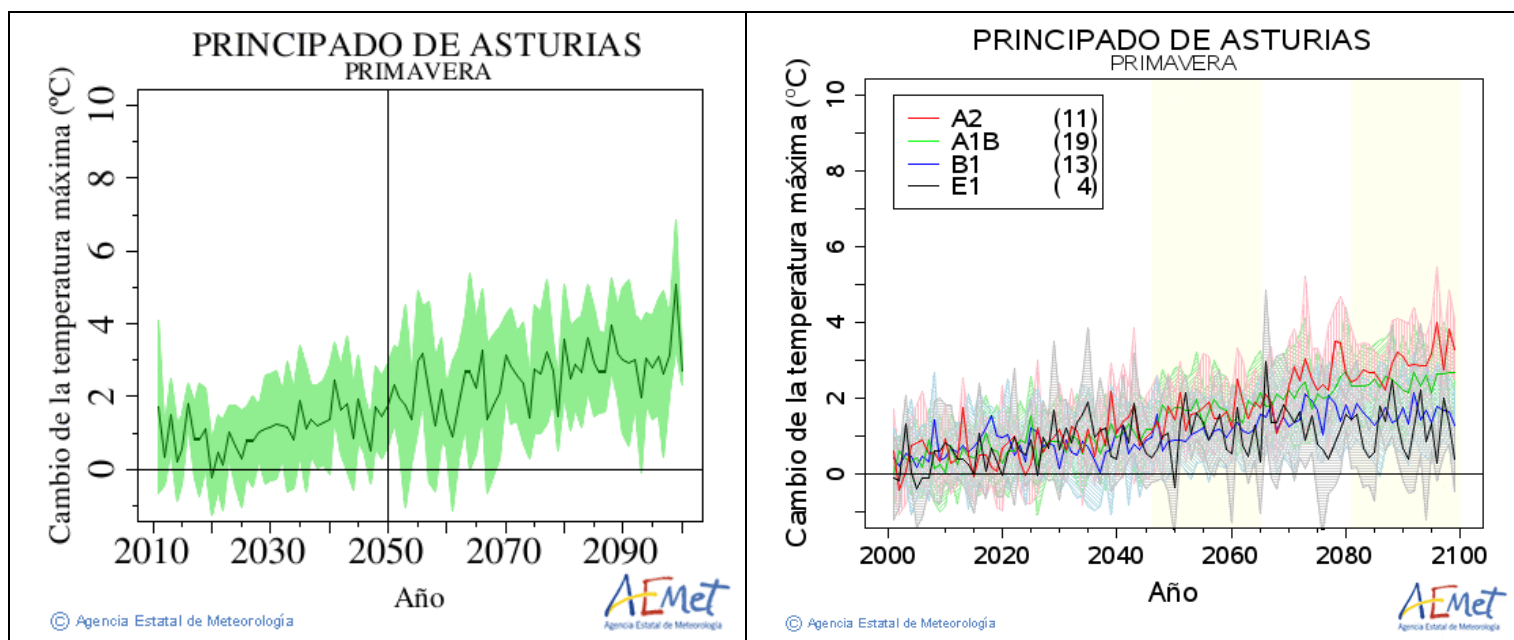

a)

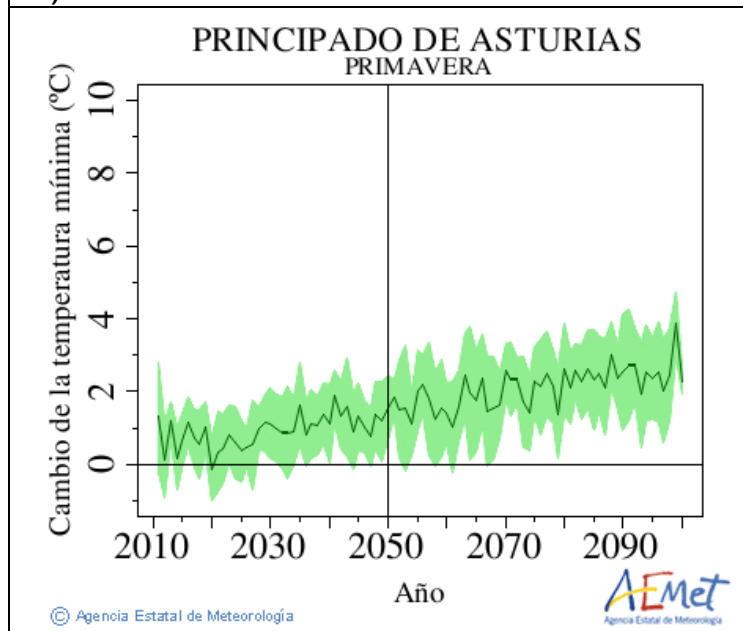

b)

c)

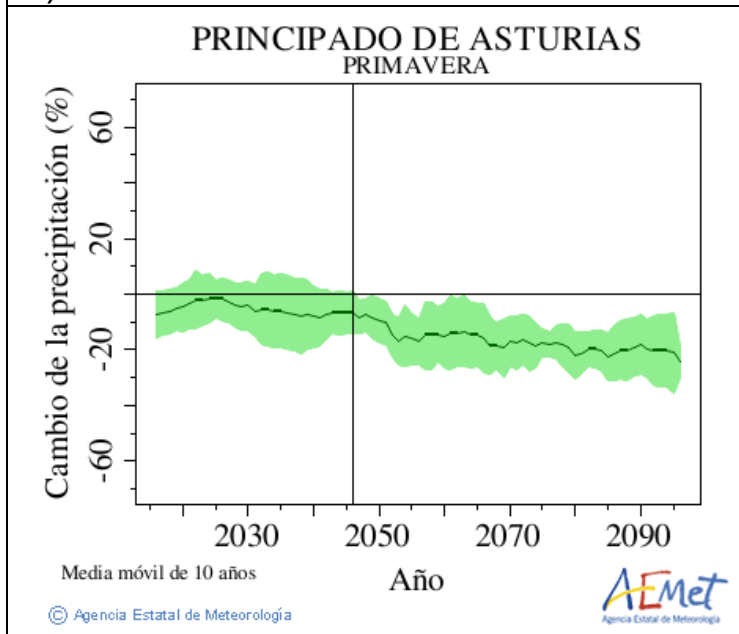

e)

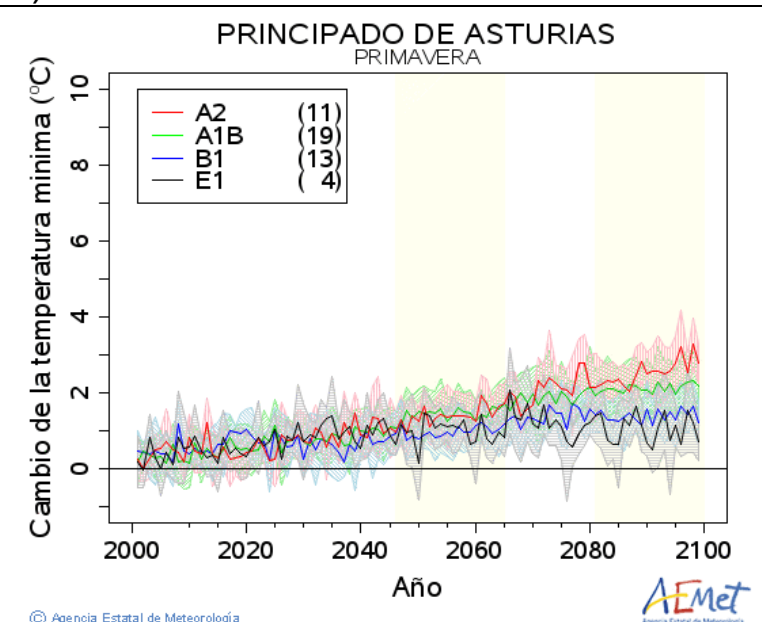

d)

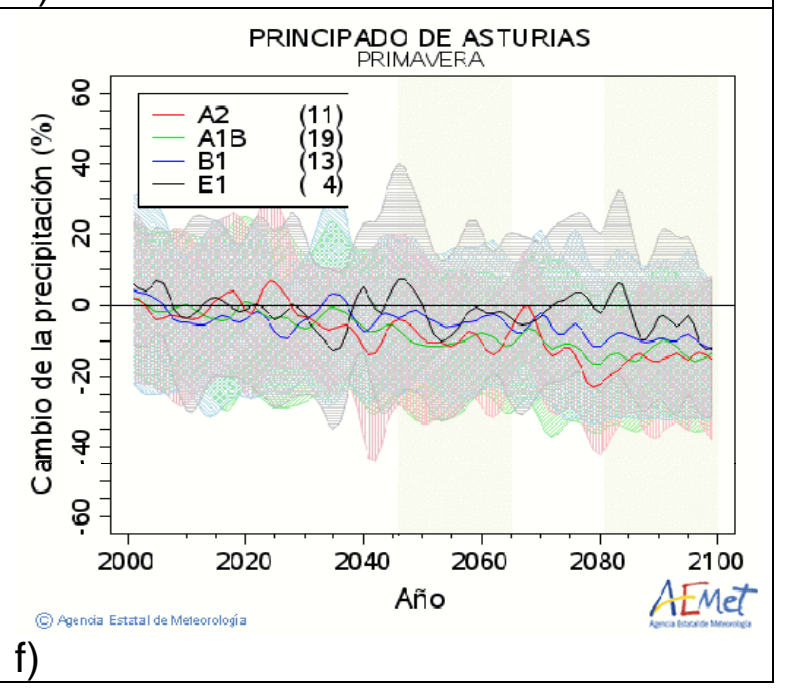

Figura A.20. Igual que la Figura A.6 para el Principado de Asturias. 

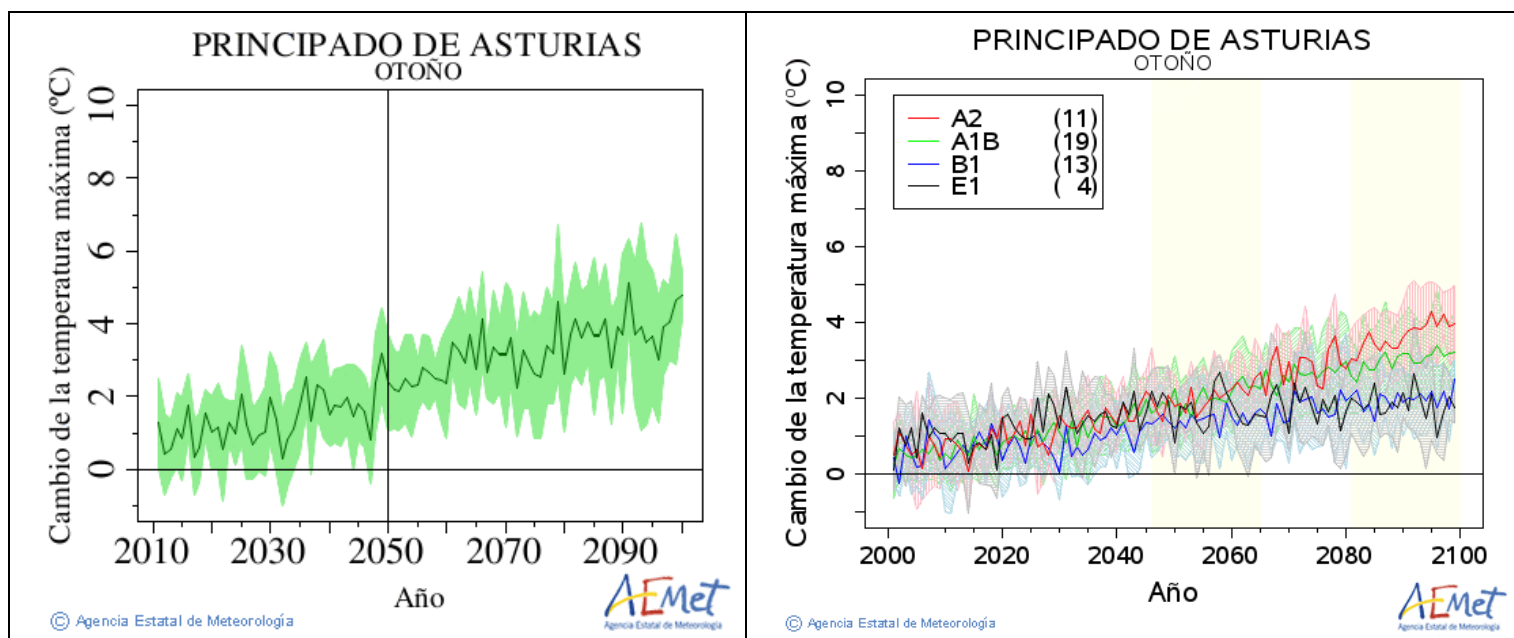

a)

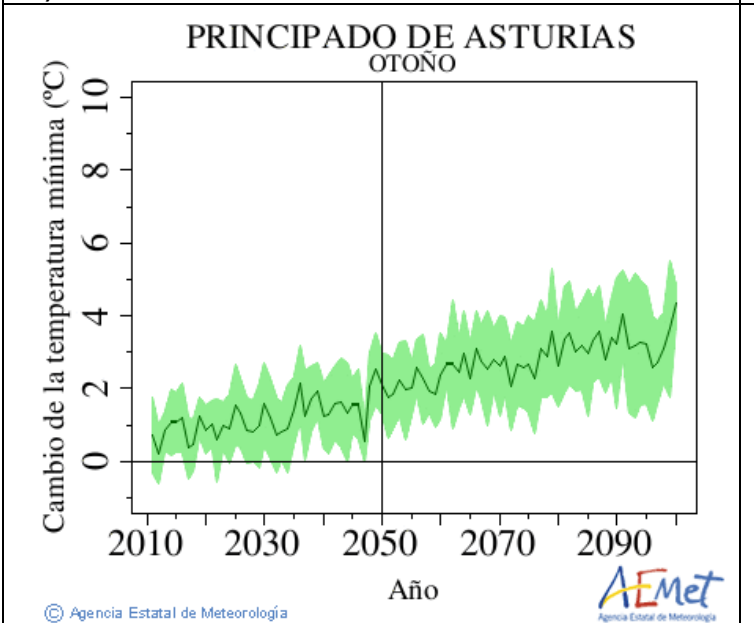
b)

c)

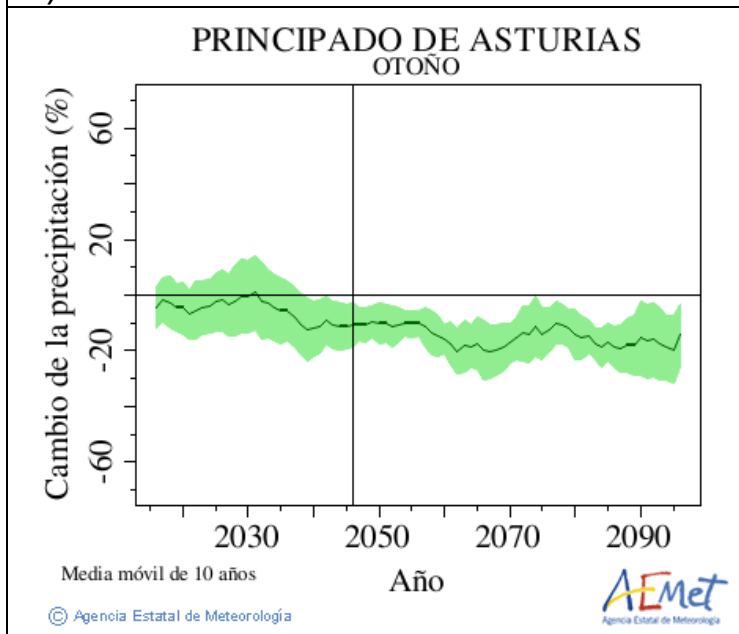

e)

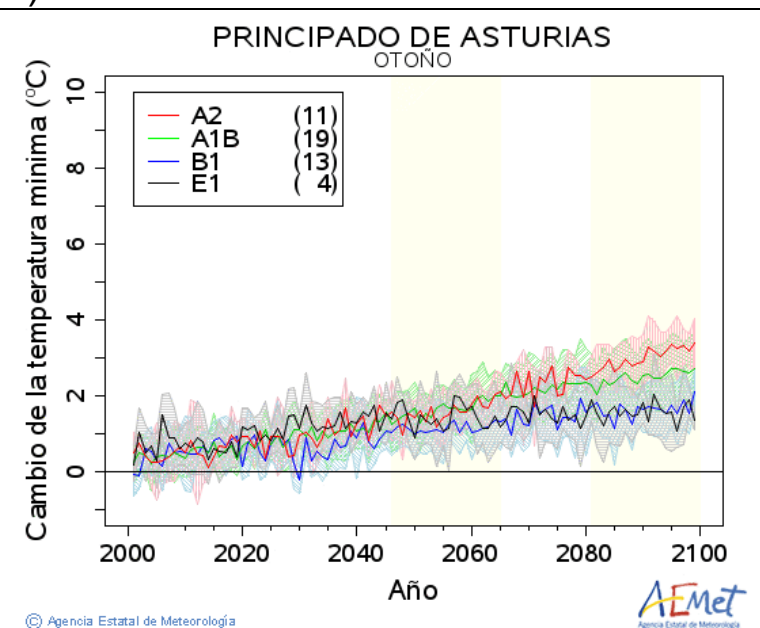

d)

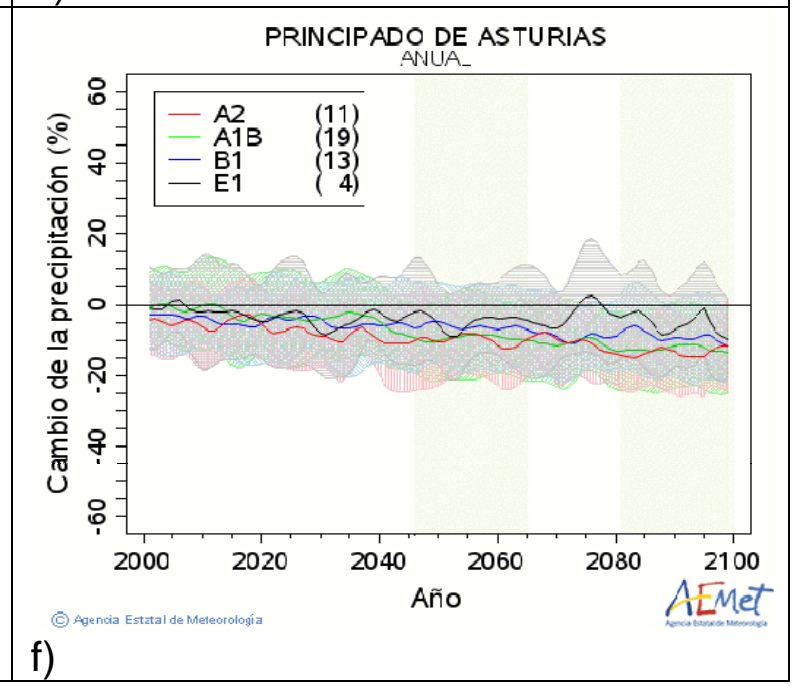

Figura A.21. Igual que la Figura A.7 para el Principado de Asturias. 
ILLES BALEARS

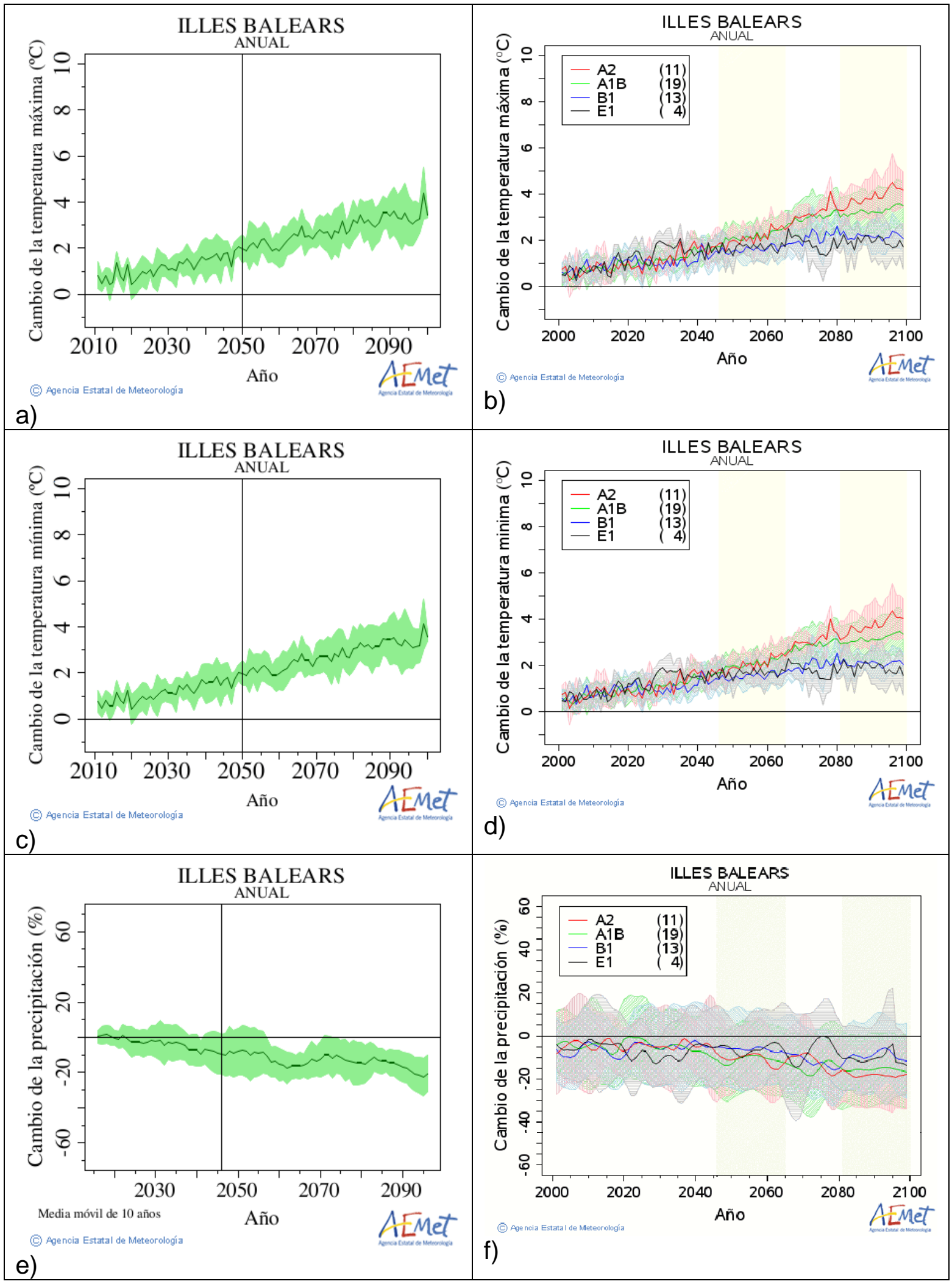

Figura A.22. Igual que la Figura A.1 para las Illes Balears. 


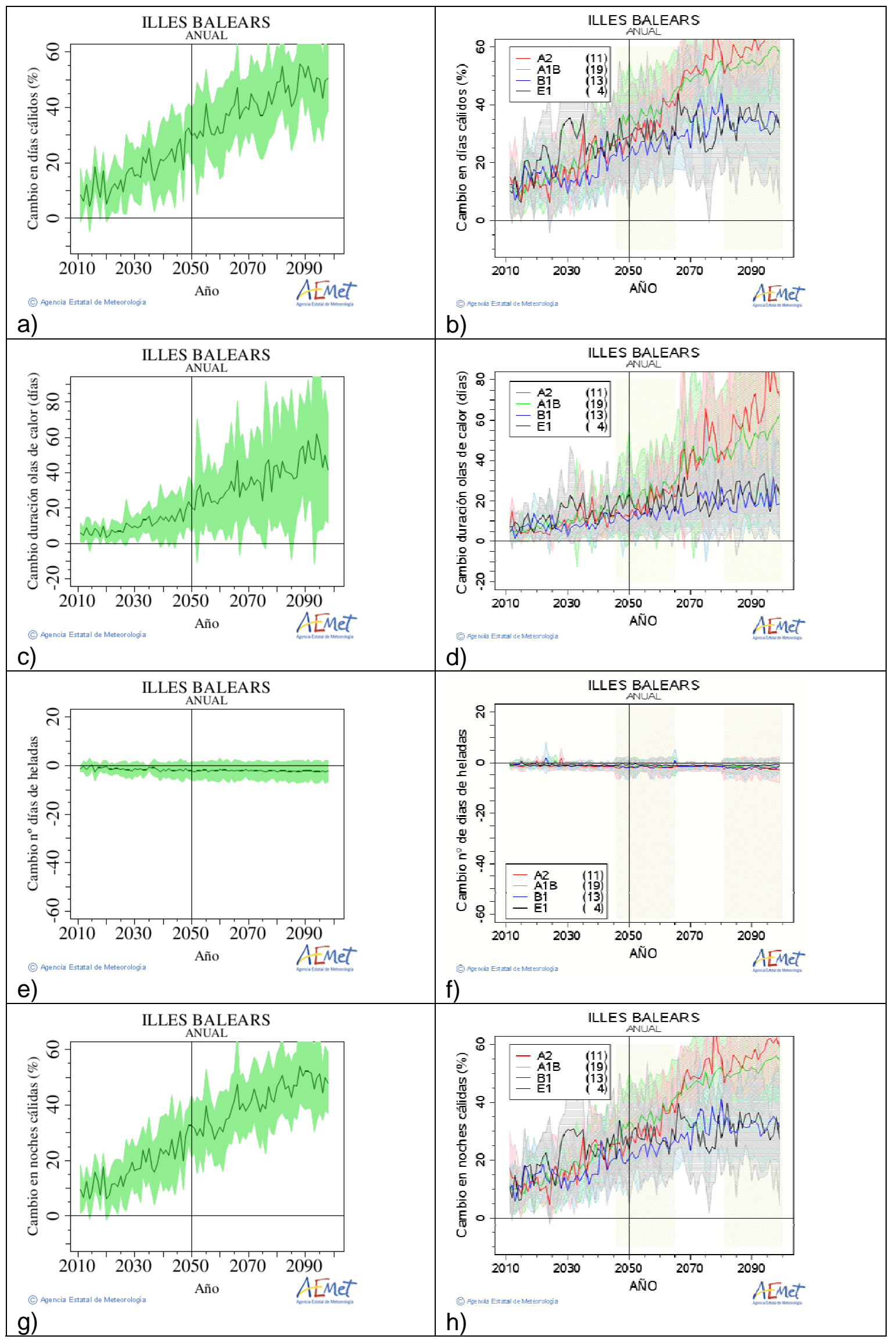

Figura A.23. Igual que la Figura A.2 para las Illes Balears. 

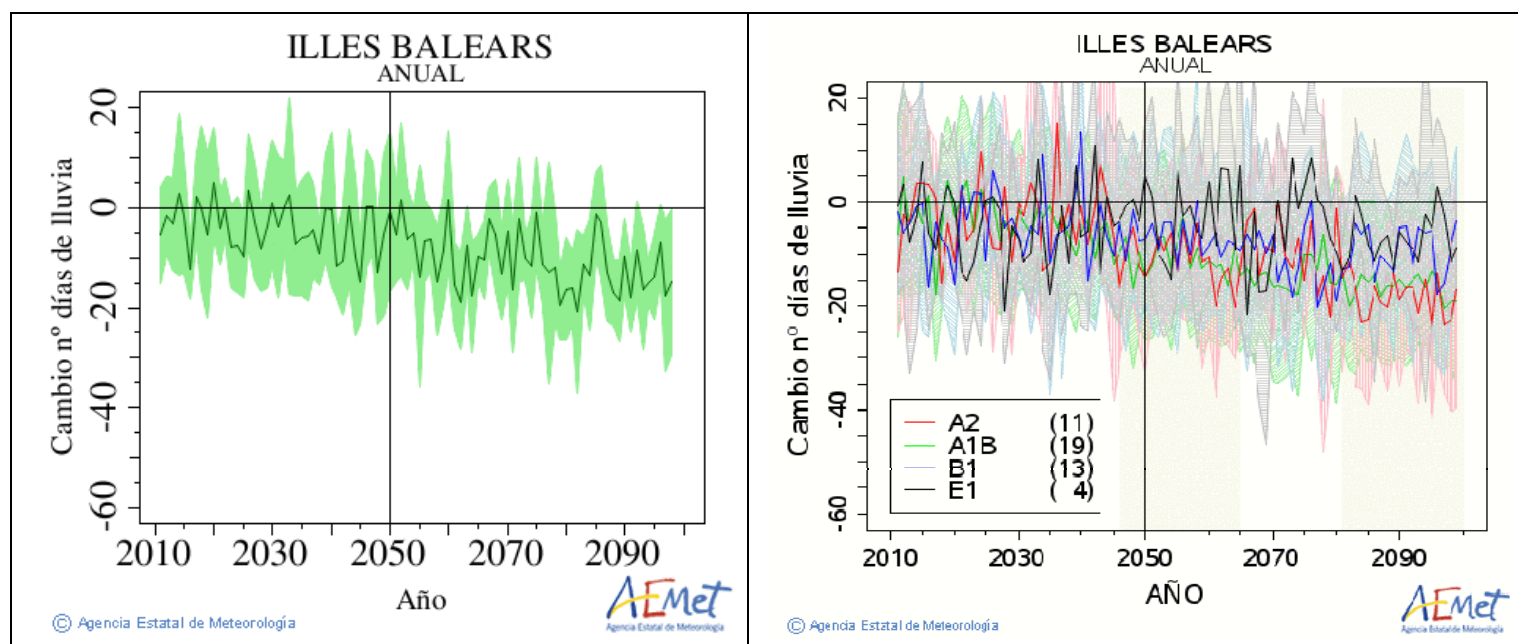

a)

b)
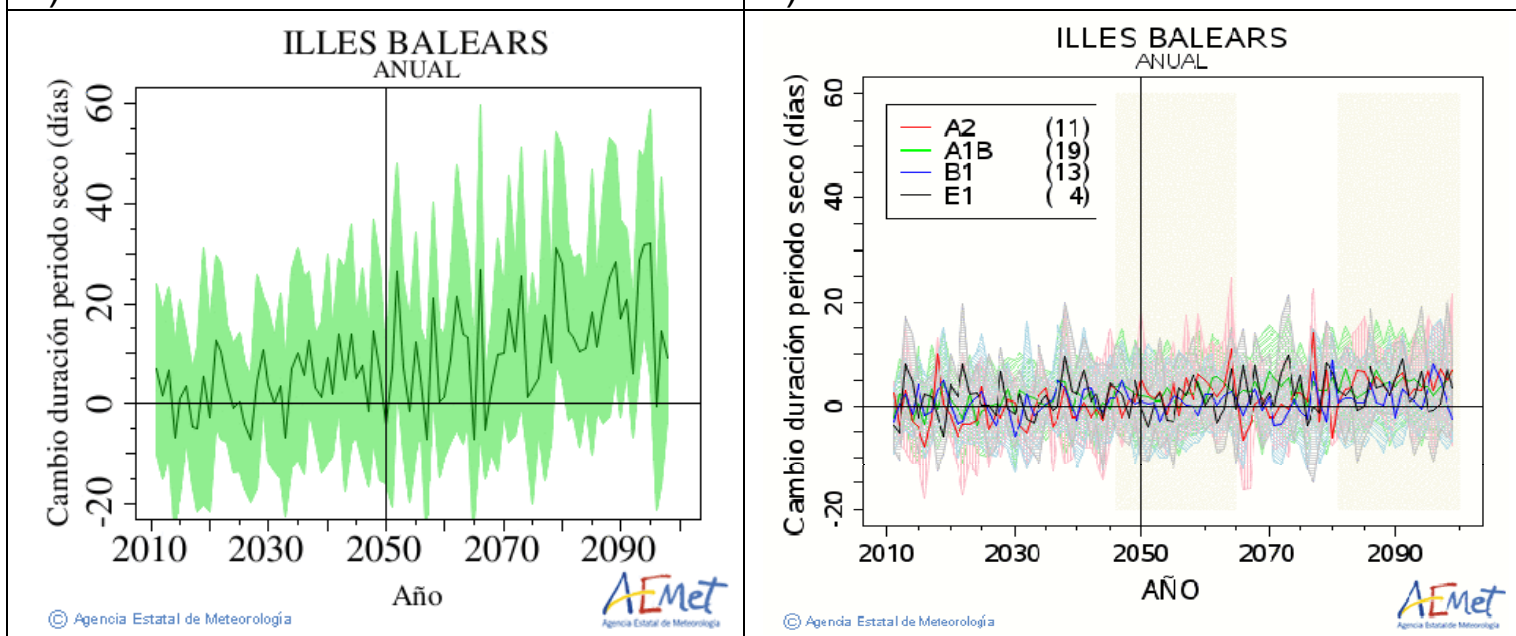

c)

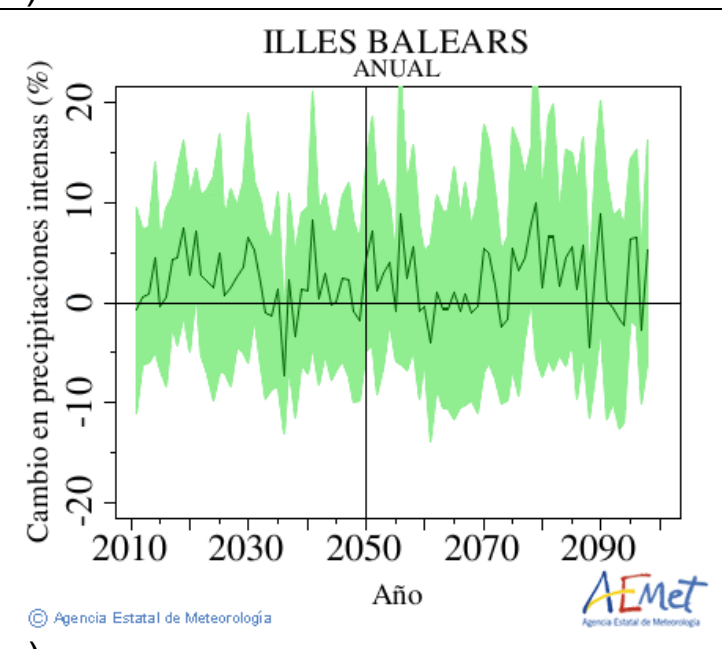

d)

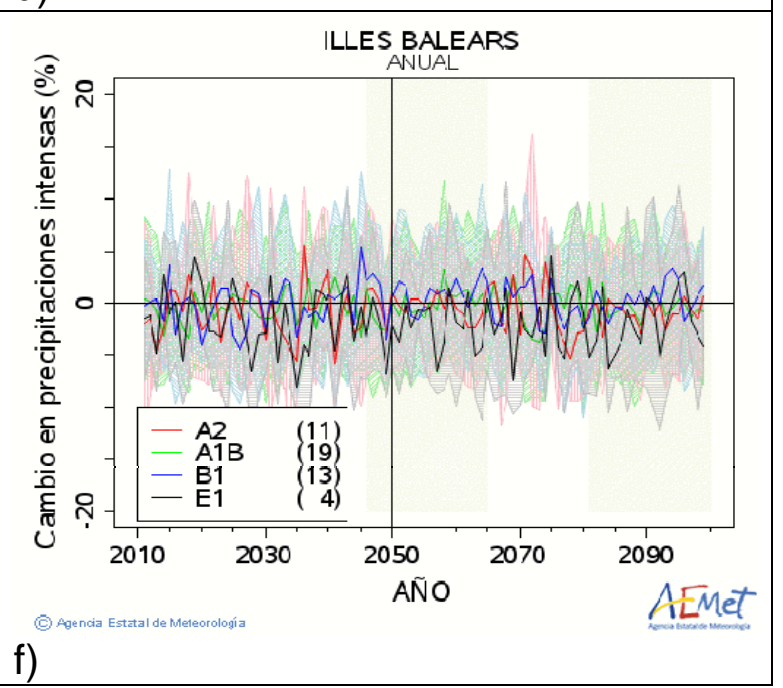

Figura A.24. Igual que la Figura A.3 para las Illes Balears. 


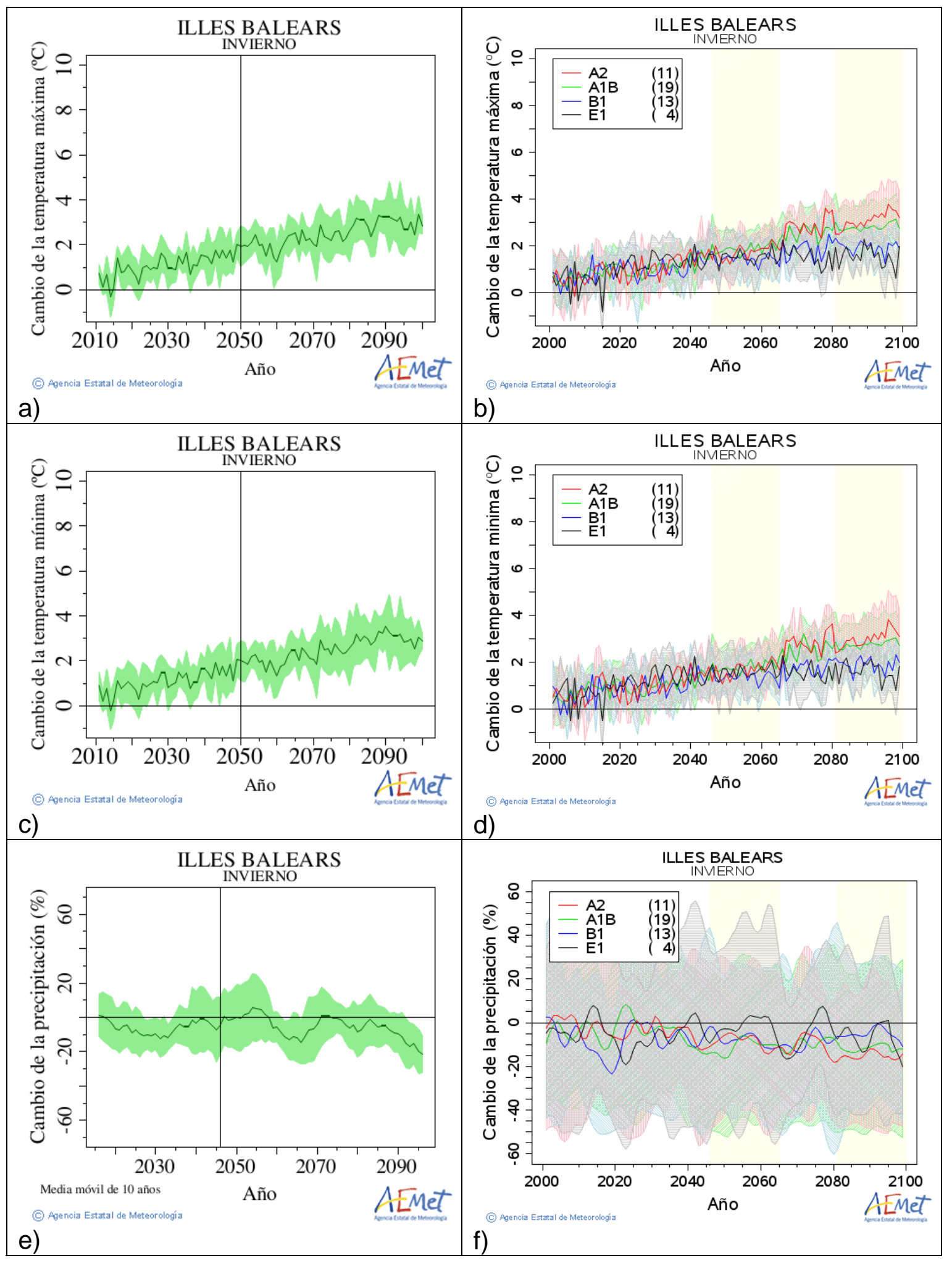

Figura A.25. Igual que la Figura A.4 para las Illes Balears. 

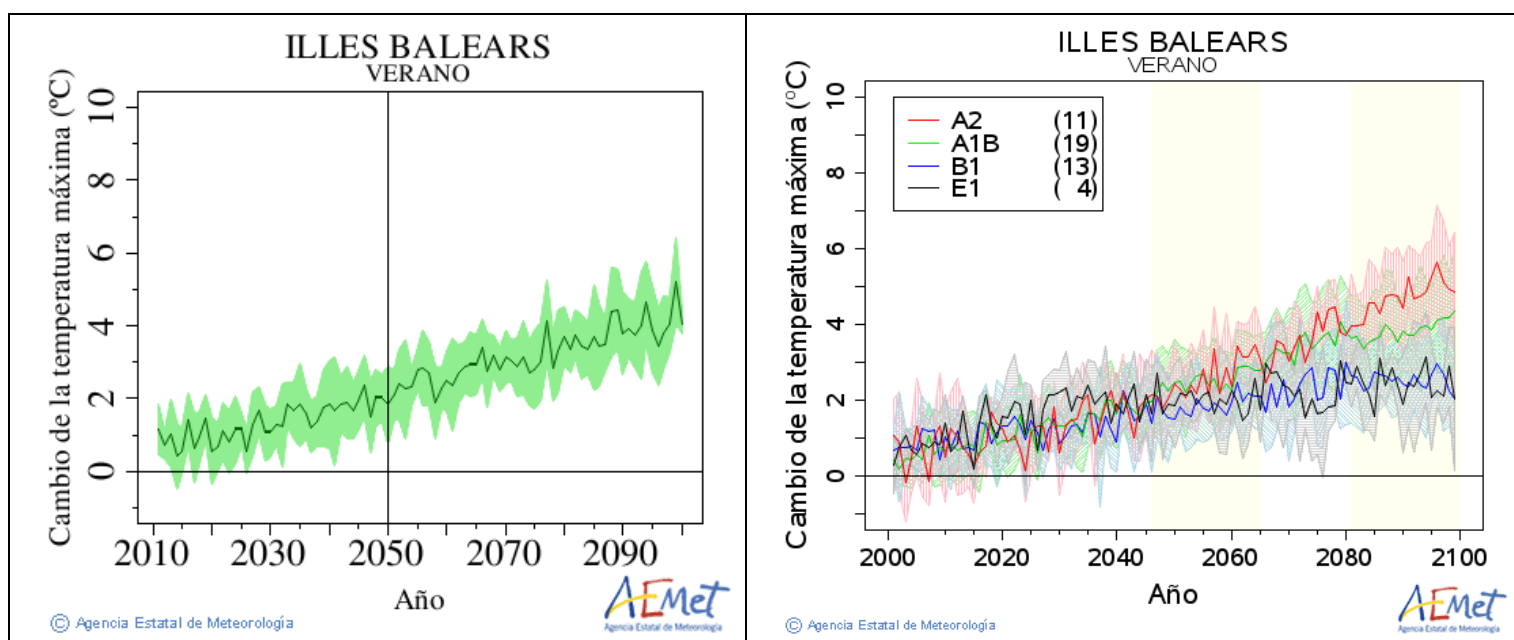

a)

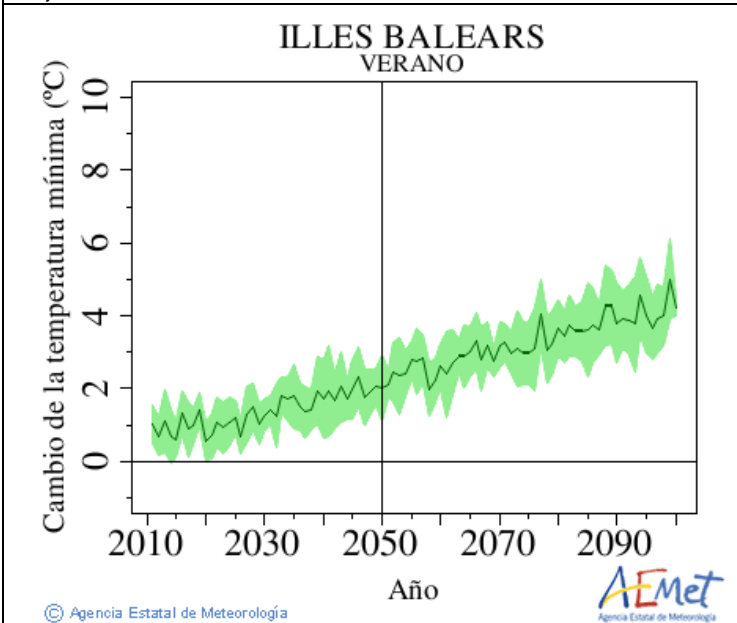

b)

c)

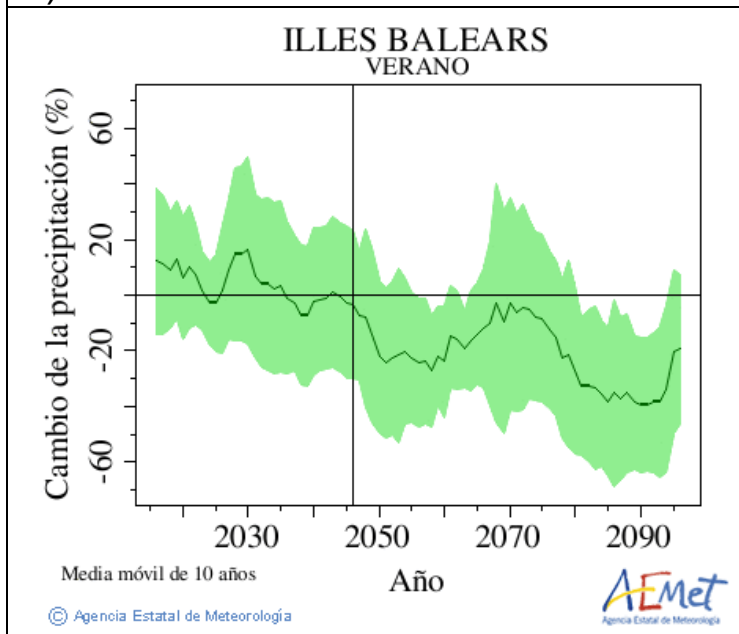

e)

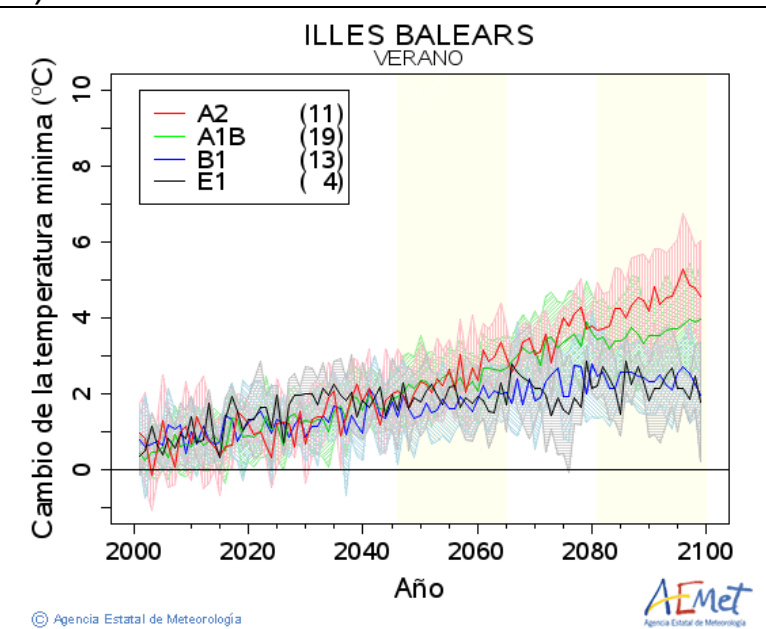

d)

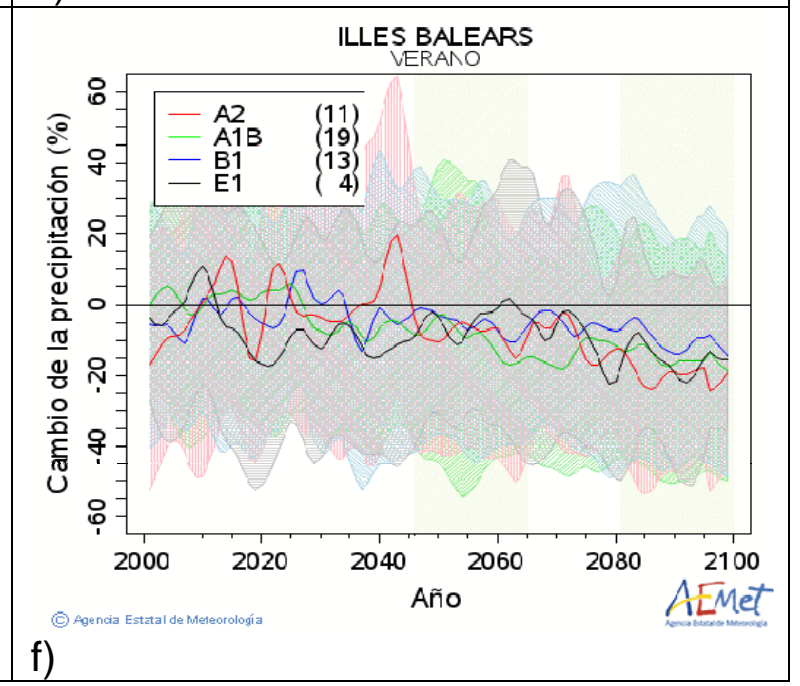

Figura A.26. Igual que la Figura A.5 para las Illes Balears. 


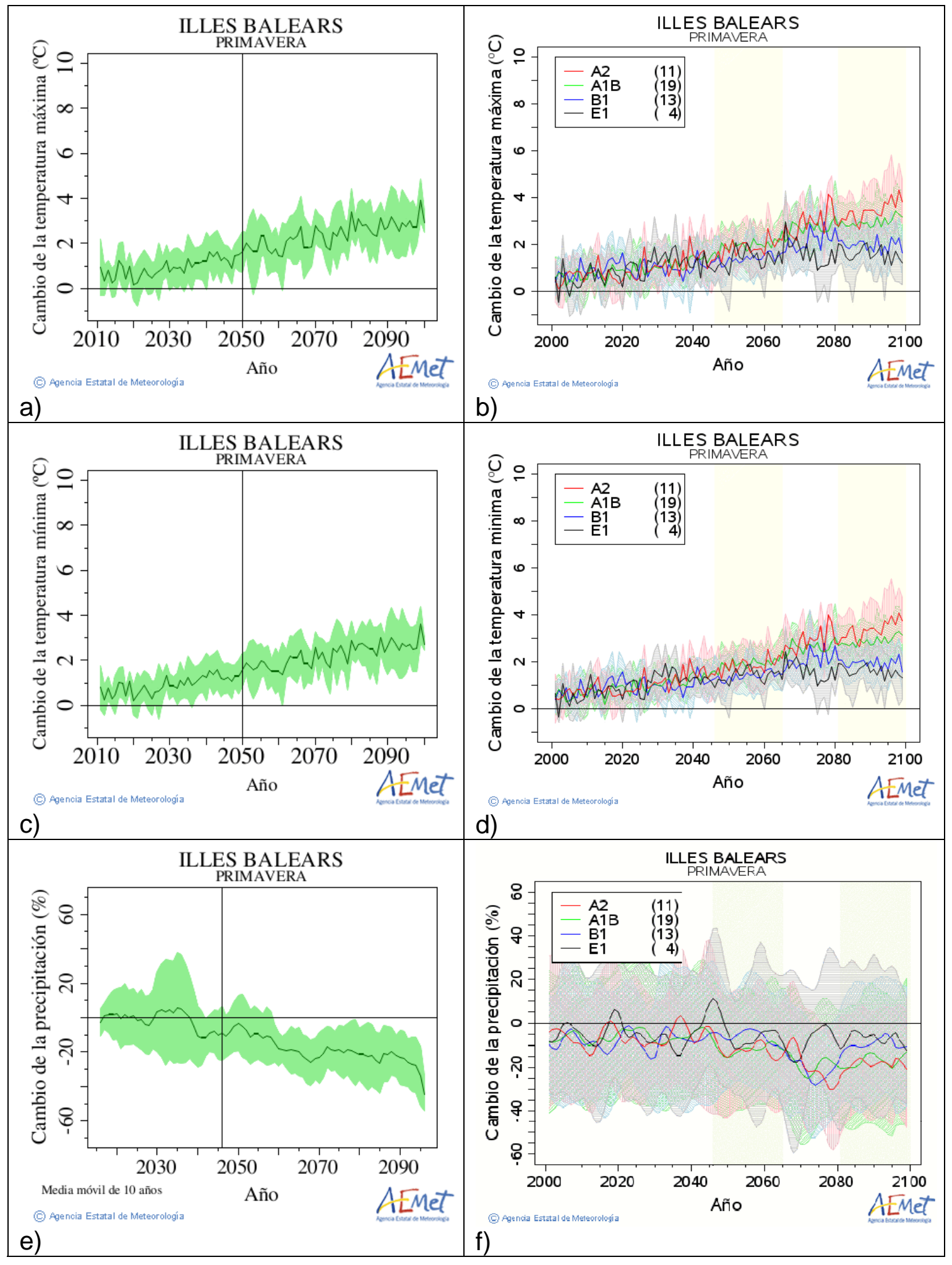

Figura A.27. Igual que la Figura A.6 para las Illes Balears.. 

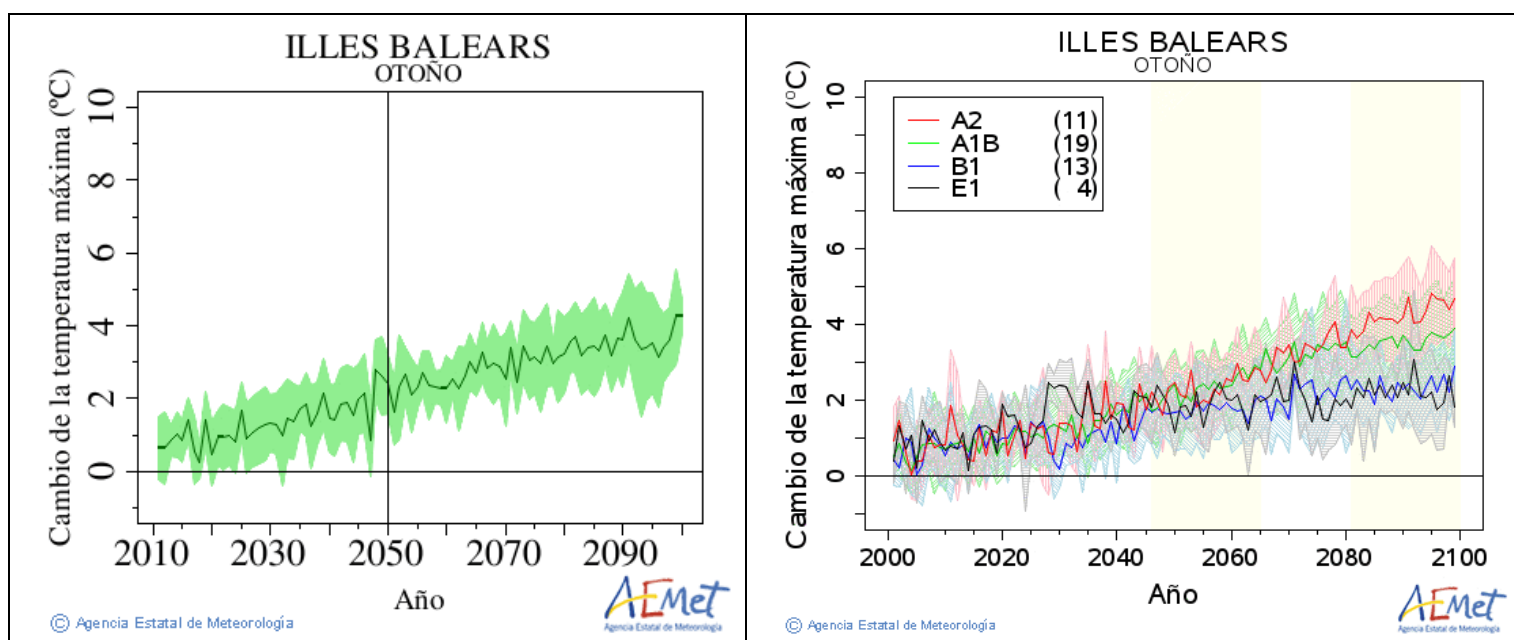

a)

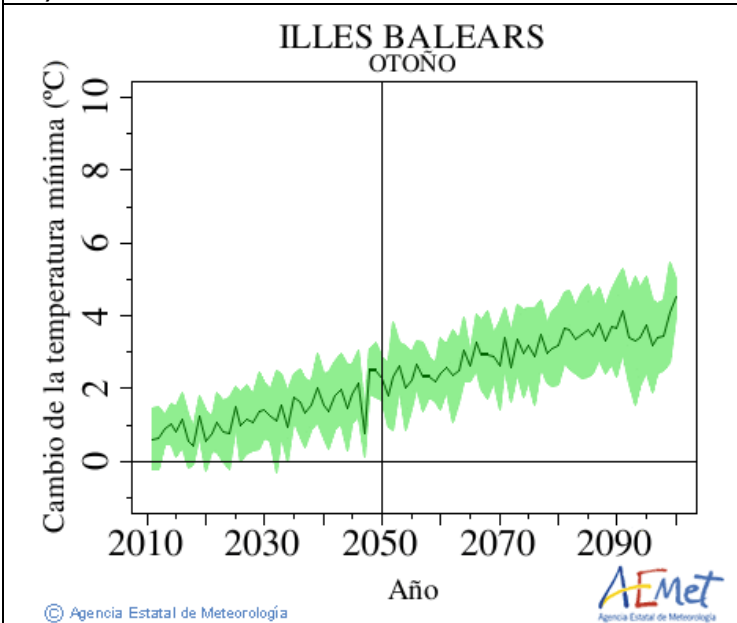

b)

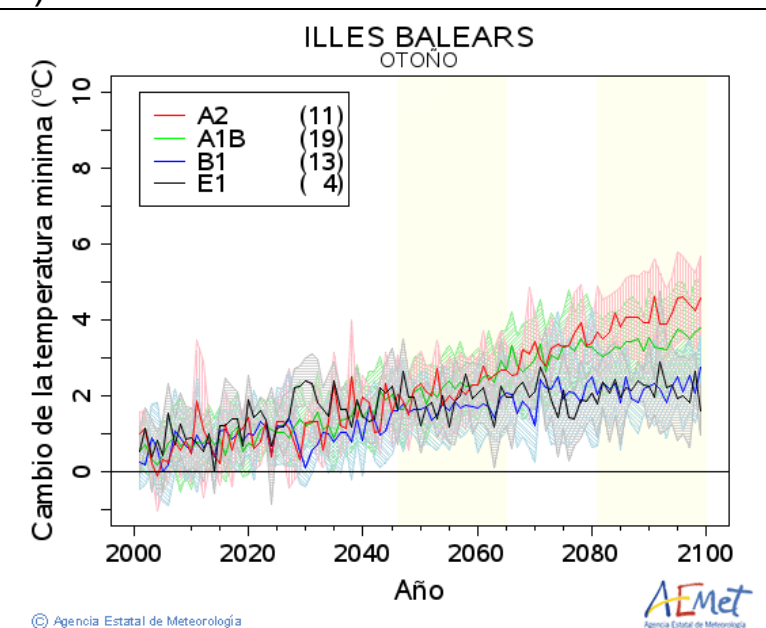

c)

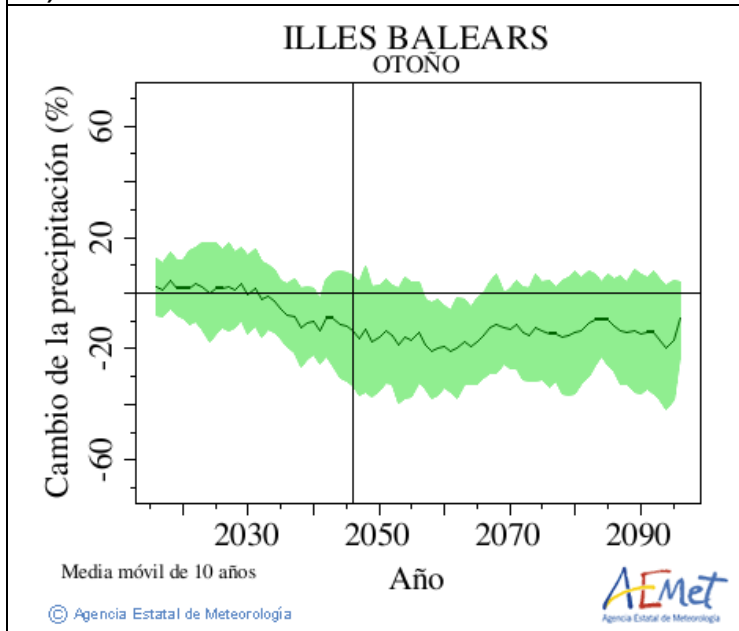

d)

e)

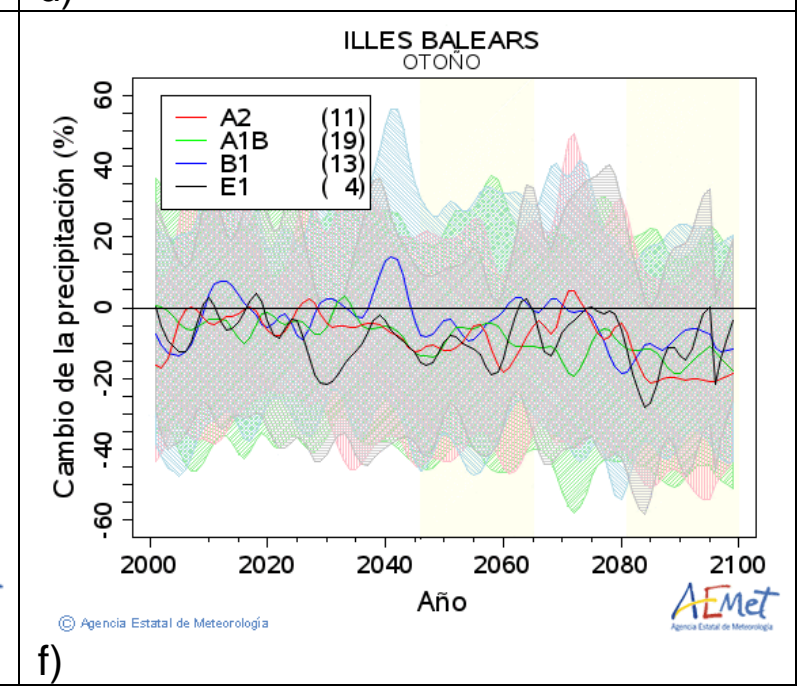

Figura A.28. Igual que la Figura A.7 para las Illes Balears. 
ISLAS CANARIAS

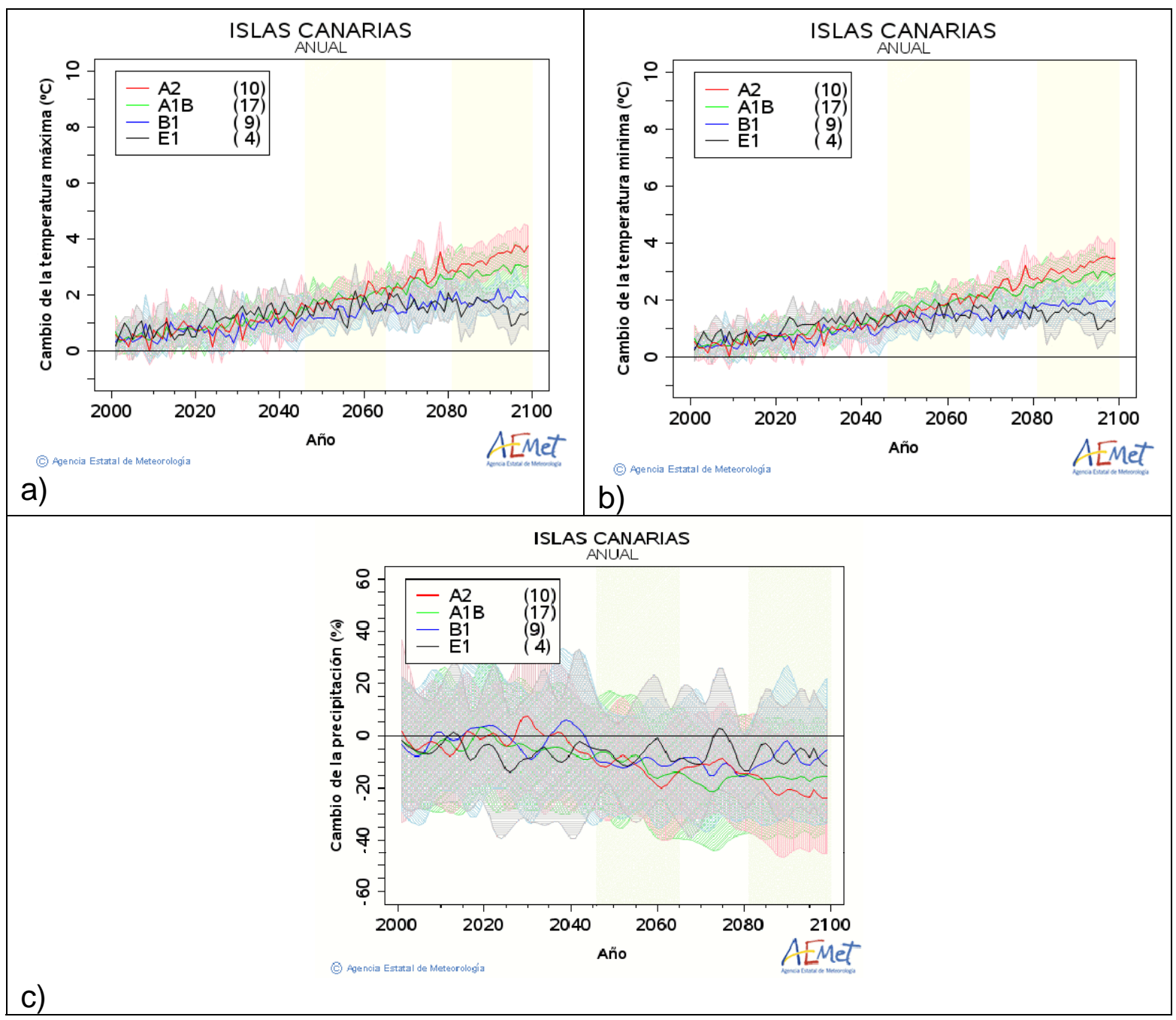

Figura A.29. Evolución temporal del valor medio anual para las Islas Caarias de: (a) temperatura máxima $\left({ }^{\circ} \mathrm{C}\right)$; (b) temperatura mínima $\left({ }^{\circ} \mathrm{C}\right)$ y $(\mathrm{c})$ tasa de precipitación en la España peninsular para el siglo XXI obtenida mediante técnicas de regionalización estadística agrupadas por escenarios de emisiones: A2-altas (rojo); A1B-medias (verde); B1-bajas (azul) y E1-fuerte mitigación (negro). Entre paréntesis se recoge el número de proyecciones realizadas para cada escenario. En línea continua (sombreada) se representa el valor promedio (incertidumbre). Las franjas verticales sombreadas indican las ventanas temporales en las que el número de regionalizaciones es máximo. 


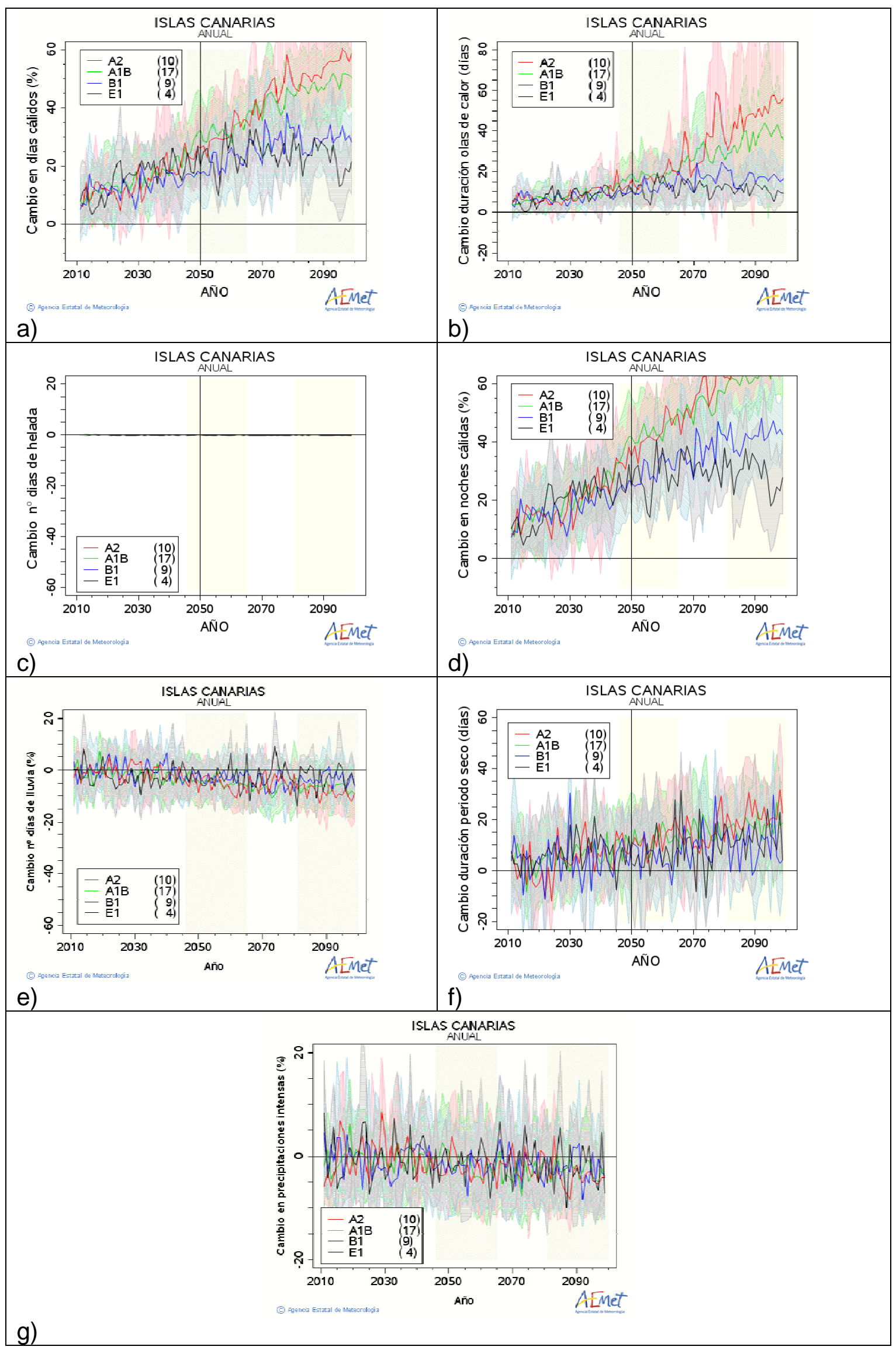

Figura A.30. Igual que la Figura A.29 para evolución temporal del valor medio anual de los índices extremos (a) DC; (b) DOC; (c) DH; (d) NC; (e) DL; (f) PS y (g) PI. 


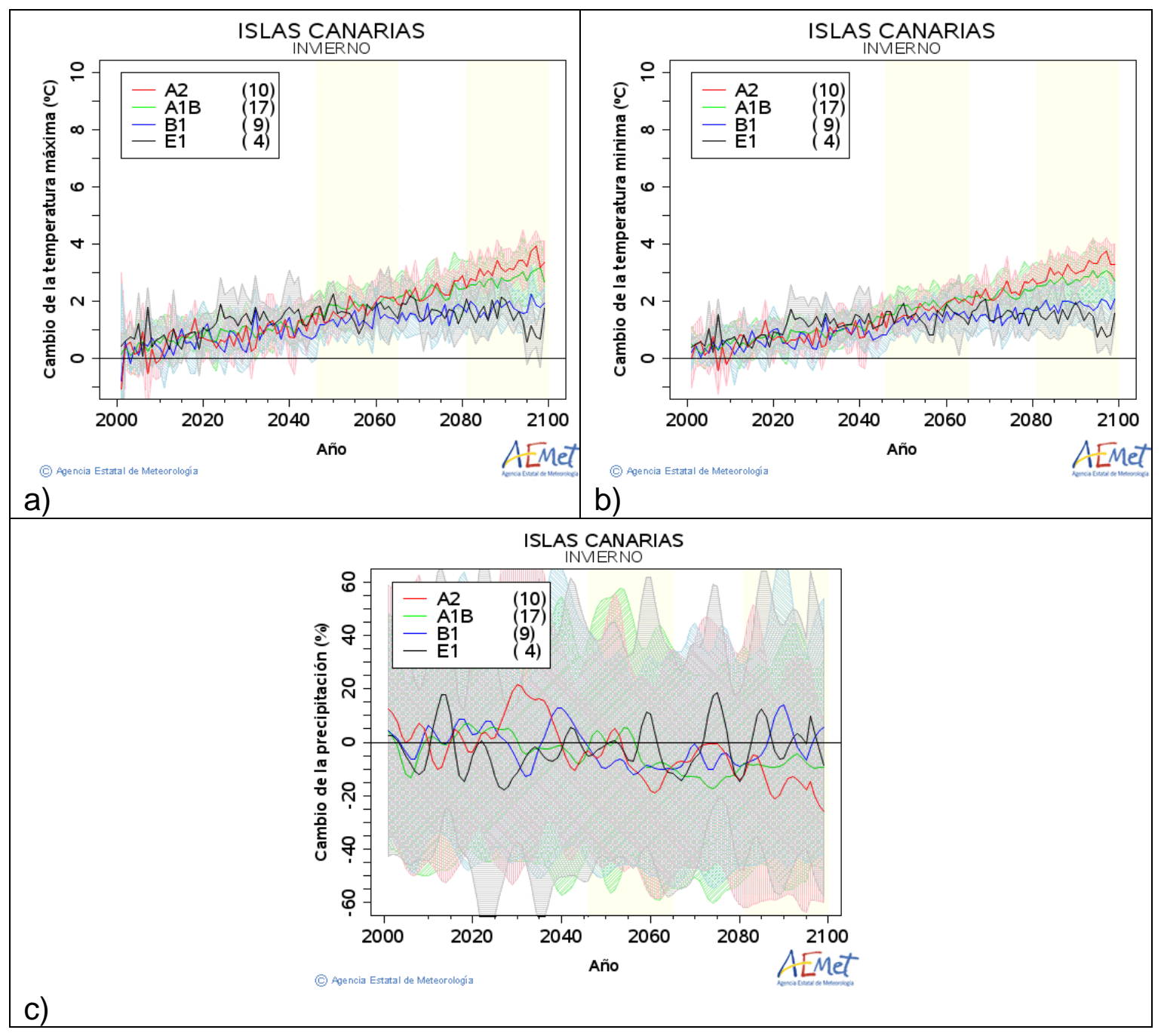

Figura A.31. Igual que la Figura A.29 para el valor medio de invierno. 


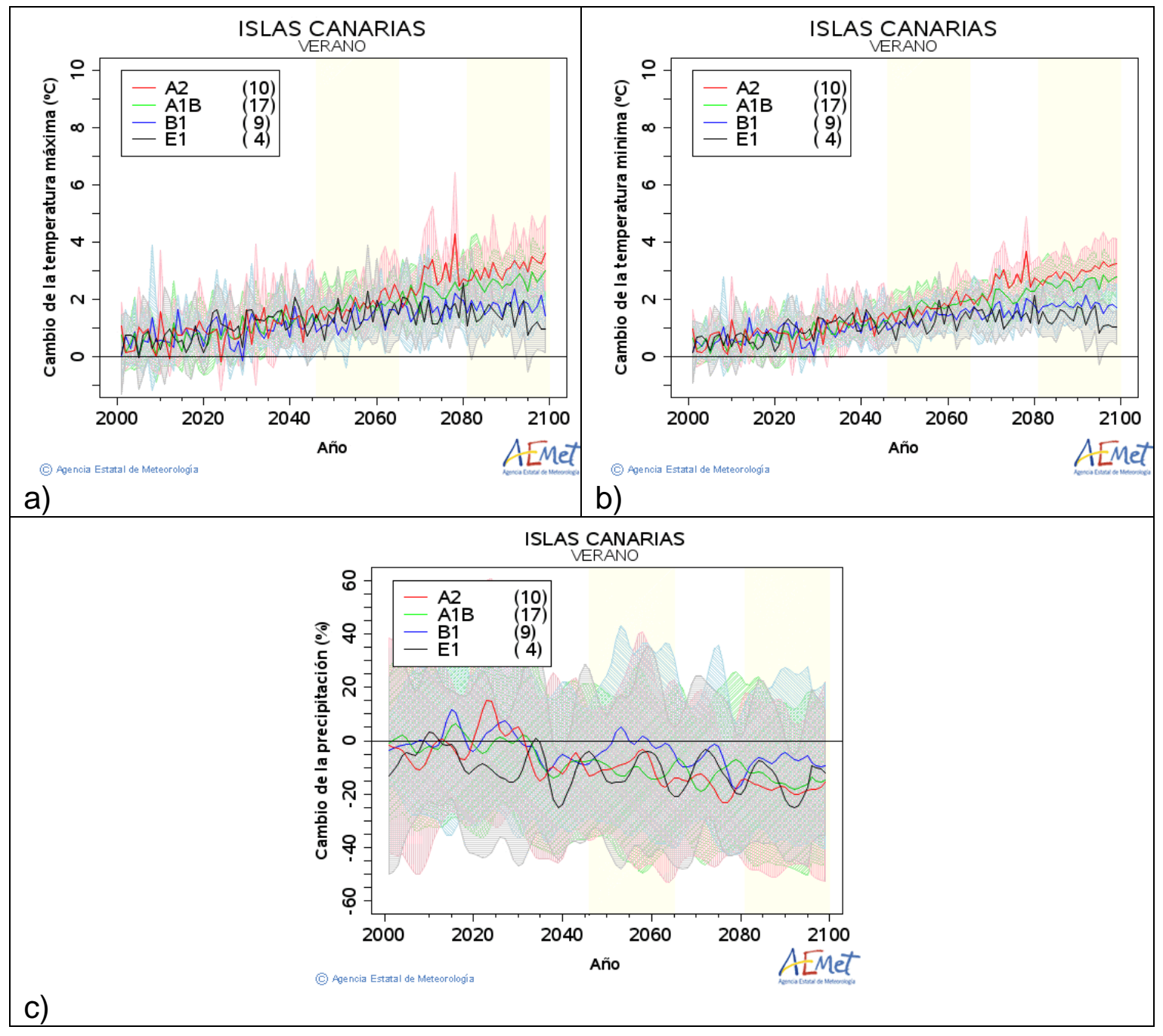

Figura A.32. Igual que la Figura A.29 para el valor medio de verano. 


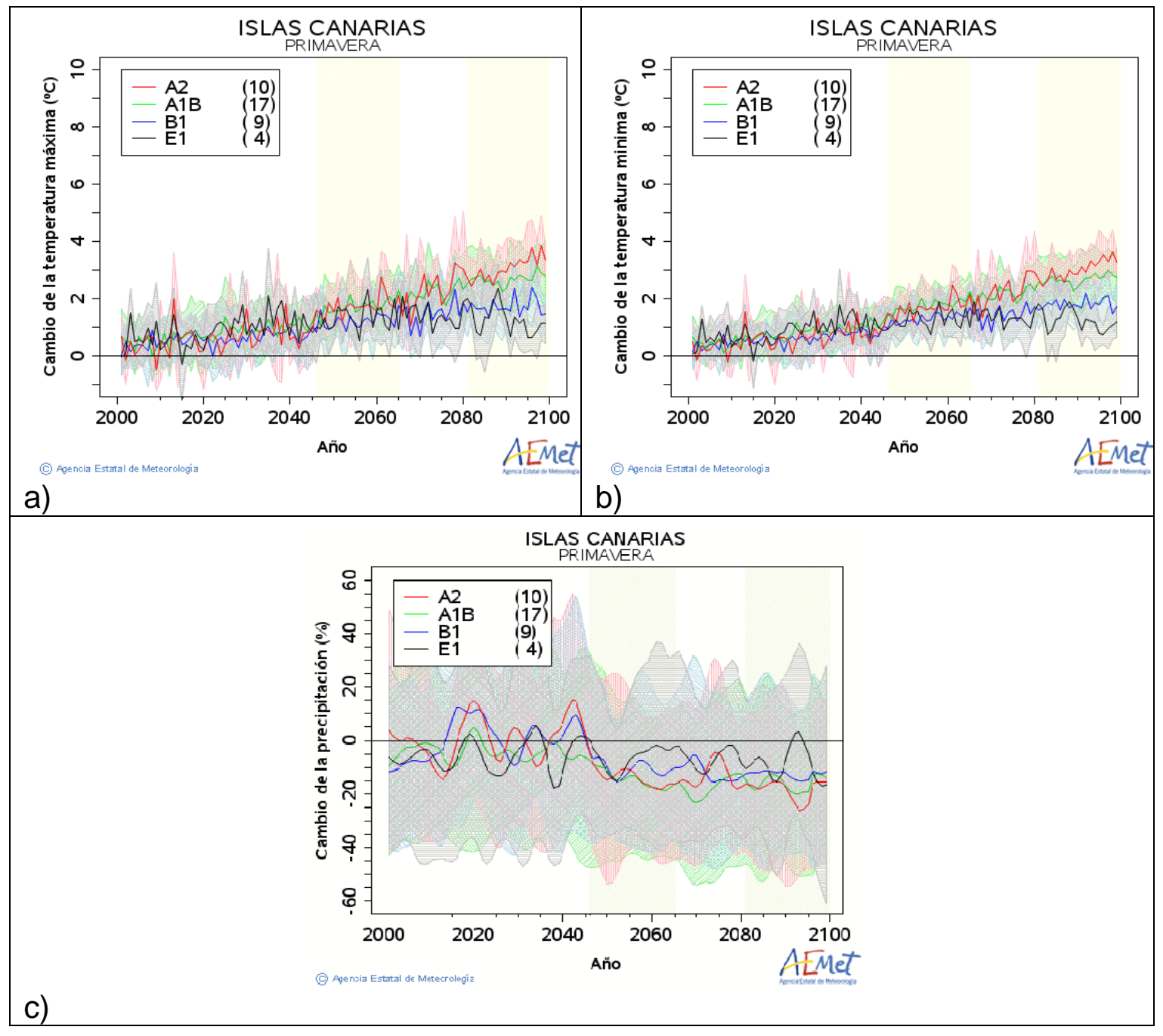

Figura A.33. Igual que la Figura A.29 para el valor medio de primavera. 


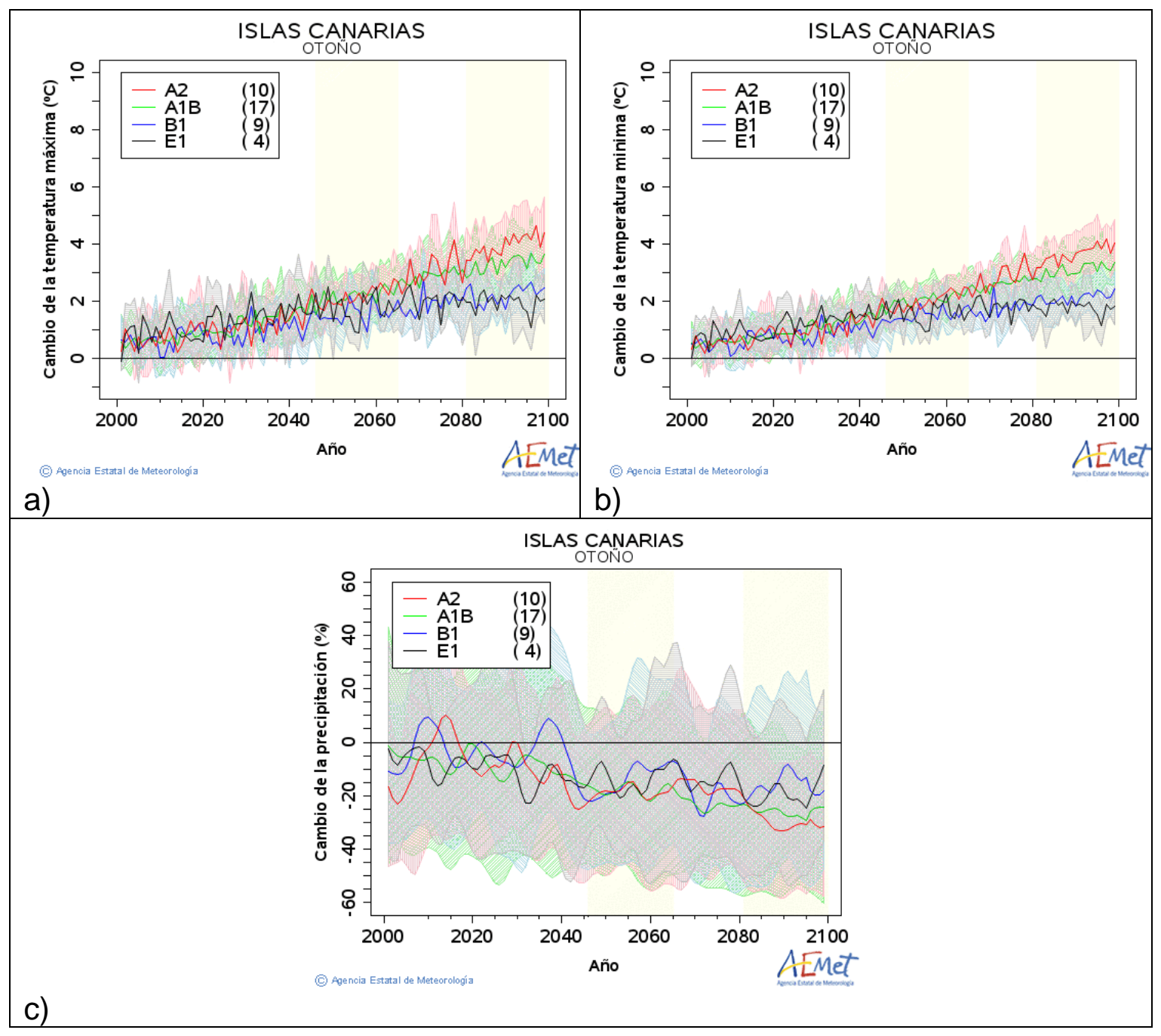

Figura A.34. Igual que la Figura A.29 para el valor medio de otoño. 
CANTABRIA

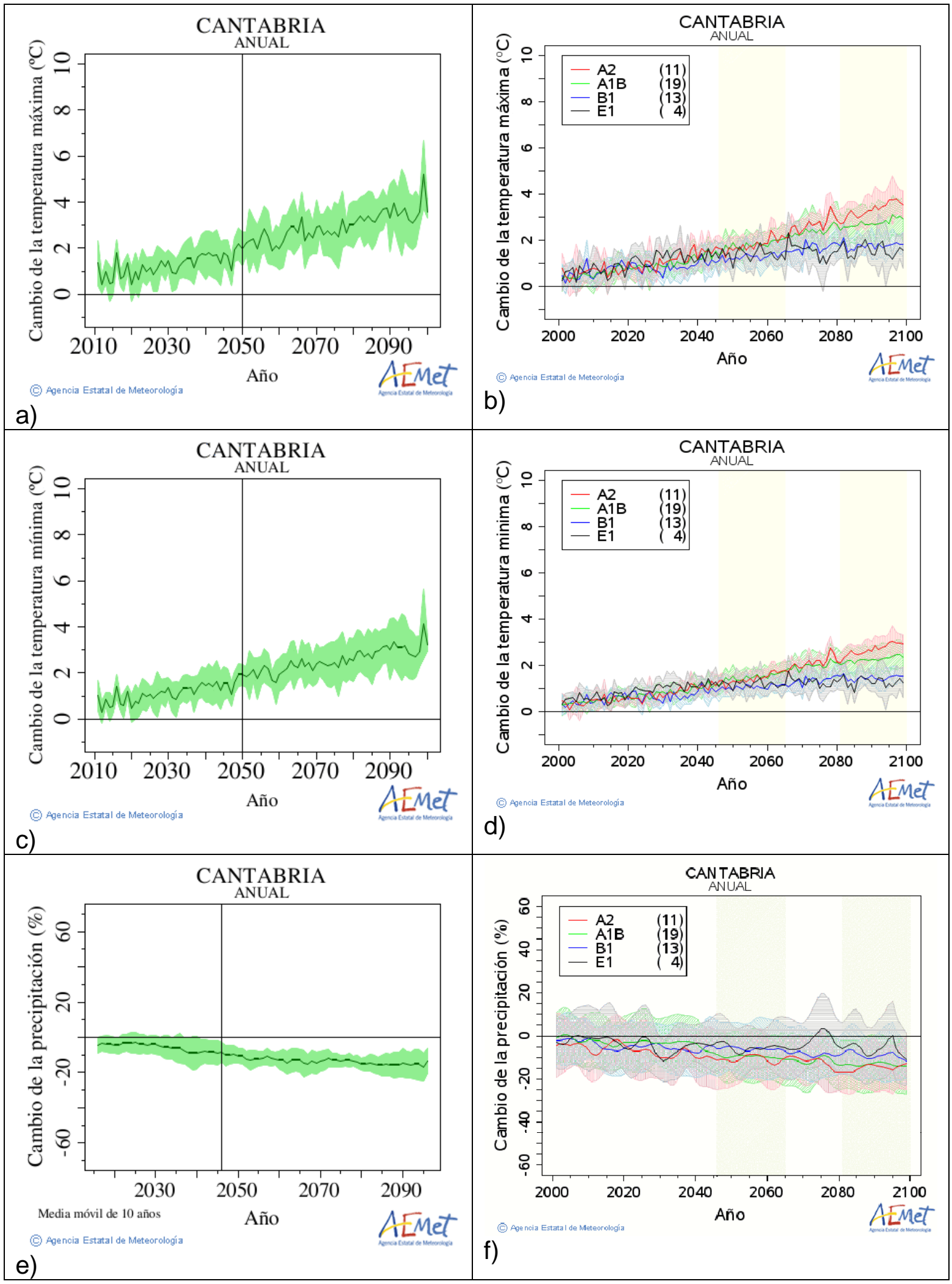

Figura A.36. Igual que la Figura A.1 para la Comunidad Autónoma de Cantabria. 


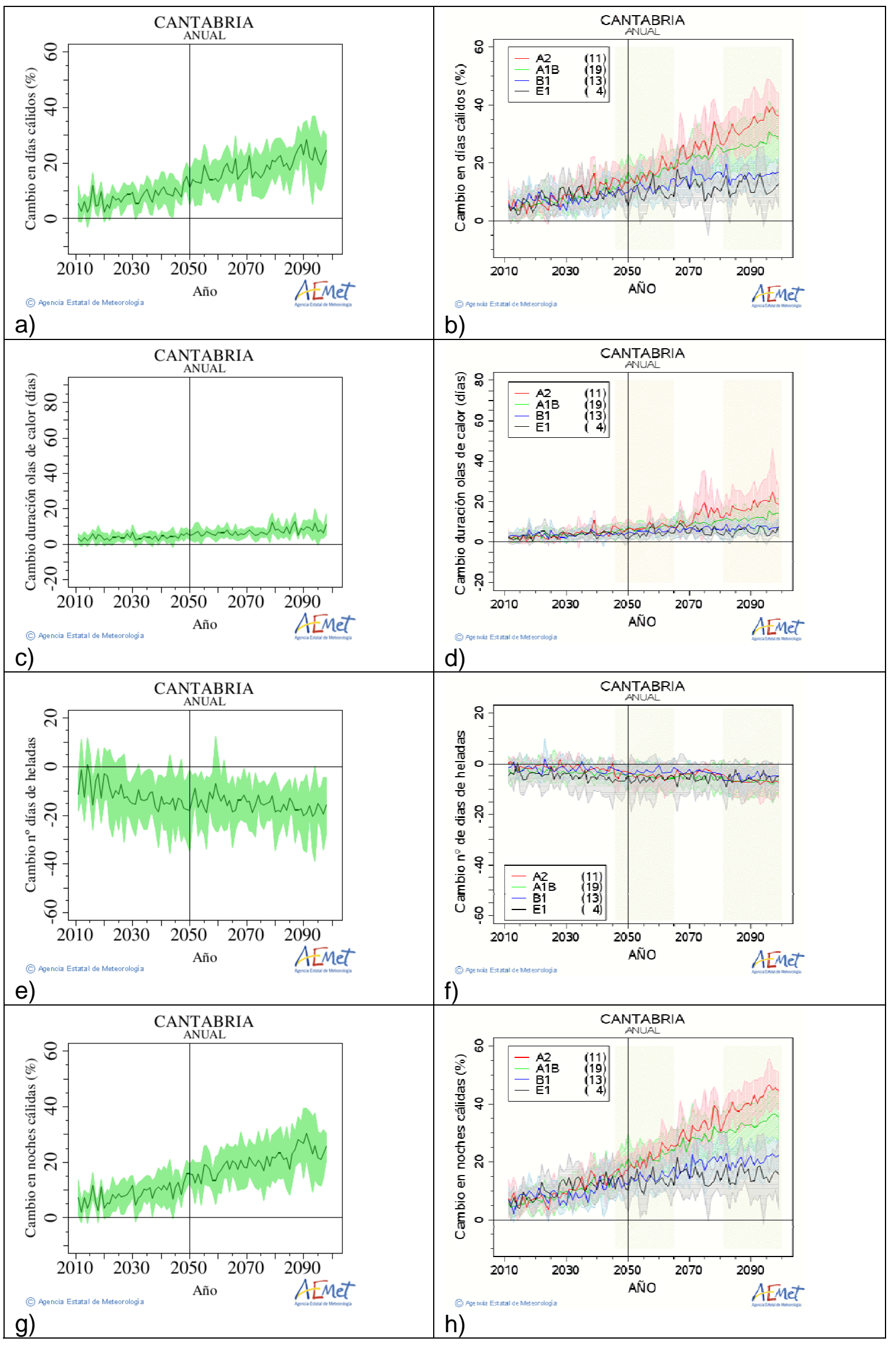

Figura A.37 Igual que la Figura A.2 para la Comunidad Autónoma de Cantabria. 

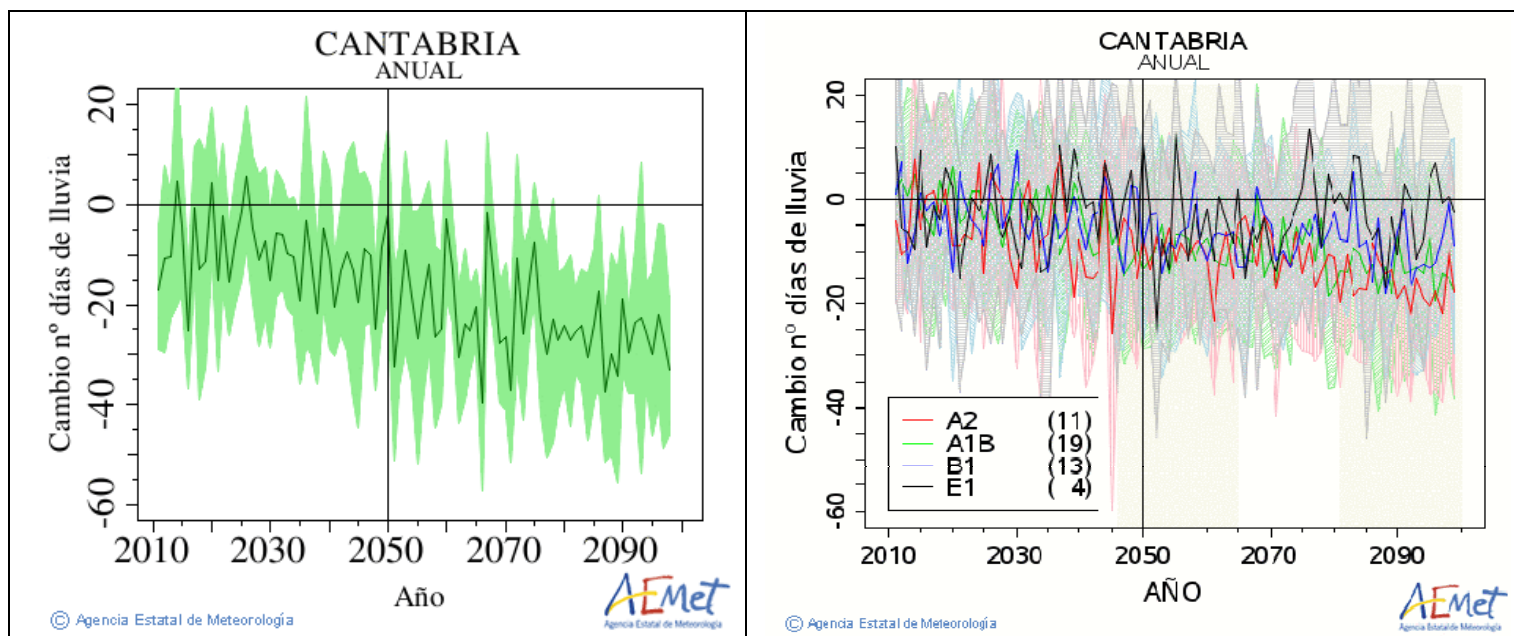

a)

b)
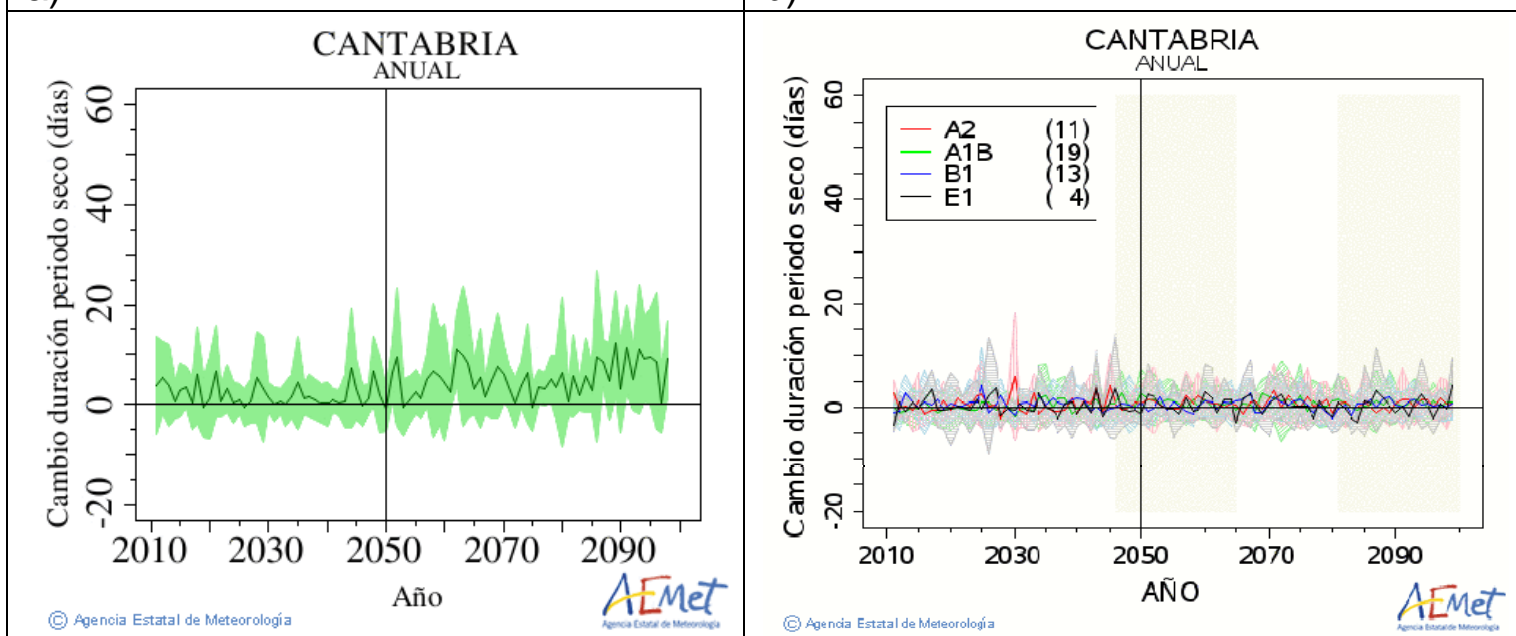

c)

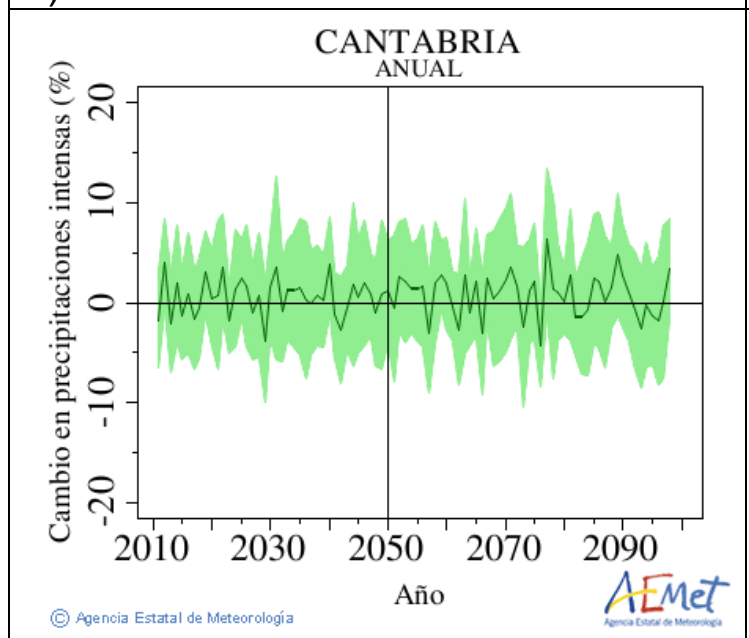

e)

d)

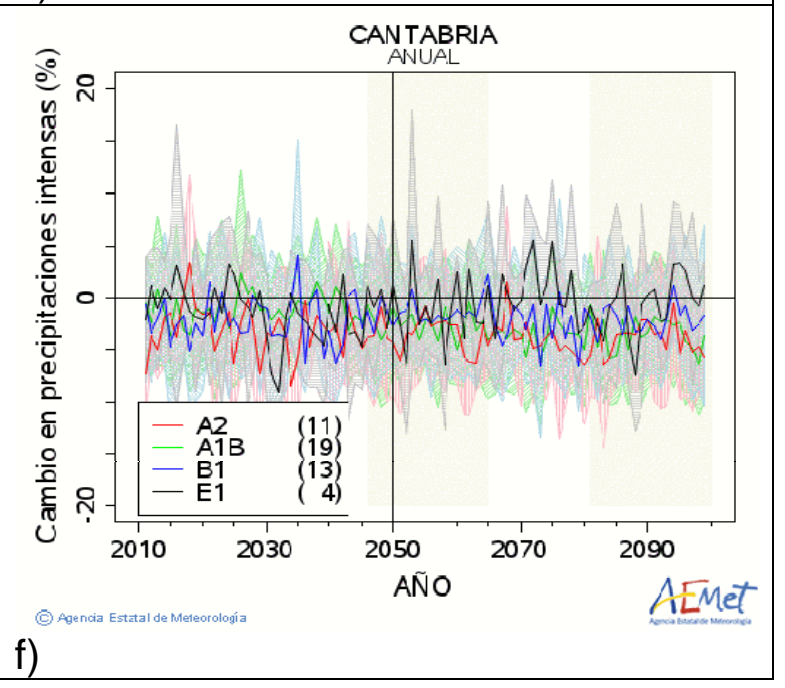

Figura A.38. Igual que la Figura A.3 para la Comunidad Autónoma de Cantabria. 


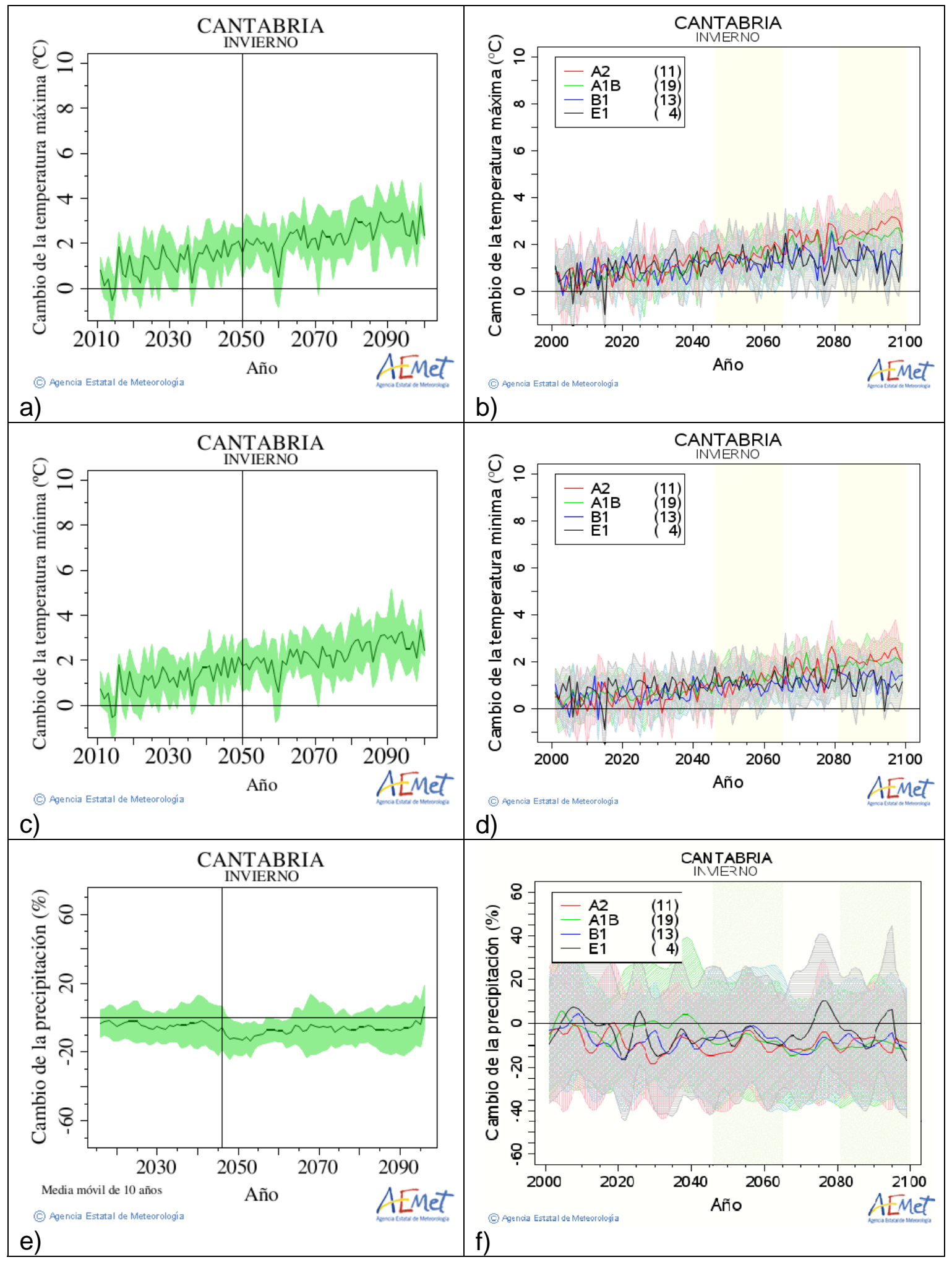

Figura A.39. Igual que la Figura A.4 para la Comunidad Autónoma de Cantabria. 

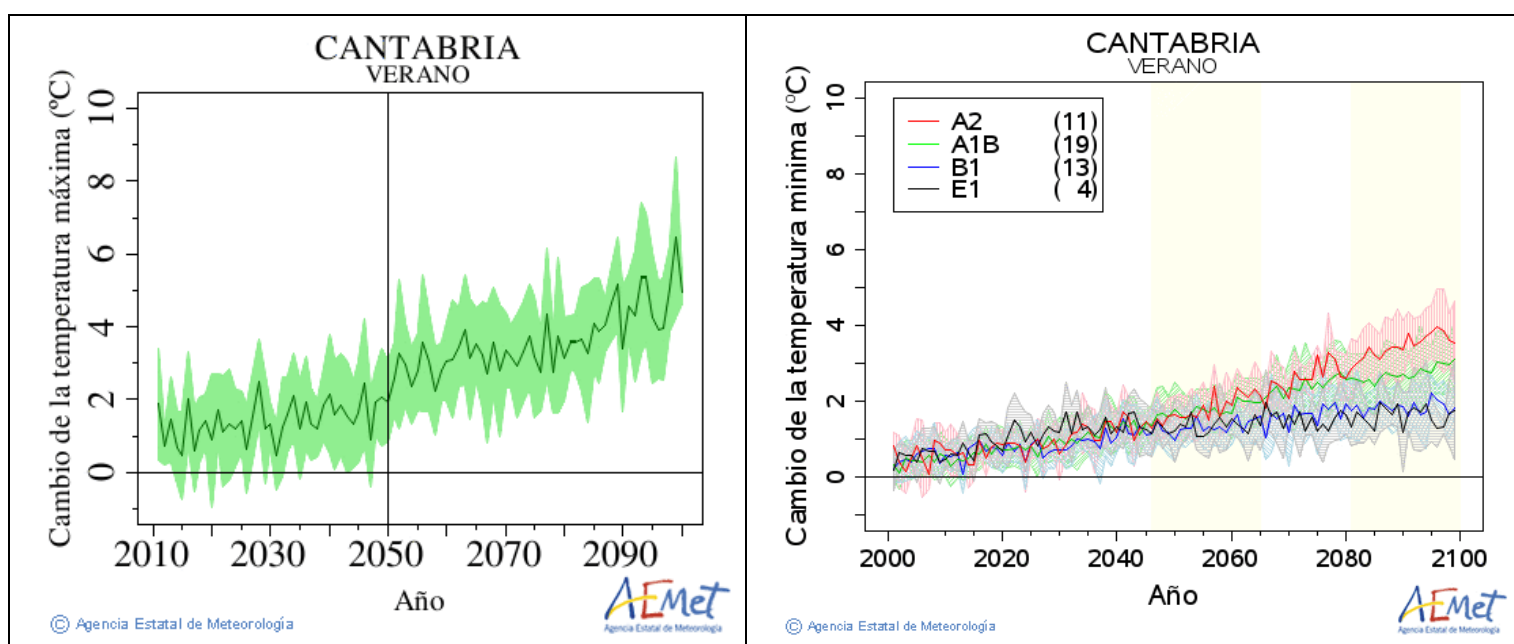

a)

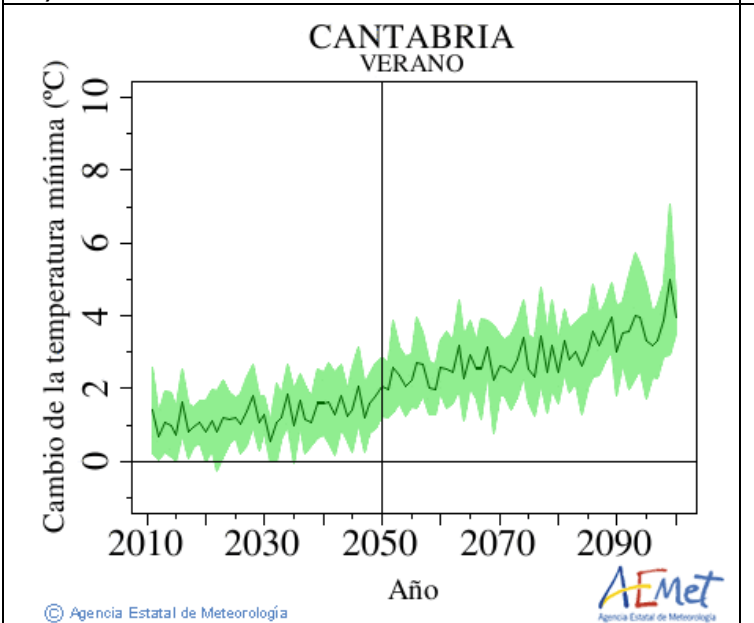

b)

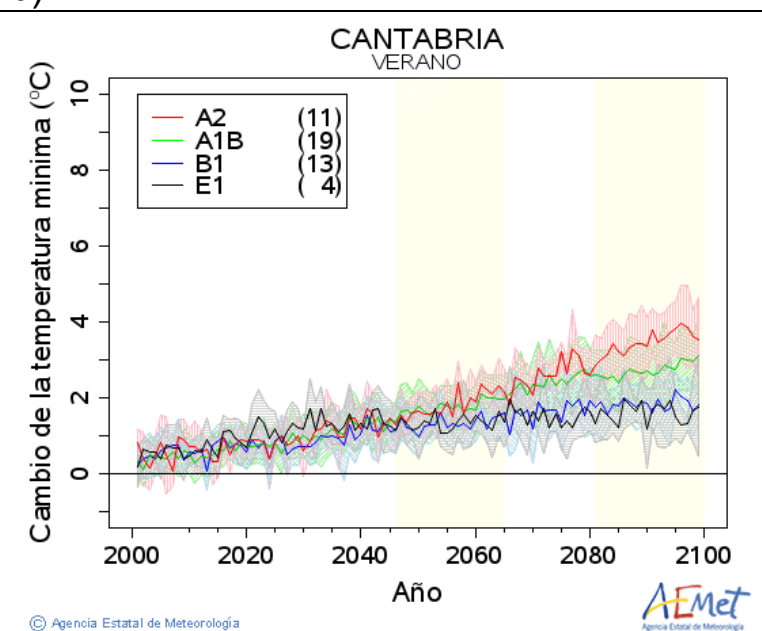

c)

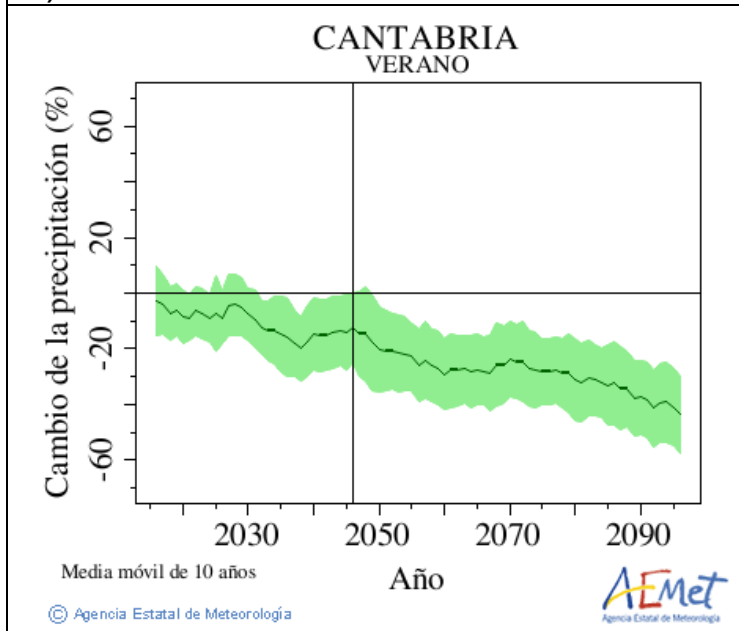

d)

e)

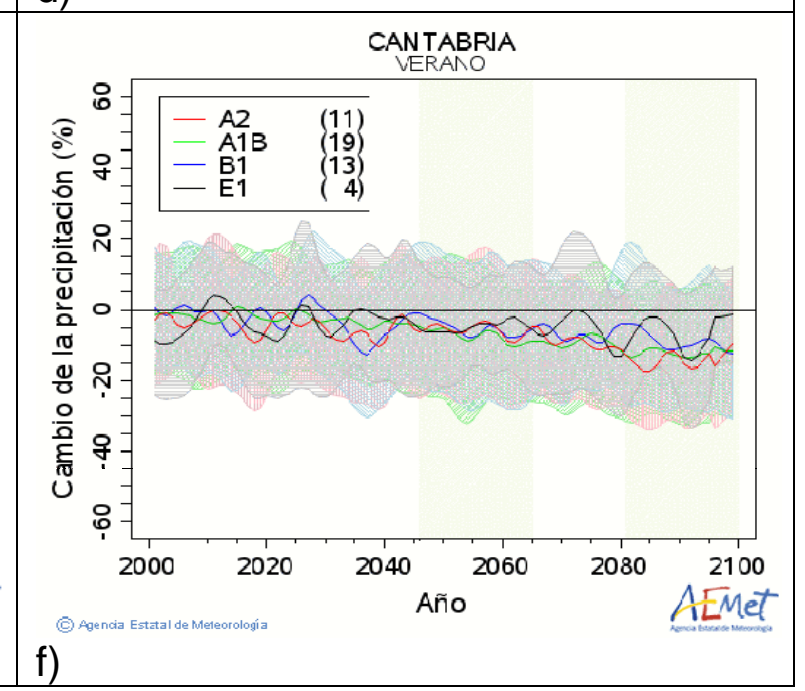

Figura A.40. Igual que la Figura A.5 para la Comunidad Autónoma de Cantabria. 


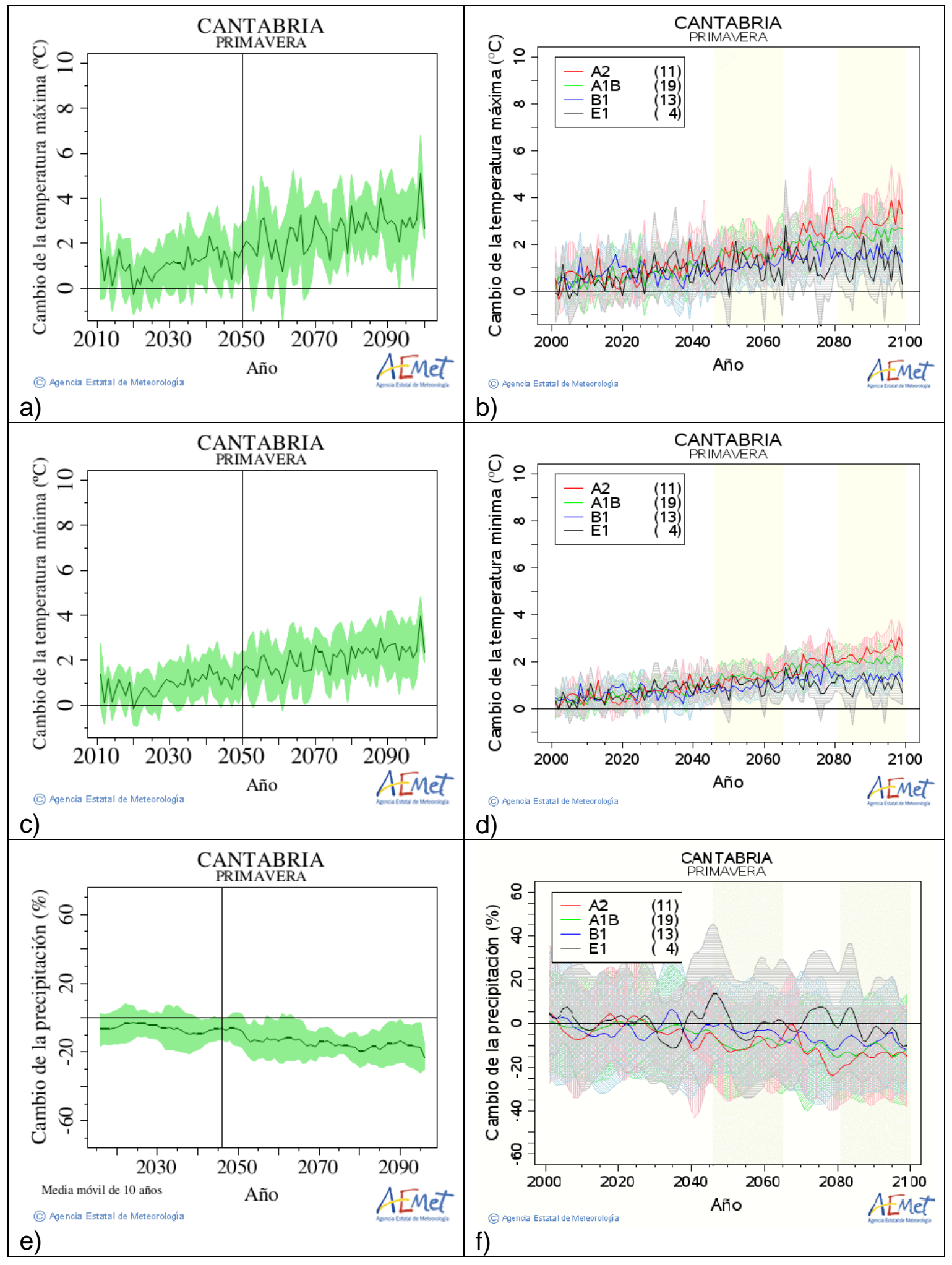

Figura A.41. Igual que la Figura A.6 para la Comunidad Autónoma de Cantabria. 

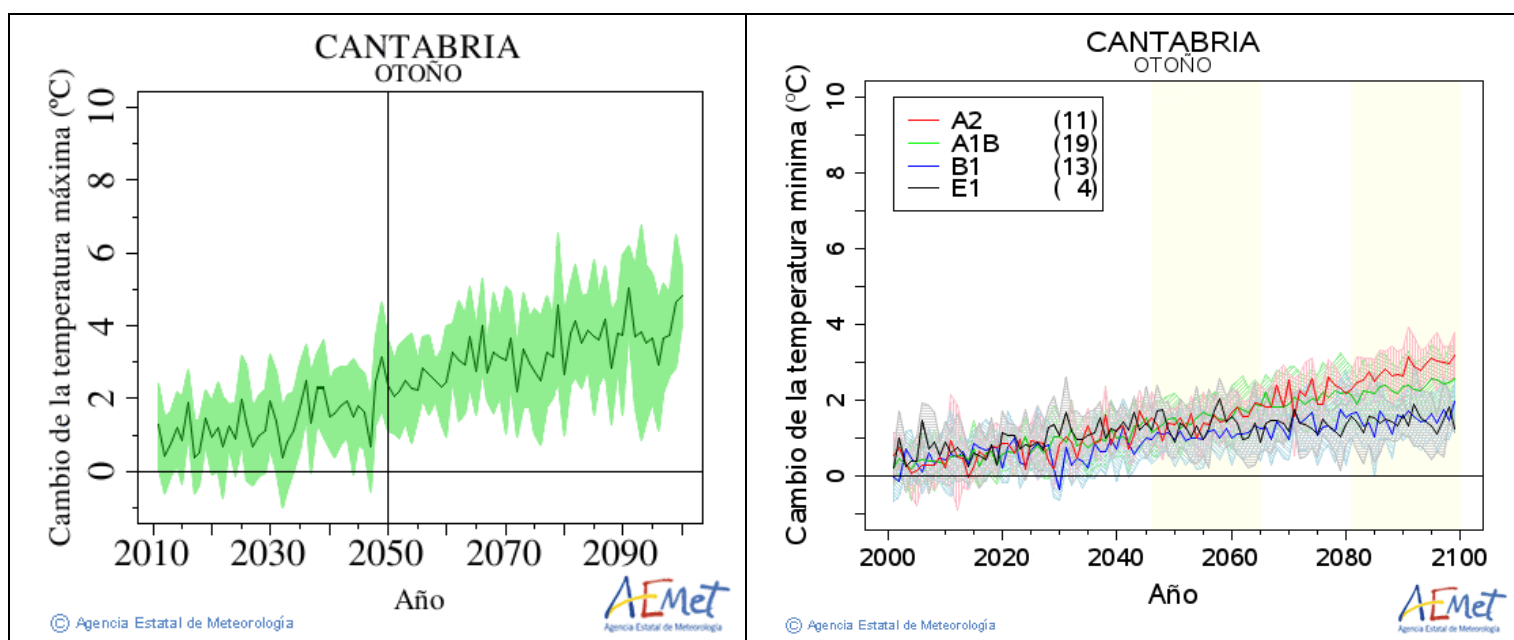

a)

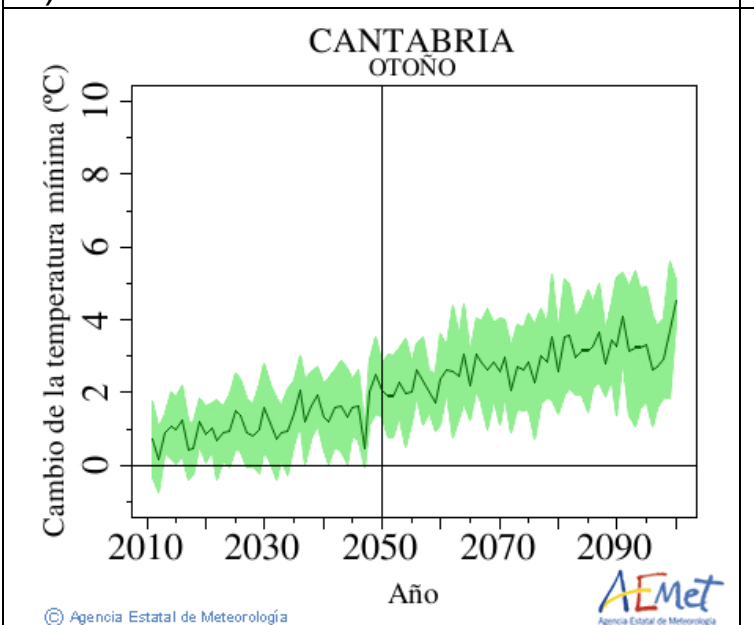

b)

c)

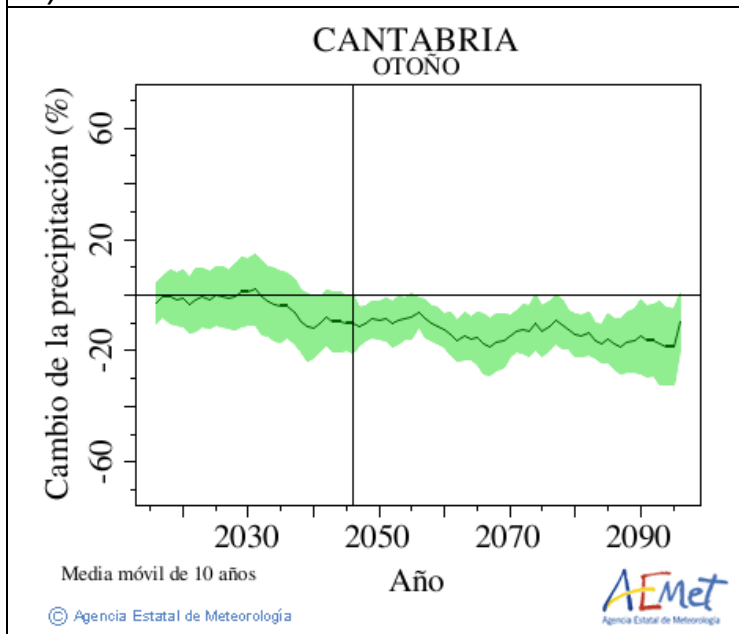

e)

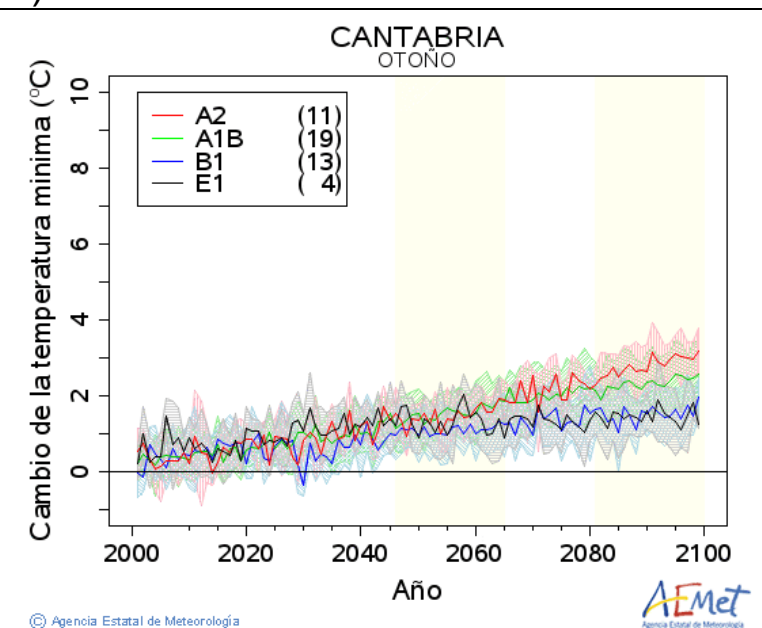

d)

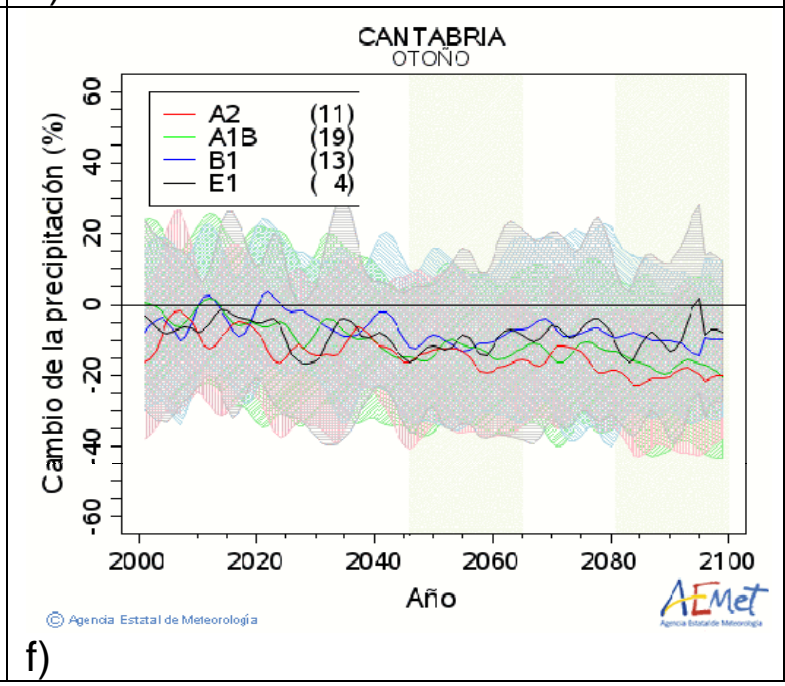

Figura A.42. Igual que la Figura A.7 para la Comunidad Autónoma de Cantabria. 
CASTILLA - LA MANCHA

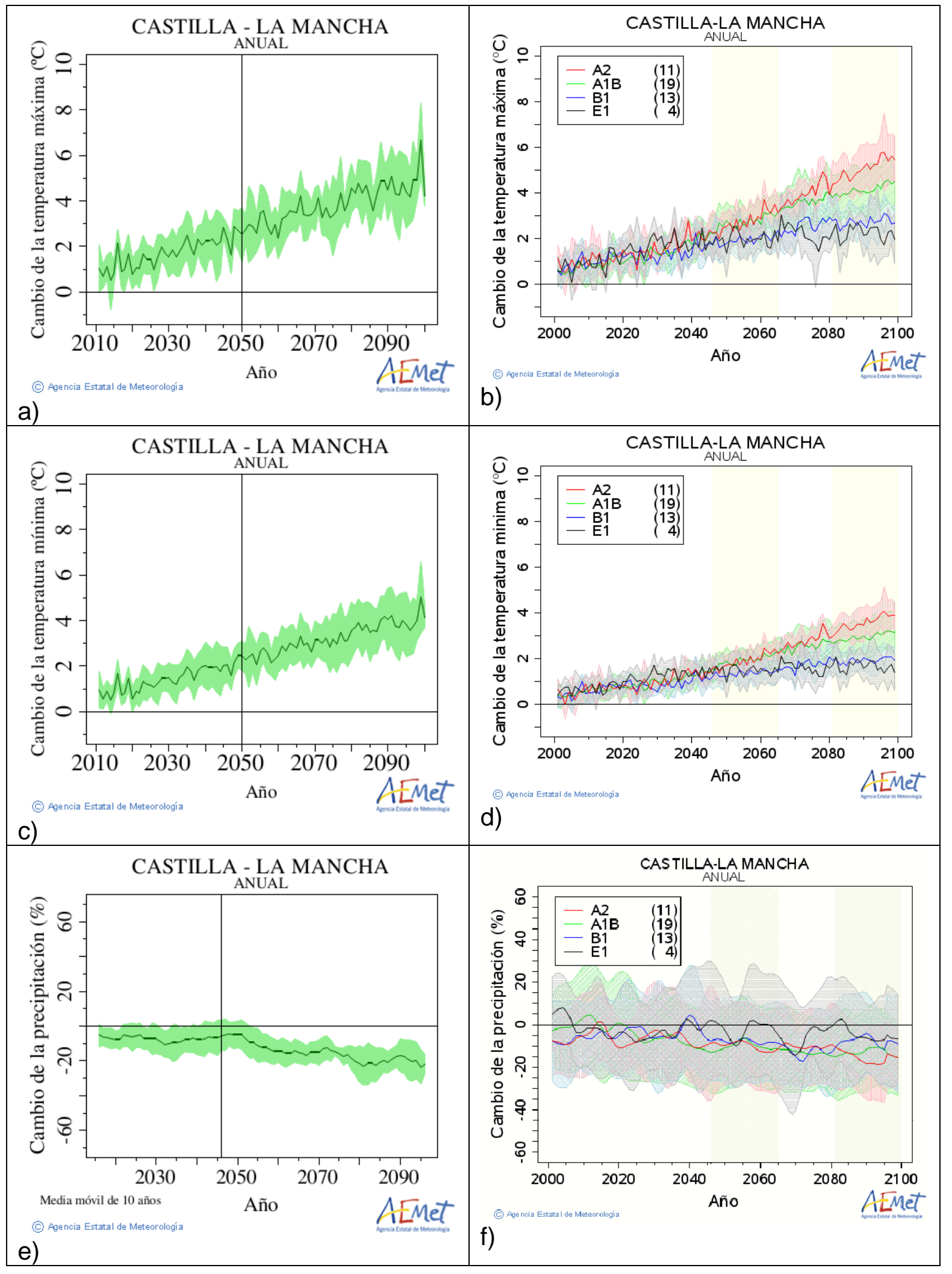

Figura A.43. Igual que la Figura A.1 para la Comunidad Autónoma de Castilla - La Mancha. 


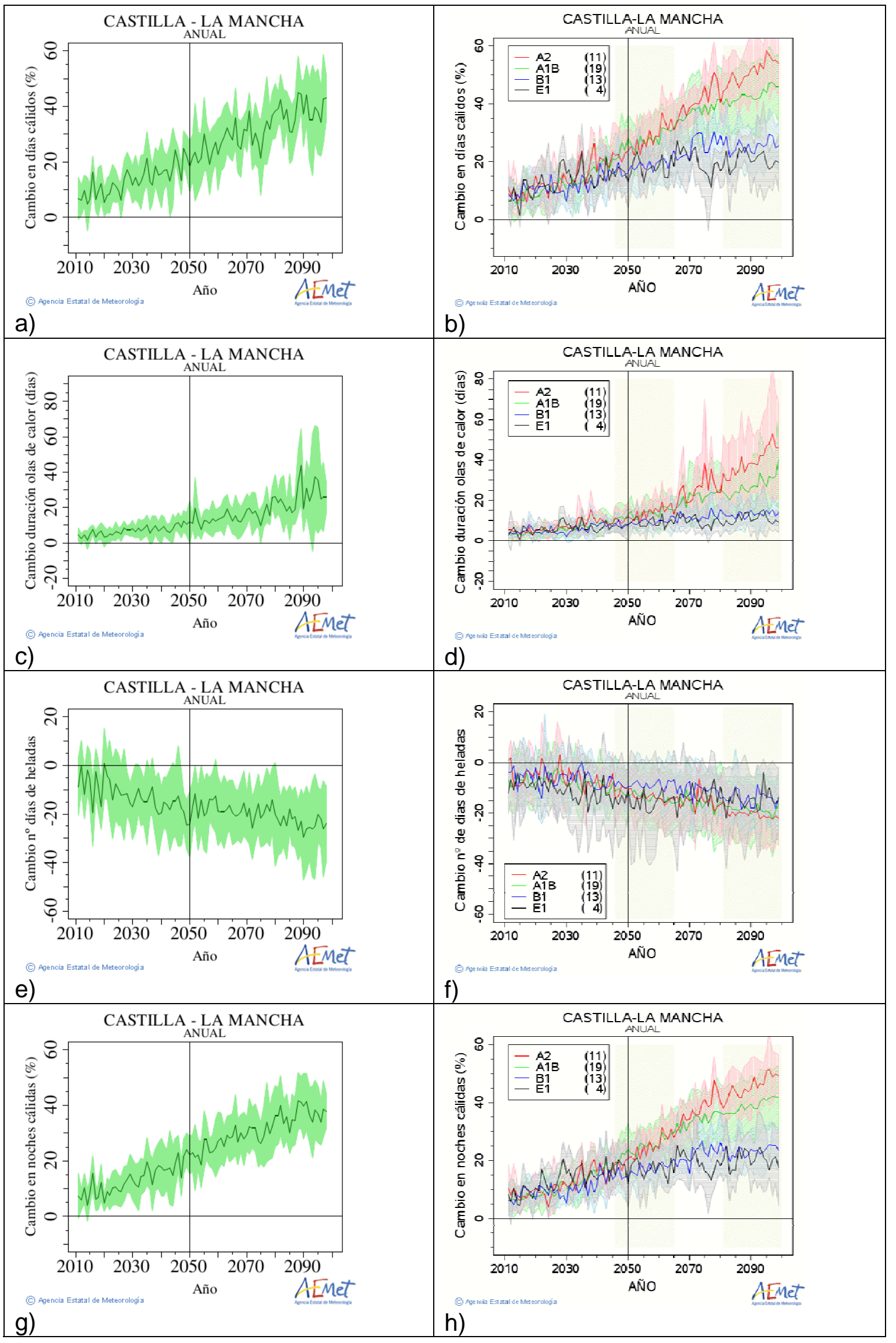

Figura A.44. Igual que la Figura A.2 para la Comunidad Autónoma de Castilla - La Mancha. 

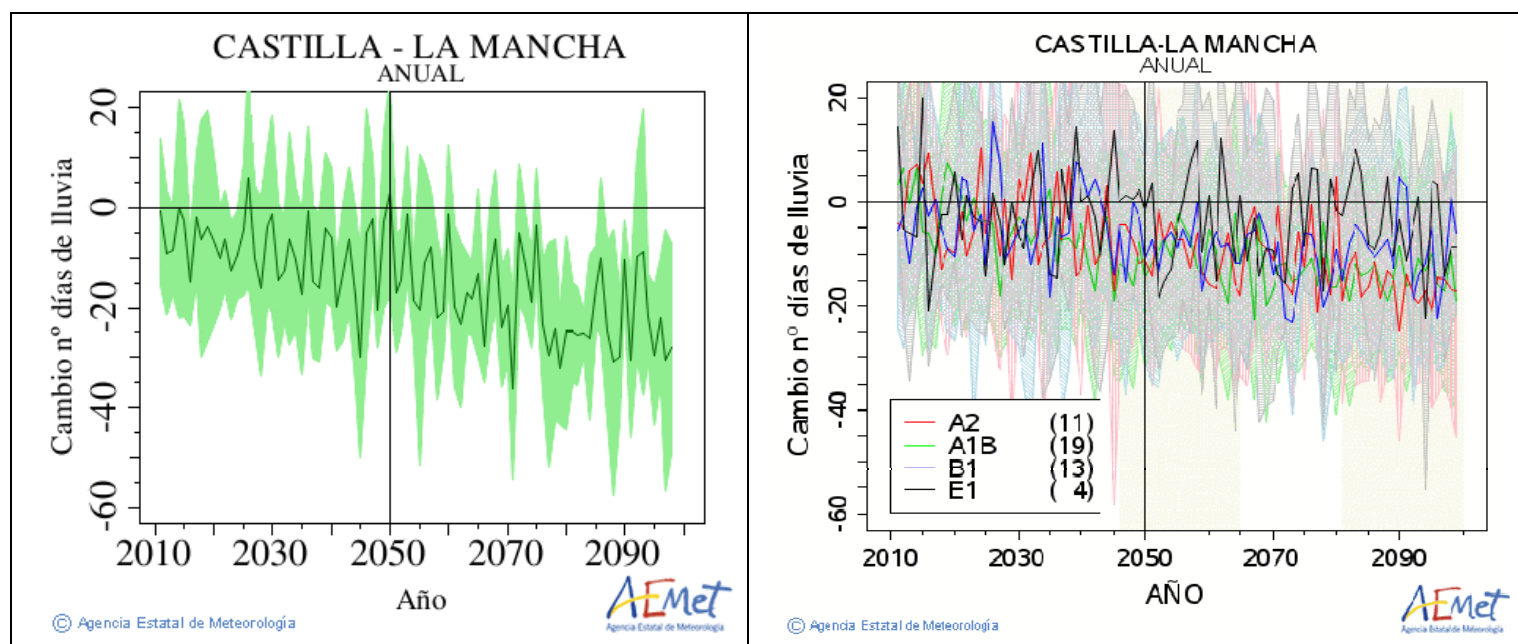

a)

b)
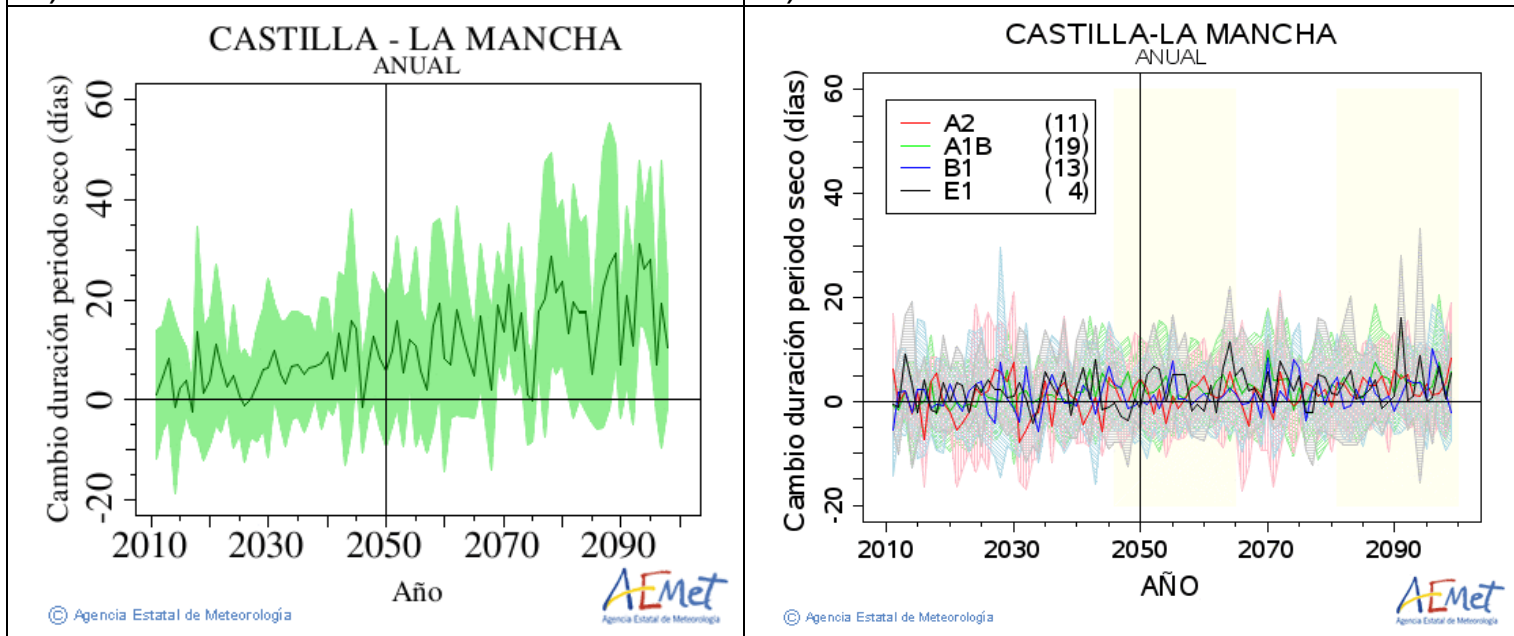

c)

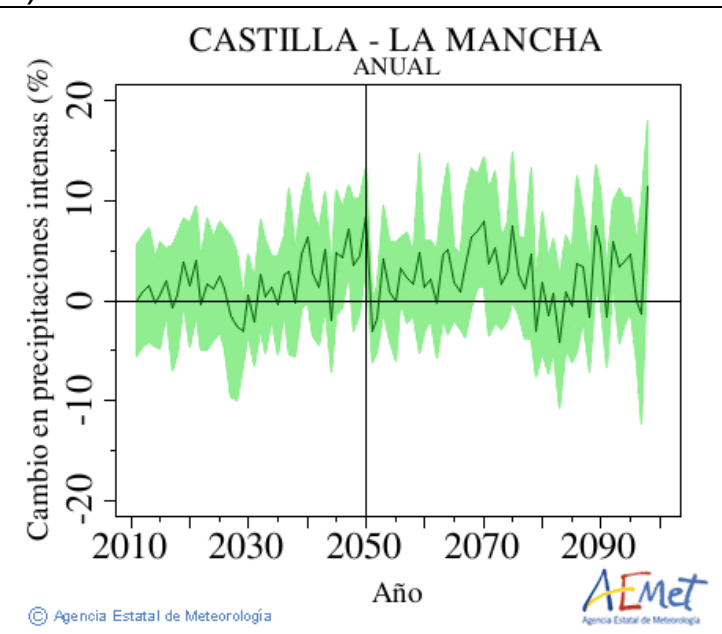

d)

e)

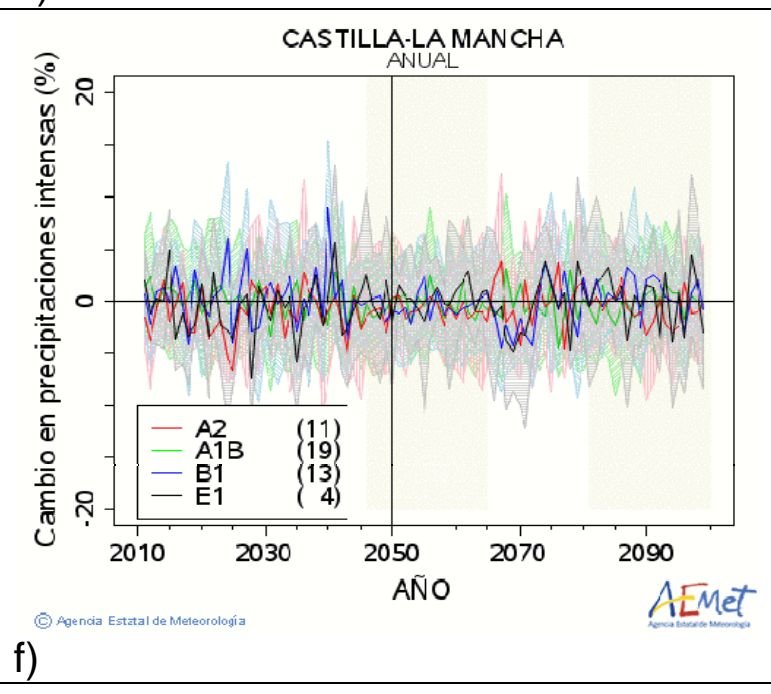

Figura A.45. Igual que la Figura A.3 para la Comunidad Autónoma de Castilla - La Mancha. 


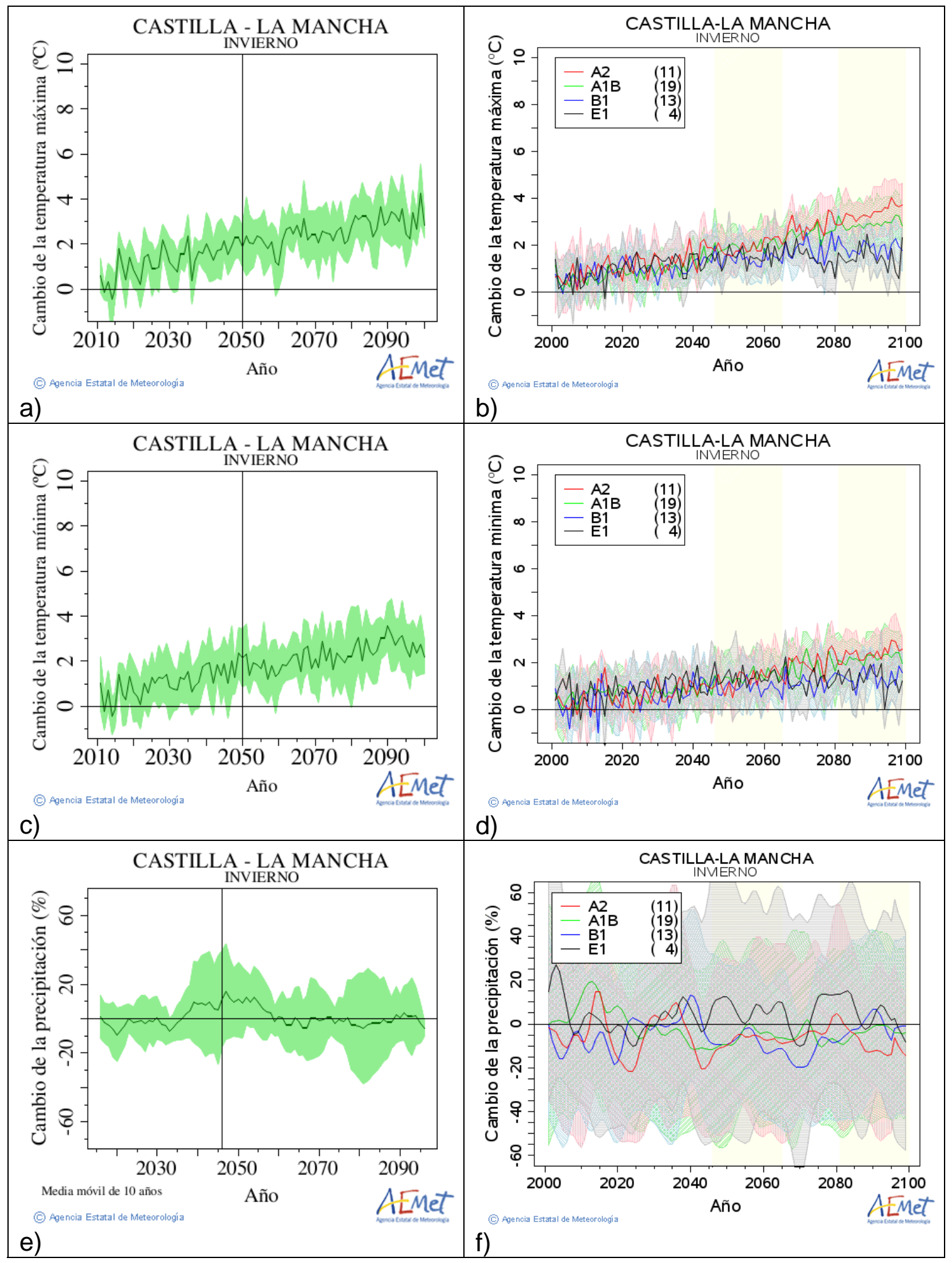

Figura A.46. Igual que la Figura A.4 para la Comunidad Autónoma de Castilla - La Mancha. 

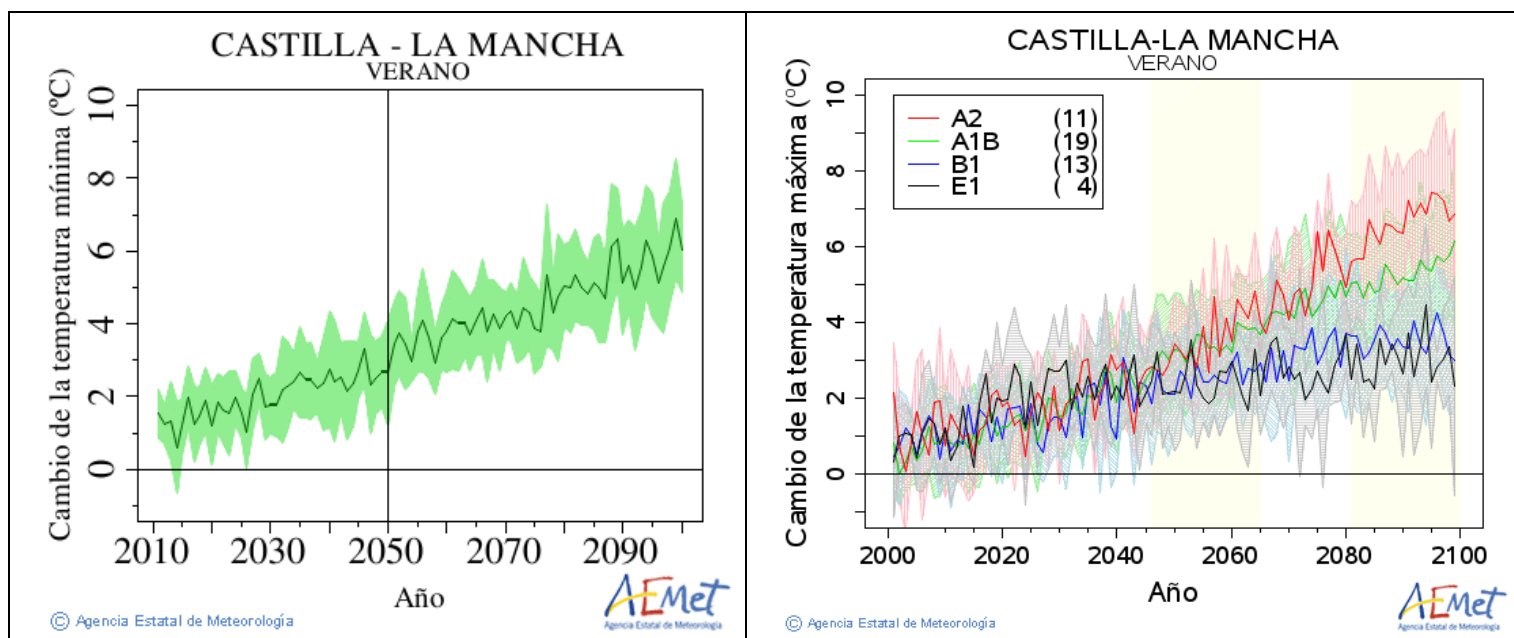

a)

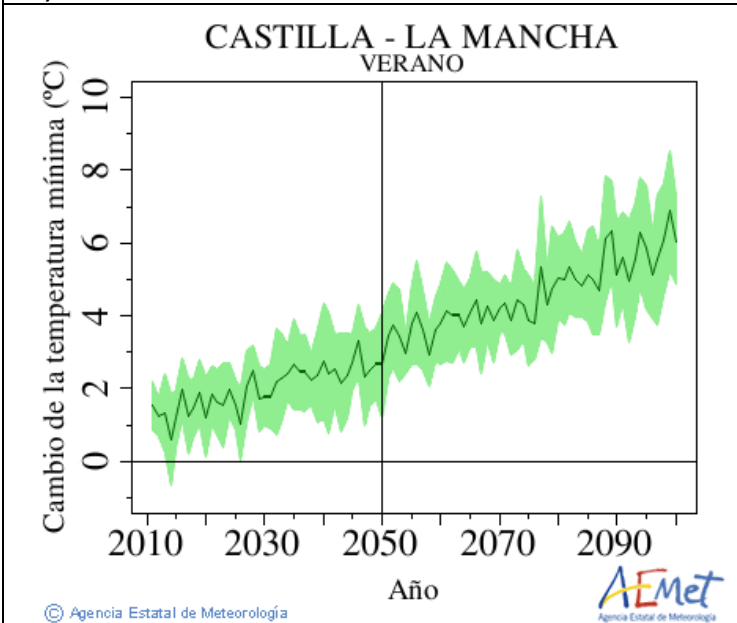

b)

c)

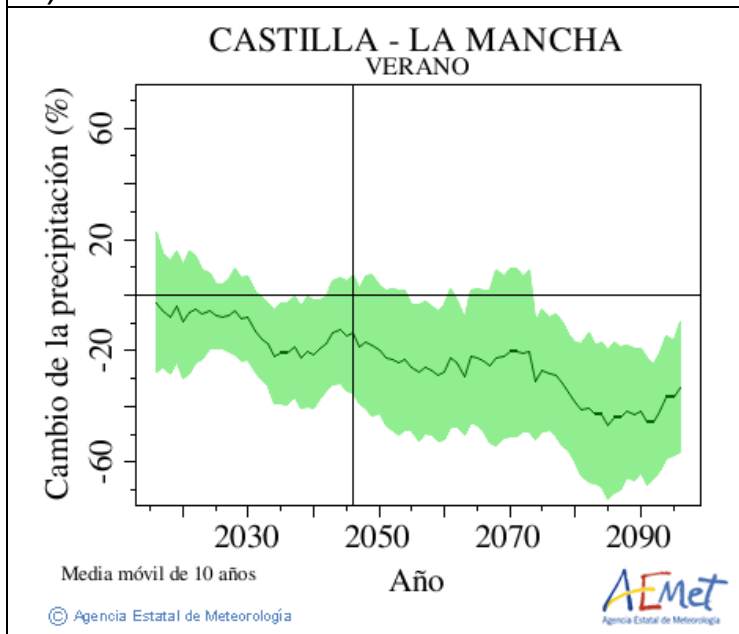

e)

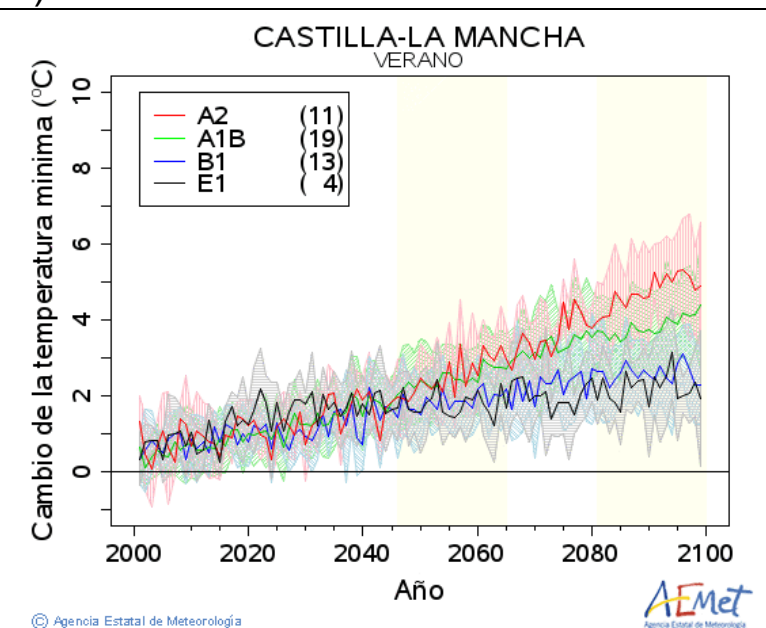

d)

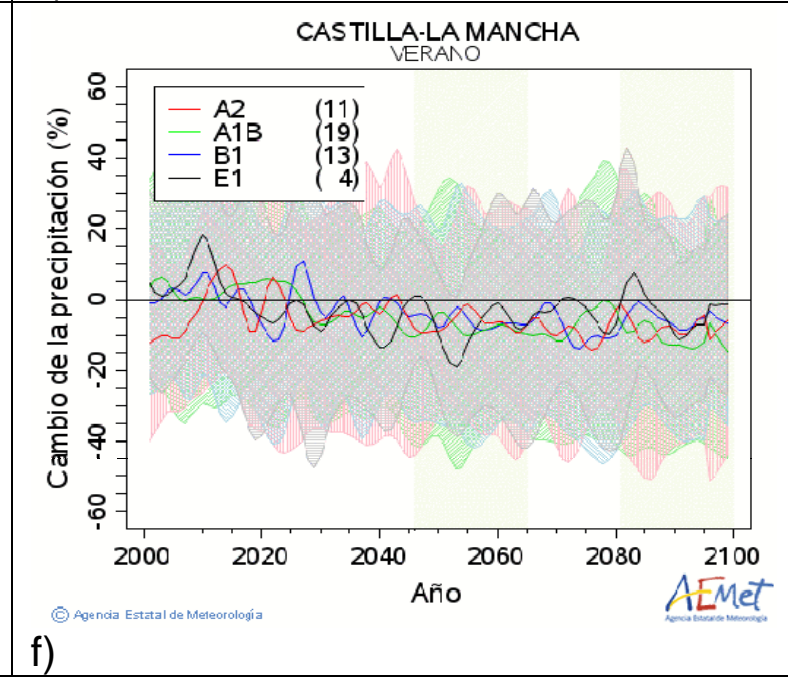

Figura A.47. Igual que la Figura A.5 para la Comunidad Autónoma de Castilla - La Mancha. 


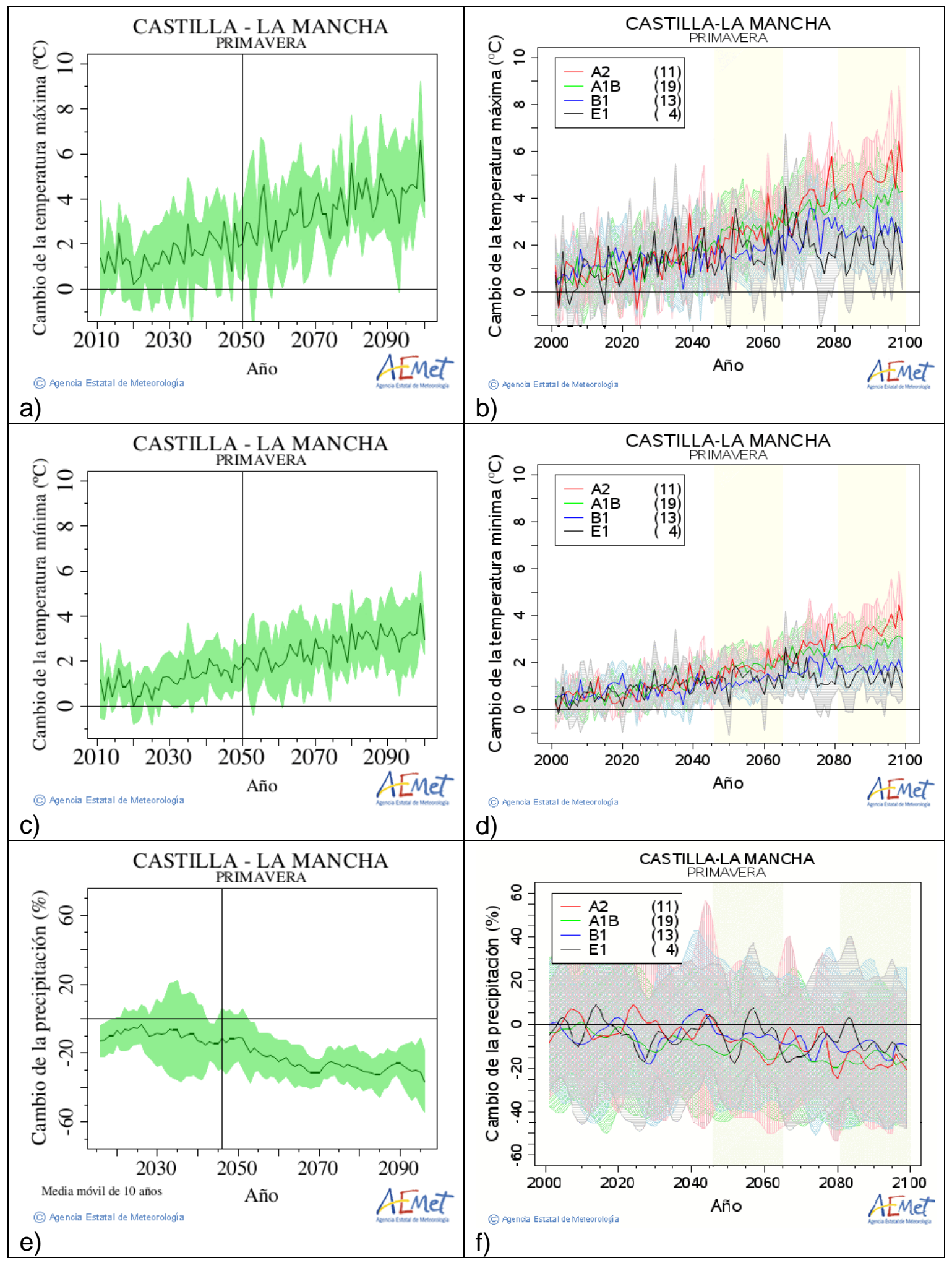

Figura A.48. Igual que la Figura A.6 para la Comunidad Autónoma de Castilla - La Mancha. 

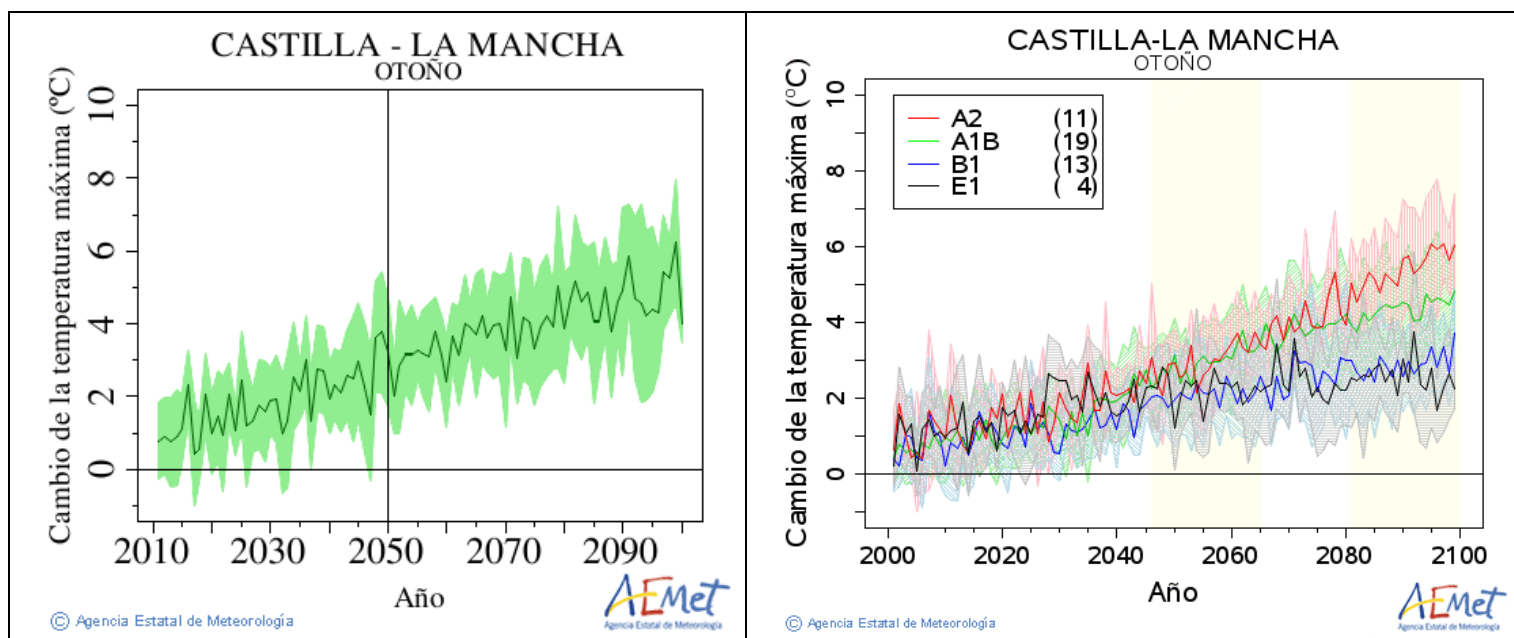

a)

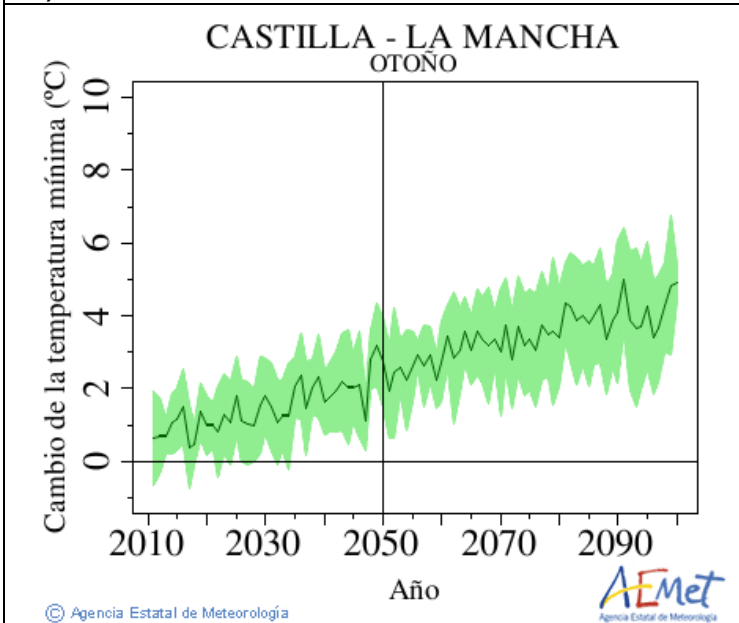

b)

c)

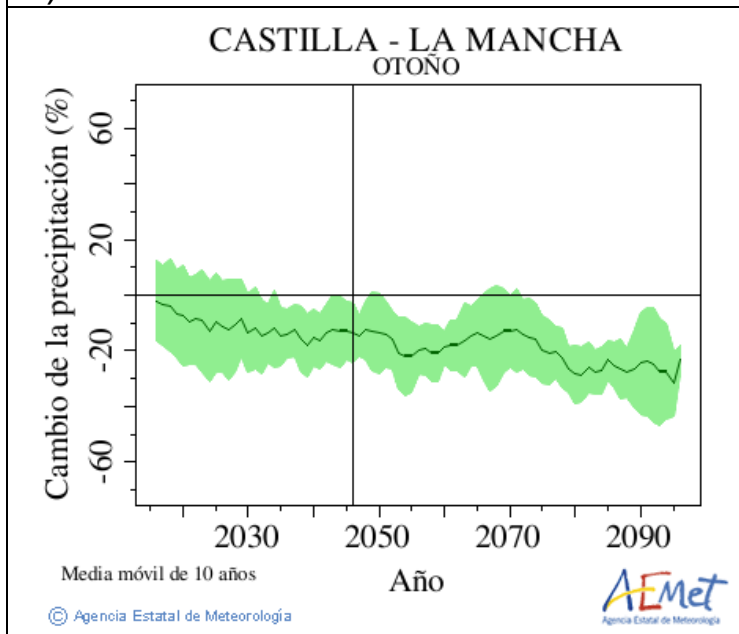

e)

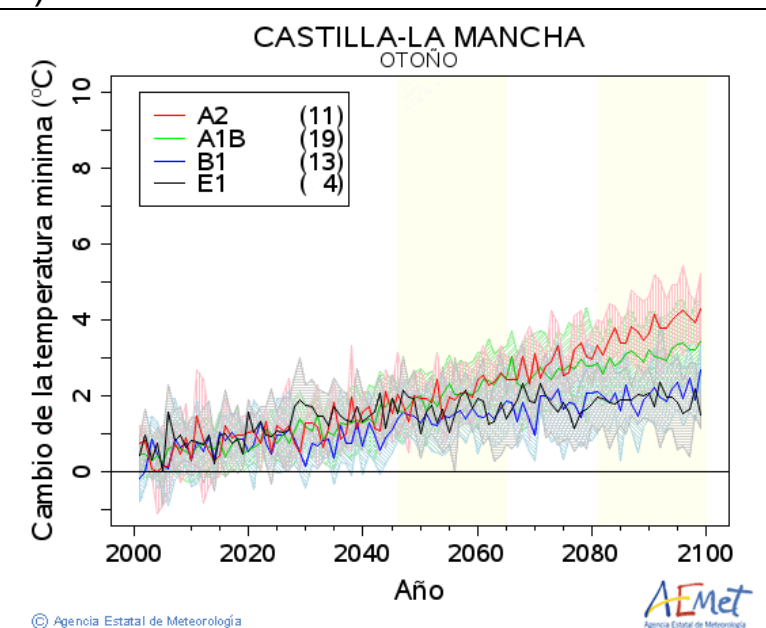

d)

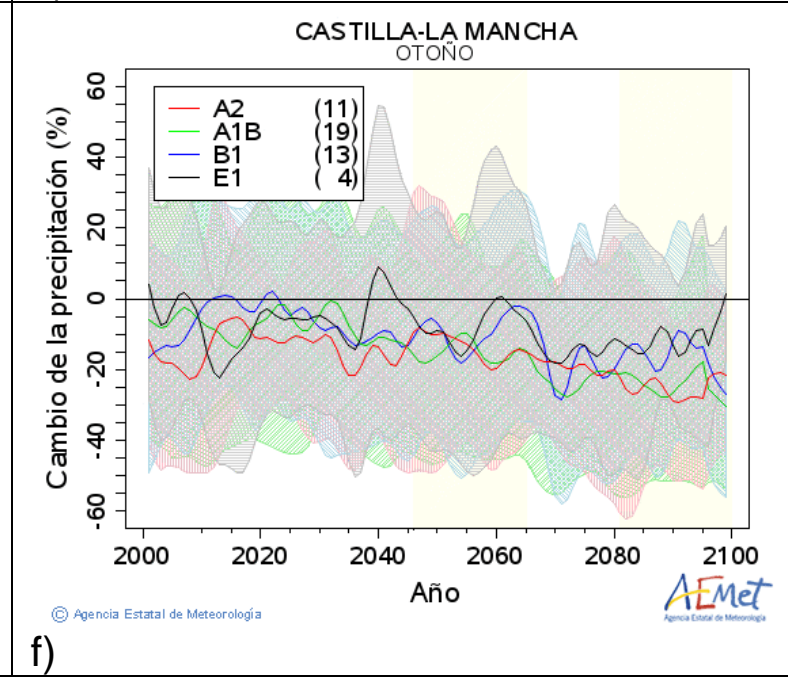

Figura A.49. Igual que la Figura A.7 para la Comunidad Autónoma de Castilla - La Mancha. 
CASTILLA - LEON

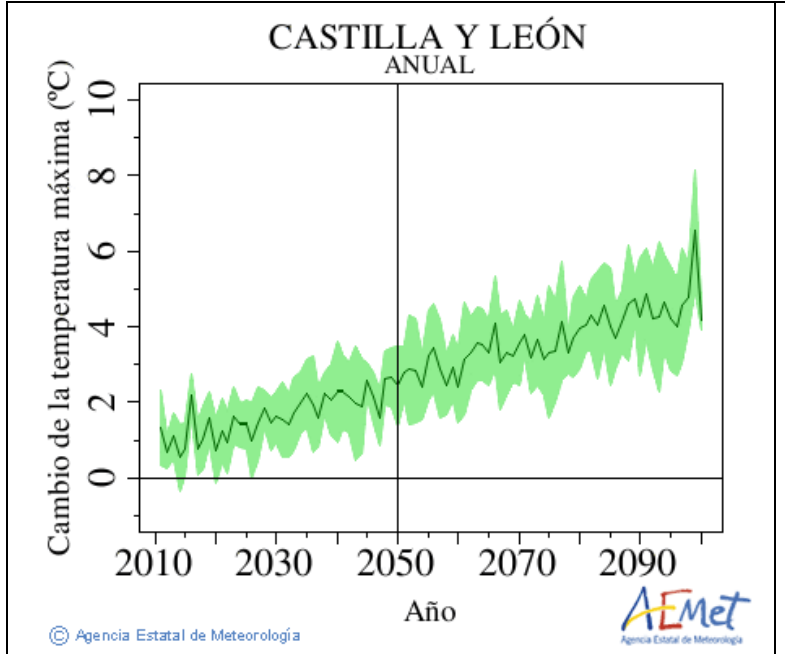

a)

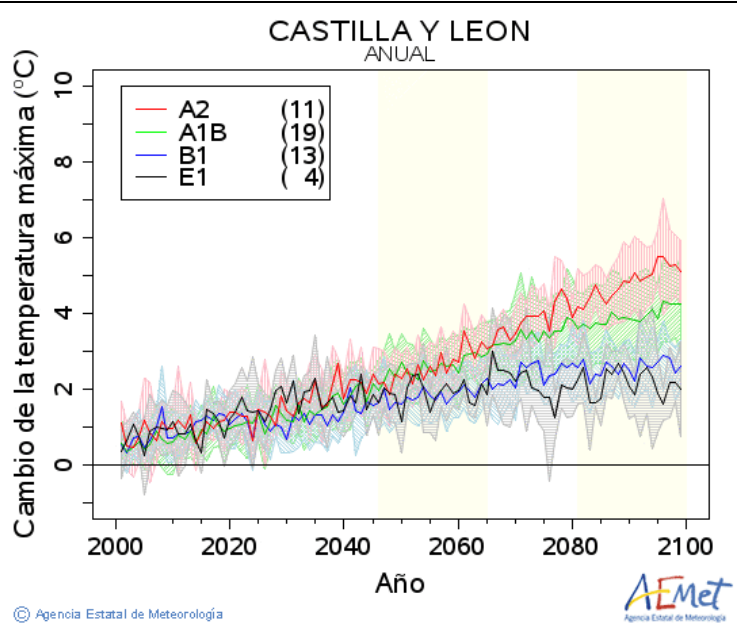

b)
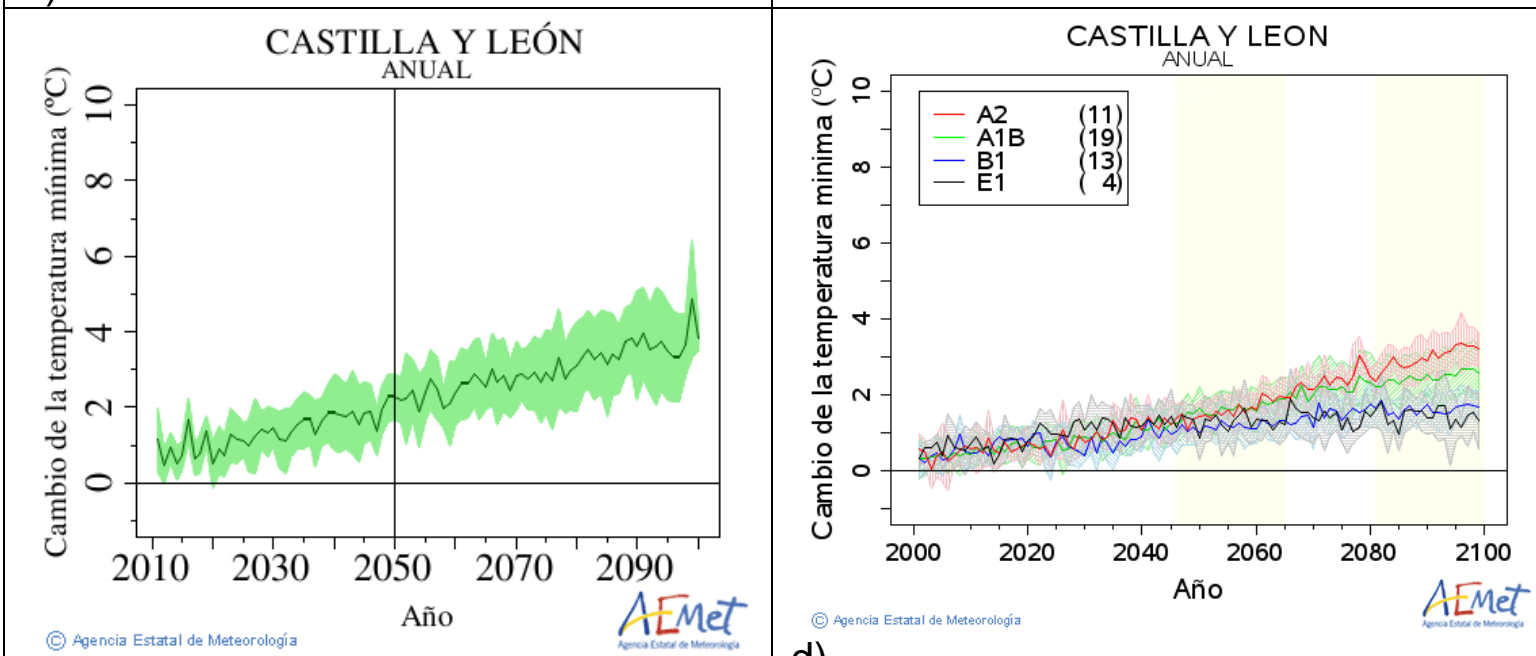

c)

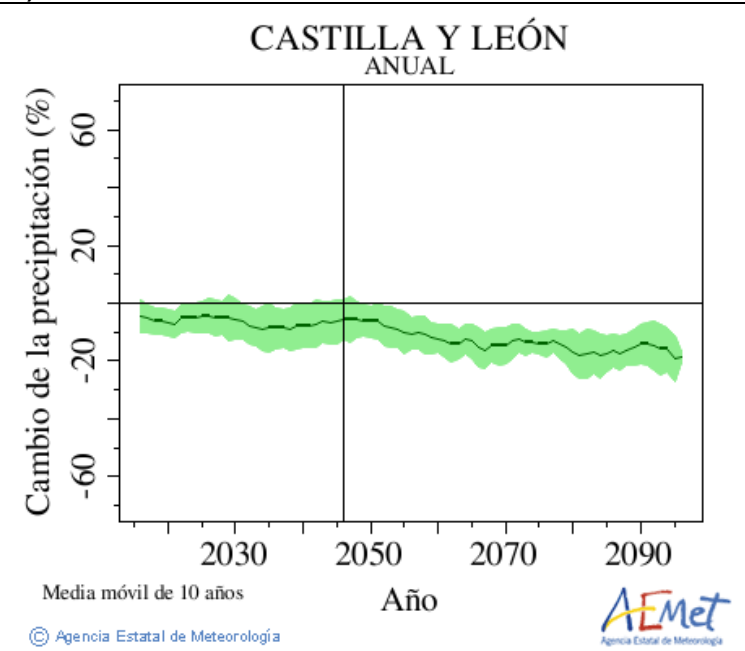

e)

d)

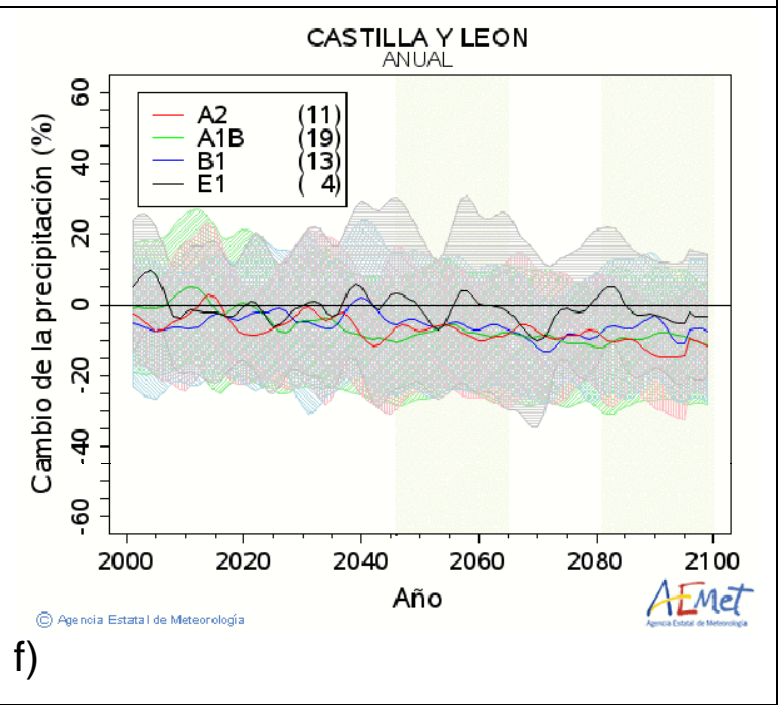

Figura A.50. Igual que la Figura A.1 para la Comunidad Autónoma de Castilla - León. 


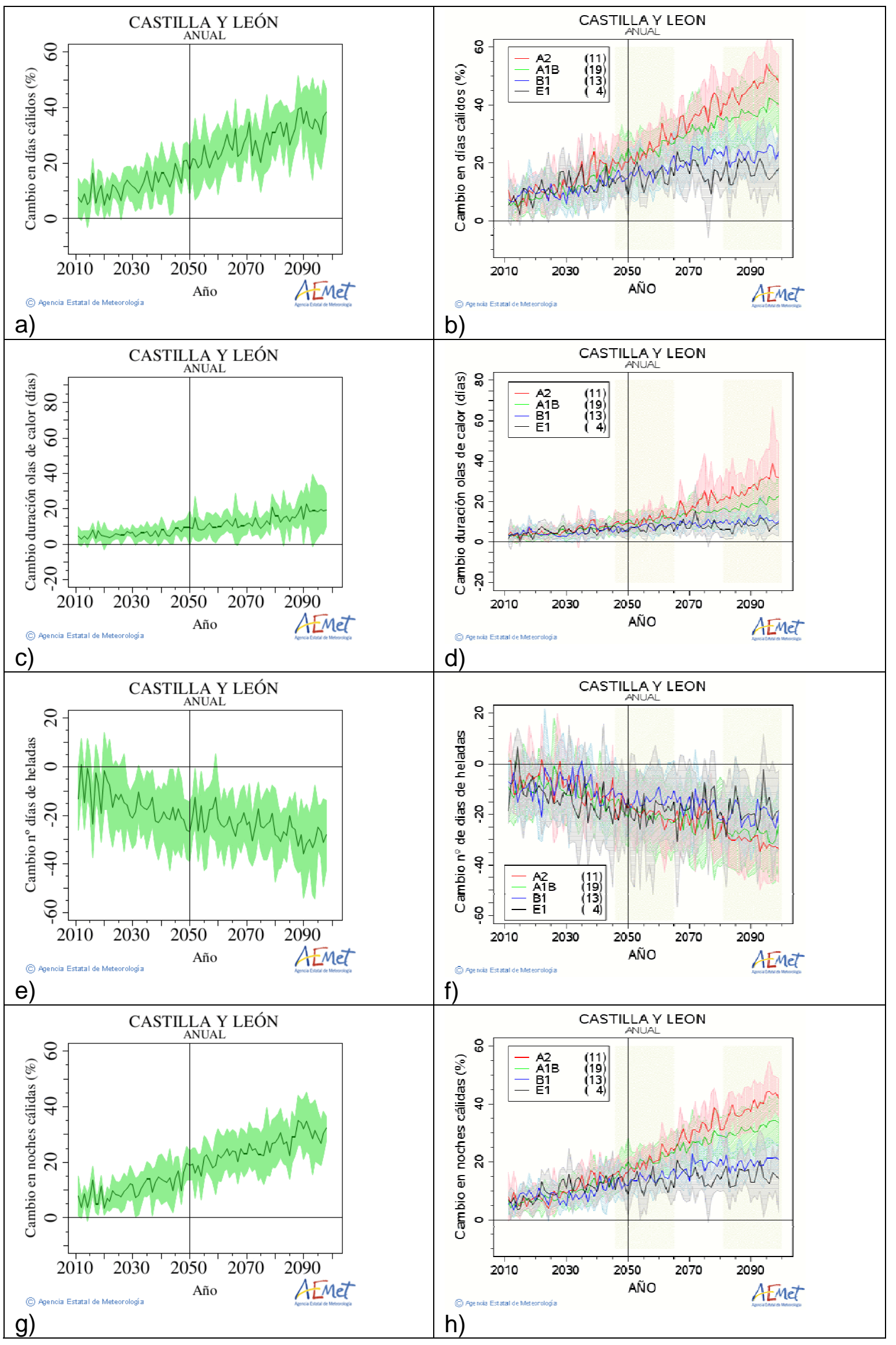

Figura A.51. Igual que la Figura A.2 para la Comunidad Autónoma de Castilla - León. 

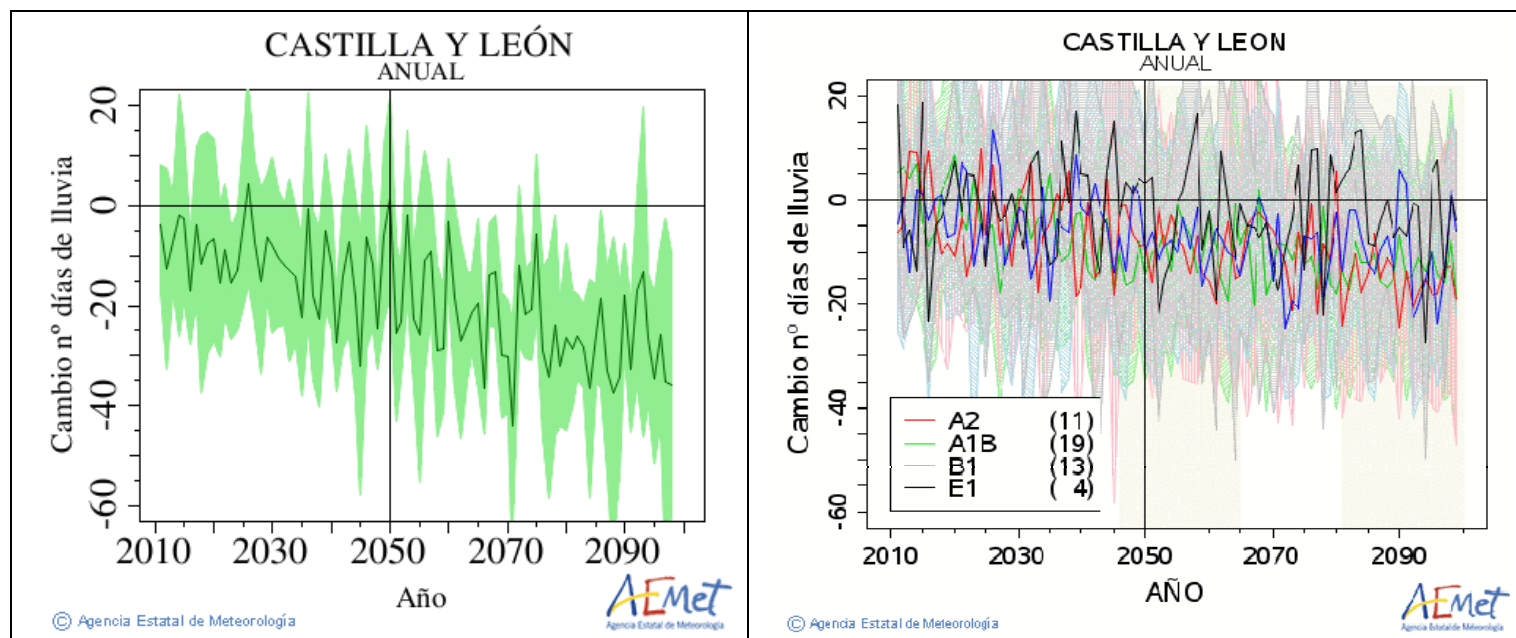

a)

b)
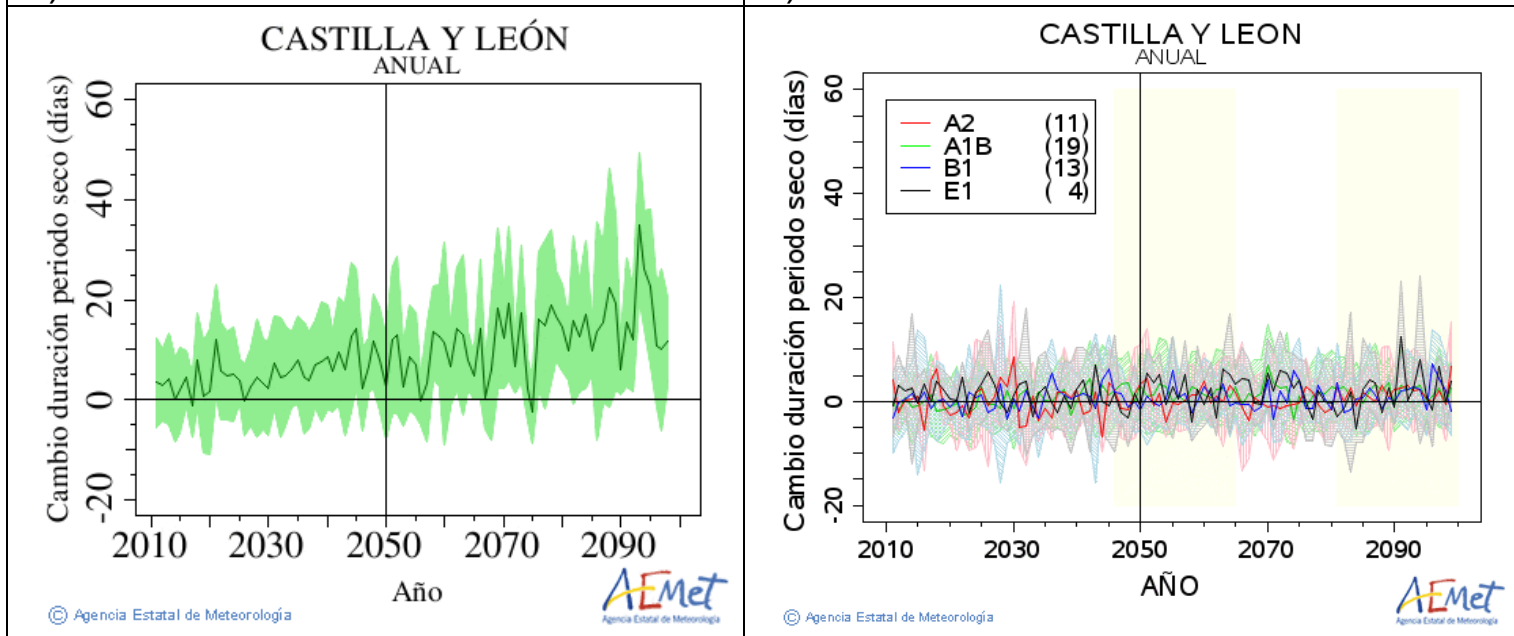

c)

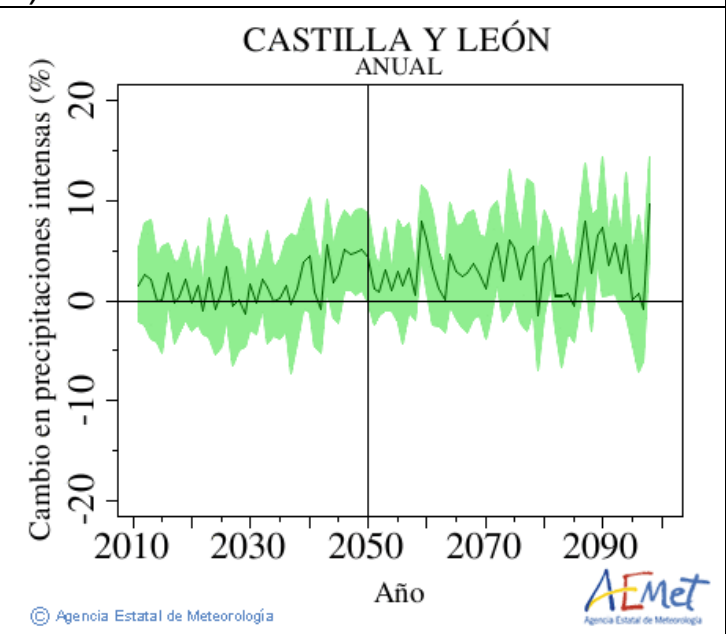

e)

d)

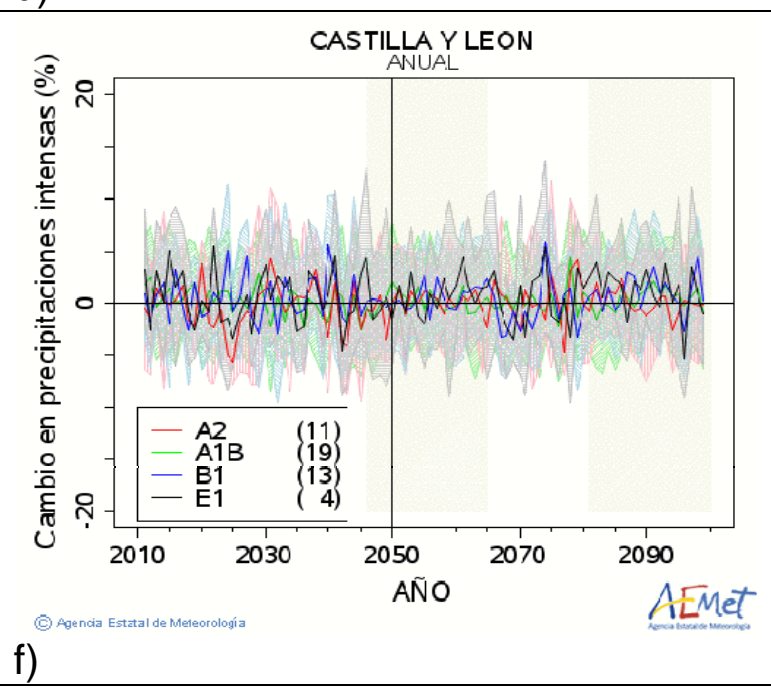

Figura A.52. Igual que la Figura A.3 para la Comunidad Autónoma de Castilla - León. 


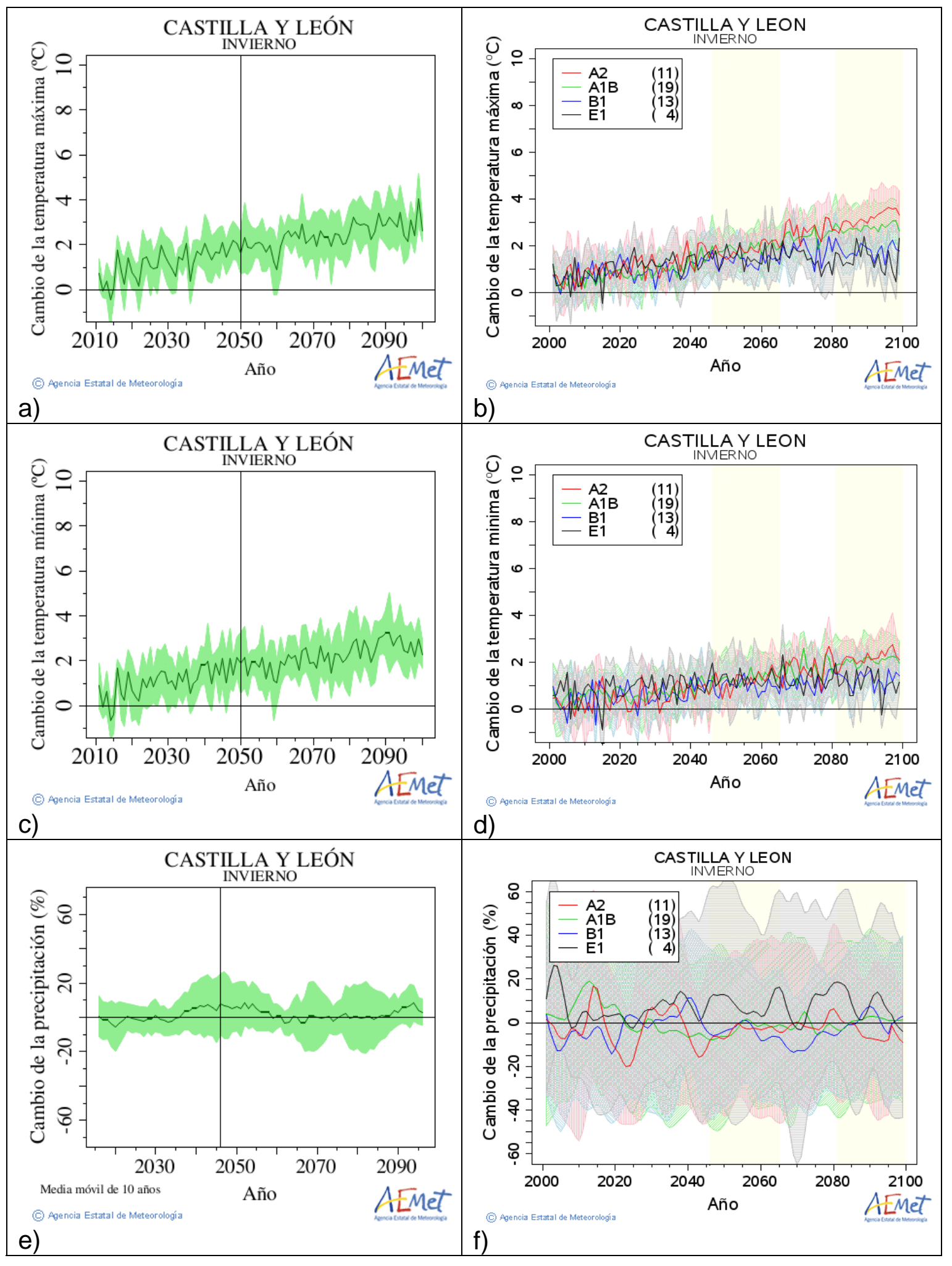

Figura A.53. Igual que la Figura A.4 para la Comunidad Autónoma de Castilla - León. 

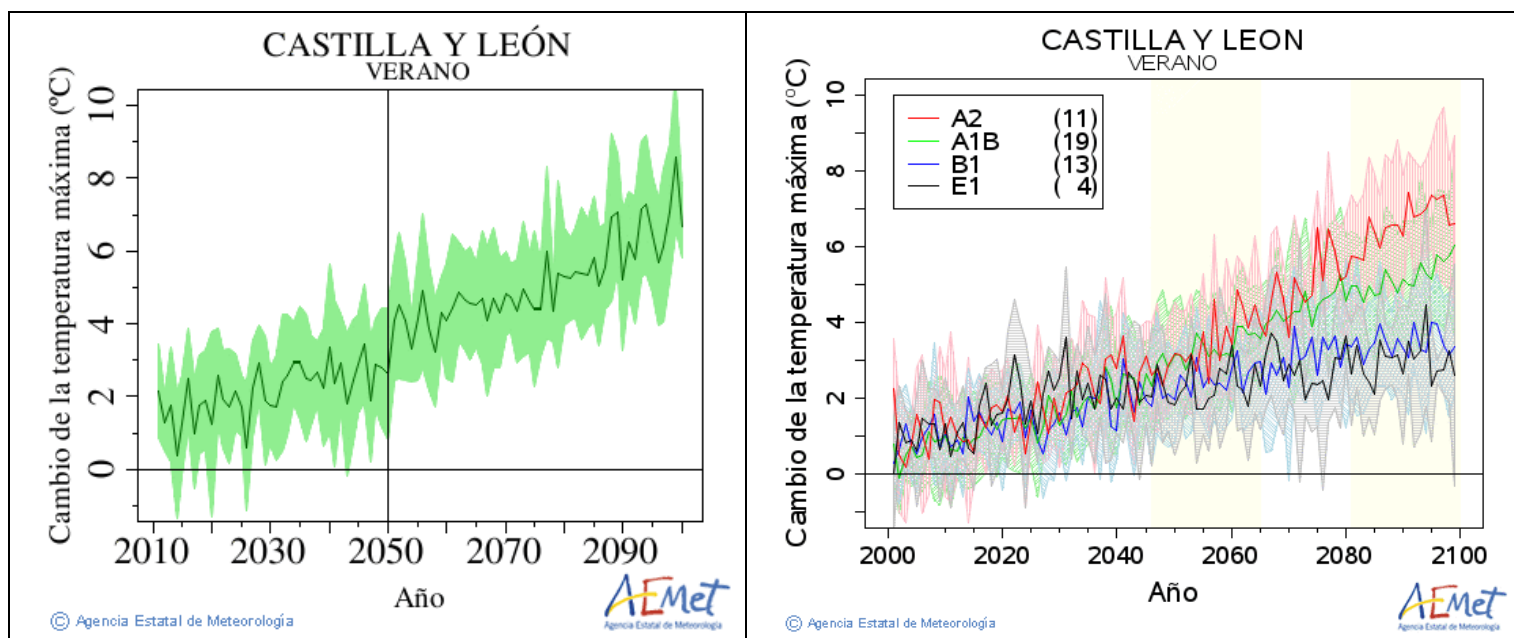

a)

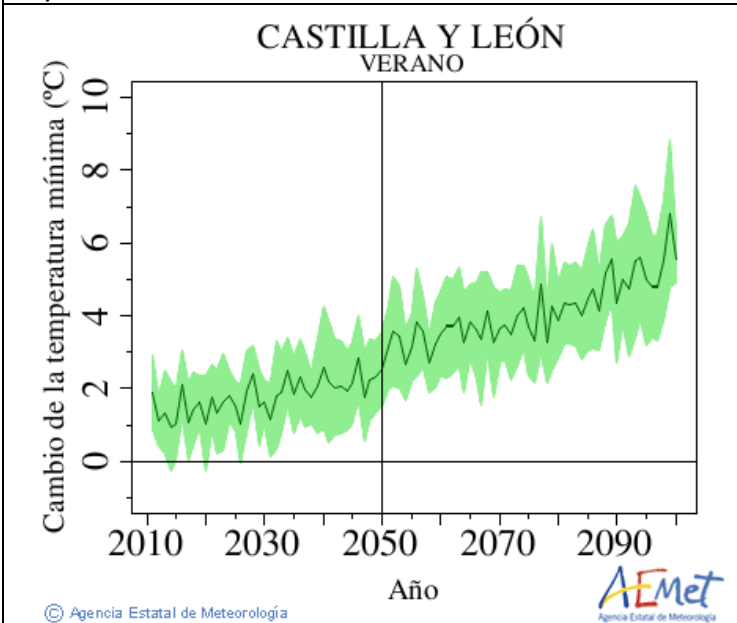

b)

c)

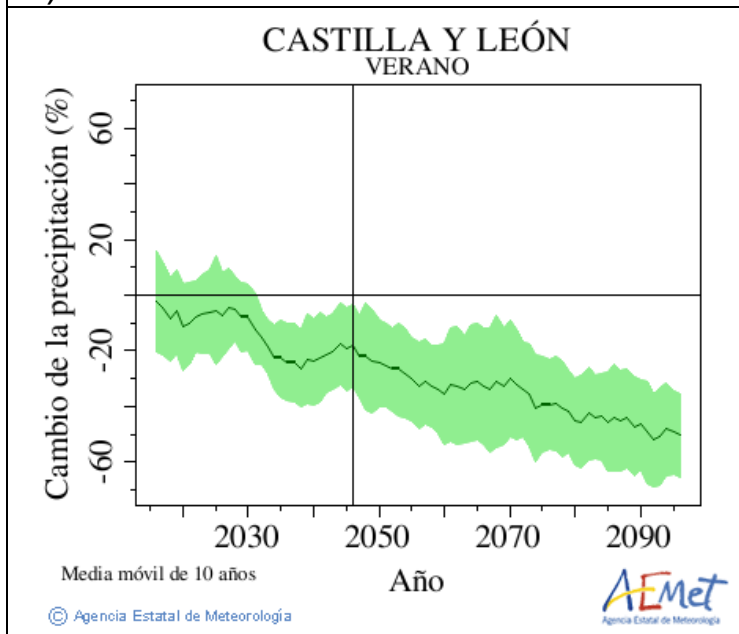

e)

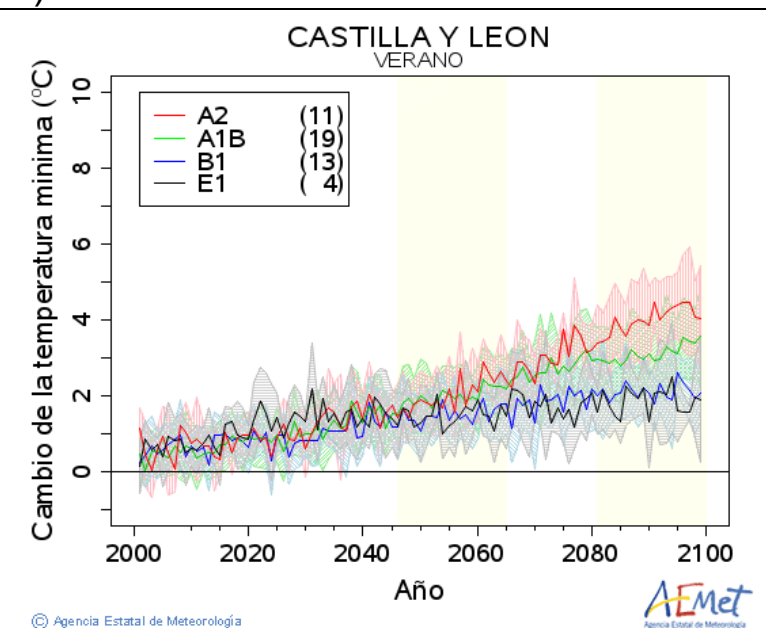

d)

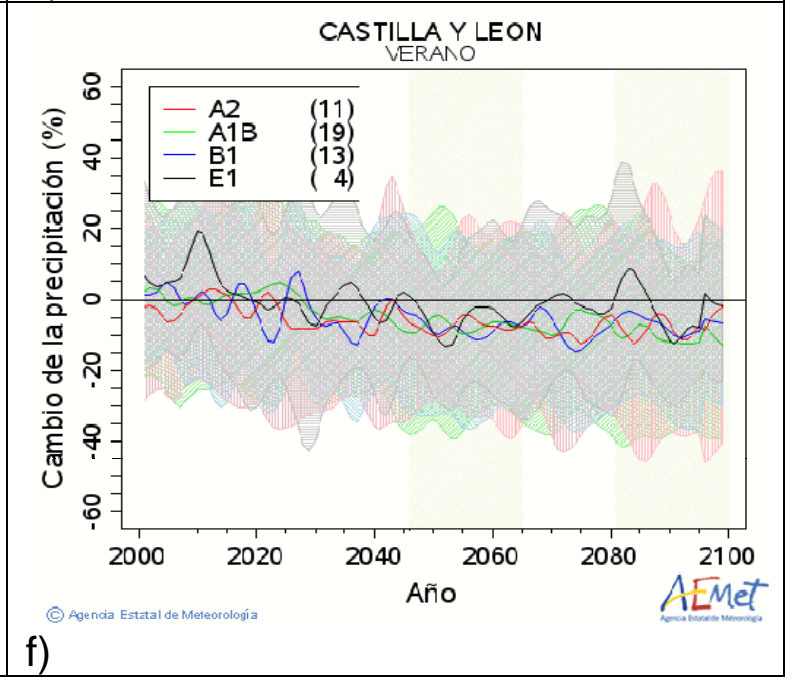

Figura A.54. Igual que la Figura A.5 para la Comunidad Autónoma de Castilla - León. 

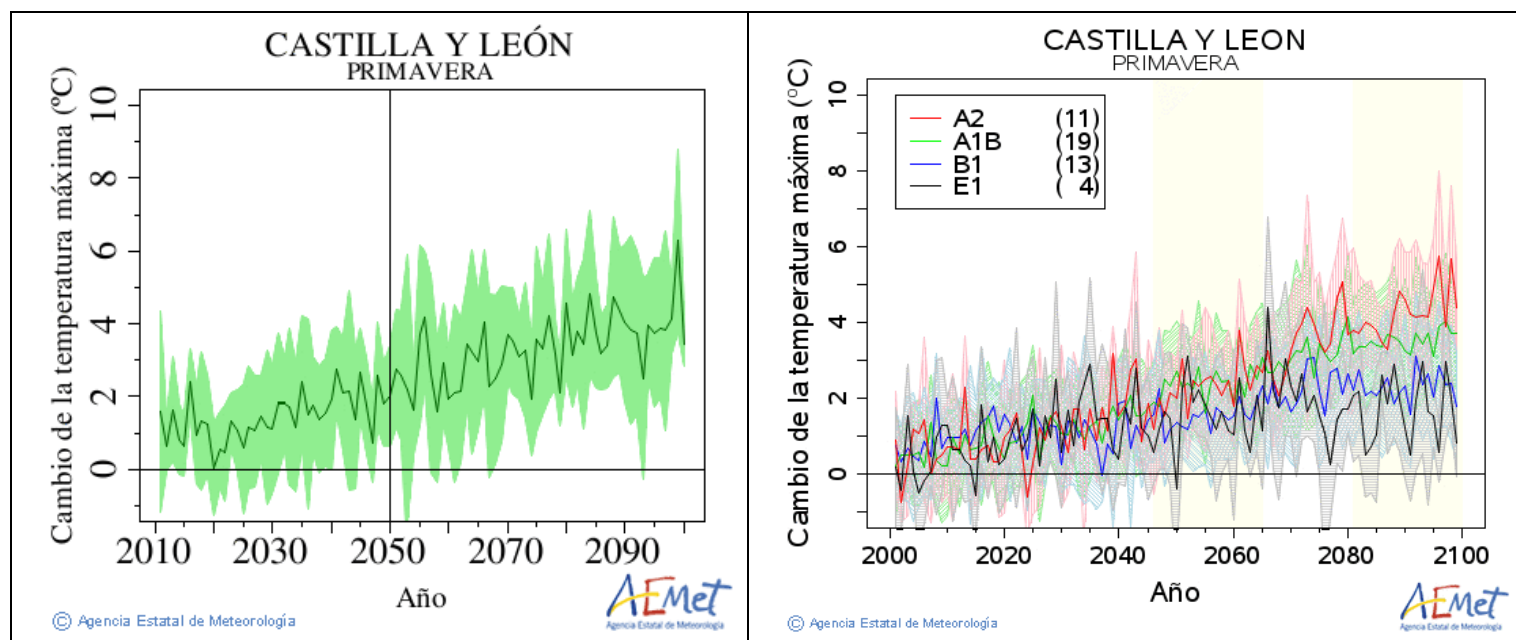

a)

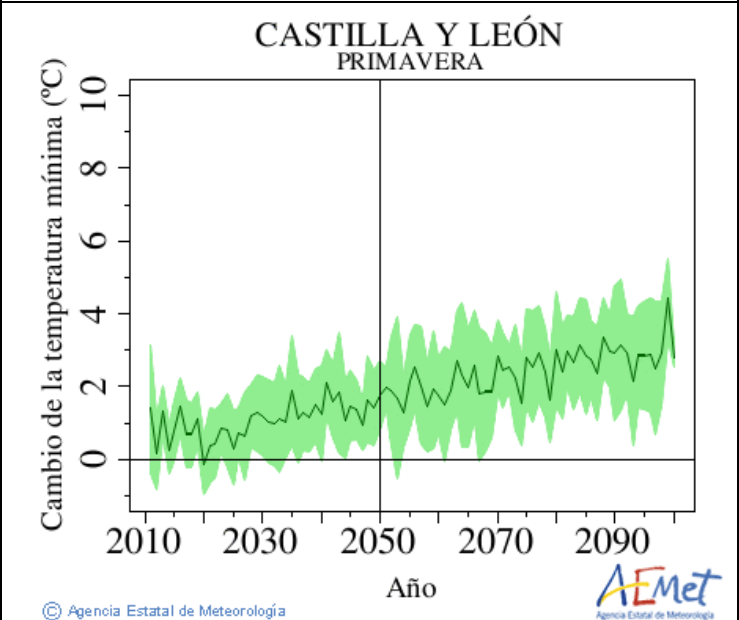

b)

c)

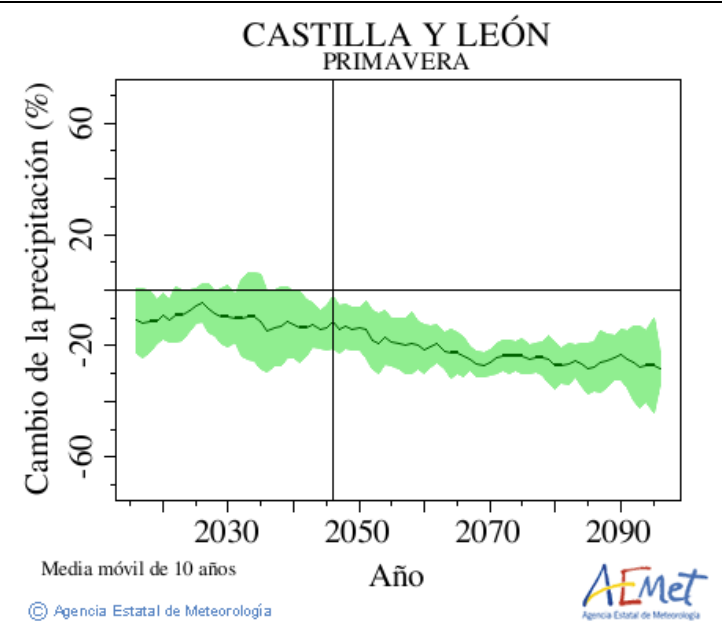

e)

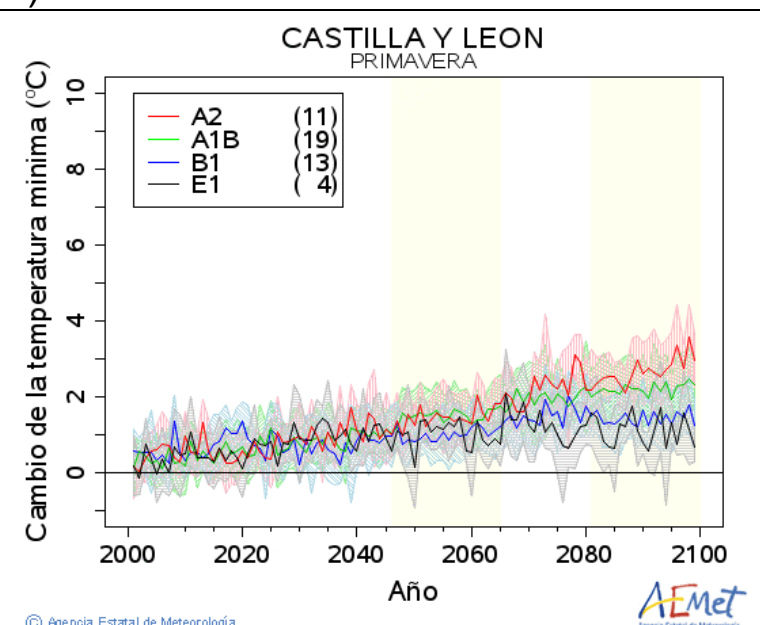

d)

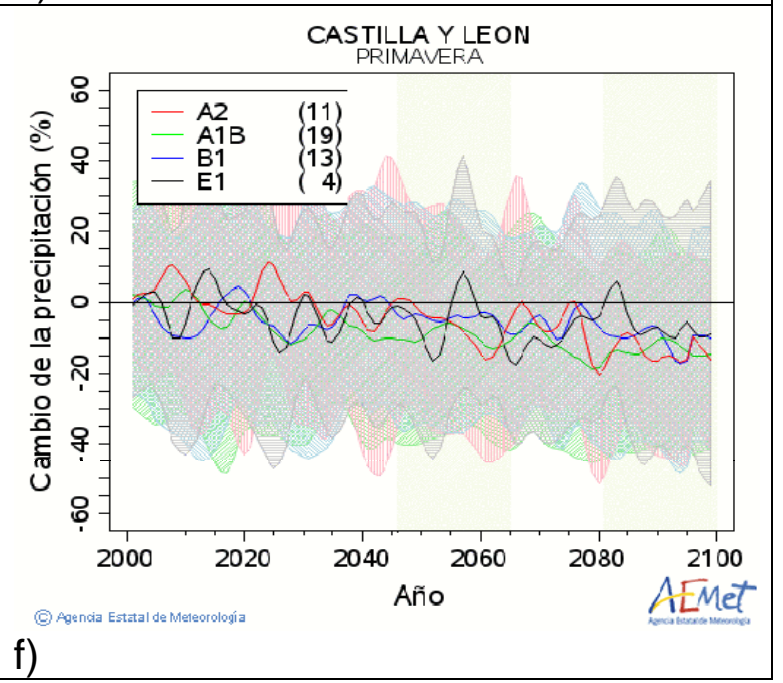

Figura A.55. Igual que la Figura A.6 para la Comunidad Autónoma de Castilla - León. 

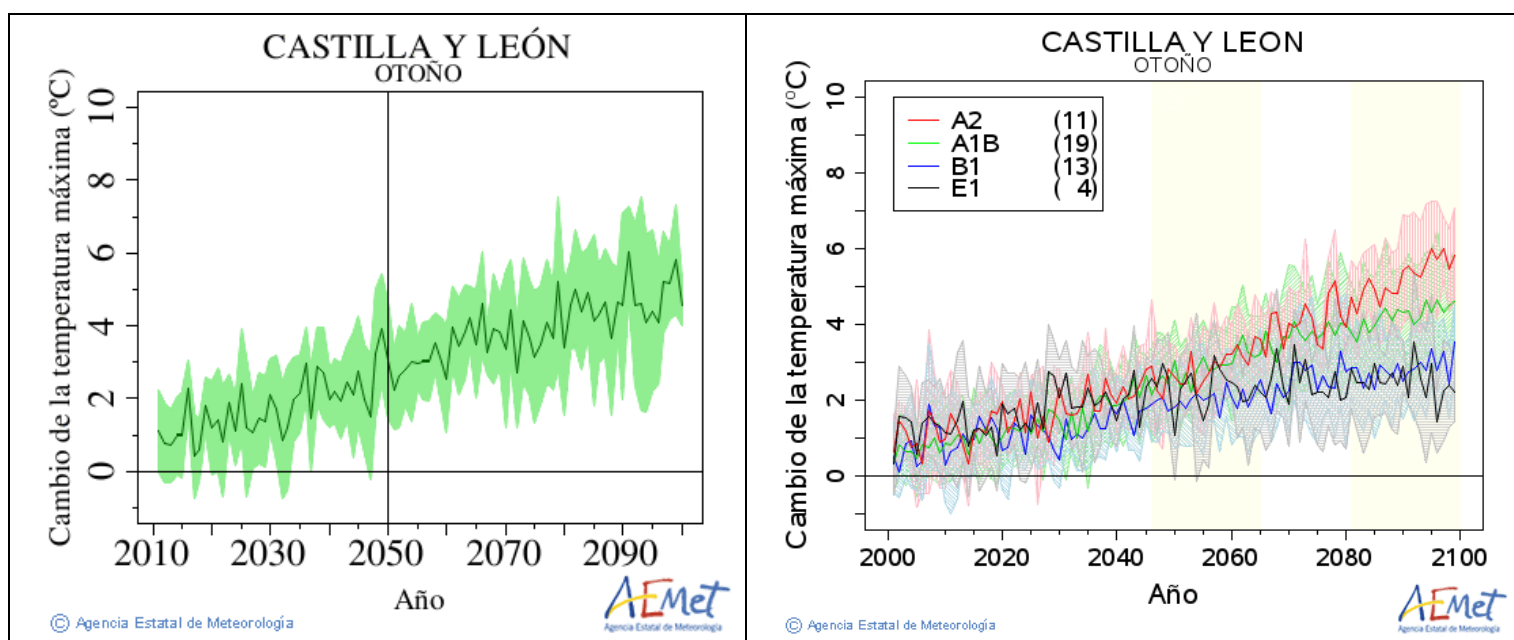

a)

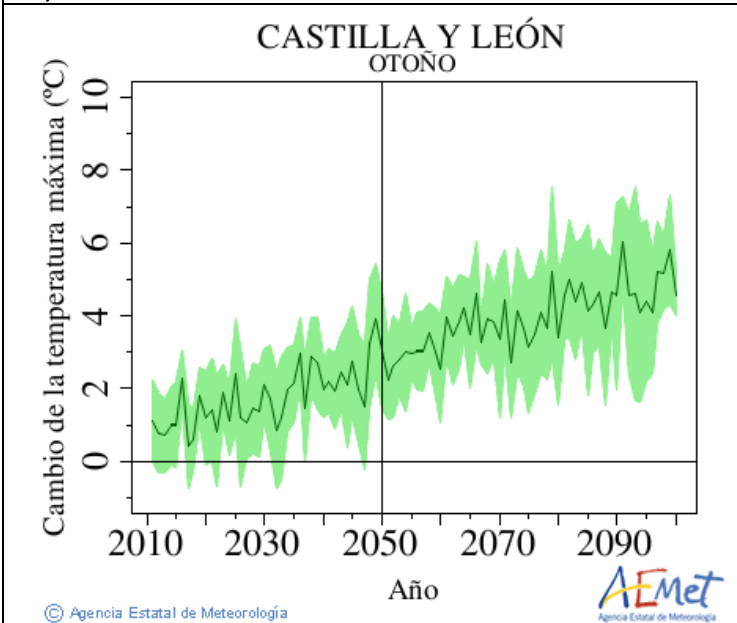

b)

c)

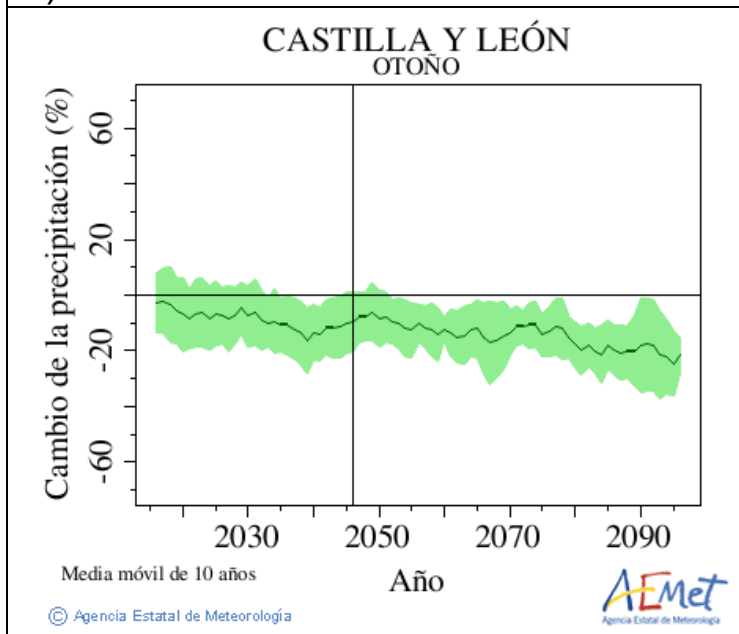

e)

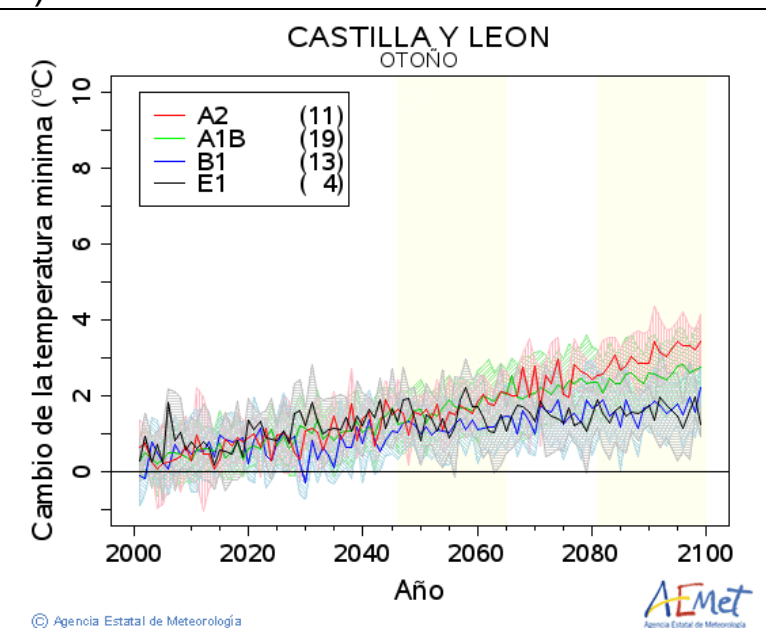

d)

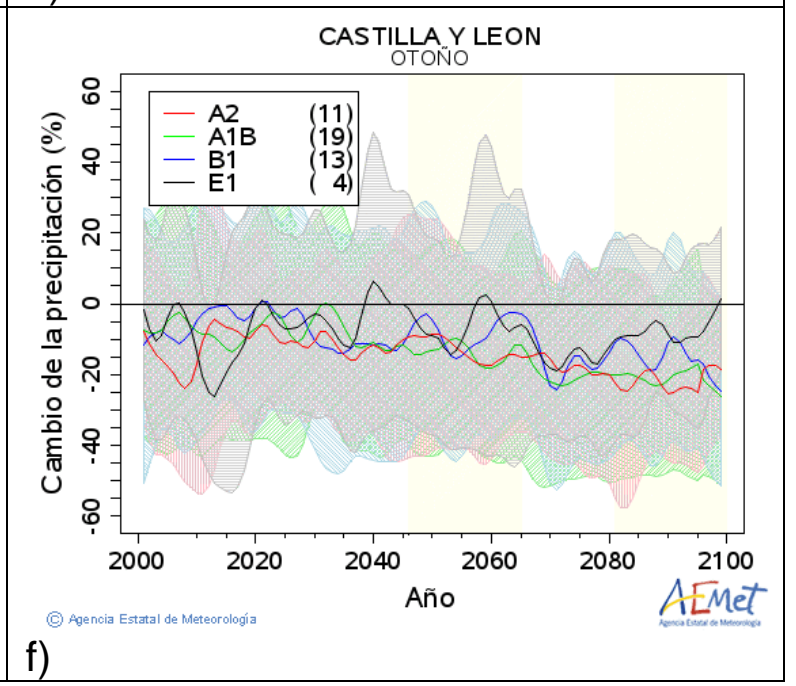

Figura A.56. Igual que la Figura A.7 para la Comunidad Autónoma de Castilla - León. 
CATALUÑA

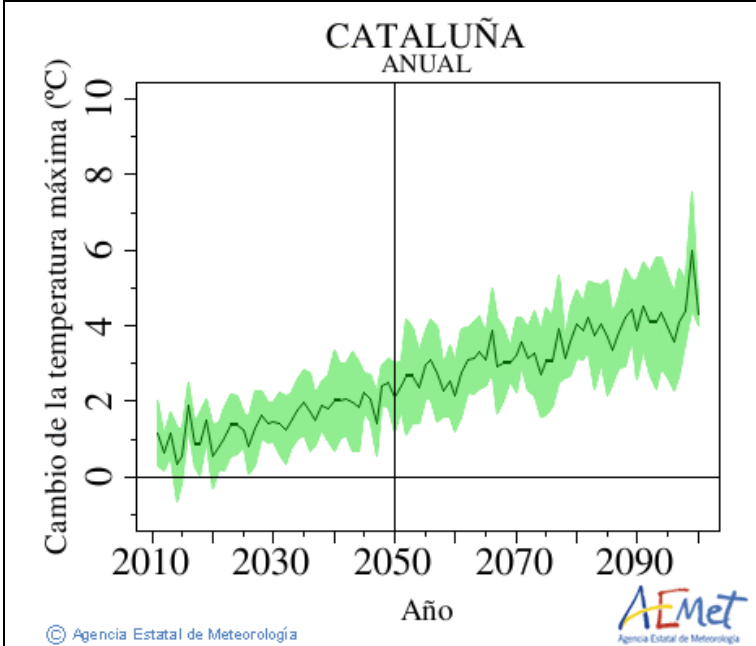

a)

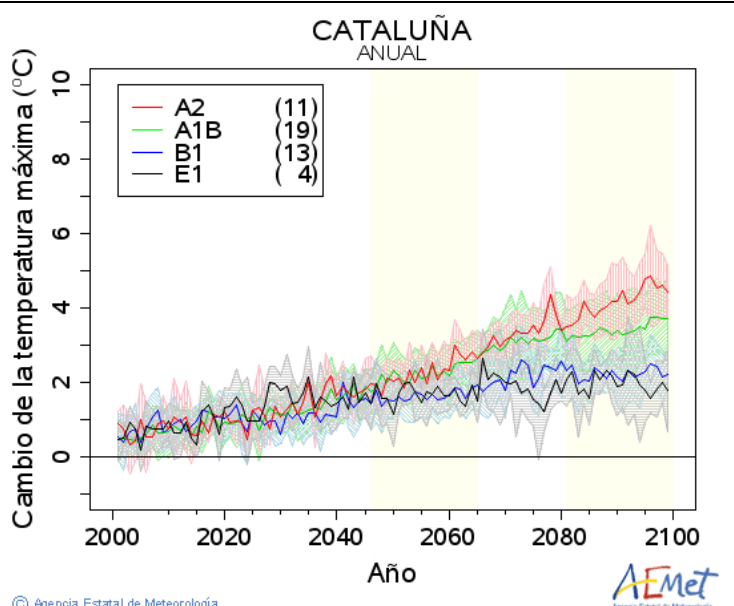

b)

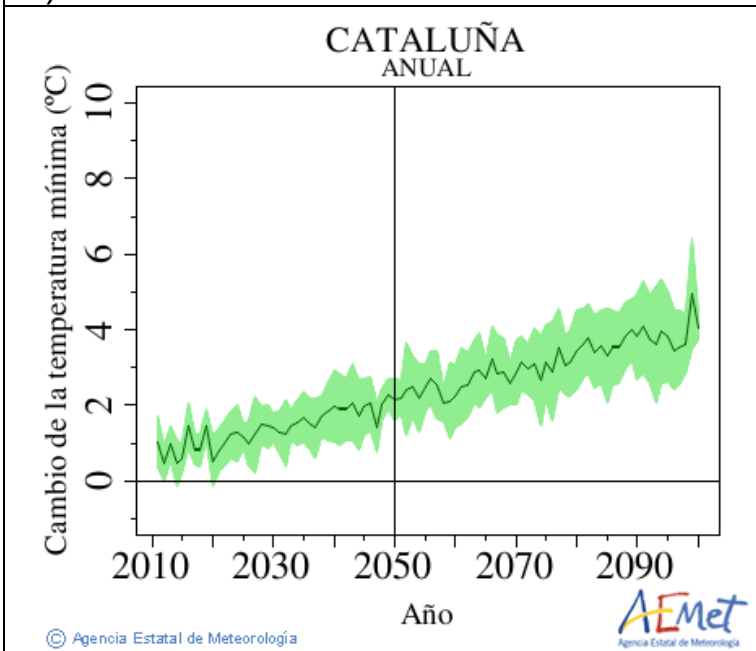

c)

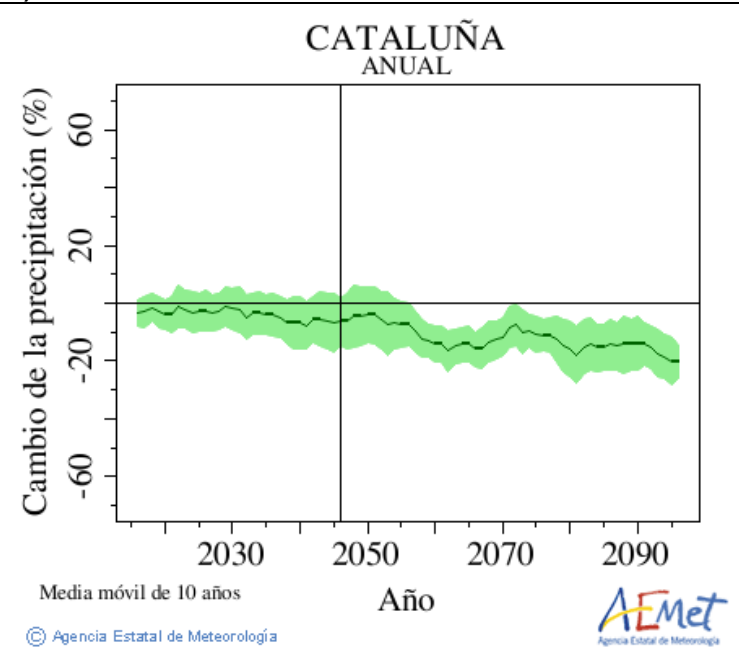

e)

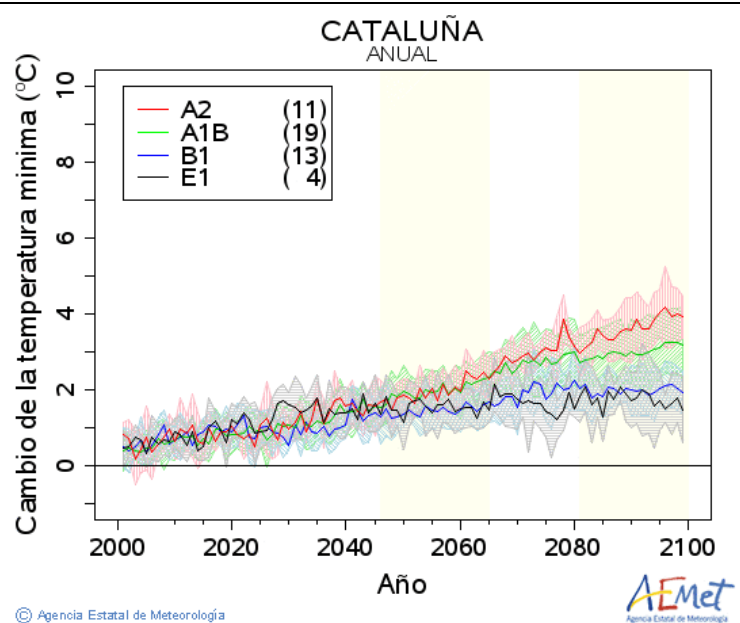

d)

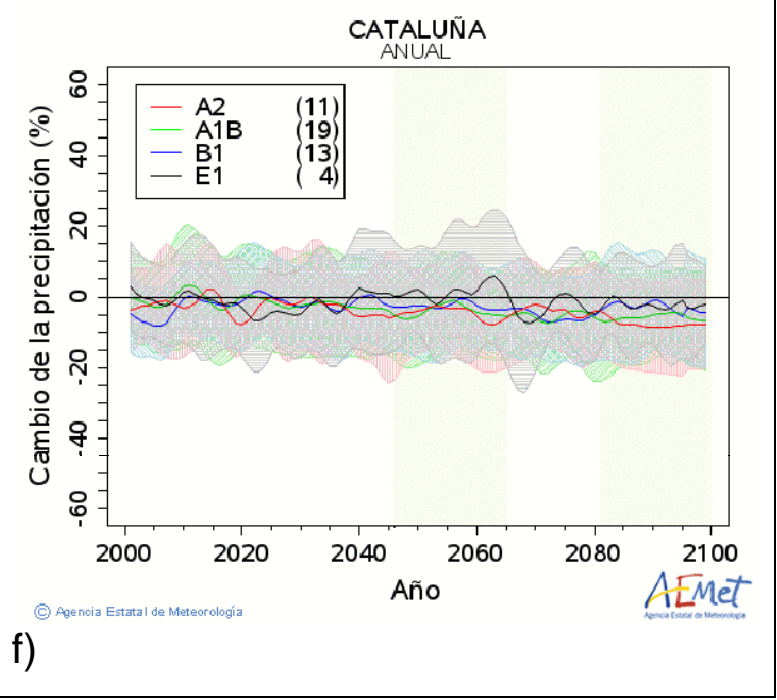

Figura A.57. Igual que la Figura A.1 para la Comunidad Autónoma de Cataluña. 


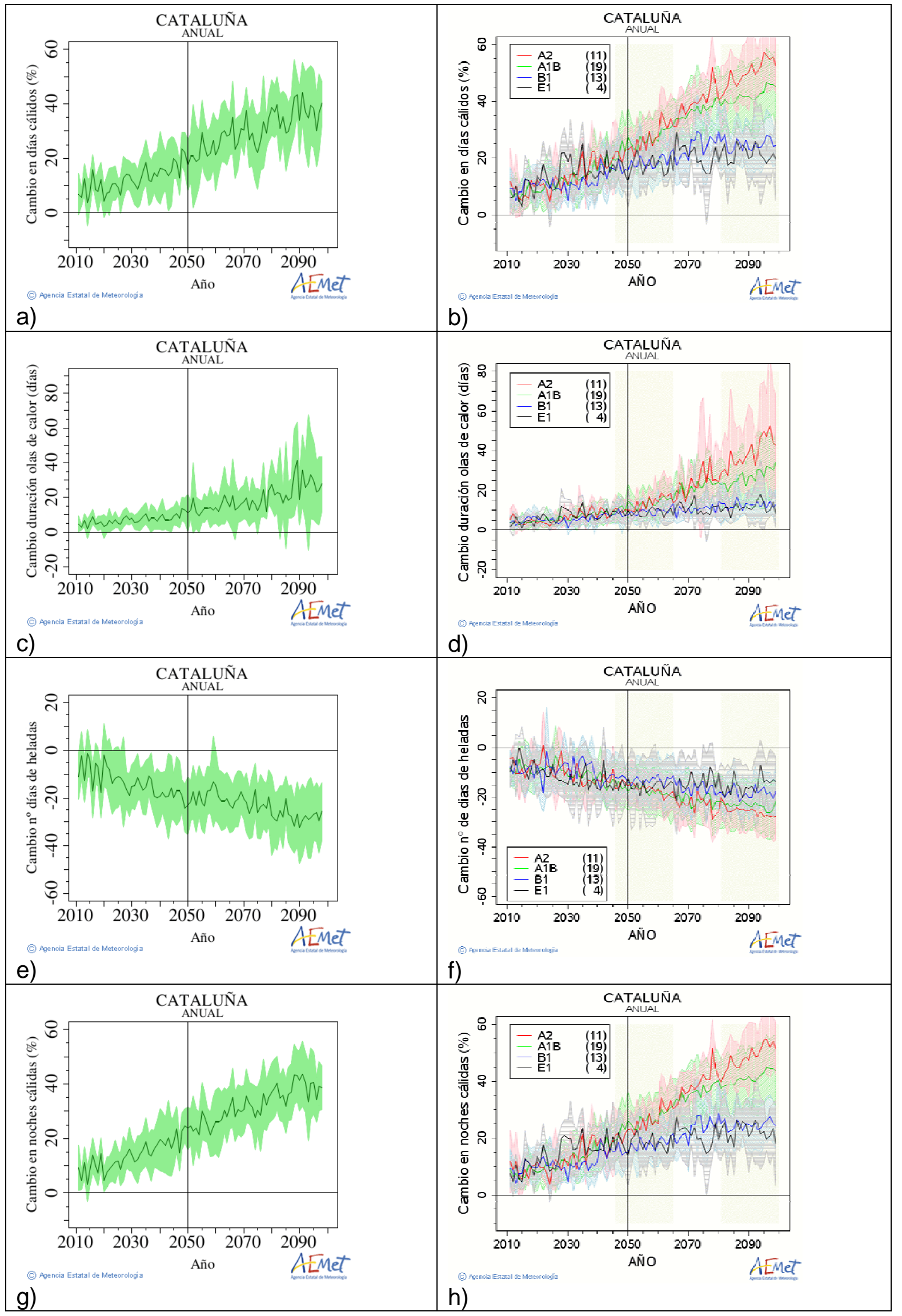

Figura A.58. Igual que la Figura A.2 para la Comunidad Autónoma de Cataluña. 


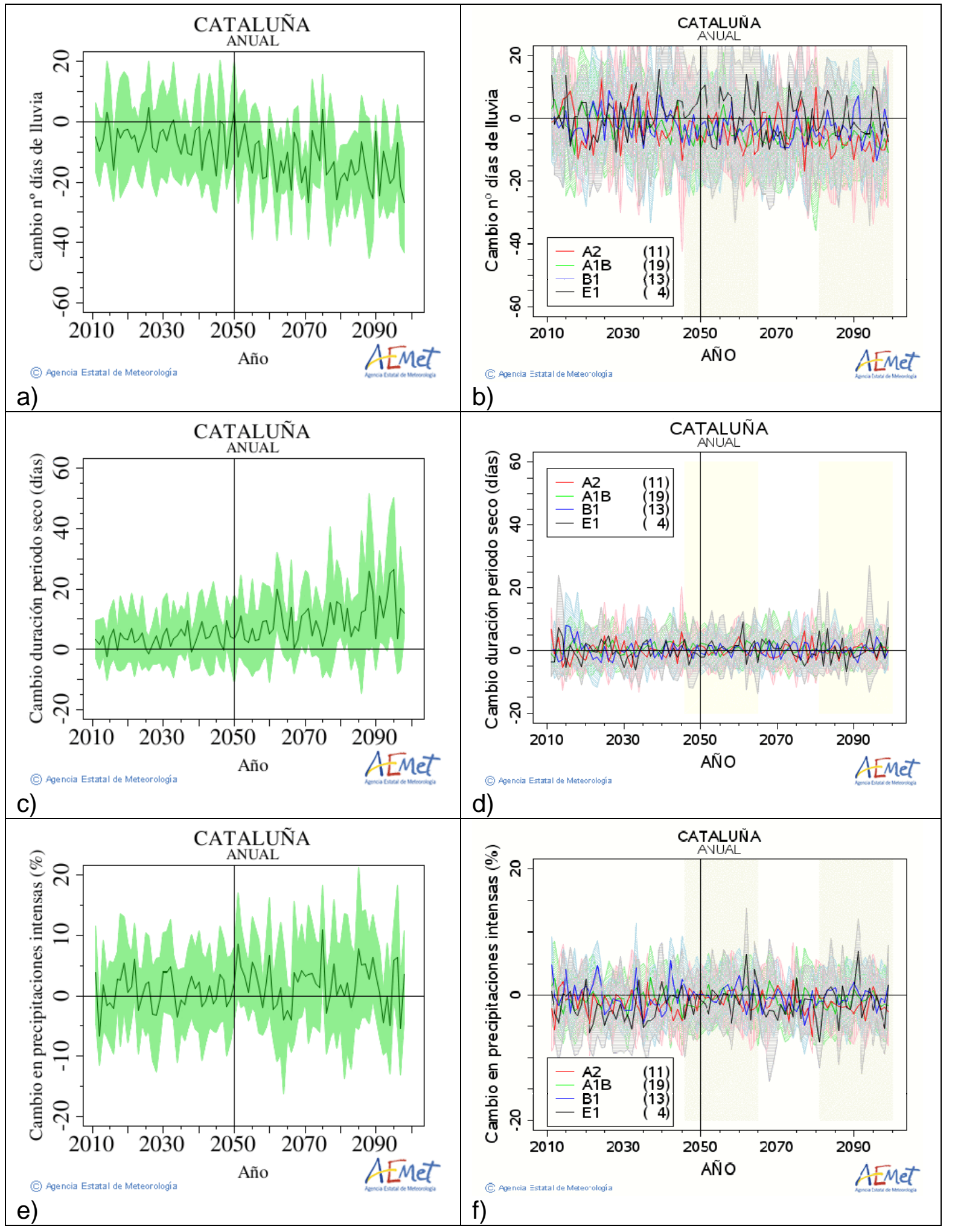

Figura A.59. Igual que la Figura A.3 para la Comunidad Autónoma de Cataluña. 


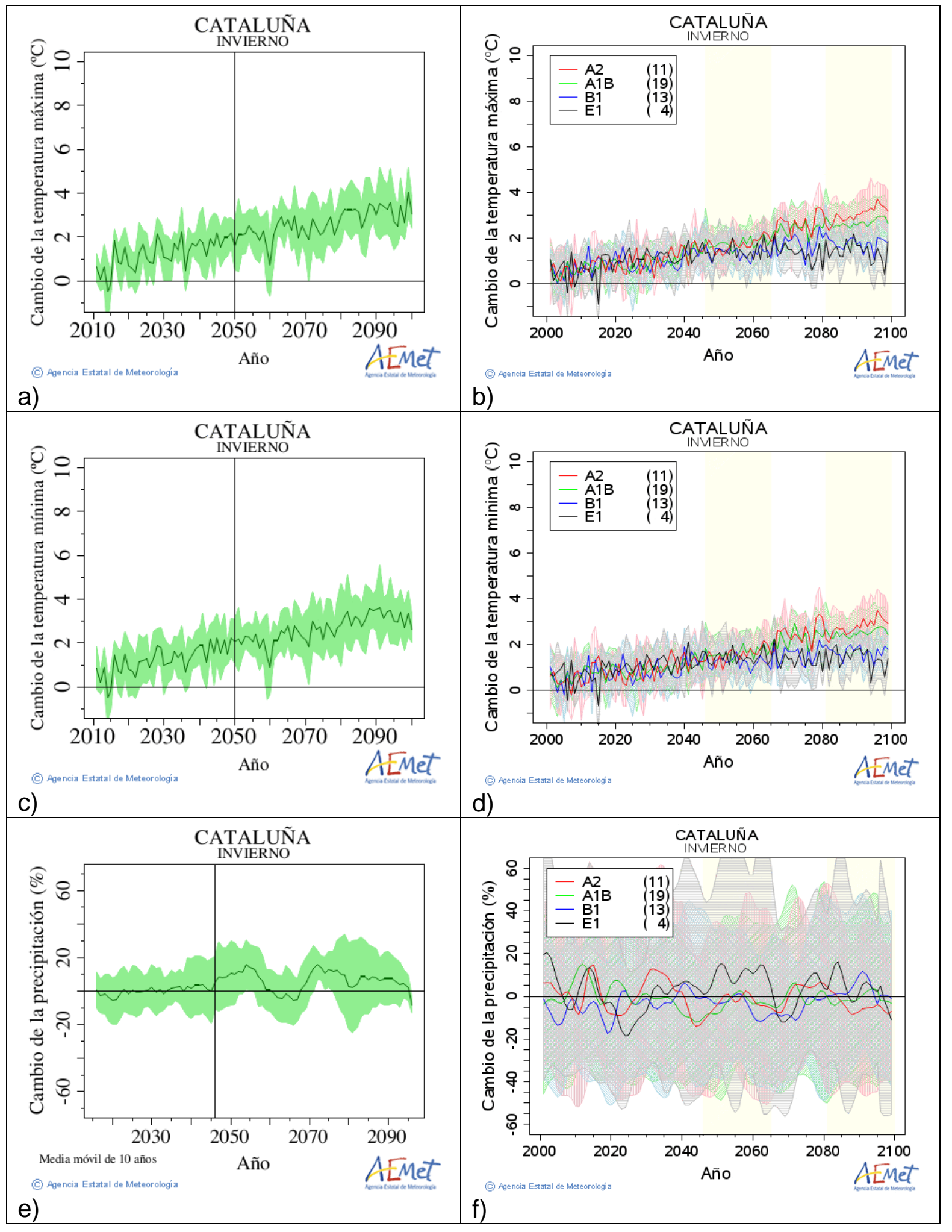

Figura A.60. Igual que la Figura A.4 para la Comunidad Autónoma de Cataluña. 


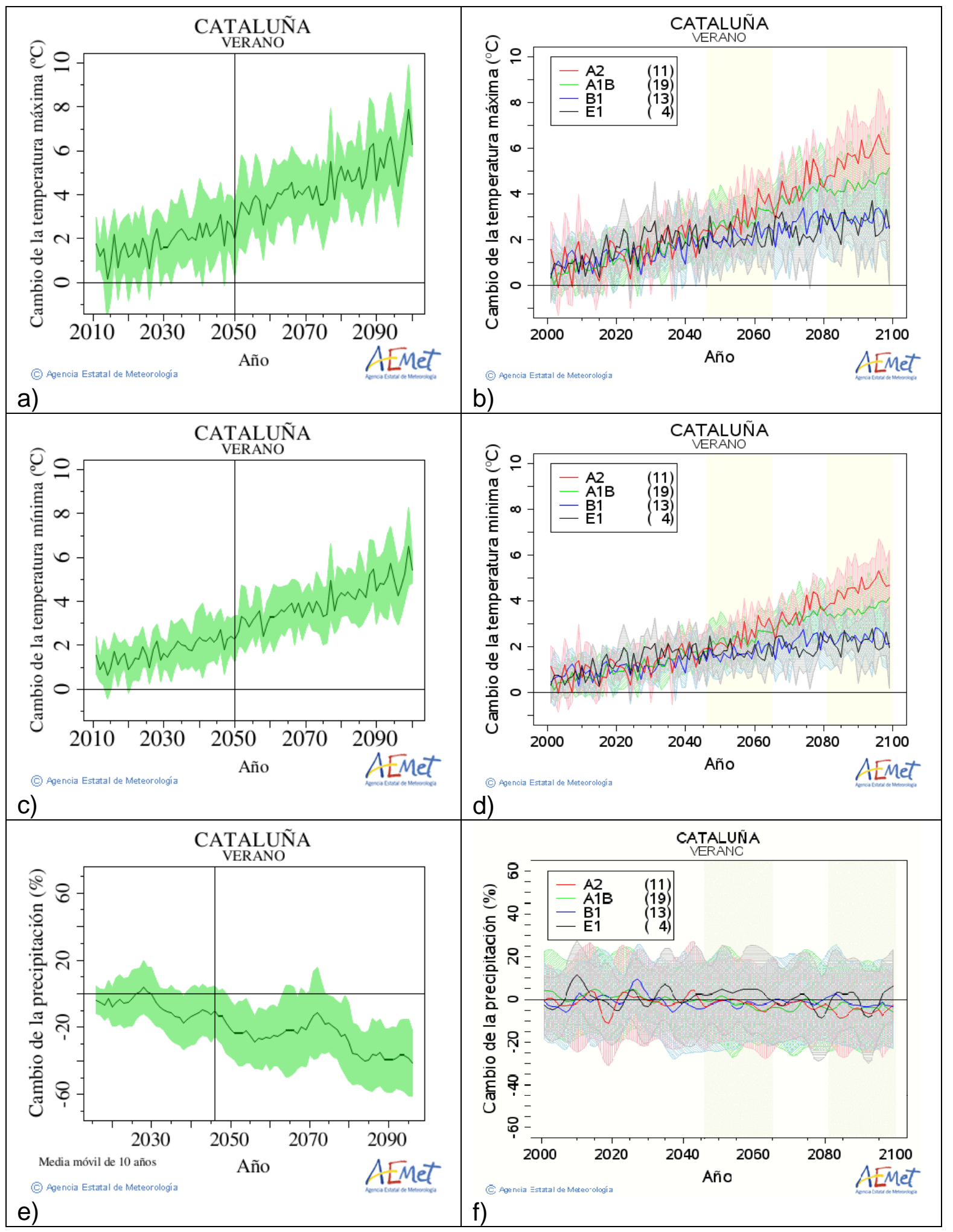

Figura A.61. Igual que la Figura A.5 para la Comunidad Autónoma de Cataluña. 


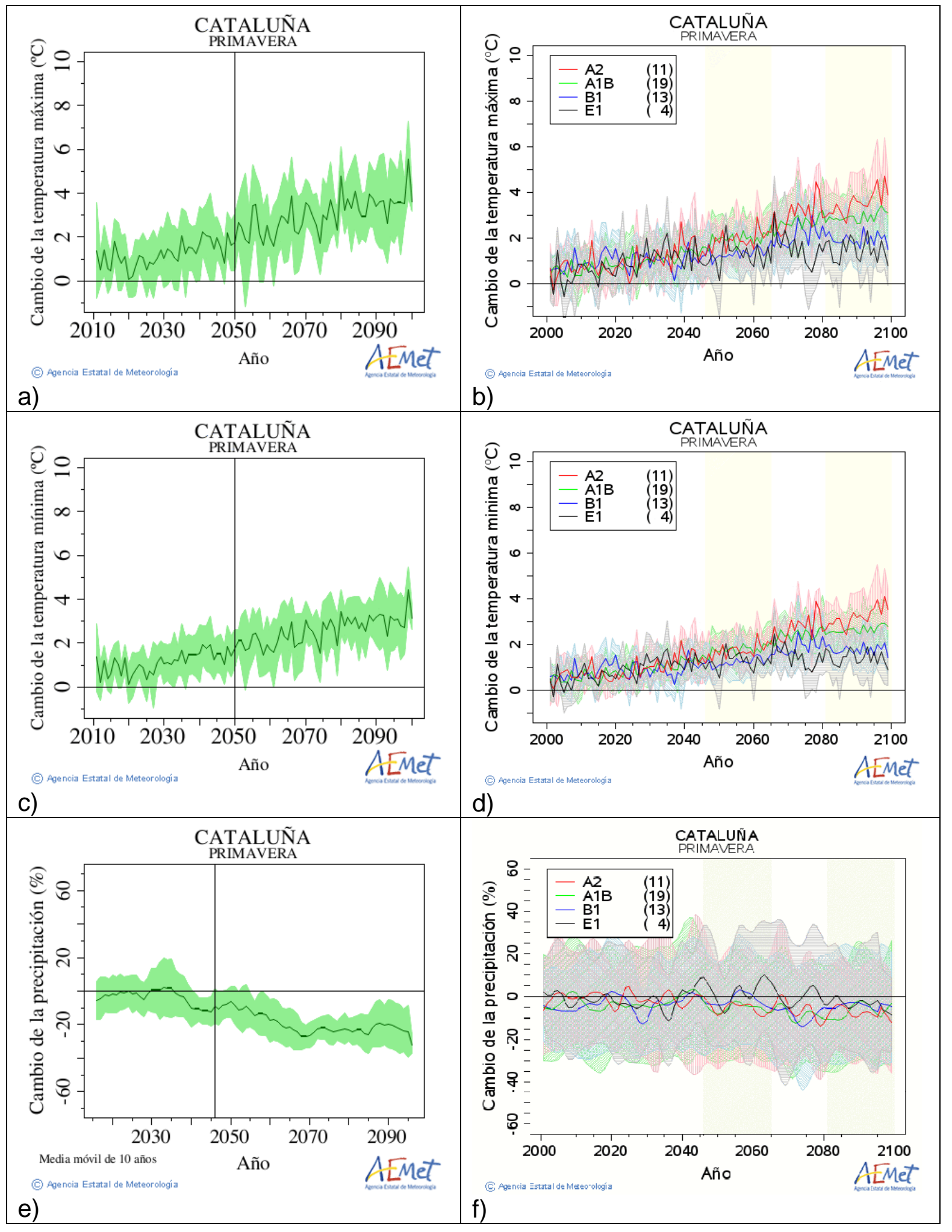

Figura A.62. Igual que la Figura A.6 para la Comunidad Autónoma de Cataluña. 


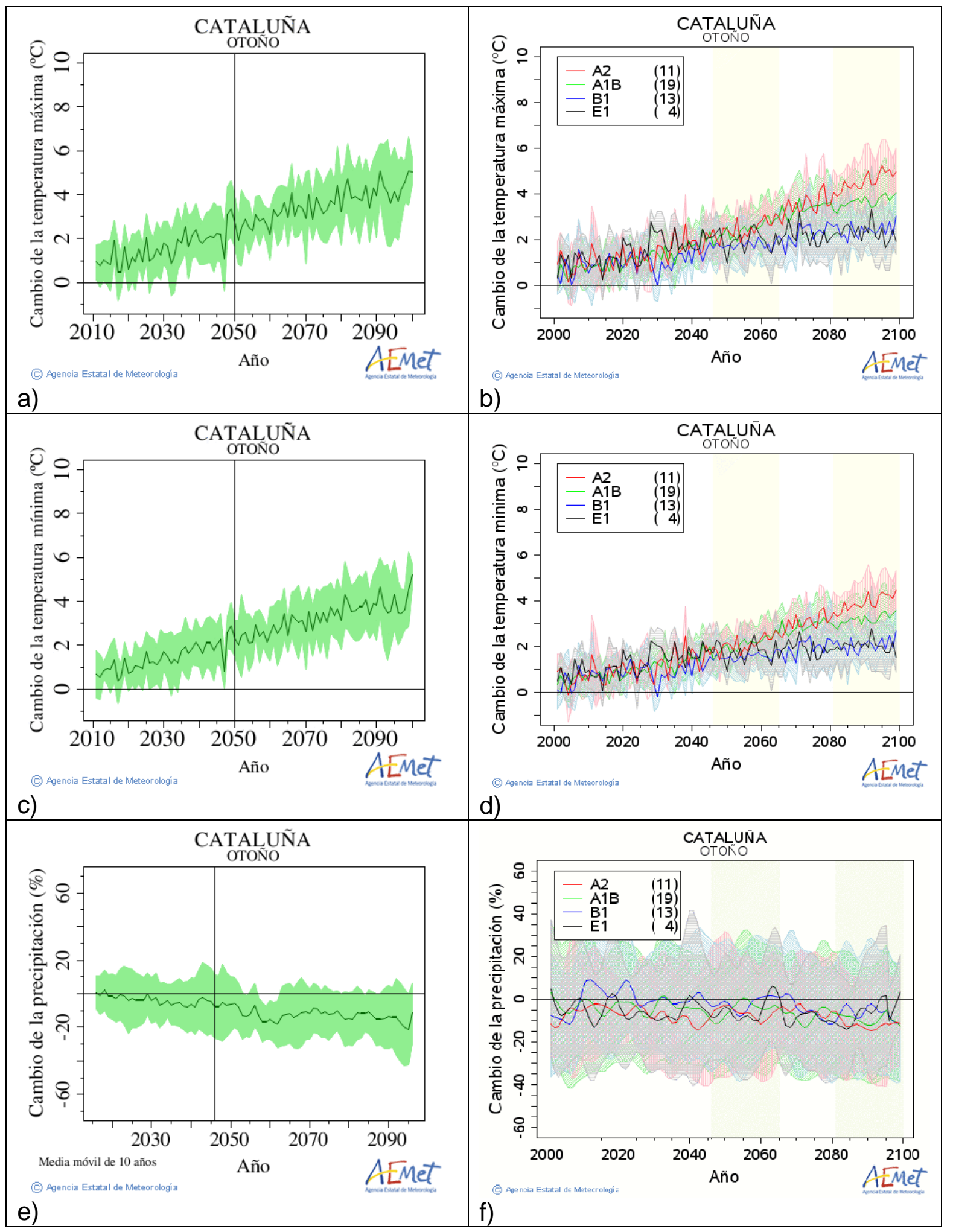

Figura A.63. I Igual que la Figura A.7 para la Comunidad Autónoma de Cataluña. 
EXTREMADURA

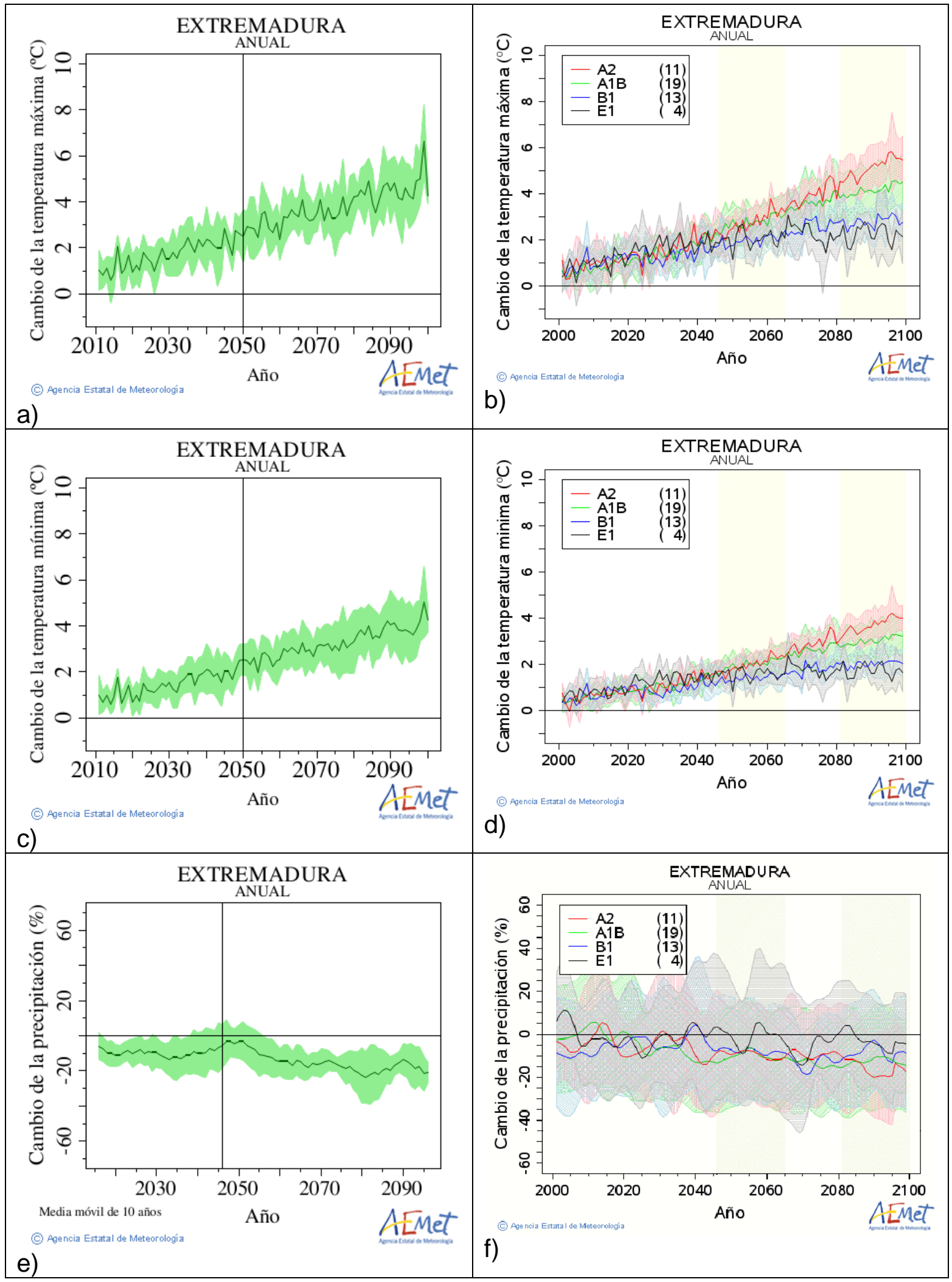

Figura A.64. Igual que la Figura A.1 para la Comunidad Autónoma de Extremadura. 


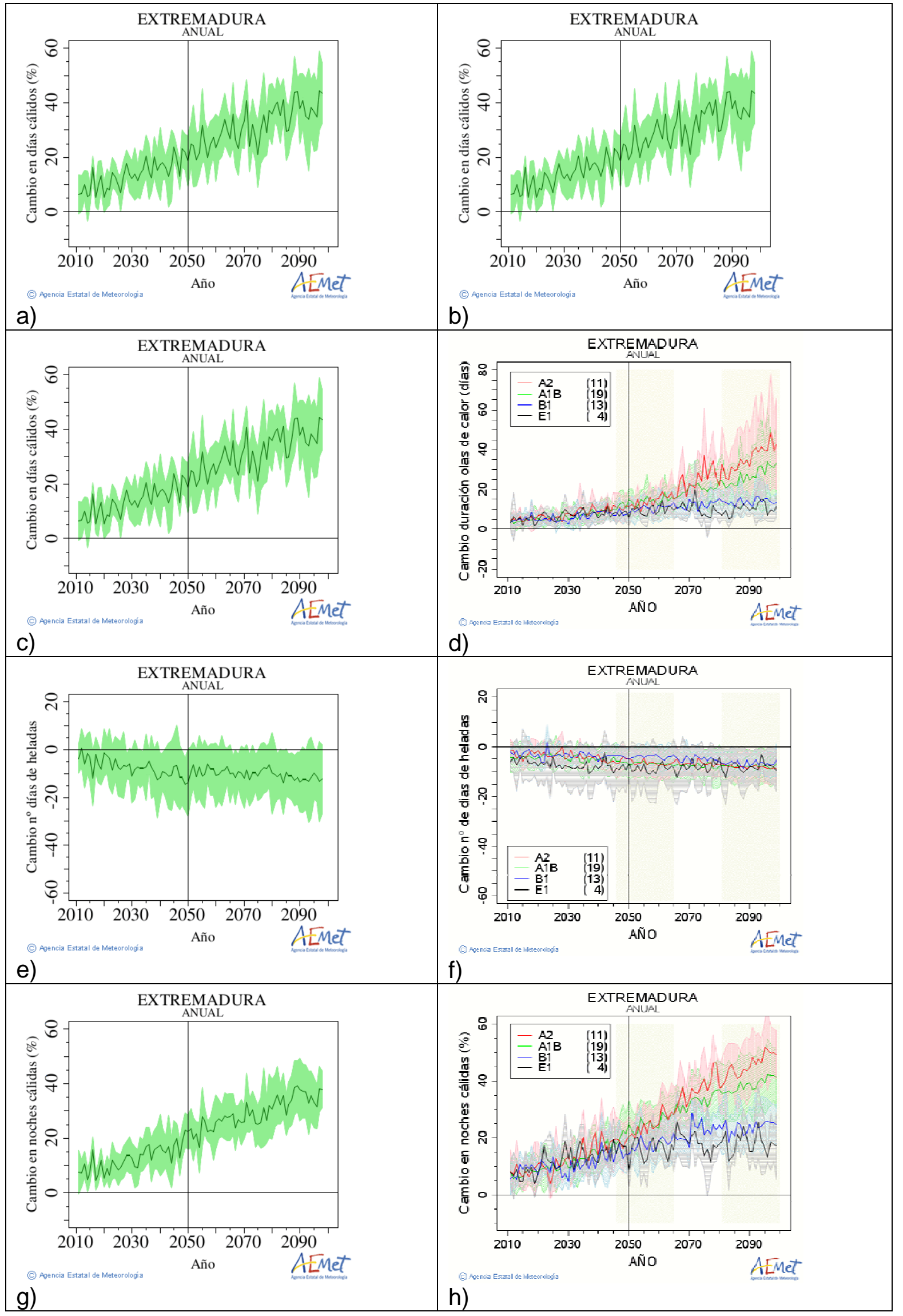

Figura A.65. Igual que la Figura A.2 para la Comunidad Autónoma de Extremadura. 


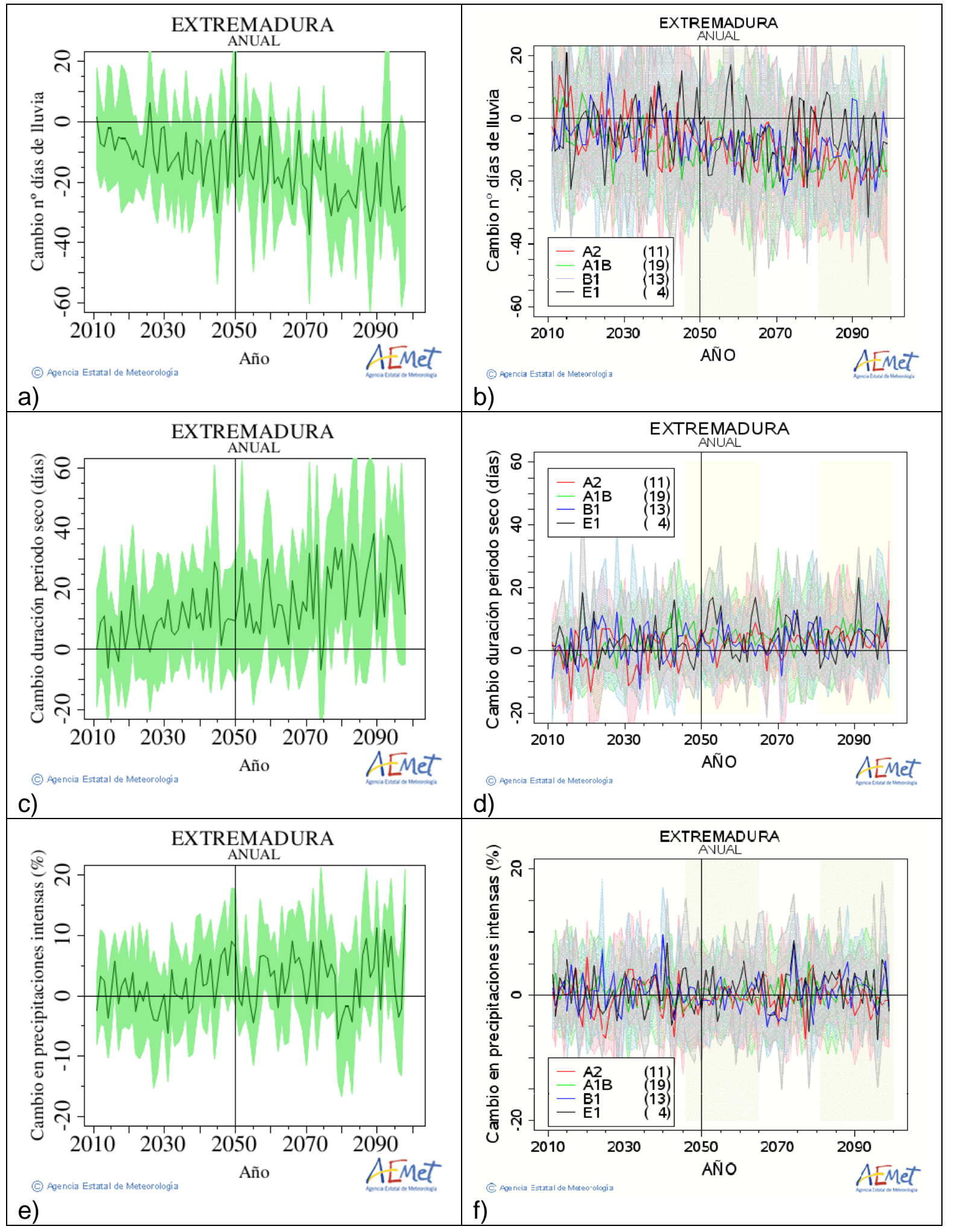

Figura A.66. Igual que la Figura A.3 para la Comunidad Autónoma de Extremadura. 


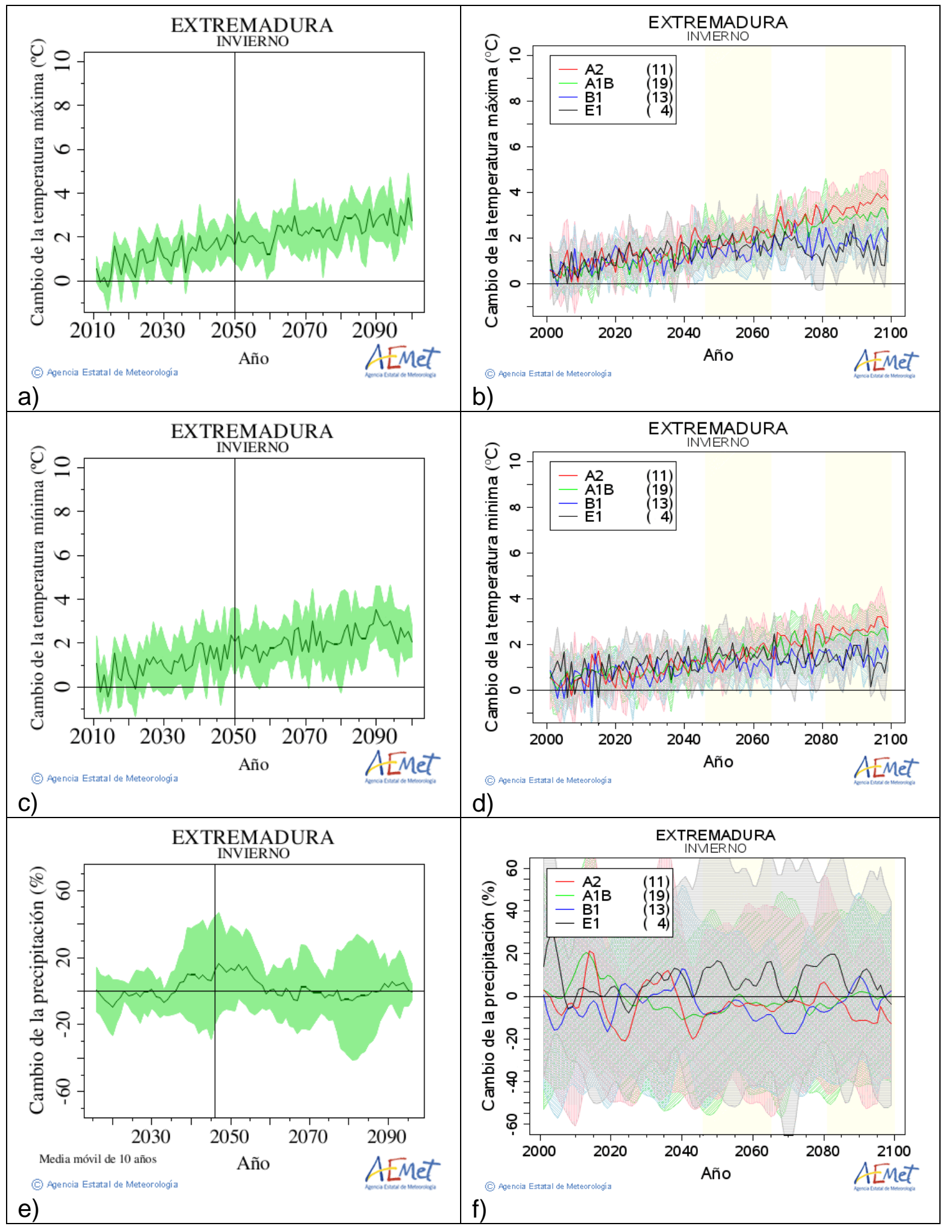

Figura A.67. Igual que la Figura A.4 para la Comunidad Autónoma de Extremadura. 


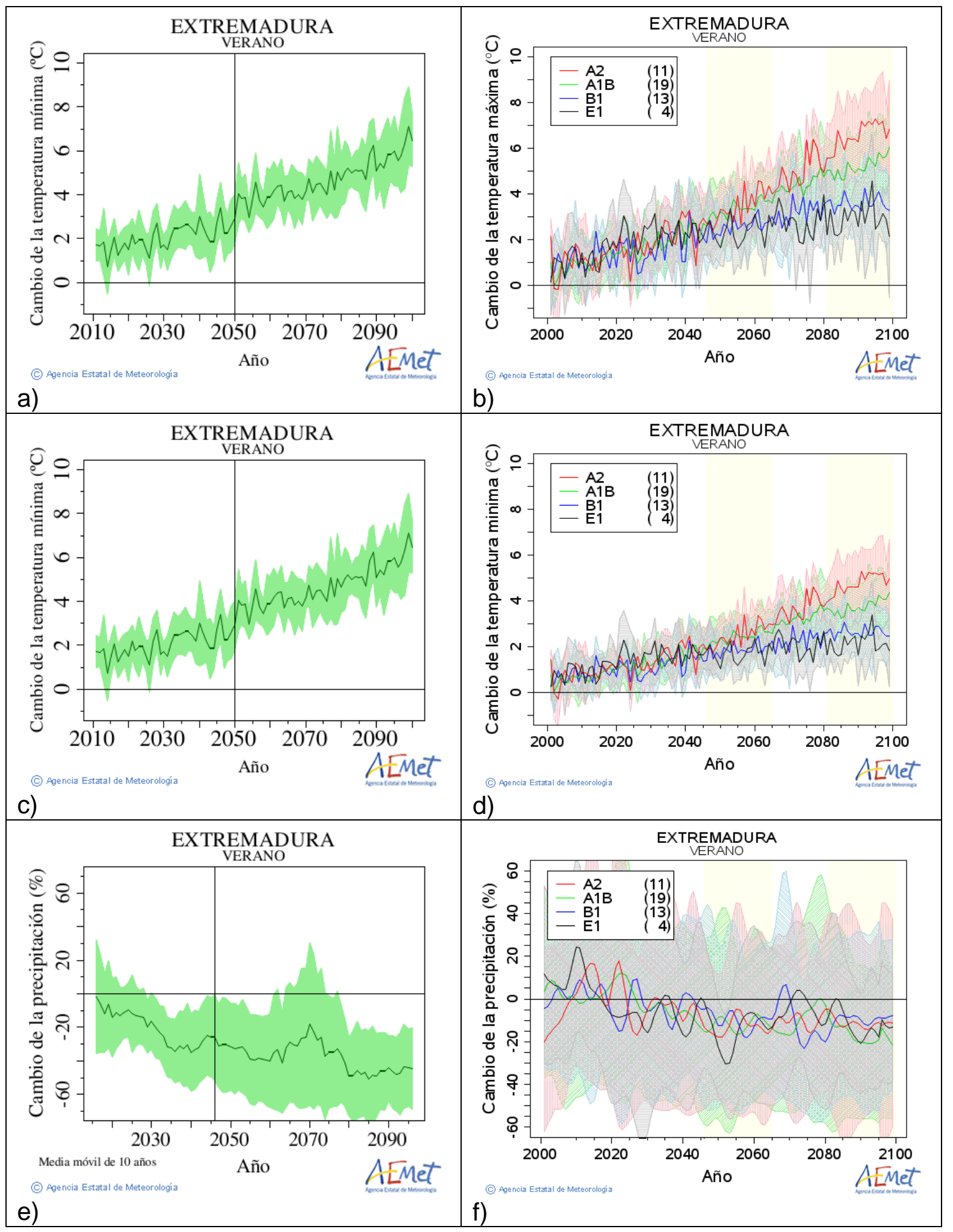

Figura A.68. Igual que la Figura A.5 para la Comunidad Autónoma de Extremadura. 


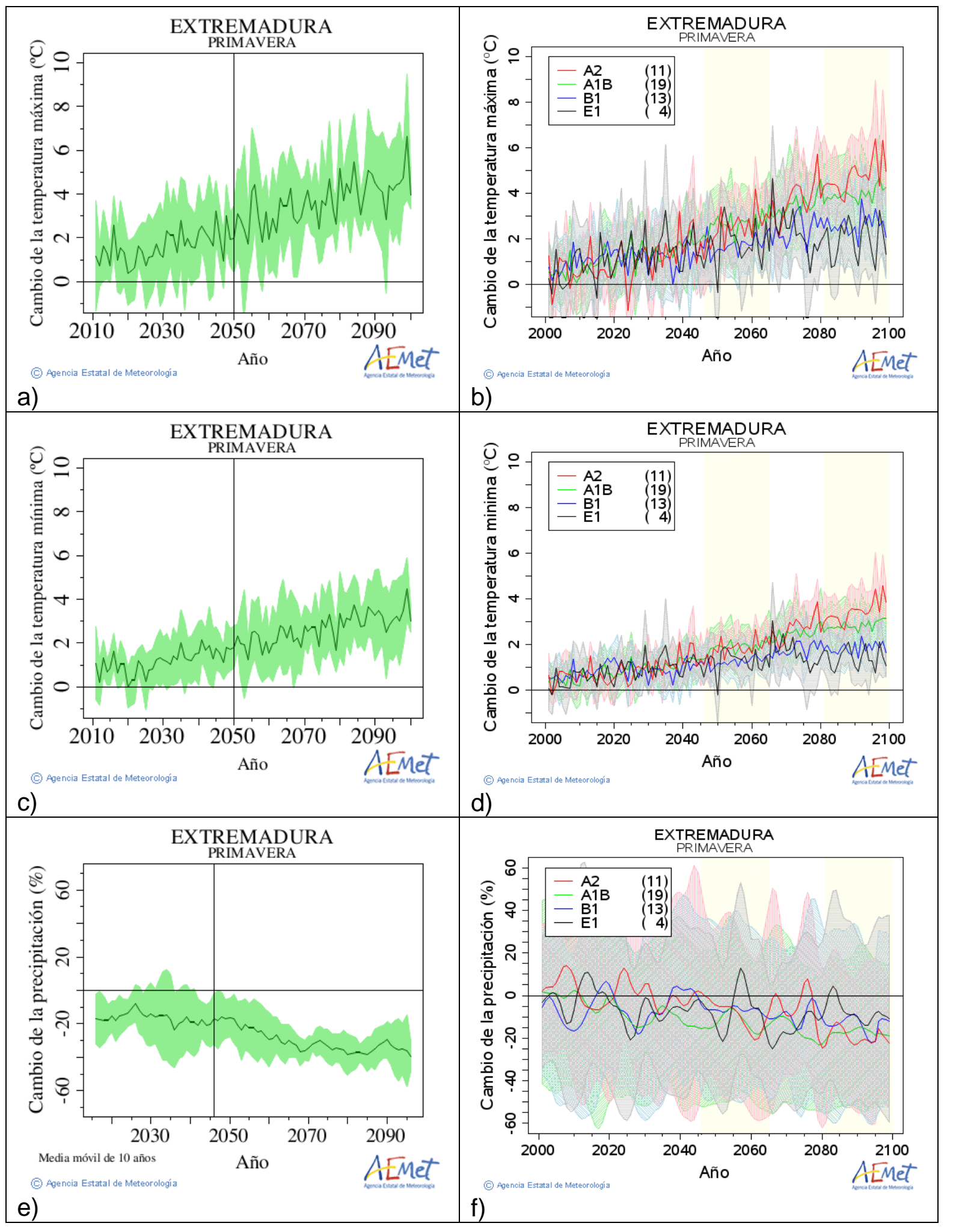

Figura A.69. Igual que la Figura A.6 para la Comunidad Autónoma de Extremadura. 


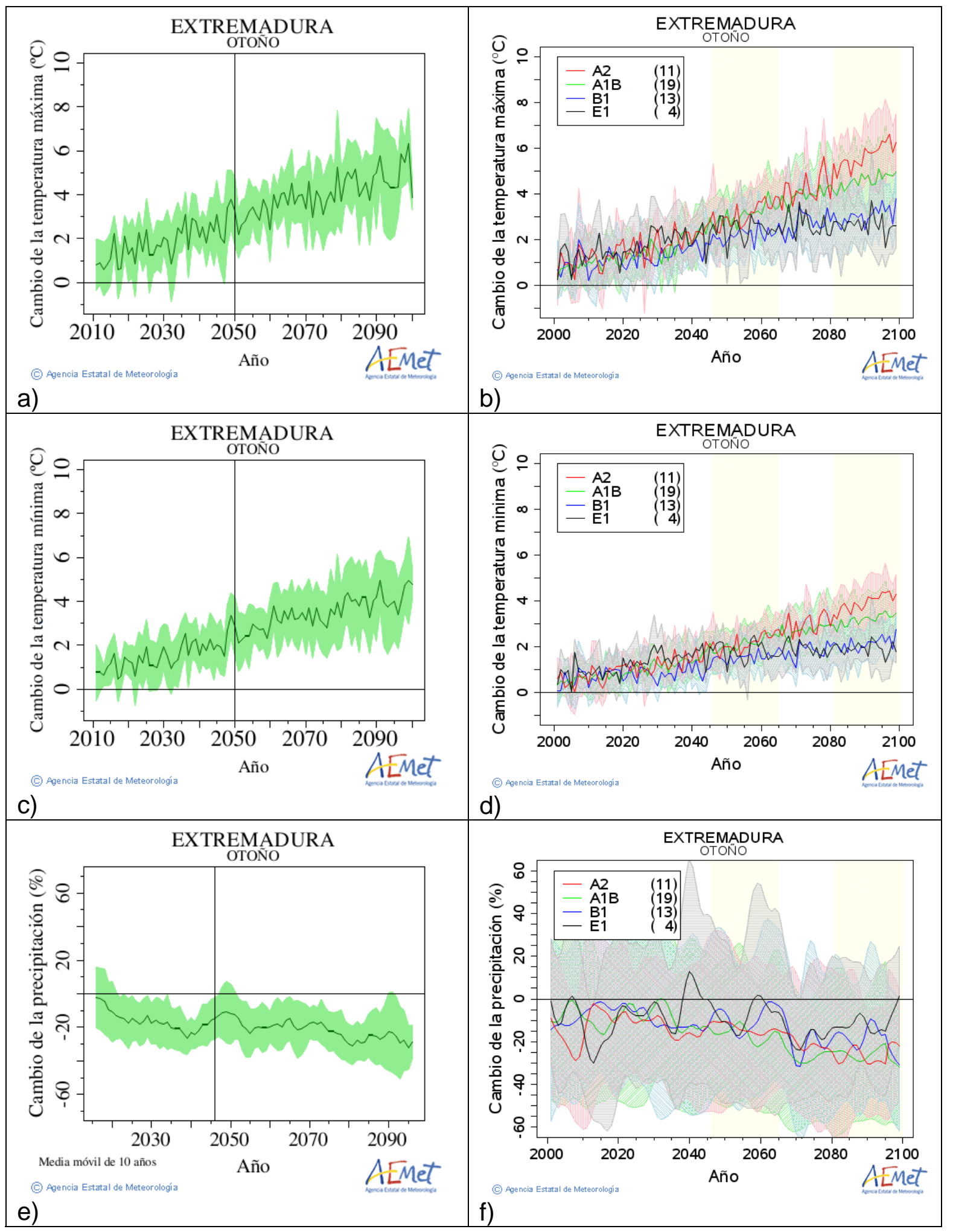

Figura A.70. Igual que la Figura A.7 para la Comunidad Autónoma de Extremadura. 
GALICIA

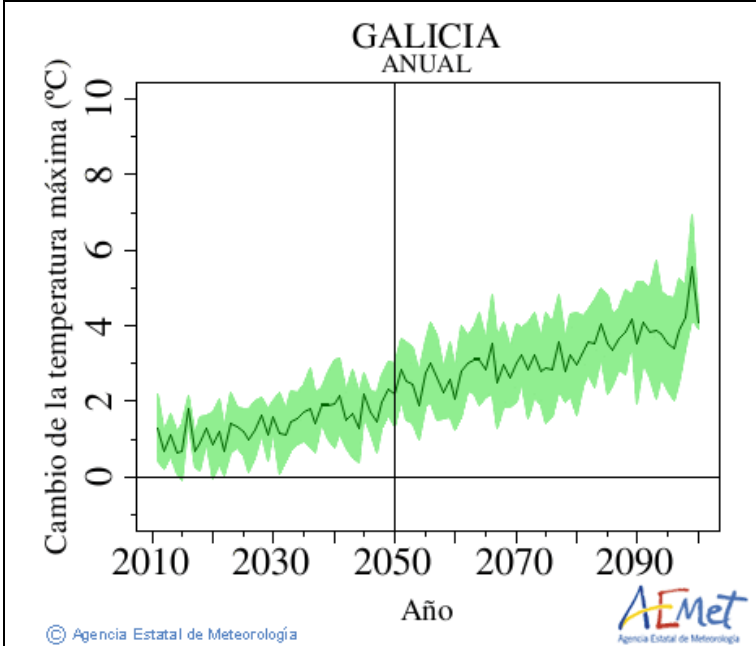

a)

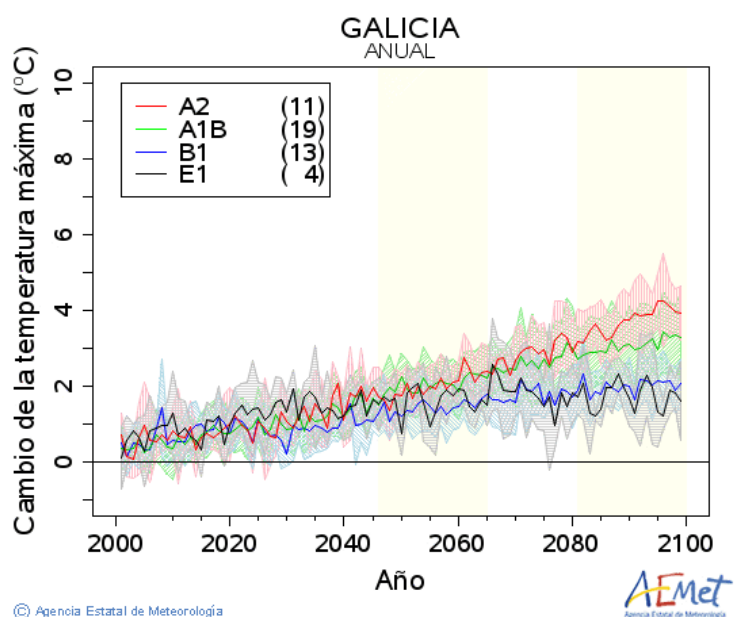

b)
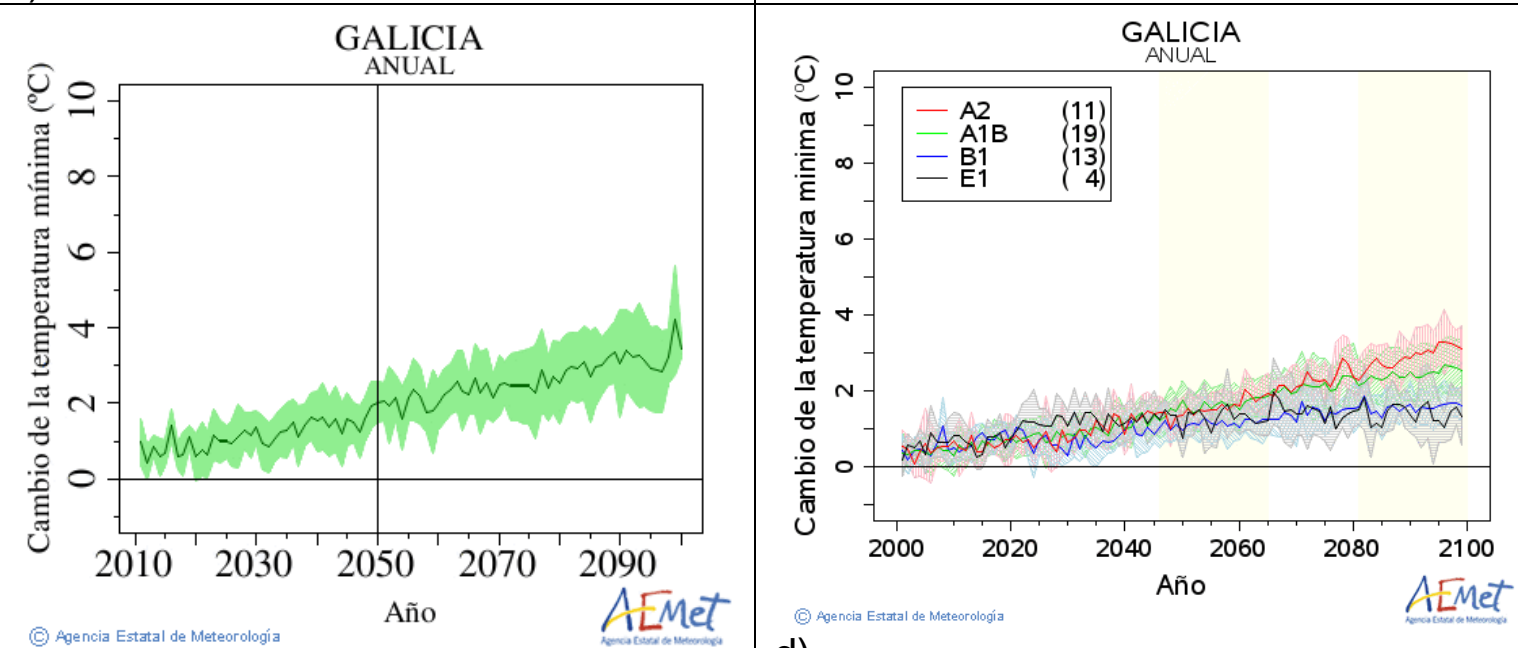

c)

d)

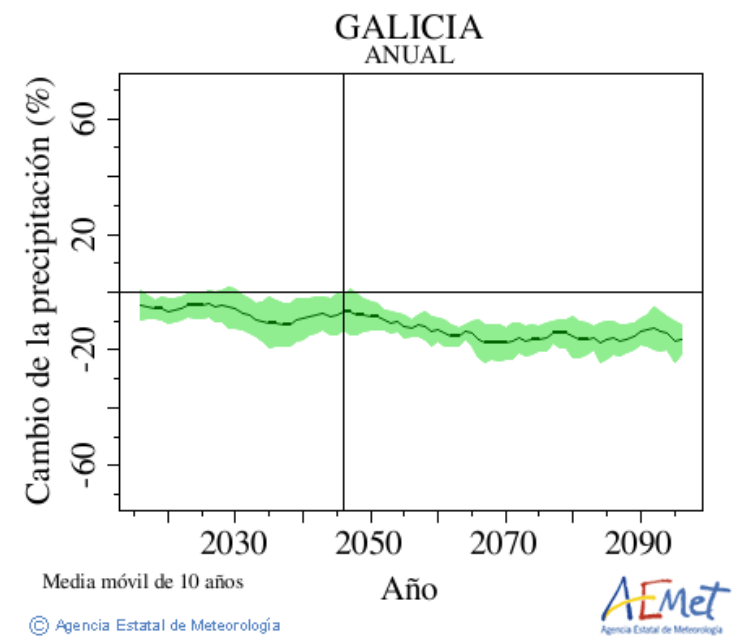

e)

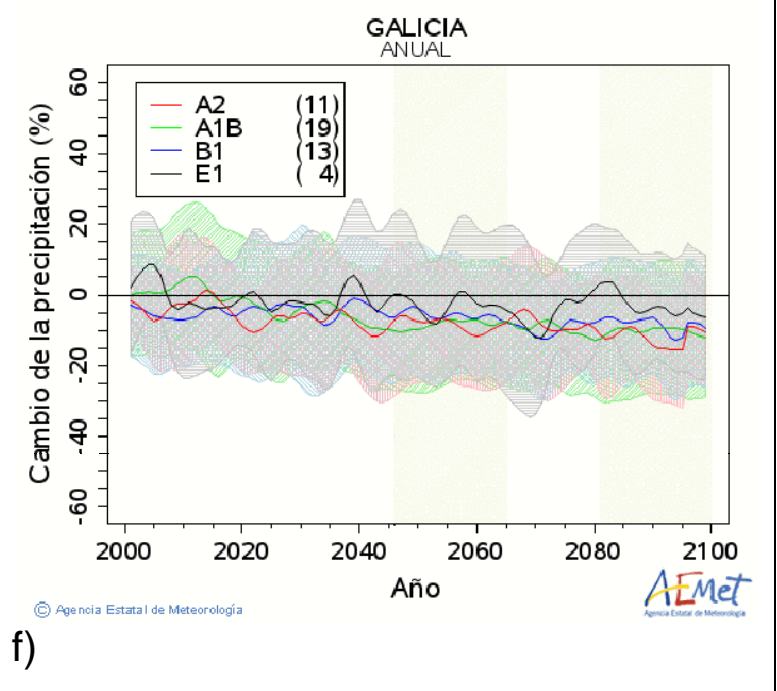

Figura A.71. Igual que la Figura A.1 para la Comunidad Autónoma de Galicia. 


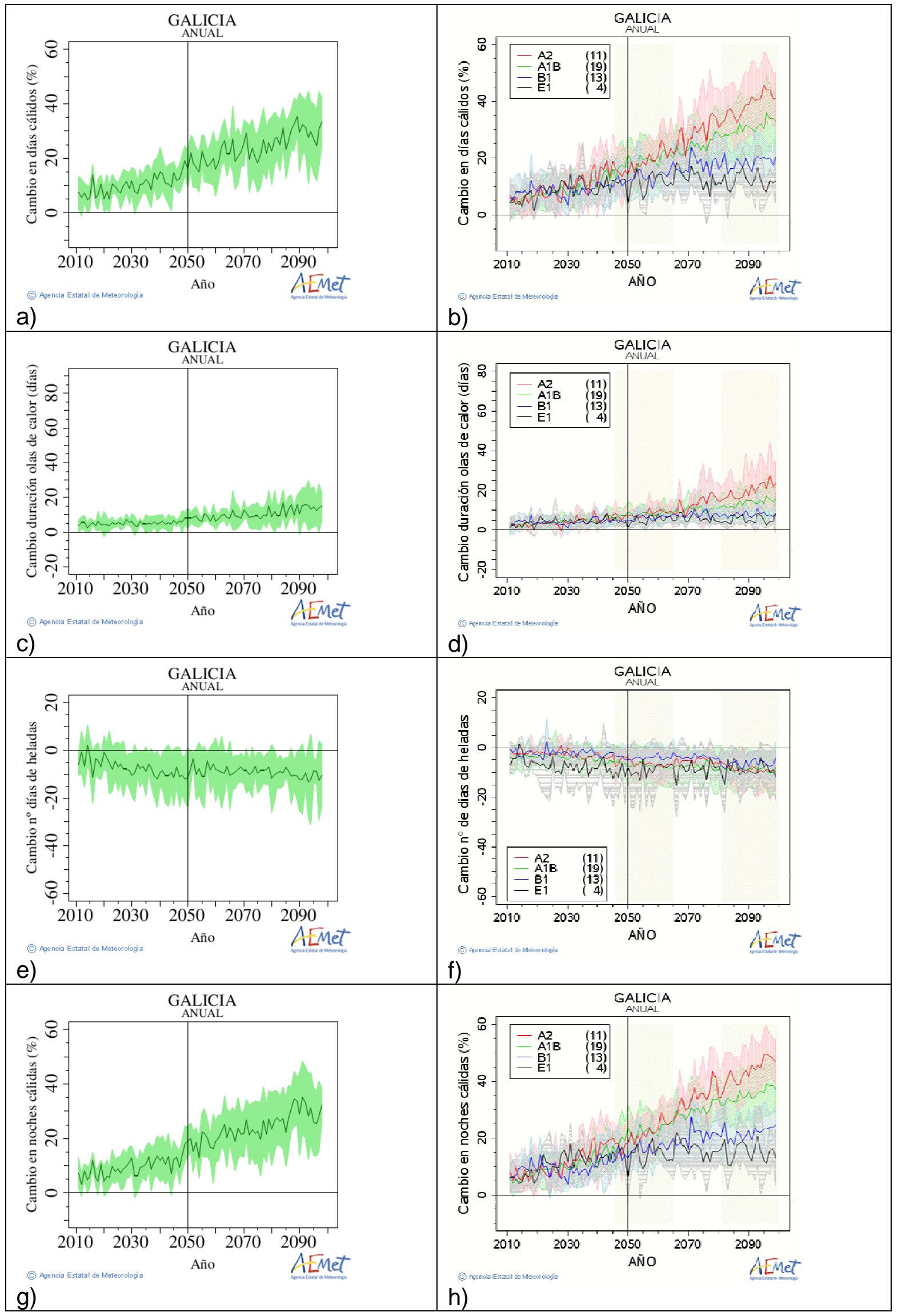

Figura A.72. Igual que la Figura A.2 para la Comunidad Autónoma de Galicia. 


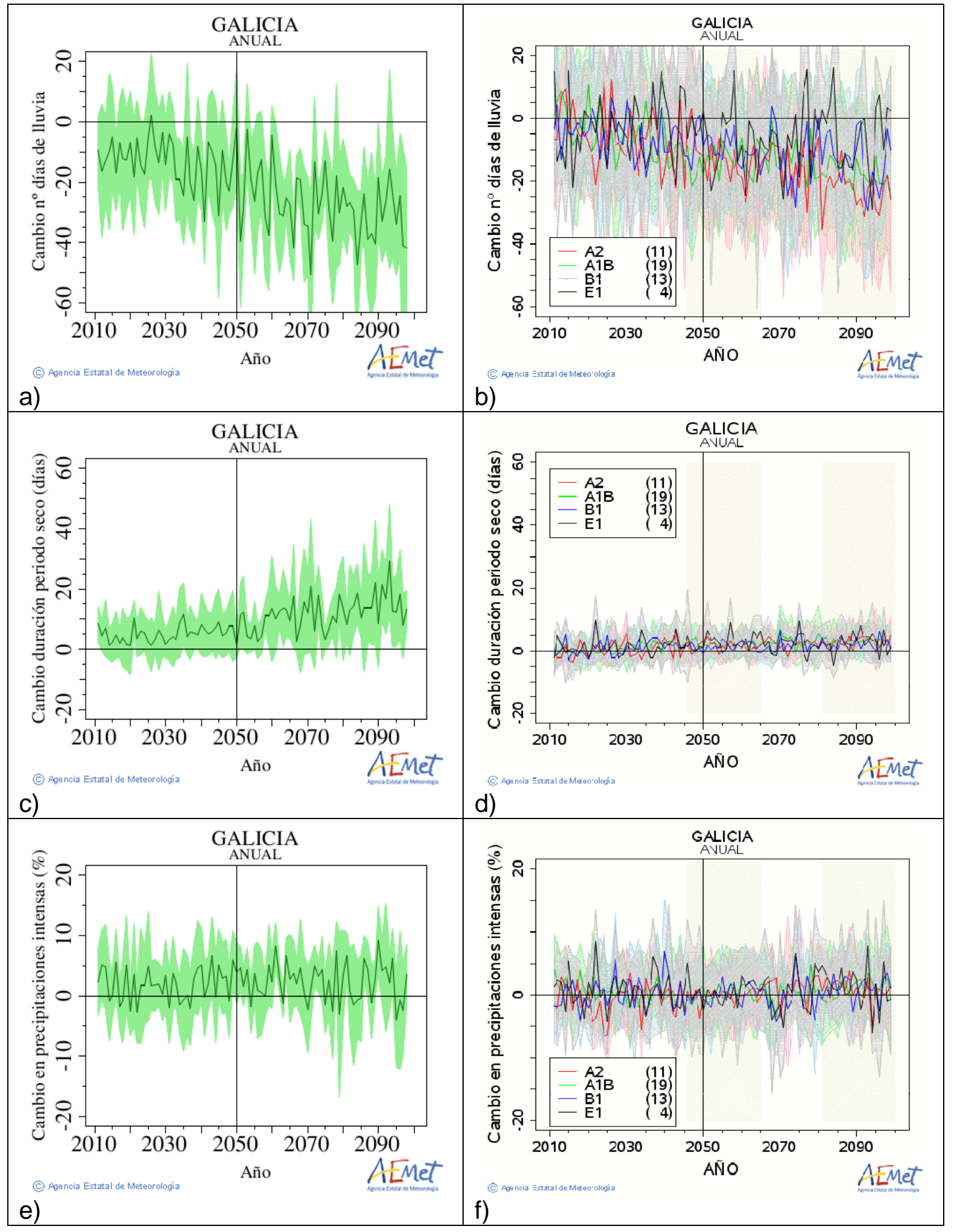

Figura A.73. Igual que la Figura A.3 para la Comunidad Autónoma de Galicia. 


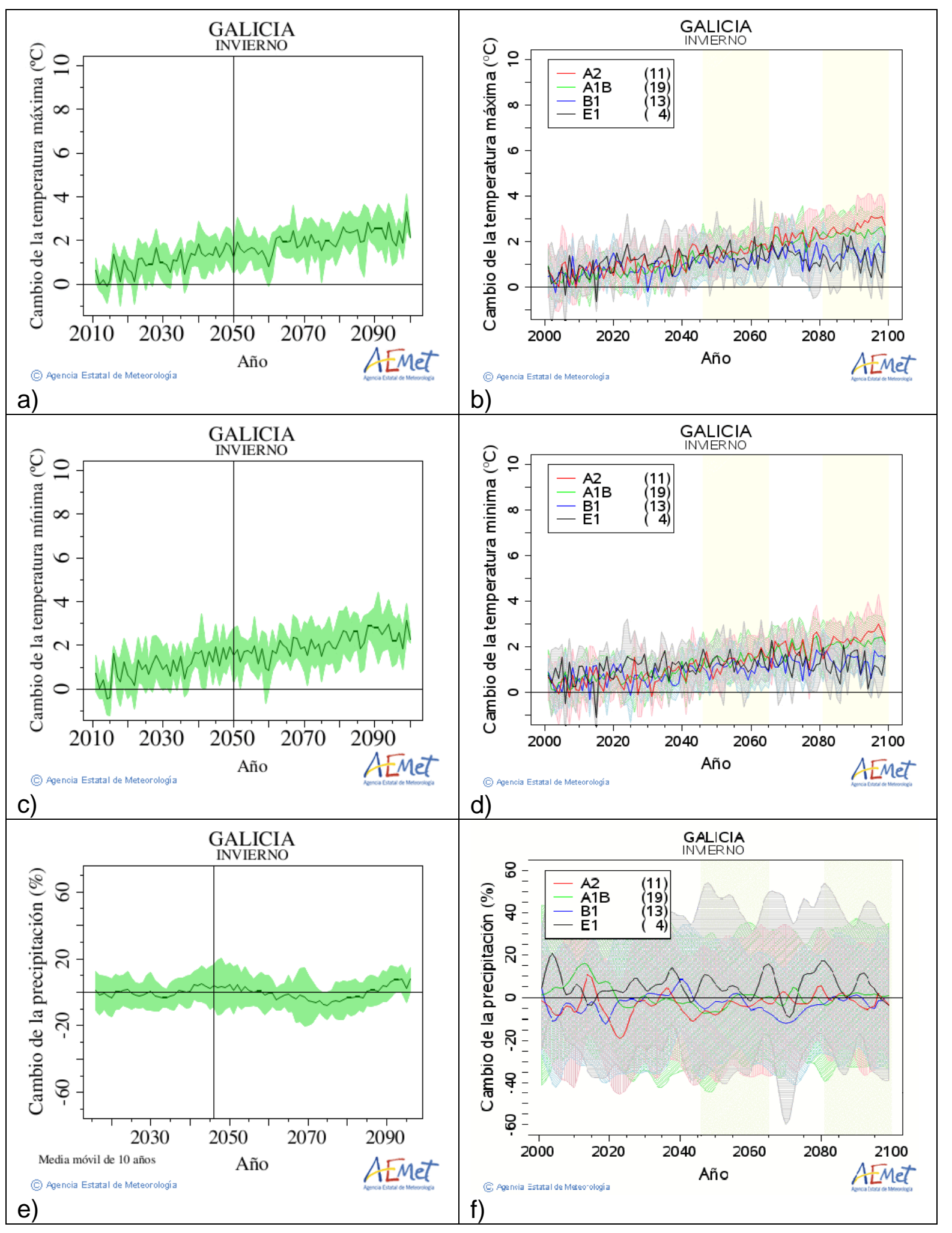

Figura A.74. Igual que la Figura A.4 para la Comunidad Autónoma de Galicia. 


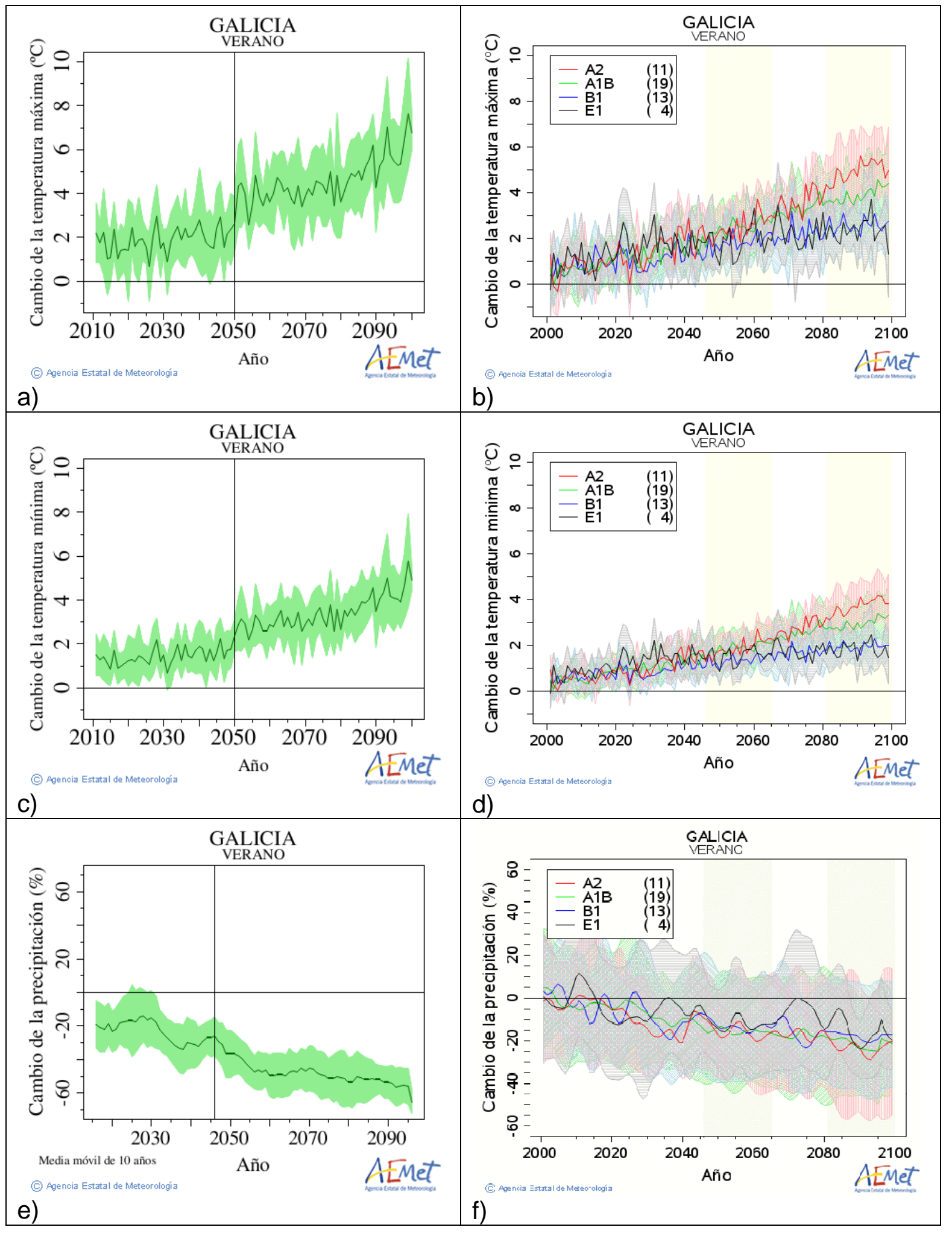

Figura A.75. Igual que la Figura A.5 para la Comunidad Autónoma de Galicia. 


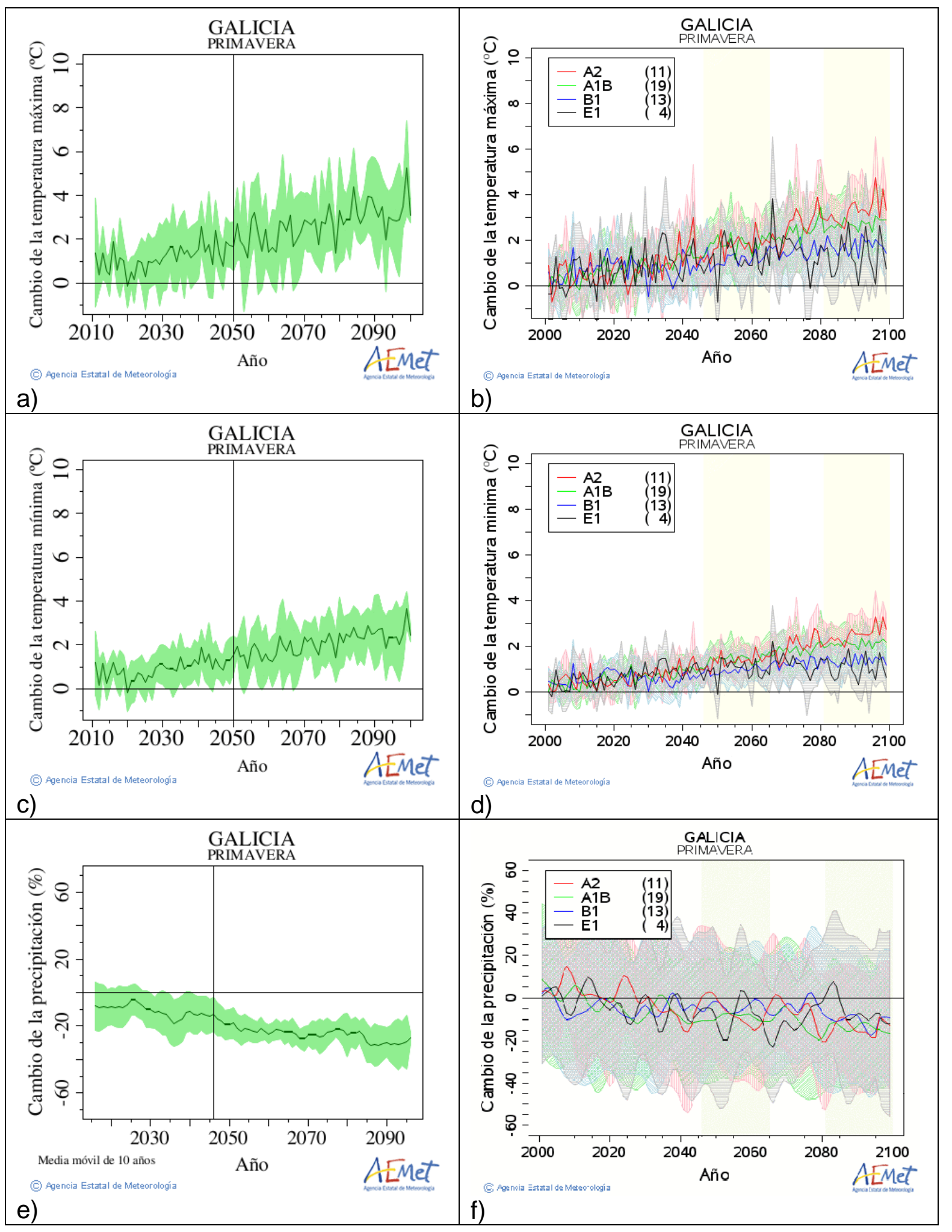

Figura A.76. Igual que la Figura A.6 para la Comunidad Autónoma de Galicia. 


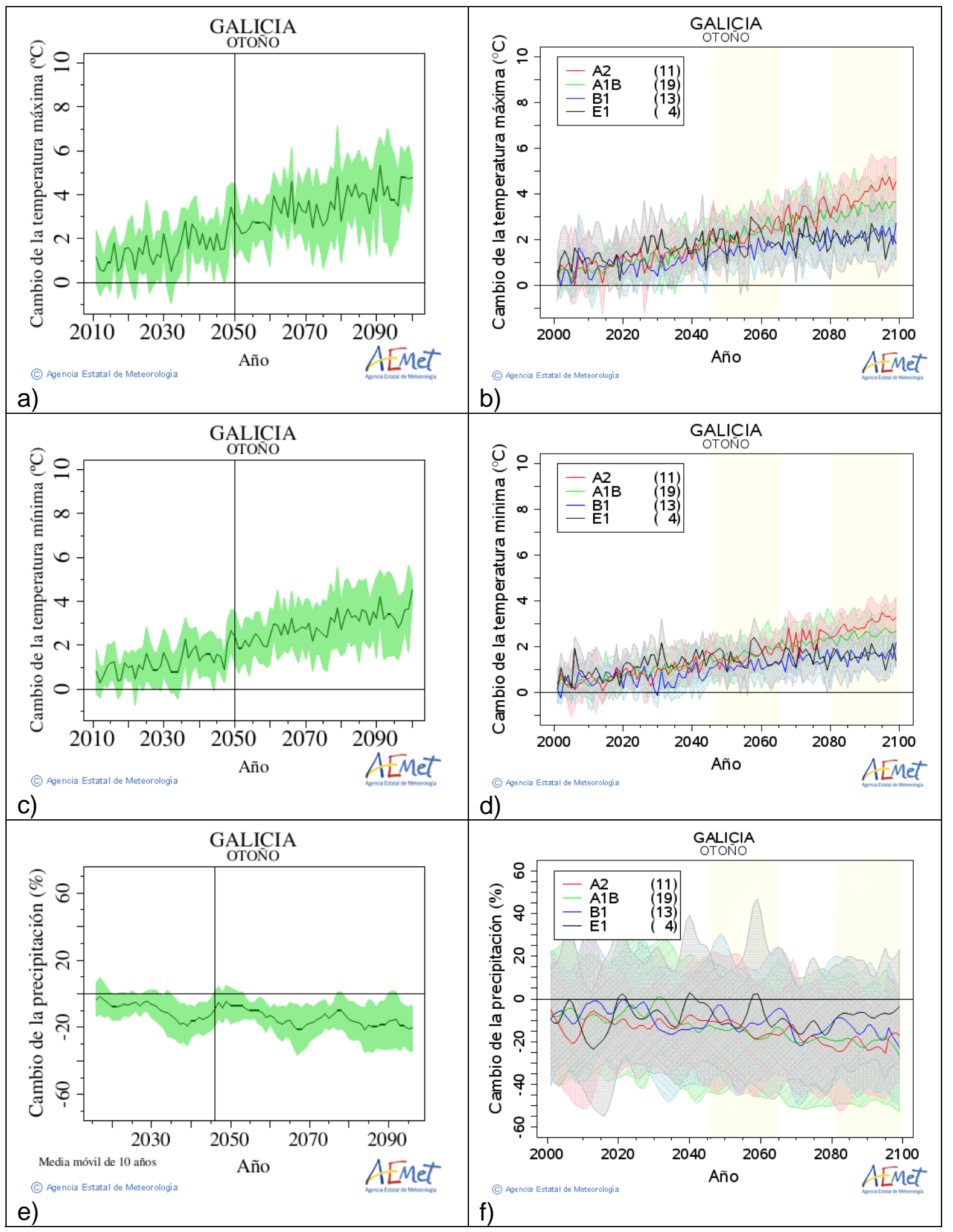

Figura A.77. Igual que la Figura A.7 para la Comunidad Autónoma de Galicia. 
LA RIOJA

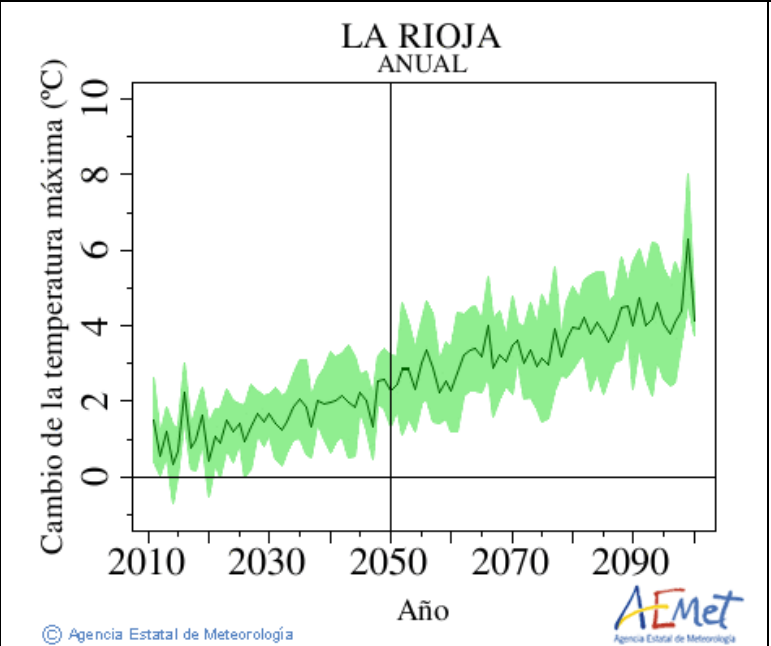

a)

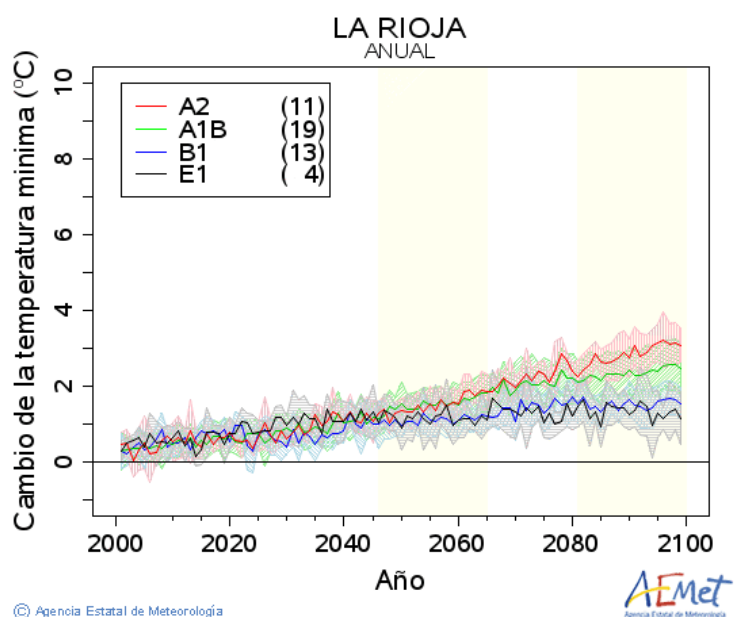

b)
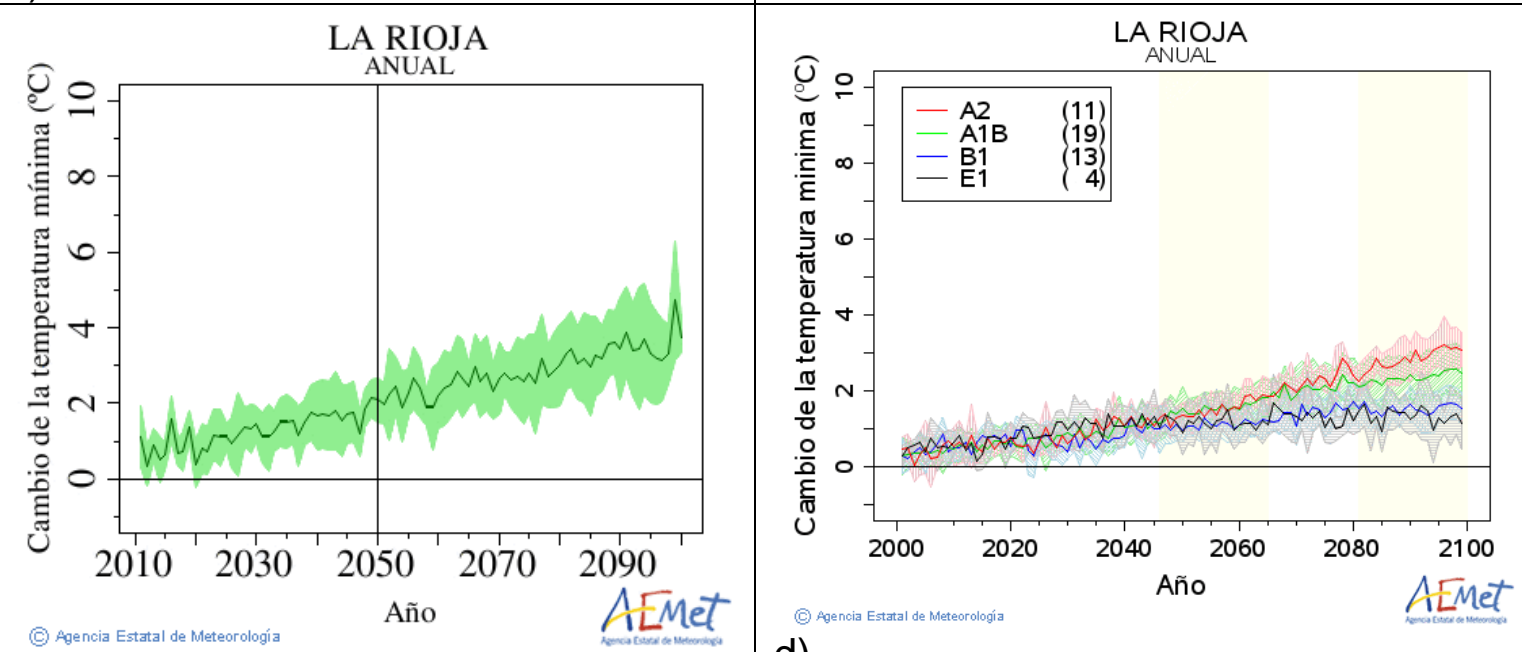

c)

d)

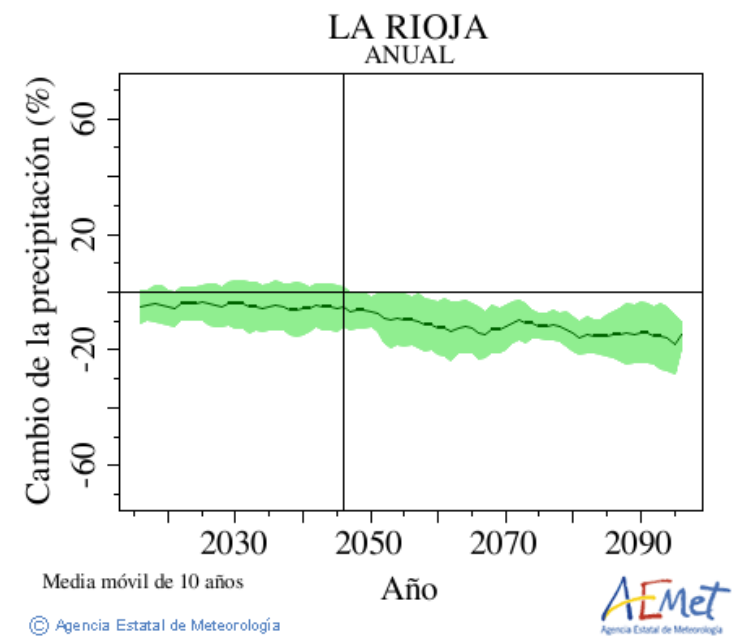

e)

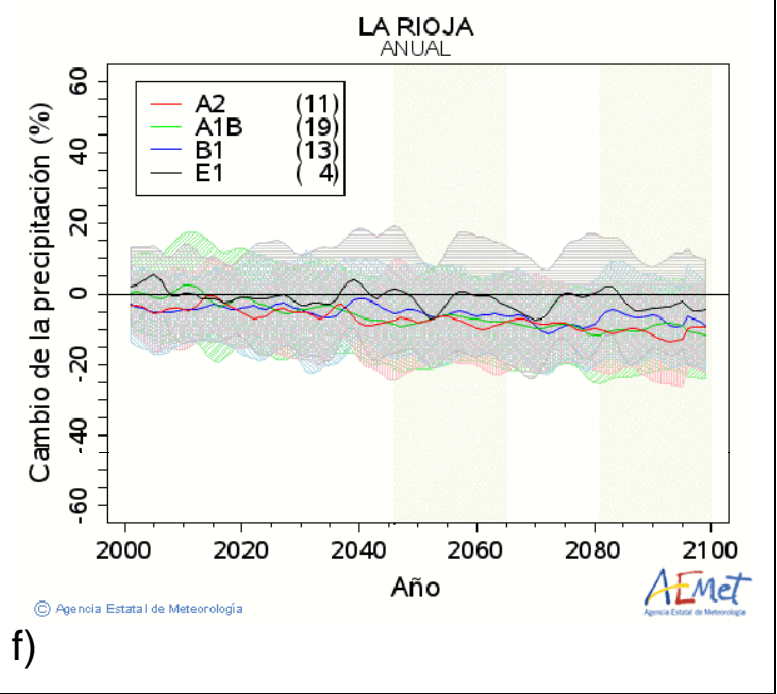

Figura A.78. Igual que la Figura A.1 para la Comunidad Autónoma de La Rioja. 


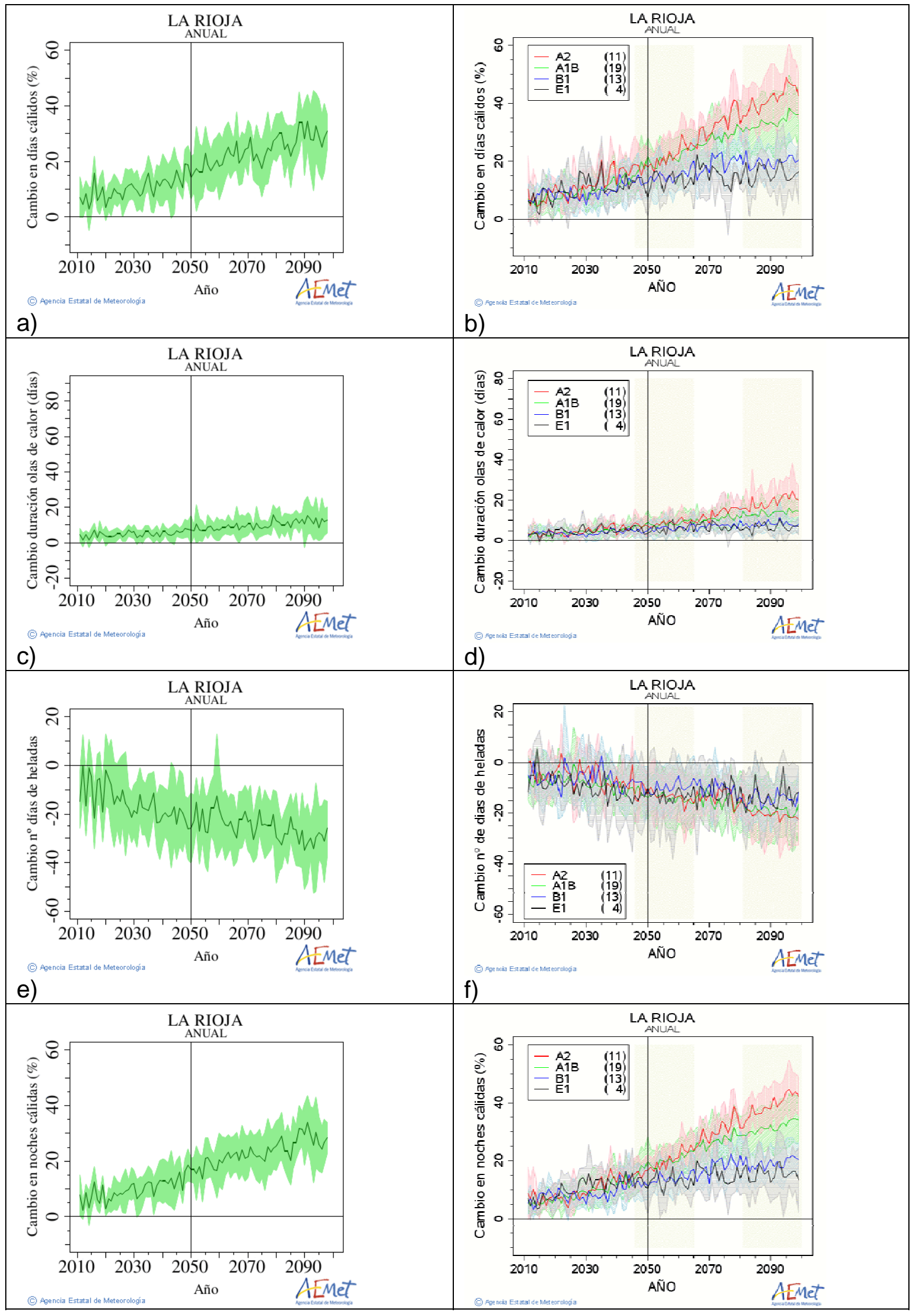

Figura A.79. Igual que la Figura A.2 para la Comunidad Autónoma de La Rioja. 


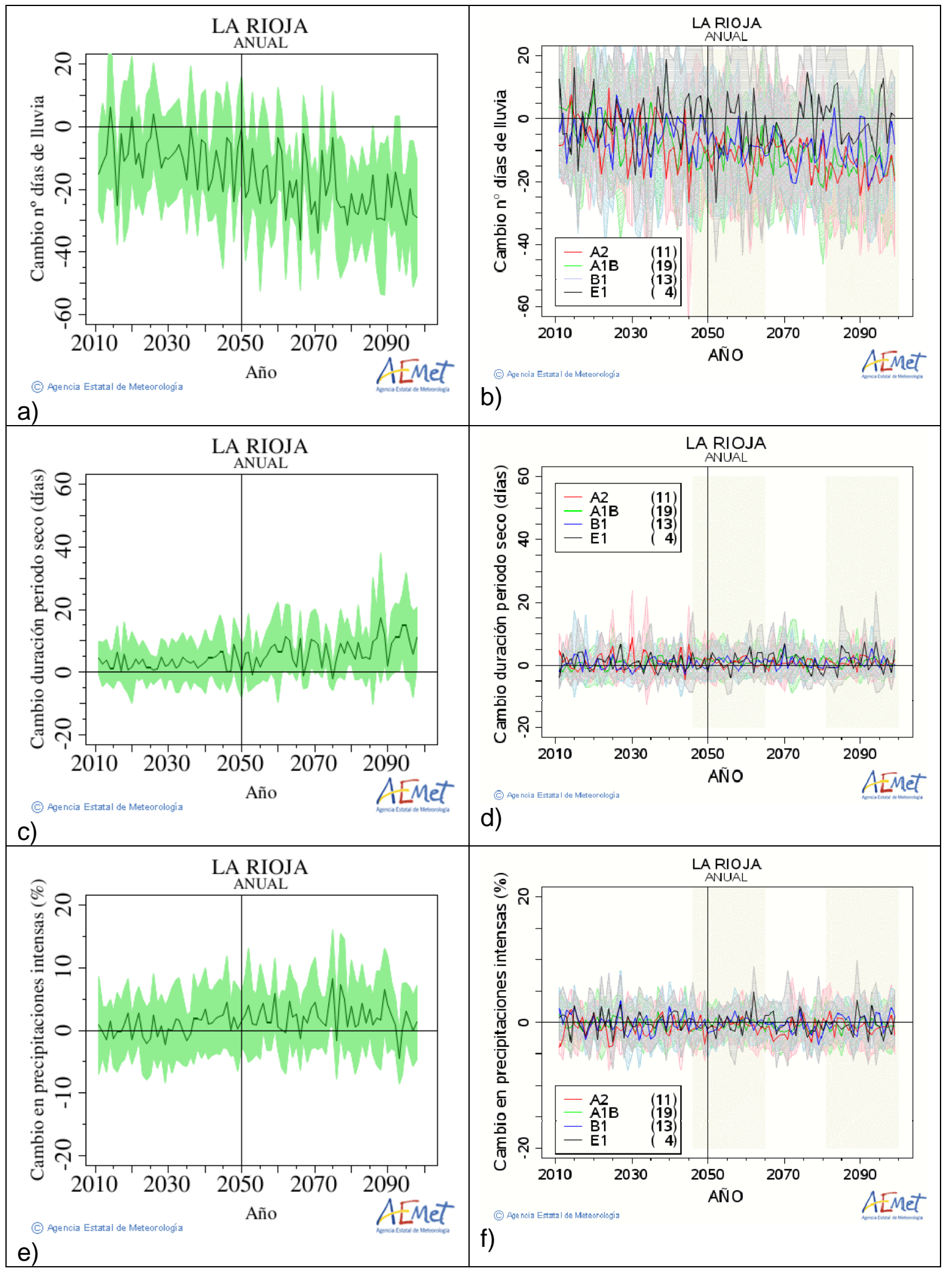

Figura A.80. Igual que la Figura A.3 para la Comunidad Autónoma de La Rioja. 


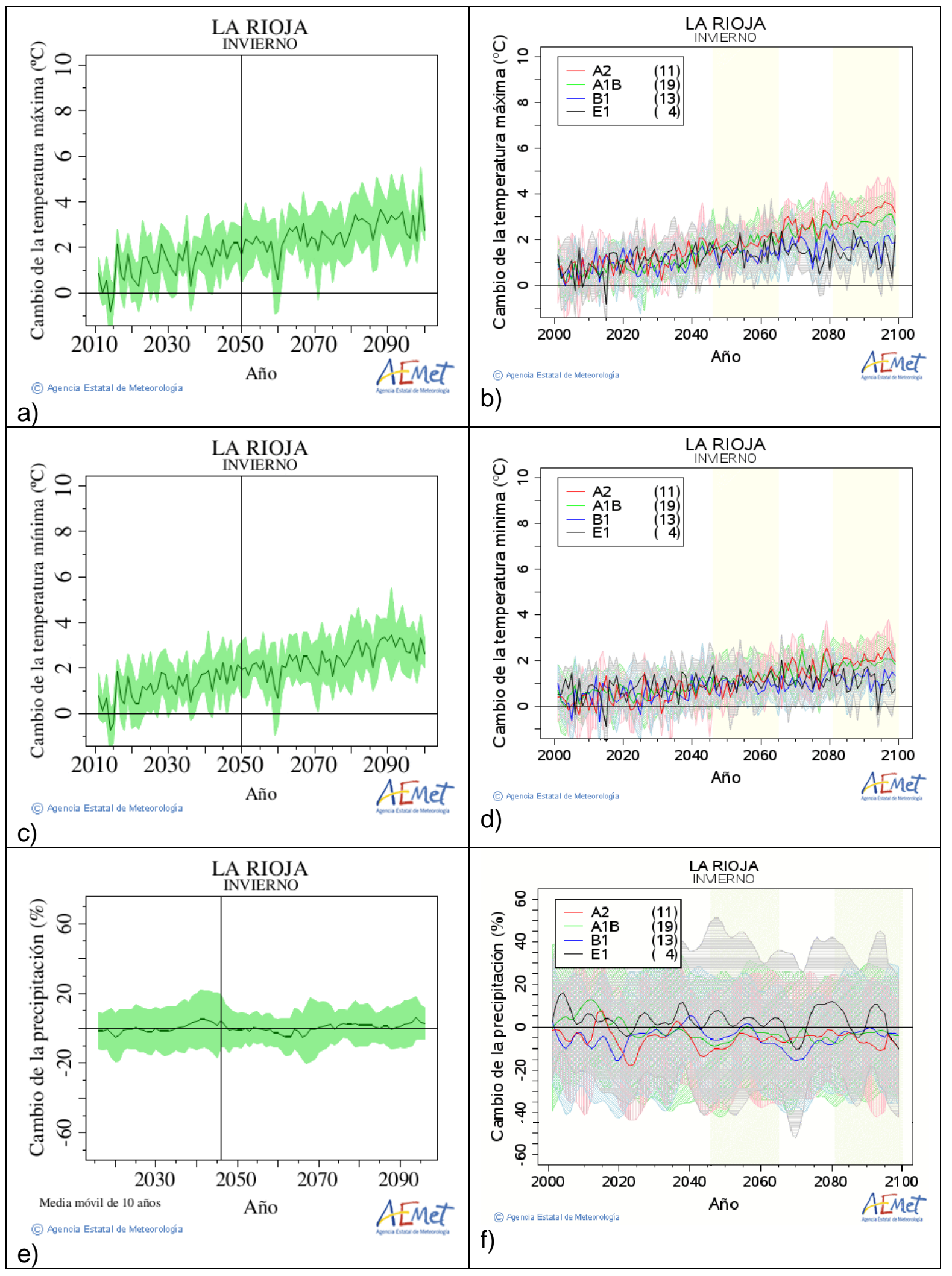

Figura A.81. Igual que la Figura A.4 para la Comunidad Autónoma de La Rioja. 


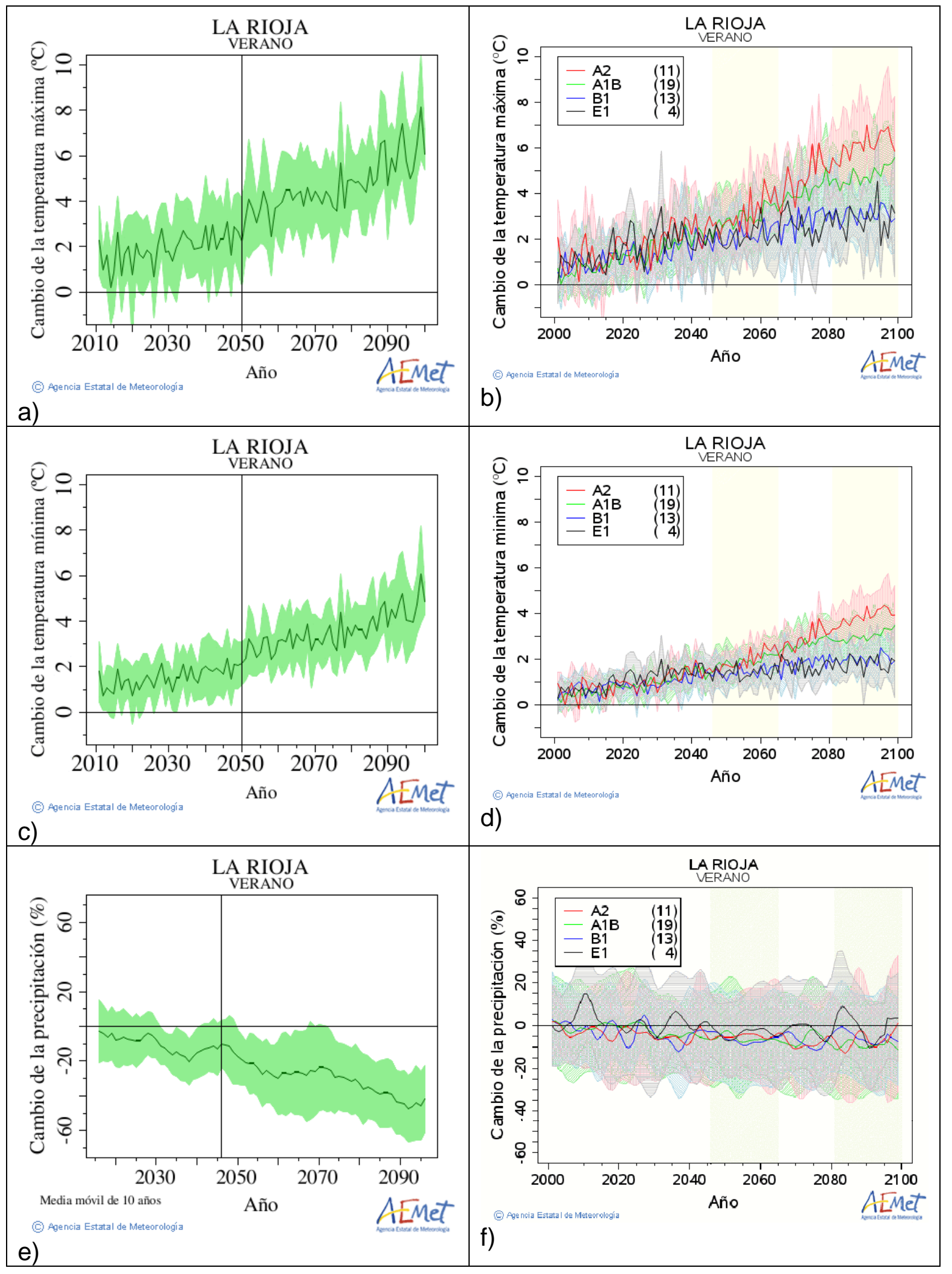

Figura A.82. Igual que la Figura A.5 para la Comunidad Autónoma de La Rioja. 


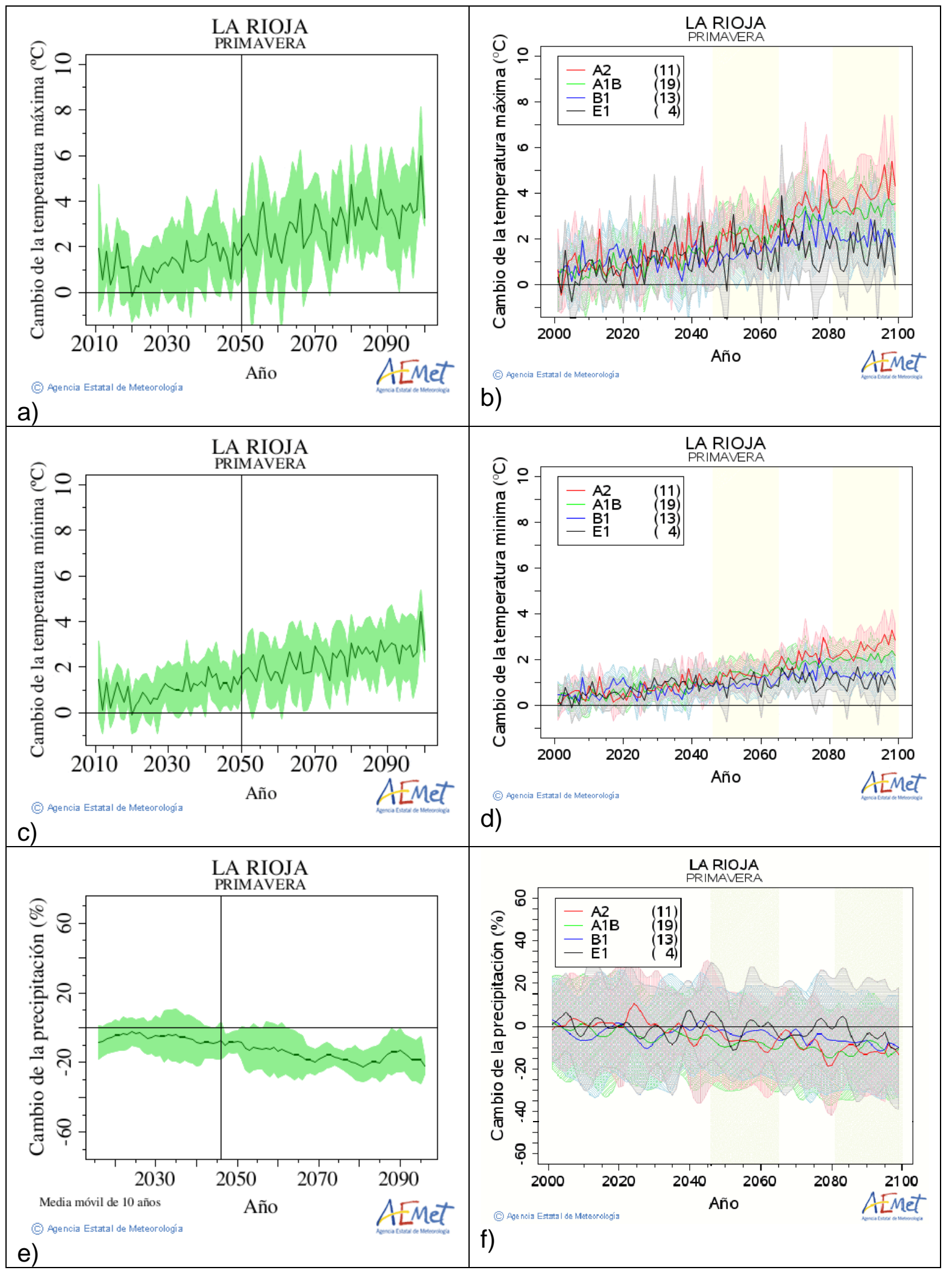

Figura A.83. Igual que la Figura A.6 para la Comunidad Autónoma de La Rioja. 


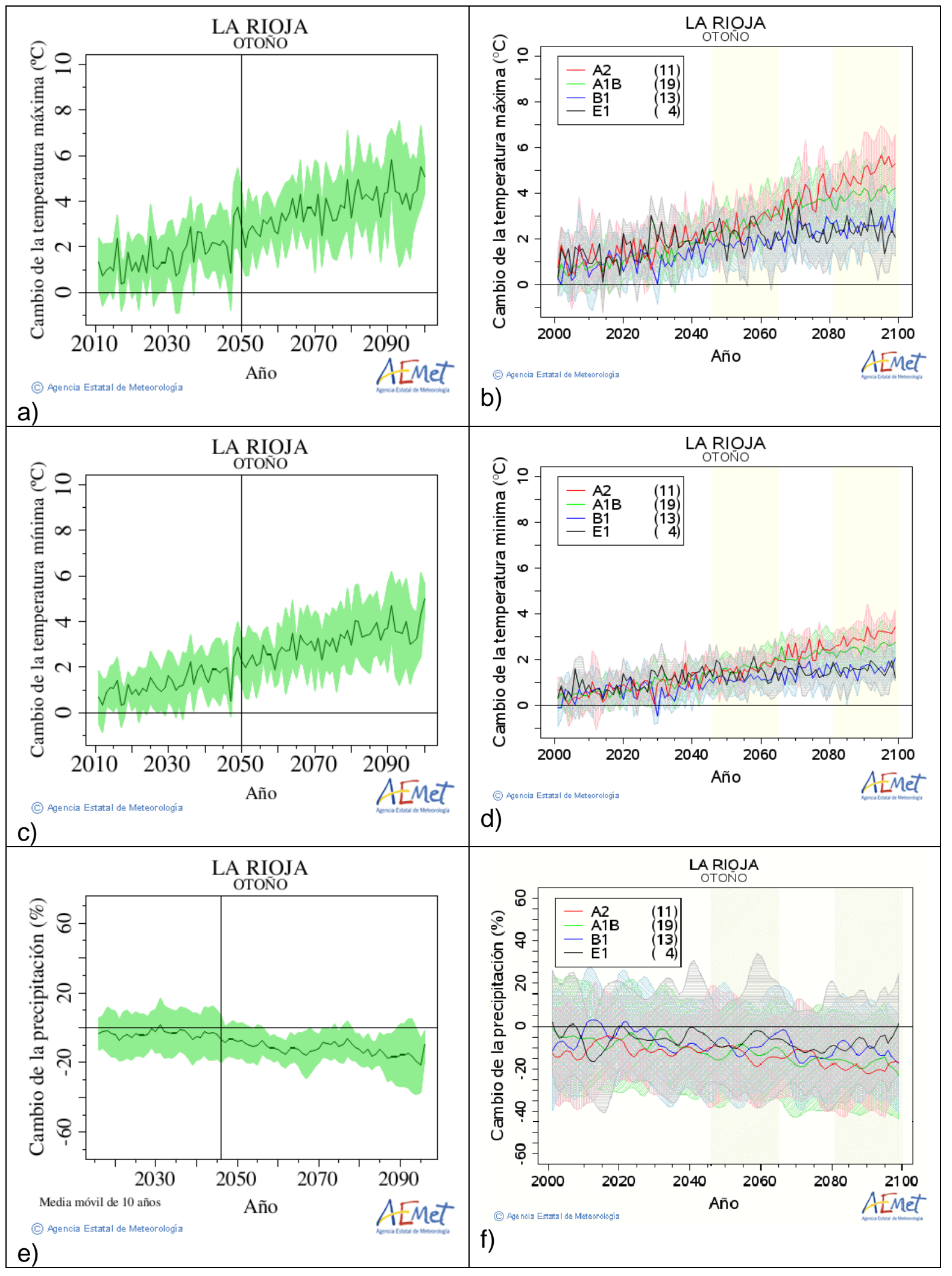

Figura A.84. Igual que la Figura A.7 para la Comunidad Autónoma de La Rioja. 
COMUNIDAD DE MADRID

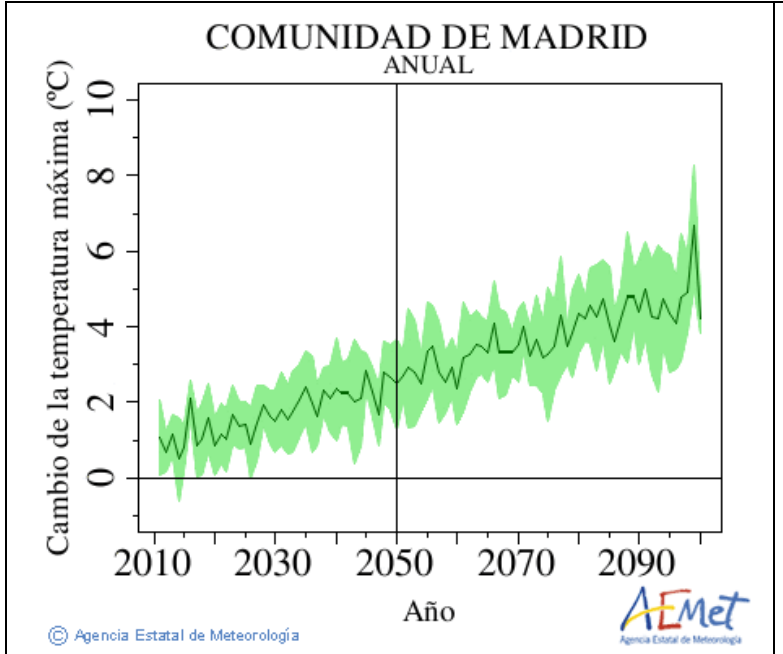

a)
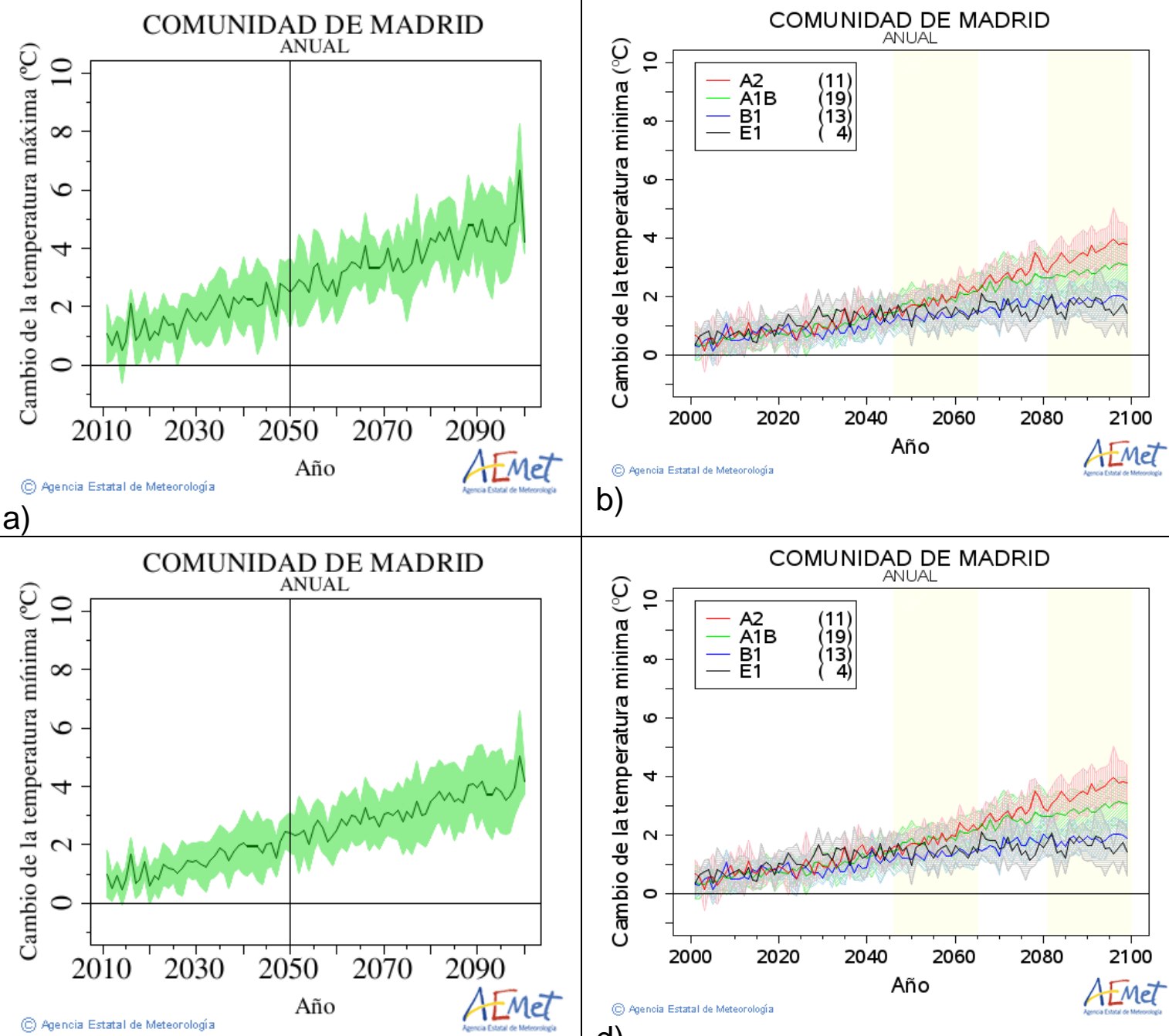

b)

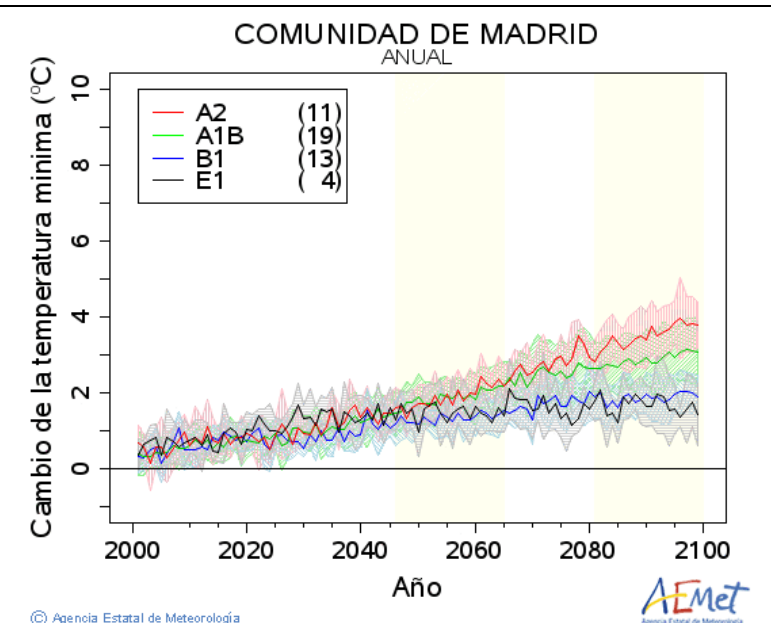

c)

d)

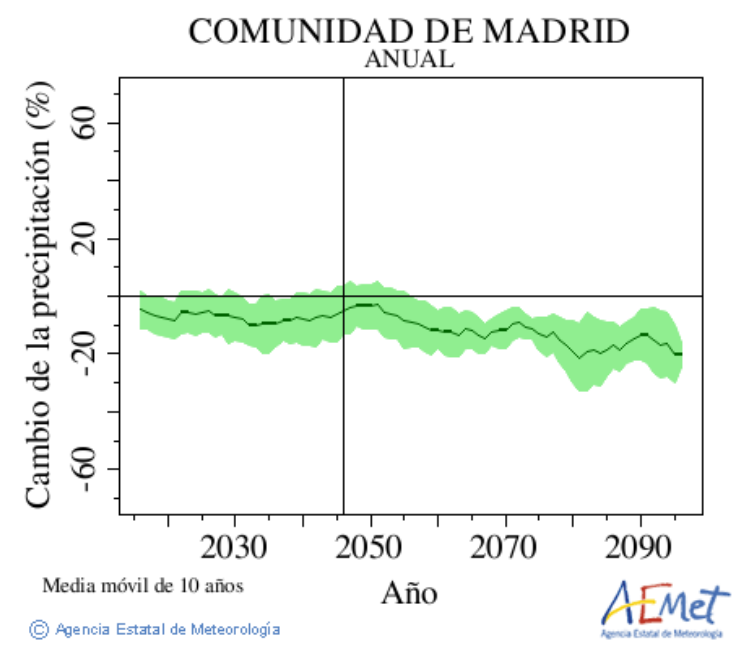

e)

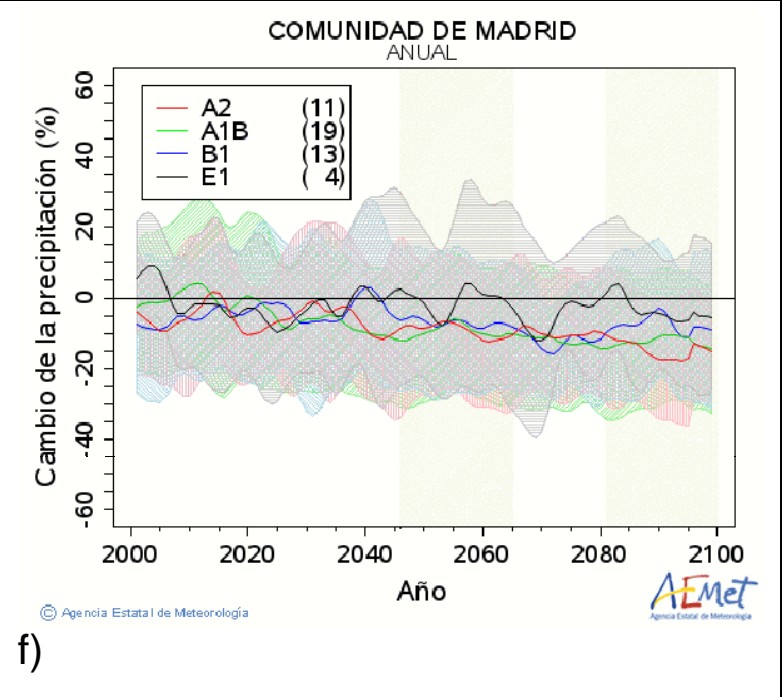

Figura A.85. Igual que la Figura A.1 para la Comunidad de Madrid. 


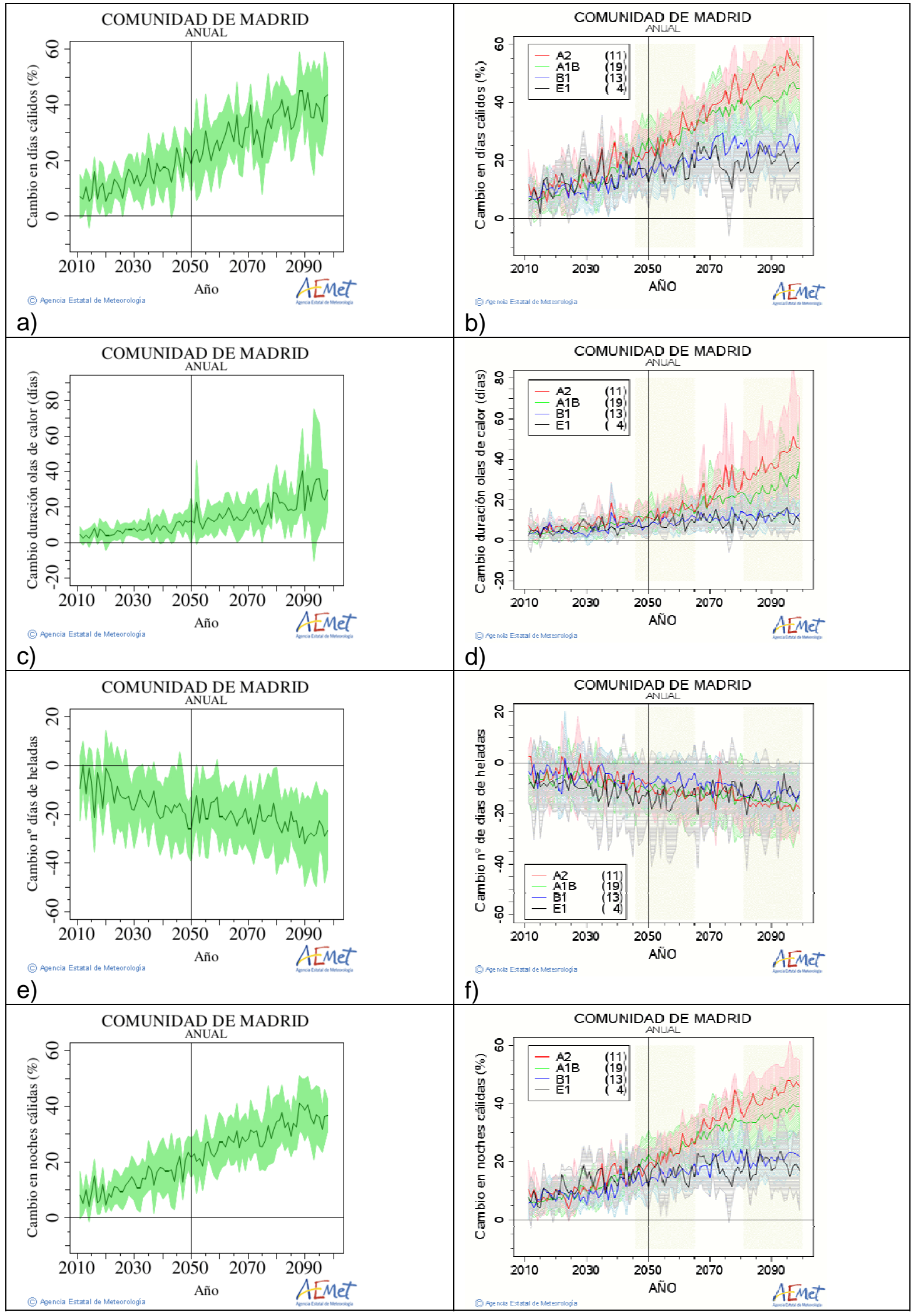

Figura A.86. Igual que la Figura A.2 para la Comunidad de Madrid. 


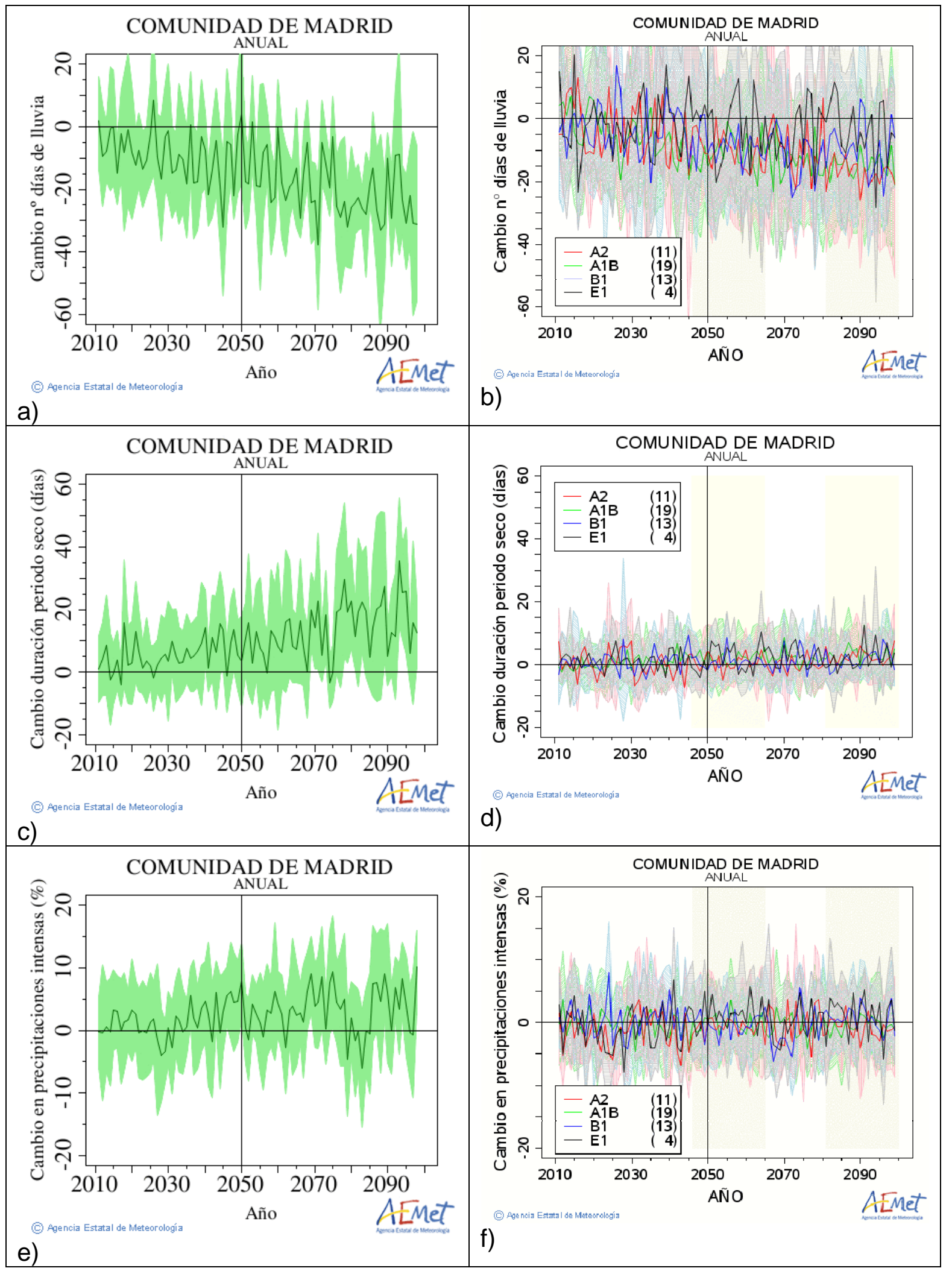

Figura A.87. Igual que la Figura A.3 para la Comunidad de Madrid. 


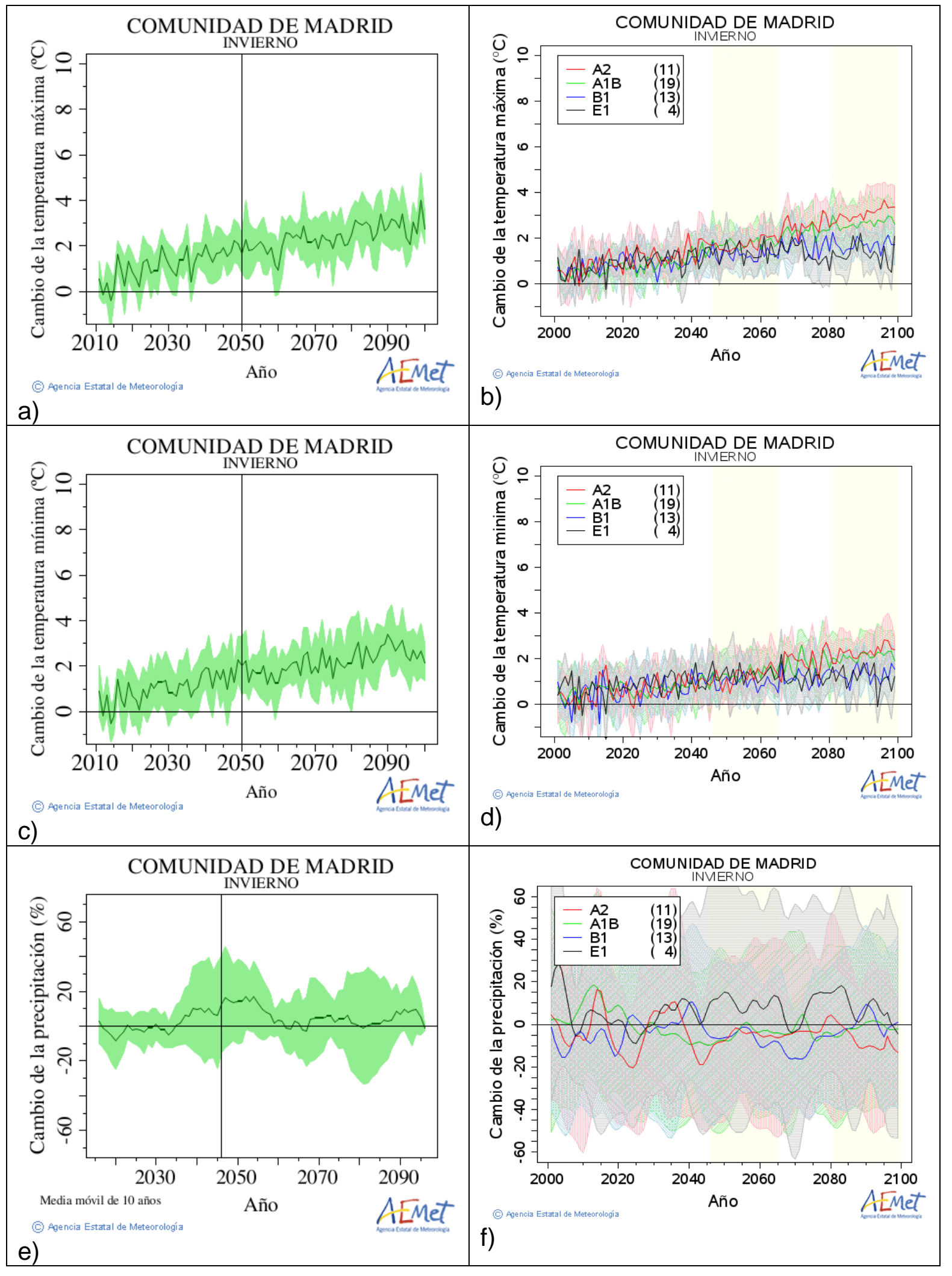

Figura A.88. Igual que la Figura A.4 para la Comunidad de Madrid. 


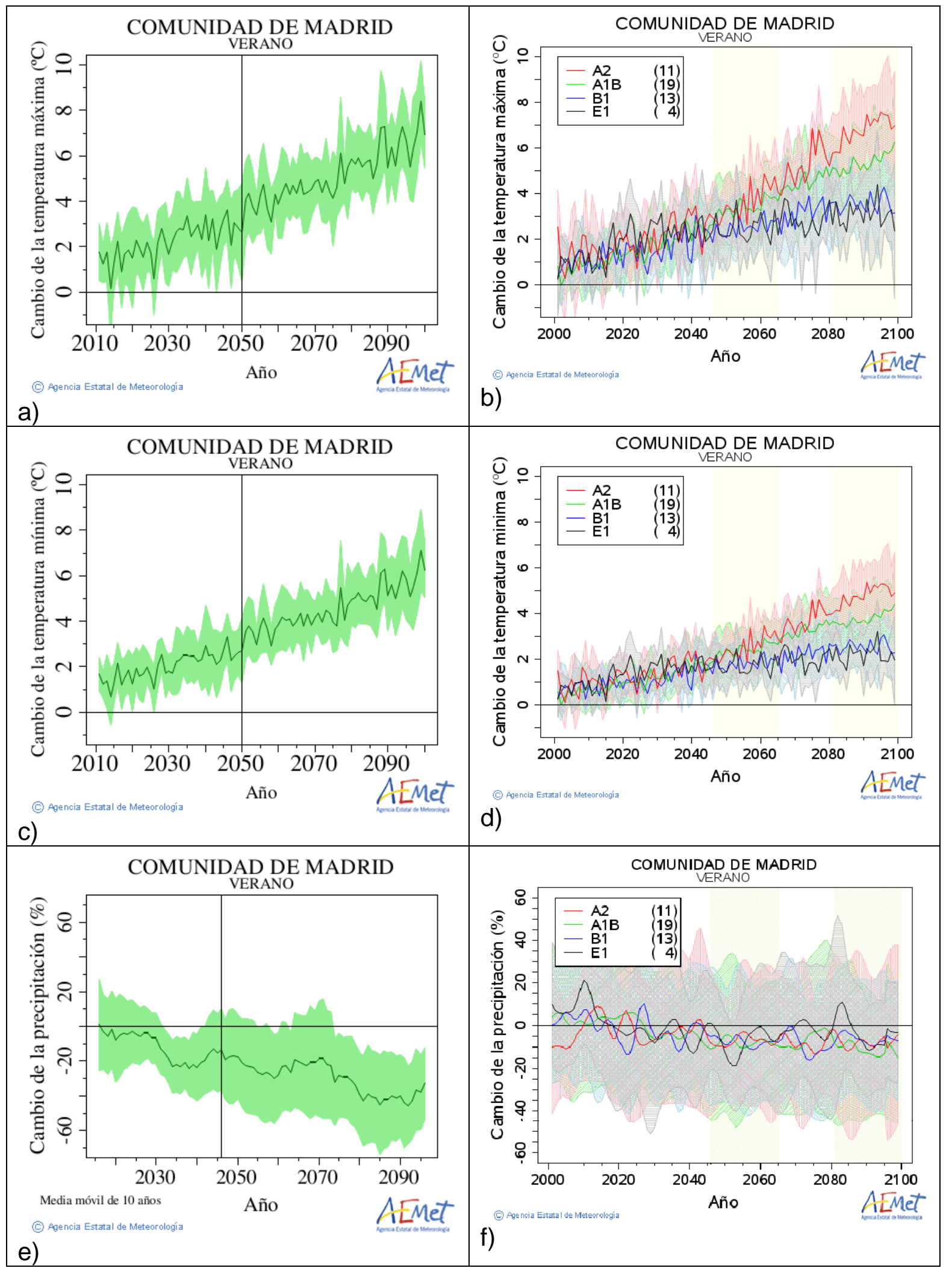

Figura A.89. Igual que la Figura A.5 para la Comunidad de Madrid. 


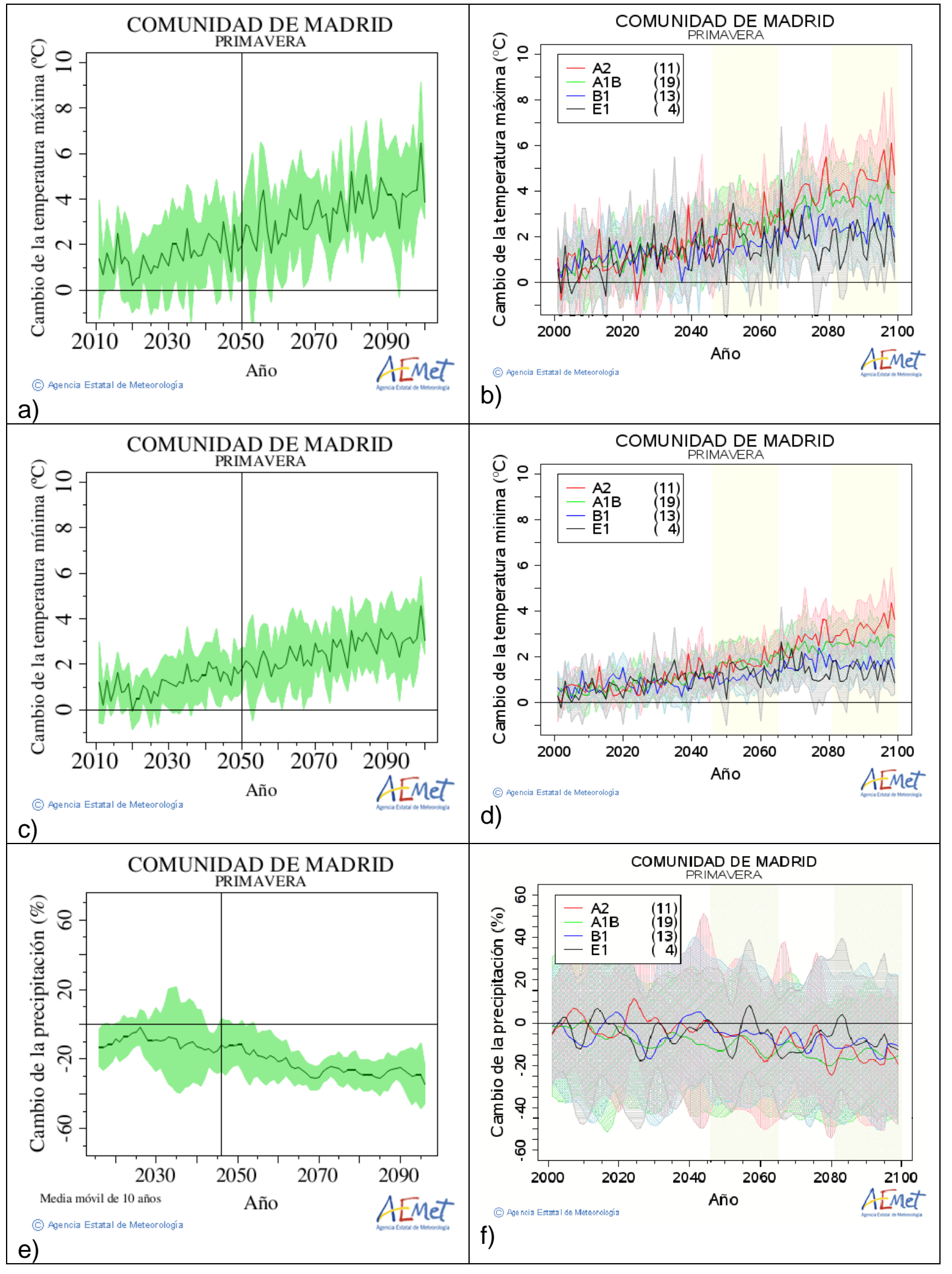

Figura A.90. Igual que la Figura A.6 para la Comunidad de Madrid. 


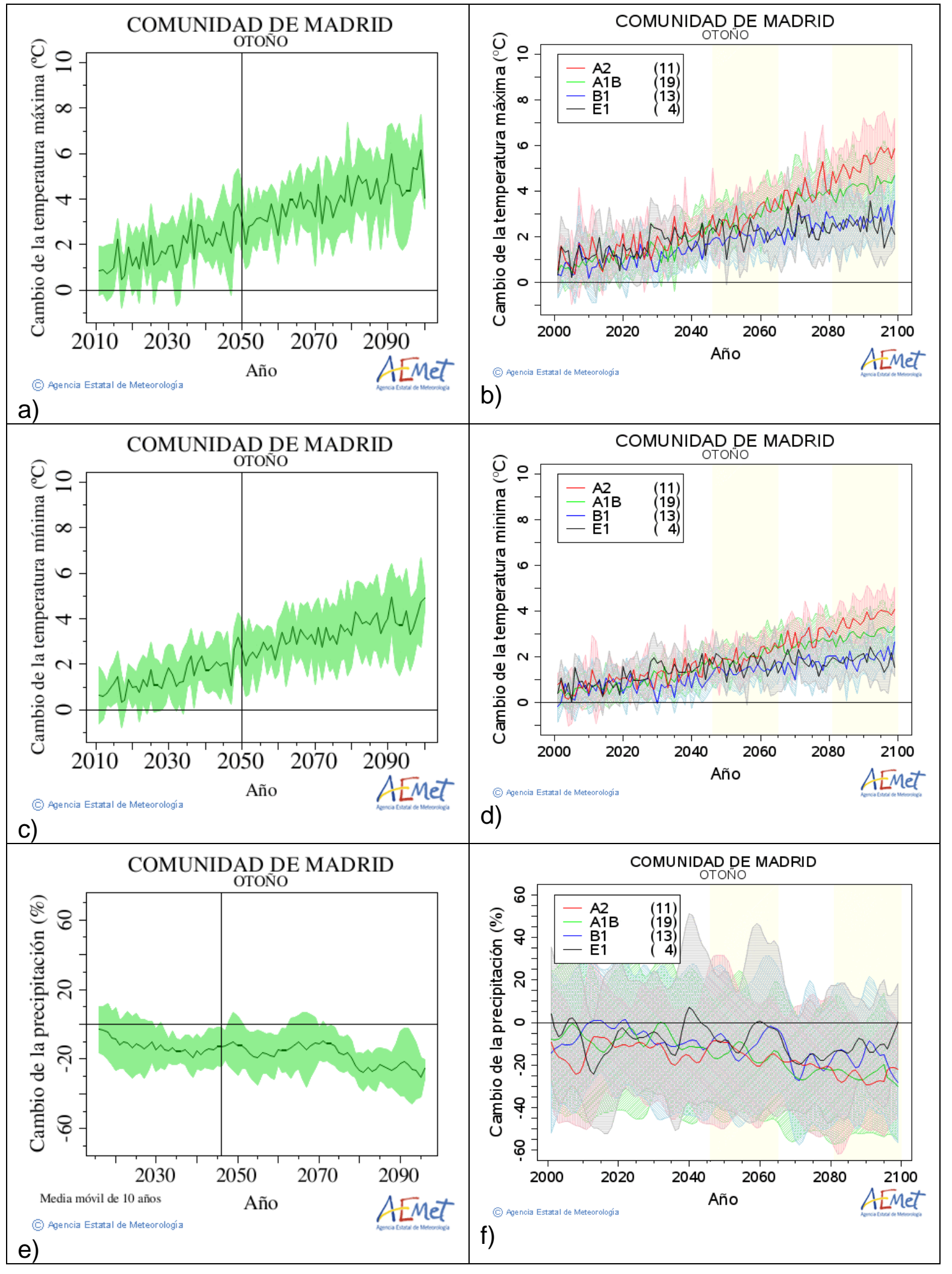

Figura A.91. Igual que la Figura A.7 para la Comunidad de Madrid. 
REGIÓN DE MURCIA

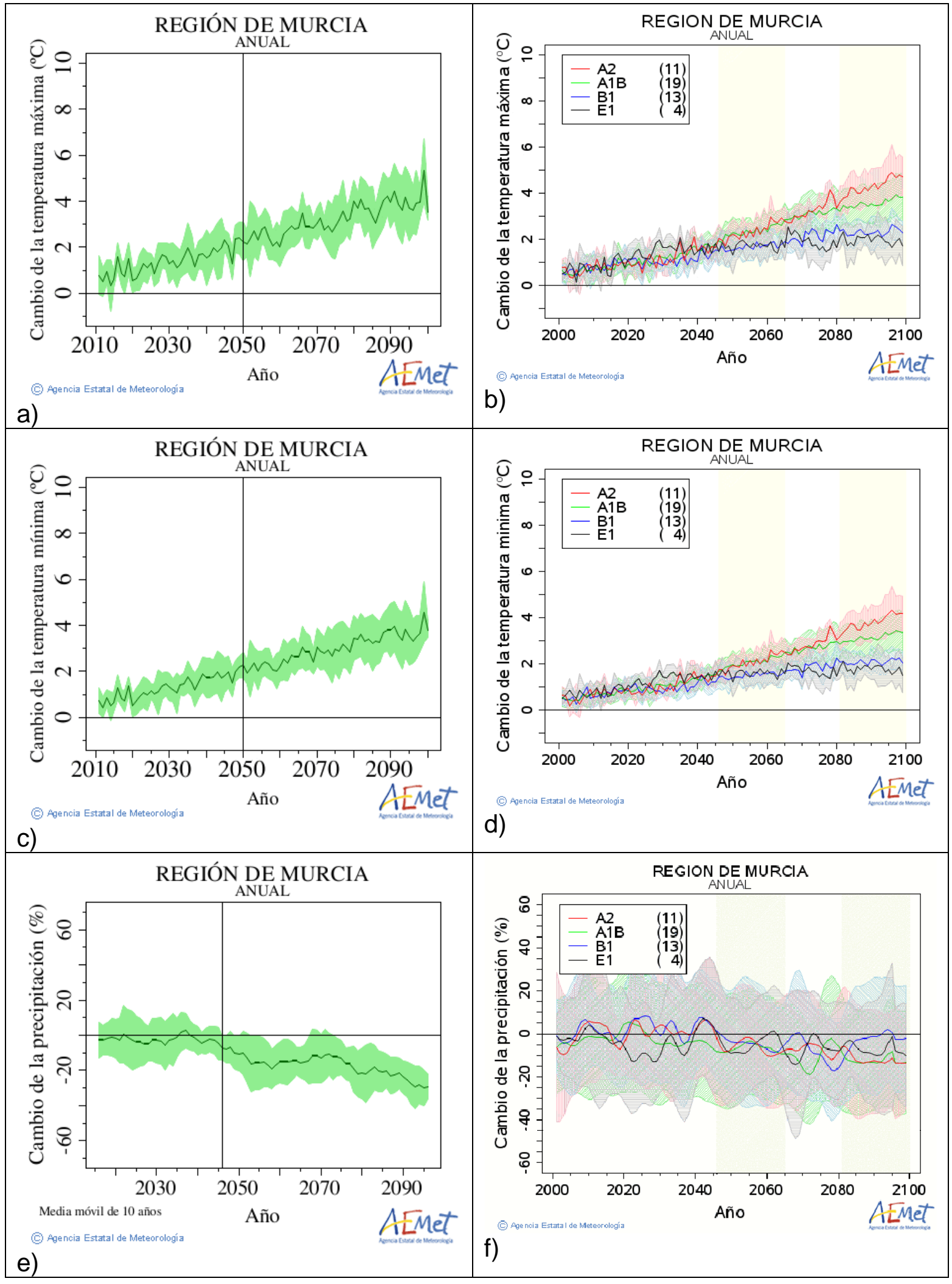

Figura A.92. Igual que la Figura A.1 para la Región de Murcia. 


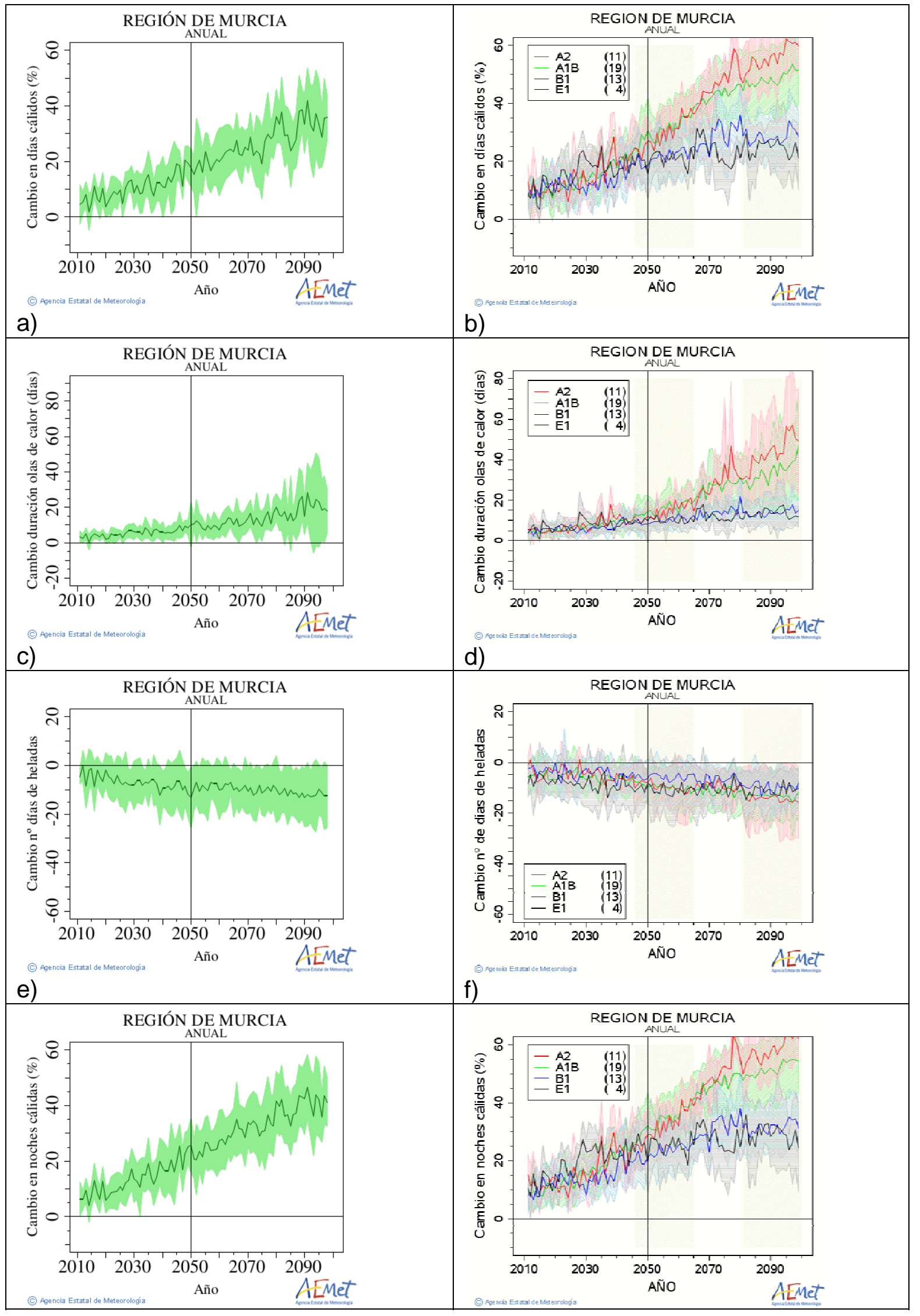

Figura A.93. Igual que la Figura A.2 para la Región de Murcia. 


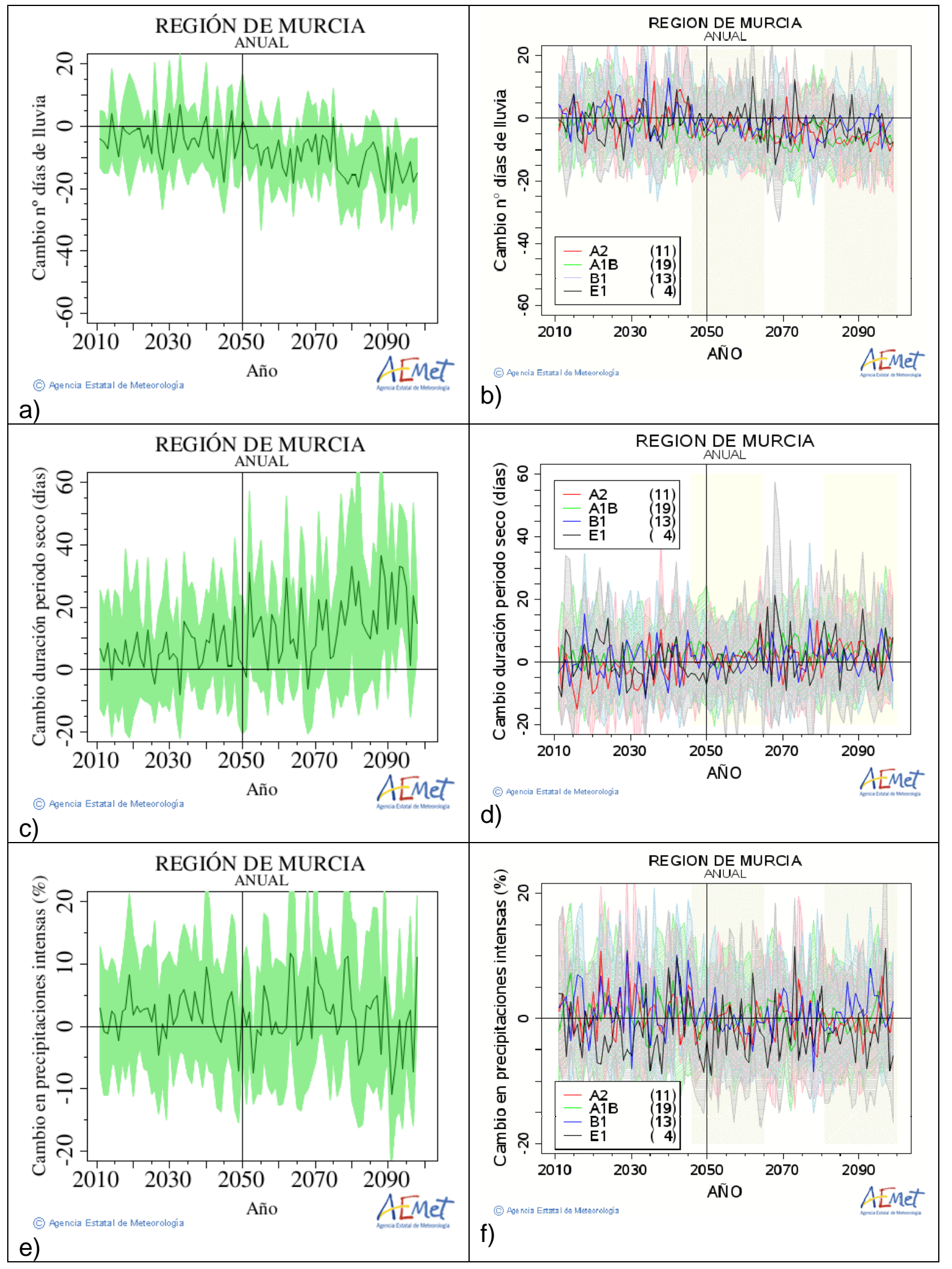

Figura A.94. Igual que la Figura A.3 para la Región de Murcia. 


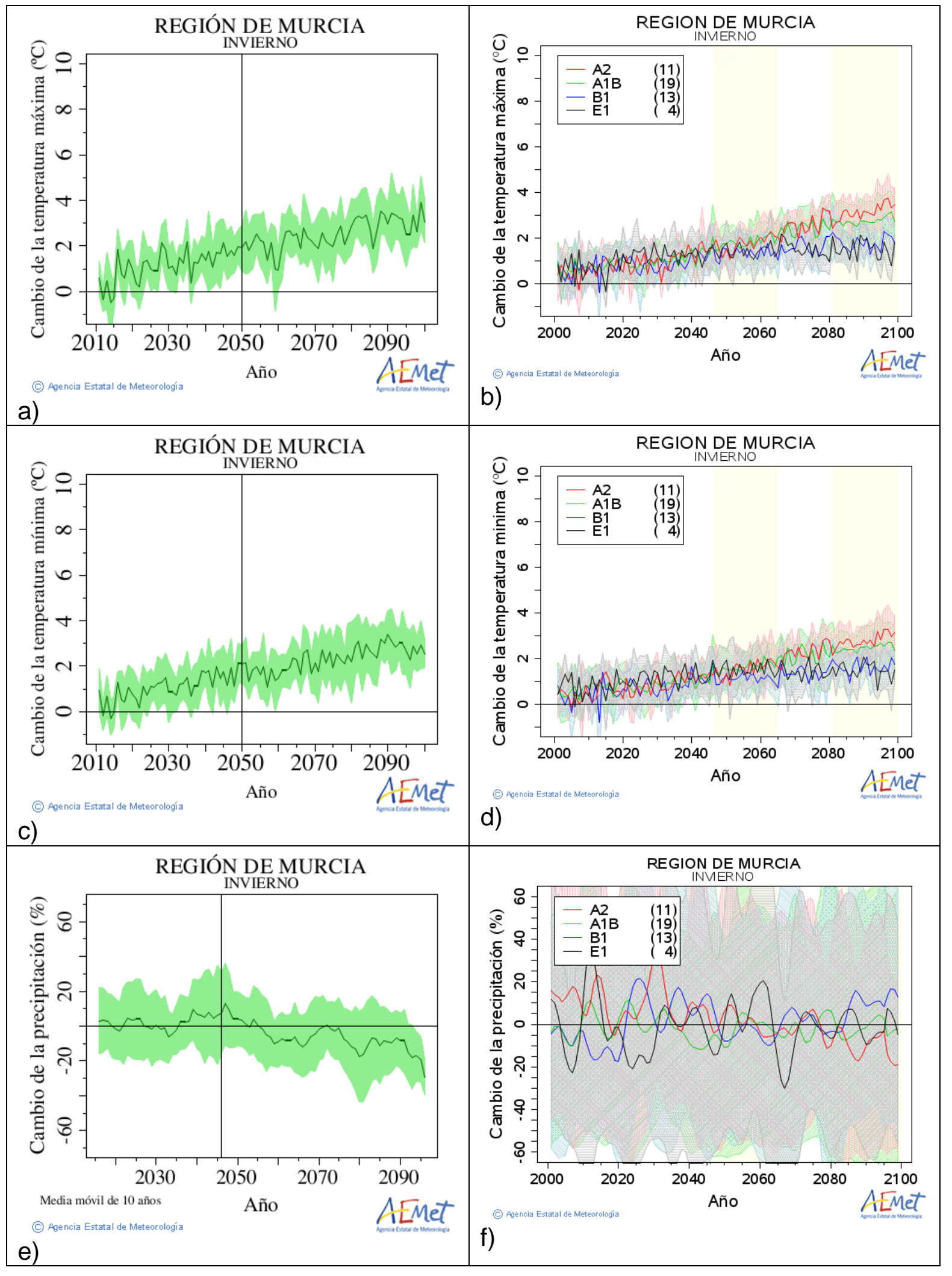

Figura A.95. Igual que la Figura A.4 para la Región de Murcia. 


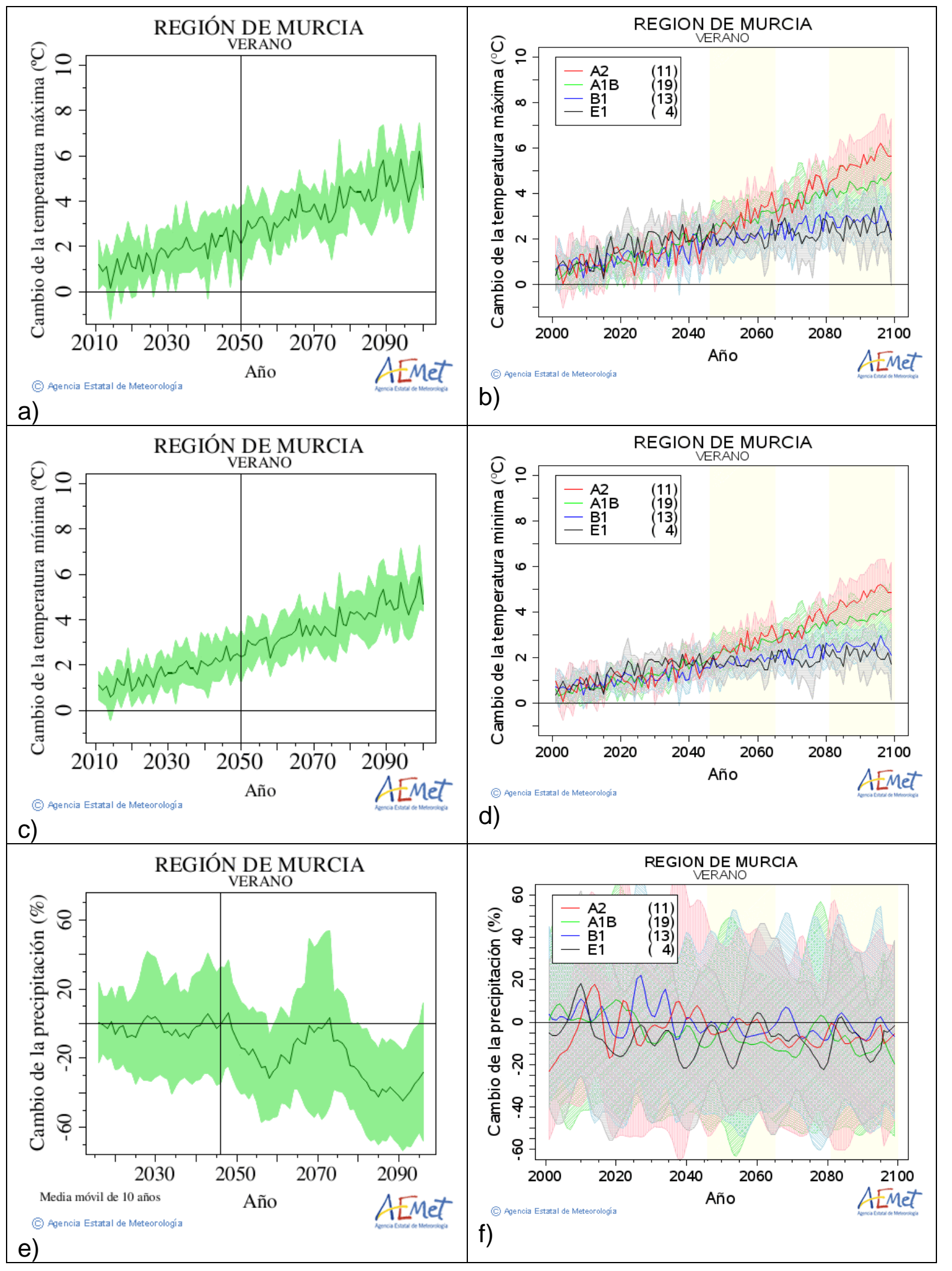

Figura A.96. Igual que la Figura A.5 para la Región de Murcia. 


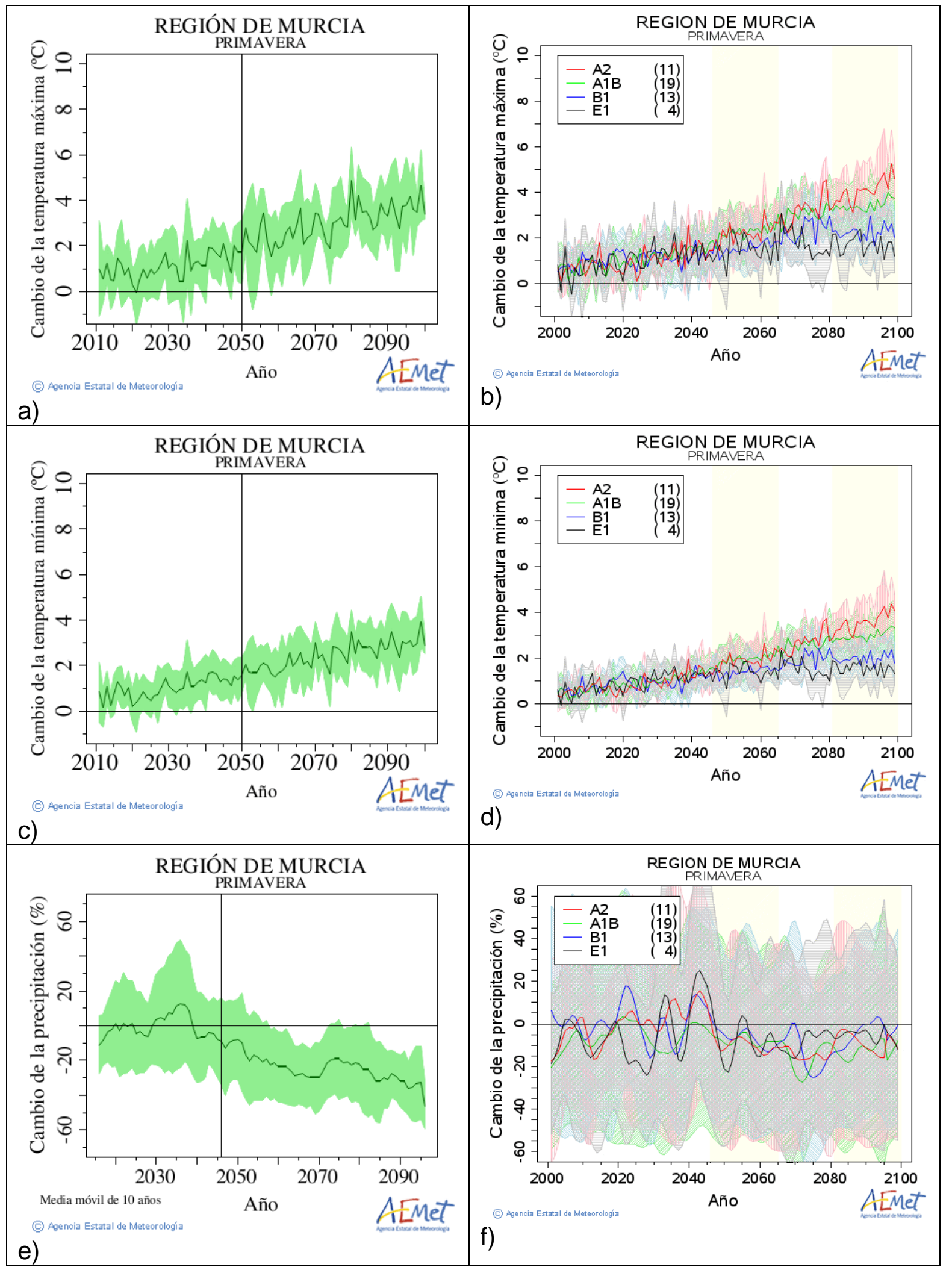

Figura A.97. Igual que la Figura A.6 para la Región de Murcia. 


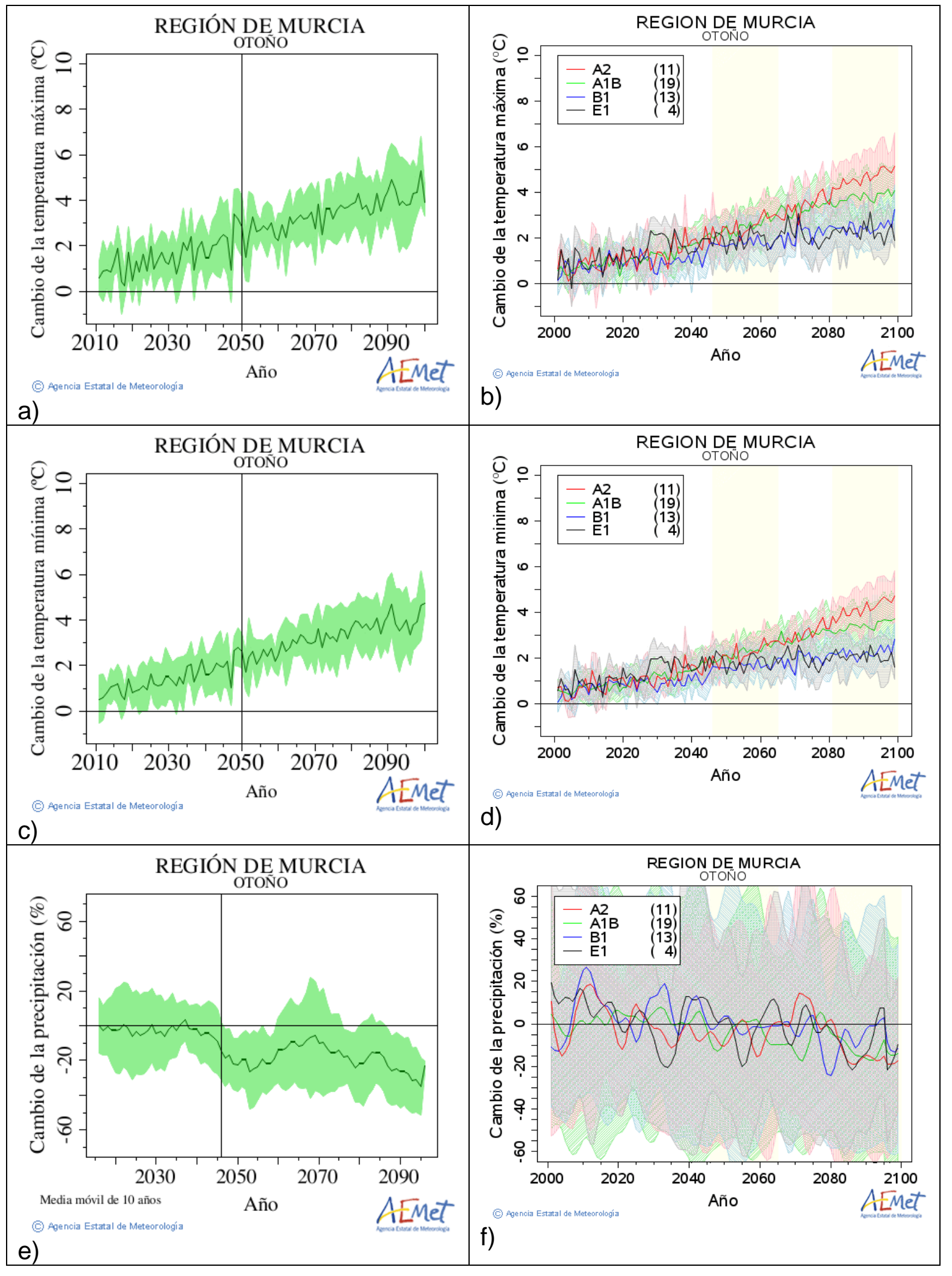

Figura A.98. Igual que la Figura A.7 para la Región de Murcia. 


\section{COMUNIDAD FORAL DE NAVARRA}

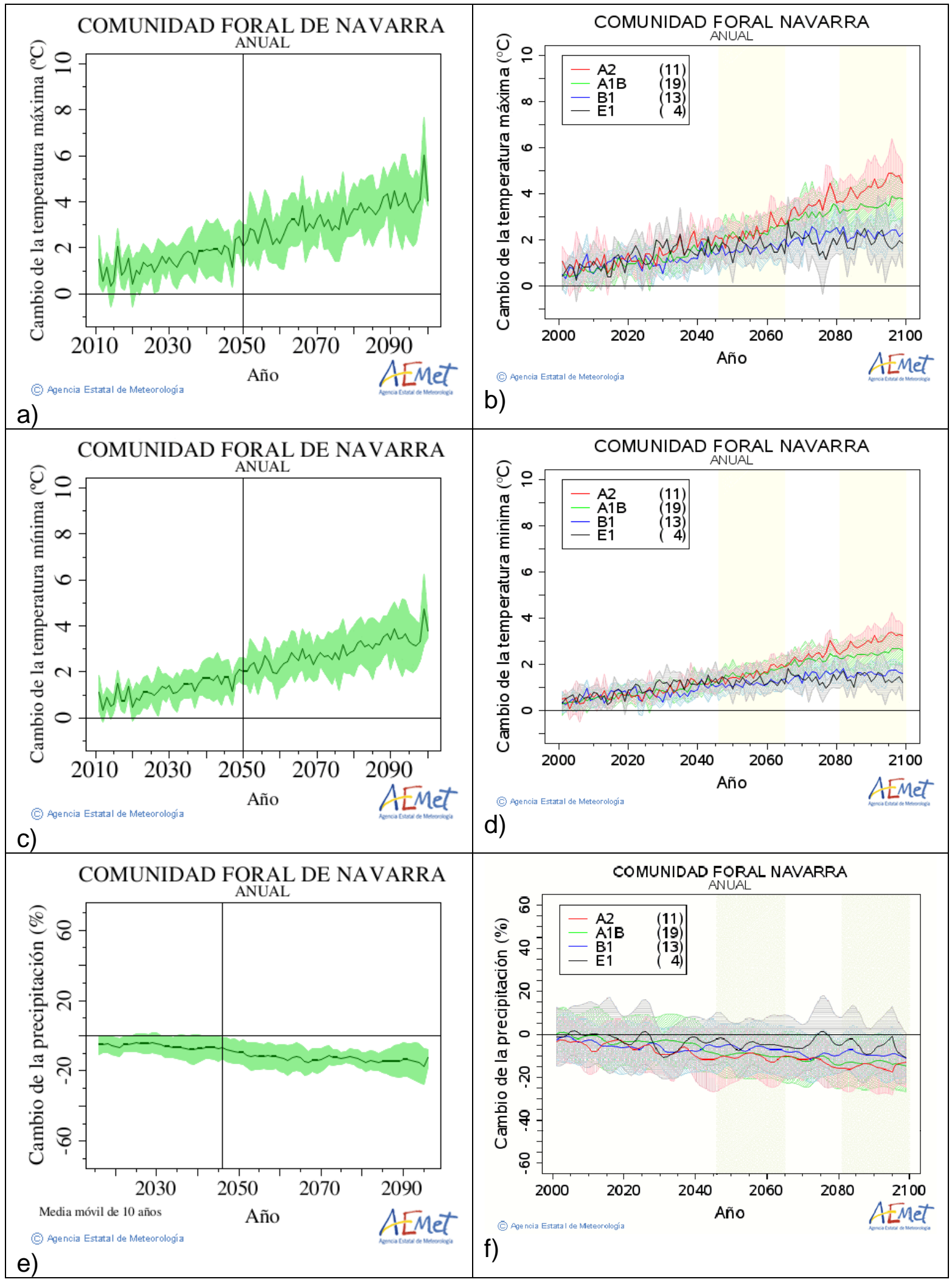

Figura A.99. Igual que la Figura A.1 para la Comunidad Foral de Navarra. 


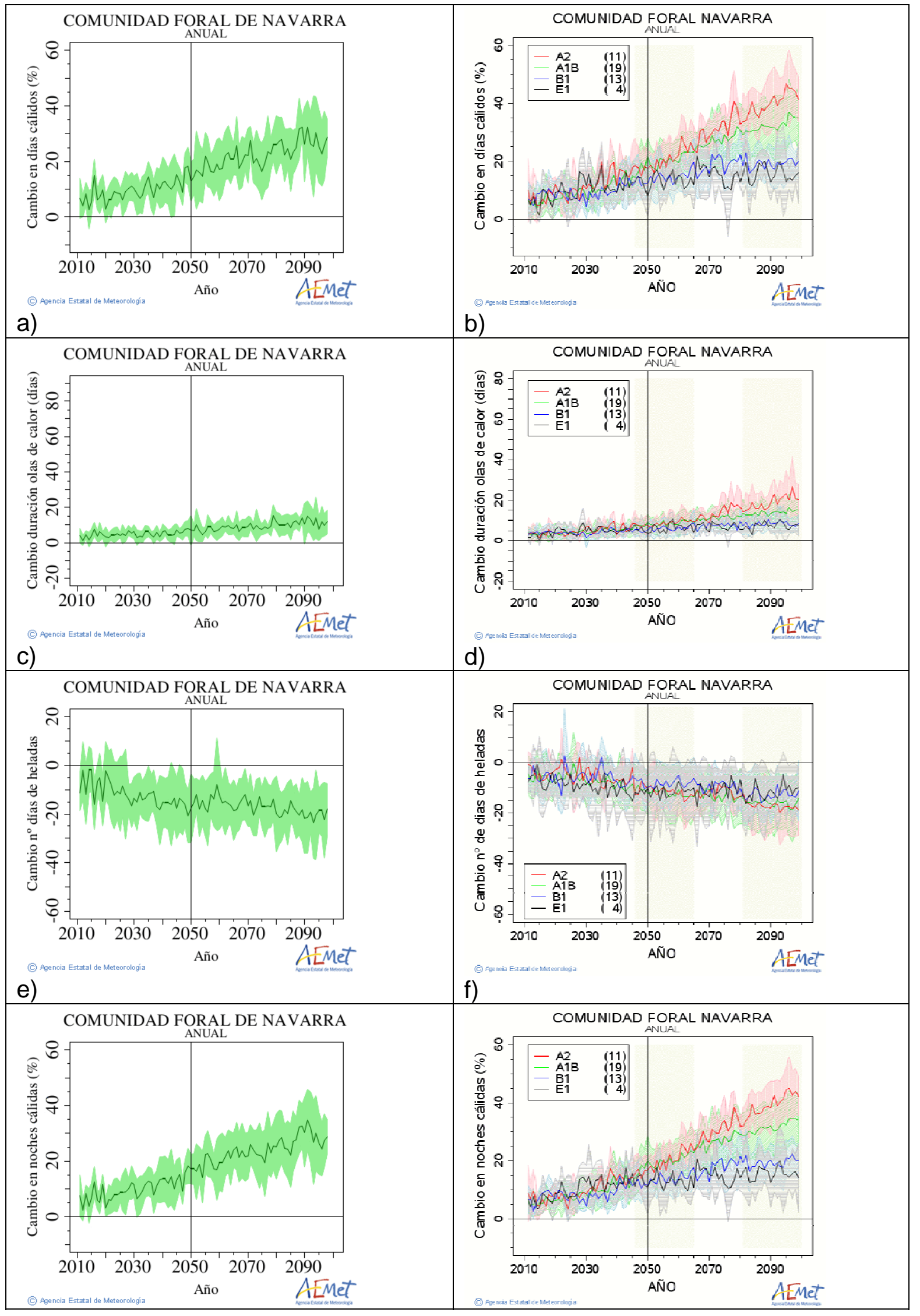

Figura A.100. Igual que la Figura A.2 para la Comunidad Foral de Navarra. 


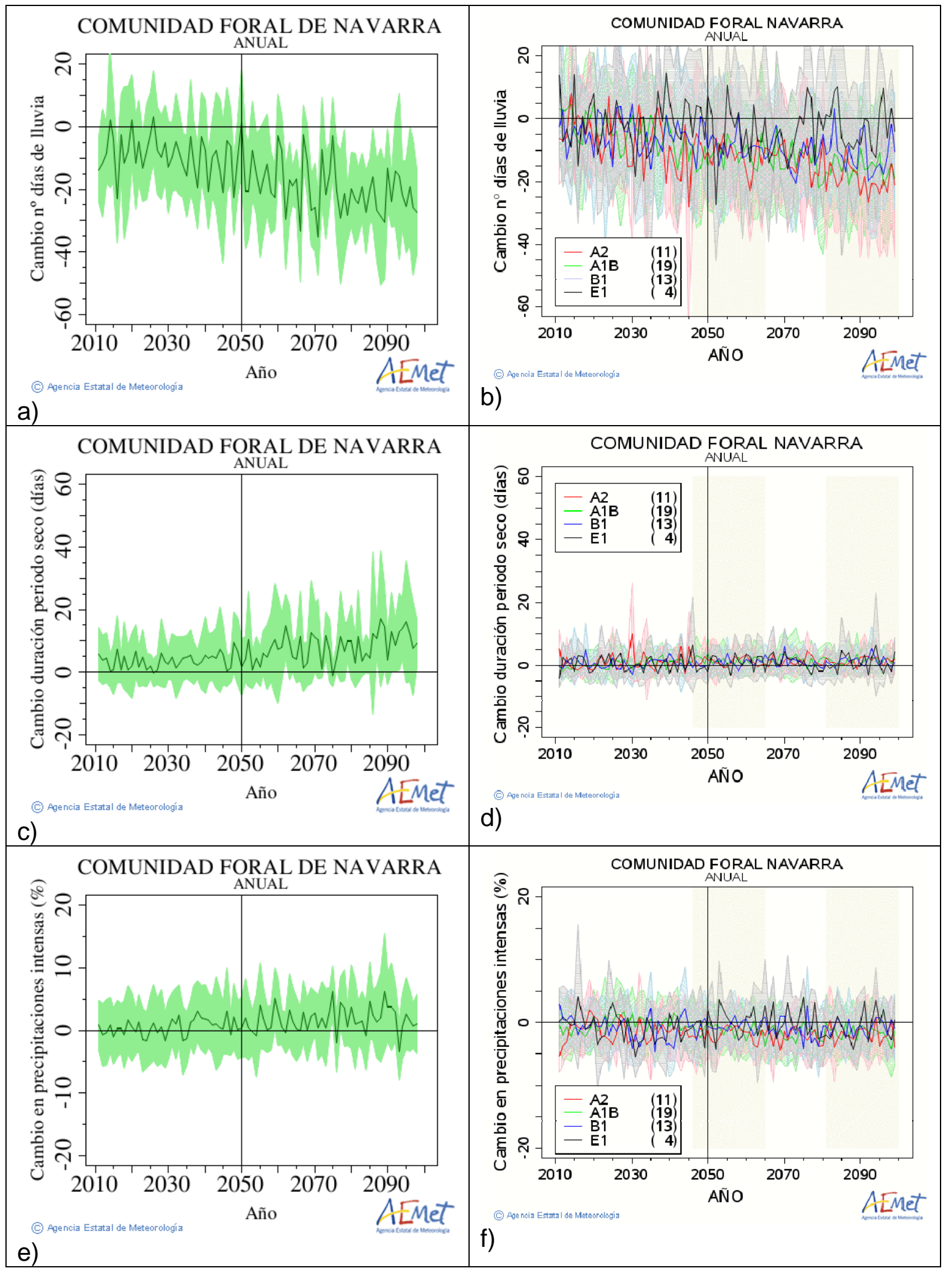

Figura A.101. Igual que la Figura A.3 para la Comunidad Foral de Navarra. 


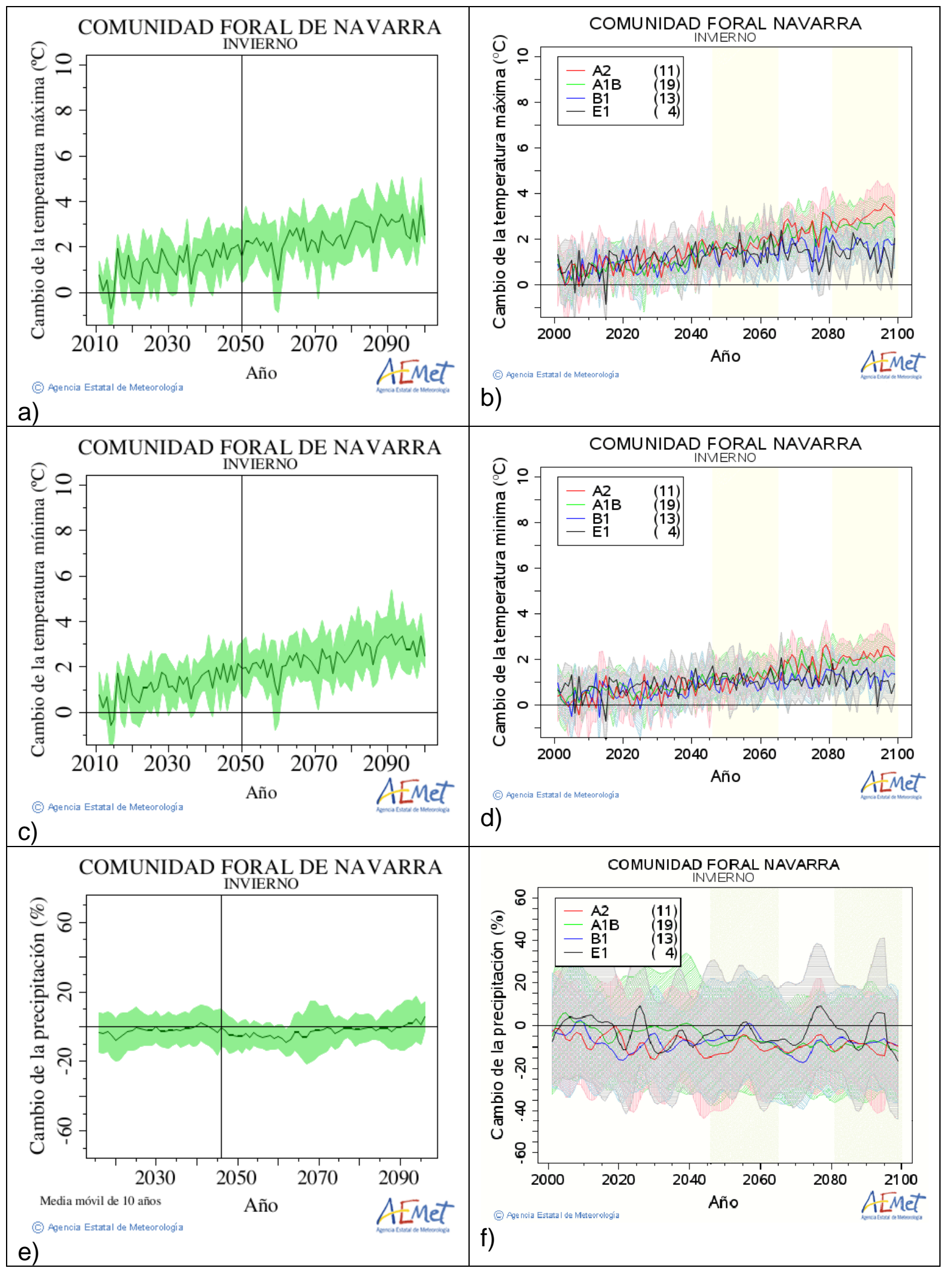

Figura A.102. Igual que la Figura A.4 para la Comunidad Foral de Navarra. 


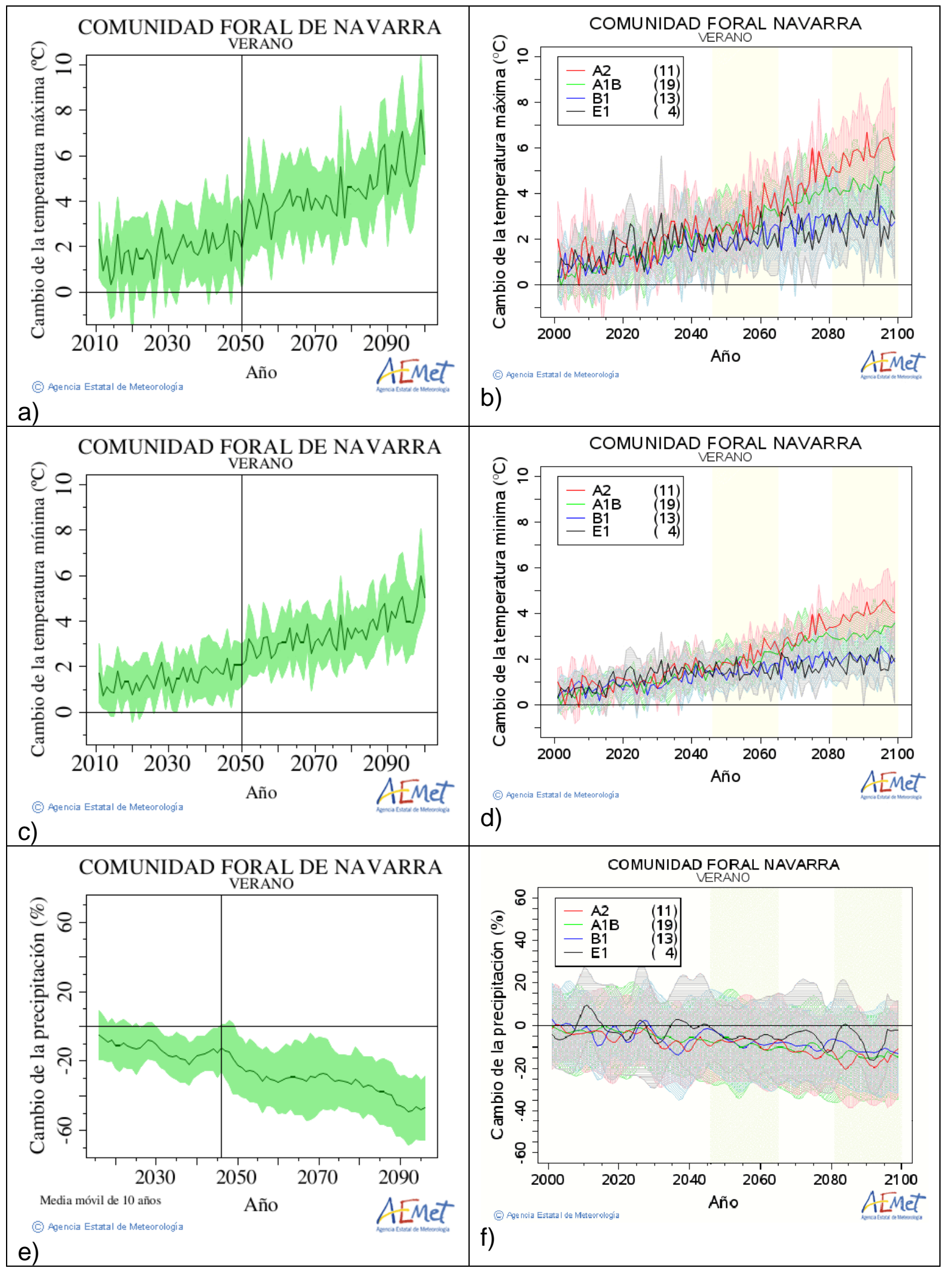

Figura A.103. Igual que la Figura A.5 para la Comunidad Foral de Navarra. 


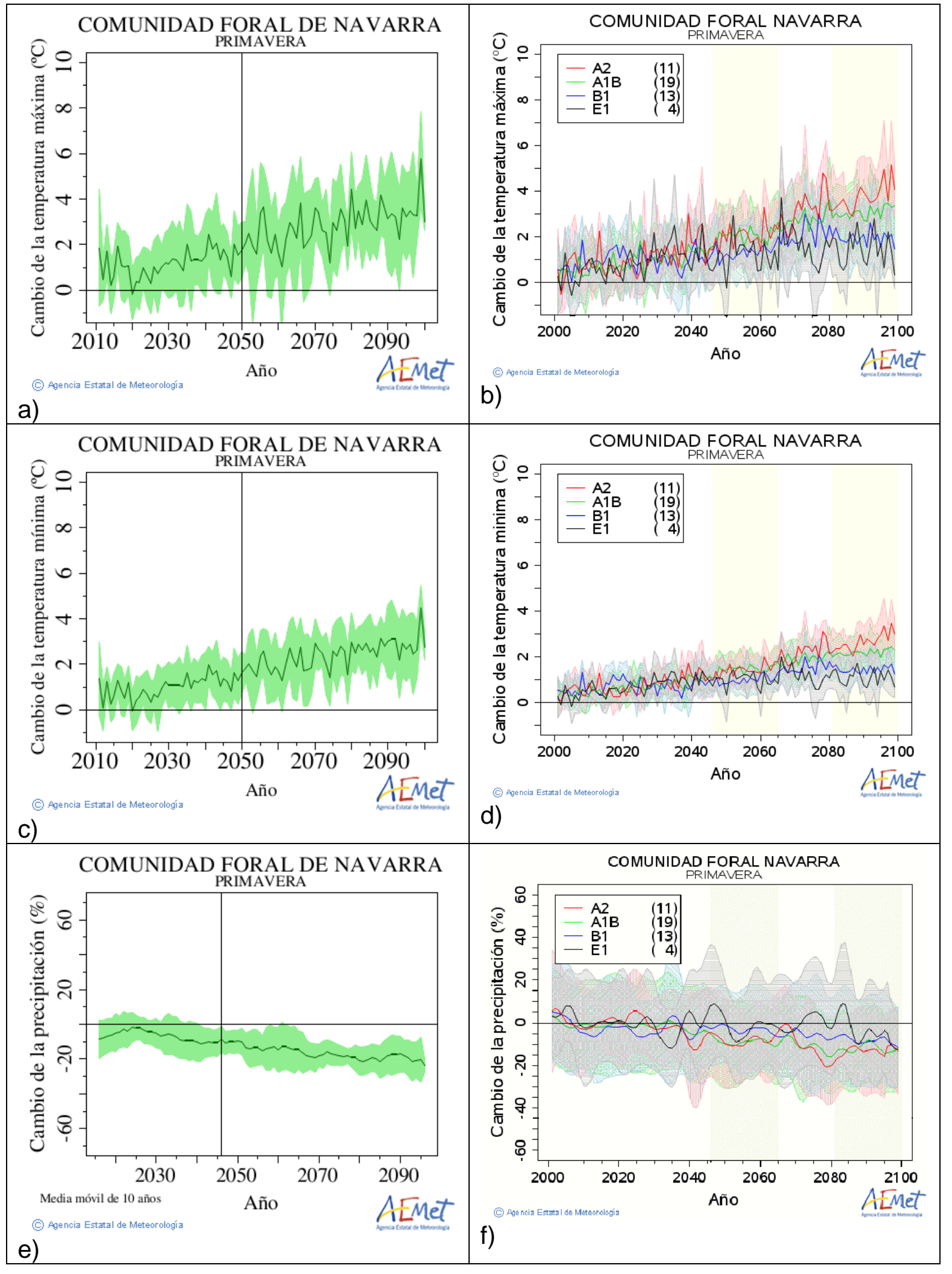

Figura A.104. Igual que la Figura A.6 para la Comunidad Foral de Navarra. 


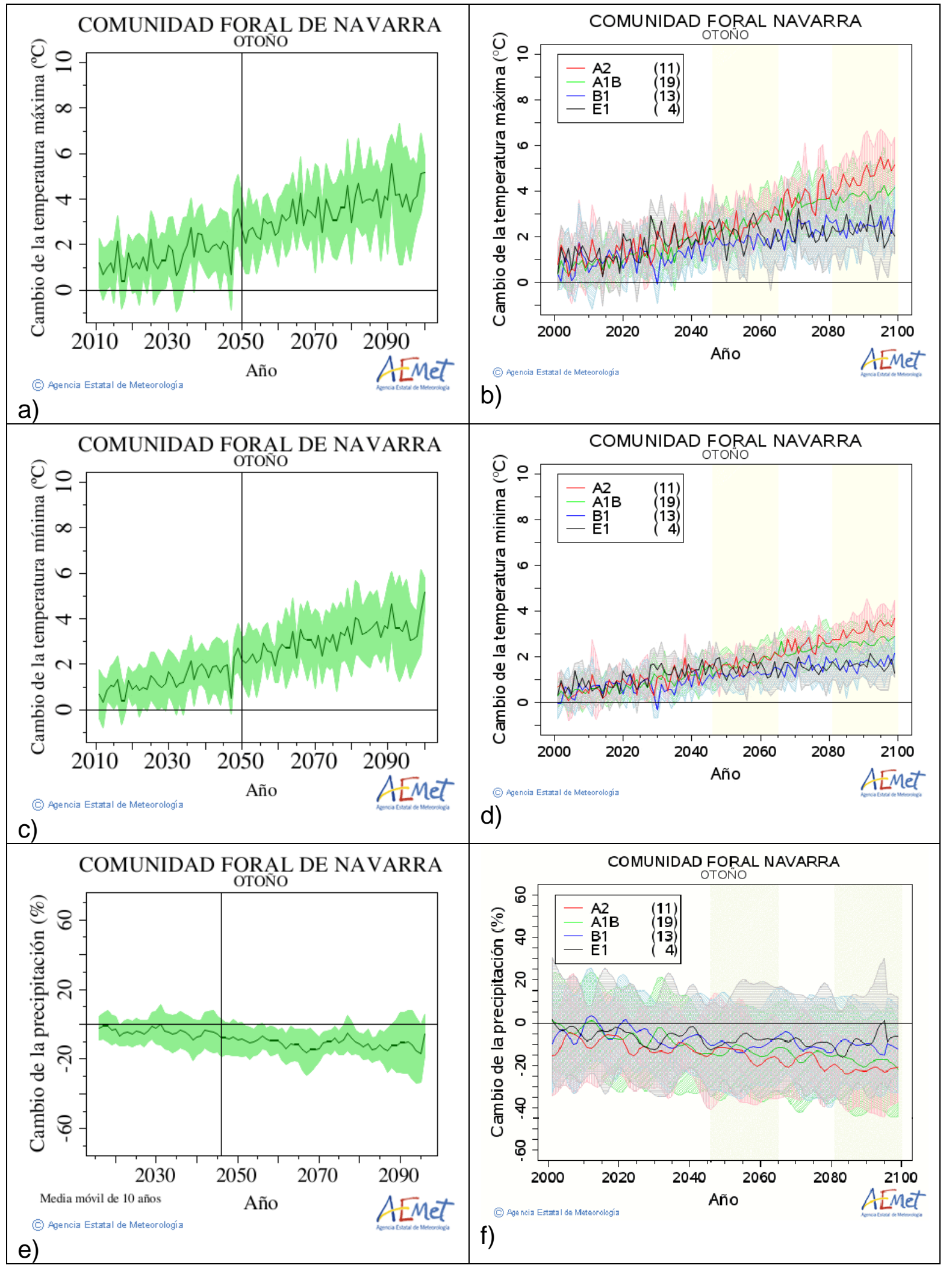

Figura A.105. Igual que la Figura A.7 para la Comunidad Foral de Navarra.. 
PAÍS VASCO

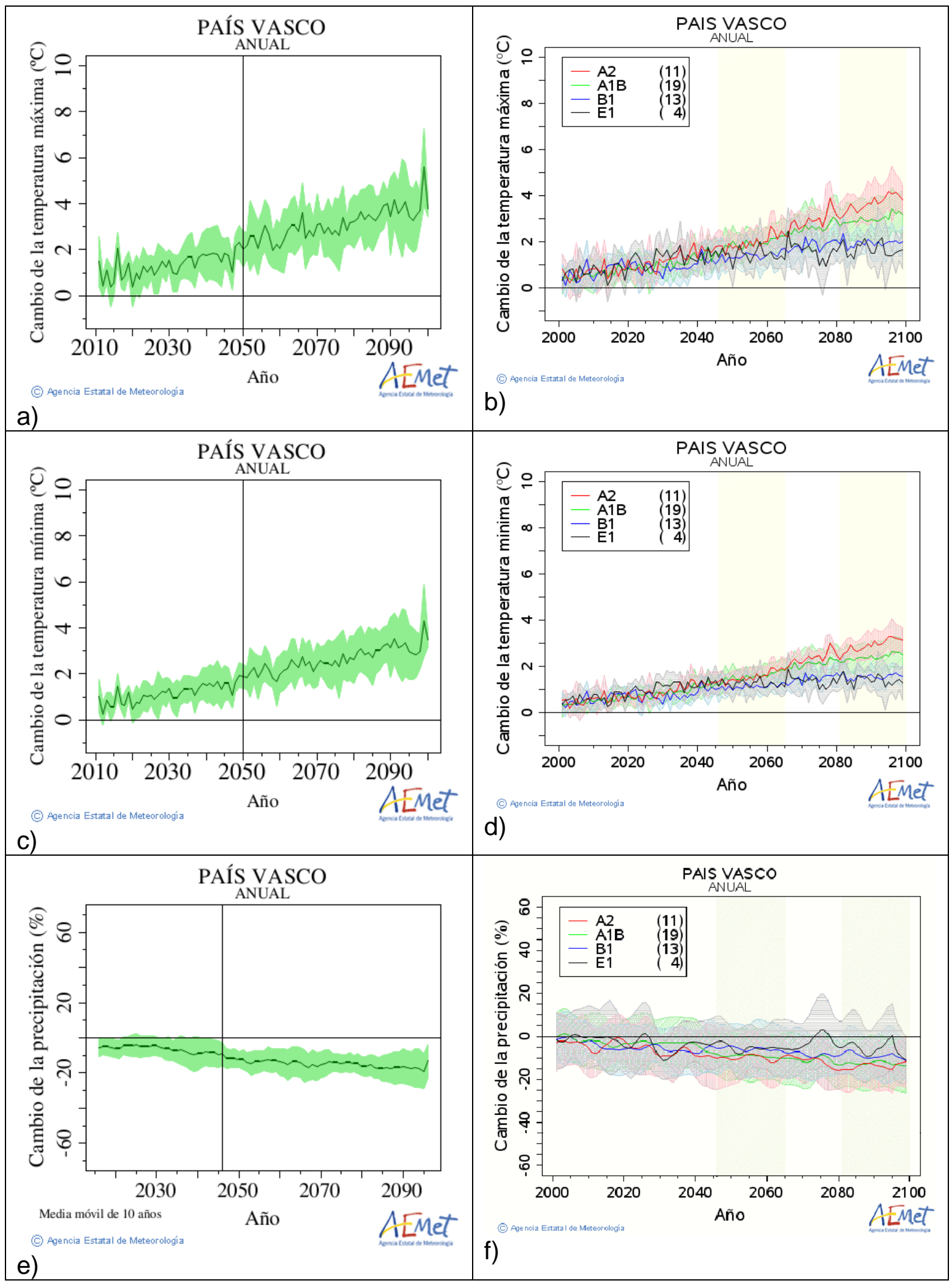

Figura A.106. Igual que la Figura A.1 para el País Vasco. 


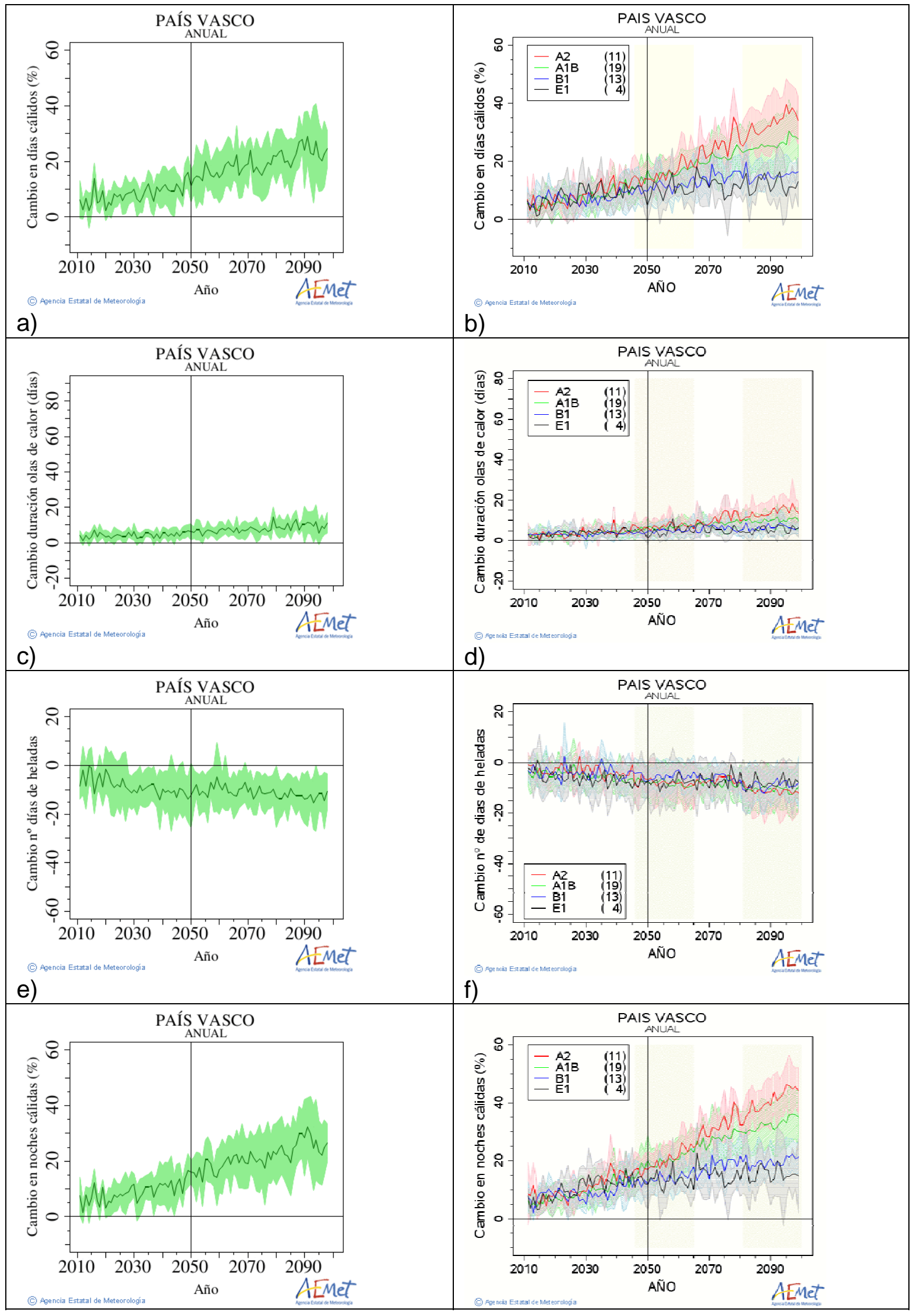

Figura A.107. Igual que la Figura A.2 para el País Vasco. 


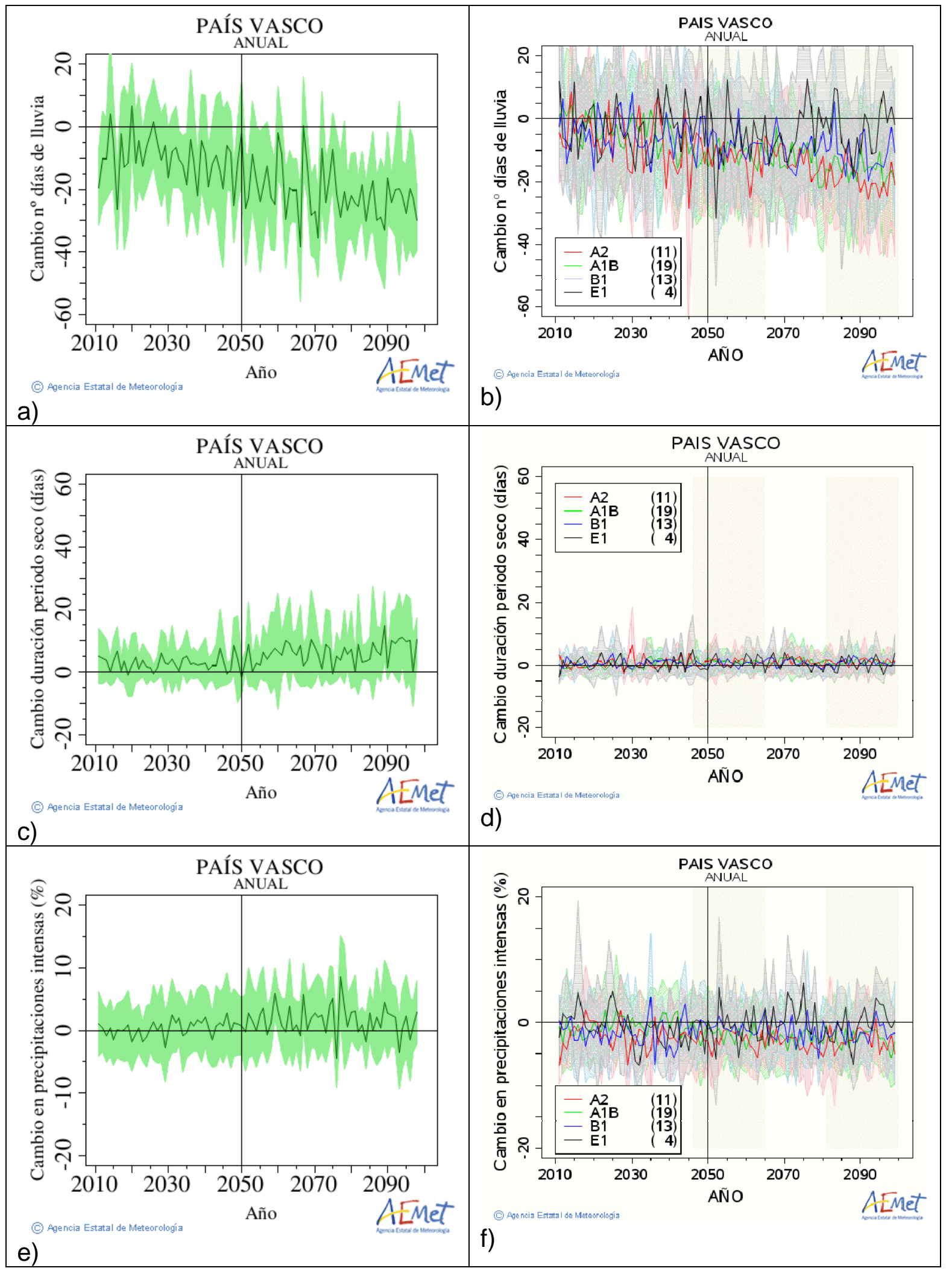

Figura A.108. Igual que la Figura A.3 para el País Vasco. 


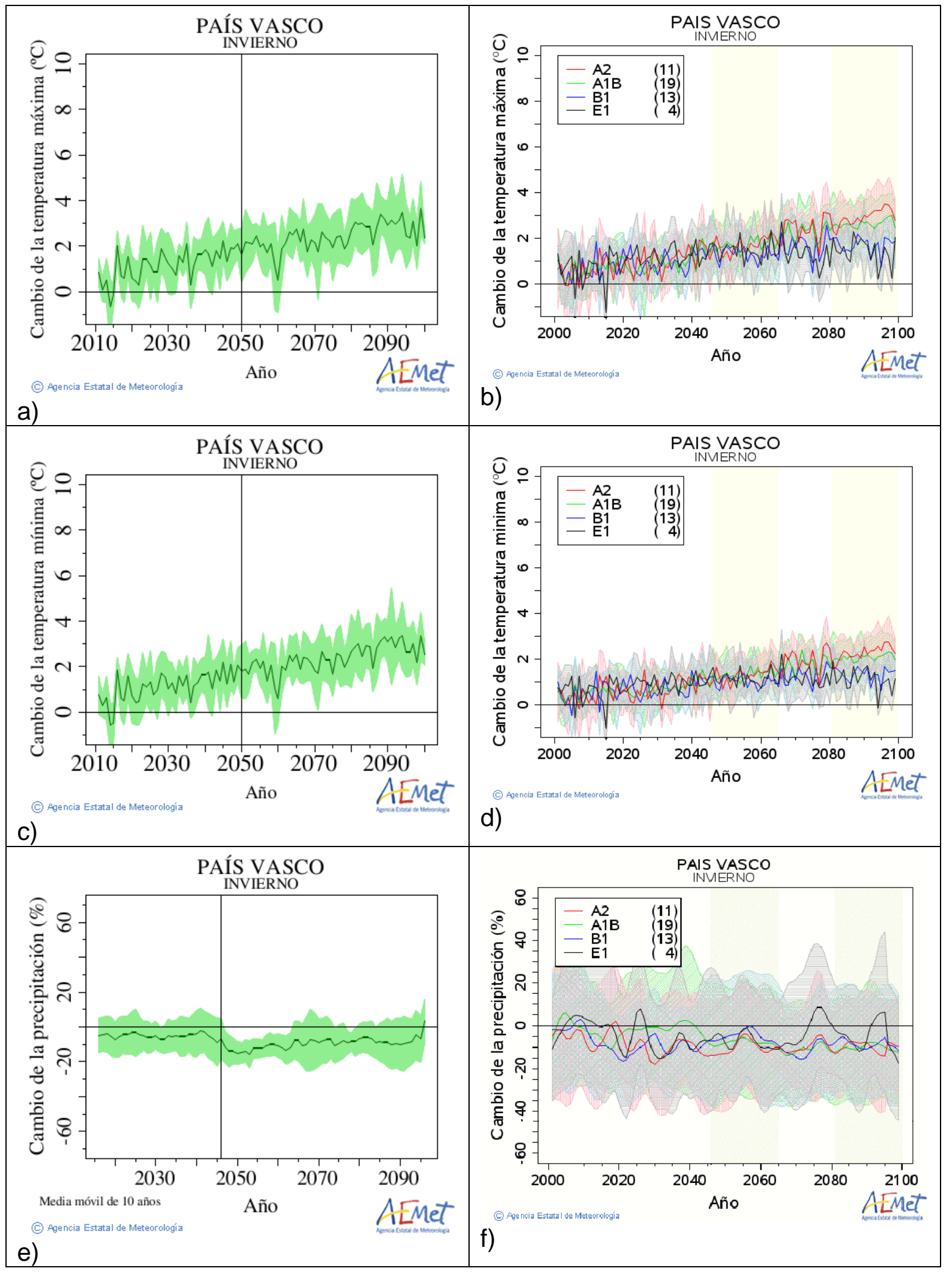

Figura A.109. Igual que la Figura A.4 para el País Vasco. 


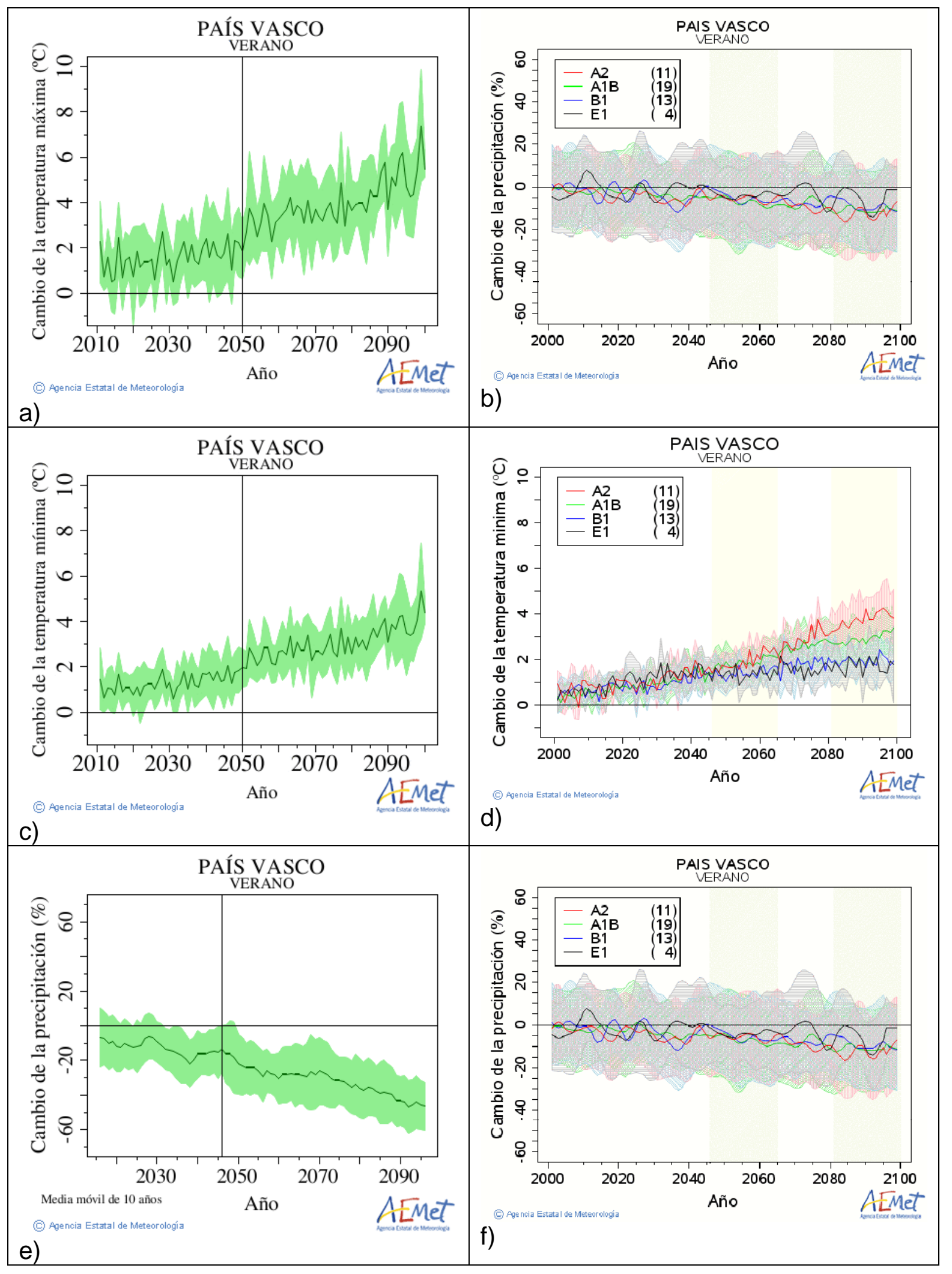

Figura A.110. Igual que la Figura A.5 para el País Vasco. 


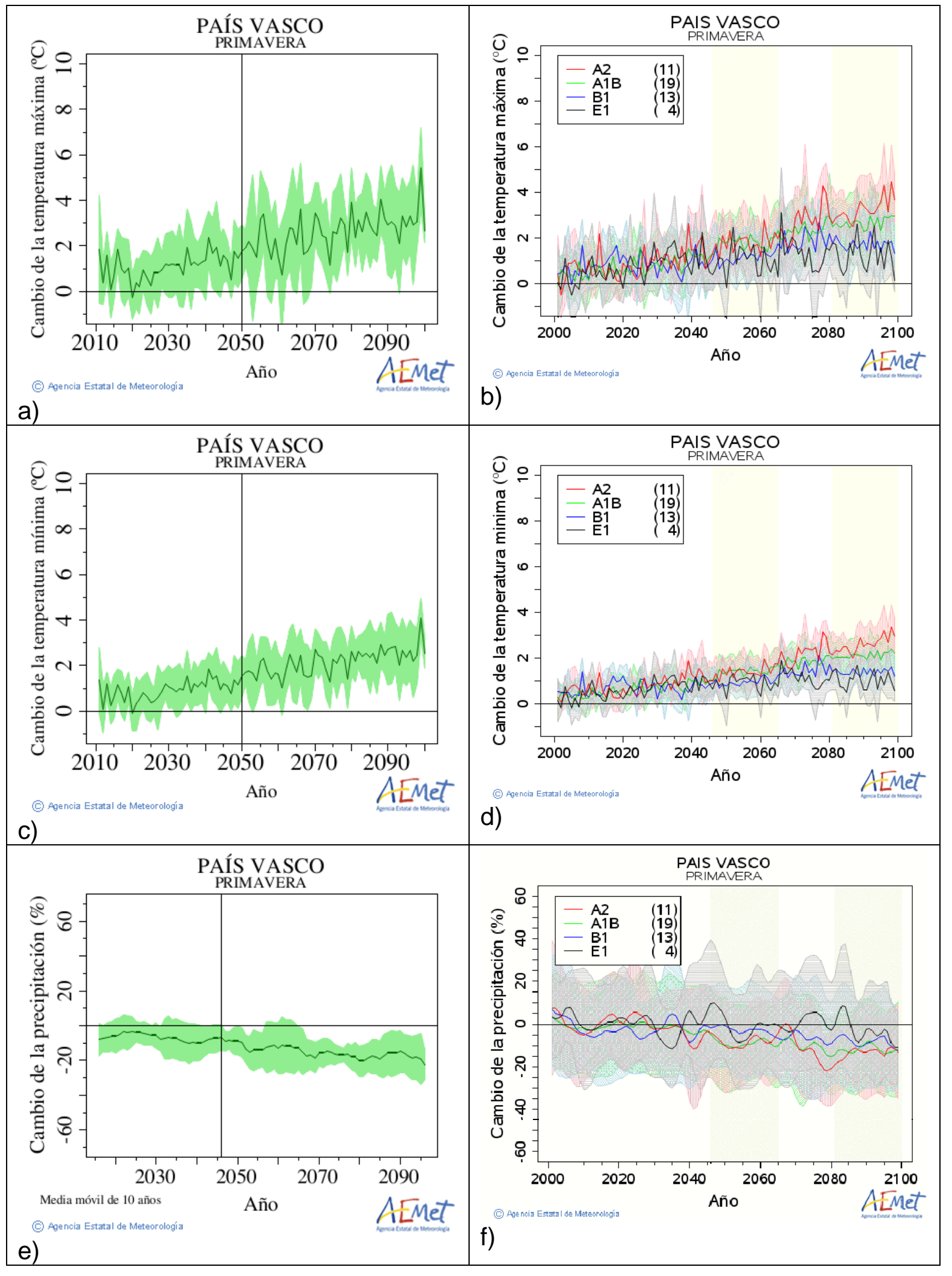

Figura A.111. Igual que la Figura A.6 para el País Vasco. 


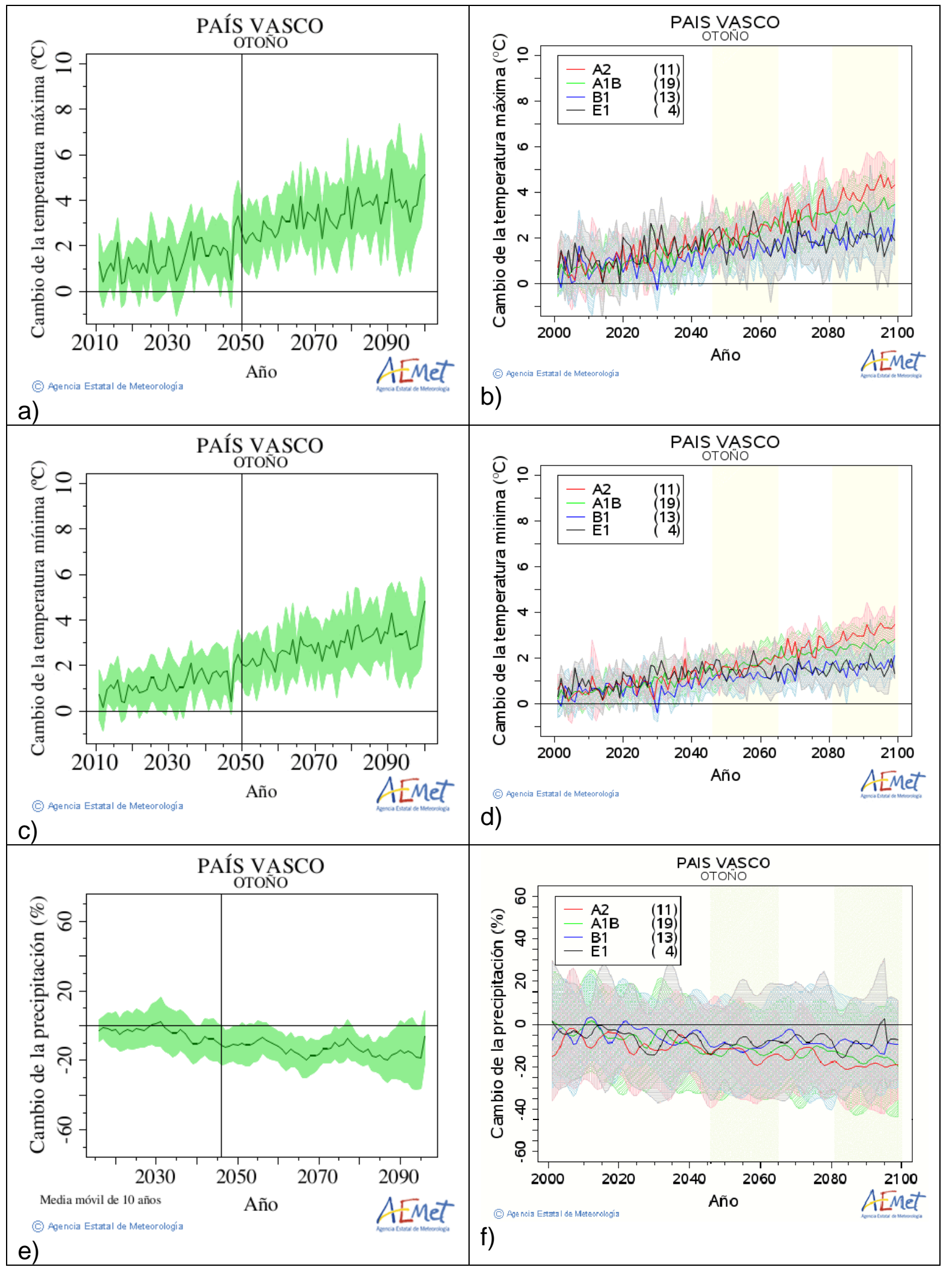

Figura A.112. Igual que la Figura A.7 para el País Vasco. 
COMUNIDAD VALENCIANA

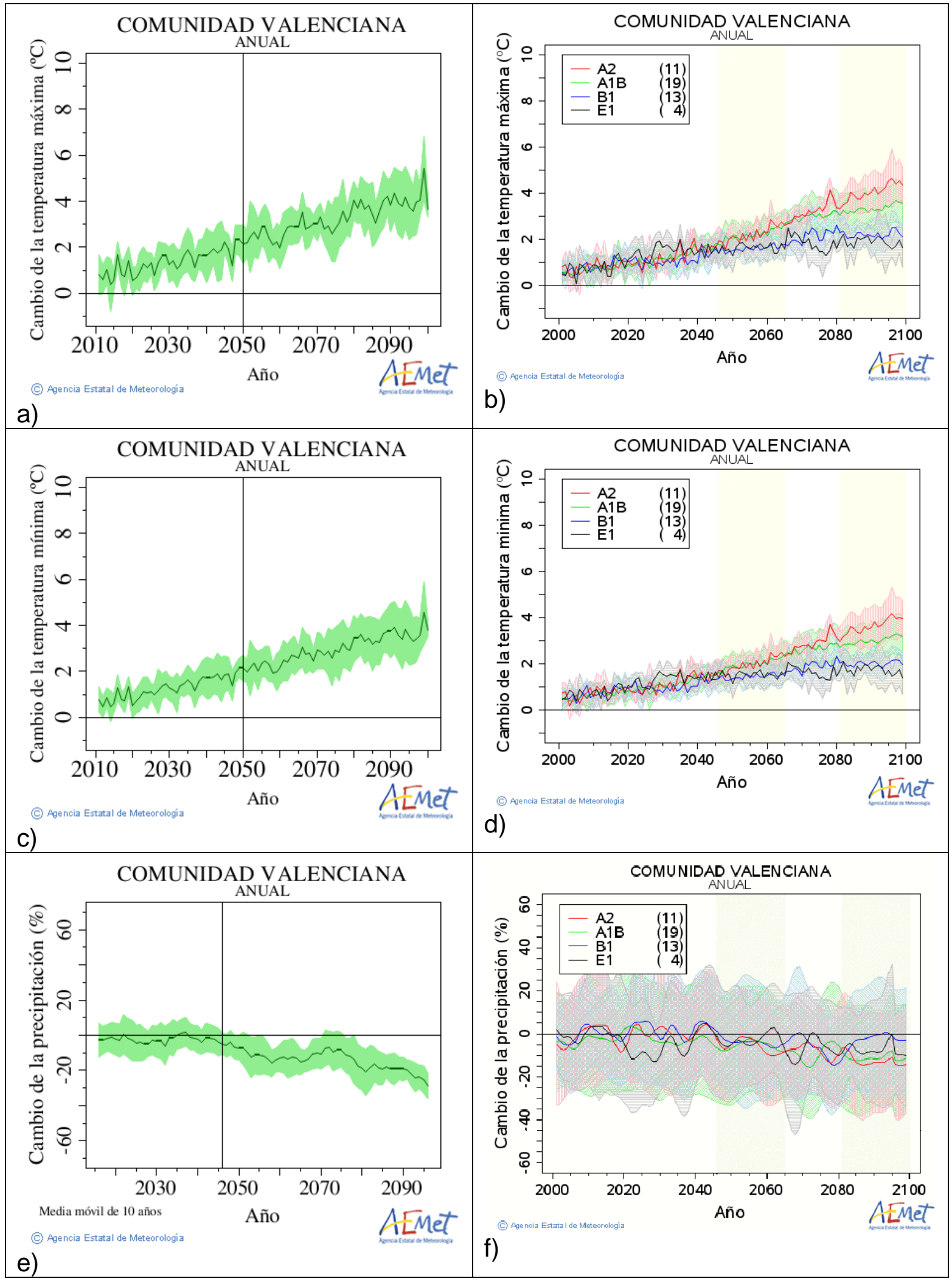

Figura A.113. Igual que la Figura A.1 para la Comunidad Valenciana. 


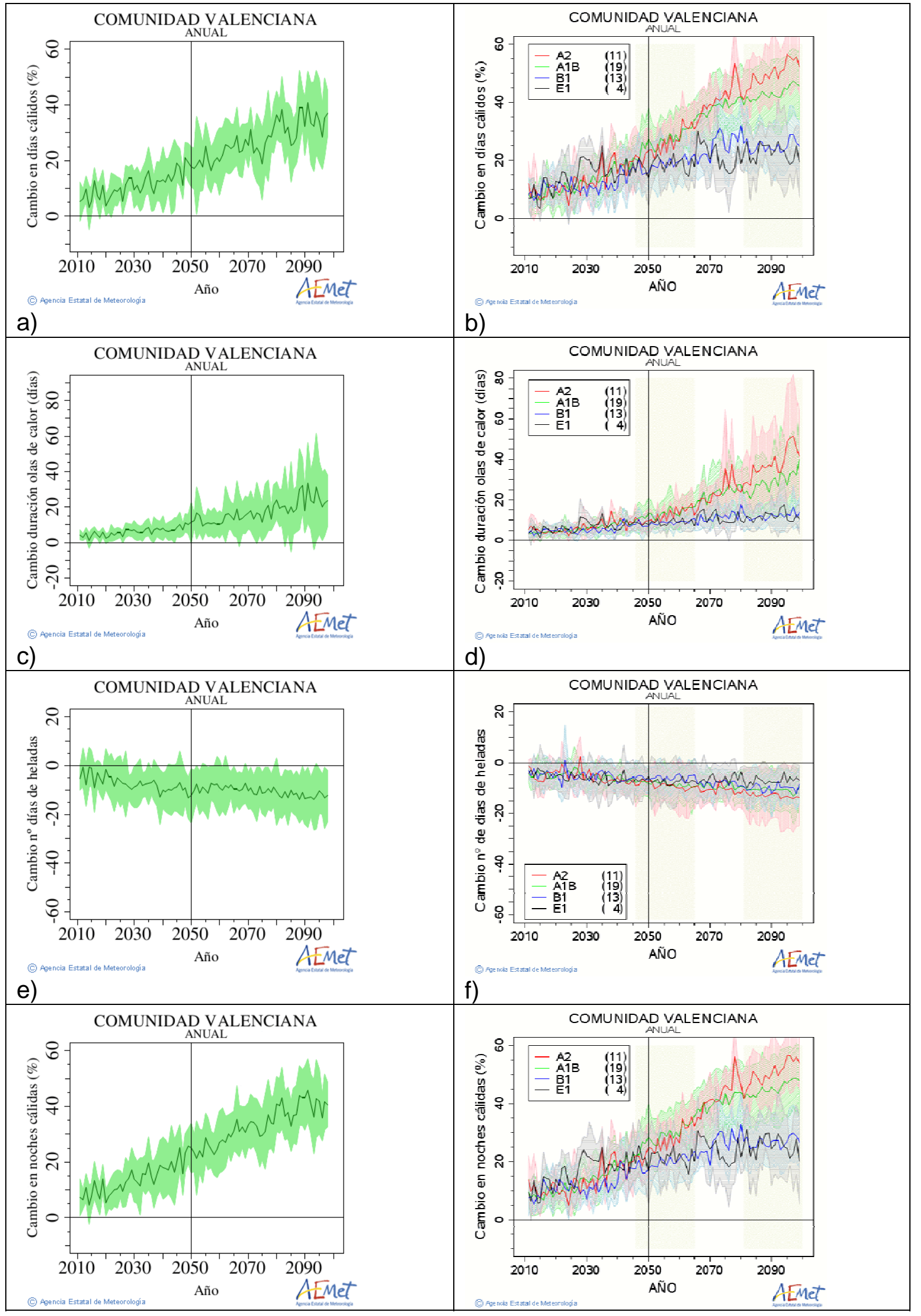

Figura A.114. Igual que la Figura A.2 para la Comunidad Valenciana. 


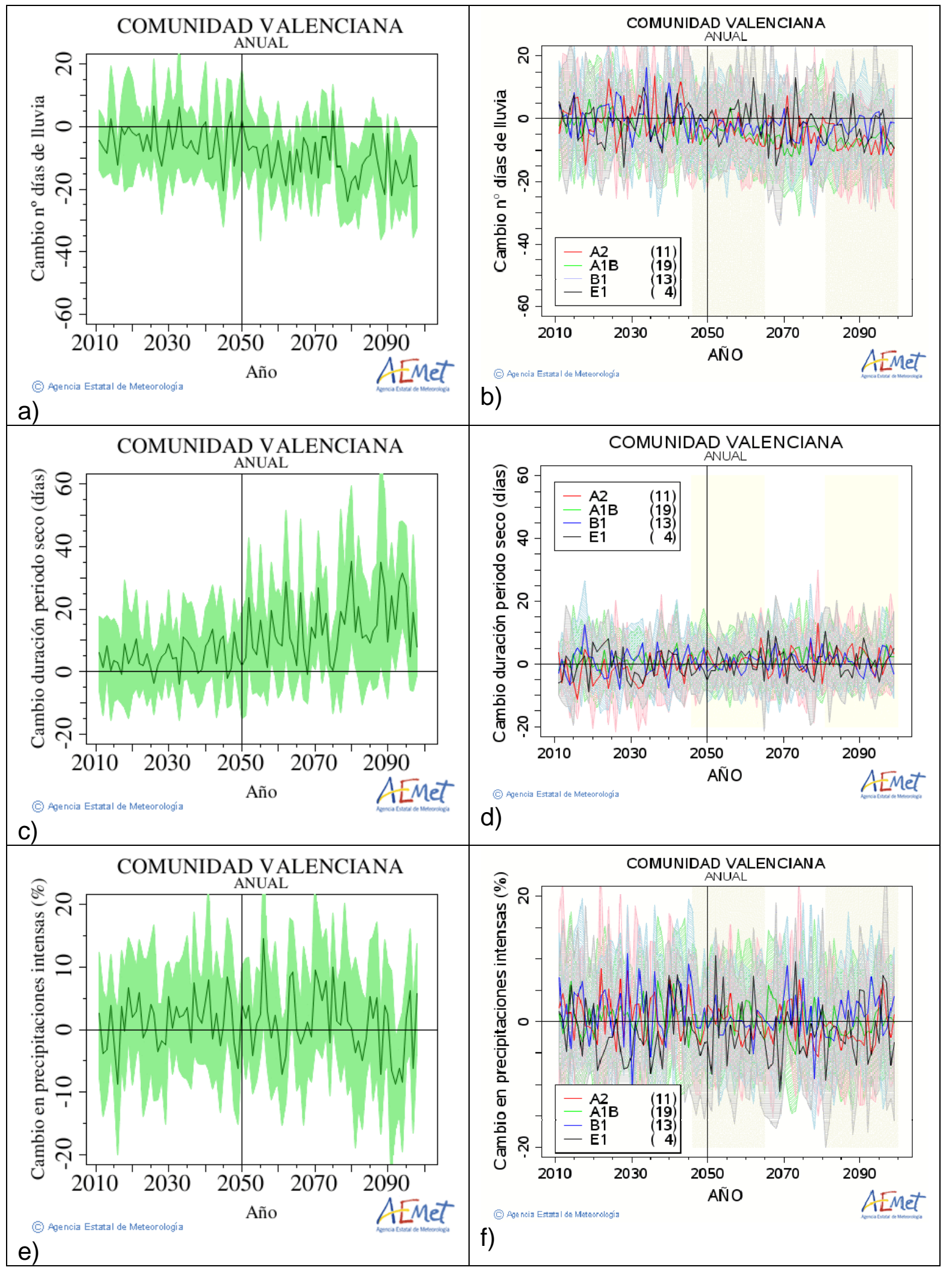

Figura A.115. Igual que la Figura A.3 para la Comunidad Valenciana. 


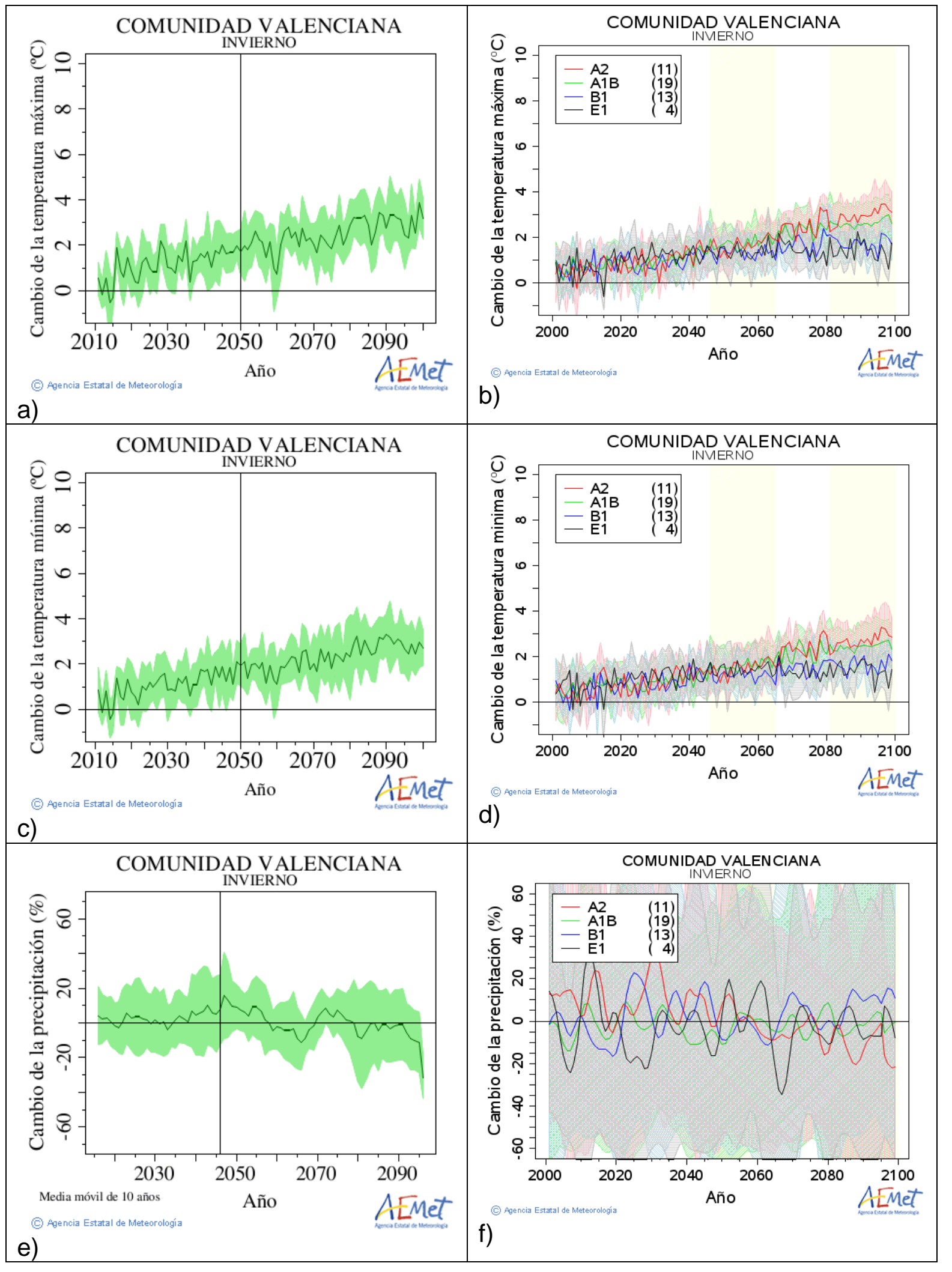

Figura A.116. Igual que la Figura A.4 para la Comunidad Valenciana. 


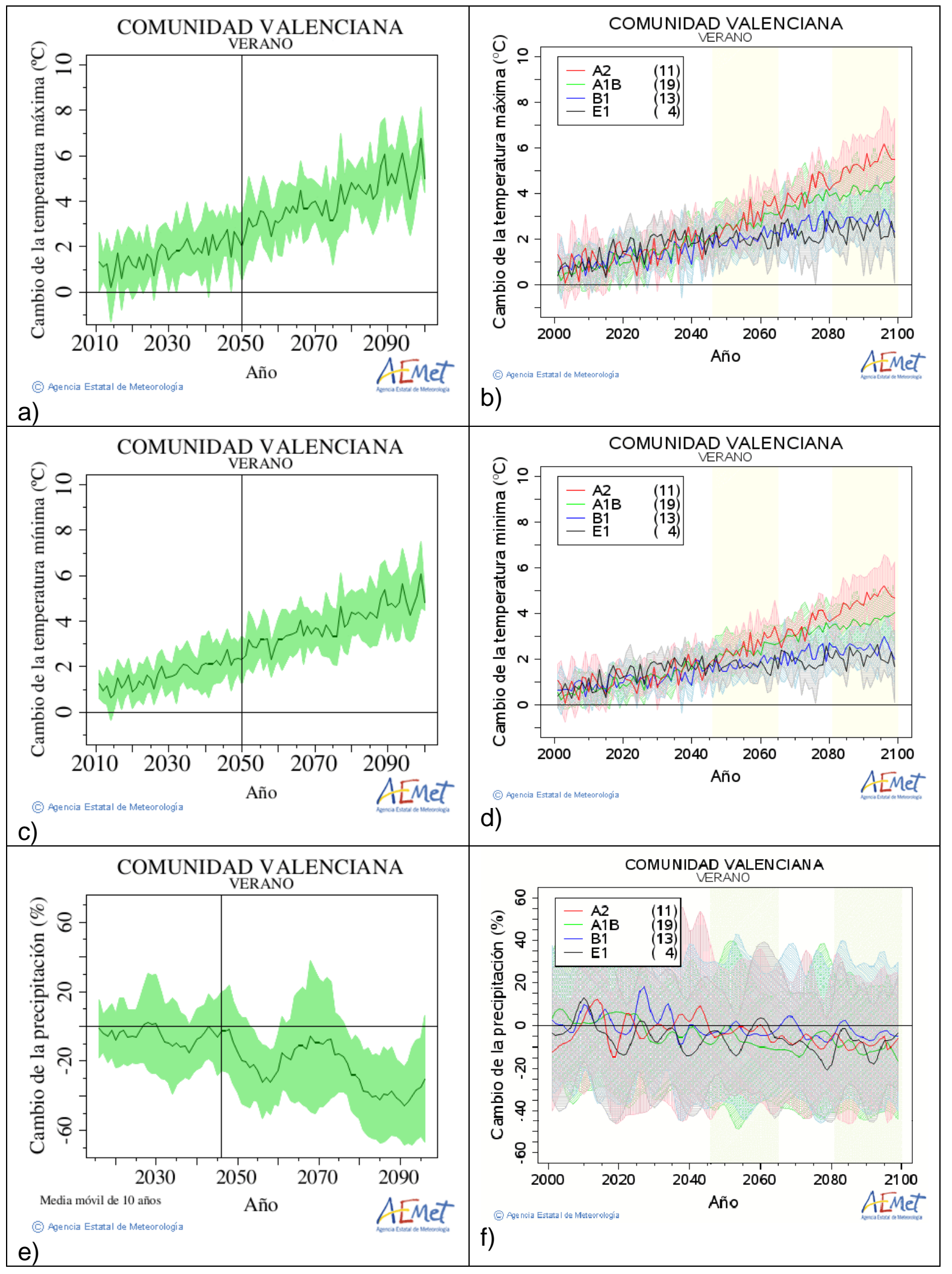

Figura A.117. Igual que la Figura A.5 para la Comunidad Valenciana. 


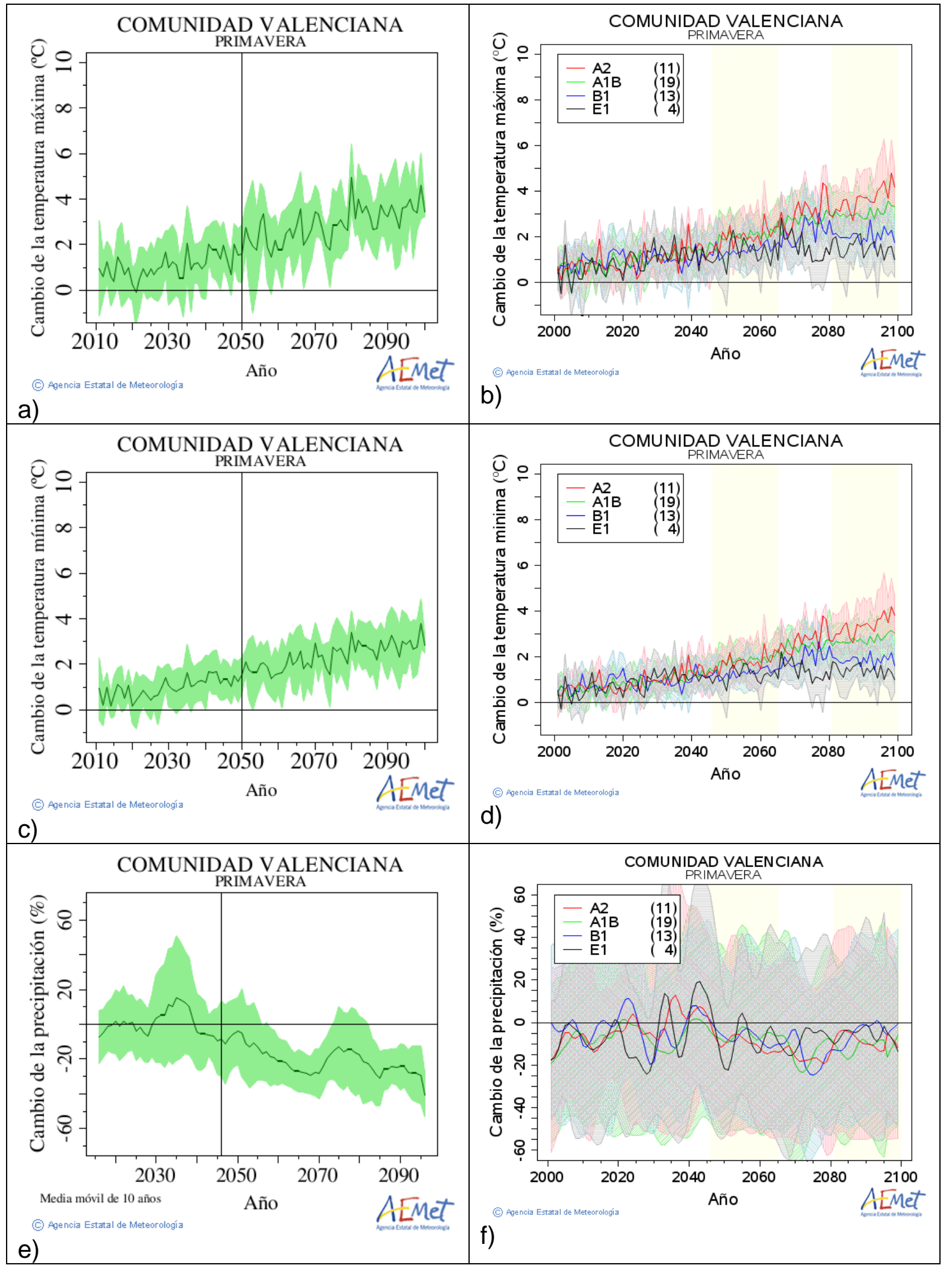

Figura A.118. Igual que la Figura A.6 para la Comunidad Valenciana. 


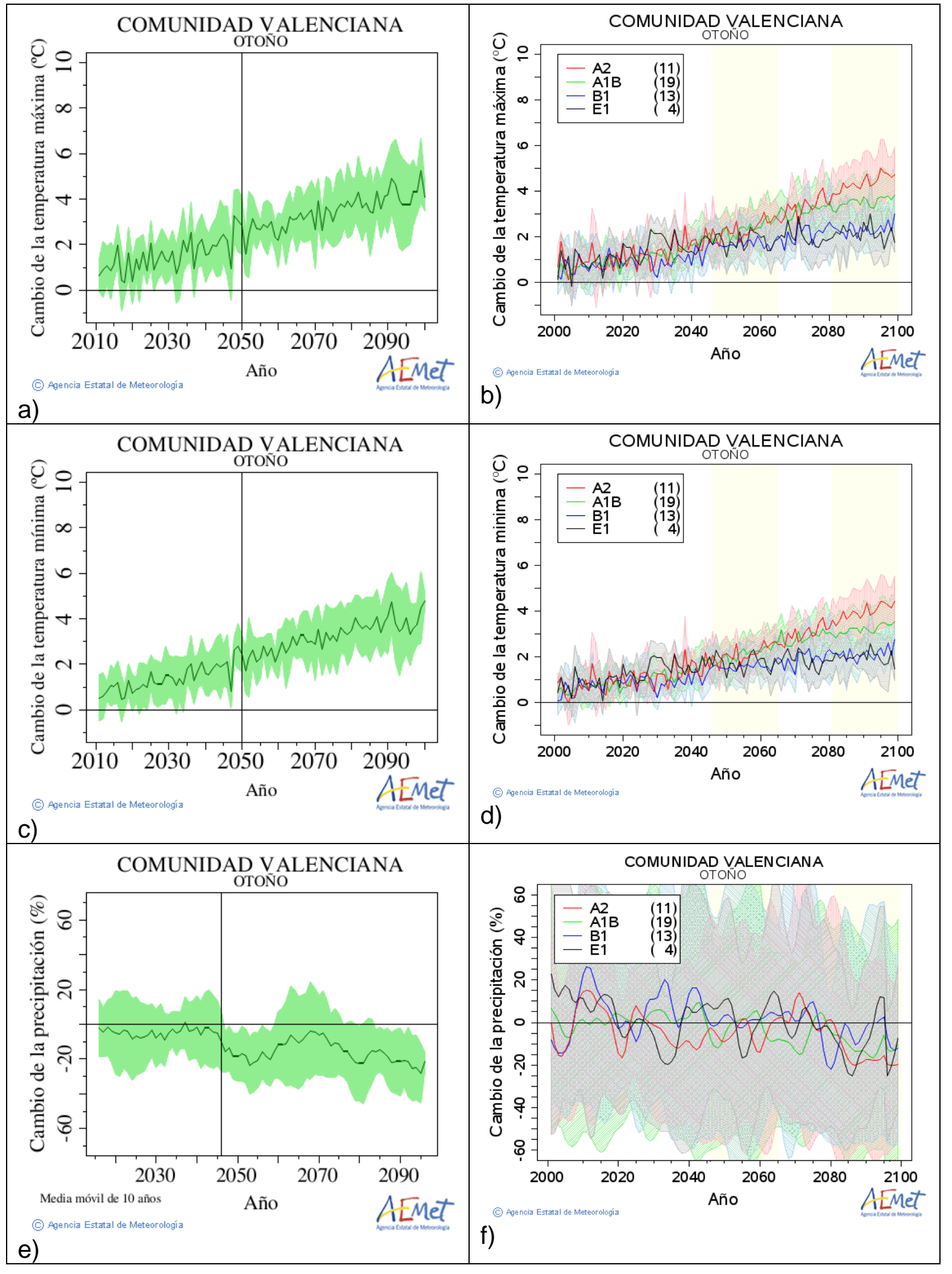

Figura A.119. Igual que la Figura A.7 para la Comunidad Valenciana. 


\section{ESPAÑA PENINSULAR}
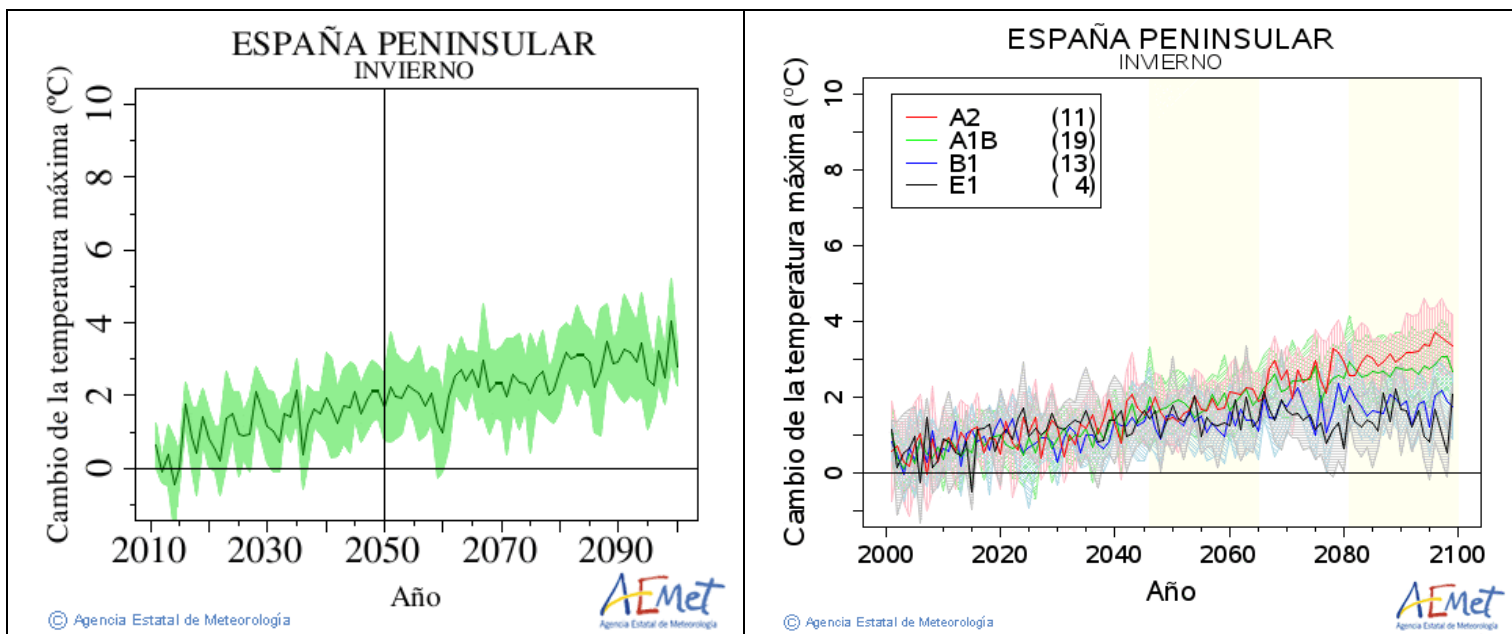

a)

b)
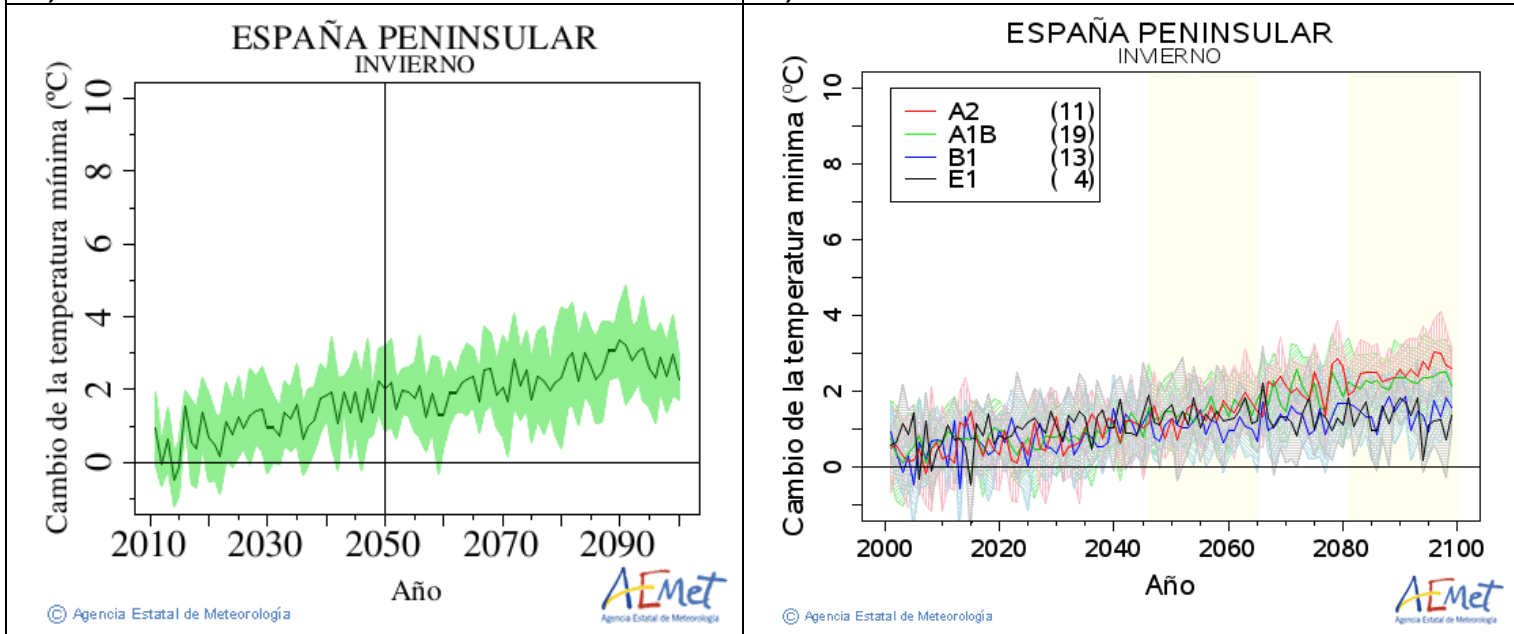

c)

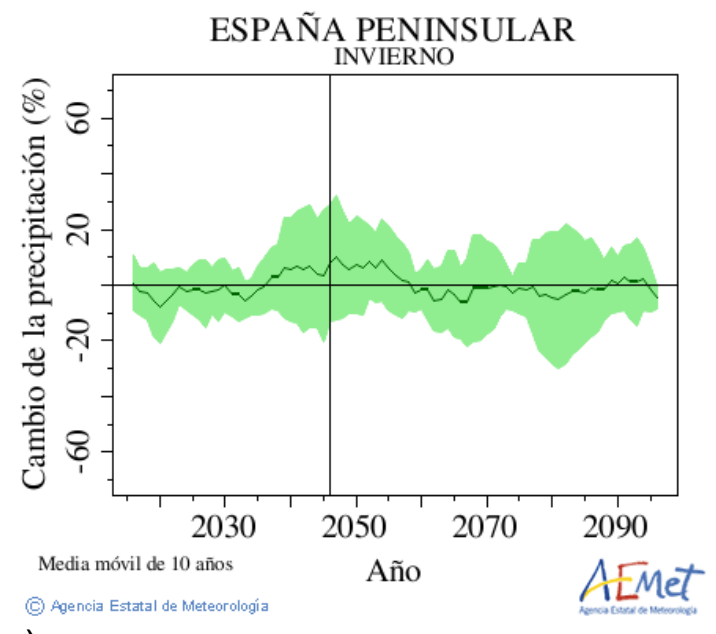

d)

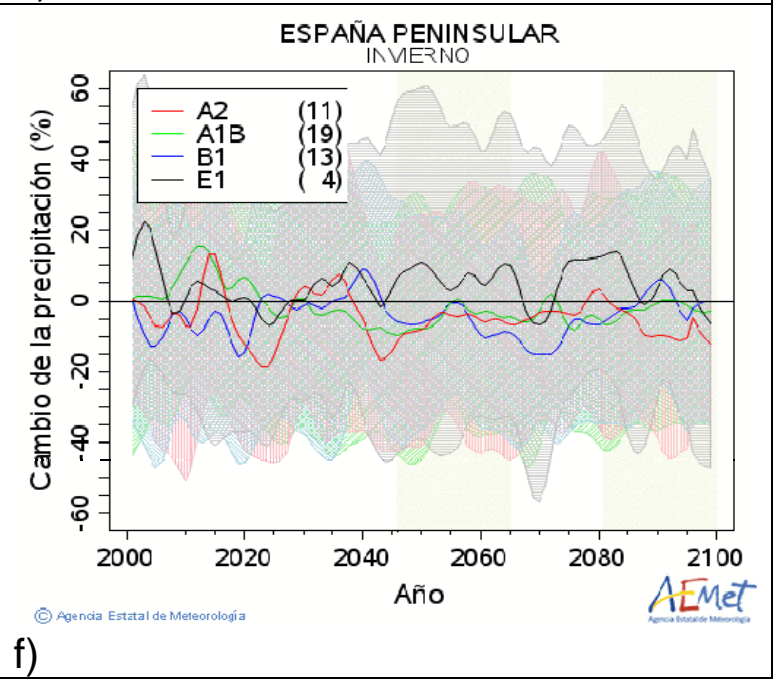

Figura A.120. Igual que la Figura A.4 para España peninsular. 


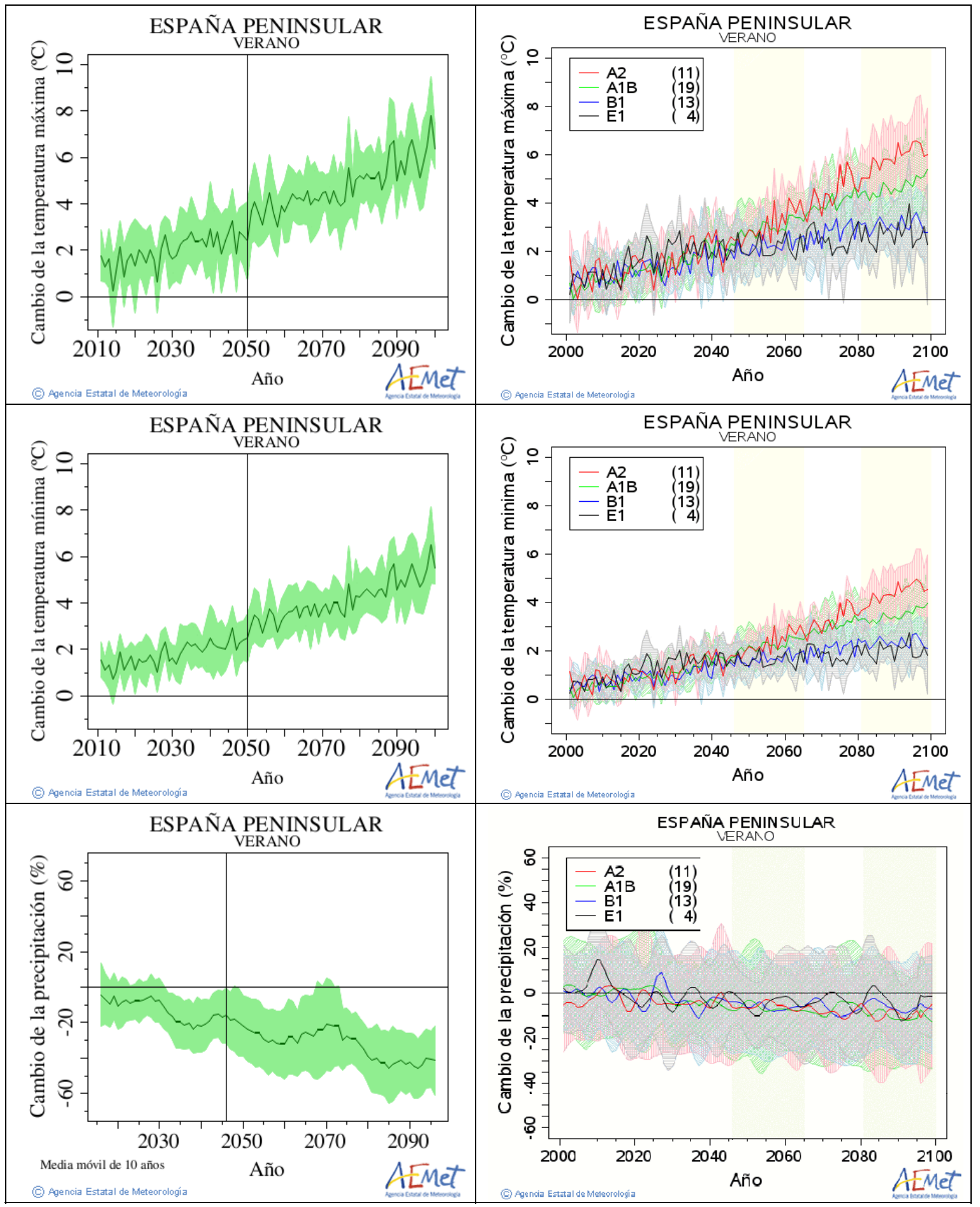

Figura A.121. Igual que la Figura A.5 para España peninsular. 


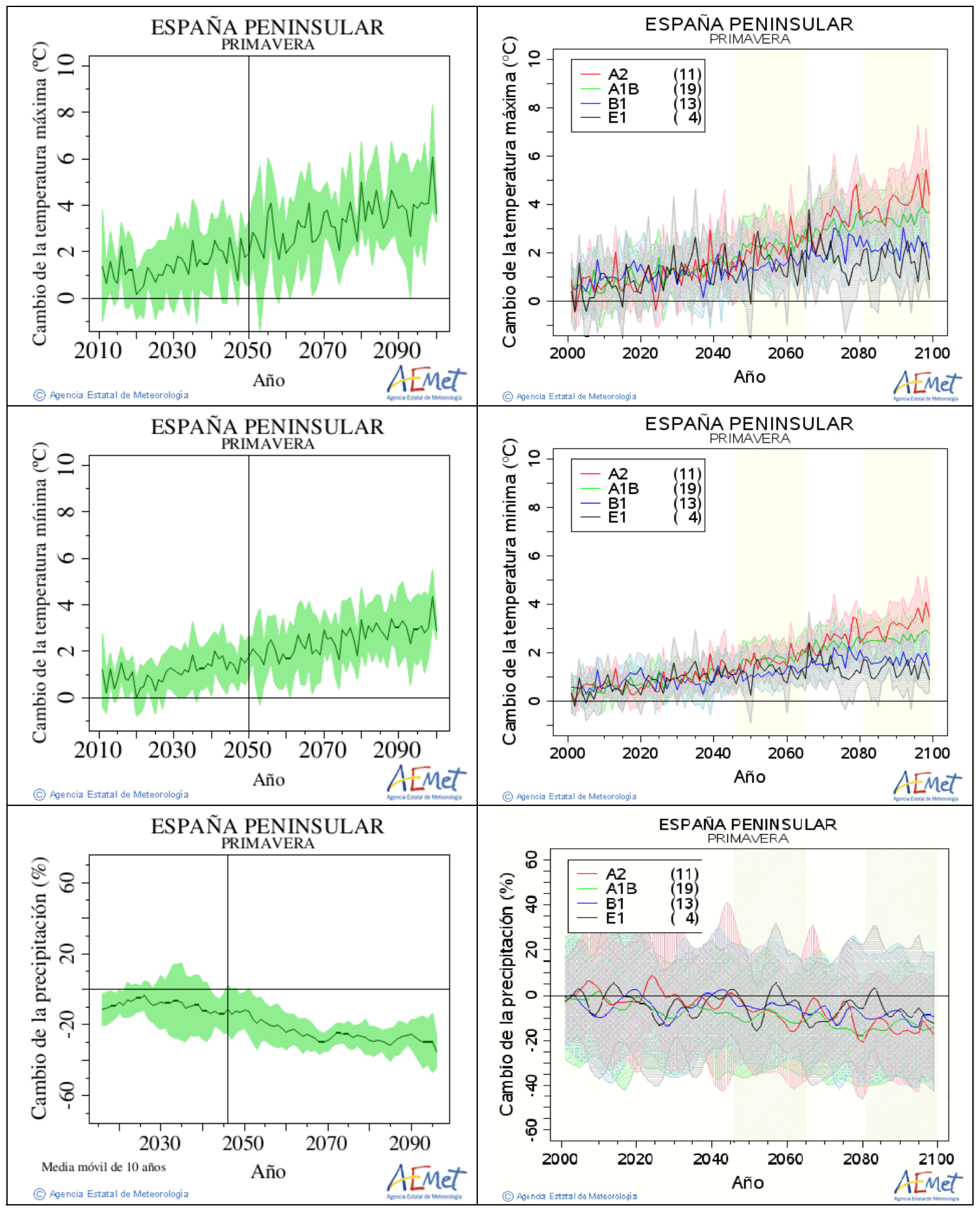

Figura A.122. Igual que la Figura A.6 para España peninsular. 


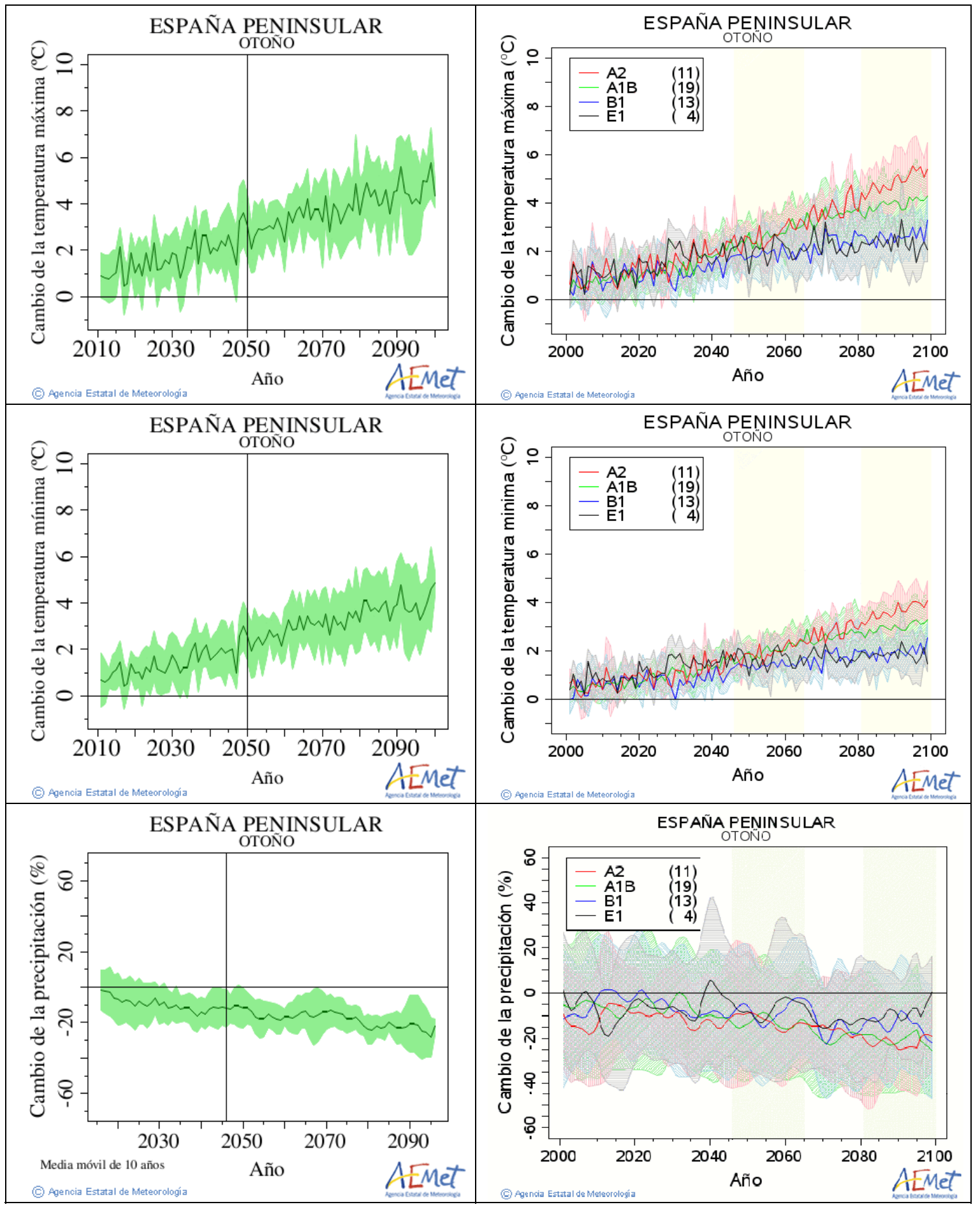

Figura A.123. Igual que la Figura A.7 para España peninsular. 

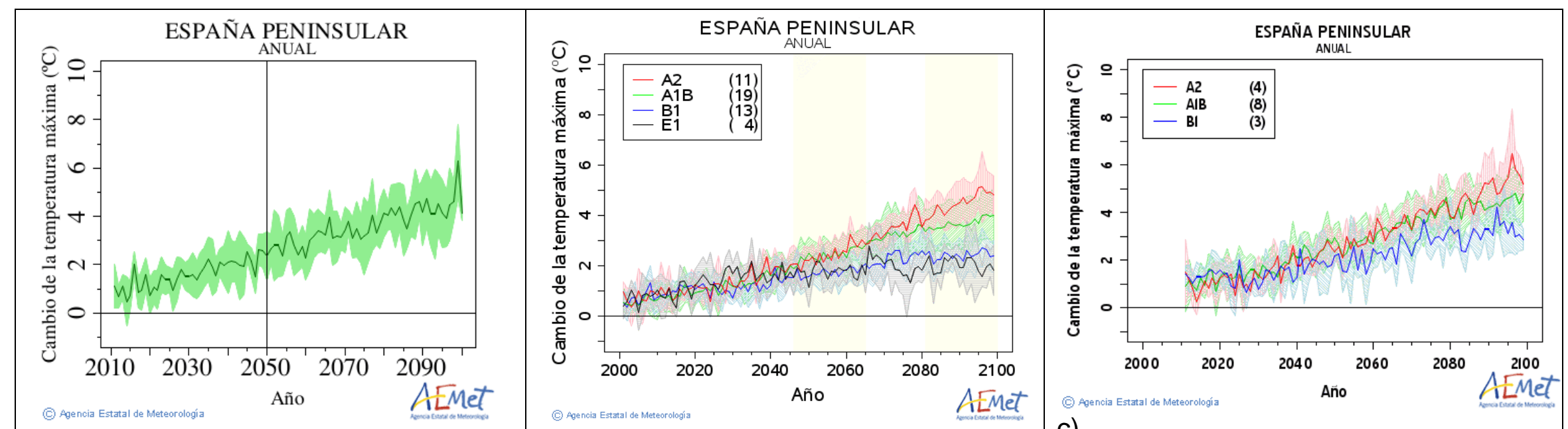

a) b)
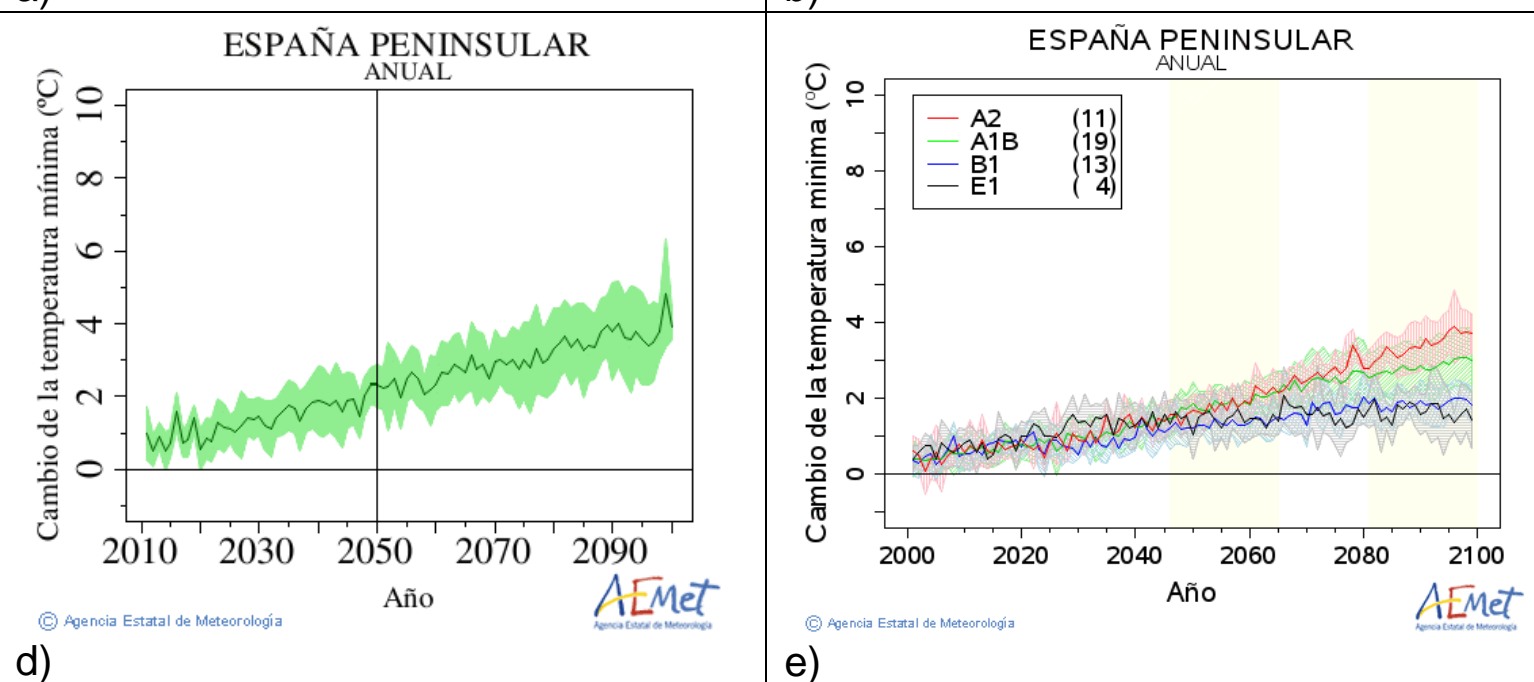

c)

d)

\begin{abstract}
e)
\end{abstract}

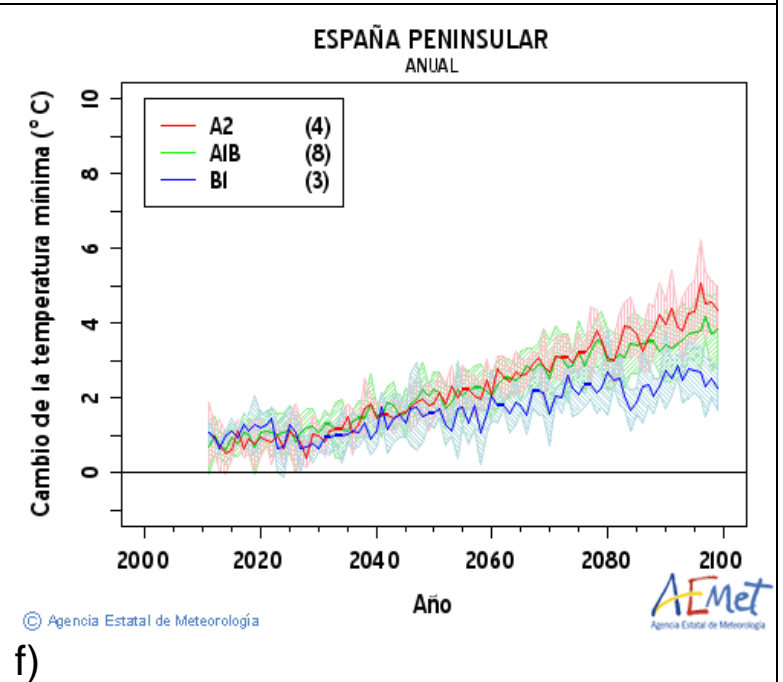

Figura A.124. Fila superior: Cambio en la evolución temporal del valor medio anual para España peninsular de temperatura máxima obtenida mediante técnicas de regionalización: (a) dinámica; (b) estadística-análogos y (c) estadística-sdsm agrupadas por escenarios de emisiones Entre paréntesis se recoge el número de proyecciones realizadas para cada escenario. En línea continua (sombreada) se representa el valor promedio (incertidumbre). Fila inferior: Igual para la temperatura mínima mediante regionalización (d) dinámica; (e) estadística-análogos y (f) estadística-sdsm. 


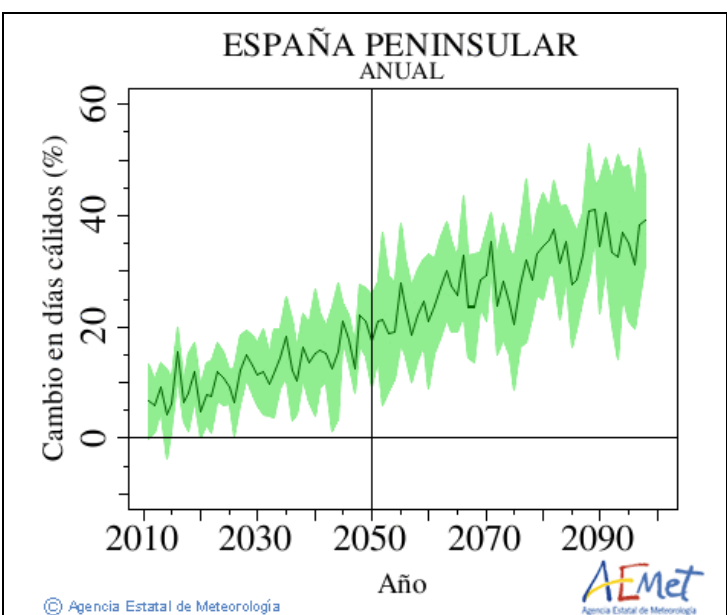

a)

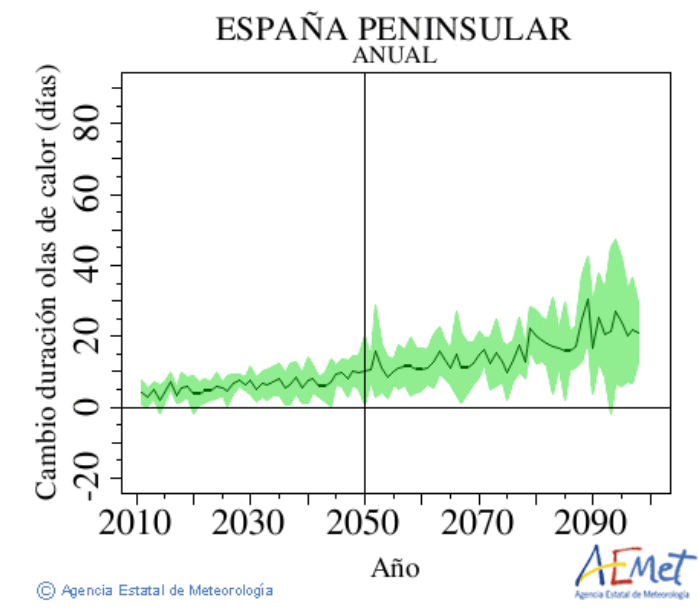

d)

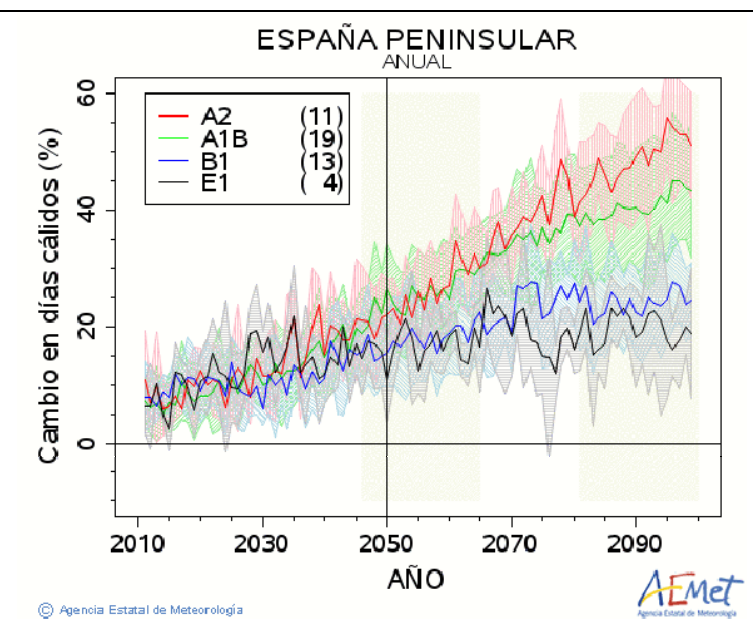

b)

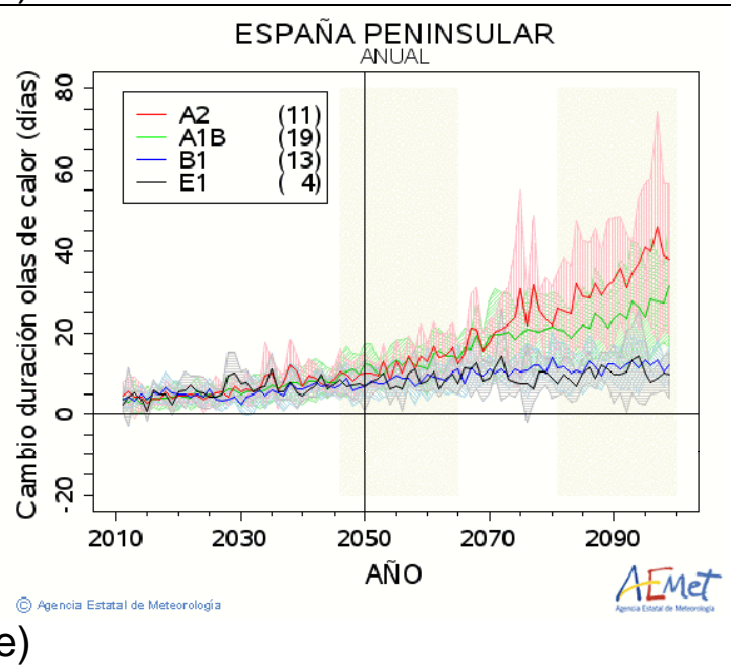

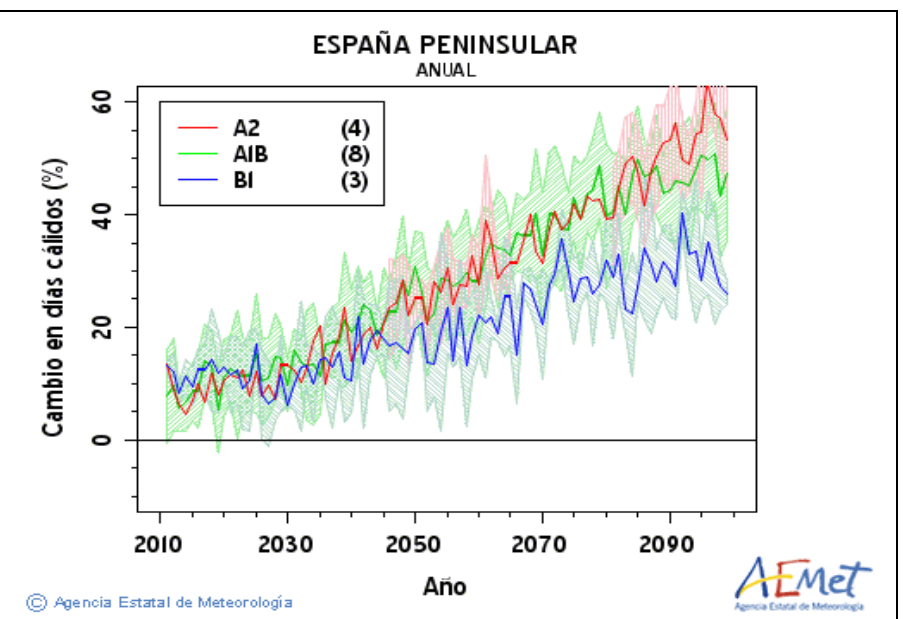

c)

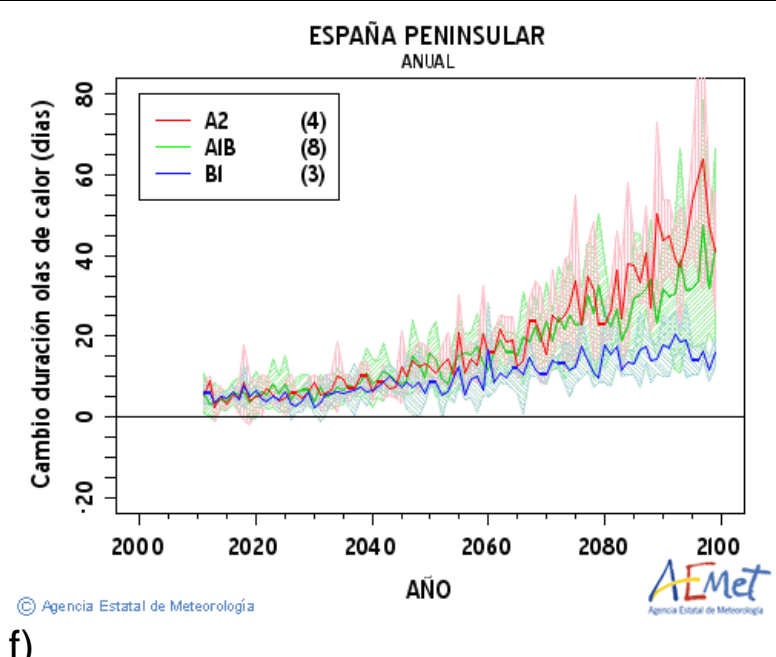

Figura A.125. Igual que la Figura A.124 para los índices extremos DC (fila superior) y DOC (fila inferior). 

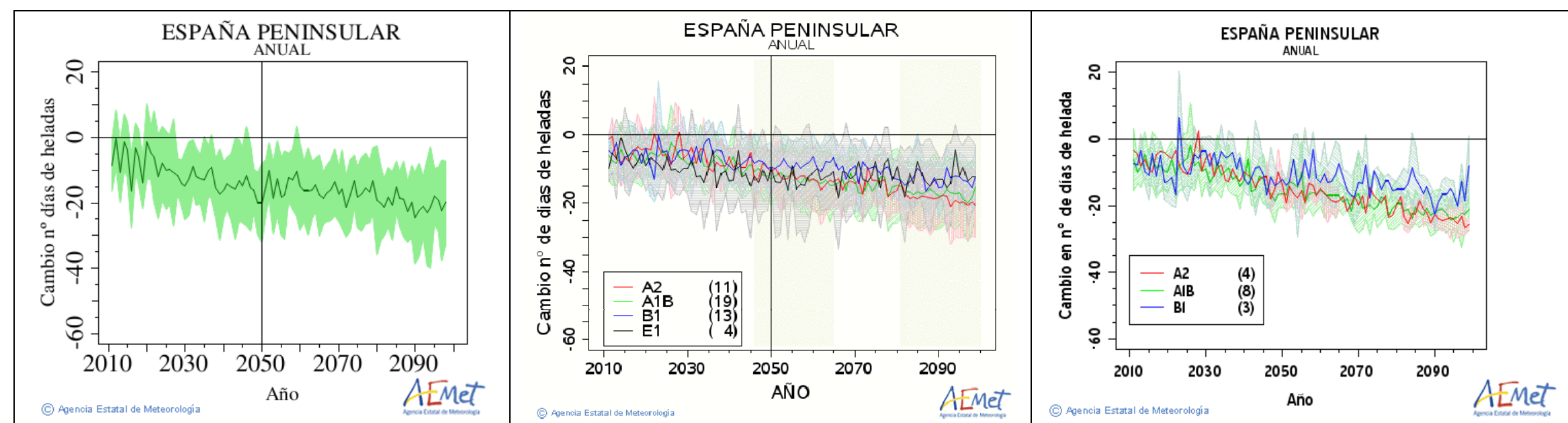

a)

b)
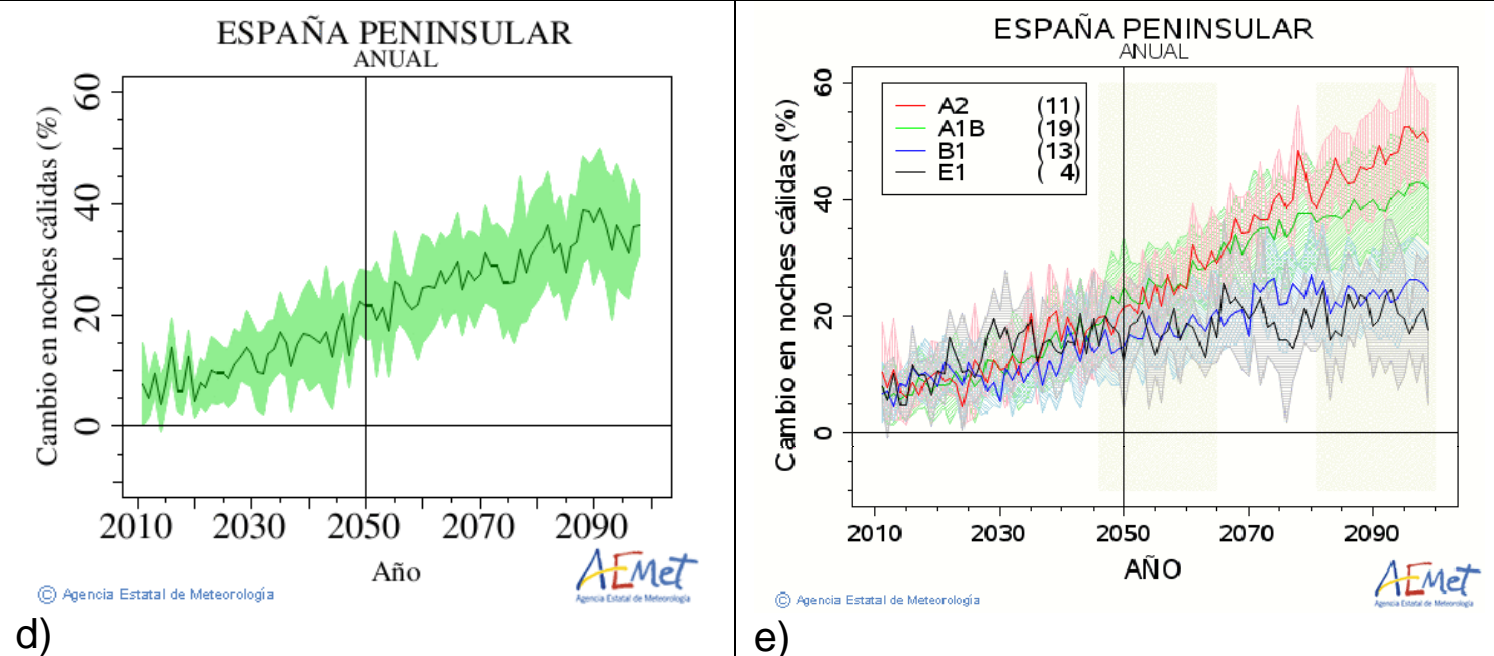

c)

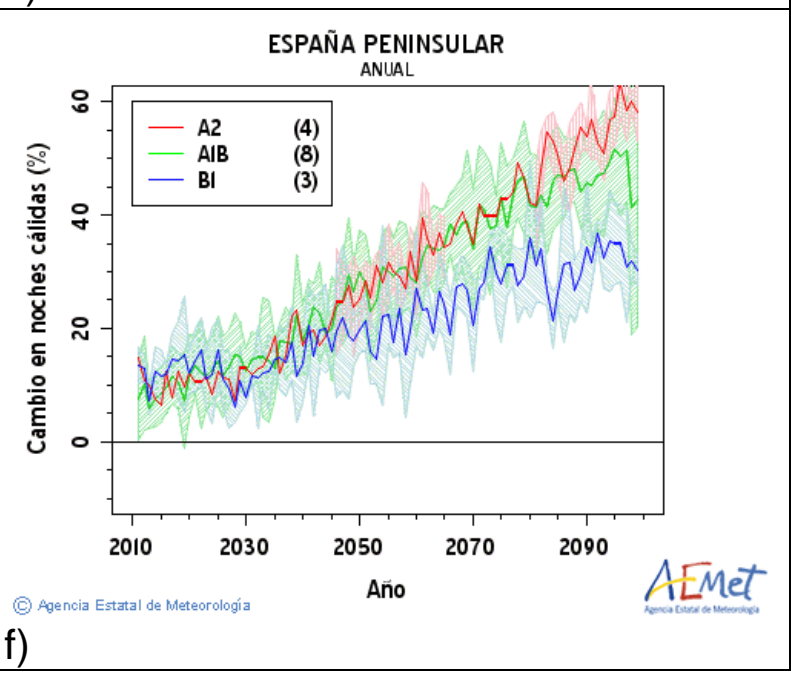

Figura A.126. Igual que la Figura A.124 para Igual que la Figura A.124 para los índices extremos DH (fila superior) y NC (fila inferior). 

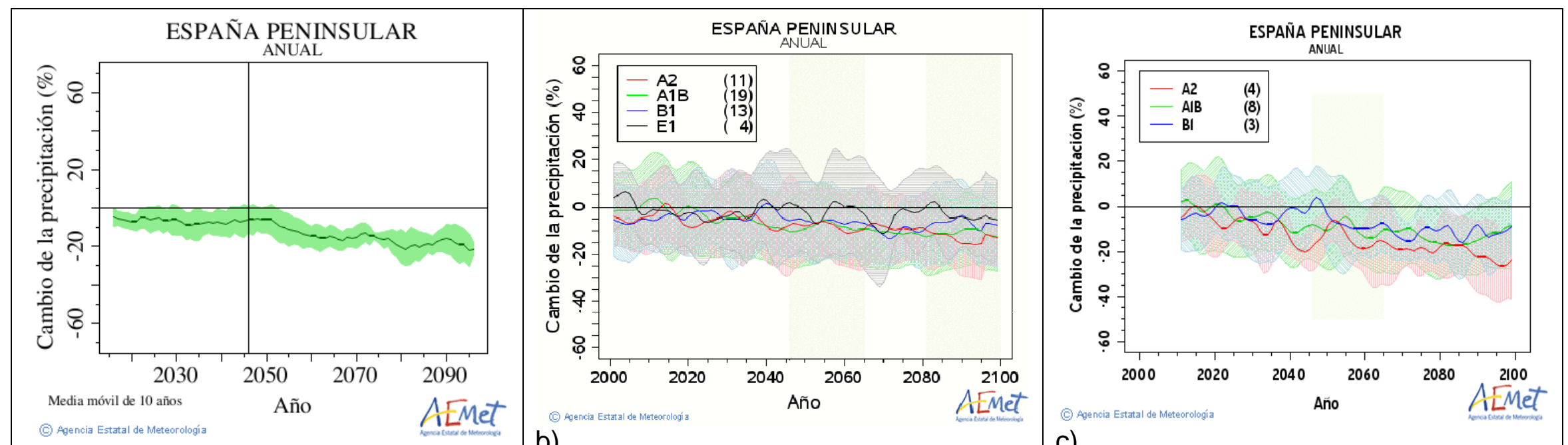

a)

b)

c)

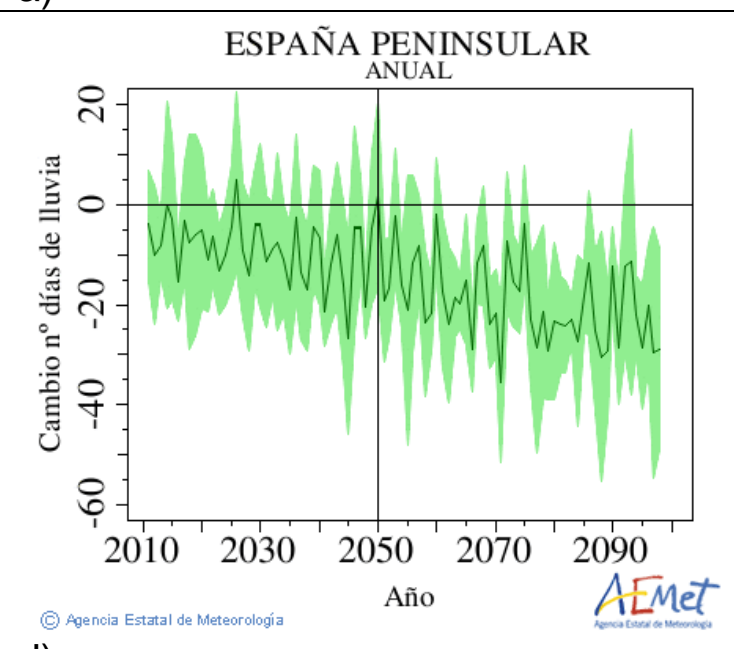

d)
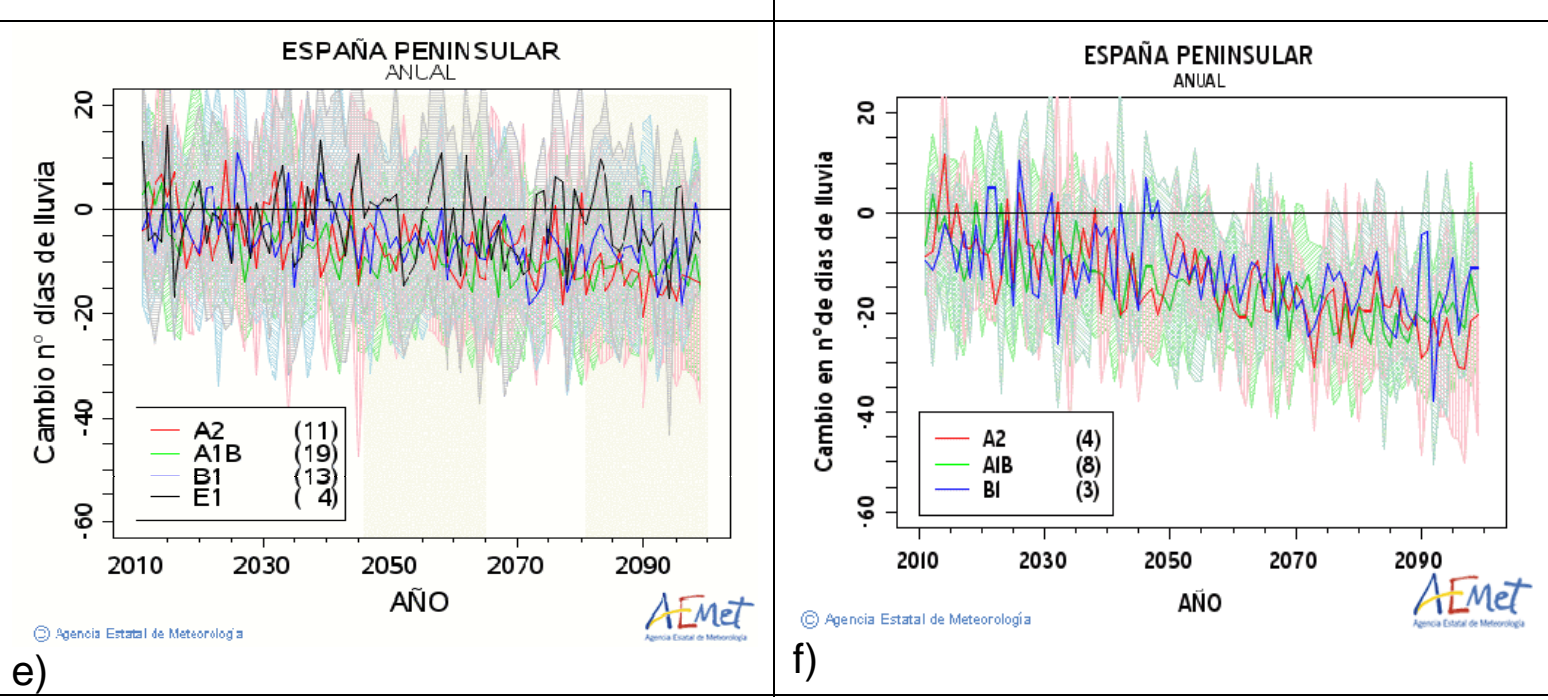

Figura A.127. Igual que la Figura A.124 para el cambio en la precipitación media (fila superior) y el índice extremo DL (fila inferior). 


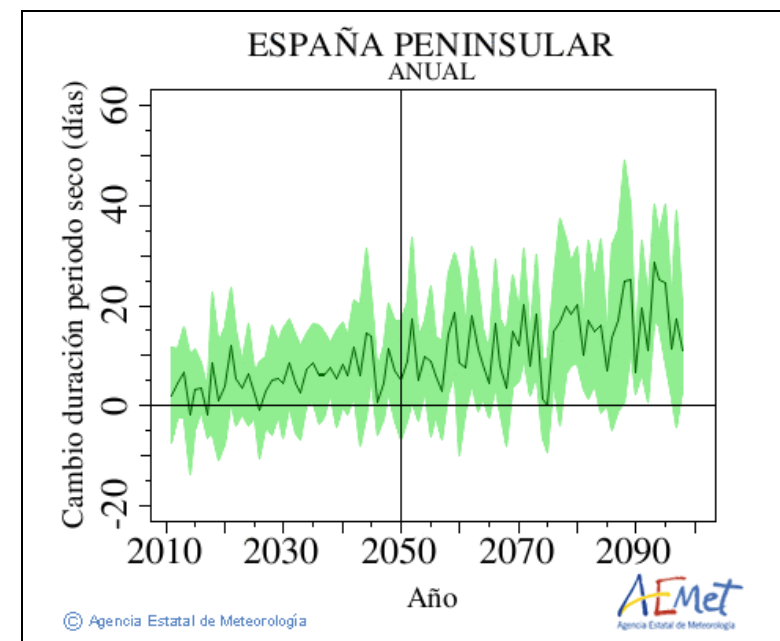

a)

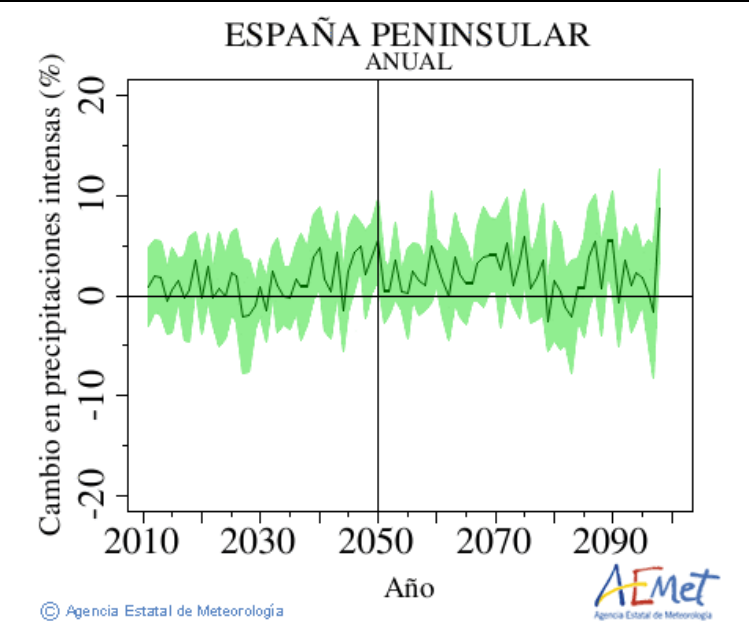

d)

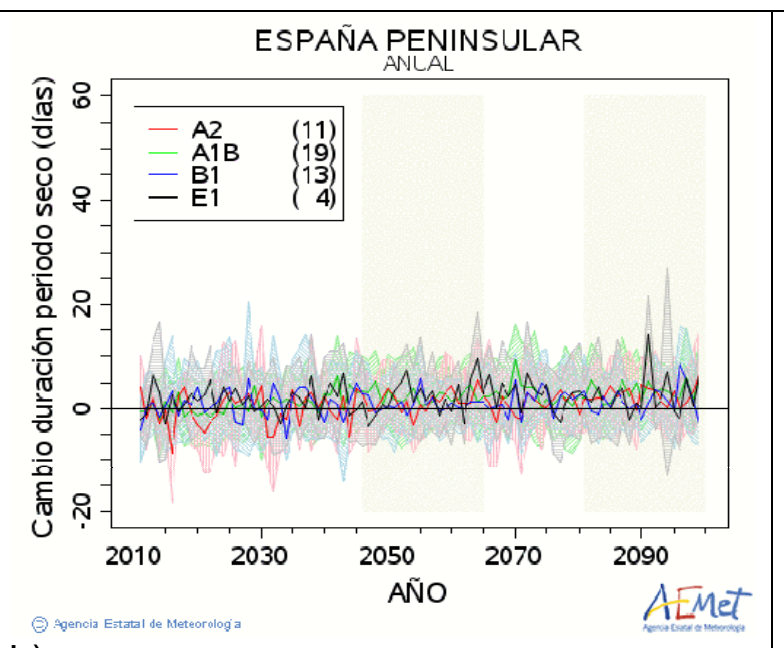

b)

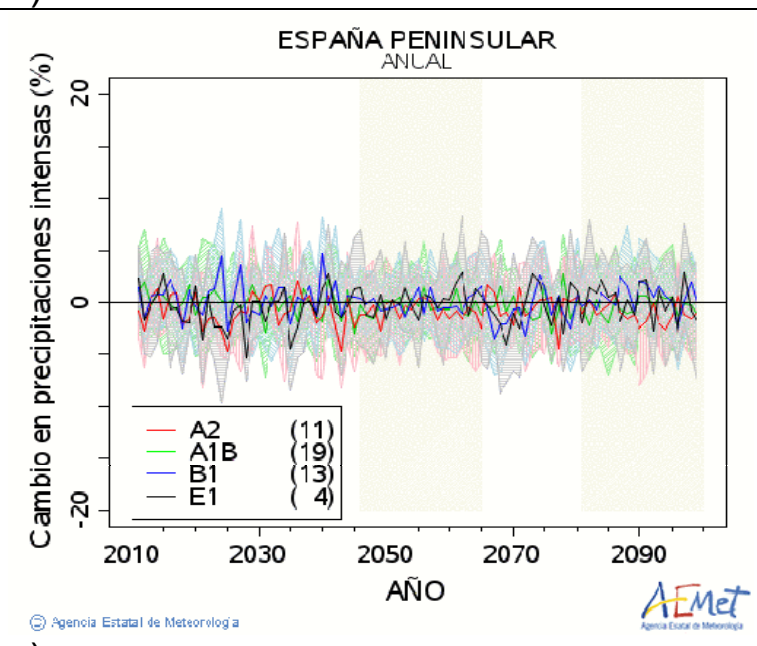

e)

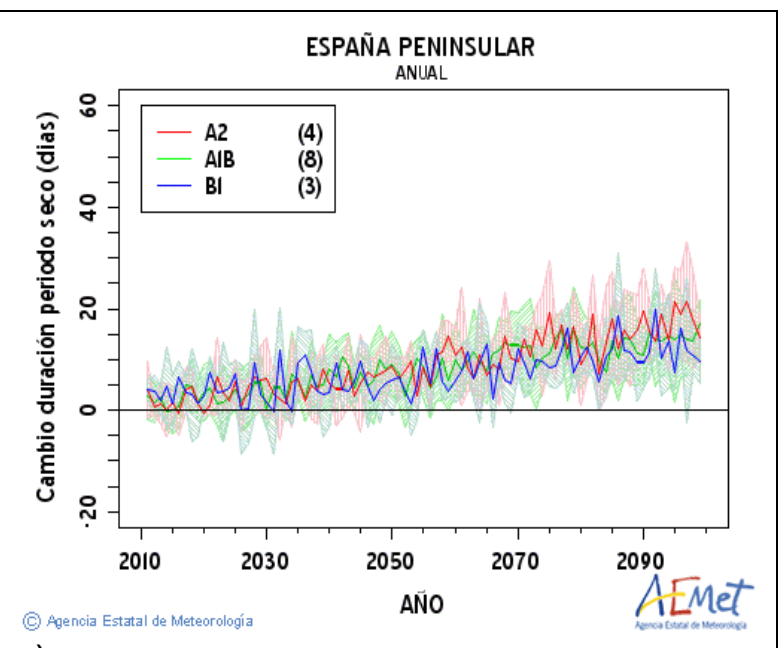

c)

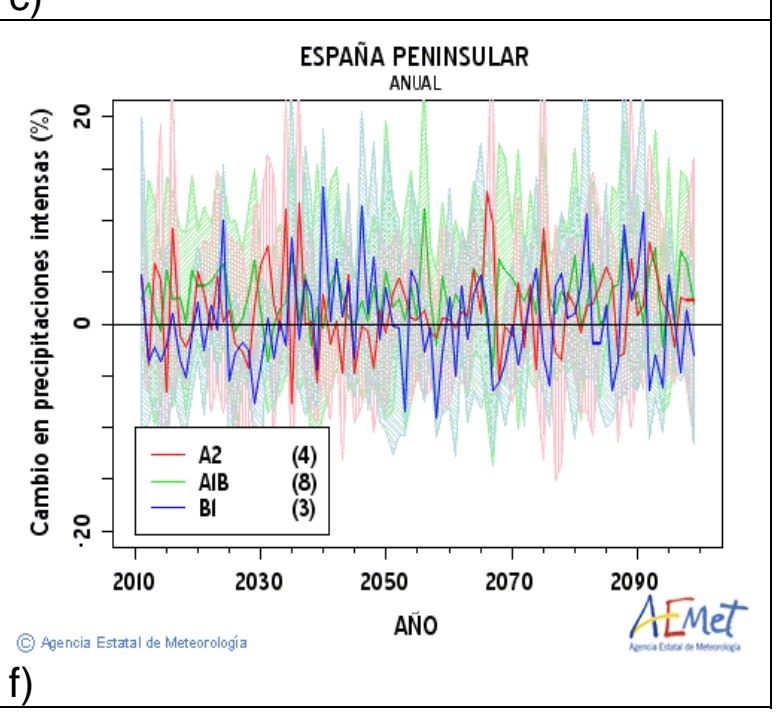

Figura A.128. Igual que la Figura A.124 para los índices extremos PS (fila superior) y PI (fila inferior). 


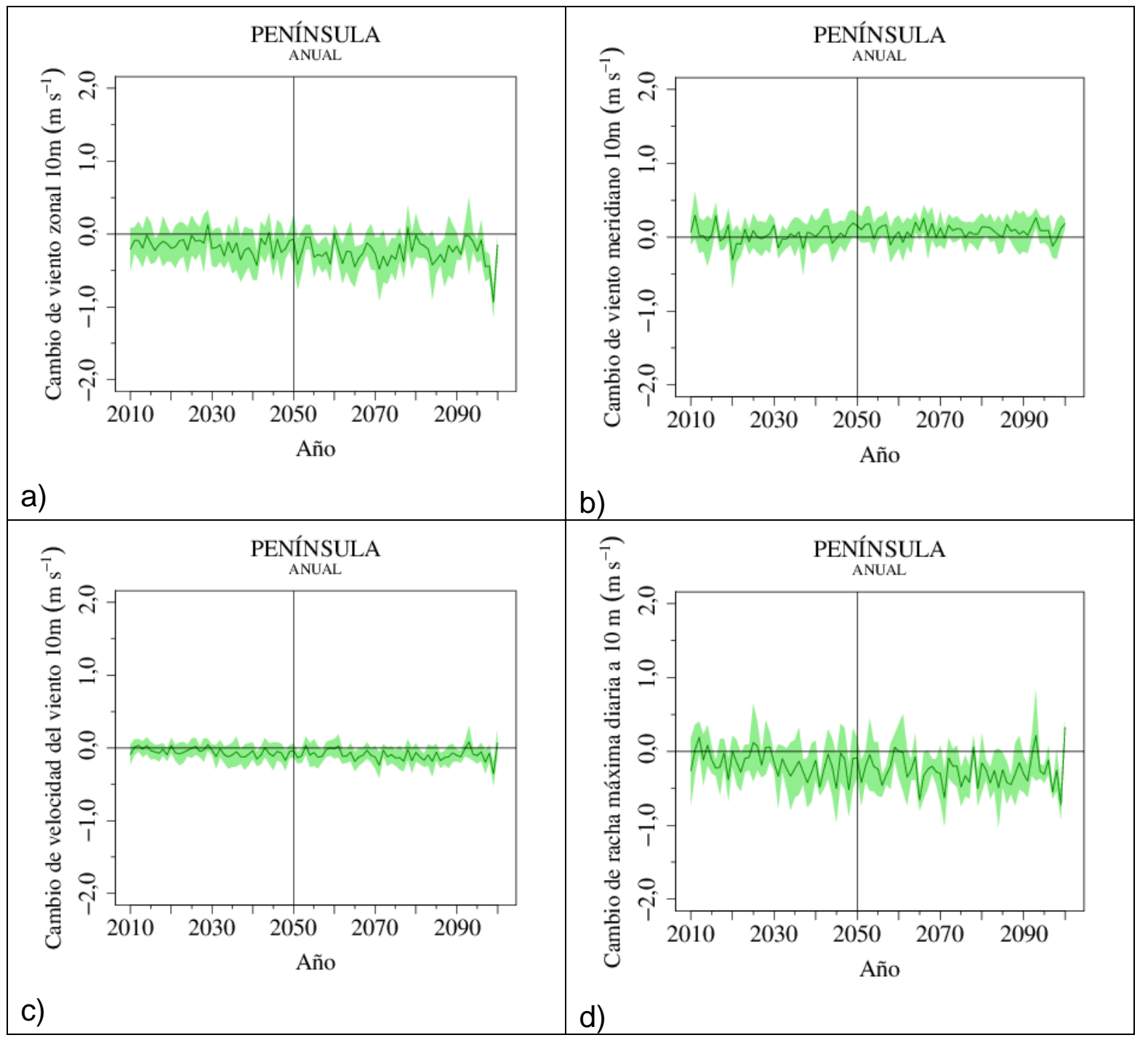

Figura A.129. Evolución temporal del valor medio anual en España peninsular obtenida mediante técnicas de regionalización estadística de: (a) componente U10 (m $\left.\mathbf{s}^{-1}\right)$; (b) componente V10 ( $\left.\mathrm{m} \mathrm{s}^{-1}\right)$; (c) velocidad W10 ( $\left.\mathrm{m} \mathrm{s}^{-1}\right)$; (d) racha máxima R10 (m s$\left.)^{-1}\right)$. En línea continua (área sombreada) se representa el valor promedio (incertidumbre). 


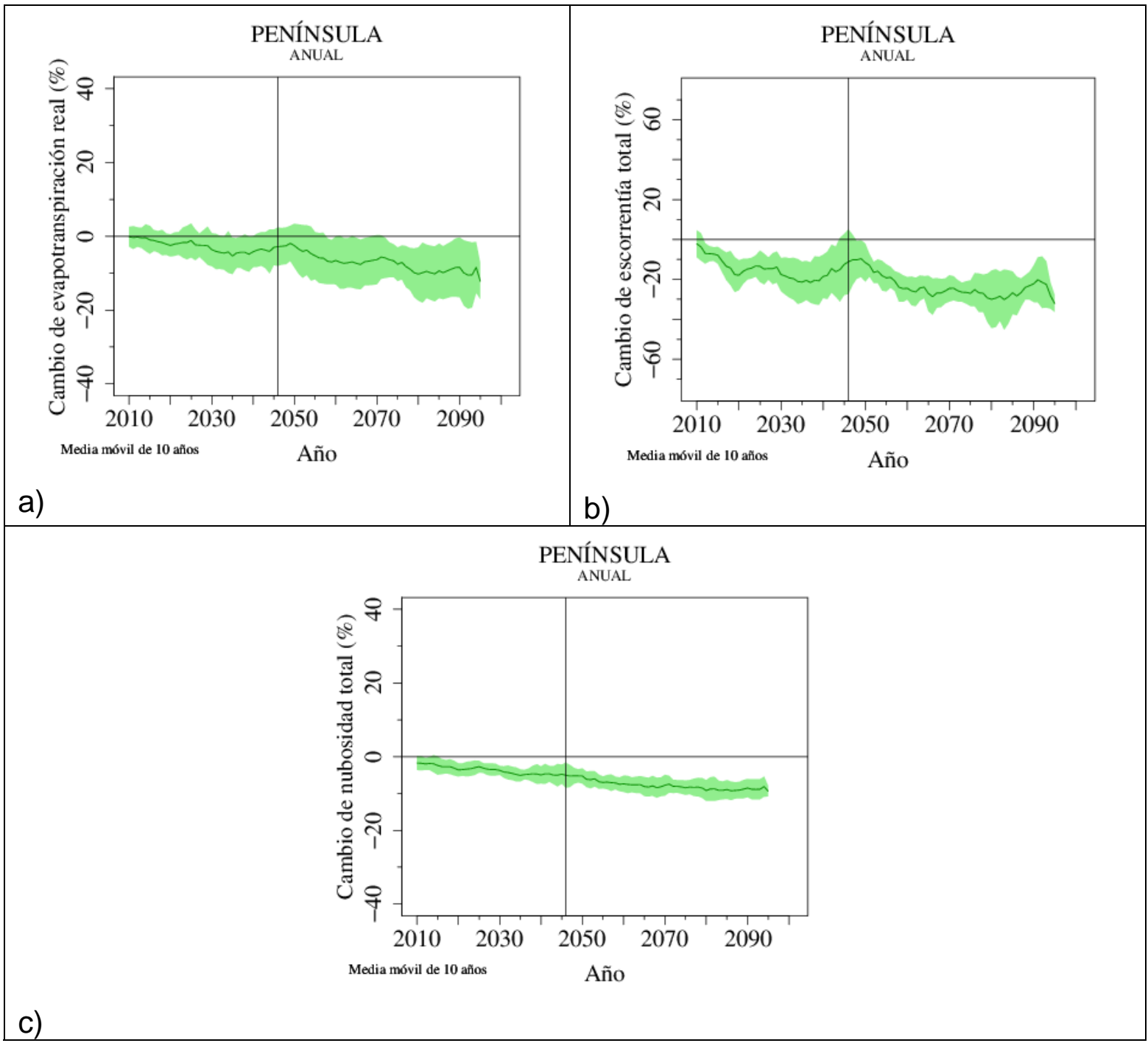

Figura A.130. Evolución temporal del valor medio anual en España peninsular obtenida mediante técnicas de regionalización estadística de: (a) tasa de $\mathbf{Q}$ filtrada con media móvil de 10 años; (b) tasa de ETR filtrada con media móvil de 10 años. En línea continua (área sombreada) se representa el valor promedio (incertidumbre). 


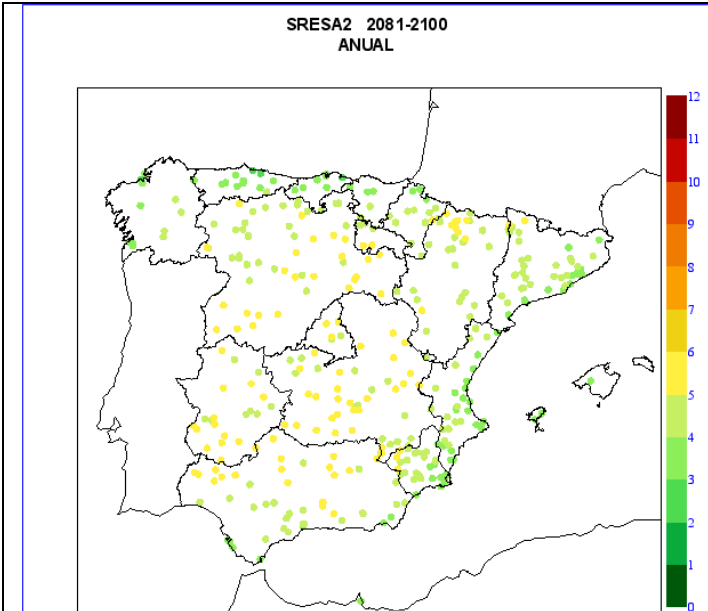

a)

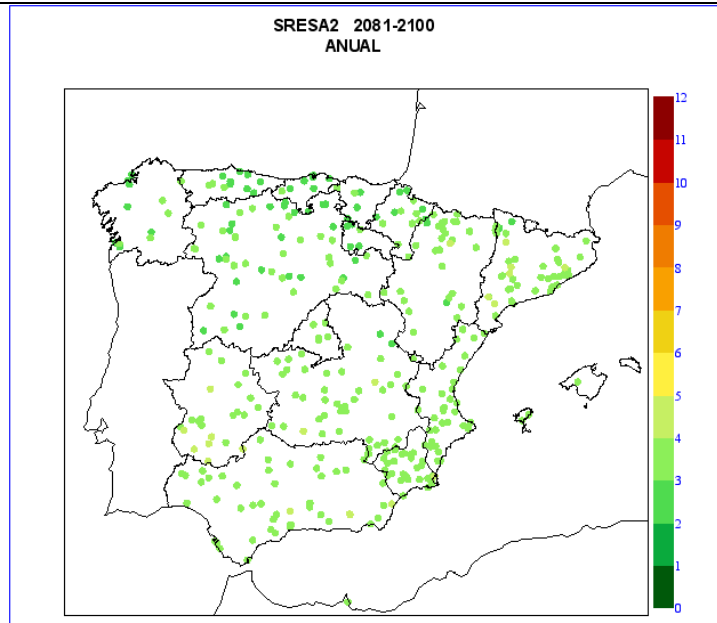

c)

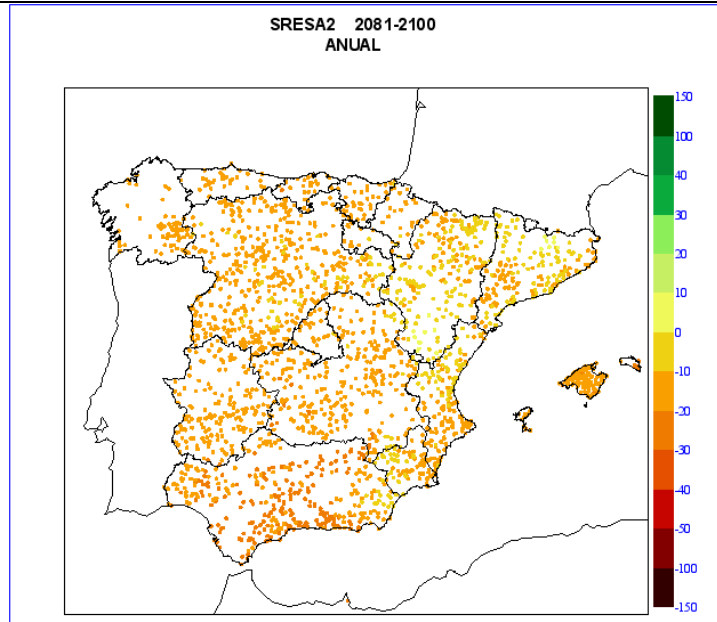

e)

Figura A.131. (a) Distribución espacial del cambio en el valor medio anual de la temperatura máxima obtenida mediante técnicas de regionalización estadística del escenario A2 para el período 2081-2098 y (b) distribución espacial de su incertidumbre; (c)-(d): Igual que (a)-(b) para la temperatura mínima y (e)-(f) Igual que (a)-(b) para la precipitación. b)
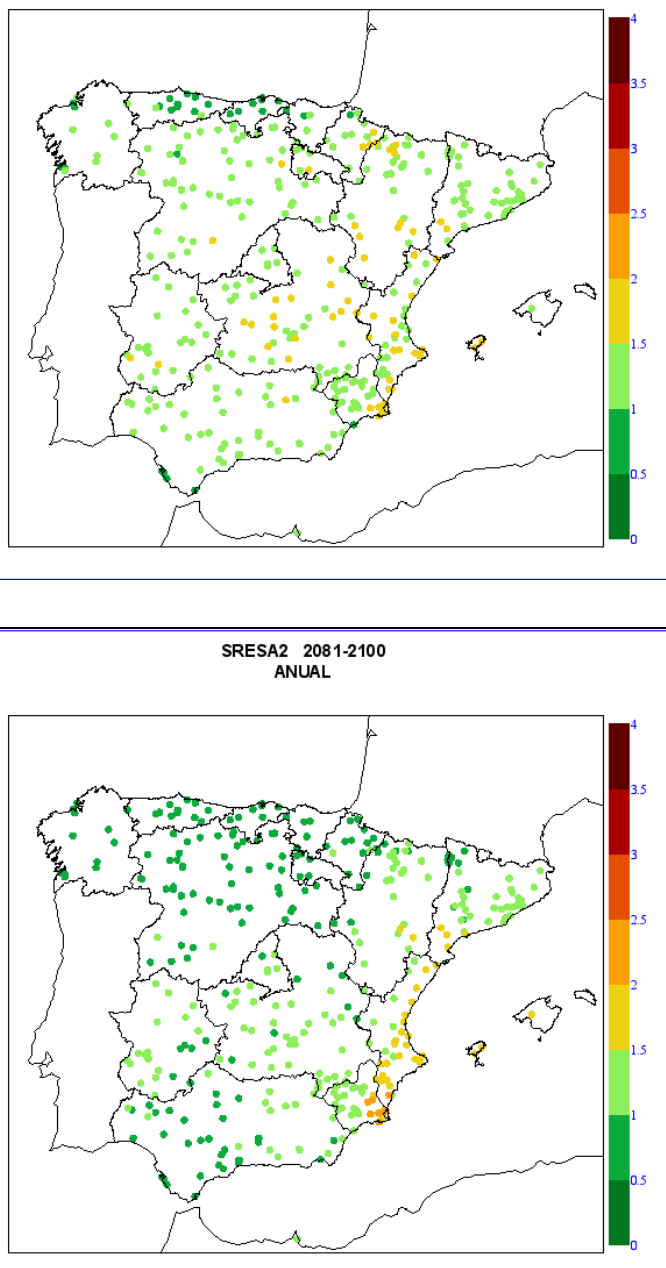

d)

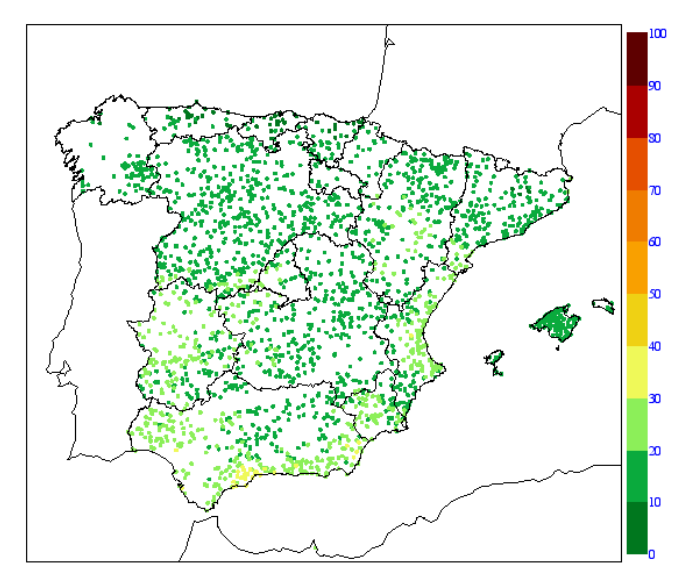

f) SRESA2 2081-2100
ANUAL

RESA2 2081-2100 ANUAL SA2 2081-2100 ANUAL

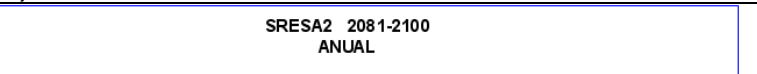




\section{Anexo B}

\section{Lista de acrónimos}

AEMET: Agencia Estatal de Meteorología.

AMIP: Atmospheric Model Intercomparison Project.

AOGCM: Coupled Atmosphere-Ocean Circulation Models.

CCAA: Comunidades Autónomas.

CNRM: Centre National de Recherches Meteorologiques.

CMIP: Coupled Model Intercomparison Project.

CMIP3: Coupled Model Intercomparison Project phase 3.

$\mathrm{CTL}$ : tasa de cobertura nubosa total.

CTRL: escenario de Control.

DC: porcentaje de Días Cálidos.

DH: número de Días de Helada.

DL: número de Días de Lluvia.

DMI: Danish Meteorological Institute.

DOC: Duración de Olas de Calor.

ENSEMBLES: ENSEMBLE-based Predictions of Climate Changes and their Impacts. 
ES1: ENSEMBLES-Stream1.

ES2: ENSEMBLES-Stream2.

ETHZ: Swiss Institute of Technology.

ETR: Evapotranspiración Real.

GEI: Gases de Efecto Invernadero.

ICTP: Abdus Salam International Centre for Theoretical Physics.

IPCC-AR3: Third Assessment Report of the Intergovernmental Panel on Climate Change.

IPCC-AR4: Fourth Assessment Report of the Intergovernmental Panel on Climate Change.

KNMI: Koninklijk Nederlands Meteorologisch Instituut.

MC: estimación por Mínimos Cuadrados.

MCGAO: Modelos de Circulación General Acoplados Atmósfera-Océano.

MCR: Modelos Climáticos Regionales.

MetoHC: Hadley Center/UK Met Office.

Met.NO: The Norwegian Meteorological Institute.

MPI-M: Max Planck Institute for Meteorology.

MSAE: Minimización de la Suma de los Errores Absolutos.

NC: porcentaje de Noches Cálidas.

NCEP/NCAR: National Centers for Environmental Prediction/ National Center for Atmospheric Research.

PCP: Precipitación.

PS: duración de Períodos Secos.

PI: tasa de ocurrencia de Precipitaciones Intensas.

PNACC: Plan Nacional de Adaptación al Cambio Climático.

OMM: Organización Meteorológica Mundial. 
Q: escorrentía total.

R10: racha máxima diaria del viento a 10m.

SDSM: Statistical DownScaling Method.

SMHI: Swedish Meteorological and Hydrological Institute.

SRES: Special Report on Emissions Scenarios.

SXXI: Siglo XXI.

SXX: Siglo XX.

TI: Temperatura minima.

TX: Temperatura maxima.

UCLM: Universidad de Castilla la Mancha.

U10: componente zonal del viento a 10m.

V10: componente meridiana del viento a $10 \mathrm{~m}$.

W10: velocidad media del viento a $10 \mathrm{~m}$. 



\section{Anexo C}

\section{Referencias}

Alexander, L. V., Zhang, X., Peterson, T. C., Caesar, J., Gleason, B., Klein Tank, A. M. G., Haylock, M., Collins, D., Trewin, B., Rahimzadeh, F., Tagipour, A., Rupa Kumar, K., Revadekar, J., Griffiths, G., Vincent, L., Stephenson, D. B., Burn, J., Aguilar, E., Brunet, M., Taylor, M., New, M., Zhai, P., Rusticucci, M., Vazquez - Aguirre, J. L. 2006. Global observed changes in daily climate extremes of temperature and precipitation, Journal Geophysical Research, 111, D05109.

Andrews T, Gregory JM, Webb MJ, Taylor KE. 2012. Forcing, feedbacks and climate sensitivity in CMIP5 coupled atmosphere ocean climate models. Geophysical Research Letters, 39(9).

Bärring, L., Fortuniak, K., 2009. Multi-indices analysis of southern Scandinavian storminess 1780-2005 and links to interdecadal Variations in the NW Europe-North Sea region. International Journal Climatology, 29: 373-384.

Barros, A.P., W. Hwu, 2002. Study of land-atmosphere interactions during summertime rainfall using a mesoscale model. Journal Geophysical Research, 107 (D14), 4227.

Beniston, M., Stephenson, D. B., Christensen, O. B., Ferro, C. A. T., Frei, C. and coauthors. 2007. Future extreme events in European climate: an exploration of regional climate model projections. Climate Change 81, 71-95. 
Betts R.A, Boucher, O., Collins, M., Cox, P. M., Falloon, P. D., Gedney, N., Hemming, DL, Huntingford, C., Jones, CD., Sexton, DMH, Webb, M. J. 2007. Projected increase in continental runoff due to plant responses to increasing carbon dioxide. Nature 448, 10371041.

Bony, S., Dufresne, J.-L., Le Treut, H., Morcrette, J.-J., Senior, C. 2004.On dynamic and thermodynamic components of cloud changes. Climate Dynamics, 22: 71-86.

Bony, S., Dufresne, J.-L. 2005. Marine boundary layer clouds at the heart of tropical cloud feedback uncertainties in climate models, Geophysical Research Letters, 32, L20806.

Bony, S., R Colman, V M Kattsov, R P Allan, C S Bretherton, J-L Dufresne, A Hall, S Hallegatte, M M Holland, W Ingram, D A Randall, B J Soden, G Tselioudis, M J Webb, 2006. How well do we understand and evaluate climate change feedback processes? Journal Climate, 19, 3445-3482.

Borén, R., Ribalaygua, J., Balairón, L. 1995. Método Analógico de Simulación de Escenarios Climáticos a escala Comarcal. Informe Técnico $n^{\circ} 4$. Servicio de Variabilidad y Predicción del Clima, INM, Madrid.

Braganza, K., Karoly, D. J., Arblaster, J. M. 2004. Diurnal temperature range as an index of global climate change during the twentieth century, Geophysical Research Letters, 31, L13217.

Brunet, M., Saladié, O., Jones, P., Sigró, J., Aguilar, E., Moberg, A., Lister, D., Walther, A., López, D., Almarza, C. 2006. The development of a new dataset of Spanish daily adjusted temperature series (SDATS) (1850-2003). International Journal Climatology, 26(13), 17771802.

Brunet, M., Casado, M.J., Castro, M., Galán, P., López, J.A., Martín, J.M., Pastor, A., Petisco, E., Ramos, P., Ribalaygua, J., Rodríguez, E., Sanz, I., Torres, L. 2009. Generación de escenarios regionalizados de cambio climático para España. Ministerio de Medio Ambiente Medio Rural y Marino. 158 pp,

Calbó, J., Sanchez-Lorenzo, A. 2009. Cloudiness climatology in the Iberian Peninsula from three global gridded datasets (ISCCP, CRU TS 2.1, ERA-40), Theoretical Applied Climatology 96, 105-115.

Christensen JH, Christensen OB, Lopez P, van Meijgaard E, Botzet B. 1996. The HIRHAM4: regional atmospheric climate model. DMI Scientific Report, 96-4

Colman, R. 2003. A comparison of climate feedbacks in general circulation models. Climate Dynamics, 20, 865-873.

Costa, A.C., Santos, J.A. Pinto, J.G. 2012. Climate change scenarios for precipitation extremes in Portugal, Theoretical Applied Climatology, 108, 1-2, 217.

Della-Marta, P.M., Mathis, H., Frei, C., Liniger, M.A., Klein, J., Appenzeller, C. 2009. The return period of wind storms over Europe. International Journal Climatology, 29:437-459. 
Delworth, T.L. T. L., Broccoli, A. J., Rosati, A., Stouffer, R. J., Balaji, V., Beesley, J. A., ... \& Zhang, R. 2006. GFDL's CM2 Global Coupled Climate Models. Part I: Formulation and simulation characteristics. Journal Climate, 19, 643-674.

Diansky, N.A., Volodin, E.M. 2002. Simulation of the present-day climate with a coupled atmosphere-ocean general circulation model. Izvestiya Atmospheric and Oceanic Physics., 38, 732-747 (English translation)

Dufresne JL. 2009. ENSEMBLES STREAM2 IPSLCM4_v2 20C3M run1, daily values. CERA database. World Data Center for Climate, Hamburg.

Elguindi, N. , Rauscher, S. A., Giorgi, F. 2013. Historical and future changes in maximum and minimum temperature records over Europe, Climate Change, 117, 1-2, 415.

Fischer, E. M. and Schär, C. 2009. Future changes in daily summer temperature variability: driving processes and role for temperature extremes. Climate Dynamics, 33, 917-935.

Fischer EM, Schär C. 2010. Consistent geographical patterns of changes in high-impact European heatwaves. Nature Geoscience, 3(6), 398-403.

Flato, G.M., G.J. Boer, W.G. Lee, N.A. McFarlane, D. Ramsden, M.C. Reader, and A.J. Weaver, 2000. The Canadian Centre for Climate Modelling and Analysis global coupled model and its climate. Climate Dynamics, 16, 451-467.

Flato, G.M., 2005. The Third Generation Coupled Global Climate Model (CGCM3) (and included links to the description of the AGCM3 atmospheric model). http://www.cccma.bc.ec.gc.ca/models/cgcm3.shtml.

Font I. 2000. Climatología de España y Portugal, $2^{\mathrm{a}}$ Ed., Ediciones Universidad de Salamanca, Salamanca, Spain, 422 pp.

Furevik, T., Bentsen, M., Drange, H., Kindem, I. K. T., Kvamstø, N. G., Sorteberg, A. 2003. Description and evaluation of the Bergen climate model: ARPEGE coupled with MICOM. Climate Dynamics, 21, 27-51.

Gallego, M. C., R. M. Trigo, J. M. Vaquero, M. Brunet, J. A. García, J. Sigró, and M. A. Valente. 2011. Trends in frequency indices of daily precipitation over the Iberian Peninsula during the last century, Journal Geophysical Research, 116, D02109.

Gershunov, A., Barnett, T., Cayan, D., Tubbs, T., L Goddard. 2000. Predicting and downscaling ENSO impacts on intraseasonal precipitation statistics in California: The 1997/98 event. Journal of Hydrometeorology Vol. 1: 201-209.

Gibelin AL, Déqué M. 2003. Anthropogenic climate change over the Mediterranean region simulated by a global variable resolution model. Climate Dynamics, 20, 327-339.

Giorgi, F. 2001. Emerging Patterns of simulated regional climatic changes for the 21st century due to anthropogenic forcings. Geophysical Research Letters, 28, 3317-3320 
Giorgi, F, 2006. Climate change hot-spots. Geophysical Research Letters, L08707.

Giorgi, F, Coppola E., 2007. European climate-change oscillation (ECO). Geophysical Research Letters, L21703.

Hertig, E., Seubert, S., Paxian, A., Vogt , G. Paeth, H., Jacobeit, J. 2013. Changes of total versus extreme precipitation and dry periods until the end of the twenty-first century: statistical assessments for the Mediterranean area. Theoretical Applied Climatology, 111,120.

Houghton, J. T., Y. Ding, D. J. Griggs, M. Noguer, P. J. van der Linden, X. Dai, K. Maskell, and C. A. Johnson, Eds. 2001. Climate Change 2001: The Scientific Basis. Cambridge University Press, 944 pp.

Huebener H, Koerper J. 2008. ENSEMBLES STREAM2 EGMAM2 20C3M run1, daily values. CERA database. World Data Center for Climate, Hamburg.

Hungate, B. A., Reichstein, M., Dijkstra, P., Johnson, D., Hymus, G., Tenhunen, J. D., ... , Drake, B. G. 2002. Evapotranspiration and soil water content in a scrub - oak woodland under carbon dioxide enrichment. Global Change Biology, 8(3), 289-298.

Jacob, D. 2001. A note to the simulation of the annual and inter-annual variability of the water budget over the Baltic Sea drainage basin. Meteorology and Atmospheric Physics, $77,61-73$.

Jacob, D, Podzun R. 1997. Sensitivity studies with the regional climate model REMO. Meteorology and Atmospheric Physics, 63,119-129.

Jaeger, EB, Anders I, Lüthi D, Rockel B, Schär C, Seneviratne SI. 2008. Analysis of ERA40-driven CLM simulations for Europe. Meteorologische Zeitschrift, 17,349-367.

Jones, P.D., A. Moberg, 2003. Hemispheric and large-scale surface air temperature variations: An extensive revision and an update to 2001. Journal Climate, 16, 206-223.

Johns, TC. 2009. ENSEMBLES STREAM2 METO-HCHADGEM2A O20C3M run1, daily values. CERA database. World Data Center for Climate, Hamburg

Kjellström, E, Bärring L, Gollvik S, Hansson U., Jones, C. 2005. A 140-year simulation of European climate with the new version of the Rossby Centre regional atmospheric climate model (RCA3). Reports Meteorology and Climatology, 108, Swedish Meteorological and Hydrological Institute, Norrköping, Sweden.

Kalnay, E., M. Kanamitsu, R. Kistler, D. Collins, D. Deaven, L. Gandin, M. Iredell, S. Saha, G. White, J. Woolen, Y. Zhu, M. Chelliah, W. Ebisuzaki, W. Higgins, J. Janowiak, K. C. Mo, C. Kopelewski, J. Wang, A. Leetmaa, R. Reynolds, R. Jeene and D. Jsoseph. 1996. The NCEP/NCAR 40-years Reanalysis, Project. Bulletin American Meteorological Society, 77(3), 437-471. 
Kharin, V. V. and Zwiers, F. W. 2004. Estimating extremes in transient climate change simulations. Journal Climate, 18, 1156-1173.

Kharin, V. V., Zwiers F. W., Zhang X. and Hegerl, G. C. 2007. Changes in temperature and precipitation extremes in the IPCC ensemble of global coupled model simulations. Journal Climate, 20, 1419-1444.

Kittel T, Giorgi F, Meehl G., 1998. Intercomparison of regional biases and doubled CO2 sensitivities of coupled atmosphere-ocean general circulation model experiments. Climate Dynamics, 14, 1-5.

Kilsby C.G., 1998. Predicting rainfall statistics in England and Wales using atmospheric circulation variables. International Journal Climatology, 18, 523-539.

Kjellström E, Bärring L, Gollvik S, Hansson U, Jones C, Samuelsson P, Rummukainen M, Ullersig A, Willen U, Wyser K. 2005. A 140-year simulation of European climate with the new version of the Rossby Centre regional atmospheric climate model (RCA3). Reports Meteorology and Climatology, 108, SMHI, SE-60176 Norrköping, Sweden, 54 pp.

Knote, C., Heinemann, G., Rockel, B., 2010. Changes in weather extremes: assesment of return values using high resolution climate simulations at convection-resolving scale. Meteorologische Zeitschrift, 19(1), 11-23.

Leckebusch, G. C., Koffi, B., Ulbrich, U., Pinto, J., Spangehl, T., Zacharias, S. 2006. Analysis of frequency and intensity of winter storm events in Europe on synoptic and regional scales from a multi-model perspective. Climate Research, 31, 59-74.

Legutke, S., Voss, R. 1999. The Hamburg atmosphere-ocean coupled climate circulation model ECHO-G. DKRZ Technical report no. 18, Deutsches Klimarechenzentrum, Hamburg, Germany.

Lenderink G, van den Hurk B, van Meijgaard E, van Ulden A, Cujipers H. 2003. Simulation of present-day climate in RACMO2: first results and model developments. KNMI Technical Report, 252, 24.

Libro Digital del Agua. 2008. Ministerio de Agricultura, Alimentación y Medio Ambiente. Gobierno de España.

Long, S. P., Ainsworth, E. A., Leakey, A. D. B., Nösberger, J. and Ort, D. R. 2006. Food for thought: lower-than-expected crop yield stimulation with rising $\mathrm{CO} 2$ concentrations. Science, 312, 1918-1921.

Mace, G. G., Benson, S., Kato, S. 2006. Cloud radiative forcing at the Atmospheric Radiation Measurement Program Climate Research Facility: 2. Vertical redistribution of radiant energy by clouds. Journal Geophysical Research, 111, D11S91. 
Meehl, G. A., C. Covey, T. Delworth, M. Latif, B. Mcavaney, J. F. B. Mitchell, R. J. Stouffer Y K. E. Taylor. 2007. THE WCRP CMIP3 Multimodel Dataset: A New Era in Climate Change Research, Bulletin American Meteorological Society, 88, 1383-1394.

Meehl GA, Stocker TF, Collins WD, Friedlingstein P, Gaye AT, Gregory JM, Kitoh A, Knutti R, Murphy JM, Noda A, Raper SCB, Watterson IG, Weaver AJ, Zhao ZC. 2007. Global climate projections. Cambridge University Press, Cambridge. Climate Change 2007: the physical science basis. Contribution of working group I to the fourth assessment report of the intergovernmental panel on climate change.

Nakicenovic, N., Alcamo, J., Davis, G., De Vries, B., Fenhann, J., Gaffin, S., ... \& Dadi, Z. 2000. IPCC Spetial Report on Emissions Scenarios. Cambridge University Press, UK. 599 pp.

Narula S.C. y Wellington J. F., 1977. An algorithm for linear regression with minimum sum of absolute errors. Applied Statistics, 26,106-111.

Niehörster, F. 2008. ENSEMBLES EGMAM 20C3M run1, daily values. CERA database. World Data Center for Climate, Hamburg.

Nikulin, G., Kjellström, E., Hansson, U., Strandberg, G., Ullerstig, A. 2011. Evaluation and future projections of temperature, precipitation and wind extremes over Europe in an ensemble of regional climate simulations. Tellus A, 63, 41-55.

Petisco, S.E., Martín, J.M., Gil, D. 2005. Método de estima de precipitación mediante "downscaling". Nota técnica $\mathrm{n}^{\circ} 11$ del Servicio de Variabilidad y Predicción del Clima, INM, Madrid.

Probst, P., Rizzi, R., Tosi, E., Lucarini, V., Maestri, T. 2012. Total cloud cover from satellite observations and climate models, Atmospheric Research, 107, 161-170.

Pryor, S. C., Barthelmie, R. J. 2003. Long term trends in near surface flow over the Baltic. International Journal Climatology, 23, 271-289.

Pryor SC, Barthelmie R.J., Schoof, J.T. 2005a. Inter-annual variability of wind indices across Europe. Wind Energy, 9(1-2), 27-38.

Pryor, S. C., Schoof, J. T., Barthelmie, R. J. 2005b. Empirical downscaling of wind speed probability distributions. Journal Geophysical Research, 110, D19109.

Pryor, S.C., Schoof JT, Barthelmie, R.J., Schoof, J.T. 2009. Climate change impacts on wind speeds and wind energy density in northern Europe: empirical downscaling of multiple AOGCMs. Climate Research, 29, 183-198.

Pryor, S.C., Ledolter J., 2010. Addendum to: wind speed trends over the contigous USA. Journal Geophysical Research, 115, D10103. 
Pryor, S.C., Barthelmie, R.J., Clausen, N.E., Drews, M., MacKellar, N., Kjellström, E. 2012. Analyses of possible changes in intense and extreme wind speeds over northern Europe under climate change scenarios. Climate Dynamics, 38, 189-208.

Przybylak, R. 2000. Diurnal temperature range in the Arctic and its relation to hemispheric and Arctic circulation patterns, International Journal Climatology, 20, 231-253.

Räisänen, J., Hansson, U., Ullerstig, A., Döscher, R., Graham, L. P. 2003. GCM driven simulations of recent and future climate with the Rossby Centre coupled atmosphereBaltic Sea regional climate model RCAO. Reports Meteorology and Climatology Volume 101, Swedish Meteorological and Hydrological Institute, SE-601 76 Norrköping, Sweden, 61.

Randall D, Wood R, Bony S, Colman R, Fichefet T, Fyfe J, Kattsov V, Pitman A, Shukla J, Srinivasan J, Stouffer R, Sumi A, Taylor K. 2007. Climate models and their evaluation, Cambridge University Press, Cambridge. Climate Change 2007: the physical science basis. Contribution of working group I to the fourth assessment report of the intergovernmental panel on climate change.

Rockel, B., Woth, K. 2007. Extremes of near surface wind speed over Europe and their future changes as estimated from an ensemble of RCM simulations. Climatic Change, 81, 267-280.

Roeckner, E. 1996. The atmospheric general circulation model ECHAM 4: Model description and simulation of present day climate. Max-Planck-Institut für Meteorologie, Rep. 218, 90 pp.

Roeckner, E., Bäuml, G., Bonventura, L., Brokopf, R., Esch, M., Giorgetta, M., ... \& Tompkins, A. 2003. The Atmospheric General Circulation Model ECHAM5. Part I: Model Description. MPI Report 349, Max Planck Institute for Meteorology, Hamburg, Germany, $127 \mathrm{pp}$

Roeckner, E., Brokopf, R., Esch, M., Giorgetta, M., Hagemann, S., Kornblueh, L., ... \& Schulzweida, U. 2006. Sensitivity of simulated climate to horizontal and vertical resolution in the ECHAM5 atmosphere model. Journal of Climate, 19(16), 3771-3791.

Roeckner, E. 2007. ENSEMBLES ECHAM5-MPI-OM 20C3M run1, daily values. CERA database. World Data Center for Climate, Hamburg.

Roeckner, E. 2008. ENSEMBLES STREAM2 ECHAM5C-MPIOM 20C3M run1, daily values. CERA database. World Data Center for Climate, Hamburg.

Rossow, W. B., Schiffer, R. A. 1999. Advances in understanding clouds from ISCCP, Bulletin American Meteorological Society, 80, 2261- 2288.

Rossow, W. B., Dueñas, E. N. 2004. The International Satellite Cloud Climatology Project (ISCCP) web site, Bulletin American Meteorological Society, 85, 167-172.

Rowell, D. P. 2005. A scenario of European climate change for the late twenty-first century: seasonal means and interannual variability. Climate Dynamics, 25, 837-849. 
Royer, JF. 2006. ENSEMBLES CNRM-CM3 20C3M run1, daily values. CERA database. World Data Center for Climate, Hamburg.

Royer, JF. 2008. ENSEMBLES STREAM2 CNRM-CM33 20C3M run1, daily values. CERA database. World Data Center for Climate, Hamburg

Russell, G.L., J.R. Miller, and D. Rind, 1995. A coupled atmosphere-ocean model for transient climate change studies. Atmosphere.-Ocean, 33, 683-730.

Sánchez E, Gallardo C, Gaertner MA, Arribas A, Castro M. 2004. Future climate extreme events in the Mediterranean simulated by a regional climate model: a first approach. Global Planet Change 44:163-180

Sánchez-Gomez E., S. Somot, M. Deque. 2009. Ability of an ensemble of regional climate models to reproduce weather regimes over Europe-Atlantic during the period 1961-2000. Climate Dynamics, 33, 723-736.

Schär C., P. L. Vidale, D. Lüthi, C. Frei, C. Häerli, M. A. Linie and C. Appenzeler, 2004. The role of increasing temperature variability in European summer heatwaves. Nature, 427, 332-336.

Schulzweida, U., Kornblue, L., Quast, R. 2012. Climate Data Operators. CDO- User's Guide. https://code.zmaw.de/embedded/cdo/1.5.5/cdo.html

Sillmann J, Roeckner E. 2008. Indices for extreme events in projections of anthropogenic climate change. Climatic Change, 86, 83-104.

Soden, B.J., Held, I. M. 2006. An assessment of climate feedbacks in coupled oceanatmosphere models. Journal Climate, 19, 3354-3360.

Steppeler J, Dom G, Schättler U, Bitzer HW, Grassmann A, Damrath U, Gregoric G. 2003. Mesogamma scale forecasts using the nonhydrostatic model LM. Meteorology and Atmospheric Physics, 82(1-4), 75-96.

Trenberth KE, Jones PD, Ambenje P, Bojariu R, Easterling D, Klein, Tank A, Parker D, Rahimzadeh F, Renwick JA, Rusticucci M, Soden B, Zhai P., 2007. Observations: surface and atmospheric climate change. In: Solomon S, Qin S, Manning M et al (eds) Climate change 2007: the physical science basis. Contribution of Working Group I to the Fourth Assessment Report of the Intergovernmental Panel on Climate Change. Cambridge University Press, Cambridge.

Tsushima, Y, Ringer, MA, Webb, MJ, Williams, KD. 2012. Quantitative evaluation of the seasonal variations in climate model cloud regimes. Climate Dynamics. 1-18.

Van der Linden, P, Mitchell, JFB. 2009. ENSEMBLES: Climate Change and its Impacts: Summary of research and results from the ENSEMBLES project. Met Office Hadley Centre, FitzRoy Road, Exeter EX1 3PB, UK. 160pp. 
Van Ulden, AP, Van Oldenborgh, GJ. 2006. Large scale atmospheric circulation biases and changes in global climate model simulation and their importance for climate change in Central Europe. Atmospheric Chemistry Physics, 6, 863-881.

Vose, R. S., Easterling, D. R., Gleason, B. 2005. Maximum and minimum temperature trends for the globe: An update through 2004, Geophysical Research Letters, 32, L23822.

Wang, Y., Leung, L. R., McGREGOR, J. L., Lee, D. K., Wang, W. C., Ding, Y., Kimura, F. 2004. Regional climate modeling: progress, challenges, and prospects. Journal of the Meteorological Society of Japan. Ser. II, 82(6), 1599-1628.

Warren, S. G., Eastman, R. M., Hahn, C. J. 2007. A survey of changes in cloud cover and cloud types over land from surface observations, 1971-96, Journal Climate, 20, 717-738.

Webb, M.J., Senior, C.A., Sexton, D.M.H., Ingram, W.J., Williams, K.D., Ringer, M.A., McAvaney, B.J., Colman, R., Soden, B.J., Gudgel, R., Knutson, T., Emori, S., Ogura, T., Tsushima, Y., Andronova, N., Li, B., Musat, I., Bony, S., Taylor, K.E. 2006. On the contribution of local feedback mechanisms to the range of climate sensitivity in two GCM ensembles. Climate Dynamics, 27,17-38.

Weilicki, B. A., Barkstrom, B. R., Baum, B. A., Charlock, T. P., Green, R. N., Kratz, D. P., ..., Welch, R. M. 1998. Clouds and the Earth's radiant energy system (CERES): Algorithm overview. Geoscience and Remote Sensing, IEEE Transactions on, 36(4), 1127- 1141.

Wilby R.L., Dawson C.W., Barrow E.M., 2002. SDSM - a decision support tool for the assessment of regional climate change impacts. Environmental Modelling Software, 17, 145-157.

Wilby, R. L., Charles, S. P., Zorita, E., Timbal, B., Whetton, P., Mearns, L. O. 2004. Guidelines for use of climate scenarios developed from statistical downscaling methods. IPCC task group on data and scenario support for impacts and climate analysis.

Wilby, R., Dawson, C. 2004. Using SDSM version 3.1- A decision support tool for the assessment of regional climate change impacts. England, UK.

Williams, K.D., Tselioudis, G. 2007. GCM intercomparison of global cloud regimes: present-day evaluation and climate change response. Climate Dynamics, 29, 231-250.

Yan Z, Bate S, Chandler R, Ishram V, Wheater H., 2002. An analysis of daily maximum wind speed in northwestern Europe using generalized linear models. Journal Climate, 15, 2073-2088.

Yao, M.-S., Del Genio, A. D. 2002. Effects of cloud parameterization on the simulation of climate changes in the GISS GCM. Part II: Sea surface temperature and cloud feedbacks. Journal Climate, 15, 2491-2503.

Yukimoto, S., et al., 2001: The new Meteorological Research Institute global oceanatmosphere coupled GCM (MRI-CGCM2)--Model climate and variability. Papers in Meteorology and Geophysics, 51, 47-88. 
A EMet 